\title{
Organization and transcription analyses of the immunoglobulin genes in cattle and horses
}

\author{
Dissertation \\ to obtain the Ph.D. degree \\ in the Ph.D. Program for Agricultural Sciences in Göttingen (PAG) \\ at the Faculty of Agricultural Sciences, \\ Georg-August-University Göttingen, Germany
}

\author{
presented by \\ Stefanie Walther \\ born in Sangerhausen, Germany
}

Göttingen, May 2016 
D7

1. Name of supervisor: Prof. Dr. Dr. Claus-Peter Czerny

2. Name of co-supervisor: Prof. Dr. Sven König

Date of dissertation: May 12, 2016 


\section{Table of Contents}

Summary

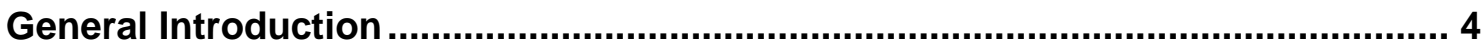

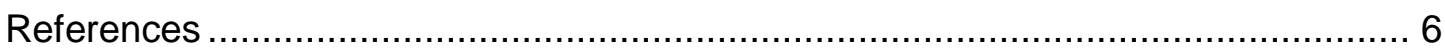

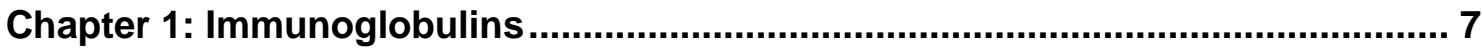

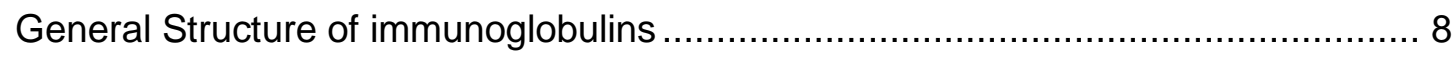

Immunoglobulin heavy chains.............................................................. 10

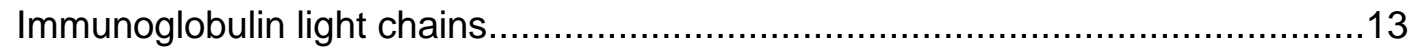

Development of the immunoglobulin repertoire .............................................. 16

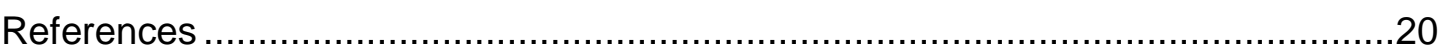

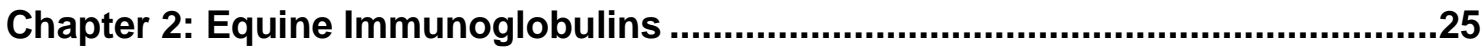

Equine immunoglobulins and organization of immunoglobulin genes..................26

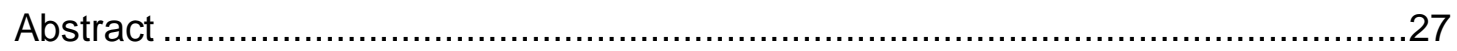

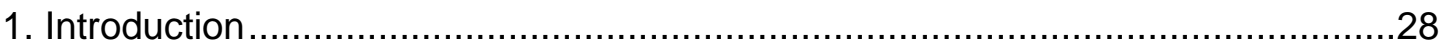

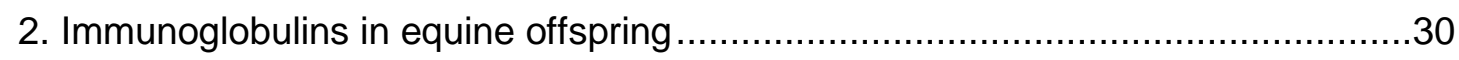

3. The equine immunoglobulin heavy chain gene locus ......................................31

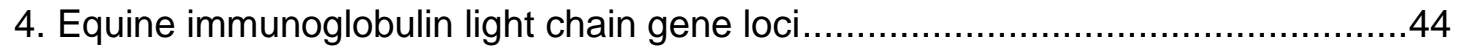

5. Transcription analyses of heavy and light chain genes ..................................55

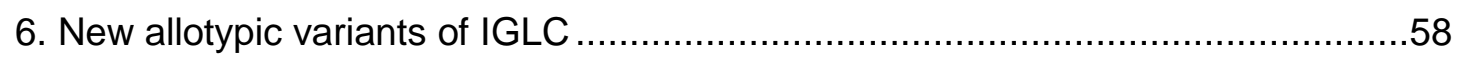

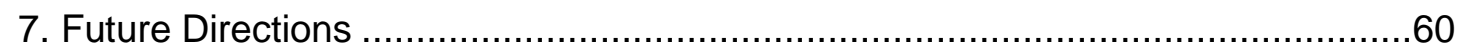

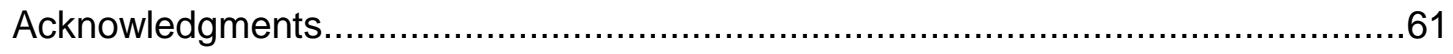

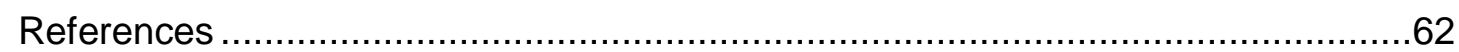

Transcriptional analysis of equine $\lambda$-light chains in the horse breeds Rhenish-

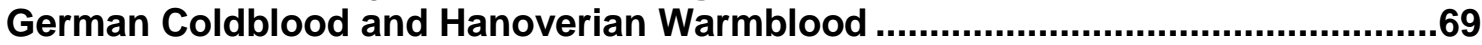

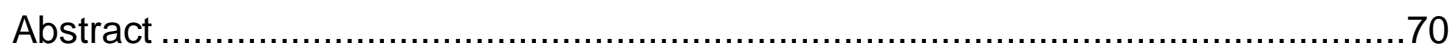

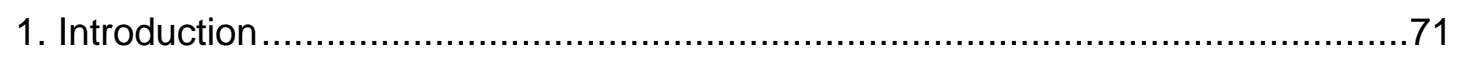

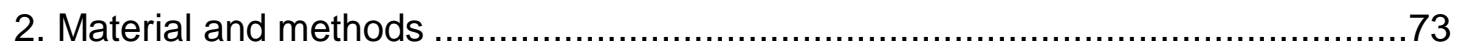

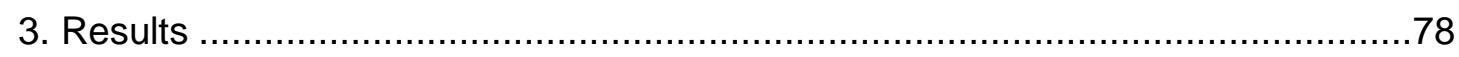

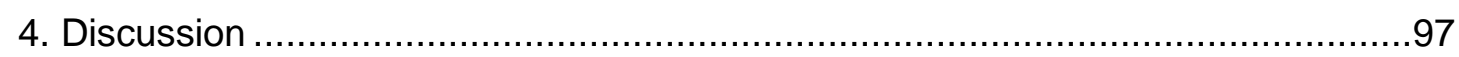

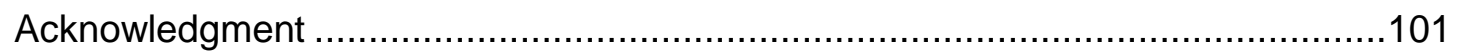

Appendix A. Supplementary data .......................................................... 101

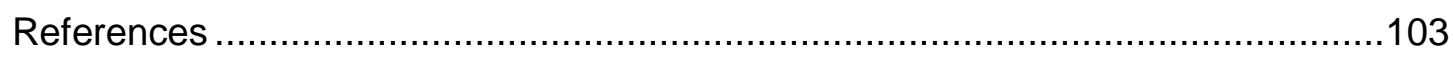




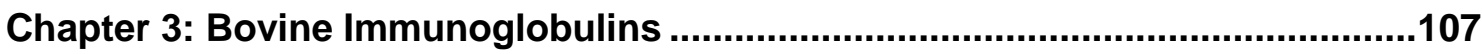

Bovine immunoglobulin heavy and light chains.............................................108

Bovine immunoglobulin heavy chain gene locus .............................................108

The bovine immunoglobulin light chain gene loci ................................................115

Development of $B$ cells and the bovine antibody repertoire...............................119

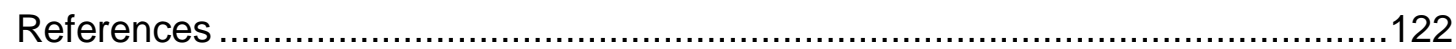

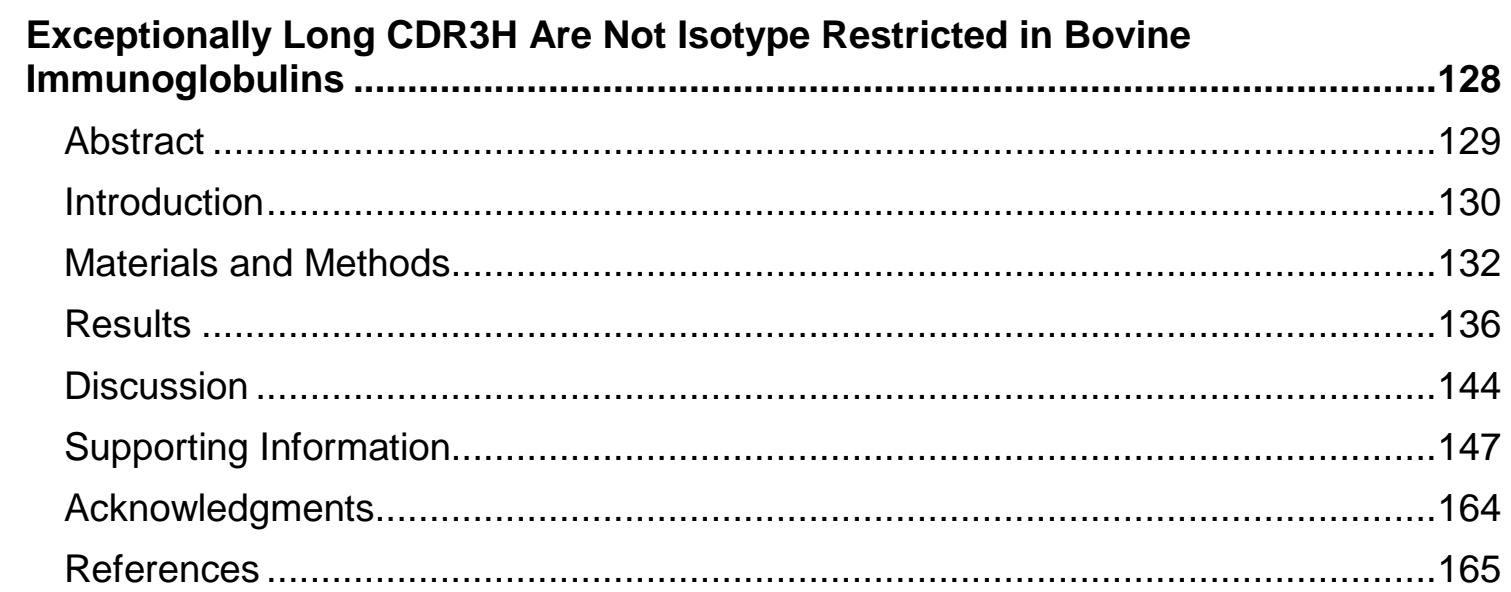

Development of a bioinformatics framework for the detection of gene conversion and the analysis of combinatorial diversity in immunoglobulin heavy chains in four cattle breeds .............................................................................................169

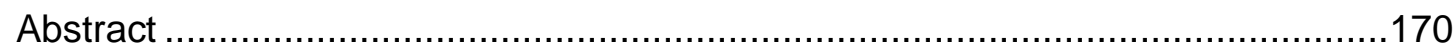

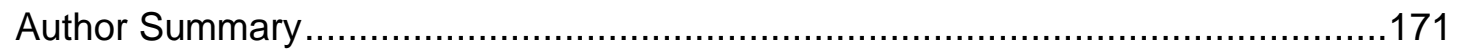

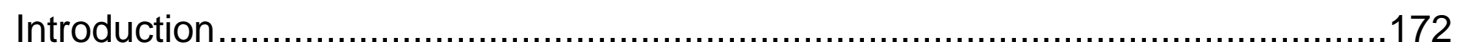

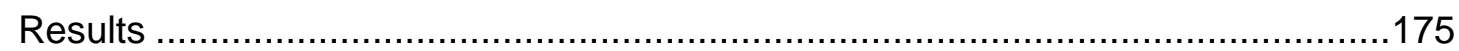

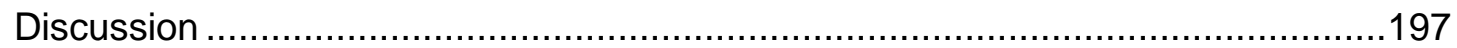

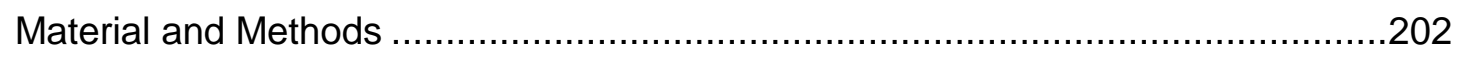

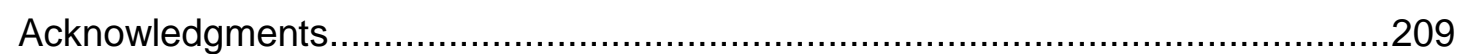

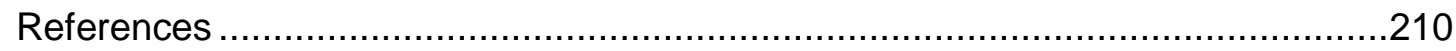

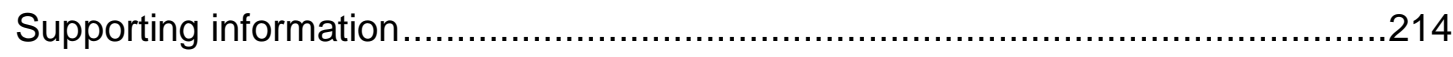

Chapter 4: General Discussion ......................................................................231

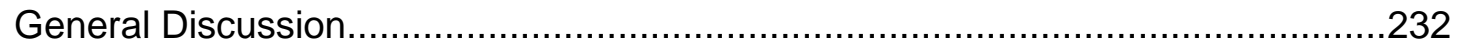

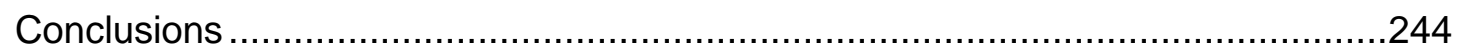

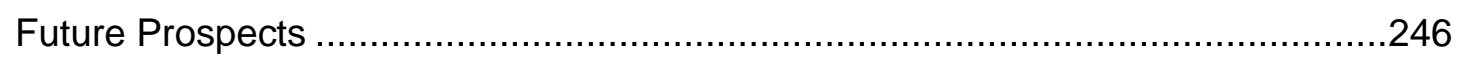

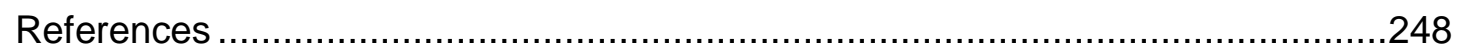

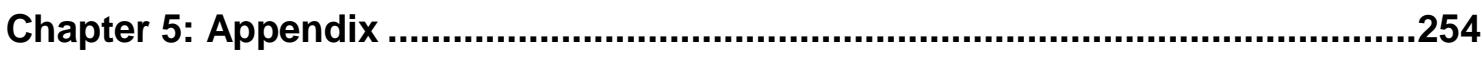

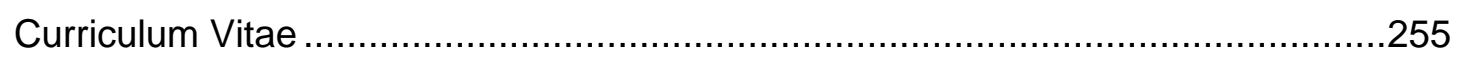




\section{Summary}

Initial studies on genetic aspects of immunoglobulins were performed on humans and mice but were successfully applied to various other animals such as chicken, rabbit, swine, cattle, and horses, too. Especially in cattle and horses, fundamental research in immunoglobulin genetics still needs more attention to complete previous information such as the number of available gene segments, gene families, and allotypes of different isotypes of the immunoglobulin heavy and light chains. Results will enable the analysis and generation of synthetic recombinant antibodies, as well as an alternating treatment of infectious diseases to prevent resistance to antibiotics.

As reviewed in the first publication, the understanding of the organization of equine immunoglobulin genes has increased significantly in the recent years. For equine heavy chains, 52 immunoglobulin heavy chain variable gene segments (IGHV), 40 immunoglobulin heavy chain diversity gene segments (IGHD), 8 immunoglobulin heavy chain joining gene segments (IGHJ) and 11 immunoglobulin heavy chain constant region genes (IGHC) are present. Seven of these IGHCs are gamma chain genes. Sequence diversity is increasing between fetal, neonatal, foal and adult age. The kappa light chain contains 60 immunoglobulin kappa light chain variable gene segments (IGKV), 5 immunoglobulin kappa light chain joining gene segments (IGKJ) and 1 immunoglobulin kappa light chain constant region gene (IGKC), whereas there are 144 immunoglobulin lambda light chain variable gene segments (IGLV), 7 immunoglobulin lambda light chain joining gene segments (IGLJ), and 7 immunoglobulin lambda light chain constant region genes (IGLC) for the lambda light chain, which is expressed predominantly in horses. A decrease in IGLVs is noted during age development, although nucleotide diversity and significant differences in gene usage increased. A standardization of the existing nomenclature of immunoglobulin genes is suggested.

The first experimental study focused on the identification of allotypic variants of equine IGLC and differences in the expression of IGLV within and between the two horse breeds Rhenish-German Coldblood (RGC) and Hanoverian Warmblood (HW). The two breeds differ in stud book size and breeding goals. After PCR amplification of cDNA and subcloning, 120 samples per breed were isolated and sequenced. Statistical analysis of transcription frequencies were performed applying non-parametric tests. The significant majority of the sequences represented IGLC6/7 in both breeds, 
whereas IGLC1, IGLC4, and IGLC5 occurred in significant different frequencies per breed. Five allotypic IGLC1 variants, four allotypic IGLC5 variants, and three allelic as well as two allotypic IGLC6/7 variants were identified in breed specific proportions. Eleven out of 144 known IGLV segments were transcribed of which IGLV15 and IGLV17 were preferred significantly. IGLV25 displayed significant differences in the rearrangement between both breeds. In addition, the pseudogenes IGLV101\% and IGLV74 $\Psi$ were also identified. Rearrangements with IGLC genes showed significant differences for IGLV15 in both breeds, whereas IGLV25 also revealed significant differences between the breeds. The transcriptional orientation of the functional segments had no influence on the occurrence of the IGLV.

The second experimental study carried out in cattle dealt with two main topics. On the one hand it focused on the third complementarity determining region of the bovine heavy chain $(\mathrm{CDR} 3 \mathrm{H})$ whose exceptional length previously was described as a specificity of bovine $\lg G$ and $\lg M$. On the other hand, the genomic organization of the immunoglobulin heavy chain locus was analyzed with a special focus on the number of IGHV. After isotype-specific cDNA-PCR, subcloning of 20 DNA plasmids per immunoglobulin isotype and sequence analyzes of the variable regions, we proved the existence of exceptionally long $\mathrm{CDR} 3 \mathrm{H}$ in all five bovine isotypes. The sequences of $\mathrm{CDR} 3 \mathrm{H}$ belong to three distinct groups and possess $\leq 10,12$ to 31 or $\geq 48$ amino acid residues. Hydrophilic amino acid residues dominated in long and intermediate long $\mathrm{CDR} 3 \mathrm{H}$, while short $\mathrm{CDR} 3 \mathrm{H}$ possessed hydrophobic amino acid residues, too. All sequences with exceptionally long CDR3H were related to the germline IGHV10. Further, the germline IGHD2, with $148 \mathrm{bp}$ in size, contributes to those CDR3H.

The genomic organization of the bovine immunoglobulin heavy-chain locus was analyzed using the current genome assembly, Bos taurus NCBI build 6.1. A main locus was identified on BTA21. Additional exons coding for immunoglobulin heavy chain variable (IGHV), diversity (IGHD), and joining (IGHJ) segments, as well as for the constant regions of different isotypes, were localized on BTA7, BTA8, BTA20, and on unplaced contigs, too. Altogether, 36 IGHV were detected of which 13 are putatively functional. For the phylogenetic analysis, the complete nucleotide sequences of the 36 bovine IGHV segments were aligned with one member of the human IGHV families 1 to 7. Results proved the existence of two bovine IGHV families (boVH1, boVH2). The boVH1 comprises all functional segments. This study substantially improved the understanding of the generation of immunoglobulin diversity in cattle. 
The third study aimed to gain more insight into the combinatorial diversity, somatic hypermutations and putative gene conversions of $\lg G$ in the four cattle breeds Aubrac, German Simmental, German Black Pied, and Holstein Friesian. For the more detailed analysis of rearranged bovine heavy chain immunoglobulin variable regions, a new bioinformatics framework was developed by combining and refining widely used alignment algorithms. Immunoglobulin heavy chains possessing exceptionally long CDR3Hs can now be analyzed specifically, as well as the dominantly transcribed IGHV, IGHD, and IGHJ segments and their recombination.

The use of 15 different IGHV segments, 21 IGHD segments, and 2 IGHJ segments was investigated with significant different transcription levels within the breeds. There are preferred rearrangements within the 3 groups of $\mathrm{CDR} 3 \mathrm{H}$ lengths. In sequences of group 1 ( $\leq 10 \mathrm{aa}$ ) and 3 ( $\geq 48 \mathrm{aa}$ ) a lower number of recombinations were observed than in sequences of group 2 (11-47 aa). The combinatorial diversity revealed 162 significantly different rearrangements of germline IGHV, IGHD, and IGHJ segments. The few preferably rearranged gene segments within group $3 \mathrm{CDR} 3 \mathrm{H}$ regions are supposed to indicate specialized antibodies because this length is unique in cattle.

The main result of this study enabled by the new bioinformatics framework, is the strong evidence for gene conversion as a rare event using pseudogenes fulfilling all definitions for this particular diversification mechanism.

In conclusion, this thesis contributes to a more detailed understanding of the expressed immunoglobulin repertoire in cattle and horses. Breed and husbandry conditions are supposed to influence the repertoire significantly. This thesis also highlights that the bovine heavy chain diversity is not restricted to the use of a limited number of germline genes although there are preferred rearrangements within the three groups of CDR3H lengths. These results will be of future importance in analyzing seroconversion data after infection or vaccination, as well as determining breed specific differences to select healthy, robust animals. 


\section{General Introduction}

Immunoglobulin genetics focuses on the special genetic aspects of immunoglobulins. As relatively few research groups work on immunogenetics, fundamental research is still necessary. Consequently no definite numbers of germline gene segments leading to immunoglobulins, gene families and allotypic variants are known in many species. Nevertheless, in the last few decades, this field of research has attracted higher attention because of its increasing importance in the regional or global eradication and monitoring of several infectious diseases. The availability of new high throughput technologies and descending prices facilitate and advance the experimental work flow in analyzing the entire immunoglobulin repertoire. Initial studies were performed on humans and mice but successful techniques were applied to various other animals such as chicken, rabbit, cattle, and horses, too. Especially in cattle and horses, investigation in immunoglobulin genetics still needs more attention to complete previous information about fundamentals such as the number of available gene segments, gene families, and allotypes of different isotypes of the immunoglobulin heavy and light chains. Results will contribute to the analysis and generation of synthetic recombinant species-specific antibodies. The production of antibodies from animals may be replaced and first of all, the results will enable an alternating treatment of infectious diseases to avoid antibiotics and resulting resistance of antigens. Side effects of conventional therapeutics might be excluded and higher antigen specificity will be achieved. Therefore, recombinant antibodies and antibody fragments are important tools for research, diagnostics and therapy (Hust et al. 2002; Hust and Dubel 2004). A widely used method for the selection of recombinant antibody fragments is the phage display (Smith 1985; Taussig et al. 2007). Further, monoclonal antibodies have been used successfully for the therapeutic treatment of many disorders, including inflammatory and putative autoimmune diseases as they bind to cell-specific antigens and mediate immune response (Hohlfeld and Wekerle 2005). By adding an appropriate constant domain, a promising antibody fragment is converted into any antibody isotype, for example lgG from different species (Moutel et al. 2009).

This present thesis was performed to contribute to a more detailed understanding of immunoglobulin diversity in cattle and horses. One of the major scopes was to investigate the bovine germline heavy chain gene segments in silico, which represent the theoretical immunoglobulin repertoire. The expressed repertoire was then 
investigated in four different cattle breeds by transcriptional analyses and statistical methods. Further, the bovine specific mechanism of exceptionally long CDR3H contributing to diversity was proven in all five immunoglobulin isotypes. Gene conversion using pseudogenes was indicated for the first time in bovine heavy chains. In addition, gene segments of transcribed equine lambda light chains were evaluated statistically in two different horse breeds. The previous findings of horse immunoglobulins were collated and reviewed.

The objectives of this dissertation were:

1. Equine immunoglobulins and organization of immunoglobulin genes

2. Transcriptional analysis of equine $\lambda$ light chains in the horse breeds RhenishGerman Coldblood and Hanoverian Warmblood

3. Exceptionally long CDR3H are not isotype restricted in bovine immunoglobulins

4. Development of a bioinformatics framework for the detection of gene conversion and the analysis of combinatorial diversity in immunoglobulin heavy chains in four cattle breeds 


\section{References}

Hohlfeld R, Wekerle H. 2005. Drug insight: using monoclonal antibodies to treat multiple sclerosis. Nature clinical practice Neurology 1: 34-44.

Hust M, Dubel S. 2004. Mating antibody phage display with proteomics. Trends in biotechnology 22: 8-14.

Hust M, Maiss E, Jacobsen HJ, Reinard T. 2002. The production of a genus-specific recombinant antibody (scFv) using a recombinant potyvirus protease. Journal of virological methods 106: 225-233.

Moutel S, El Marjou A, Vielemeyer O, Nizak C, Benaroch P, Dubel S, Perez F. 2009. A multi-Fc-species system for recombinant antibody production. BMC biotechnology 9: 14.

Smith GP. 1985. Filamentous fusion phage: novel expression vectors that display cloned antigens on the virion surface. Science 228: 1315-1317.

Taussig MJ, Stoevesandt O, Borrebaeck CA, Bradbury AR, Cahill D, Cambillau C, de Daruvar A, Dubel S, Eichler J, Frank R et al. 2007. ProteomeBinders: planning a European resource of affinity reagents for analysis of the human proteome. Nature methods 4: 13-17. 


\section{Chapter 1:}

Immunoglobulins 


\section{General Structure of immunoglobulins}

The three-dimensional structure of immunoglobulins was determined by crystallization. Immunoglobulins (Ig) are described as a Y-shaped tetramer composed of four polypeptide chains containing two identical heavy chains (IGH) and two identical light chains (IGL) that are covalently connected by disulfide bonds (Figure 1) (Edelman 1973). In addition, non-covalent interactions contribute to the connection of IGH and IGL. While an IGH has a molecular mass of about $50 \mathrm{kDa}$, the molecular mass of an IGL is of about $25 \mathrm{kDa}$.

Both the IGH and IGL chains are further divided functionally and genetically into variable and constant domains that show a similar structural folding (Marchalonis et al. 2002; Ramsland and Farrugia 2002). Each chain possesses one variable domain (IGHV, IGLV), while the number of constant domains depends on the chain type and isotype. The variable domains of both chains are located at the arms of the Y-shaped structure (Figure 1). The variability, mediated by the first 110 amino-terminal amino acids, accounts for competent and highly specific antigen binding, whereby both the heavy and light chain variable region work together (Ramsland and Farrugia 2002; Torres et al. 2007). At the carboxyl terminus of the IGH, at least two constant regions (IGHC2 and IGHC3) are connected to the arms of the $Y$ shaped structure. The IGHC3 regions of both IGH interact, but steric hindrance by carbohydrate side chains inhibits the interaction of the IGHC2 (Ramsland and Farrugia 2002).

In addition to flexible regions or hinge regions between the IGHC1 and IGHC2 connection between variable and constant domain is adjustable, too. Both torsion and bending are possible and enable simultaneous binding of antigen structures with different distances, as well as the interaction with signal proteins to enable effector mechanisms (Porter 1973; Ramsland and Farrugia 2002).

The proteases papain and pepsin cleave the polypeptide chain at specific amino acids generating fragments of different sizes (Porter 1973). While cleavage with papain occurs at the carboxyl terminal side of the disulfide bonds within the hinge region of an $\lg \mathrm{G}$ resulting in two fragments, cleavage at the amino terminal side of the disulfide bonds using papain generates three fragments. The resulting fragments are named according to their characteristic features such as antigen binding $\left(F\left(a b^{c}\right)_{2}\right.$ fragment $)$ and the ability to crystallize (Fc fragment) (Yamaguchi et al. 1995). $F(a b)_{2}$ fragments contain the complete IGL, as well as the variable domain and the first constant region (IGHC1) of the heavy chain. They may still bind antigens. IGHC2 and IGHC3 belong to 
the easy crystallizable Fc fragment. These regions mediate effector mechanisms after antigen binding.

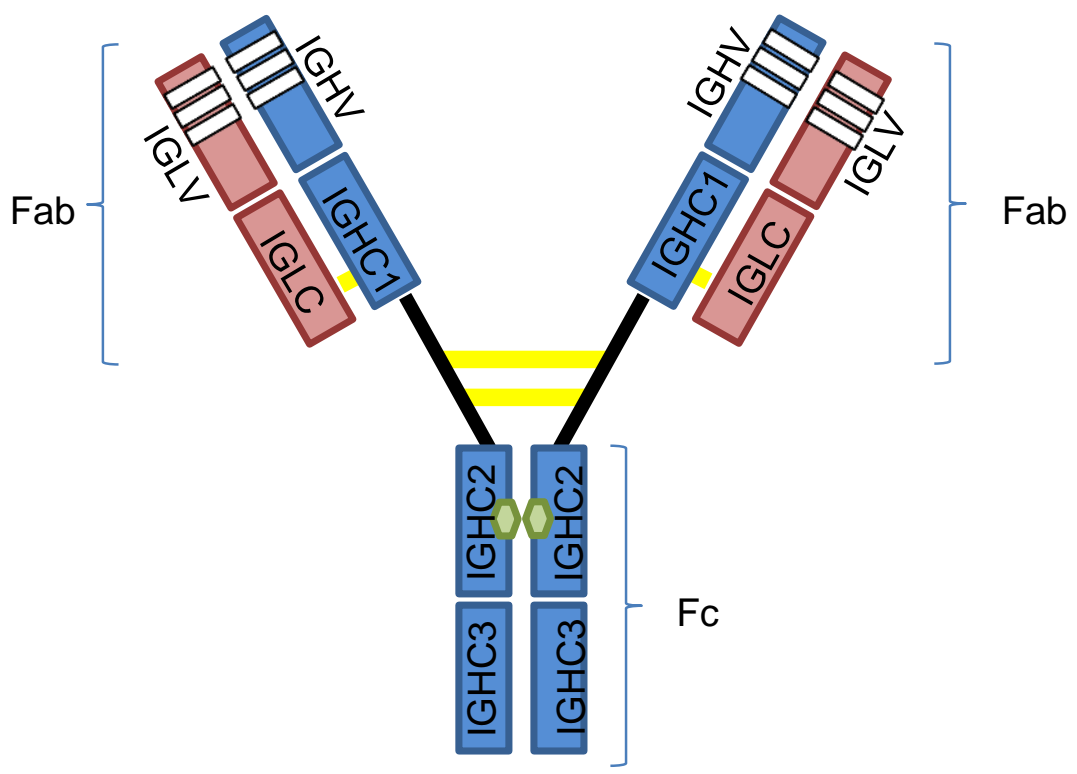

Figure 1: Structure of an immunoglobulin $\mathrm{G}(\mathrm{IgG})$

The heavy chains are shown in blue, the light chains are red. IGLV and IGHV designate the variable regions of light and heavy chain. White boxes within IGLV and IGHV show the complementarity determining regions. The constant regions of light and heavy chain are marked by IGLC and IGHC1 to IGHC3. The hinge region is shown in black, disulfide bonds are colored in yellow. The antigen binding fragments are named Fab and Fc denominates the easy crystallizable fragment. Carbohydrate side chains are colored in green.

The secondary and tertiary structure of all domains is similar but there are differences between variable and constant domains (Ramsland and Farrugia 2002). Both domains consist of seven stacked antiparallel beta-strands that form a beta-barrel (Figure 2). At the end of each beta-strand and change of direction flexible turns are generated. Variable domains possess one more turn than constant domains. The three turns of each the IGH and IGL variable domain generate the area for antigen binding and consist of hypervariable regions contributing to the diversity of the immunoglobulin repertoire as these regions are characterized by extraordinary variability. They are located at restricted areas at the tip of the arms of the Y-shaped molecule (Figure 1, 2). The three-dimensional structure of their amino acid motif is complementary to the three-dimensional structure of the antigen epitope and is called complementarity determining region (CDR1-3, Figure 2) (Wu and Kabat 1970; Decanniere et al. 2000; Ramsland and Farrugia 2002). The length of the CDRs varies. Especially the CDR3 of the heavy chain is highly variable as described in cattle (Walther et al. 2013). 
Depending on the amino acid sequence, electrostatic interactions, hydrogen bonds, Van-der-Waals-forces, and hydrophobic interactions also contribute to antigen binding (Braden and Poljak 1995; Braden et al. 1998). The complete specificity for antigens is generated by the combination of IGH and IGL.

The three hypervariable regions are interspersed by four less variable parts called framework regions (FR1-4). Their amino acid sequences within the variable and constant domain are very similar and they are responsible for stability and structure (Ramsland and Farrugia 2002).
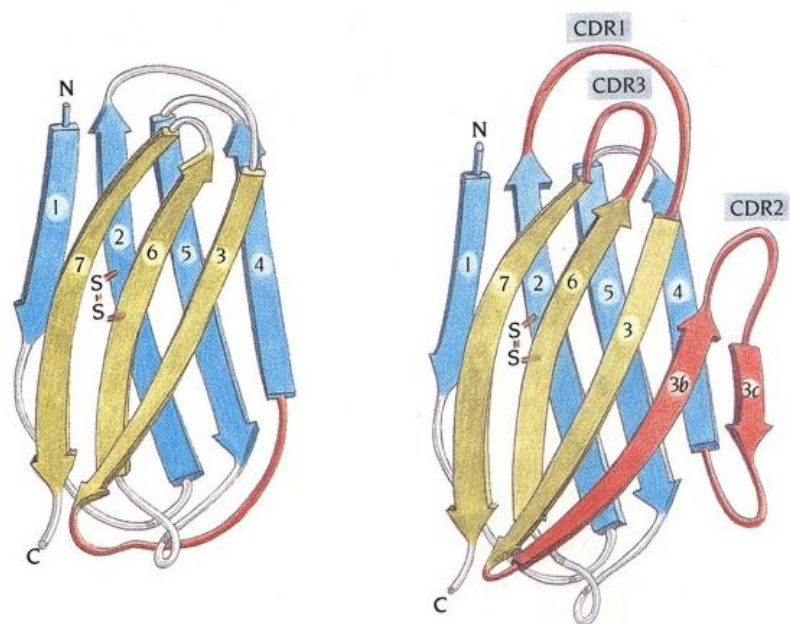

Figure 2: Secondary structure of the constant (left) and variable (right) domains

There are three hypervariable loops (CDR1-3) within the IGHV and the IGLV domains that account for most of the structural variability of the binding site. The CDRs are colored in red. The $\beta$-strands build the framework. The insertion of two $\beta$-strands (3b and $3 \mathrm{c}$ ) linked by a loop containing the CDR2 in the variable domain is also marked red. (Branden 1991)

\section{Immunoglobulin heavy chains}

One immunoglobulin heavy chain is composed of one variable domain and a varying number of constant domains. The variable region is composed of three different gene segments that were randomly joined together during B-cell development and finally possesses around 110 amino acid residues. These gene segments are the variable (IGHV), diversity (IGHD), and joining (IGHJ) segments existing in multiple copies at the heavy chain locus (Figure 3) (Taussig 1988). Numbers of the gene segments are species specific. For instance in human, 123-129 IGHV, 27 IGHD, and 9 IGHJ segments are known, whereby not all of these segments are functional (Lefranc 2001). Non-functional gene segments are called pseudogenes. Mutations leading to premature stop codons prevent the formation of functional proteins. Further, changes within sequence regions necessary for gene recombination such as promotor or 
recombination signal sequences lead to potentially functional gene segments, named open reading frame (ORF) (Lefranc 1998).

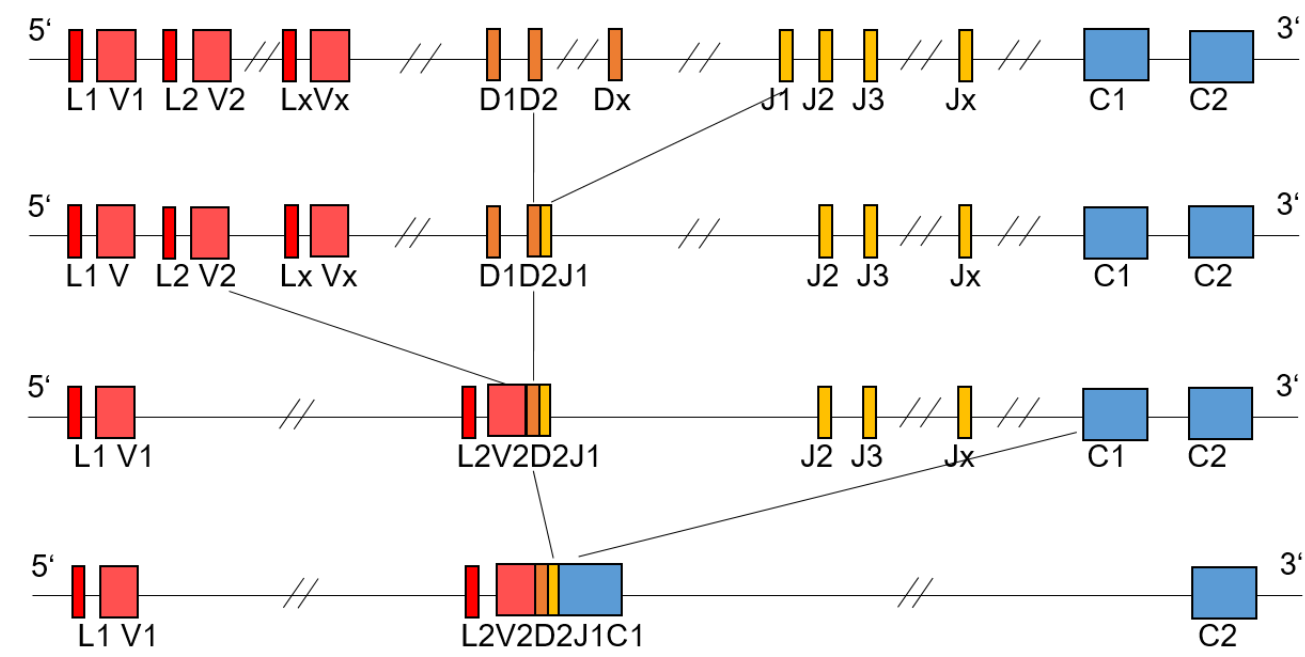

Figure 3: Genomic organization and recombination of the heavy chain gene segments The immunoglobulin heavy chain variable (V1-x, light red), diversity (D1-x, orange), and joining ( $\mathrm{J} 1-\mathrm{x}$, dark yellow) gene segments, as well as the constant region genes (C1-2, blue) are organized in separated clusters on the genome. The recombination of single gene segments occurs at random in multiple steps and results in the deletion of gene segments between the recombined ones.

In general, the immunoglobulin heavy chain gene segments are located on a single chromosome. However, for instance in cattle there is evidence for gene segments located outside the major locus which are called orphan genes (Walther et al. 2013). Each IGHV is preceded by a leader sequence and they are grouped together upstream of an IGHD cluster. Downstream of IGHD, a separate cluster of IGHJ follows. The constant region genes are located 3' of the IGHJ cluster (Figure 3). Each IGHC corresponds to a different isotype.

The different variable gene segments are divided into several families where the members show sequence identities of at least $80 \%$ as recommended for mice (Brodeur and Riblet 1984). Families are further combined to clans. Families within one clan are more similar than families of different clans (Kirkham et al. 1992; Ota and Nei 1994). The variable gene segments of reptiles, amphibians, and mammals belong to the same three clans. During evolution, gene duplication and diversifications led to the variable gene segments known so far.

The number of constant domains in the immunoglobulin molecule depends on the isotype of which five different ones are described in humans, as well as in i.e. mice, cattle, and horses (Berens et al. 1997). These isotpyes are known as $\lg M, \lg D$, $\lg G$, 
$\lg E$, and $\lg A$ (Figure 4), whereby they are encoded by $\mu, \delta, \gamma, \varepsilon$, and $\alpha$ genes (Woof and Burton 2004). IgM and $\lg D$ are coexpressed in naïve B-cells due to alternative splicing of mRNA or class switch recombination in artiodactyls. Activation by antigen contact leads to a switch of isotypes which is also called class switch recombination.

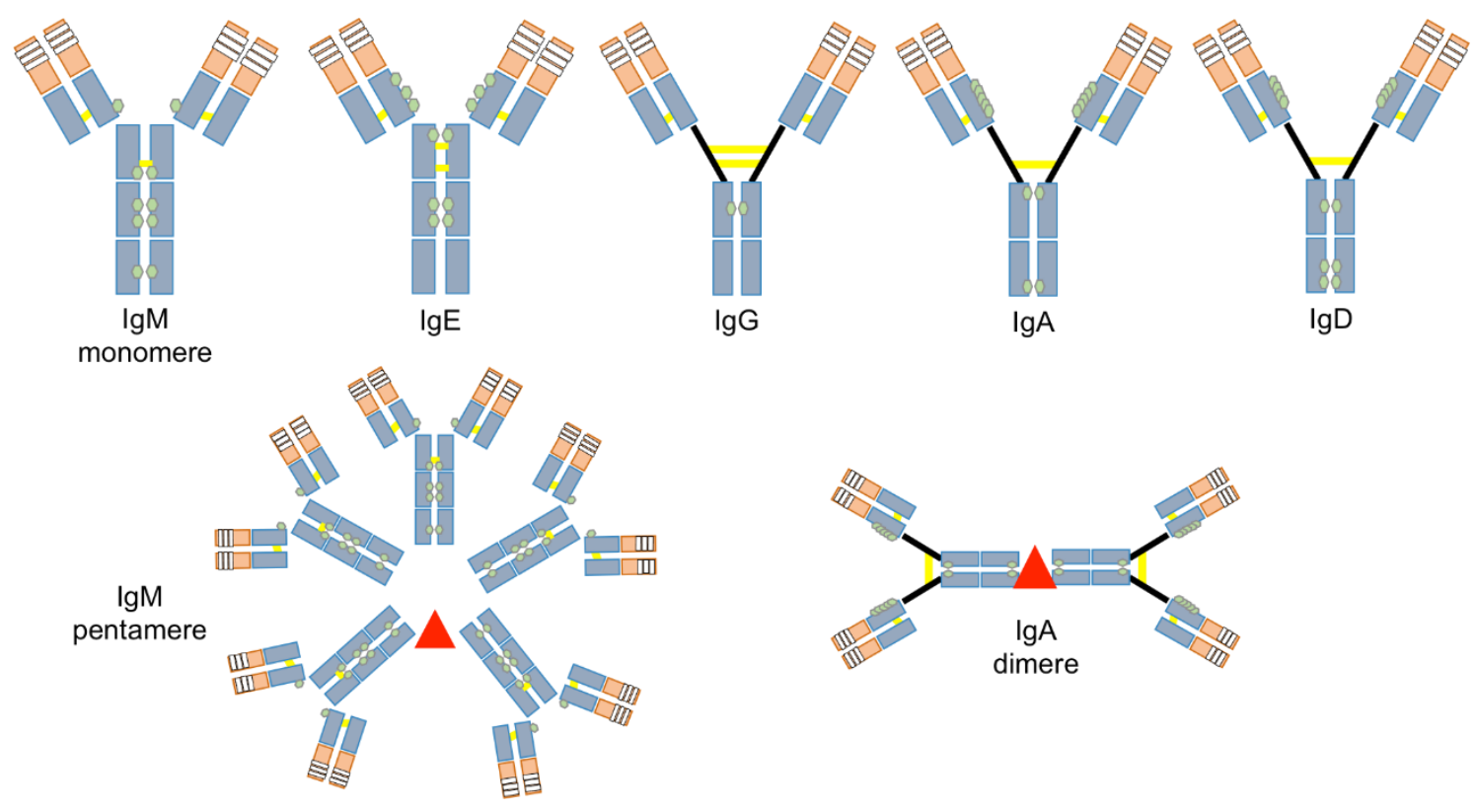

Figure 4: The 5 immunoglobulin isotypes and their multimers

The immunoglobulin variable domains are shown in light red, the constant region genes are shown in blue. The white boxes within the variable domains indicate the complementarity determining regions. Green spots symbolize carbohydrate side chains. Disulfide bonds are shown in yellow and the hinge region is black. The red triangle indicates an additional $15 \mathrm{kDa}$ polypeptide chain contributing to polymerization.

Heavy chains of the $\alpha-, \delta-$, and $\gamma$-isotype possess a constant region composed of three domains (IGHC1, IGHC2, IGHC3) whereas the $\mu$ - and $\varepsilon$-isotypes have an additional fourth constant region (IGHC4). While $\lg A, \lg D$, and $\lg G$ possess a flexible hinge region, $\lg \mathrm{M}$ and $\lg \mathrm{E}$ achieve flexibility by bending of the antibody binding fragments (Mousavi et al. 1998; Janeway 2001). Furthermore, isotypes vary in the number of disulfide bonds between the chains, connected oligosaccharids, and length of the hinge region. Different sizes and compositions are characteristic for each isotpye. Consequently, $\alpha$ and $\gamma$ possess 450 amino acids, whereas $\delta$ contains approximately 500 amino acids, and $\mu$ and $\varepsilon$ are composed of 550 amino acids. Further, the antibody classes may occur as monomers (membrane bound in all isotypes), dimers, or multimers (Fudenberg and Warner 1970; Mestecky 1972). Secreted IgM and IgA may 
occur as pentamers in plasma or dimers in mucous secretions, respectively (Figure 4). The IGHCs are responsible for complement activation, Fc receptor binding, serum half-life, and flexibility or stabilization of the variable region (Ravetch and Kinet 1991; Woof and Burton 2004).

\section{Immunoglobulin light chains}

Light chains contribute to antigen binding and enlarge variability of antibodies. They enable the expression of the heavy chains in pre-B-cells and therefore are responsible for the expression of B-cell receptors, as well as of secreted antibodies (Meffre et al. 2001). Immunoglobulin light chain constant regions support antigen recognition, stabilize the variable region, and are associated to the first constant region of heavy chain isotypes by specific amino acid residues that form an interdomain interface and contribute to non-covalent binding, as well as they contribute to covalent binding due to disulfide bonds (Padlan et al. 1986; Chen et al. 2008).

In mammals, two isotypes of IGL exist, which are called kappa (K) and lambda $(\lambda)$ (Korngold and Lipari 1956). As there is no shared origin for the light chain isotypes, they are polyphyletic (Sitnikova and Su 1998). The ratio of the isotypes depends on the species. A $\mathrm{k}: \lambda$ ratio of 2:1 is found in human and swine, mice possess a ratio of 20:1, whereas in cattle and horse ratios of $1: 20$ and 1:13 are described. Consequently, in cattle and horses $\lambda$-light chains are predominantly expressed (Home et al. 1992; Arun et al. 1996; Butler 1998). Exceptions from these immunoglobulins with either $\mathrm{K}$ - or $\lambda$-light chain are found in chicken, camel, and shark where solely $\lambda$-light chains are expressed or antibodies without any light chains and heavy chain homodimers were found (Ford et al. 1994; Wernery 2001; Saini et al. 2003). In Xenopus laevis an additional IGL of $\sigma$-isotype was described (Klein et al. 2002). The light chain isotypes can be distinguished by specific conserved amino acid motifs (Das et al. 2008). Hence, this isotype occurs in more than $90 \%$ of horse serum antibodies (Gibson 1974).

The light chain isotypes comprises of 211 to 217 amino acid residues (Janeway 2001). Characteristic amino acid motifs within the framework regions of the variable domain allow differentiation of the three IGL isotypes (Das et al. 2008). Distinctive features are the additional three amino acids in FR3 in $\sigma$-isotype compared to $\mathrm{K}$ - and $\lambda$-light chains, 22 amino acids within FR1 in $\lambda$-isotypes and 23 amino acids building FR1 in the $\mathrm{K}$-isotypes. Further, amino acids Ser and Thr are distinguished at position 7 using the Ensembl annotation which is based on the IMGT nomenclature (Das et al. 2008). Also 
the amino acid residue at position 53 differentiates $\mathrm{k}$ - and $\lambda$-light chains ( $\mathrm{k}$ : Phe/Tyr vs. $\lambda$ : Ala/Gly). A conserved amino acid motif in $\lambda$-isotype is Asp/Glu/Ala/Asp, which is missing in the $\sigma$ - and $\mathrm{K}$-isotypes. Beside the differences within the variable gene segments, differences within the joining gene segments are described $(\sigma$ : Ser 4 , Ser 7; $\lambda$ and $\mathrm{k}$ : Gly 4, Thr 7; $\mathrm{k}$ : Thr 2, Glu-Ile-Lys/Glu-Leu-Lys 10-12; $\lambda$ and $\sigma:$ Thr-ValLeu/Thr-Val-Thr und Ile-Val-Thr 10-12). Specific amino acids at the positions 14, 32, 34, 79, and 91 using the Ensembl annotation enable to discriminate the constant regions of $\sigma$-light chains from $\mathrm{k}$ - and $\lambda$-light chains, while particular amino acids at positions $17,56,60,65$, and 102 are responsible for the differentiation of $\mathrm{K}-$ and $\lambda$-isotypes (Das et al. 2008).

Genes coding for light chain isotypes are located on different chromosomes. While $\mathrm{K}$ - and $\lambda$-light chains show similar differences in their sequences compared to the $\sigma$-light chains, the genomic organization of joining gene segments and constant region genes of $\sigma$-and $k$-light chains is analogical and differs from the organization found for $\lambda$ (Das et al. 2008). Joining gene segments and constant region genes in $\sigma$ - and $\mathrm{k}$-loci have an own cluster, whereas in the $\lambda$-locus joining gene segments and constant region genes cluster pairwise (Figure 5 ).

Hitherto no functional differences were described between the light chain isotypes although they appear in connection to specific diseases (Das et al. 2008). For instance, allotypic markers of human light chains were associated with the susceptibility to different infectious diseases (Pandey et al. 1995; Pandey 2000; Giha et al. 2009). 
A

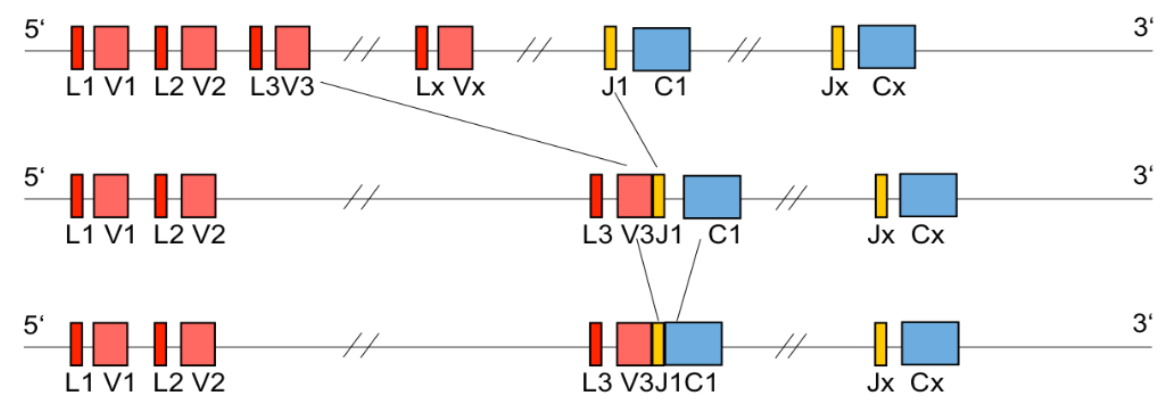

B

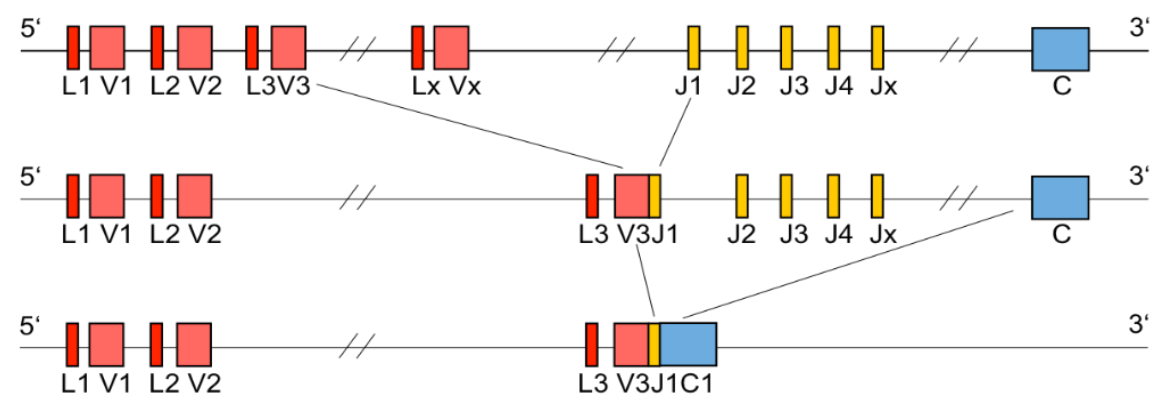

Figure 5: Genomic organization and recombination of the light chain gene segments

The immunoglobulin light chain variable (V1-x, light red), diversity (D1-x, orange), and joining ( $\mathrm{J} 1-\mathrm{x}$, dark yellow) gene segments, as well as the constant region genes ( $\mathrm{C} / \mathrm{Cx}$, blue) are organized in separated clusters on the genome. (A) Lambda light chain genes, the joining and constant region genes occur pairwise. (B) Kappa light chain genes, joining gene segments and the constant region gene are separated. The recombination of single gene segments occurs at random in multiple steps and results in the deletion of gene segments between the recombined ones. 


\section{Development of the immunoglobulin repertoire}

The vertebrate immune system is able to produce a large diversity of antibodies with different specificities from a relatively modest number of gene segments (Parng et al. 1996). Therefore, immunoglobulins are a major component of the humoral immunity (Saini et al. 2007). Immunoglobulins are produced by B-cells, whereas each of these cells is specific for one antigen. Consequently, the immunoglobulin repertoire at a certain point in time is restricted by the number of B-cells and depends on antigen contacts (Janeway CA Jr 2001).

In one individual, the repertoire of immunoglobulins is immense. Immunoglobulins are produced by B-lymphocytes and plasma cells and may be membrane-bound (B-cell receptors) or secreted proteins (antibodies), which become diversified additionally. The development of the whole immunoglobulin repertoire depends on different primary and secondary mechanisms. The original repertoire is generated by the combinatorial diversity due to heavy and light chain pairing, random gene rearrangements, as well as the junctional diversity, which introduces insertions and deletions of nucleotides at the recombination site. Antigen contact and effector functions of the expressed antibodies further increase the primary repertoire. This secondary part of diversification is based on somatic hypermutations, gene conversion, and class switch recombination.

During the early development of lymphatic progenitor B-cells, variable and constant domains are joined together by somatic recombinations of separate heavy and light chain variable (IGHV, IGLV), diversity (IGHD), joining (IGHJ, IGLJ), and constant (IGHC, IGLC) germline components (Tonegawa 1983). In B-lymphocytes, the heavy chain rearrangement precedes the rearrangement of the light chains (Alt et al. 1981). Thus, recombination process starts within the heavy chain locus in the pro-B-cells. The separate gene segments rearrange together to form one complete variable domain exon. This process depends on recombination signal sequences (RSS) consisting of two conserved parts, the heptamer and the nonamer that are separated by a 12 or 23 bp spacer. The heptamer is directly connected to the gene segment. The spacer length is specific for the segment type e.g. the spacer following the IGLV of the $\lambda$-light chain (IGVL) has $23 \mathrm{bp}$, whereas the nonamer and the heptamer of the joining segment (IGJL) are separated by a 12 bp spacer. Spacer lengths between heptamer and nonamer of the IGLV and IGLJ of the K-light chains (IGVK and IGKJ) possess 12 bp and $23 \mathrm{bp}$, respectively. Due to the spacer length, the heptamer and nonamer bind to the protein complex catalyzing the somatic recombination (Kim et al. 2015; 
Lapkouski et al. 2015). This process follows the $12 / 23$ rule and uses gene segments located on the same chromosome. During the process of recombination, the DNA located between the two joined segments is deleted (Sakano et al. 1979; Akira et al. 1987). After effective rearrangement, a $\mu$-chain is expressed and associates with surrogate light chains. These surrogate light chains simulate the variable and constant region of the light chains that are not expressed yet at this step in B-cell development. Their expression is caused by the transcription factors E2A and EBF. This first checkpoint induces the completion of the heavy chain rearrangement and results in allelic exclusion (Loffert et al. 1996; Melchers et al. 1999; Vettermann and Schlissel 2010). Consequently, only one of the two alleles is expressed in one pro-B-cell. Subsequently, pro-B-cells divide and result in a large number of pro-B-cells that contain the same heavy chain and develop into pre-B-cells. The rearrangement of the light chain genes starts and is repeated until a productive light chain emerges. In case of unsuccessful recombinations of one light chain isotype, the rearrangement may also switch to the second light chain isotype. This process is called light chain rescue to prevent cell death. Also during rearrangements in the light chain genes, allelic exclusion and isotype exclusion occur. Therefore, just one light chain isotype is transcribed in one B-cell (Arakawa et al. 1996; Loffert et al. 1996; Melchers et al. 1999). Finally, the associated $\mu$ - and light chains are expressed as B-cell receptors on the surface of immature B-cells. Before these immature cells leave the bone marrow for the periphery, they undergo several types of negative selection, such as clonal deletion (Nemazee and Burki 1989), receptor editing (Gay et al. 1993; Tiegs et al. 1993), clonal anergy (Goodnow et al. 1988), or apoptosis to avoid autoreactivity (Levine et al. 2000).

Beside the random combination of different variable, diversity, and joining gene segments a junctional diversity occurs during rearrangements by the insertion or deletion of nucleotides within the joining area of IGHV-IGHD, IGHD-IGHJ, IGKV-IGKJ, and IGLV-IGLJ, respectively. The inserted nucleotides are called $\mathrm{N}$ - and P-nucleotides that are characterized by the addition of non-encoded $(\mathrm{N}-)$ or palindromic ( $\mathrm{P}-$ ) nucleotides catalyzed by the enzymes terminal deoxynucleotidyltransferase, as well as RAG-proteins and the artemis enzyme complex. Further, exonucleases may delete nucleotides. These mechanisms result in an increased variability of nucleotides and amino acid residues within the CDR3 of both heavy and light chains, as well as in length differences. Following, the identification of the originating IGHD is sometimes difficult or may even be impossible in some cases. 
The secondary diversification mechanisms, somatic hypermutation, gene conversion, and class switch recombination introduce changes into the sequence of functional, secreted antibodies (Figure 6). All these mechanisms are initiated on single stranded DNA by the activation induced cytidine deaminase (AID) (Di Noia and Neuberger 2002; Petersen-Mahrt et al. 2002; Bransteitter et al. 2003; Chaudhuri et al. 2003).

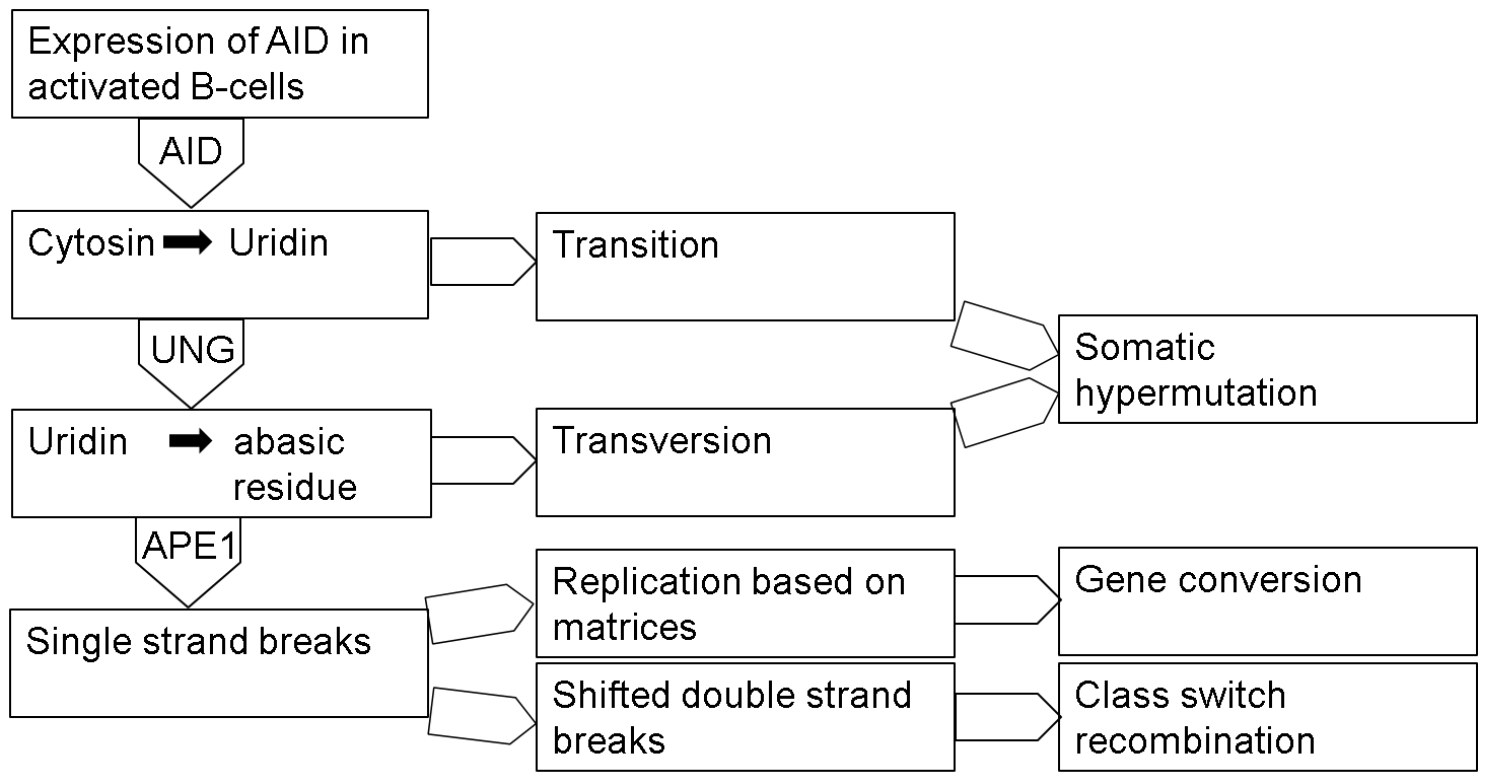

Figure 6: Secondary diversification mechanisms

AID: activation induced cytidine deaminase; UNG: uracil DNA glycosylase; APE1: apurinic/ apyrimidinic endonuclease 1 (modified from "Janeway Immunologie", (Murphy 2009))

Deamination of cytidine to uracil by AID results in transition mutations, one kind of somatic hypermutation. A second kind of somatic hypermutation, transversion mutation, is generated by the base excision enzyme uracil DNA glycosylase (UNG), which deletes the uracil generated by AID. Somatic hypermutation occurs in B-cells located in peripheric lymphoid tissues after stimulation by an antigen and generates point mutations within the complete exon of the variable region of both the heavy and light chains (Muramatsu et al. 2000). While silent mutations accumulate also in FRs, mutations affecting amino acid substitutions and protein structure are mainly found in the CDRs (Maizels 2005; Neuberger 2008). Certain major hotspots targeting somatic hypermutation are known. For instance, a cytosine $(\mathrm{C})$ residue is more likely to be mutated if it is part of a WRCY motif $(\mathrm{W}=\mathrm{A}$ or $\mathrm{T}, \mathrm{R}=\mathrm{A}$ or $\mathrm{G}, \mathrm{Y}=\mathrm{T}$ or $\mathrm{C})$ and also WA motifs ( $\mathrm{Li}$ et al. 2004; Wang et al. 2010). If this leads to improved affinity for antigens, the resulting affinity maturation of cells expressing such immunoglobulins leads to 
further expansion. After deamination of cytidine by AID and deletion of uracil by UNG an abasic residue exists. This abasic residue is exised by the apurinic/ apyrimidinic endonuclease 1 (APE1) leading to a single strand break which is assumed to result in matrix-based replication and gene conversion or shifted double strand brakes and class switch recombination, respectively. Gene conversion also affects the complete variable regions. Parts of pseudogene sequences replace the original sequence, which is assumed to be a homology based repair mechanism characteristically found in chicken and rabbits. This mechanism increases antibody diversification in species with small number of germline gene segments for the variable region such as chicken, sheep, rabbits, cattle, and is assumed in horses (Reynaud et al. 1985; Reynaud et al. 1987; Reynaud et al. 1989; Becker and Knight 1990; Reynaud et al. 1995; Parng et al. 1996; Sun et al. 2010). Class switch recombination concerns the constant region. Hence, the same heavy chain variable region associates with different IGHC during one immune response. Once a B-cell was stimulated by an antigen, CD40 and toll like receptors provide the activation for $B$ cells to undergo class switch recombination. For this purpose, toll like receptors on the surface of major $B$ cells respond to microbial products such as lipopolysaccharides and CpG-enriched DNA. Both ligands (lipopolysaccharides and CpG DNA) of toll like receptors stimulate cell proliferation, AID expression and class switch recombination, as well as differentiation into antibody secreting cells by signals transduced through the toll like receptors (Edry et al. 2008; Pone et al. 2012). During class switch recombination, the primary $\lg M$ is replaced by an alternative IGHC isotype resulting in an increased functional diversity of the immunoglobulin molecule. This process is directed by repetitive nucleotide sequences (switch-regions). For instance, common elements are GGGGT, GGGCT, or GAGCT within the introns upstream of the IGHC and downstream of the IGHJ. Switch regions possess tandem repeats of short consensus elements that function as hotspot target for the AID. Class switches are supposed to occur by a non-homologous end joining mechanism. Cytokines produced by T-helper cells and dendritic cells regulate this intrachromosomal deletional recombination by inducing transcription form promotors located upstream to the acceptor switch region. Consequently, cytokines target the class switch recombination to a specific isotype (Stavnezer et al. 2008). In mice, IL-4 induces the switch to $\lg G 1$ and $\lg E$, while TGF- $\beta$ induces the switch to $\lg G 2 b$ and $\lg A$ (Stavnezer and Amemiya 2004). Class switch recombination always results in functional immunoglobulins. 


\section{References}

Akira S, Okazaki K, Sakano H. 1987. Two pairs of recombination signals are sufficient to cause immunoglobulin V-(D)-J joining. Science 238: 1134-1138.

Alt F, Rosenberg N, Lewis S, Thomas E, Baltimore D. 1981. Organization and reorganization of immunoglobulin genes in A-MULV-transformed cells: rearrangement of heavy but not light chain genes. Cell 27: 381-390.

Arakawa H, Shimizu T, Takeda S. 1996. Re-evaluation of the probabilities for productive arrangements on the kappa and lambda loci. International immunology 8: 91-99.

Arun SS, Breuer W, Hermanns W. 1996. Immunohistochemical examination of lightchain expression (lambda/kappa ratio) in canine, feline, equine, bovine and porcine plasma cells. Zentralblatt fur Veterinarmedizin Reihe A 43: 573-576.

Becker RS, Knight KL. 1990. Somatic diversification of immunoglobulin heavy chain VDJ genes: evidence for somatic gene conversion in rabbits. Cell 63: 987-997.

Berens SJ, Wylie DE, Lopez OJ. 1997. Use of a single VH family and long CDR3s in the variable region of cattle Ig heavy chains. International immunology 9: 189199.

Braden BC, Goldman ER, Mariuzza RA, Poljak RJ. 1998. Anatomy of an antibody molecule: structure, kinetics, thermodynamics and mutational studies of the antilysozyme antibody D1.3. Immunological reviews 163: 45-57.

Braden BC, Poljak RJ. 1995. Structural features of the reactions between antibodies and protein antigens. FASEB journal : official publication of the Federation of American Societies for Experimental Biology 9: 9-16.

Branden CT, J. 1991. Introduction to Protein Structure. Garland Publishing.

Bransteitter R, Pham P, Scharff MD, Goodman MF. 2003. Activation-induced cytidine deaminase deaminates deoxycytidine on single-stranded DNA but requires the action of RNase. Proceedings of the National Academy of Sciences of the United States of America 100: 4102-4107.

Brodeur $\mathrm{PH}$, Riblet R. 1984. The immunoglobulin heavy chain variable region (Igh-V) locus in the mouse. I. One hundred Igh-V genes comprise seven families of homologous genes. European journal of immunology 14: 922-930.

Butler JE. 1998. Immunoglobulin diversity, B-cell and antibody repertoire development in large farm animals. Rev Sci Tech 17: 43-70.

Chaudhuri J, Tian M, Khuong C, Chua K, Pinaud E, Alt FW. 2003. Transcriptiontargeted DNA deamination by the AID antibody diversification enzyme. Nature 422: 726-730.

Chen L, Li M, Li Q, Yang X, An X, Chen Y. 2008. Characterization of the bovine immunoglobulin lambda light chain constant IGLC genes. Veterinary immunology and immunopathology 124: 284-294.

Das S, Nikolaidis N, Klein J, Nei M. 2008. Evolutionary redefinition of immunoglobulin light chain isotypes in tetrapods using molecular markers. Proceedings of the National Academy of Sciences of the United States of America 105: 1664716652.

Decanniere K, Muyldermans S, Wyns L. 2000. Canonical antigen-binding loop structures in immunoglobulins: more structures, more canonical classes? Journal of molecular biology 300: 83-91.

Di Noia J, Neuberger MS. 2002. Altering the pathway of immunoglobulin hypermutation by inhibiting uracil-DNA glycosylase. Nature 419: 43-48. 
Chapter 1: Immunoglobulins

Edelman GM. 1973. Antibody structure and molecular immunology. Science 180: 830840.

Edry E, Azulay-Debby H, Melamed D. 2008. TOLL-like receptor ligands stimulate aberrant class switch recombination in early B cell precursors. International immunology 20: 1575-1585.

Ford JE, Home WA, Gibson DM. 1994. Light chain isotype regulation in the horse. Characterization of Ig kappa genes. Journal of immunology 153: 1099-1111.

Fudenberg HH, Warner NL. 1970. Genetics of immunoglobulins. Advances in human genetics 1: 131-209.

Gay D, Saunders T, Camper S, Weigert M. 1993. Receptor editing: an approach by autoreactive $\mathrm{B}$ cells to escape tolerance. The Journal of experimental medicine 177: 999-1008.

Gibson D. 1974. Structural studies on normal horse immunoglobulin light chains. Detection of k-type N-terminal sequences. Biochemistry 13: 2776-2785.

Giha HA, Nasr A, Iriemenam NC, Arnot D, Troye-Blomberg M, Theander TG, Berzins K, EIGhazali G, Pandey JP. 2009. Antigen-specific influence of $G M / K M$ allotypes on $\lg G$ isotypes and association of $\mathrm{GM}$ allotypes with susceptibility to Plasmodium falciparum malaria. Malaria journal 8: 306.

Goodnow CC, Crosbie J, Adelstein S, Lavoie TB, Smith-Gill SJ, Brink RA, PritchardBriscoe H, Wotherspoon JS, Loblay $\mathrm{RH}$, Raphael K et al. 1988. Altered immunoglobulin expression and functional silencing of self-reactive $B$ lymphocytes in transgenic mice. Nature 334: 676-682.

Home WA, Ford JE, Gibson DM. 1992. L chain isotype regulation in horse. I. Characterization of Ig lambda genes. Journal of immunology 149: 3927-3936.

Janeway CA Jr TP, Walport M, et al. . 2001. The generation of diversity in immunoglobulins. . in Immunobiology: The Immune System in Health and Disease New York: Garland Science.

Janeway CA, Jr. 2001. How the immune system protects the host from infection. Microbes and infection / Institut Pasteur 3: 1167-1171.

Kim MS, Lapkouski M, Yang W, Gellert M. 2015. Crystal structure of the V(D)J recombinase RAG1-RAG2. Nature 518: 507-511.

Kirkham PM, Mortari F, Newton JA, Schroeder HW, Jr. 1992. Immunoglobulin VH clan and family identity predicts variable domain structure and may influence antigen binding. EMBO J 11: 603-609.

Klein SL, Strausberg RL, Wagner L, Pontius J, Clifton SW, Richardson P. 2002. Genetic and genomic tools for Xenopus research: The NIH Xenopus initiative. Dev Dyn 225: 384-391.

Korngold L, Lipari R. 1956. Multiple-myeloma proteins. III. The antigenic relationship of Bence Jones proteins to normal gammaglobulin and multiple-myeloma serum proteins. Cancer 9: 262-272.

Lapkouski M, Chuenchor W, Kim MS, Gellert M, Yang W. 2015. Assembly Pathway and Characterization of the RAG1/2-DNA Paired and Signal-end Complexes. The Journal of biological chemistry 290: 14618-14625.

Lefranc M-PaL, G. 2001. The Immunoglobulin FactsBook, Academic Press.

Lefranc MP. 1998. IMGT (ImMunoGeneTics) locus on focus. A new section of Experimental and Clinical Immunogenetics. Experimental and clinical immunogenetics 15: 1-7.

Levine MH, Haberman AM, Sant'Angelo DB, Hannum LG, Cancro MP, Janeway CA, Jr., Shlomchik MJ. 2000. A B-cell receptor-specific selection step governs immature to mature B cell differentiation. Proceedings of the National Academy of Sciences of the United States of America 97: 2743-2748. 
Li Z, Woo CJ, Iglesias-Ussel MD, Ronai D, Scharff MD. 2004. The generation of antibody diversity through somatic hypermutation and class switch recombination. Genes Dev 18: 1-11.

Loffert D, Ehlich A, Muller W, Rajewsky K. 1996. Surrogate light chain expression is required to establish immunoglobulin heavy chain allelic exclusion during early B cell development. Immunity 4: 133-144.

Maizels N. 2005. Immunoglobulin gene diversification. Annu Rev Genet 39: 23-46.

Marchalonis JJ, Jensen I, Schluter SF. 2002. Structural, antigenic and evolutionary analyses of immunoglobulins and $\mathrm{T}$ cell receptors. Journal of molecular recognition : JMR 15: 260-271.

Meffre E, Milili M, Blanco-Betancourt C, Antunes H, Nussenzweig MC, Schiff C. 2001. Immunoglobulin heavy chain expression shapes the $\mathrm{B}$ cell receptor repertoire in human B cell development. The Journal of clinical investigation 108: 879-886.

Melchers F, ten Boekel E, Yamagami T, Andersson J, Rolink A. 1999. The roles of preB and $B$ cell receptors in the stepwise allelic exclusion of mouse $\operatorname{lgH}$ and $L$ chain gene loci. Seminars in immunology 11: 307-317.

Mestecky J. 1972. Structure of antibodies. Journal of oral pathology 1: 288-300.

Mousavi M, Rabbani H, Pilstrom L, Hammarstrom L. 1998. Characterization of the gene for the membrane and secretory form of the $\operatorname{lgM}$ heavy-chain constant region gene ( $\mathrm{C} \mathrm{mu}$ ) of the cow (Bos taurus). Immunology 93: 581-588.

Muramatsu M, Kinoshita K, Fagarasan S, Yamada S, Shinkai Y, Honjo T. 2000. Class switch recombination and hypermutation require activation-induced cytidine deaminase (AID), a potential RNA editing enzyme. Cell 102: 553-563.

Murphy KT, P.; Walport, M. 2009. Janeway Immunologie, Spektrum Akademischer Verlag Heidelberg

Nemazee DA, Burki K. 1989. Clonal deletion of B lymphocytes in a transgenic mouse bearing anti-MHC class I antibody genes. Nature 337: 562-566.

Neuberger MS. 2008. Antibody diversification by somatic mutation: from Burnet onwards. Immunol Cell Biol 86: 124-132.

Ota T, Nei M. 1994. Divergent evolution and evolution by the birth-and-death process in the immunoglobulin VH gene family. Molecular biology and evolution 11: 469482.

Padlan EA, Cohen GH, Davies DR. 1986. Antibody Fab assembly: the interface residues between $\mathrm{CH} 1$ and $\mathrm{CL}$. Molecular immunology 23: 951-960.

Pandey JP. 2000. Immunoglobulin GM and KM allotypes and vaccine immunity. Vaccine 19: 613-617.

Pandey JP, Elson LH, Sutherland SE, Guderian RH, Araujo E, Nutman TB. 1995. Immunoglobulin kappa chain allotypes (KM) in onchocerciasis. The Journal of clinical investigation 96: 2732-2734.

Parng CL, Hansal S, Goldsby RA, Osborne BA. 1996. Gene conversion contributes to Ig light chain diversity in cattle. Journal of immunology 157: 5478-5486.

Petersen-Mahrt SK, Harris RS, Neuberger MS. 2002. AID mutates E. coli suggesting a DNA deamination mechanism for antibody diversification. Nature 418: 99-103.

Pone EJ, Xu Z, White CA, Zan H, Casali P. 2012. B cell TLRs and induction of immunoglobulin class-switch DNA recombination. Front Biosci (Landmark Ed) 17: 2594-2615.

Porter RR. 1973. Structural studies of immunoglobulins. Science 180: 713-716.

Ramsland PA, Farrugia W. 2002. Crystal structures of human antibodies: a detailed and unfinished tapestry of immunoglobulin gene products. Journal of molecular recognition : JMR 15: 248-259.

Ravetch JV, Kinet JP. 1991. Fc receptors. Annual review of immunology 9: 457-492. 
Reynaud CA, Anquez V, Dahan A, Weill JC. 1985. A single rearrangement event generates most of the chicken immunoglobulin light chain diversity. Cell 40: 283-291.

Reynaud CA, Anquez V, Grimal H, Weill JC. 1987. A hyperconversion mechanism generates the chicken light chain preimmune repertoire. Cell 48: 379-388.

Reynaud CA, Dahan A, Anquez V, Weill JC. 1989. Somatic hyperconversion diversifies the single Vh gene of the chicken with a high incidence in the D region. Cell 59: 171-183.

Reynaud CA, Garcia C, Hein WR, Weill JC. 1995. Hypermutation generating the sheep immunoglobulin repertoire is an antigen-independent process. Cell 80: 115-125.

Saini SS, Farrugia W, Muthusamy N, Ramsland PA, Kaushik AK. 2007. Structural evidence for a new IgG1 antibody sequence allele of cattle. Scandinavian journal of immunology 65: 32-38.

Saini SS, Farrugia W, Ramsland PA, Kaushik AK. 2003. Bovine IgM antibodies with exceptionally long complementarity-determining region 3 of the heavy chain share unique structural properties conferring restricted $\mathrm{VH}+$ Vlambda pairings. International immunology 15: 845-853.

Sakano H, Huppi K, Heinrich G, Tonegawa S. 1979. Sequences at the somatic recombination sites of immunoglobulin light-chain genes. Nature 280: 288-294.

Sitnikova T, Su C. 1998. Coevolution of immunoglobulin heavy- and light-chain variable-region gene families. Mol Biol Evol 15: 617-625.

Stavnezer J, Amemiya CT. 2004. Evolution of isotype switching. Seminars in immunology 16: 257-275.

Stavnezer J, Guikema JE, Schrader CE. 2008. Mechanism and regulation of class switch recombination. Annual review of immunology 26: 261-292.

Sun Y, Wang C, Wang Y, Zhang T, Ren L, Hu X, Zhang R, Meng Q, Guo Y, Fei J et al. 2010. A comprehensive analysis of germline and expressed immunoglobulin repertoire in the horse. Developmental and comparative immunology 34: 10091020.

Taussig MJ. 1988. Molecular genetics of immunoglobulins. Immunol Supp/ 1: 7-15.

Tiegs SL, Russell DM, Nemazee D. 1993. Receptor editing in self-reactive bone marrow B cells. The Journal of experimental medicine 177: 1009-1020.

Tonegawa S. 1983. Somatic generation of antibody diversity. Nature 302: 575-581.

Torres M, Fernandez-Fuentes N, Fiser A, Casadevall A. 2007. The immunoglobulin heavy chain constant region affects kinetic and thermodynamic parameters of antibody variable region interactions with antigen. The Journal of biological chemistry 282: 13917-13927.

Vettermann C, Schlissel MS. 2010. Allelic exclusion of immunoglobulin genes: models and mechanisms. Immunological reviews 237: 22-42.

Walther S, Czerny CP, Diesterbeck US. 2013. Exceptionally long CDR3H are not isotype restricted in bovine immunoglobulins. PloS one 8: e64234.

Wang M, Rada C, Neuberger MS. 2010. Altering the spectrum of immunoglobulin V gene somatic hypermutation by modifying the active site of AID. The Journal of experimental medicine 207: 141-153.

Wernery U. 2001. Camelid immunoglobulins and their importance for the new-born--a review. Journal of veterinary medicine $B$, Infectious diseases and veterinary public health 48: 561-568.

Woof JM, Burton DR. 2004. Human antibody-Fc receptor interactions illuminated by crystal structures. Nature reviews Immunology 4: 89-99.

Wu TT, Kabat EA. 1970. An analysis of the sequences of the variable regions of Bence Jones proteins and myeloma light chains and their implications for antibody complementarity. The Journal of experimental medicine 132: 211-250. 
Yamaguchi Y, Kim H, Kato K, Masuda K, Shimada I, Arata Y. 1995. Proteolytic fragmentation with high specificity of mouse immunoglobulin G. Mapping of proteolytic cleavage sites in the hinge region. Journal of immunological methods 181: 259-267. 


\section{Chapter 2:}

\section{Equine Immunoglobulins}

The nature and extent of my contribution to the work was the following:

1. Equine immunoglobulins and organization of immunoglobulin genes

\begin{tabular}{|c|l|}
\hline Nature of contribution & Extent of contribution \\
\hline 1. Scientific design & $70 \%$ \\
\hline 2. Laboratory work & - \\
\hline 3. Evaluation & $70 \%$ \\
\hline 4. Scientific Writing & $80 \%$ \\
\hline
\end{tabular}

2. Transcriptional analysis of equine $\lambda$ light chains in the horse breeds RhenishGerman Coldblood and Hanoverian Warmblood

\begin{tabular}{|c|l|}
\hline Nature of contribution & Extent of contribution \\
\hline 1. Scientific design & $50 \%$ \\
\hline 2. Laboratory work & - \\
\hline 3. Evaluation & $70 \%$ \\
\hline 4. Scientific Writing & $70 \%$ \\
\hline
\end{tabular}




\section{Equine immunoglobulins and organization of immunoglobulin genes}

Stefanie Walther ${ }^{a}$, Tamara V. Rusitzka ${ }^{a}$, Ulrike S. Diesterbeck ${ }^{b}$, Claus-Peter Czerny ${ }^{a *}$

aDepartment of Animal Sciences, Institute of Veterinary Medicine, Division of Microbiology and Animal Hygiene, Faculty of Agricultural Sciences, GeorgAugust University Göttingen, Burckhardtweg 2, 37077 Göttingen, Germany

bBuilding 33, 33 North Drive, Bethesda, MD 20892

${ }^{*}$ Corresponding author:

Claus-Peter Czerny

Tel.: +495513933375

Fax: +495513913513

E-mail: cczerny@gwdg.de

Postal address: Prof. Claus-Peter Czerny, DVM, PhD

Department of Animal Sciences,

Georg-August University Göttingen

Burckhardtweg 2

37077 Göttingen, Germany

Published in "Developmental and Comparative Immunology"

DOI: 10.1016/j.dci.2015.07.017

URL: http://www.sciencedirect.com/science/article/pii/S0145305X15300227 


\section{Abstract}

Our understanding of how equine immunoglobulin genes are organized has increased significantly in recent years. For equine heavy chains, 52 IGHV, 40 IGHD, 8 IGHJ and 11 IGHC are present. Seven of these IGHCs are gamma chain genes. Sequence diversity is increasing between fetal, neonatal, foal and adult age. The kappa light chain contains 60 IGKV, 5 IGKJ and 1 IGKC, whereas there are 144 IGLV, 7 IGLJ, and 7 IGLC for the lambda light chain, which is expressed predominantly in horses. Significant transcriptional differences for IGLV and IGLC are identified in different breeds. Allotypic and allelic variants are observed for IGLC1, IGLC5, and IGLC6/7, and two IGLV pseudogenes are also transcribed. During age development, a decrease in IGLVs is noted, although nucleotide diversity and significant differences in gene usage increased. The following paper suggests a standardization of the existing nomenclature of immunoglobulin genes.

Keywords: horse, immunoglobulin genes, immunoglobulin heavy and light chains, allotype, diversity, equine developmental stages 
Chapter 2: Equine immunoglobulins and organization of immunoglobulin genes

\section{Introduction}

Equine immunoglobulins have played a key role throughout the history of human and veterinary immunology. Since the $19^{\text {th }}$ century, horses have been important blood donors for serum therapy in heterospecific hosts. In the early $20^{\text {th }}$ century, common horse serum or serum from immunized horses was produced, especially for the treatment and prophylaxis of diphtheria in humans (Bingel, 1918; Daniels, 1921). Later, horses assisted with the production of sera not only for the treatment of diphtheria but also for the treatment of other human infectious diseases such as tuberculosis, tetanus, and pneumonia (Behring von, 1918; Cole and Moore, 1917; GlatmanFreedman and Casadevall, 1998; Winau and Winau, 2002). Albert Calmette employed this method to produce antivenoms against poisonous bites from snakes and spiders (Calmette, 1896; Hawgood, 1999). Today, equine immunoglobulins are also used to support immunosuppression after organ or stem cell transplantation, or to manage autoimmune diseases in humans (Leleu et al., 2006; Zand, 2006). However, repeated systemic injections of hyperimmune sera or polyclonal and monoclonal antibodies from different species resulted in serum sickness and therefore, were not a feasible option for repeated therapy (Lang et al., 2000; Theakston et al., 2003). Early studies on the structure and function of equine immunoglobulins have already generated a considerable amount of data on the characterization of equine immunoglobulin genes and their genomic organization (Helms and Allen, 1970; Hill and Cebra, 1965; Pahud and Mach, 1972; Rockey, 1967; Sandor et al., 1964a; Vaerman et al., 1971; Wagner, 2006; Weir et al., 1966; Zolla and Goodman, 1968). As is known for humans and mice (Edelman, 1973), and nearly all jawed vertebrates, equine immunoglobulins are heterotetramers with two identical heavy and light chains. Both of them can be divided functionally and genetically into a variable region and a constant region. The variable regions are created by the random fusion of germline variable (V), diversity (D), and joining gene segments $(\mathrm{J})$ that are combined with a constant region gene (Tonegawa, 1983), which are found in species-specific numbers in the genome. The immunoglobulin diversity depends on several processes of combinatorial and junctional diversity due to the imprecise joining of the single gene segments and non-templated or palindromic nucleotide insertions between two adjacent gene segments, as well as somatic hypermutations. In addition, several secondary mechanisms such as gene conversion and isotype switch may increase diversity further. The resulting variable regions of the heavy and light chains together are responsible for antigen binding. 
Highly specialized, complementary determining regions form a perfect counterpart of the antigen epitope and are stabilized by conserved framework regions (Kabat and Wu, 1991). The repertoire of immunoglobulins in one individual is immense because they are produced by B-lymphocytes and plasma cells and may be membrane-bound (B-cell receptors) or secreted proteins (antibodies), which additionally become diversified. In B-lymphocytes, the heavy chain rearrangement precedes the rearrangement of the light chains (Alt et al., 1981). In mammals there are two types of light chains - the lambda and kappa light chains - which are expressed in species-specific ratios. In contrast to humans and mice, where the kappa isotype dominates in serum antibodies, the lambda isotype is predominantly found in cattle and horses (Almagro et al., 1998; Arun et al., 1996). In addition to what is already known about the general structural features of equine immunoglobulins and their function, most recent studies on diversity provide the scientific basis for the production of highly specific and effective recombinant antibodies or antibody libraries. 


\section{Immunoglobulins in equine offspring}

Evidently, as early as in the equine fetus, an initial B-cell repertoire is developed despite the lack of exogenous antigenic stimulation (Tallmadge et al., 2009). Corresponding antibodies such as $\operatorname{lgM}$, IgG1, and IgG4/7 are detectable on a limited scale at birth. Nevertheless, newborn foals are immunocompetent but do not possess an effective humoral immunity to infections and, therefore, depend on the absorption of maternal colostral immunoglobulins by specialized cells lining the small intestine (Jenvey et al., 2012). During the first $6 \mathrm{~h}$ after birth, absorption is highest and decreases gradually within $24 \mathrm{~h}$ (Franz et al., 1998). After this time, absorption of antibodies is no longer possible (McGuire and Crawford, 1973) leading to the rapid decrease of $\lg A$ and $\lg G$ levels in the mare's milk for the first days after parturition. Failure of passive transfer (FPT) - meaning the insufficient transfer of immunoglobulins via the mare's colostrum in the first 12-24 $\mathrm{h}$ after birth - results in a considerably higher risk of sepsis, bacteremia and localized infections (Haas et al., 1996; Koterba et al., 1984). Both the foal and the dam may suffer from FPT, which could be attributed to poor colostrum quality, lack of colostrum ingestion, poor intestinal absorption or a combination of these factors (Drogoul et al., 2008). At least $60 \mathrm{~g}$ of $\mathrm{lg} / \mathrm{l}$ are regarded as a sufficient quality colostrum (Drogoul et al., 2008). Nevertheless $23 \%$ to $32 \%$ of mares produce colostrum of low qualities (LeBlanc et al., 1992). With up to $70 \%$, IgG is the main isotype present (Turtinen and Allen, 1982). An average of about $100 \mathrm{~g} \mathrm{lgG}$ is secreted per lactation. Furthermore, colostrum comprises $20 \% \operatorname{lgA}$ that shows limited absorption but has a local protective local function within the digestive tract of newborn foals (Sedlinska et al., 2006). Similarly, horse type and breed are known to have an influence on the overall Ig concentration. Arabian and Quarter Horse mares show better colostrum qualities than Thoroughbred and Standardbred (Leblanc and Tran, 1987). Six hours after delivery, Haflinger exhibit higher whey protein amounts than Arabian and Trotter, but these quantities decrease more rapidly (Civardi et al., 2002). Age and rank of lactation, nutrition and body condition of mares, as well as vaccination programs, season, and temperature may be further variables concerning colostrum quality (Drogoul et al., 2008).

After maternal colostrum antibodies disappear and the appropriate antigenic stimulation is provided, the active antibody synthesis begins in the foal. The immune system starts the production of IgM followed by the other Ig classes (Wagner, 2006). 
Chapter 2: Equine immunoglobulins and organization of immunoglobulin genes

Endogenous $\lg G$ and $\lg A$ synthesis begins within the first four weeks of life. Stable levels were reached by 8 weeks of age $(\lg G a), 12$ weeks of age $(\lg G(T)$ and $\lg A)$, and 51 weeks of age (lgGb) (Holznagel et al., 2003).

\section{The equine immunoglobulin heavy chain gene locus}

In silico analyses identified the heavy chain locus on two unplaced contigs, which are called Un0011 and Un0038 (Fig. 1a and b, Tables 1-3). The nomenclature used in previous studies to denominate Ig heavy and light chain gene segments varied, although they conformed to current definitions by the international ImMunoGeneTics (IMGT) information system, as well as to the WHO-IUIS Nomenclature Subcommittee for immunoglobulins and T-cell receptors (Hara et al., 2012; Lefranc, 2001b, 2007; Sun et al., 2010; Tallmadge et al., 2014; Tallmadge et al., 2013). Nevertheless, in the designation system (Tallmadge et al., 2014) used most recently, pseudogenes and open reading frames are not indicated precisely. This led us to propose a supplemented taxonomical designation for all known lg heavy and light chain genes investigated in the most recent studies (Sun et al., 2010; Tallmadge et al., 2013; Wagner et al., 2006), which is shown in Tables 1-10 and in Figs 1-3. The gene segments IGHV/IGLV/IGKV, IGHD, IGHJ/IGLJ/IGKJ, IGHC, IGLC, and IGKC (without superscript letters) are potentially functional variable gene segments. Superscript ORF was used to indicate variable gene segments with open reading frames that have either a defect in splicing sites, recombination signal sequence (RSS) and/or regulatory elements, and/or changes to the conserved amino acids, and therefore have been suggested to lead to incorrect folding (Lefranc, 1998). Superscript $P$ indicates pseudovariable gene segments. The genes were named according to the subgroup they belong to (Sun et al., 2010) and their number within this subgroup. The former ' $\mathrm{VH} 1$ ' was renamed 'IGHV1S1' to designate sequence 1 of subgroup 1 . Based on $>75 \%$ nucleotide identity 28 subgroups were established for the 40 IGHD genes and 2 subgroups were established for the 8 IGHJ genes. The classification of variable genes followed previous research. In Sun et al. (2010) and Tallmadge et al. (2013) sequences with at least $75 \%$ identity were grouped to the same family (Giudicelli and Lefranc, 1999). Their genes were named accordingly (Tallmadge et al., 2013). However, for future analyses we suggest to use $80 \%$ nucleotide identity as already recommended in 1984 for mouse immunoglobulin genes (Brodeur and Riblet, 1984). The IGHC 
nomenclature also conforms to IMGT. Positions identified on several contigs are listed as well. The contigs are Un0011/NW_001876796, *Un0388/NW_001871527,

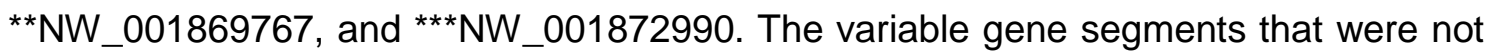
classified into subgroups because they were too divergent or truncated are marked with n.c. (Sun et al., 2010).

(A) Un0011

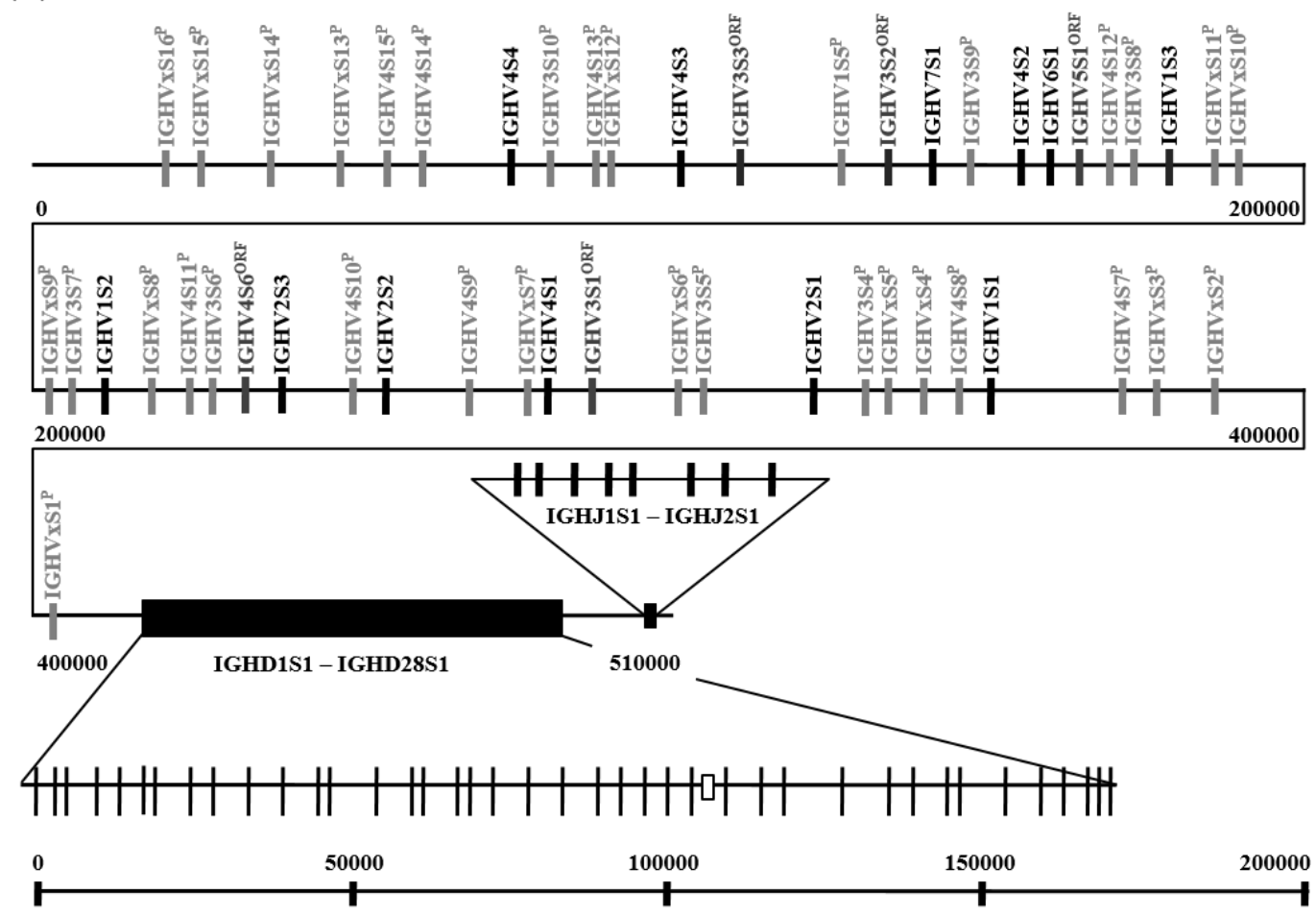

(B) Un0388

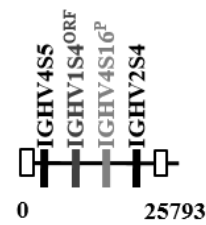

(C) Map of the equine IGHC region

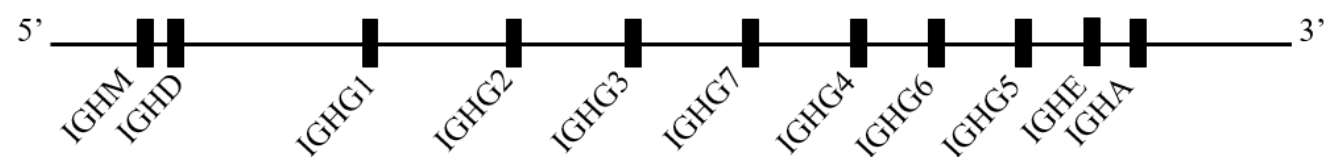


Fig. 1: Map of the equine heavy chain gene segments

(A) Within the scaffold Un0011 (NW_001876796), IGHV, IGHD and IGHJ gene clusters span a $510 \mathrm{~kb}$ region. (B) Scaffold Un0388 (NW_001871527) contains two potentially functional IGHVs (IGHV4S5 and IGHV2S4, according to the supposed designation), one IGHV ORF (IGHV1S4ORF) and one IGHV $\Psi$ (IGHV4S16P). (C) Map of the IGHC region of the horse, indicating the order of the eleven IGHC genes (adapted from Wagner et al., 2004). Boxes indicate IGHC genes. This map is adapted from Wagner et al. (2004) and Sun et al. (2010) to ensure that the newly proposed nomenclature (Tables 1-4) is designated to the respective positions on the locus. IGHV without superscript letters are potentially functional variable gene segments. Superscript ORF indicates variable gene segments with open reading frames that either have a defect in splicing sites, RSS and/or regulatory elements, and/or changes to the conserved amino acids, and therefore have been suggested to lead to incorrect folding (Lefranc, 1998). Superscript $P$ indicates pseudo-variable gene segments.

The heavy chain contig Un0011 contains 50 variable gene segments (IGHV), 40 diversity gene segments (IGHD), as well as eight joining gene segments (IGHJ). Twelve out of 50 IGHV were described as functional, whereas 33 IGHV were defined as pseudogenes. Five open reading frames (ORF) were also described by Sun and coworkers. Two additional functional IGHV, as well as one ORF and one pseudogene were identified on Un0038 (Sun et al., 2010). All IGHV, IGHJ, and IGHC show the same transcriptional orientation (Fig. 1a, b). Most of the IGHV segments are flanked by 23 bp-spaced RSSs at their 3'ends, except IGHV7S1, IGHVxS1 ${ }^{\mathrm{P}}, \mathrm{IGHV} \mathrm{IS}^{\mathrm{P}}$, IGHVxS7P, IGHVxS8 ${ }^{P}, I G H V 3 S 7^{P}$, and IGHV1S5P, which either lack the nonamer or carry spacers shorter than $23 \mathrm{bp}$. The segments IGHV1S6 and IGHV4S17 were identified on the unplaced contigs NW_001869767 and NW_001872990, respectively (Table 1) (Tallmadge et al., 2013). IGHJ2S1 and IGHJ1S4 have 22 bp-spaced RSSs at their 5'ends. The remaining 6 IGHJs show 23 bp-spaced RSSs. All the IGHDs have 12 bp-spaced RSSs on both sides (Sun et al., 2010). With 40 IGHD identified, horses belong the mammalian species that possess the most IGHD. For instance, in guinea pig and the African elephant 41 and 87 IGHD gene segments were identified so far (Guo et al., 2011; Guo et al., 2012).

The equine immunoglobulin heavy chain constant region gene locus was localized on chromosome 24 (ECA24qter) and comprises 11 genes. All five isotypes known from humans were also identified in horses (Fig. 1c, Table 4). The entire equine IGHC region is located on a minimum of two overlapping clones from the CHORI-241 Horse Bacterial Artificial Chromosome library, suggesting that the size of the IGHC region is 250-350 kb (Wagner et al., 2004). 
Chapter 2: Equine immunoglobulins and organization of immunoglobulin genes

Table 1: Nomenclature of the equine IGHV gene segments

\begin{tabular}{|c|c|c|c|c|}
\hline $\begin{array}{l}\text { IGHV } \\
\text { subgroup }\end{array}$ & $\begin{array}{l}\text { Designation } \\
\text { Sun et al. } 2010\end{array}$ & $\begin{array}{l}\text { Designation } \\
\text { Tallmadge et al. } \\
2013\end{array}$ & $\begin{array}{l}\text { Proposed } \\
\text { designation }\end{array}$ & $\begin{array}{l}\text { Genome } \\
\text { Position }\end{array}$ \\
\hline 1 & $\mathrm{VH} 1$ & IGHV1S1 & IGHV1S1 & $349128-349423$ \\
\hline 1 & VH6 & IGHV1S2 & IGHV1S2 & $211197-211492$ \\
\hline 1 & $\mathrm{VH} 7$ & IGHV1S3 & IGHV1S3 & $175891-176186$ \\
\hline 1 & VHORF6 & IGHV1S4 & IGHV1S4 ${ }^{\mathrm{ORF}}$ & $7876-8169^{\star}$ \\
\hline 1 & pVH24 & IGHV1S5 & IGHV1S5 ${ }^{P}$ & $126998-127283$ \\
\hline 1 & Novel Vha & IGHV1S6 & IGHV1S6 & $38420-38913^{\star *}$ \\
\hline 2 & $\mathrm{VH} 2$ & IGHV2S1 & IGHV2S1 & $322988-323280$ \\
\hline 2 & VH4 & IGHV2S2 & IGHV2S2 & $254382-254674$ \\
\hline 2 & VH5 & IGHV2S3 & IGHV2S3 & $237777-238067$ \\
\hline 2 & $\mathrm{VH} 14$ & IGHV2S4 & IGHV2S4 & $19736-20028^{*}$ \\
\hline 3 & VHORF1 & IGHV3S1 & IGHV3S1 $1^{\mathrm{ORF}}$ & 283617-283909 \\
\hline 3 & VHORF4 & IGHV3S2 & IGHV3S2 ${ }^{\mathrm{ORF}}$ & $132488-132804$ \\
\hline 3 & VHORF5 & IGHV3S3 & IGHV3S3 ORF & $110030-110316$ \\
\hline 3 & $\mathrm{pVH} 8$ & IGHV3S4 & IGHV3S4 $4^{P}$ & 330696-330991 \\
\hline 3 & pVH9 & IGHV3S5 & IGHV3S5 ${ }^{P}$ & $303932-304217$ \\
\hline 3 & $\mathrm{pVH} 14$ & IGHV3S6 & IGHV3S6 ${ }^{P}$ & $227389-227680$ \\
\hline 3 & pVH17 & IGHV3S7 & IGHV3S7P & $203930-204210$ \\
\hline 3 & $\mathrm{pVH} 21$ & IGHV3S8 & IGHV3S8P & $172321-172616$ \\
\hline 3 & $\mathrm{pVH} 23$ & IGHV3S9 & IGHV3S9P & $147144-147434$ \\
\hline 3 & $\mathrm{pVH} 27$ & IGHV3S10 & IGHV3S10 & $79642-79940$ \\
\hline 4 & VH3 & IGHV4S1 & IGHV4S1 & $280198-280496$ \\
\hline 4 & VH9 & IGHV4S2 & IGHV4S2 & $156258-156556$ \\
\hline 4 & VH11 & IGHV4S3 & IGHV4S3 & $100632-100927$ \\
\hline 4 & $\mathrm{VH} 12$ & IGHV4S4 & IGHV4S4 & $75777-76072$ \\
\hline 4 & $\mathrm{VH} 13$ & IGHV4S5 & IGHV4S5 & $4967-5265^{\star}$ \\
\hline 4 & VHORF2 & IGHV4S6 & IGHV4S6 $6^{\mathrm{ORF}}$ & $232063-232361$ \\
\hline 4 & $\mathrm{pVH} 4$ & IGHV4S7 & IGHV4S7P & $370921-371206$ \\
\hline 4 & pVH5 & IGHV4S8 & IGHV4S8 ${ }^{P}$ & $344891-345187$ \\
\hline 4 & $\mathrm{pVH} 12$ & IGHV4S9 & IGHV4S9P & 266901-267198 \\
\hline 4 & $\mathrm{pVH} 13$ & IGHV4S10 & IGHV4S $10^{P}$ & 248659-248968 \\
\hline 4 & $\mathrm{pVH} 15$ & IGHV4S11 & IGHV4S $11^{\mathrm{P}}$ & $223950-224266$ \\
\hline 4 & $\mathrm{pVH} 22$ & IGHV4S12 & IGHV4S12P & $169462-169754$ \\
\hline 4 & pVH26 & IGHV4S13 & IGHV4S13P & 87064-87357 \\
\hline 4 & $\mathrm{pVH} 28$ & IGHV4S14 & IGHV4S14 & 59569-59865 \\
\hline 4 & pVH29 & IGHV4S15 & IGHV4S15 & $54540-54838$ \\
\hline 4 & $\mathrm{pVH} 34$ & IGHV4S16 & IGHV4S16 & $13567-13866^{*}$ \\
\hline 4 & Novel VH & IGHV4S17 & IGHV4S17 & $11760-12258^{* \star *}$ \\
\hline 5 & VHORF3 & IGHV5S1 & IGHV5S1 $1^{\mathrm{ORF}}$ & $165239-165534$ \\
\hline 6 & VH8 & IGHV6S1 & IGHV6S1 & $159437-159729$ \\
\hline
\end{tabular}


Chapter 2: Equine immunoglobulins and organization of immunoglobulin genes

\begin{tabular}{|c|c|c|c|c|}
\hline $\begin{array}{l}\text { IGHV } \\
\text { subgroup }\end{array}$ & $\begin{array}{l}\text { Designation } \\
\text { Sun et al. } 2010\end{array}$ & $\begin{array}{l}\text { Designation } \\
\text { Tallmadge et al. } \\
2013\end{array}$ & $\begin{array}{l}\text { Proposed } \\
\text { designation }\end{array}$ & $\begin{array}{l}\text { Genome } \\
\text { Position }\end{array}$ \\
\hline 7 & VH10 & IGHV7S1 & IGHV7S1 & $141209-141509$ \\
\hline n.c. & $\mathrm{pVH} 1$ & & $I G H V x S 1^{P}$ & $400324-400623$ \\
\hline n.c. & $\mathrm{pVH} 2$ & & $I G H V x S 2^{P}$ & $385845-386119$ \\
\hline n.c. & pVH3 & & $I G H V x S 3^{P}$ & $375741-376041$ \\
\hline n.c. & pVH6 & & $I G H V x S 4^{P}$ & $339915-340211$ \\
\hline n.c. & $\mathrm{pVH} 7$ & & $I G H V x S 5^{P}$ & $333740-334025$ \\
\hline n.c. & $\mathrm{pVH} 10$ & & $1 G H V x S 6^{P}$ & $301302-301579$ \\
\hline n.c. & $\mathrm{pVH} 11$ & & $I G H V x S 7^{P}$ & $277258-277526$ \\
\hline n.c. & $\mathrm{pVH} 16$ & & $I G H V x S 8^{P}$ & $217225-217491$ \\
\hline n.c. & $\mathrm{pVH} 18$ & & $I G H V x S 9^{P}$ & $200550-200817$ \\
\hline n.c. & pVH19 & & IGHVxS10 & $188124-188419$ \\
\hline n.c. & $\mathrm{pVH} 20$ & & $\mathrm{IGHV} \times \mathrm{S} 11^{\mathrm{P}}$ & $185173-185466$ \\
\hline n.c. & pVH25 & & $\mathrm{IGHVxS} 12^{\mathrm{P}}$ & $89740-89991$ \\
\hline n.c. & $\mathrm{pVH} 30$ & & $\mathrm{IGHV} x \mathrm{~S} 13^{\mathrm{P}}$ & $47308-47605$ \\
\hline n.c. & $\mathrm{pVH} 31$ & & IGHVxS14P & $35699-35979$ \\
\hline n.c. & $\mathrm{pVH} 32$ & & IGHVxS15 & $24565-24859$ \\
\hline n.c. & $\mathrm{pVH} 33$ & & IGHVxS16 ${ }^{P}$ & 19976-20184 \\
\hline
\end{tabular}

Un0011 NW_001876796

*Un0388=NW__001871527

**NW 001869767

${ }^{* * *} \mathrm{NW}$-001872990 
Chapter 2: Equine immunoglobulins and organization of immunoglobulin genes

Table 2: Nomenclature of the equine IGHD gene segments

\begin{tabular}{|c|c|c|c|c|}
\hline $\begin{array}{l}\text { IGHD } \\
\text { subgroup }\end{array}$ & $\begin{array}{l}\text { Designation } \\
\text { Sun et al. } 2010\end{array}$ & $\begin{array}{l}\text { Designation } \\
\text { Tallmadge et al. } \\
2013\end{array}$ & $\begin{array}{l}\text { Proposed } \\
\text { designation }\end{array}$ & $\begin{array}{l}\text { Genome } \\
\text { Position }\end{array}$ \\
\hline 1 & $\mathrm{DH} 1$ & IGHD1S1 & IGHD1S1 & $415757-415787$ \\
\hline 2 & $\mathrm{DH} 2$ & IGHD2S1 & IGHD2S1 & $417275-417293$ \\
\hline 3 & $\mathrm{DH} 3$ & IGDH3S1 & IGDH3S1 & $417450-417497$ \\
\hline 4 & $\mathrm{DH} 4$ & IGHD4S1 & IGHD4S1 & $419955-419986$ \\
\hline 5 & $\mathrm{DH} 5$ & IGHD5S1 & IGHD5S1 & $422080-422110$ \\
\hline 5 & $\mathrm{DH} 10$ & IGHD5S2 & IGHD5S2 & $430784-430814$ \\
\hline 5 & $\mathrm{DH} 15$ & IGHD5S3 & IGHD5S3 & $441806-441836$ \\
\hline 5 & $\mathrm{DH} 21$ & IGHD5S4 & IGHD5S4 & $453012-453042$ \\
\hline 5 & $\mathrm{DH} 25$ & IGHD5S5 & IGHD5S5 & $459989-460019$ \\
\hline 5 & $\mathrm{DH} 28$ & IGHD5S6 & IGHD5S6 & $466318-466348$ \\
\hline 6 & $\mathrm{DH} 6$ & IGHD6S1 & IGHD6S1 & $423268-423286$ \\
\hline 7 & $\mathrm{DH} 7$ & IGHD7S1 & IGHD7S1 & $423733-423761$ \\
\hline 7 & $\mathrm{DH} 17$ & IGHD7S2 & IGHD7S2 & $445696-445724$ \\
\hline 8 & $\mathrm{DH} 8$ & IGHD8S1 & IGHD8S1 & $426759-426777$ \\
\hline 8 & $\mathrm{DH} 16$ & IGHD8S2 & IGHD8S2 & $443012-443030$ \\
\hline 8 & $\mathrm{DH} 19$ & IGHD8S3 & IGHD8S3 & $447486-447504$ \\
\hline 9 & $\mathrm{DH} 9$ & IGHD9S1 & IGHD9S1 & $428333-428363$ \\
\hline 10 & $\mathrm{DH} 11$ & IGHD10S1 & IGHD10S1 & $433564-433589$ \\
\hline 11 & $\mathrm{DH} 12$ & IGHD11S1 & IGHD11S1 & $435422-435455$ \\
\hline 12 & $\mathrm{DH} 13$ & IGHD12S1 & IGHD12S1 & $436872-436890$ \\
\hline 12 & $\mathrm{DH} 37$ & IGHD12S2 & IGHD12S2 & $486726-486744$ \\
\hline 13 & DH14 & IGHD13S1 & IGHD13S1 & $439594-439631$ \\
\hline 14 & $\mathrm{DH} 18$ & IGHD14S1 & IGHD14S1 & $446390-446423$ \\
\hline 15 & $\mathrm{DH} 20$ & IGHD15S1 & IGHD15S1 & $450401-450433$ \\
\hline 15 & $\mathrm{DH} 27$ & IGHD15S2 & IGHD15S2 & $463956-463987$ \\
\hline 16 & $\mathrm{DH} 22$ & IGHD16S1 & IGHD16S1 & $454814-454843$ \\
\hline 17 & $\mathrm{DH} 23$ & IGHD17S1 & IGHD17S1 & $456420-456444$ \\
\hline 17 & $\mathrm{DH} 26$ & IGHD17S2 & IGHD17S2 & $461177-461201$ \\
\hline 18 & $\mathrm{DH} 24$ & IGHD18S1 & IGHD18S1 & $457832-457861$ \\
\hline 19 & $\mathrm{DH} 29$ & IGHD19S1 & IGHD19S1 & $467519-467540$ \\
\hline 20 & $\mathrm{DH} 30$ & IGHD20S1 & IGHD20S1 & $471601-471618$ \\
\hline 20 & $\mathrm{DH} 40$ & IGHD20S2 & IGHD20S2 & $490706-490721$ \\
\hline 21 & $\mathrm{DH} 31$ & IGHD21S1 & IGHD21S1 & $475562-475586$ \\
\hline 22 & $\mathrm{DH} 32$ & IGHD22S1 & IGHD22S1 & $477123-477153$ \\
\hline 23 & $\mathrm{DH} 33$ & IGHD23S1 & IGHD23S1 & $478925-478957$ \\
\hline 24 & DH34 & IGHD24S1 & IGHD24S1 & $480174-480192$ \\
\hline 25 & DH35 & IGHD25S1 & IGHD25S1 & $483056-483084$ \\
\hline 26 & $\mathrm{DH} 36$ & IGHD26S1 & IGHD26S1 & $485482-485512$ \\
\hline
\end{tabular}


Chapter 2: Equine immunoglobulins and organization of immunoglobulin genes

\begin{tabular}{lllll}
\hline $\begin{array}{l}\text { IGHD } \\
\text { subgroup }\end{array}$ & $\begin{array}{l}\text { Designation } \\
\text { Sun et al. 2010 }\end{array}$ & $\begin{array}{l}\text { Designation } \\
\text { Tallmadge et al. } \\
\mathbf{2 0 1 3}\end{array}$ & $\begin{array}{l}\text { Proposed } \\
\text { designation }\end{array}$ & $\begin{array}{l}\text { Genome } \\
\text { Position }\end{array}$ \\
\hline 27 & DH38 & IGHD27S1 & IGHD27S1 & $489459-489477$ \\
28 & DH39 & IGHD28S1 & IGHD28S1 & $489958-489978$ \\
\hline Un0011 NW_001876796 & & &
\end{tabular}

Table 3: Nomenclature of the equine IGHJ gene segments

\begin{tabular}{lllll}
\hline $\begin{array}{l}\text { IGHJ } \\
\text { subgroup }\end{array}$ & $\begin{array}{l}\text { Designation } \\
\text { Sun et al. 2010 }\end{array}$ & $\begin{array}{l}\text { Designation } \\
\text { Tallmadge et al. } \\
\mathbf{2 0 1 3}\end{array}$ & $\begin{array}{l}\text { Proposed } \\
\text { designation }\end{array}$ & $\begin{array}{l}\text { Genome } \\
\text { Position }\end{array}$ \\
\hline $\mathbf{1}$ & JH1 & IGHJ1S1 & IGHJ1S1 & $504144-504197$ \\
$\mathbf{1}$ & $\mathrm{JH3}$ & IGHJ1S2 & IGHJ1S2 & $504660-504713$ \\
$\mathbf{1}$ & $\mathrm{JH} 4$ & IGHJ1S3 & IGHJ1S3 & $505003-505050$ \\
$\mathbf{1}$ & $\mathrm{JH} 5$ & IGHJ1S4 & IGHJ1S4 & $505280-505330$ \\
$\mathbf{1}$ & $\mathrm{JH} 6$ & IGHJ1S5 & IGHJ1S5 & $505855-505908$ \\
$\mathbf{1}$ & $\mathrm{JH} 7$ & IGHJ1S6 & IGHJ1S6 & $506275-506331$ \\
$\mathbf{1}$ & $\mathrm{JH} 8$ & IGHJ1S7 & IGHJ1S7 & $506729-506782$ \\
$\mathbf{2}$ & $\mathrm{JH} 2$ & IGHJ2S1 & IGHJ2S1 & $504350-504402$ \\
\hline
\end{tabular}


Chapter 2: Equine immunoglobulins and organization of immunoglobulin genes

Table 4: Nomenclature of the equine IGHC genes

\begin{tabular}{|c|c|c|c|c|}
\hline $\begin{array}{l}\text { Original } \\
\text { nomen-clature }\end{array}$ & $\begin{array}{l}\text { Current } \\
\text { desig-nation }\end{array}$ & Gene & Allel & $\begin{array}{l}\text { Genome Position } \\
\text { on NW_001876796 }\end{array}$ \\
\hline \multirow[t]{4}{*}{$\lg M$} & $\lg M$ & IGHM & M_02 & $519420-524201$ \\
\hline & & & & $514993-518108$ \\
\hline & & & M_01 & $516522-517025$ \\
\hline & & & & $515374-515717$ \\
\hline \multirow[t]{2}{*}{$\lg D$} & $\lg \mathrm{D}$ & IGHD & & $531198-534510$ \\
\hline & & & & $526129-527334$ \\
\hline \multirow[t]{6}{*}{ IgGa } & $\lg G 1$ & IGHG1 & & $576506-576800$ \\
\hline & & & & $577273-577607$ \\
\hline & & & & $577685-578019$ \\
\hline & & & G1_01 & $576506-578019$ \\
\hline & $\lg G 2$ & IGHG2 & & $608720-610787$ \\
\hline & & & & $625782-627849$ \\
\hline \multirow[t]{6}{*}{$\lg G(T)$} & $\lg G 3$ & IGHG3 & G3_01 & $651171-651465$ \\
\hline & & & & $651958-652292$ \\
\hline & & & & $652361-652695$ \\
\hline & & & G3_02 & $650866-653171$ \\
\hline & $\lg G 7$ & IGHG7 & G7_01 & 674467-676688 \\
\hline & & & G7_02 & $674945-676422$ \\
\hline \multirow[t]{3}{*}{$\operatorname{lgGb}$} & $\operatorname{lgG} 4$ & IGHG4 & G4_02 & 674954-676422 \\
\hline & & & & $651171-651701$ \\
\hline & & & G4_01 & $674827-676691$ \\
\hline \multirow[t]{2}{*}{ IgGc } & $\lg G 6$ & IGHG6 & & 698724-700204 \\
\hline & & & & $651171-651723$ \\
\hline $\lg G(T)$ & $\lg G 5$ & IGHG5 & & $730626-732347$ \\
\hline \multicolumn{5}{|l|}{ IgGB } \\
\hline \multirow[t]{11}{*}{$\lg E$} & $\lg E$ & IGHE & E_01 & $754864-756464$ \\
\hline & & & E_02 & $756129-756464$ \\
\hline & & & & $755315-755639$ \\
\hline & & & & $755732-756058$ \\
\hline & & & & $754864-755155$ \\
\hline & & & E_03 & $756129-756585$ \\
\hline & & & & $755732-756058$ \\
\hline & & & E_04 & $756129-756551$ \\
\hline & & & & $755732-756058$ \\
\hline & & & & $755315-755639$ \\
\hline & & & & $754858-755155$ \\
\hline \multirow[t]{2}{*}{$\lg A$} & $\lg A$ & IGHA & & $766888-768383$ \\
\hline & & & & $768662-769379$ \\
\hline
\end{tabular}




\section{1. $\lg M$}

$\lg \mathrm{M}$ is the first isotype that appears phylogenetically, as well as ontogenetically. It is the first immunoglobulin produced by fetuses. This isotype is responsible for the primary immune response. IgM may be bound to the membrane as a monomer or secreted as a pentameric molecule with high avidity. $\lg M$, as well as $\lg G$, is present in the plasma (Tallmadge et al., 2009).

The equine IGHM locus spans 1472 bp coding for 451 amino acid residues (Fig. 1c, Table 4). This isotype exhibits six potential N-glycosylation sites. Similar to IgE, no separated hinge region was identified. To enable flexibility of this istopye, the second constant region may act as hinge. The third and fourth constant regions of $\lg M$ are conserved even between different species, which indicates a specific role of $\operatorname{lgM}$ in complement activation (Schrenzel et al., 1997).

\section{2. $\lg D$}

Although the importance of this isotype remains unclear and a receptor-like function is supposed, constant region genes coding for $\lg D$ are not restricted to primates and rodents. After successful description of the $\lg D$ isotype in cattle, sheep and swine, $\lg D$ was also identified in horses (Wagner et al., 2004; Zhao et al., 2002). The equine IgD locus was identified $5 \mathrm{~kb}$ downstream of the genes coding for IgM (Fig. 1c, Table 4). The entire genomic locus spans about $9.1 \mathrm{~kb}$ and contains eight exons that are: $\mathrm{CH} 1$, two exons coding for the hinge region, $\mathrm{CH} 2, \mathrm{CH} 3$, one exon for the secreted molecule, as well as two exons for the transmembrane anchor; the first of which contains a specific splicing site. For $\lg D$, the secreted form consists of 391 amino acid residues and the transmembrane form of 418 amino acid residues. The coding nucleotide sequences of $\mathrm{CH} 1$ to $\mathrm{CH} 3$ exons show sequence identities of $64 \%$ to human and porcine $\lg D$ genes and $61 \%$ to bovine and ovine $\lg D$ genes. In analogy to the human $\lg \mathrm{D}$, there is no switch-region between the genes coding for $\lg \mathrm{M}$ and $\lg \mathrm{D}$, indicating that there is an alternative splice mechanism (Wagner et al., 2004). In contrast, a c-switch region has only been identified for cattle and pig (Zhao et al., 2002; Zhao et al., 2003). 
3.3. $\lg G$

The $\mathrm{IgG}$ isotype is considered to be the most versatile immunoglobulin in all mammals and birds. Only in the porcine immune system has a similarly high number of eleven genomic constant region genes representing six putative subclasses been described (Fig. 1c, Table 4) (Butler et al., 2009). IgG performs all functions of a typical immunoglobulin molecule such as antigen binding, complement fixation, and binding to various cells like phagocytic cells, lymphocytes, platelets, mast cells, and basophils. It contributes about $75 \%$ of the antibodies. The isotype was defined at a biochemical and serological level and the different genomic elements were characterized. The subclasses $\lg G a, \lg G b$, $\lg G$ c, $\lg G(T)$, and $\lg G(B)$ were classified (Klinman et al., 1965; Rockey, 1967; Rockey et al., 1964; Sandor et al., 1964b; Sheoran and Holmes, 1996; Widders et al., 1986) according to their antigenic differences and their serological and electrophoretic properties. Restriction enzyme analyses of the genomic DNA of horse lambda phage clones, deletion analyses of heterohybridomas, and nucleotide sequencing of individual IGHC genes revealed six constant region genes in the equine haploid genome (Overesch et al., 1998; Wagner et al., 2006). The characterization of the complete IGHC region and the analysis of 34 overlapping genomic clones from the CHORI-241 BAC library revealed seven constant region genes of equine IgG (Table 4) and constituted the current repertoire of IgG IGHCs based on their sequences, phylogeny, and homologous structures (Wagner et al., 2002; Wagner et al., 2004). Multiple duplications, gene conversions, and crossovers are supposed to explain these seven IgG isotypes, which are unique in all species investigated. IgG4 and IgG7 seem to be the latest duplication events in evolution as their sequences show $96 \%$ identity. As available reagents are insufficient for differentiation, isotypes lgG2 and IgG7 could not yet be diversified in serum and other body fluids (Wagner et al., 2006). The individual subclasses possess specific roles in protective immunity. Thus, the FcyR and complement-binding isotypes IgG1, IgG4, and IgG7 (former IgGa and lgGb) contribute to the protection against several equine pathogens such as equine influenza virus (Breathnach et al., 2006; Nelson et al., 1998), Streptococcus equi (Sheoran et al., 1997) and Rhodococcus equi (Lopez et al., 2002). In addition, systemic and mucosal IgG responses were described to play an important role in limiting the spread and severity of equine herpes virus 1 (Kydd et al., 2006). Although IgG4 and IgG7 represent the dominating serum antibodies, these isotypes are rarely found in clinically healthy horses (Keggan et al., 2013). 
Chapter 2: Equine immunoglobulins and organization of immunoglobulin genes

All these seven IgG subclasses were expressed in vivo (Wagner et al., 2004). Based on these insights, the first recombinant versions of all seven equine $\lg G$ subclasses (mouse $\lambda$-light chains, horse IGHG1-7) were expressed in Chinese Hamster Ovary cells to analyze their individual physical and biological properties (Lewis et al., 2008). In contrast to these first recombinant equine immunoglobulins, which were expressed for functional analyses, seven distinct monoclonal equine antibodies (lgM, $\lg G 1$, $\lg G 3$, $\lg G 4 / 7, \lg G 5, \lg G 6$, and $\lg E$ ) were produced in equine-murine heterohybridomas to be used for quantification of isotypes in diagnostic testing and immunological research (Keggan et al., 2013).

All recombinant IgGs were $\mathrm{N}$-glycosylated and maintained the affinity for their antigen. Heavy and light chains assembled in intact IgG were stabilized by interchain disulfide bridges except in a small proportion of lgG4 and 7. Complete lgG molecules possessed molecular weights of approximately $150 \mathrm{kDa}$. Nevertheless, differences in complement activation, Fc-receptor binding and the bacterial protein binding capacity between the IgG isotypes were observed. IgG3 showed O-glycosylation in addition to $\mathrm{N}$-glycans. As its hinge region is rich in Proline at position -1 and +3 relative to Threonine residues, which are known sites for O-glycan attachment, O-glycans may be attached to the hinge region of the equine IgG3 (Lewis et al., 2008; Wilson et al., 1991). In IgG4, IgG6, and IgG7 the Cystein equivalent to the human Cys131 was missing within the $\mathrm{CH} 1$ region. Disulfide bond formation - with a Cystein at position 220 in the upper hinge region - is assumed to support the formation of intact IgG. Amino acid substitutions at key positions (234-239, 251-154, 270, 311, 314, 318, 320, $322,329,331,380,382,428,432-436,438)$ of the $\operatorname{lgG}$ molecule, which is responsible for binding to protein A and G, were described (Lewis et al., 2008). IgG1, IgG3, IgG4, IgG5, and IgG7 elicited strong respiratory burst activity in equine peripheral blood leukocytes (PBL), indicating an efficient interaction with FcyR. In contrast, IgG2 and IgG6 induced little or no response. Apparently, they are unable to interact with FcyR. An amino acid residue motif at the N-terminus of the $\mathrm{CH} 2$ domain (Leu234-Leu235Gly236-Gly237-Pro238-Ser239) seemed to be involved in Fc-receptor binding. In complement activation, $\lg \mathrm{G} 1$, $\lg G 3$, IgG4, and $\lg \mathrm{g} 7$ showed positive reactions, whereas IgG2, IgG5, and IgG6 failed. Responsible C1q binding sites appeared not to be conserved between different mammalian species, but there was a Lysine corresponding to human and mouse Lysine at position 322, which was found in all complement activating equine $\lg G$, as well as in $\lg G 6$, but Lys322 was replaced by Serine in lgG2 and lgG5 (Keggan et al., 2013; Lewis et al., 2008). 
Chapter 2: Equine immunoglobulins and organization of immunoglobulin genes

Evidently, IgG plays an important role both in serum and mucosal compartments in horses. Therefore, the summarized considerations appear to be relevant to systemic and mucosal vaccination strategies. IgG antibodies of the subclasses $\lg$ G1, IgG3, lgG4/lgG7 should be induced by vaccines to achieve maximal protection via FcyR and complement-mediated elimination mechanisms. Less effective protection is provided by vaccines that trigger only lgG2, IgG5 or IgG6 antibodies (Lewis et al., 2008).

\section{4. $\lg E$}

$\lg \mathrm{E}$ is the least common serum immunoglobulin isotype (Oethinger et al., 1997). Besides the immune response against parasites, $\lg E$ is also known to be responsible for type 1 allergies and resulting diseases like summer eczema, urticaria, COPD (recurrent airway obstruction) or head shaking (Wagner et al., 2006). For primary investigations, equine $\lg E$ was prepared from the serum of foals suffering from endoparasitic infections (Suter and Fey, 1981). Compared to humans, horses revealed a high concentration of $\mathrm{IgE}$ in serum (Wagner et al., 2006). The constant region gene is located between the constant region genes coding for $\lg G 5$ and $\lg \mathrm{A}$, and it contains four exons. Similar to $\lg M$, there is no separated hinge region for $\lg E$ and the second constant region enables flexibility. The switch region is located 2-4 kb upstream of the constant region gene. Three haplotypes are described differing in two SNPs, which result in two amino acid residue substitutions (Fig. 1c, Table 4) (Navarro et al., 1995; Wagner et al., 2006). The polymorphic character of the $\lg E$ genes may influence effector functions, binding mast cells and basophils, and finally the level of allergic reactions.

\section{5. $\lg A$}

$\lg \mathrm{A}$ dominates in the mucosal immune response of respiratory, gastrointestinal and genitourinary tracts (Souza et al., 2010). In body fluids such as milk, nasal secretions and saliva, it appears as a dimeric secreted molecule and is called secretory $\lg A$. In serum it shows itself in monomeric form (Sheoran et al., 1997; Wagner et al., 2003). IgA produced by $\mathrm{B} 1$ lymphocytes represents a T-lymphocyte independent source of $\lg \mathrm{A}$, which recognizes commensal bacteria. IgA produced by $\mathrm{B} 2$ lymphocytes in the 
Chapter 2: Equine immunoglobulins and organization of immunoglobulin genes

mucosal-associated lymphoid tissues represents T-dependent secreted IgA, e.g. directed against exotoxins (Macpherson et al., 2000).

The IgA locus possesses three exons, two introns, and one hinge region. This hinge region is an extension of the $\mathrm{CH} 2$ sequence. Within the equine IGHC region, IGHA is the most 3'located gene (Fig. 1c, Table 4). The switch region is located $1.4 \mathrm{~kb}$ upstream of the constant region genes coding for $\lg \mathrm{A}$. Phylogenetic comparisons show a common cluster with the IgA genes of humans, dogs, swine, cattle, and sheep. In contrast to rabbits possessing 13 subclasses of $\lg A$ and humans with two subclasses of $\lg A$, horses have only one IgA class (Burnett et al., 1989; Mestecky et al., 2004; Wagner et al., 2003).

Investigations concerning the concentration of $\lg \mathrm{A}$ in serum showed significantly lower IgA quantities in sport horses when compared to extensively reared horses (Souza et al., 2010). Comparable amounts of IgA were found in the colostrum of Warmblood and Standardbred mares after parturition (Kohn et al., 1989). In milk, the reduced IgA concentration is likely due to passively transferred $\lg A$, which was observed in a similar way for $\lg G$ (Jenvey et al., 2012). Once the window for absorbing maternal $\lg A$ has passed, IgA levels in the mare's milk persisted but the significance of this observation is yet to be fully established. However, investigations in human neonates demonstrated that $\lg \mathrm{A}$ and other factors within breast milk may provide an ongoing contribution to local passive protection and immunological development (Corthesy, 2007). Equine serum and secretory $\lg A$ was quantified in Shetland ponies 182 days after parturition. While $\lg G$ levels decreased with time after parturition, $\lg A$ levels increased until $\lg A$ was the predominant immunoglobulin (McGuire and Crawford, 1972). In contrast, the quantity of $\lg A$ in colostrum and milk from Thoroughbred mares rapidly decreased during the first 19 days after parturition, and fluctuated at this level until day 60 p.p. (Jenvey et al., 2012). 
Chapter 2: Equine immunoglobulins and organization of immunoglobulin genes

\section{Equine immunoglobulin light chain gene loci}

As known from other mammals, horses also express two isotypes of light chains ( $\lambda$ and к) differentiated by isotype-specific amino acid residue motifs, antigenic properties, and chemical structure. In contrast to other mammals, $95 \%$ of the equine serum antibodies combine $\lambda$-light chains with the heavy chains, although the reason for this phenomenon is not yet clear (Gibson, 1974; Home et al., 1992). The assumption that amino acid residue substitutions in IGKC result in structures with reduced binding efficiency to the heavy chains was not verified (Ford et al., 1994; Sun et al., 2010). Therefore, a correlation between the number of IGLV gene segments and the preferred usage of a particular isotype is supposed. The $\lambda$-light chain locus was mapped on equine autosome 8 (ECA8) and the gene segments of the $\kappa$-light chain were located on ECA15 (Das et al., 2008).

First studies on the organization of the equine light chain loci revealed a restricted number of variable gene segments for both isotypes. The assembly of variable, joining, and constant region genes was also found to be similar to other species (Ford et al., 1994; Home et al., 1992). Today 144 IGLV, 7 IGLJ and 7 IGLC are identified within $1310 \mathrm{~kb}$ on ECA8 (Fig. 2, Tables 5-7). Each of the 7 IGLJ is preceded by one IGLC and there are two clusters of IGLV possessing different transcriptional orientations. Within each IGLV cluster there are functional genes and pseudogenes (Sun et al., 2010). Similarly, pseudogenes are described in different species such as chicken, where it is already known that pseudogenes are used for gene conversion (Reynaud et al., 1985).

As there were differences in the nomenclature, we also suggest a supplemented designation for the light chain gene segments (Tables 5-7). The name of Ig lambda light chain variable (IGLV) and joining (IGLJ) gene segments were assigned according to IMGT nomenclature guidelines; the international ImMunoGeneTics information system (www.imgt.org). The genes were named according to the subgroup they belong to (Sun et al., 2010) and their number within this subgroup. Furthermore, to denote open reading frames or pseudogenes, sequence names were complemented by superscript ORF and $P$, respectively. The former ' $V \lambda 1$ ' was renamed 'IGLV1S1' to designate sequence 1 of subgroup 1. In analogy, IGLJ and IGLC genes were designated IGLJ1S1 through IGLJ7S1 and IGLC1S1 through IGLC7S1, instead of "J $\lambda 1$ " and "C $\lambda 1$ ", which is consistent with the human nomenclature of Ig lambda genes 
(Hara et al., 2012; Lefranc, 2001a; Sun et al., 2010; Tallmadge et al., 2014). Positions identified on several contigs are also listed. The contigs are NW_001867428.1 and chromosome 8 (Sun et al., 2010). Superscript P indicates pseudo-variable gene segments. The variable gene segments that were not classified to subgroups because they were too divergent or truncated were marked with n.c. (Sun et al., 2010).

According to the supposed nomenclature, which is in analogy to the heavy and $\lambda$-light chain, IGLJ5S1, IGLJ6S1, and IGLJ7S1 show identical sequences. IGLC2S1P is defined as pseudogene due to premature stop codons, as well as IGLJ2S1 ${ }^{\mathrm{P}}$, IGLJ3S1P, and IGLJ4S1 ${ }^{(P)}$ (Sun et al., 2010). Among the 144 IGLV segments identified by Sun et al. (2010), 108 have RSS heptamers and nonamers, 9 lack conserved nonamers and the remaining segments lack both heptamers and nonamers. IGLV3S1, IGLV9S1, IGLV9S2, and IGLV9S3 ${ }^{\circ R}$ have 22-spaced RSS and the remaining potentially functional and ORF-IGLVs carry 23-spaced RSS. The spacer length is more varied among the 76 IGLVP segments, which have the intact RSSs. As many as 55 IGLVP segments maintain 23 bp-spaced RSSs, whereas 16 IGLVP segments carry 22 bp-spaced RSSs. The IGLVxS36 ${ }^{P}, I$ IGLVS4 ${ }^{P}$ and IGLVxS59P have spacer lengths of 21,21 and $19 \mathrm{bp}$, respectively. All 7 IGLJ segments have $12 \mathrm{bp}$-spaced RSSs at their 5'end. 

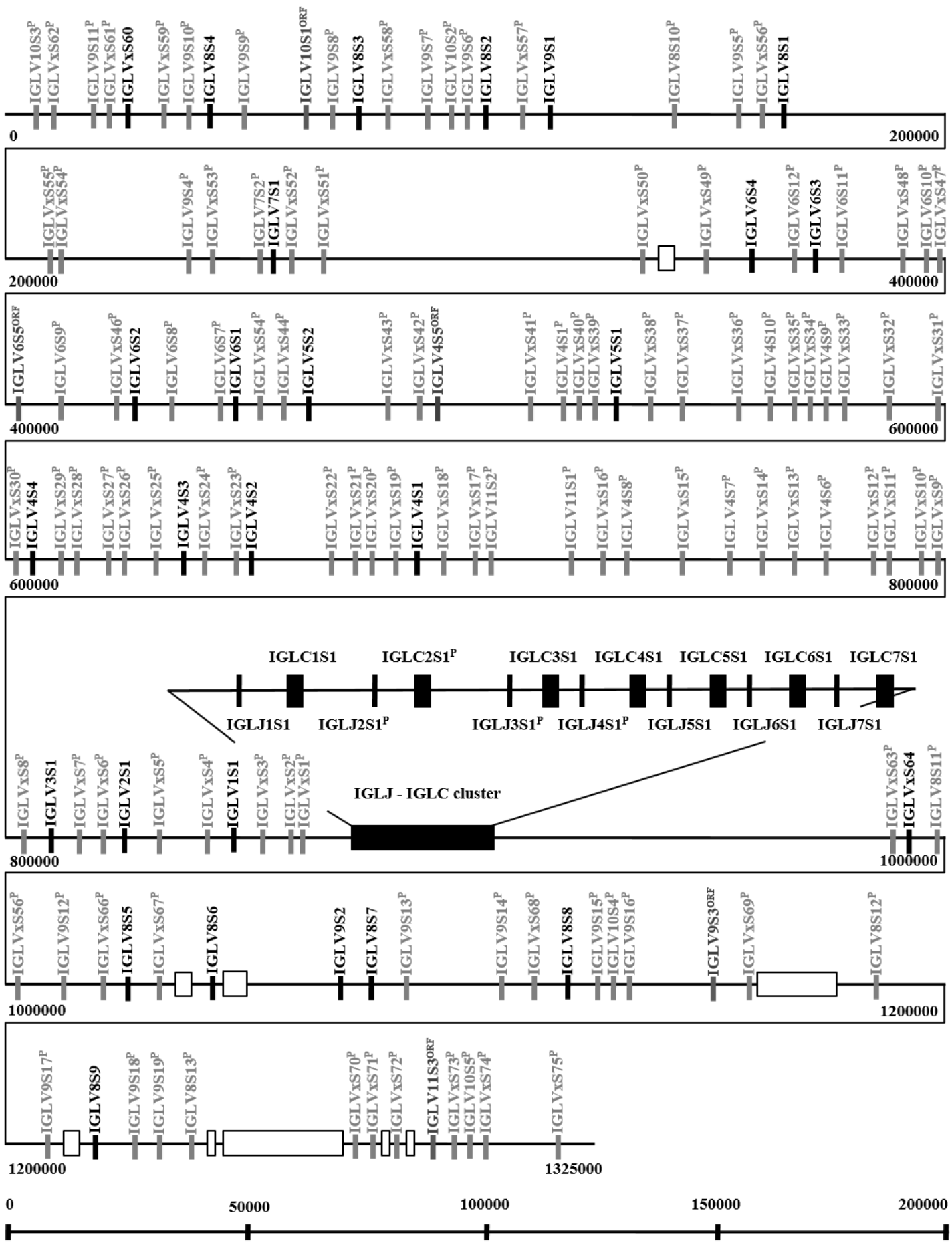

Fig. 2: Map of the equine lambda light chain gene locus

The lambda light chain genes are located on chromosome 8 spanning about $1310 \mathrm{~kb}$. The IGLJ-IGLC pairs and the IGLVs upstream of the (IGLJ-IGLC)7 show opposite transcriptional orientation to the IGLVs downstream of the (IGLJ-IGLC)7 cluster. This physical map is adapted from Sun et al. (2010) to ensure that the newly proposed nomenclature (table 4-7) is designated to the respective positions on the locus. IGLV without superscript letters are potentially functional variable gene segments. Superscript ORF indicates variable gene segments with open reading frames that either have a defect in splicing sites, RSS and/or regulatory elements, and/or changes to the conserved amino acids, and therefore have been suggested to lead to incorrect folding (Lefranc, 1998). Superscript $P$ indicates pseudo-variable gene segments. 
Chapter 2: Equine immunoglobulins and organization of immunoglobulin genes

Table 5: Nomenclature of the equine IGLV gene segments

\begin{tabular}{|c|c|c|c|c|}
\hline IGLV subgroup & $\begin{array}{l}\text { Designation } \\
\text { Sun et al. } 2010\end{array}$ & $\begin{array}{l}\text { Designation } \\
\text { Tallmadge et al. } \\
2013\end{array}$ & $\begin{array}{l}\text { Proposed } \\
\text { designation }\end{array}$ & $\begin{array}{l}\text { Genome } \\
\text { Position }\end{array}$ \\
\hline 1 & $\mathrm{~V} \wedge 1$ & IGLV1-38 & IGLV1S1 & $4171705-4171992$ \\
\hline 2 & $\mathrm{~V} \lambda 2$ & IGLV2-41 & IGLV2S1 & $4195092-4195381$ \\
\hline 3 & $\mathrm{~V} \lambda 3$ & IGLV3-44 & IGLV3S1 & $4210612-4210896$ \\
\hline 4 & V^4 & IGLV4-61 & IGLV4S1 & $4330800-4331090$ \\
\hline 4 & V入5 & IGLV4-66 & IGLV4S2 & $4366543-4366833$ \\
\hline 4 & V 16 & IGLV4-69 & IGLV4S3 & $4381050-4381340$ \\
\hline 4 & V $\lambda 7$ & IGLV4-75 & IGLV4S4 & $4411343-4411633$ \\
\hline 4 & VAORF1 & IGLV4-92 & IGLV4S5 $5^{\mathrm{ORF}}$ & $4529112-4529396$ \\
\hline 4 & $\mathrm{pV} \wedge 13$ & IGLV4-50 & IGLV4S6 ${ }^{P}$ & $4245996-4246283$ \\
\hline 4 & $\mathrm{pV} \wedge 16$ & IGLV4-53 & IGLV4S7 $7^{P}$ & $4267110-4267396$ \\
\hline 4 & $\mathrm{pV} \wedge 18$ & IGLV4-55 & IGLV4S8P & $4287903-4288193$ \\
\hline 4 & $\mathrm{p} \vee \wedge 39$ & IGLV4-80 & IGLV4S9P & $4447087-4447374$ \\
\hline 4 & $\mathrm{p} \vee \wedge 42$ & IGLV4-83 & IGLV4S $10^{P}$ & $4458683-4458970$ \\
\hline 4 & $\mathrm{p} \vee \wedge 48$ & IGLV4-90 & IGLV4S11 ${ }^{P}$ & $4502561-4502854$ \\
\hline 5 & V入8 & IGLV5-87 & IGLV5S1 & $4489461-4489759$ \\
\hline 5 & V入9 & IGLV5-95 & IGLV5S2 & $4554617-4554901$ \\
\hline 6 & $V \lambda 10$ & IHLV6-98 & IHLV6S1 & $4571250-4571546$ \\
\hline 6 & V $\lambda 11$ & IGLV6-101 & IGLV6S2 & $4590316-4590612$ \\
\hline 6 & V^12 & IGLV6-109 & IGLV6S3 & $4648068-4648364$ \\
\hline 6 & $V \lambda 13$ & IGLV6-111 & IGLV6S4 & $4660775-4661062$ \\
\hline 6 & VAORF2 & IGLV6-104 & IGLV6S5 $5^{\mathrm{ORF}}$ & $4614091-4614386$ \\
\hline 6 & pV入54 & IGLV6-99 & IGLV6S7 $7^{P}$ & $4574617-4574913$ \\
\hline 6 & $\mathrm{pV} \wedge 55$ & IGLV6-100 & IGLV6S8P & $4584807-4585103$ \\
\hline 6 & $\mathrm{p} \vee \wedge 57$ & IGLV6-103 & IGLV6S9P & $4607689-4607985$ \\
\hline 6 & pV入59 & IGLV6-106 & IGLV6S10 & $4624835-4625128$ \\
\hline 6 & $\mathrm{p} \vee \wedge 61$ & IGLV6-108 & IGLV6S $11^{\mathrm{P}}$ & $4643773-4644068$ \\
\hline 6 & $\mathrm{p} \vee \lambda 62$ & IGLV6-110 & IGLV6S12P & $4654126-4654437$ \\
\hline 7 & V $\lambda 14$ & IGLV7-116 & IGLV7S1 & $4763155-4763451$ \\
\hline 7 & $\mathrm{p} \vee \wedge 67$ & IGLV7-117 & IGLV7S2P & 4766554-4766835 \\
\hline 8 & $V \wedge 15$ & IGLV8-122 & IGLV8S1 & $4855083-4855381$ \\
\hline 8 & $\mathrm{~V} \wedge 17$ & IGLV8-128 & IGLV8S2 & $4916905-4917203$ \\
\hline 8 & V 118 & IGLV8-133 & IGLV8S3 & $4944794-4945086$ \\
\hline 8 & Vर19 & IGLV8-137 & IGLV8S4 & $4975350-4975639$ \\
\hline 8 & $\mathrm{~V} \wedge 22$ & IGLV8-28 & IGLV8S5 & $3994214-3994503$ \\
\hline 8 & $\mathrm{~V} \wedge 23$ & IGLV8-26 & IGLV8S6 & $3976833-3977125$ \\
\hline 8 & $\mathrm{~V} \wedge 25$ & IGLV8-24 & IGLV8S7 & $3942211-3942500$ \\
\hline 8 & $\mathrm{~V} \lambda 26$ & IGLV8-20 & IGLV8S8 & $3902029-3902327$ \\
\hline 8 & $\mathrm{~V} \wedge 27$ & IGLV8-12 & IGLV8S9 & $3799404-3799702$ \\
\hline 8 & $\mathrm{pV} \lambda 74$ & IGLV8-125 & IGLV8S10 $10^{P}$ & $4878404-4878692$ \\
\hline
\end{tabular}


Chapter 2: Equine immunoglobulins and organization of immunoglobulin genes

\begin{tabular}{|c|c|c|c|c|}
\hline IGLV subgroup & $\begin{array}{l}\text { Designation } \\
\text { Sun et al. } 2010\end{array}$ & $\begin{array}{l}\text { Designation } \\
\text { Tallmadge et al. } \\
2013\end{array}$ & $\begin{array}{l}\text { Proposed } \\
\text { designation }\end{array}$ & $\begin{array}{l}\text { Genome } \\
\text { Position }\end{array}$ \\
\hline 8 & $\mathrm{pV} \wedge 89$ & IGLV8-32 & IGLV8S11 $11^{P}$ & $4023156-4023449$ \\
\hline 8 & $\mathrm{pV} \lambda 101$ & IGLV8-14 & IGLV8S12 & $3838136-3838430$ \\
\hline 8 & $\mathrm{pV} \lambda 105$ & IGLV8-9 & IGLV8S13 & $3781419-3781717$ \\
\hline 9 & V入16 & IGLV9-126 & IGLV9S1 & $4904149-4904473$ \\
\hline 9 & $V \wedge 24$ & IGLV9-25 & IGLV9S2 & $3948638-3948962$ \\
\hline 9 & VAORF4 & IGLV9-16 & IGLV9S3 ORF & $3870582-3870906$ \\
\hline 9 & $\mathrm{pV} \wedge 69$ & IGLV9.119 & IGLV9S4P & $4780685-4780996$ \\
\hline 9 & $\mathrm{pV} \wedge 73$ & IGLV9-124 & IGLV9S5 ${ }^{P}$ & $4866218-4866534$ \\
\hline 9 & pV入76 & IGLV9-129 & IGLV9S6 ${ }^{P}$ & $4922628-4922938$ \\
\hline 9 & $\mathrm{p} \vee \lambda 78$ & IGLV9-131 & IGLV9S7P & $4929757-4930046$ \\
\hline 9 & $\mathrm{p} \vee \lambda 80$ & IGLV9-134 & IGLV9S8P & $4950548-4950857$ \\
\hline 9 & $\mathrm{pV} \wedge 81$ & IGLV9-136 & IGLV9S9P & $4968911-4969235$ \\
\hline 9 & $\mathrm{pV} \wedge 82$ & IGLV9-138 & IGLV9S10 $10^{P}$ & $4981125-4981435$ \\
\hline 9 & $\mathrm{pV} \wedge 85$ & IGLV9-142 & IGLV9S11 $11^{P}$ & 4999541-4999848 \\
\hline 9 & $\mathrm{pV} \wedge 91$ & IGLV9-30 & IGLV9S12 & $4006944-4007268$ \\
\hline 9 & $\mathrm{pV} \wedge 94$ & IGLV9-23 & IGLV9S13 & $3936435-3936744$ \\
\hline 9 & pV^95 & IGLV9-22 & IGLV9S14 & $3914834-3915157$ \\
\hline 9 & $\mathrm{p} \vee \wedge 97$ & IGLV9-19 & IGLV9S15 & $4780685-4780996$ \\
\hline 9 & pV^99 & IGLV9-17 & IGLV9S16 $6^{P}$ & $4866218-4866534$ \\
\hline 9 & $\mathrm{pV} \lambda 102$ & IGLV9-13 & IGLV9S17 $17^{P}$ & $3812261-3812584$ \\
\hline 9 & $\mathrm{pV} \wedge 103$ & IGLV9-11 & IGLV9S18 & $3793651-3793937$ \\
\hline 9 & $\mathrm{pV} \lambda 104$ & IGLV9-10 & IGLV9S19 & $3788296-3788612$ \\
\hline 10 & VגORF3 & IGLV10-135 & IGLV10S1 $1^{\mathrm{ORF}}$ & $4954678-4954976$ \\
\hline 10 & pV $\lambda 77$ & IGLV10-130 & IGLV10S2P & $4926370-4926670$ \\
\hline 10 & $\mathrm{p} \vee \wedge 87$ & IGLV10-144 & IGLV10S3 ${ }^{P}$ & $5012699-5012989$ \\
\hline 10 & $\mathrm{pV} \wedge 98$ & IGLV10-18 & IGLV10S4P & $3892471-3892771$ \\
\hline 10 & $\mathrm{pV} \lambda 110$ & IGLV10-3 & IGLV10S5 $5^{P}$ & $3723141-3723424$ \\
\hline 11 & $\mathrm{pV} \wedge 20$ & IGLV11-140 & IGLV11S1 ${ }^{P}$ & $4300066-4300348$ \\
\hline 11 & $\mathrm{pV} \wedge 21$ & IGLV11-33 & IGLV11S2 & $4316629-4316892$ \\
\hline 11 & V^ORF5 & IGLV11-5 & IGLV11S3ORF & $3730193-3730489$ \\
\hline n.c. & $\mathrm{pV} \lambda 1$ & & $\mid G L V x S 1^{P}$ & $4157587-4157847$ \\
\hline n.c. & $\mathrm{p} \vee \wedge 2$ & & IGLVxS2P & $4160537-4160835$ \\
\hline n.c. & $\mathrm{pV} \lambda 3$ & & IGLVxS3 ${ }^{P}$ & $4166903-4167176$ \\
\hline n.c. & $\mathrm{pV} \wedge 4$ & & IGLVxS4P & $4178429-4178741$ \\
\hline n.c. & $\mathrm{pV} \wedge 5$ & & $\operatorname{IGLVxS5^{P}}$ & $4188046-4188311$ \\
\hline n.c. & $\mathrm{pV} \wedge 6$ & & IGLVxS6 ${ }^{P}$ & 4199645-4199909 \\
\hline n.c. & $\mathrm{pV} \lambda 7$ & & IGLVxS7P & $4205575-4205866$ \\
\hline n.c. & $\mathrm{pV} \wedge 8$ & & IGLVxS8 ${ }^{P}$ & $4216947-4217233$ \\
\hline
\end{tabular}


Chapter 2: Equine immunoglobulins and organization of immunoglobulin genes

\begin{tabular}{|c|c|c|c|c|}
\hline IGLV subgroup & $\begin{array}{l}\text { Designation } \\
\text { Sun et al. } 2010\end{array}$ & $\begin{array}{l}\text { Designation } \\
\text { Tallmadge et al. } \\
2013\end{array}$ & $\begin{array}{l}\text { Proposed } \\
\text { designation }\end{array}$ & $\begin{array}{l}\text { Genome } \\
\text { Position }\end{array}$ \\
\hline n.c. & $\mathrm{pV} \wedge 9$ & & IGLVxS9P & $4221861-4222159$ \\
\hline n.c. & $\mathrm{p} \vee \wedge 10$ & & IGLVxS10 & $4226486-4226798$ \\
\hline n.c. & $\mathrm{pV} \wedge 11$ & & $\operatorname{IGLVxS} 11^{P}$ & $4232825-4233111$ \\
\hline n.c. & $\mathrm{pV} \wedge 12$ & & IGLVxS12 & $4236991-4237275$ \\
\hline n.c. & $\mathrm{pV} \wedge 14$ & & IGLVxS13 & $4252560-4252850$ \\
\hline n.c. & $\mathrm{pV} \wedge 15$ & & IGLVxS14 & $4259340-4259638$ \\
\hline n.c. & $\mathrm{pV} \wedge 17$ & & IGLVxS15 & $4276218-4276516$ \\
\hline n.c. & $\mathrm{p} \vee \lambda 19$ & & IGLVxS16 & $4293600-4293868$ \\
\hline n.c. & $\mathrm{pV} \wedge 22$ & & IGLVxS17 & $4320273-4320548$ \\
\hline n.c. & $\mathrm{pV} \wedge 23$ & & IGLVxS18 & $4327257-4327515$ \\
\hline n.c. & $\mathrm{pV} \wedge 24$ & & IGLVxS19P & $4336476-4336776$ \\
\hline n.c. & $\mathrm{p} \vee \lambda 25$ & & IGLVxS20 & $4341721-4341986$ \\
\hline n.c. & $\mathrm{pV} \lambda 26$ & & IGLVxS2 $21^{P}$ & $4345368-4345651$ \\
\hline n.c. & $\mathrm{pV} \wedge 27$ & & IGLVxS22 & $4350798-4351060$ \\
\hline n.c. & $\mathrm{pV} \wedge 28$ & & IGLVxS23 ${ }^{P}$ & $4370580-4370835$ \\
\hline n.c. & $\mathrm{p} \vee \wedge 29$ & & IGLVxS24 & $4377206-4377464$ \\
\hline n.c. & $\mathrm{p} \vee \wedge 30$ & & IGLVxS25 & $4387212-4387524$ \\
\hline n.c. & $\mathrm{p} \vee \wedge 31$ & & IGLVxS26 ${ }^{P}$ & $4393759-4394024$ \\
\hline n.c. & $\mathrm{pV} \wedge 32$ & & IGLVxS27 $7^{P}$ & $4397408-4397690$ \\
\hline n.c. & $\mathrm{p} \vee \wedge 33$ & & IGLVxS28 & $4403625-4403886$ \\
\hline n.c. & $\mathrm{pV} \wedge 34$ & & IGLVxS29P & $4407546-4407804$ \\
\hline n.c. & $\mathrm{pV} \wedge 35$ & & IGLVxS30 & $4417549-4417861$ \\
\hline n.c. & $\mathrm{pV} \wedge 36$ & & IGLVxS31 ${ }^{P}$ & $4423822-4424107$ \\
\hline n.c. & $\mathrm{p} \vee \wedge 37$ & & IGLVxS32 & $4433296-4433588$ \\
\hline n.c. & $\mathrm{pV} \wedge 38$ & & IGLVxS33 & $4444562-4444847$ \\
\hline n.c. & $\mathrm{p} \vee \wedge 40$ & & IGLVxS34 & $4450690-4451002$ \\
\hline n.c. & $\mathrm{p} \vee \wedge 41$ & & IGLVxS35 & $4454114-4454375$ \\
\hline n.c. & $\mathrm{pV} \wedge 43$ & & IGLVxS36 ${ }^{P}$ & $4464714-4465026$ \\
\hline n.c. & $\mathrm{pV} \wedge 44$ & & IGLVxS37 & $4476525-4476807$ \\
\hline n.c. & $\mathrm{p} \vee \wedge 45$ & & IGLVxS38 & $4483271-4483580$ \\
\hline n.c. & $\mathrm{pV} \wedge 46$ & & IGLVxS39P & $4495317-4495629$ \\
\hline n.c. & $\mathrm{p} \vee \wedge 47$ & & IGLVxS40 & $4498517-4498783$ \\
\hline n.c. & $\mathrm{pV} \wedge 49$ & & IGLVxS41 ${ }^{P}$ & $4508307-4508617$ \\
\hline n.c. & $\mathrm{pV} \wedge 50$ & & IGLVxS42P & $4532982-4533235$ \\
\hline n.c. & $\mathrm{p} \vee \wedge 51$ & & IGLVxS43 & $4539033-4539326$ \\
\hline n.c. & pV入52 & & IGLVxS44 & $4560252-4560551$ \\
\hline n.c. & $\mathrm{pV} \wedge 53$ & & IGLVxS45 & \\
\hline n.c. & $\mathrm{p} \vee \wedge 56$ & & IGLVxS46 & $4595924-4596217$ \\
\hline
\end{tabular}


Chapter 2: Equine immunoglobulins and organization of immunoglobulin genes

\begin{tabular}{|c|c|c|c|c|}
\hline IGLV subgroup & $\begin{array}{l}\text { Designation } \\
\text { Sun et al. } 2010\end{array}$ & $\begin{array}{l}\text { Designation } \\
\text { Tallmadge et al. } \\
2013\end{array}$ & $\begin{array}{l}\text { Proposed } \\
\text { designation }\end{array}$ & $\begin{array}{l}\text { Genome } \\
\text { Position }\end{array}$ \\
\hline n.c. & $\mathrm{pV} \vee 58$ & & IGLVxS47 & $4621790-4622080$ \\
\hline n.c. & $\mathrm{p} \vee \lambda 60$ & & IGLVxS48 & $4629113-4629400$ \\
\hline n.c. & $\mathrm{p} \vee \lambda 63$ & & IGLVxS49P & $4670998-4671281$ \\
\hline n.c. & $\mathrm{p} \vee \lambda 64$ & & IGLVxS50 & $4684017-4684281$ \\
\hline n.c. & $\mathrm{pV} \wedge 65$ & & IGLVxS51 ${ }^{P}$ & $4753071-4753353$ \\
\hline n.c. & $\mathrm{p} \vee \wedge 66$ & & IGLVxS52 & $4760021-4760299$ \\
\hline n.c. & $\mathrm{p} \vee \lambda 68$ & & IGLVxS53 & $4775528-4775824$ \\
\hline n.c. & $\mathrm{p} \vee \wedge 70$ & & IGLVxS54 & $4807475-4807769$ \\
\hline n.c. & $\mathrm{pV} \wedge 71$ & & IGLVxS55 & 4808989-4809266 \\
\hline n.c. & $\mathrm{p} \vee \wedge 72$ & & IGLVxS56 & $4860864-4861172$ \\
\hline n.c. & $\mathrm{pV} \wedge 75$ & & IGLVxS57P & $4910505-4910786$ \\
\hline n.c. & pV入79 & & IGLVxS58 ${ }^{P}$ & $4938347-4938661$ \\
\hline n.c. & $\mathrm{p} \vee \wedge 83$ & & IGLVxS59P & $4985429-4985745$ \\
\hline n.c. & V 120 & & IGLVxS60 & $4994608-4994903$ \\
\hline n.c. & $\mathrm{p} \vee \wedge 84$ & & IGLVxS61 & $4998412-4998621$ \\
\hline n.c. & $\mathrm{pV} \wedge 86$ & & IGLVxS62 & $5010496-5010800$ \\
\hline n.c. & $\mathrm{p} \vee \wedge 88$ & & IGLVxS63 & 4033346-4033647 \\
\hline n.c. & $\mathrm{V} \wedge 21$ & & IGLVxS64 & $4031616-4031911$ \\
\hline n.c. & $\mathrm{p} \vee \lambda 90$ & & IGLVxS65 & $4017794-4018083$ \\
\hline n.c. & $\mathrm{p} \vee \lambda 92$ & & IGLVxS66 & $4000617-4000857$ \\
\hline n.c. & $\mathrm{p} \vee \lambda 93$ & & IGLVxS67 & 3988326-3988631 \\
\hline n.c. & $\mathrm{pV} \wedge 96$ & & IGLVxS68 & 3908433-3908722 \\
\hline n.c. & $\mathrm{pV} \wedge 100$ & & IGLVxS69P & $3864249-3864531$ \\
\hline n.c. & $\mathrm{p} \vee \lambda 106$ & & IGLVxS70 & $3745800-3746092$ \\
\hline n.c. & $\mathrm{p} \vee \wedge 107$ & & IGLVxS71 ${ }^{P}$ & $3742171-3742365$ \\
\hline n.c. & $\mathrm{pV} \lambda 108$ & & IGLVxS72 & 3738392-3738672 \\
\hline n.c. & $\mathrm{p} \vee \lambda 109$ & & IGLVxS73 & $3726480-3726695$ \\
\hline n.c. & $\mathrm{pV} \lambda 111$ & & IGLVxS74 & $3719722-3720011$ \\
\hline n.c. & $\mathrm{pV} \lambda 112$ & & IGLVxS75 & $3703267-3703501$ \\
\hline
\end{tabular}


Chapter 2: Equine immunoglobulins and organization of immunoglobulin genes

Table 6: Nomenclature of the equine IGLJ gene segments

\begin{tabular}{lllll}
\hline $\begin{array}{l}\text { IGLJ } \\
\text { subgroup }\end{array}$ & $\begin{array}{l}\text { Designation } \\
\text { Sun et al. 2010 }\end{array}$ & $\begin{array}{l}\text { Designation } \\
\text { Tallmadge et al. } \\
\mathbf{2 0 1 3}\end{array}$ & $\begin{array}{l}\text { Proposed } \\
\text { designation }\end{array}$ & $\begin{array}{l}\text { Genome } \\
\text { Position }\end{array}$ \\
\hline 1 & $\mathrm{~J} \lambda 1$ & IGLJ1 & IGLJ1S1 & $4146337-4146302^{\mathrm{a}}$ \\
2 & $\mathrm{~J} \lambda 2$ & IGLJ2 & IGLJ2S1 & $4141033-4140996^{\mathrm{a}}$ \\
3 & $\mathrm{~J} \lambda 3$ & IGLJ3 & IGLJ3S1P & $4135576-4135541^{\mathrm{a}}$ \\
4 & $\mathrm{~J} \lambda 4$ & IGLJ4 & IGLJ4S1 & $4132448-4132410^{\mathrm{a}}$ \\
5 & $\mathrm{~J} \lambda 5$ & IGLJ5 & IGLJ5S1 & $4129086-4129050^{\mathrm{a}}$ \\
6 & $\mathrm{~J} \lambda 6$ & IGLJ6 & IGLJ6S1 & $4125743-4125707^{\mathrm{a}}$ \\
7 & $\mathrm{~J} \lambda 7$ & IGLJ7 & IGLJ7S1 & $4122272-4122236^{\mathrm{a}}$ \\
\hline${ }^{\mathrm{a}}$ Contig_NW_001867428.1 & & &
\end{tabular}

Table 7: Nomenclature of the equine IGLC genes

\begin{tabular}{lllll}
\hline $\begin{array}{l}\text { IGLC } \\
\text { subgroup }\end{array}$ & $\begin{array}{l}\text { Designation } \\
\text { Sun et al. 2010 }\end{array}$ & $\begin{array}{l}\text { Designation } \\
\text { Tallmadge et al. } \\
\mathbf{2 0 1 3}\end{array}$ & $\begin{array}{l}\text { Proposed } \\
\text { designation }\end{array}$ & $\begin{array}{l}\text { Genome } \\
\text { Position }\end{array}$ \\
\hline 1 & $\mathrm{C} \lambda 1$ & IGLC1 & IGLC1S1 & $4144802-4144483^{\mathrm{a}}$ \\
2 & $\mathrm{pC} \lambda 2$ & IGLC2 & IGLC2S1 & $4139692-4139371^{\mathrm{a}}$ \\
3 & $\mathrm{C} \lambda 3$ & IGLC3 & IGLC3S1 & $4134292-4133973^{\mathrm{a}}$ \\
4 & $\mathrm{C} \lambda 4$ & IGLC4 & IGLC4S1 & $4131021-4130702^{\mathrm{a}}$ \\
5 & $\mathrm{C} \lambda 5$ & IGLC5 & IGLC5S1 & $4127666-4127347^{\mathrm{a}}$ \\
6 & $\mathrm{C} \lambda 6$ & IGLC6 & IGLC6S1 & $4124320-4124002^{\mathrm{a}}$ \\
7 & $\mathrm{C} \lambda 7$ & IGLC7 & IGLC7S1 & $4120851-4120531^{\mathrm{a}}$ \\
\hline${ }^{\mathrm{a}}$ Contig_NW_001867428.1 & & &
\end{tabular}

The $\lambda$-light chain locus shows a unique feature of two IGLV clusters compared to this locus in all other species analyzed, whereas the $\kappa$-light chain genes are arranged as usual in one cluster. In the $820 \mathrm{~kb}$ locus, there is one cluster of 60 IGKV, one cluster of five IGKJ, and a single IGKC (Fig. 3, Tables 8-10) (Sun et al., 2010). Moreover, the functional and nonfunctional IGKV are located within one locus. All IGKV segments have 12 bp-spaced RSSs at their 3'ends except IGKV1S21 ${ }^{\mathrm{P}}$, which possesses an 11 bp spacer and additional 8 IGKVP segments where RSSs are lacking completely. IGKJ1S1, IGKJ2S1, and IGKJ4S1 have 23 bp-spaced RSSs at their 5'ends in contrast to IGKJ3S1, which has a 20 bp-spaced RSS. IGKJ5S1 ${ }^{P}$ is nonfunctional as there is no conserved heptamer within the RSS (Sun et al., 2010). Transcriptional orientations in both directions are found without grouping. The single IGKC is separated from the IGKJ by a 2926 bp intron. IGKJ5S1 ${ }^{\mathrm{P}}$ has no heptamer in the recombination signal sequence (RSS) and is therefore described as pseudogene. In IGKJ3S1, the spacer of the RSS shows $20 \mathrm{bp}$ instead of $23 \mathrm{bp}$, which does not result in reduced recombination frequency (Ford et al., 1994; Sun et al., 2010). 

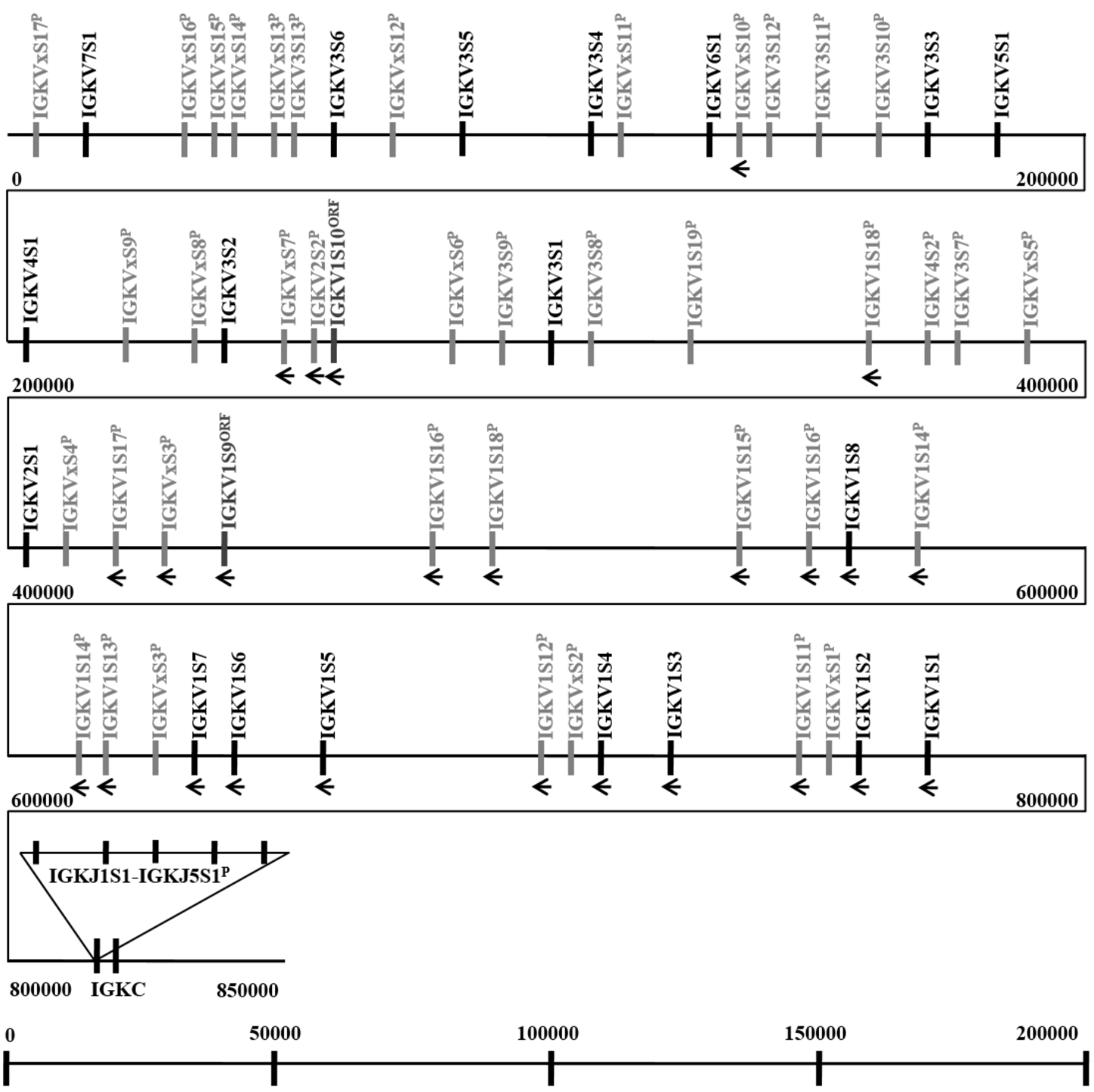

Fig. 3: Physical map of the equine kappa light chain gene locus

$820 \mathrm{~kb}$ of the kappa light chain genes are located on chromosome 15. The potentially functional IGKVs, 2 ORFs and 15 pseudogenes show opposite transcriptional orientation to the (IGKJ)5-IGKC cluster. Arrows below the vertical lines directed ' 5 ' indicate transcriptional orientation. This physical map is adapted from Sun et al. (2010) to ensure that the newly proposed nomenclature (table 3 ) is designated to the respective positions on the locus. IGKV without superscript letters are potentially functional variable gene segments. Superscript ORF indicates variable gene segments with open reading frames that either have a defect in splicing sites, RSS and/or regulatory elements, and/or changes to the conserved amino acids, and therefore have been suggested to lead to incorrect folding (Lefranc, 1998). Superscript $P$ indicates pseudo-variable gene segments. 
Chapter 2: Equine immunoglobulins and organization of immunoglobulin genes

Table 8: Nomenclature of the equine IGKV gene segments

\begin{tabular}{|c|c|c|c|}
\hline $\begin{array}{l}\text { IGKV } \\
\text { subgroup }\end{array}$ & $\begin{array}{l}\text { Designation } \\
\text { Sun et al. } 2010\end{array}$ & $\begin{array}{l}\text { Proposed } \\
\text { designation }\end{array}$ & $\begin{array}{l}\text { Genome } \\
\text { Position }\end{array}$ \\
\hline 1 & Vk1 & IGKV1S1 & $17071497-17071783$ \\
\hline 1 & Vк2 & $\operatorname{lgKV} 1 S 2$ & $17057542-17057828$ \\
\hline 1 & Vk3 & IGKV1S3 & $17023418-17023704$ \\
\hline 1 & Vk4 & IGKV1S4 & $17010212-17010498$ \\
\hline 1 & Vk5 & IGKV1S5 & $16958378-16958664$ \\
\hline 1 & Vк6 & IGKV1S6 & $16941855-16942141$ \\
\hline 1 & Vk7 & IGKV1S7 & $16933657-16933943$ \\
\hline 1 & Vк8 & IGKV1S8 & $16855771-16856057$ \\
\hline 1 & VkORF1 & IGKV1S9ORF & $16739610-16739895$ \\
\hline 1 & VkORF2 & IGKV1S10 ORF & $16560248-16560552$ \\
\hline 1 & $\mathrm{pV \kappa 2}$ & IGKV1S11 ${ }^{P}$ & $17046559-17046846$ \\
\hline 1 & $\mathrm{pVk} 4$ & IGKV1S12P & $16998516-16998803$ \\
\hline 1 & $\mathrm{p} \vee к 6$ & IGKV1S13P & $16917360-16917646$ \\
\hline 1 & $\mathrm{pVk} 7$ & IGKV1S14P & $16912894-16913180$ \\
\hline 1 & pУк8 & IGKV1S15 & $16868993-16869281$ \\
\hline 1 & $\mathrm{p} \vee \mathrm{k} 9$ & IGKV1S16 $6^{P}$ & $16849772-16850060$ \\
\hline 1 & $\mathrm{pVk} 10$ & IGKV1S17P & $16835919-16836203$ \\
\hline 1 & $\mathrm{pVk} 11$ & IGKV1S18 & $16789755-16790042$ \\
\hline 1 & $\mathrm{pVk} 12$ & IGKV1S19P & $16778531-16778817$ \\
\hline 1 & $\mathrm{pVk} 13$ & IGKV1S20P & $16728653-16728934$ \\
\hline 1 & $\mathrm{pVk} 14$ & IGKV1S21 ${ }^{\mathrm{P}}$ & $16720475-16720760$ \\
\hline 1 & $\mathrm{pVk} 19$ & IGKV1S22P & $16659096-16659390$ \\
\hline 1 & $\mathrm{pVk20}$ & IGKV1S23P & $16626800-16627104$ \\
\hline 2 & Vк9 & IGKV2S1 & $16700635-16700951$ \\
\hline 2 & $\mathrm{pVk24}$ & IGKV2S2P & $16557308-16557612$ \\
\hline 3 & Vk10 & IGKV3S1 & $16599670-16599971$ \\
\hline 3 & Vk11 & IGKV3S2 & $16540025-16540326$ \\
\hline 3 & Vk14 & IGKV3S3 & $16470843-16471144$ \\
\hline 3 & Vk16 & IGKV3S4 & $16407261-16407562$ \\
\hline 3 & Vk17 & IGKV3S5 & $16383431-16383732$ \\
\hline 3 & Vk18 & IGKV3S6 & $16360142-16360443$ \\
\hline 3 & $\mathrm{pVk} 17$ & IGKV3S7P & $16676005-16676305$ \\
\hline 3 & $\mathrm{pVk} 21$ & IGKV3S8P & $16608233-16608528$ \\
\hline 3 & $\mathrm{pVk22}$ & IGKV3S9P & $16591859-16592160$ \\
\hline 3 & pУк28 & IGKV3S10P & $16461689-16461983$ \\
\hline 3 & pVк29 & IGKV3S11 $1^{\mathrm{P}}$ & $16450171-16450478$ \\
\hline 3 & pУк30 & IGKV3S12 & $16441110-16441414$ \\
\hline 3 & pVк34 & IGKV3S13P & $16351930-16352220$ \\
\hline 4 & Vk12 & IGKV4S1 & $16502696-16502982$ \\
\hline 4 & $\mathrm{pVk} 18$ & IGKV4S2 ${ }^{P}$ & $16672345-16672638$ \\
\hline 5 & Vk13 & IGKV5S1 & $16484542-16484828$ \\
\hline 6 & Vk15 & IGKV6S1 & $16431518-16431804$ \\
\hline 7 & $V_{k} 19$ & IGKV7S1 & $16313053-16313339$ \\
\hline
\end{tabular}


Chapter 2: Equine immunoglobulins and organization of immunoglobulin genes

\begin{tabular}{|c|c|c|c|}
\hline $\begin{array}{l}\text { IGKV } \\
\text { subgroup }\end{array}$ & $\begin{array}{l}\text { Designation } \\
\text { Sun et al. } 2010\end{array}$ & $\begin{array}{l}\text { Proposed } \\
\text { designation }\end{array}$ & $\begin{array}{l}\text { Genome } \\
\text { Position }\end{array}$ \\
\hline n.c. & $\mathrm{pV} \mathrm{k} 1$ & $I G K V x S 1^{P}$ & $17053257-17053545$ \\
\hline n.c. & $\mathrm{p \bigvee k} 3$ & IGKVxS2P & $17004266-17004536$ \\
\hline n.c. & pУк5 & IGKVxS3P & $16927468-16927746$ \\
\hline n.c. & pVk15 & IGKVxS4P & $16709612-16709911$ \\
\hline n.c. & $\mathrm{pVk} 16$ & $I G K V x S 5^{P}$ & $16689071-16689357$ \\
\hline n.c. & $\mathrm{pVk23}$ & IGKVxS6P & $16581856-16582113$ \\
\hline n.c. & pVk25 & IGKVxS7P & $16550552-15550840$ \\
\hline n.c. & pVk26 & IGKVxS8P & $16534333-16534598$ \\
\hline n.c. & pVk27 & IGKVxS9P & $16520693-16520979$ \\
\hline n.c. & pVk31 & IGKVxS10 & $16434887-16435177$ \\
\hline n.c. & $\mathrm{pVk} 32$ & IGKVxS11 ${ }^{P}$ & $16413133-16413356$ \\
\hline n.c. & pVk33 & IGKVxS12 & $16370408-16370629$ \\
\hline n.c. & pVk35 & IGKVxS13 & $16348990-16349239$ \\
\hline n.c. & pVk36 & IGKVxS14 & $16342798-16343043$ \\
\hline n.c. & pVk37 & IGKVxS15 & $16338745-16339014$ \\
\hline n.c. & pVк38 & IGKVxS16 & $16332882-16333152$ \\
\hline n.c. & pVк39 & IGKVxS17 & $16305235-16305453$ \\
\hline
\end{tabular}

IGKV: Sun et al., 2010, Chromosom 15, NW_001867379.1

Table 9: Nomenclature of the equine IGKJ gene segments

\begin{tabular}{llll}
\hline $\begin{array}{l}\text { IGKJ } \\
\text { subgroup }\end{array}$ & $\begin{array}{l}\text { Designation } \\
\text { Sun et al. 2010 }\end{array}$ & $\begin{array}{l}\text { Proposed } \\
\text { designation }\end{array}$ & $\begin{array}{l}\text { Genome } \\
\text { Position }\end{array}$ \\
\hline 1 & JK1 & IGKJ1S1 & $13727254-13727217^{*}$ \\
2 & JK2 & IGKJ2S1 & $13727624-13727586^{*}$ \\
3 & JK3 & IGKJ3S1 & $13727931-13727894^{*}$ \\
4 & JK4 & IGKJ4S1 & $13728269-13728232^{*}$ \\
5 & pJK5 & IGKJ5S1 & $13728570-13728534^{*}$ \\
\hline
\end{tabular}

${ }^{*}$ Contig: NW_001867379.1

Table 10: Nomenclature of the equine IGKC genes

\begin{tabular}{llll}
\hline $\begin{array}{l}\text { IGKC } \\
\text { subgroup }\end{array}$ & $\begin{array}{l}\text { Designation } \\
\text { Sun et al. 2010 }\end{array}$ & $\begin{array}{l}\text { Proposed } \\
\text { designation }\end{array}$ & $\begin{array}{l}\text { Genome } \\
\text { Position }\end{array}$ \\
\hline 1 & CK1 & IGKC1S1 & $13731499-13731821^{*}$ \\
\hline${ }^{*}$ Contig: NW_001867379.1 & & &
\end{tabular}


Chapter 2: Equine immunoglobulins and organization of immunoglobulin genes

\section{Transcription analyses of heavy and light chain genes}

\subsection{Heavy chain genes}

Newborn foals are unable to mount an effective humoral immune response despite many features of the equine immune system having been developed during fetal life. Immunohistochemical experiments showed the expression of essential B-cell genes during equine gestation. Active gene recombination and isotype switching were suggested and underpinned by limited but detectable production of $\lg M$ and $\lg G$ at birth (Tallmadge et al., 2009). After demonstrating that equine neonatal B-cells produce all known five Ig isotypes, usage frequencies of the equine variable, diversity, and joining gene segments of the heavy and light chains were first investigated in two unrelated horses (Sun et al., 2010). Both animals showed a similar usage pattern of IGHV, IGHD, and IGHJ. Out of 50 IGHV, four (IGHV4S1, IGHV2S2, IGHV2S3, and IGHV4S5) were expressed but IGHV2S2 and IGHV2S3 were used preferably. Six out of eight IGHJ were recombined. IGHJ1S1 and IGHJ2S1 were not detected, whereas IGHJ1S5 was mainly rearranged. The analysis of the usage frequencies of the 40 germline IGHD revealed the dominant frequent use of IGHD18S1. The transcription of five additional IGHDs (IGDH3S1, IGHD12S1, IGHD8S2, IGHD8S3, IGHD28S1) was not observed. Eight clones indicated the incorporation of more than one IGHD into the IGHV-IGHDIGHJ rearrangement resulting in IGHV-IGHD-IGHD-IGHJ. The second incorporated IGHD was supposed to be located 5' of the first IGHD in the germline locus (Sun et al., 2010). In addition to these studies of immunoglobulin diversity in adult horses, the level of Ig heavy chain variable region diversity was analyzed from fetal spleen, neonatal, foal, and adult horse mesenteric lymph node samples (Tallmadge et al., 2009). Interestingly, combinatorial and junctional levels of VDJ sequences in equine fetus were comparable to those of adult horses and a similar set of variable gene segments was used during fetal and post-natal life stages (Tallmadge et al., 2009; Tallmadge et al., 2013). During fetal life stage, 7 IGHV genes were identified with nucleotide identities of at least $97 \%$ compared to the donor germline sequences. Besides the gene segments IGHV2S2, IGHV2S3, IGHV1S3, IGHV4S2, IGHV4S5 and IGHV2S4, a newly identified IGHV4S17 was recognized. A reduced repertoire was observed in neonates where only 4 segments were used: IGHV2S2, IGHV2S3, IGHV2S4, and a novel IGHV1S6. In foals and adult horses, 5 and 4 IGHV were transcribed, respectively (foal: IGHV2S2, IGHV2S3, IGHV2S4, IGHV1S3, IGHV4S2; adult: IGHV2S2, IGHV2S3, 
Chapter 2: Equine immunoglobulins and organization of immunoglobulin genes

IGHV2S4, IGHV4S2). The segments IGHV2S2, IGHV2S3, and IGHV2S4 were used predominantly at all ages. Between the expressed gene segments, there are 250,000 bases suggesting that the entire IGHV locus is available throughout equine life (Tallmadge et al., 2013). Similarly, in fetal piglets the IGHV usage was described to be independent of the genome position although there is a limited number of IGHV representing the Ig repertoire, too (Butler et al., 2011; Eguchi-Ogawa et al., 2010). In contrast, the biased usage of germline IGHV in the mouse fetus was explained with different accessible positions within the IGHV locus (Jeong et al., 1988).

Analysis of the IGHD gene segments revealed that 13, 17, 15, and 20 different IGHDs were used in fetuses, neonates, foals, and adult horses. The predominant IGHD was IGHD18S1 at all ages. Four out of 8 IGHJ had an influence on diversity in all life stages, whereas IGHJ1S5 was the most common (Tallmadge et al., 2013).

VDJ diversity increased mainly during the last two thirds of gestation. Overall, there was no biased usage of a single IGHV, IGHD, or IGHJ gene segment at any age. In foals, first variations in the length of $\mathrm{CDR} 2 \mathrm{H}$ were found. Sequence diversity and length variation further increased in $\mathrm{CDR} 1 \mathrm{H}$ and $\mathrm{CDR} 2 \mathrm{H}$, and framework regions of adult horses in accordance to somatic hypermutation (Tallmadge et al., 2013). Nontemplated nucleotide insertions of about 8 nucleotides were observed at the IGHVIGHD junction in fetuses and neonates, although there was no significant increase in CDR3H length, varying from 7 to 21 amino acids in contrast to cattle (Walther et al., 2013). The length of the IGHV-IGHD and IGHD-IGHJ junctions increased significantly until birth and then remained similar in foals and adult horses. At least 5 amino acid residues were observed within the $\mathrm{CDR} 3 \mathrm{H}$ of an adult horse, whereas 25 amino acid residues were counted in the longest $\mathrm{CDR} 3 \mathrm{H}$, which was identified in an equine neonate (Tallmadge et al., 2013). In comparison, recent studies showed the existence of very short CDR3H (5-10 amino acids), midlength CDR3H (11-31 amino acids) and exceptionally long CDR3H (more than 47 amino acids) in both bovine fetuses and adult cattle. This length heterogeneity is not isotype restricted and very long CDR3H contribute to diversity by uniquely folded small domains (Koti et al., 2010; Saini et al., 1999; Saini and Kaushik, 2002; Shojaei et al., 2003; Walther et al., 2013; Wang et al., 2013). 
Chapter 2: Equine immunoglobulins and organization of immunoglobulin genes

\subsection{Light chain genes}

For the IGKV, a preferential usage pattern was also observed. IGKV1S1, IGKV1S4, and IGKV2S1 were used most often. These gene segments showed an opposite transcriptional orientation to the IGKJ and IGKC cluster. All four functional IGKJ were expressed (Sun et al., 2010).

Within the transcribed $\lambda$-light chains, IGLVs belonging to subgroup 8 were preferred. IGLV from subgroup 6 and 4 were also transcribed but less frequently. The recombined IGLVs - especially IGLV8S2 and IGLV8S7 - were located within the IGLV cluster downstream of the IGLJ-IGLC cluster and were oriented in opposite transcriptional polarity. In both animals, examined by Sun and coworkers, genomic IGLJ-IGLC were amplified, whereas IGLJ-IGLC5 could not be amplified and IGLJ-IGLC7 was amplified successfully in one of these animals. IGLJ4S $1^{(\mathrm{P})}$ was found to be functional but without the single $\mathrm{C}$-insertion, which is present in the database sequence of the equine genome project (Sun et al., 2010). Subsequent investigations of the transcription frequencies of $\lambda$-light chain genes in ten animals of Rhenish-German Coldblood (RGC) and Hanoverian Warmblood (HW), respectively, showed similar results (Hara et al., 2012). Eleven out of 144 IGLV were identified; all members of subgroup 8 as caused by the applied primers. In both breeds analyzed, the preferred use of two IGLV (IGLV8S1 and IGLV8S2) was observed. In addition, significant differences in usage frequencies of the IGLV (both within and between the breeds) were noted. Two pseudogenes were also recombined (IGLV8S10 ${ }^{P}$ and IGLV8S12P). The transcriptional orientation of the IGLV had no influence on the transcription frequencies. All four functional IGLC were also transcribed. IGLC6S1/7S1 was predominantly used in both breeds. These two constant region genes could not be distinguished due to high sequence similarity. Isotypes IGLC1S1 and IGLC5S1 revealed significantly higher transcription frequencies than IGLC4S1 in RGC and showed significant usage differences in HW. New allotypic variants were observed for IGLC1S1, IGLC5S1 and IGLC6S1/7S1 (Hara et al., 2012; Tallmadge et al., 2014).

The constant gene usage was also investigated in fetal spleen, neonatal, foal, and adult horse mesenteric lymph node tissues (Tallmadge et al., 2014). In equine fetuses, IGLC1S1, IGLC4S1 and IGLC5S1 were expressed. This feature changed in neonates, where IGLC1S1 and IGLC7S1 were transcribed. Both of these IGLC were still expressed in foal and adult horses but they also showed IGLC4S1. IGLC1S1, solely 
Chapter 2: Equine immunoglobulins and organization of immunoglobulin genes

found in all four developmental stages, was not the predominantly used constant region gene.

Furthermore, the usage and diversity of the equine IGLV was analyzed in fetal spleen and mesenteric lymph nodes of neonates, foals, and adult horses (Tallmadge et al., 2014). Analysis of the transcribed IGLV revealed usage of 13 IGLV in equine fetuses; 14 IGLV were used in neonates; 11 IGLV were identified in foals; and 9 IGLV were expressed in adult horses. Out of 27 functional genomic IGLVs, 18 different were identified in this study. In addition, the pseudogene IGLV8S10P (Table 4) was also transcribed, as it had already been observed in Hanoverian Warmblood and RhenishGerman Coldblood horses (Hara et al., 2012). Interestingly, five sequences of equine fetus and neonate were not assigned to the currently defined germline IGLVs. These new IGLVs belonged to subgroups 4, 6, and 8, whereas the already known expressed IGLVs belonged to subgroups 2, 3, and 11. Within each age group, subgroup 8 was the most prevalent. Furthermore, the first allelic variants in horse IGLV were identified. Nine IGLV genes were found in only one developmental stage and 5 IGLV genes were identified in all life stages investigated. More and varying IGLVs were used during equine fetal life than in later stages. Nucleotide diversity increased significantly with time (Tallmadge et al., 2014).

\section{New allotypic variants of IGLC}

It is well known today that immunoglobulin light chains increase antibody variability and contribute to antigen binding. In addition to the use of non-functional variable gene segments due to gene conversion, genes coding for the constant regions also influence antibody diversity (Emorine et al., 1983; Moxley and Gibbs, 1992). Allotypic variants from allels of IGHCs and IGLCs originate from single nucleotide polymorphisms (SNPS) and amino acid residue substitutions. Resulting epitope variability can improve immune responses as already shown for human and cattle. Allotypic markers of human light chains were associated with the susceptibility of infectious diseases caused by Plasmodium falciparum malaria, Haemophilus influenza, and Meningococcus polysaccharides for example (Giha et al., 2009; Granoff et al., 1984; Pandey et al., 1979). For allotypes of the bovine IgG2, correlations regarding complement activation, age-dependent expression, and influences on the effector function were reported in 
binding of Haemophilus somnus immunoglobulin binding protein (IgBP) and cleavage by Tritrichomonas foetus extracellular cysteine proteinase (Bastida-Corcuera et al., 2000; Bastida-Corcuera et al., 1999a; Bastida-Corcuera et al., 1999c; Corbeil et al., 1997). Allelic variations of the bovine light chain have been described for different cattle breeds (Diesterbeck et al., 2012; Stein et al., 2012). Similarly, the constant region genes of the $\lambda$-light chains of the horse breeds RGC and HW showed five allotypic IGLC1S1 variants (IGLC1S1 ${ }^{\text {a-e }}$ ), four allotypic IGLC5S1 variants (IGLC5S1 ${ }^{\text {a-d }}$ ), and three allelic, as well as two allotypic IGLC6S1/7S1 variants (IGLC6S1/7S1 ${ }^{\text {a1-a3,b). In }}$ RGC, IGLC1S1 $1^{\mathrm{b}, \mathrm{d}}$, IGLC5S1 $1^{\mathrm{c}, \mathrm{d}}$ and IGLC6S1/7S1 ${ }^{\mathrm{a} 3, \mathrm{~b}}$ were observed, whereas IGLC1S1 ${ }^{\mathrm{c}}$ and IGLC5S1 ${ }^{\mathrm{b}}$ were detected in HW. Amino acid residue substitutions were identified inside the molecule, as well as on the solvent accessible surface. They were shown by homology-based 3D modeling. Conservative amino acid residue substitutions as well as changes of charge or hydrophobicity were identified. Replacements adjacent to the interface and within the interface to IGHC1 were supposed to potentially influence the stable connection between both the heavy and light chains (Hara et al., 2012). These investigations of the Ig lambda constant region genes in adult horses of two different breeds were complemented by the analyses of the Ig lambda joining gene segments in combination with the constant genes at different equine developmental stages (Tallmadge et al., 2014). In fetuses, the usage of germline IGLJ1S1-IGLC1S1, IGLJ4S1 ${ }^{(P)}$-IGLC4S1 and IGLJ5S1-IGLC5S1 was found. New allelic variants were identified for IGLJ1S1, IGLC1S1, IGLJ4S1 ${ }^{(P)}$ and IGLC4S1. The previously described isotype IGLC5S $1^{\text {b }}$ was also expressed. Neonatal sequences showed the transcription of IGLJ1S1-IGLC1S1, as well as IGLJ7S1IGLC7S1, whereas there were two allelic variants for IGLJ7S1-IGLC7S1. The same allelic IGLJ7S1-IGLC7S1 variants were also expressed in addition to one version of IGLJ1S1-IGLC1S1 in neonates. Both in foals and adult horses, IGLJ1S1-IGLC1S1, IGLJ4S1 ${ }^{(P)}$-IGLC4S1, and IGLJ7S1-IGLC7S1 were transcribed. Two alleles were identified for IGLC7S1 in both developmental stages. IGLC4S1 revealed two allelic versions in foal. Only IGLC1S1 was detected at all four life stages, but it was not the most frequently used gene at any stage (Tallmadge et al., 2014). 
Chapter 2: Equine immunoglobulins and organization of immunoglobulin genes

\section{Future Directions}

Since the late $19^{\text {th }}$ century, horse antibodies in terms of anti-sera were closely connected with the medication of human diseases. Today equine antibody applications come more and more to the fore in order to prevent or treat equine infectious diseases. Amongst others they can be regarded as potential alternatives to antibiotic therapy in the near future.

Starting with the germline repertoire, it is, therefore, necessary to investigate the fundamental immunoglobulin genetics underlying the equine immunoglobulin immune response.

Recent analyzes already revealed individual and breed specific differences in the genomic fundament, as well as the gene usage at discrete life stages and an increased variability throughout life (Hara et al., 2012; Sun et al., 2010; Tallmadge et al., 2014; Tallmadge et al., 2013). However, more detailed studies are required to substantiate allelic or haplotypic differences for both variable and constant region genes and link them to individuals or breeds. Such detailed maps of the germline repertoire are currently available for human and mice. In vitro analyses of bovine IgG already determined the influences of genetic variations on the immune response (BastidaCorcuera et al., 2000; Bastida-Corcuera et al., 1999a; Bastida-Corcuera et al., 1999c; Corbeil et al., 1997). Similar studies are still missing in horses as well as analyzes of their in vivo impact.

Subsequent investigations in genetic, structural, and configurational properties of equine immunoglobulins might offer opportunities for the development of new antibodybased immunotherapeutics. For instance, recombinant antibodies or antibody-fragment related products are among the fastest growing new therapeutics worldwide (Dübel, 2010). However, only few engineered species-specific antibodies have been developed against veterinary pathogens (Koti et al., 2014). The most promising outcome from latest equine immunoglobulin research might be the construction of tailor-designed antibodies based on the format of single chain fragments variable (scFv). In these antibodies VH and VL domains are linked together by, e.g., a (Gly $\left.{ }_{4} \mathrm{Ser}\right)_{3}$ linker. When naïve or immunized immunoglobulin libraries are constructed for this reason by PCR amplification with well established primer sets for the equine $\mathrm{VH}$ and $\mathrm{VL}$ domains, highly affine and neutralizing recombinant scFv molecules can be assembled by phage display against any desired viral, bacterial, and parasitic antigenic site, as well as toxins. To elongate the short half-life time and clearance of scFvs in vivo, and to 
achieve effector functions, such as opsonization, complement-dependent cytotoxicity (CDC), and antibody-dependent cellular cytotoxicity (ADCC), equine scFv-molecules can be enhanced in the same way as established for human antibodies to scFvFCGRT (FcRn) fusion proteins (Kontermann, 2011; Koti et al., 2014) or to complete $\lg G$ antibodies through genetic manipulation.

Meanwhile, X-ray crystal structure analyses of antigen/antibody binding are increasing (Saini et al., 1999; Saini and Kaushik, 2002; Wang et al., 2013) and together with immunoglobulin sequencing data, even from high-throughput next generation sequencing, they will give valuable information on antigen-/antibody interactions. Subsequent amino acid replacement, especially in the CDR-regions, may contribute to enhanced binding efficiency of equine recombinant antibody molecules.

Consequently, future investigations of the basic biology once might lead into translation of new prophylaxis and treatment options of diseases.

\section{Acknowledgments}

We are grateful to Lynne Riddles for completing the critical review of this manuscript. 
Chapter 2: Equine immunoglobulins and organization of immunoglobulin genes

\section{References}

Almagro, J.C., Hernandez, I., Ramirez, M.C., Vargas-Madrazo, E., 1998. Structural differences between the repertoires of mouse and human germline genes and their evolutionary implications. Immunogenetics 47, 355e363.

Alt, F., Rosenberg, N., Lewis, S., Thomas, E., Baltimore, D., 1981. Organization and reorganization of immunoglobulin genes in A-MULV-transformed cells: rearrangement of heavy but not light chain genes. Cell 27, 381e390.

Arun, S.S., Breuer, W., Hermanns, W., 1996. Immunohistochemical examination of light-chain expression (lambda/kappa ratio) in canine, feline, equine, bovine and porcine plasma cells. Zent. Vet. A 43, 573e576.

Bastida-Corcuera, F., Butler, J.E., Heyermann, H., Thomford, J.W., Corbeil, L.B., 2000. Tritrichomonas foetus extracellular cysteine proteinase cleavage of bovine $\lg \mathrm{G} 2$ allotypes. J. Parasitol. 86, 328e332.

Bastida-Corcuera, F.D., Butler, J.E., Yahiro, S., Corbeil, L.B., 1999a. Differential complement activation by bovine IgG2 allotypes. Vet. Immunol. Immunopathol. $71,115 \mathrm{e} 123$.

Bastida-Corcuera, F.D., Nielsen, K.H., Corbeil, L.B., 1999c. Binding of bovine IgG2a and IgG2b allotypes to protein A, protein $G$, and Haemophilus somnus IgBPs. Vet. Immunol. Immunopathol. 71, 143e149.

Behring von, E., 1918. Epidemiologie, Aetiologie und Bek€ampfung der Diphtherie. In: Beiträge zur experimentellen Therapie. Springer.

Bingel, A., 1918. Über Behandlung der Diphtherie mit gew€ohnlichem Pferdeserum. F. C. W. Vogel, Leipzig.

Breathnach, C.C., Clark, H.J., Clark, R.C., Olsen, C.W., Townsend, H.G., Lunn, D.P., 2006. Immunization with recombinant modified vaccinia Ankara (rMVA) constructs encoding the HA or NP gene protects ponies from equine influenza virus challenge. Vaccine 24, 1180e1190.

Brodeur, P.H., Riblet, R., 1984. The immunoglobulin heavy chain variable region (IghV) locus in the mouse. I. One hundred Igh-V genes comprise seven families of homologous genes. Eur. J. Immunol. 14, 922e930.

Burnett, R.C., Hanly, W.C., Zhai, S.K., Knight, K.L., 1989. The IgA heavy-chain gene family in rabbit: cloning and sequence analysis of $13 \mathrm{C}$ alpha genes. EMBO J. 8, $4041 \mathrm{e} 4047$.

Butler, J.E., Sun, X., Wertz, N., Lager, K.M., Chaloner, K., Urban Jr., J., Francis, D.L., Nara, P.L., Tobin, G.J., 2011. Antibody repertoire development in fetal and neonatal piglets XXI. Usage of most VH genes remains constant during fetal and postnatal development. Mol. Immunol. 49, 483e494.

Butler, J.E., Wertz, N., Deschacht, N., Kacskovics, I., 2009. Porcine IgG: structure, genetics, and evolution. Immunogenetics $61,209 \mathrm{e} 230$.

Calmette, A., 1896. Venin des Serpents. Physiologie de I'Envenimation Traitment des Morsures Venimeuses par le Seárum des Animaux Vaccineás. Socieáte ád`Eáditions Scienti ques, Paris.

Civardi, G., Curadi, M.C., Orlandi, M., Cattaneo, M., Giangiacomo, R., 2002. Capillar electrophoresis (CE) applied to analysis of mare's milk. Milchwissenschaft 57, $515 \mathrm{e} 517$.

Cole, R., Moore, H.F., 1917. The production of Antipneumococcic serum. J. Exp. Med. $26,537 e 561$.

Corbeil, L.B., Gogolewski, R.P., Kacskovics, I., Nielsen, K.H., Corbeil, R.R., Morrill, J.L., Greenwood, R., Butler, J.E., 1997. Bovine IgG2a antibodies to Haemophilus somnus and allotype expression. Can. J. Vet. Res. 61, $207 e 213$. 
Chapter 2: Equine immunoglobulins and organization of immunoglobulin genes

Corthesy, B., 2007. Roundtrip ticket for secretory IgA: role in mucosal homeostasis? J. Immunol. 178, 27e32.

Daniels, J., 1921. Über die prophylaktische Wirkung des Diphtherieheilserums und des normalen antitoxinfreien Pferdeserums auf die Diphtherieinfektion des Meerschweinchens. Feller, Neubrandenburg.

Das, S., Nikolaidis, N., Klein, J., Nei, M., 2008. Evolutionary redefinition of immunoglobulin light chain isotypes in tetrapods using molecular markers. Proc. Natl. Acad. Sci. U. S. A. 105, 16647e16652.

Diesterbeck, U.S., Aboelhassan, D.M., Stein, S.K., Czerny, C.P., 2012. Detection of new allotypic variants of bovine lambda-light chain constant regions in different cattle breeds. Dev. Comp. Immunol. 36, 130e139.

Drogoul, C., Cl_ement, F., Ventorp, M., Curadi, M.C., Orlandi, M., 2008. Euine passive immune transfer trough colostrum. Horse Health Nutrition. In: 4th European Nutrition \& Health Congress, vol. 18e19, pp. 23 e27.

Dübel, S., 2010. Handbook of Therapeutic Antibodies. Weiley-Blackwell, Weinheim, Germany.

Edelman, G.M., 1973. Antibody structure and molecular immunology. Science 180, $830 \mathrm{e} 840$.

Eguchi-Ogawa, T., Wertz, N., Sun, X.Z., Piumi, F., Uenishi, H., Wells, K., Chardon, P., Tobin, G.J., Butler, J.E., 2010. Antibody repertoire development in fetal and neonatal piglets. XI. The relationship of variable heavy chain gene usage and the genomic organization of the variable heavy chain locus. J. Immunol. 184, $3734 \mathrm{e} 3742$.

Emorine, L., Dreher, K., Kindt, T.J., Max, E.E., 1983. Rabbit immunoglobulin kappa genes: structure of a germline b4 allotype J-C locus and evidence for several b4- related sequences in the rabbit genome. Proc. Natl. Acad. Sci. U. S. A. 80, 5709 e5713.

Ford, J.E., Home, W.A., Gibson, D.M., 1994. Light chain isotype regulation in the horse. Characterization of Ig kappa genes. J. Immunol. 153, 1099 e1111.

Franz, L.C., Landon, J.C., Lopes, L.A., Marinho, L.A., Sarma, C., Bruemmer, J., Squires, E.L., 1998. Oral and intravenous immunglobulin therapy in neonatal foals. Equine Vet. Sci. 18, $742 \mathrm{e} 748$.

Gibson, D., 1974. Structural studies on normal horse immunoglobulin light chains. Detection of k-type N-terminal sequences. Biochemistry 13, $2776 \mathrm{e} 2785$.

Giha, H.A., Nasr, A., Iriemenam, N.C., Arnot, D., Troye-Blomberg, M., Theander, T.G., Berzins, K., ElGhazali, G., Pandey, J.P., 2009. Antigen-specific influence of $\mathrm{GM} / \mathrm{KM}$ allotypes on $\mathrm{IgG}$ isotypes and association of GM allotypes with susceptibility to Plasmodium falciparum malaria. Malar. J. 8, 306.

Giudicelli, V., Lefranc, M.P., 1999. Ontology for immunogenetics: the IMGTONTOLOGY. Bioinformatics 15, 1047e1054.

Glatman-Freedman, A., Casadevall, A., 1998. Serum therapy for tuberculosis revisited: reappraisal of the role of antibody-mediated immunity against Mycobacterium tuberculosis. Clin. Microbiol. Rev. 11, 514e532.

Granoff, D.M., Pandey, J.P., Boies, E., Squires, J., Munson Jr., R.S., Suarez, B., 1984. Response to immunization with Haemophilus influenzae type b polysaccharidepertussis vaccine and risk of Haemophilus meningitis in children with the $\mathrm{Km}(1)$ immunoglobulin allotype. J. Clin. Investig. 74, $1708 \mathrm{e} 1714$.

Guo, Y., Bao, Y.,Wang, H., Hu, X., Zhao, Z., Li, N., Zhao, Y., 2011. A preliminary analysis of the immunoglobulin genes in the african elephant (Loxodonta africana). PLoS One 6, e16889. 
Chapter 2: Equine immunoglobulins and organization of immunoglobulin genes

Guo, Y., Bao, Y., Meng, Q., Hu, X., Meng, Q., Ren, L., Li, N., Zhao, Y., 2012. Immunoflobulin genomics in the guinea pig (Cavia porcellus). PLoS One 7, e39298.

Haas, S.D., Bristol, F., Card, C.E., 1996. Risk factors associated with the incidence of foal mortality in an extensively managed mare herd. Can. Vet. J. 37, 91e95.

Hara, S., Diesterbeck, U.S., Konig, S., Czerny, C.P., 2012. Transcriptional analysis of equine lambda-light chains in the horse breeds Rhenish-German Coldblood and Hanoverian Warmblood. Vet. Immunol. Immunopathol. 145, 50e65.

Hawgood, B.J., 1999. Doctor Albert Calmette 1863e1933: founder of antivenomous serotherapy and of antituberculous BCG vaccination. Toxicon 37, 1241e1258.

Helms, C.M., Allen, P.Z., 1970. Studies on equine immunoglobulins, II. Antigenic interrelationships among horse $\lg \mathrm{G}(\mathrm{T})$ and antipneumococcal gamma-1component. J. Immunol. 105, $1253 \mathrm{e} 1263$.

Hill, W.C., Cebra, J.J., 1965. Horse anti-SI immunoglobulins. I. Properties of gammaM-antibody. Biochemistry 4, $2575 \mathrm{e} 2584$.

Holznagel, D.L., Hussey, S., Mihalyi, J.E., Wilson, W.D., Lunn, D.P., 2003. Onset of immunoglobulin production in foals. Equine Vet. J. 35, 620e622.

Home, W.A., Ford, J.E., Gibson, D.M., 1992. L chain isotype regulation in horse. I. Characterization of Ig lambda genes. J. Immunol. 149, 3927 e3936.

Jenvey, C., Caraguel, C., Howarth, G.B., Riley, C.B., 2012. Identification of periparturient mare and foal associated predictors of post parturient immunoglobulin A concentrations in thoroughbred foals. Equine Vet. J. Suppl. $73 e 77$.

Jeong, H.D., Komisar, J.L., Kraig, E., Teale, J.M., 1988. Strain-dependent expression of VH gene families. J. Immunol. 140, $2436 \mathrm{e} 2441$.

Kabat, E.A.,Wu, T.T., 1991. Identical V region amino acid sequences and segments of sequences in antibodies of different specificities. Relative contributions of $\mathrm{VH}$ and VL genes, minigenes, and complementarity-determining regions to binding of antibody-combining sites. J. Immunol. 147, 1709e1719.

Keggan, A., Freer, H., Rollins, A., Wagner, B., 2013. Production of seven monoclonal equine immunoglobulins isotyped by multiplex analysis. Vet. Immunol. Immunopathol. 153, 187e193.

Klinman, N.R., Rockey, J.H., Karush, F., 1965. Equine antihapten antibody li. The gamma-G(7s-gamma) components and their specific interaction. Int. J. Cancer Suppl. 47, 51e60.

Kohn, C.W., Knight, D., Hueston,W., Jacobs, R., Reed, S.M., 1989. Colostral and serum $\lg$, IgA, and $\lg M$ concentrations in Standardbred mares and their foals at parturition. J. Am. Vet. Med. Assoc. 195, 64e68.

Kontermann, R.E., 2011. Strategies for extended serum half-life of protein therapeutics. Curr. Opin. Biotechnol. 22, 868e876.

Koterba, A.M., Brewer, B.D., Tarplee, F.A., 1984. Clinical and clinicopathological characteristics of the septicaemic neonatal foal: review of 38 cases. Equine Vet. J. 16, 376e382.

Koti, M., Kataeva, G., Kaushik, A.K., 2010. Novel atypical nucleotide insertions specifically at $\mathrm{VH}-\mathrm{DH}$ junction generate exceptionally long $\mathrm{CDR} 3 \mathrm{H}$ in cattle antibodies. Mol. Immunol. 47, $2119 \mathrm{e} 2128$.

Koti, M., Saini, S.S., Sachan, A., Kaushik, A., 2014. Engineered bovine antibodies in the development of novel therapeutics, immunomodulators and vaccines. Antibodies 3, 205e214.

Kydd, J.H., Townsend, H.G., Hannant, D., 2006. The equine immune response to equine herpesvirus-1: the virus and its vaccines. Vet. Immunol. Immunopathol. $111,15 \mathrm{e} 30$. 
Chapter 2: Equine immunoglobulins and organization of immunoglobulin genes

Lang, J., Kamga-Fotso, L., Peyrieux, J.C., Blondeau, C., Lutsch, C., Forrat, R., 2000. Safety and immunogenicity of a new equine tetanus immunoglobulin associated with tetanus-diphtheria vaccine. Am. J. Trop. Med. Hyg. 63, 298 e305.

Leblanc, M.M., Tran, T., 1987. Relationship among colostral electrolytes, colostral IgG concentration and absortion of colostral IgG by foals. J. Reprod. Fertil. 35, $735 \mathrm{e} 736$.

LeBlanc, M.M., Tran, T., Baldwin, J.L., Pritchard, E.L., 1992. Factors that influence passive transfer of immunoglobulins in foals. J. Am. Vet. Med. Assoc. 200, $179 \mathrm{e} 183$.

Lefranc, M.P., 1998. IMGT (ImMunoGeneTics) locus on focus. A new section of experimental and clinical immunogenetics. Exp. Clin. Immunogenet. 15.

Lefranc, M.P., 2001a. IMGT, the international ImMunoGeneTics database. Nucleic Acids Res. 29, 207 e209.

Lefranc, M.P., 2001b. Nomenclature of the human immunoglobulin lambda (IGL) genes. Exp. Clin. Immunogenet. 18, $242 \mathrm{e} 254$.

Lefranc, M.P., 2007. WHO-IUIS nomenclature subcommittee for immunoglobulins and T cell receptors report. Immunogenetics 59, 899e902.

Leleu, X., Terriou, L., Duhamel, A., Moreau, A.S., Andrieux, J., Dupire, S., Coiteux, V., Berthon, C., Micol, J.B., Guieze, R., Facon, T., Bauters, F., 2006. Long-term outcome in acquired aplastic anemia treated with an intensified dose schedule of horse antilymphocyte globulin in combination with androgens. Ann. Hematol. 85, $711 \mathrm{e} 716$.

Lewis, M.J., Wagner, B., Woof, J.M., 2008. The different effector function capabilities of the seven equine $\operatorname{lgG}$ subclasses have implications for vaccine strategies. Mol. Immunol. 45, 818e827.

Lopez, A.M., Hines, M.T., Palmer, G.H., Alperin, D.C., Hines, S.A., 2002. Identification of pulmonary T-lymphocyte and serum antibody isotype responses associated with protection against Rhodococcus equi. Clin. Diagn. Lab. Immunol. 9, $1270 \mathrm{e} 1276$.

Macpherson, A.J., Gatto, D., Sainsbury, E., Harriman, G.R., Hengartner, H., Zinkernagel, R.M., 2000. A primitive T cell-independent mechanism of intestinal mucosal IgA responses to commensal bacteria. Science 288, 2222e2226.

McGuire, T.C., Crawford, T.B., 1972. Identification and quantitation of equine serum and secretory immunoglobulin A. Infect. Immun. 6, 610e615.

McGuire, T.C., Crawford, T.B., 1973. Passive immunity in the foal: measurement of immunoglobulin classes and specific antibody. Am. J. Vet. Res. 34, 1299e1303.

Mestecky, J., Jackson, S., Moldoveanu, Z., Nesbit, L.R., Kulhavy, R., Prince, S.J., Sabbaj, S., Mulligan, M.J., Goepfert, P.A., 2004. Paucity of antigen-specific IgA responses in sera and external secretions of HIV-type 1-infected individuals. AIDS Res. Hum. Retrovir. 20, 972e988.

Moxley, G., Gibbs, R.S., 1992. Polymerase chain reaction-based genotyping for allotypic markers of immunoglobulin kappa shows allelic association of $\mathrm{Km}$ with kappa variable segment. Genomics 13, 104e108.

Navarro, P., Barbis, D.P., Antczak, D., Butler, J.E., 1995. The complete cDNA and deduced amino acid sequence of equine IgE. Mol. Immunol. 32, 1 e8.

Nelson, K.M., Schram, B.R., McGregor, M.W., Sheoran, A.S., Olsen, C.W., Lunn, D.P., 1998. Local and systemic isotype-specific antibody responses to equine influenza virus infection versus conventional vaccination. Vaccine 16, $1306 \mathrm{e} 1313$.

Oethinger, M., Wand-Wurttenberger, A., Hengel, H., 1997. Mirkobiologie und Immunologie. Gustav Fischer Verlag, Ulm. 
Overesch, G.,Wagner, B., Radbruch, A., Leibold,W., 1998. Organisation of the equine immunoglobulin constant heavy chain genes. II. Equine cgamma genes. Vet. Immunol. Immunopathol. 66, $273 \mathrm{e} 287$.

Pahud, J.J., Mach, J.P., 1972. Equine secretory IgA and secretory component. Int. Arch. Allergy Appl. Immunol. 42, 175e186.

Pandey, J.P., Fudenberg, H.H., Virella, G., Kyong, C.U., Loadholt, C.B., Galbraith, R.M., Gotschlich, E.C., Parke Jr., J.C., 1979. Association between immunoglobulin allotypes and immune responses to Haemophilus influenzae and Meningococcus polysaccharides. Lancet 1, 190e192.

Reynaud, C.A., Anquez, V., Dahan, A., Weill, J.C., 1985. A single rearrangement event generates most of the chicken immunoglobulin light chain diversity. Cell 40, $283 \mathrm{e} 291$.

Rockey, J.H., 1967. Equine antihapten antibody. The subunits and fragments of antibeta-lactoside antibody. J. Exp. Med. 125, 249 e275.

Rockey, J.H., Klinman, N.R., Karush, F., 1964. Equine antihapten antibody. I. 7s Beta2a- and 1os Gamma-1- globulin components of purified anti-beta-lactoside antibody. J. Exp. Med. 120, 589e609.

Saini, S.S., Allore, B., Jacobs, R.M., Kaushik, A., 1999. Exceptionally long CDR3H region with multiple cysteine residues in functional bovine $\lg M$ antibodies. Eur. J. Immunol. 29, $2420 \mathrm{e} 2426$.

Saini, S.S., Kaushik, A., 2002. Extensive CDR3H length heterogeneity exists in bovine foetal VDJ rearrangements. Scand. J. Immunol. 55, $140 \mathrm{e} 148$.

Sandor, G., Korach, S., Mattern, P., 1964a. 7 S globulin, immunologically identical to $19 \mathrm{~S}$ gamma-1-(beta-2-)-M-globulin, a new protein of horse serum. Nature 204, $795 \mathrm{e} 796$.

Schrenzel, M.D., King, D.P., McKnight, M.L., Ferrick, D.A., 1997. Characterization of horse (Equus caballus) immunoglobulin mu chain-encoding genes. Immunogenetics 45, 386e393.

Sedlinska, M., Krejci, J., Vyskocil, M., Kudiackova, H., 2006. Postnatal development of blood serum concentrations of immunoglobulin $\lg G, \lg A$ and $\lg M$ isotypes of suckling foals. Acta Vet. Brno 75, $175 \mathrm{e} 182$.

Sheoran, A.S., Holmes, M.A., 1996. Separation of equine IgG subclasses (IgGa, IgGb and $\lg G(T)$ ) using their differential binding characteristics for staphylococcal protein A and streptococcal protein G. Vet. Immunol. Immunopathol. 55, 33e43.

Sheoran, A.S., Sponseller, B.T., Holmes, M.A., Timoney, J.F., 1997. Serum and mucosal antibody isotype responses to M-like protein (SeM) of Streptococcus equi in convalescent and vaccinated horses. Vet. Immunol. Immunopathol. 59, $239 \mathrm{e} 251$.

Shojaei, F., Saini, S.S., Kaushik, A.K., 2003. Unusually long germline DH genes contribute to large sized CDR3H in bovine antibodies. Mol. Immunol. 40, 61 e67.

Souza, C.M., Miotto, B.A., Bonin, C.P., Camargo, M.M., 2010. Lower serum IgA levels in horses kept under intensive sanitary management and physical training. Animal 4, 2080e2083.

Stein, S.K., Diesterbeck, U.S., Aboelhassan, D.M., Czerny, C.P., 2012. Comparison of joining and constant kappa-light chain regions in different cattle breeds. Anim. Genet. 43, 776e780.

Sun, Y., Wang, C., Wang, Y., Zhang, T., Ren, L., Hu, X., Zhang, R., Meng, Q., Guo, Y., Fei, J., Li, N., Zhao, Y., 2010. A comprehensive analysis of germline and expressed immunoglobulin repertoire in the horse. Dev. Comp. Immunol. 34, 1009 e1020.

Suter, M., Fey, H., 1981. Isolation and characterization of equine IgE. Zent. Vet. B 28, $414 \mathrm{e} 420$. 
Tallmadge, R.L., McLaughlin, K., Secor, E., Ruano, D., Matychak, M.B., Flaminio, M.J., 2009. Expression of essential B cell genes and immunoglobulin isotypes suggests active development and gene recombination during equine gestation. Dev. Comp. Immunol. 33, 1027 e1038.

Tallmadge, R.L., Tseng, C.T., Felippe, M.J., 2014. Diversity of immunoglobulin lambda light chain gene usage over developmental stages in the horse. Dev. Comp. Immunol. 46, 171e179.

Tallmadge, R.L., Tseng, C.T., King, R.A., Felippe, M.J., 2013. Developmental progression of equine immunoglobulin heavy chain variable region diversity. Dev. Comp. Immunol. 41, 33e43.

Theakston, R.D., Warrell, D.A., Griffiths, E., 2003. Report of a WHO workshop on the standardization and control of antivenoms. Toxicon 41, 541e557.

Tonegawa, S., 1983. Somatic generation of antibody diversity. Nature 302, 575e581.

Turtinen, L.W., Allen, G.P., 1982. Identification of the envelope surface glycoproteins of equine herpesvirus type 1. J. Gen. Virol. 63, 481e485.

Vaerman, J.P., Querinjean, P., Heremans, J.F., 1971. Studies on the IgA system of the horse. Immunology $21,443 e 454$.

Wagner, B., 2006. Immunoglobulins and immunoglobulin genes of the horse. Dev. Comp. Immunol. 30, 155e164.

Wagner, B., Greiser-Wilke, I., Antczak, D.F., 2003. Characterization of the horse (Equus caballus) IGHA gene. Immunogenetics 55, 552e560.

Wagner, B., Greiser-Wilke, I., Wege, A.K., Radbruch, A., Leibold, W., 2002. Evolution of the six horse IGHG genes and corresponding immunoglobulin gamma heavy chains. Immunogenetics 54, 353e364.

Wagner, B., Miller, D.C., Lear, T.L., Antczak, D.F., 2004. The complete map of the Ig heavy chain constant gene region reveals evidence for seven IgG isotypes and for IgD in the horse. J. Immunol. 173, 3230e3242.

Wagner, B., Miller, W.H., Morgan, E.E., Hillegas, J.M., Erb, H.N., Leibold, W., Antczak, D.F., 2006. IgE and IgG antibodies in skin allergy of the horse. Vet. Res. 37, $813 \mathrm{e} 825$.

Walther, S., Czerny, C.P., Diesterbeck, U.S., 2013. Exceptionally long CDR3H are not isotype restricted in bovine immunoglobulins. PLoS One 8, e64234.

Wang, F., Ekiert, D.C., Ahmad, I., Yu, W., Zhang, Y., Bazirgan, O., Torkamani, A., Raudsepp, T., Mwangi, W., Criscitiello, M.F., Wilson, I.A., Schultz, P.G., Smider, V.V., 2013. Reshaping antibody diversity. Cell 153, $1379 \mathrm{e} 1393$.

Weir, R.C., Porter, R.R., Givol, D., 1966. Comparison of the C-terminal amino-acid sequence of two horse immunoglobulins $\lg G$ and $\lg G(T)$. Nature 212, $205 \mathrm{e} 206$.

Widders, P.R., Stokes, C.R., Bourne, F.J., 1986. Investigation of the antigenic relationship between equine IgG and IgGT. Vet. Immunol. Immunopathol. 13, $255 \mathrm{e} 259$.

Wilson, I.B., Gavel, Y., von Heijne, G., 1991. Amino acid distributions around O-linked glycosylation sites. Biochem. J. 275 (Pt 2), 529e534.

Winau, F.,Winau, R., 2002. Emil von Behring and serum therapy. Microbes Infect. 4, $185 \mathrm{e} 188$.

Zand, M.S., 2006. B-cell activity of polyclonal antithymocyte globulins. Transplantation 82, 1387e1395.

Zhao, Y., Kacskovics, I., Pan, Q., Liberles, D.A., Geli, J., Davis, S.K., Rabbani, H., Hammarstrom, L., 2002. Artiodactyl IgD: the missing link. J. Immunol. 169, $4408 \mathrm{e} 4416$.

Zhao, Y., Kacskovics, I., Rabbani, H., Hammarstrom, L., 2003. Physical mapping of the bovine immunoglobulin heavy chain constant region gene locus. J. Biol. Chem. $278,35024 \mathrm{e} 35032$. 
Chapter 2: Equine immunoglobulins and organization of immunoglobulin genes

Zolla, S., Goodman, J.W., 1968. An aggregating immunoglobulin in hyperimmune equine anti-pneumococcal sera. J. Immunol. 100, 880e897. 


\section{Transcriptional analysis of equine $\lambda$-light chains in the horse breeds Rhenish-German Coldblood and Hanoverian Warmblood}

Stefanie Hara ${ }^{1}$, Ulrike S. Diesterbeck ${ }^{1 *}$, Sven König $^{2}$, Claus-Peter Czerny ${ }^{1}$

1Department of Animal Sciences, Institute of Veterinary Medicine, Division of Microbiology and Animal Hygiene, Faculty of Agricultural Sciences, Georg-August University Göttingen, Burckhardtweg 2, 37077 Göttingen, Germany

${ }^{2}$ Department of Animal Breeding, University of Kassel, Nordbahnhofstr. 1a, 37213

Witzenhausen, Germany

${ }^{*}$ Corresponding author:

U. S. Diesterbeck

Tel.: +49551393375

Fax: +495513913513

E-mail: udieste@gwdg.de

Postal address: Dr. Ulrike Diesterbeck

Department of Animal Sciences,

Georg-August University Göttingen

Burckhardtweg 2

37077 Göttingen, Germany

Published in "Veterinary Immunology and Immunopathology"

DOI: $10.1016 /$ j.vetimm.2011.10.006

URL: http://www.sciencedirect.com/science/article/pii/S0165242711004132 


\begin{abstract}
The present study analyzed equine $\lambda$-light chain genes (IGLV and IGLC) transcribed in the horse breeds Rhenish-German Coldblood (RGC) and Hanoverian Warmblood (HW). Primers were generated for the major expressed IGLV subgroup 8. The significant majority of the sequences represented IGLC6/7. In RGC, IGLC1 and IGLC5 were observed in significant higher frequencies than IGLC4. In HW, significant differences were obtained for the transcription of IGLC1 and IGLC5. IGLC4 was not determined in this breed. Five allotypic IGLC1 variants, four allotypic IGLC5 variants,

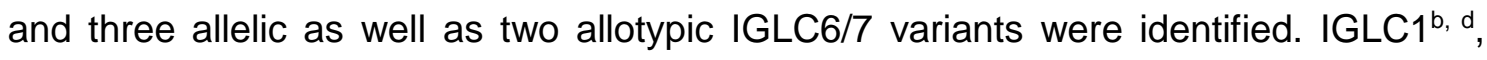
IGLC5 ${ }^{\mathrm{c}, \mathrm{d}}$, and IGLC6/7 ${ }^{\mathrm{a} 3, \mathrm{~b}}$ were detected in RGC while IGLC1 ${ }^{\mathrm{c}}$ and IGLC5 ${ }^{\mathrm{b}}$ were solely found in HW. Furthermore, 11 out of 144 known IGLV-segments were transcribed of which IGLV15 and IGLV17 were preferred significantly. IGLV25 displayed significant differences in the rearrangement between both breeds. The classified pseudogenes IGLV101 $\Psi$ and IGLV74 $\Psi$ were also identified. Rearrangements with IGLC-genes showed significant differences for IGLV15 in both breeds, whereas IGLV25 also revealed significant differences between the breeds. The transcriptional orientation of the functional segments has no influence on the occurrence of the IGLV.
\end{abstract}

\title{
Keywords
}

Equus caballus; horse; immunoglobulin; immunoglobulin light chains; allotype 


\section{Introduction}

In jawed vertebrates, immunoglobulins (Ig) are usually described as a Y-shaped tetramer composed of two identical heavy chains (IGH) and two identical light chains (IGL) (Edelman, 1973). Both chains are comprised of variable and constant domains. During differentiation of B-lymphocytes they are joined together by combinatorial assembly of separate heavy and light chain variable $\left(\mathrm{V}_{\mathrm{H}+\mathrm{L}}\right)$, diversity $\left(\mathrm{D}_{H}\right)$, joining $\left(\mathrm{J}_{\mathrm{H}+\mathrm{L}}\right)$, and constant $\left(\mathrm{C}_{\mathrm{H}+\mathrm{L}}\right)$ germline components (Tonegawa, 1983). While IGH are encoded by a single locus, mammals express 2 isotypes of IGL, which are called kappa ( $\mathrm{K}$ ) and lambda $(\lambda)$ (Korngold and Lipari, 1956). In comparison to IGH, evolution of IGL seems to be more complex. Sequences similar to $\mathrm{K}$ light chains described in different species, such as mammals and fish, gave rise to this assumption (Sitnikova and Su, 1998). In Xenopus laevis an additional IGL of $\sigma$-isotype is present (Klein et al., 2002). The light chain isotypes can be distinguished by specific conserved amino acid motifs as well as by distinct species specific usage frequencies (Das et al., 2008). For instance, in cattle, sheep, and horses $\lambda$-light chains are predominantly expressed (Arun et al., 1996; Home et al., 1992). This isotype occurs in more than $90 \%$ of horse serum antibodies (Gibson, 1974).

In several genetic investigations equine IGH constant regions (IGHC) as well as the germline $\mathrm{V}_{\mathrm{H}}, \mathrm{D}_{\mathrm{H}}$, and $\mathrm{J}_{\mathrm{H}}$ segments were mapped to Equus caballus autosome (ECA) 24 and the genomic scaffold UN0011. Eleven IGHC, $50 \mathrm{~V}_{\mathrm{H}}, 40 \mathrm{D}_{\mathrm{H}}$, and eight $\mathrm{J}_{\mathrm{H}}$ segments were described (Sun et al., 2010; Wagner, 2006). The genomic organization of equine IGL is characterized by 144 variable $\lambda$-light chain segments (IGLV) and 60 variable $\mathrm{K}$-light chain segments (IGKV). While IGKV and IGKJ-segments are organized, as it is the case, for example, in humans and mice, IGLV-segments are distributed into two clusters located up- and downstream of the IGLJ-IGLC-cluster (Kawasaki et al., 2001; Roschenthaler et al., 2000; Sun et al., 2010). The genetic information for equine $\lambda$-light chains was found to be located on ECA8. Genes for equine $\mathrm{k}$-light chains are located on ECA15. The number of genes is supposed to be responsible for the dominant $\lambda$-light chain expression (Almagro et al., 2006; Sun et al., 2010). 112 IGLV-pseudogenes $(I G L V \Psi)$ were described for the $\lambda$-locus (Sun et al., 2010). Beside IGLV, seven IGLC were described for the equine genomic $\lambda$-locus. Each was preceded by an IGLJ-segment. Genomic analyses revealed that three IGLC were functional, while in transcriptional analyses four IGLC were determined to be functional (Das et al., 2008; Sun et al., 2010). 
In general, light chains enlarge antibody variability and contribute to antigen binding. Both can be enhanced by mechanisms of somatic hypermutation and gene conversion (Arakawa and Buerstedde, 2009; Parng et al., 1996; Winstead et al., 1999; Zhou et al., 2004). Beside gene conversion utilizing nonfunctional V-gene segments, genes for the constant regions can also contribute to antibody diversity (Emorine et al., 1983; Moxley and Gibbs, 1992). Allotypic variants emerging from alleles of the constant regions of IGH and IGL as well as the resulting epitope variability can improve immune responses (Bastida-Corcuera et al., 2000; Bastida-Corcuera et al., 1999a; Bastida-Corcuera et al., 1999b; Oli et al., 2004). Similar allelic variations of the light chains have already been described for different cattle breeds (Diesterbeck et al., 2012) but not yet for horses. In addition, no structural analyses of the inter-domain interface between the first constant IGH and IGL regions are available. Comparable data were obtained by analyses of the crystal structures of two murine and two human Fabs (Padlan et al., 1986).

We investigated two horse breeds (Rhenish-German Coldblood, RGC, and Hanoverian Warmblood, HW) with different stud book sizes and breeding goals. Our interest was a comparative analysis of IGLC sequences and the identification of different alleles and putative allotypes. The locations of allotype-specific amino acid residue substitutions within the molecule were examined using comparative 3D modeling with known crystal structures. Furthermore, the usage frequency and rearrangement of IGLV-segments were examined for comparison of the $\lambda$-light chain repertoire in HW and RGC. 
Chapter 2: Transcriptional analysis of equine lambda light chains

\section{Material and methods}

\subsection{Breed selection, isolation of lymphocytes and cDNA-synthesis}

For the analysis of breed specific expression of the immunoglobulin $\lambda$-light chain the equine breeds Rhenish-German Coldblood (RGC) and Hanoverian Warmblood (HW) were chosen as these breeds differ in stud book size and breeding goals. In RGC the stud book is closed resulting in a true-breeding population. For HW the stud book is open and the breeding concept is more flexible (Hartmann et al., 2006).

Peripheral blood samples were collected from 10 randomly chosen animals per breed. All animals of one breed were located in one stable. Peripheral blood mononuclear cells (PBMC) were isolated from $20 \mathrm{ml}$ EDTA-blood using Ficoll gradients (GE Healthcare Europe $\mathrm{GmbH}$, Germany) according to the manufacturer's protocol. After staining with trypan blue viable PBMC were counted and total RNA was extracted from $1 \times 10^{7}$ cells by using the RNeasy ${ }^{\circledR}$ Mini Kit (QIAGEN, Germany). For synthesis of cDNA from $3 \mu \mathrm{g}$ of total RNA in a total volume of $20 \mu \mathrm{l} \mathrm{pd}(\mathrm{N})_{6}$-primers were used (First-Strand cDNA Synthesis Kit, GE Healthcare Europe GmbH, Germany).

\subsection{PCR amplification of the expressed immunoglobulin $\lambda$-light chain repertoire}

Complete $\lambda$-light chains were amplified using primers binding in the 5'UTR (elgL_5'UTR_for: 5'-GCCACAGAAGGCAGGACTCGG-3') and the 3'UTR (elgL_3'UTR_rev1: 5'-AGGGGTCCCGTGACAGCAGG-3'). Primer elgL_5'UTR_for hybridizes at positions 2-22 in the reference sequence L07565 (Home et al., 1992). Primer elgL_3'UTR_rev is complementary to positions 764-783 in the same reference sequence. The expected product size of the $\lambda$-light chain including leader, variable, and constant region was $782 \mathrm{bp}$. By using the elgL_5'UTR_for primer, subgroup 8-IGLV were amplified.

A total reaction volume of $50 \mu \mathrm{l}$ contained $2 \mu \mathrm{l}$ of cDNA, $200 \mu \mathrm{M}$ dNTPs (Bioline, Germany), $5 \mu$ of $10 x$ PCR buffer (75 mM Tris $\mathrm{HCl} \mathrm{pH} \mathrm{9.0;} 2 \mathrm{mM} \mathrm{MgCl}_{2} ; 50 \mathrm{mM} \mathrm{KCl}$; $\left.20 \mathrm{mM}\left(\mathrm{NH}_{4}\right)_{2} \mathrm{SO}_{4}\right), 0.4 \mu \mathrm{M}$ of each primer, and 2 units DNA polymerase (Biotools, Spain). Thermal cycling was carried out at $95^{\circ} \mathrm{C}$ for $5 \mathrm{~min}, 95^{\circ} \mathrm{C}$ for $1 \mathrm{~min}, 65^{\circ} \mathrm{C}$ for 1 $\min , 72^{\circ} \mathrm{C}$ for $2 \min$ for 35 cycles with initial denaturation at $95^{\circ} \mathrm{C}$ for 5 min and terminal elongation at $72^{\circ} \mathrm{C}$ for $10 \mathrm{~min}$. The length and purity of the PCR products was evaluated by agarose gel electrophoresis. 
Chapter 2: Transcriptional analysis of equine lambda light chains

\subsection{PCR amplification of the germline IGLJ-IGLC pairs}

To examine the presence of all predicted germline IGLJ-IGLC pairs, they were amplified from genomic DNA, which was isolated from EDTA-blood (QIAamp ${ }^{\circledR}$ DNA Blood Mini Kit, QIAGEN, Germany). Primers for the functional and transcribed IGLC were based on the breed specific sequence information derived from the analyses of the expressed $\lambda$-light chains. To generate primers for nonfunctional IGLC, sequences from Acc. No. NW_001867428.1 were used as reference. The IGLJ-IGLC pair specific primers are shown in Table 1 including sequences, start and stop positions within the reference as well as product sizes. For IGLJ5-IGLC5 and IGLJ7-IGLC7 identical reverse primers were used. IGLJ6-IGLC6 was not amplified particularly because only one difference to IGLJ7-IGLC7 concerning one nucleotide could be identified. Product size varies from $1614 \mathrm{bp}$ to $1870 \mathrm{bp}$.

\section{Table 1}

Primers for amplification of germline IGLJ-IGLC pairs. Binding positions for primers amplifiying IGLJ1-IGLC1 to IGLJ7-IGLC7 and referring to NW_001867428.1 in the equine genome assembly EquCab2.0.

\begin{tabular}{|c|c|c|c|}
\hline Primers & $\begin{array}{l}\text { Sequences } \\
5^{4}>3^{4}\end{array}$ & $\begin{array}{l}\text { Product } \\
\text { Size }\end{array}$ & Binding positions \\
\hline $\begin{array}{l}\text { elgL_J1-C1L_for } \\
\text { elgL_J1-C1L_rev }\end{array}$ & $\begin{array}{c}\text { AGAACACTCTGAGGGGGACAGT } \\
\text { TGAGACTCAGTCACCGTGGT }\end{array}$ & 1870 bp & $\begin{array}{l}4144485 \ldots 4144506 \\
4146335 \ldots 4146355\end{array}$ \\
\hline $\begin{array}{l}\text { elgL_J2-C2L_for } \\
\text { elgL_J2-C2L_rev }\end{array}$ & $\begin{array}{c}\text { CCTCTGAGGGGGACACTTTCT } \\
\text { TGTGTCACAGTGTTCATAGTTCAC }\end{array}$ & 1667 bp & $\begin{array}{l}4139378 \ldots 4139399 \\
4141021 \ldots 4141045 \\
\end{array}$ \\
\hline $\begin{array}{l}\text { elgL_J3-C3L_for } \\
\text { elgL_J3-C3L_rev }\end{array}$ & $\begin{array}{c}\text { AGCACACTCTGAGGGGGACACTTT } \\
\text { CTGTGTCACTGTGTCCTGTGC }\end{array}$ & 1614 bp & $\begin{array}{l}4133976 \ldots 4134000 \\
4135569 \ldots 4135590\end{array}$ \\
\hline $\begin{array}{l}\text { elgL_J4-C4L_for } \\
\text { elgL_J4-C4L_rev }\end{array}$ & $\begin{array}{c}\text { AAGGACACTCTGAGGGGGACAGT } \\
\text { TGCTGTATTCGGCGGAGGCAC }\end{array}$ & 1743 bp & $\begin{array}{l}4130704 \ldots 4130727 \\
4132426 \ldots 4132447\end{array}$ \\
\hline $\begin{array}{l}\text { elgL_C5L_for } \\
\text { elgL_J7-C5L_rev }\end{array}$ & $\begin{array}{l}\text { TTCCACTCTGTGGAGGTCCGTG } \\
\text { ACCTGACCATCGCAGGTGAGTC }\end{array}$ & 1635 bp & $\begin{array}{l}4127429 \ldots 4127450 \\
4129063 \ldots 4129042\end{array}$ \\
\hline $\begin{array}{l}\text { elgL_C7L_for } \\
\text { elgL_J7-C5L_rev }\end{array}$ & $\begin{array}{c}\text { TTCCACTGTGCGGAAGTCCGC } \\
\text { ACCTGACCATCGCAGGTGAGTC }\end{array}$ & 1637 bp & $\begin{array}{l}4120613 \ldots 4120633 \\
4122249 \ldots 4122228\end{array}$ \\
\hline
\end{tabular}

A total reaction volume of $50 \mu \mathrm{l}$ contained $2 \mu \mathrm{l}$ of cDNA, $200 \mu \mathrm{M}$ dNTPs (Bioline, Germany), $5 \mu$ of 10x PCR buffer (75 mM Tris- $\mathrm{HCl}$ pH 9.0; $2 \mathrm{mM} \mathrm{MgCl}_{2} ; 50 \mathrm{mM} \mathrm{KCl}$; $\left.20 \mathrm{mM}\left(\mathrm{NH}_{4}\right)_{2} \mathrm{SO}_{4}\right), 3 \%$ of DMSO; $0.4 \mu \mathrm{M}$ of each primer, and 2 units of DNA polymerase (Biotools, Spain). Thermal cycling was performed at $95^{\circ} \mathrm{C}$ for $5 \mathrm{~min}, 95^{\circ} \mathrm{C}$ for $1 \mathrm{~min}, 60^{\circ} \mathrm{C}$ for $1 \mathrm{~min}, 72^{\circ} \mathrm{C}$ for $2 \mathrm{~min}$ for 35 cycles with initial denaturation at $95^{\circ} \mathrm{C}$ for $5 \mathrm{~min}$ and terminal elongation at $72^{\circ} \mathrm{C}$ for $10 \mathrm{~min}$. Length and purity of the PCR products were evaluated by agarose gel electrophoresis. 
Chapter 2: Transcriptional analysis of equine lambda light chains

\subsection{Purification and cloning of PCR products}

For reasons of purity and amount, PCR-products were either purified by gel extraction or they were concentrated. For gel extraction the MiniElute Gel Extraction Kit was used (QIAGEN, Germany) according to the manufacturer's protocol. The QG buffer was replaced by a QX1 buffer. Samples were eluted with $13 \mu \mathrm{l}$ of $10 \mathrm{mmol} \mathrm{Tris} / \mathrm{HCl}$; $\mathrm{pH}$ 8.5. To increase the concentration of the PCR-products the DNA Clean \& Concentrator ${ }^{\mathrm{TM}}$ Kit was used (Zymo Research Corporation, CA92867 U.S.A) in accordance to the manufacturer's protocol. Purified products were sub-cloned into the $\mathrm{pCR}^{\circledR} 2.1-\mathrm{TOPO}^{\circledR}$ $3.9 \mathrm{~kb}$ TA vector and transformed into chemically competent One Shot TOP $10 \mathrm{E}$. coli cells (Invitrogen ${ }^{\mathrm{TM}}$, Germany). After blue-white selection, 12 randomly chosen white transformants, which had been generated individually from each horse, were grown in $5 \mathrm{ml}$ LB-ampicillin broth. Plasmid isolation occurred using a MiniPrep Kit (QIAGEN, Germany). In order to verify the correct insert size, plasmids were cleaved with EcoRI (New England Biolabs, Germany).

\subsection{Sequencing and sequence analysis of PCR products}

A definite number of 12 clones per animal were sequenced according to the chain-termination method (Sanger et al., 1977). The M13 (-20) Forward and M13 Reverse (Invitrogen, Germany) vector specific primers as well as the corresponding gene specific primers elgL_5'UTR_for/ elgL_3'UTR_rev were used for sequencing. The sequences were analyzed with the DNAstar program (GATC Biotech AG, Germany) and aligned by ClustalW (Thompson et al., 1994) to the nucleotide sequences from the genomic IGLV and IGLC published by Sun et al. (2010). The nomenclature for the transcribed IGLV and IGLC corresponds to this reference. The IMGT numbering system was used for numbering of amino acid residues (Lefranc et al., 2005). In order to determine the different isotypes, specific amino acid residue motifs were identified. Unique features in IGLC1 are SWK at positions 40-42, KSSSSV at positions 97-102, and VTH at positions 106-108. IGLC6/7 can only be distinguished from IGLC5 due to the combination of special motifs. In IGLC6/7 STPS, DAVTT, and TRTSAQW are characteristic at positions 1.1-3, 45-45.4, and 90-96. In contrast, IGLC5 has SAPS, DAVTN, and TRTSTEW at these positions. Identities between IGLC5 and IGLC4 are KSYSSV and VKH (positions 97-102 and 106-108). IGLC4 and IGLC1 share SWK, GAATT, and PLTPTQW at positions 40-42, 45-45.4, and 90-96. In addition, phylogenetic trees as well as sequence identity of at least $80 \%$ were used for grouping the transcribed with the genomic IGLC and IGLV. New alleles of IGLC were defined if 
Chapter 2: Transcriptional analysis of equine lambda light chains

substitutions were detected in at least two clones sequenced from one animal. Furthermore, these substitutions had to occur in at least two animals in order to exclude sequencing errors derived from reverse transcriptase and DNA polymerase amplification. Nucleotide substitutions found in more than four sequences of one animal were also defined as new allele. All alleles were compared with the equine ESTs (expressed sequence tags) database (gp/9796.11760/9796_est; 37199 sequences) using BLASTN. Breed information derived from sequences with $100 \%$ coverage and identity were also considered. In sequences received from the two horse breeds RGC and HW combinations of IGLV with IGLC-isotypes were analyzed with regard to occurrence and frequency.

\subsection{Homology-based modeling of the $\lambda$-constant region}

The amino acid sequences of the transcribed IGLC were deduced from the corresponding nucleotide sequences. By using the PHYRE server (Kelley and Sternberg, 2009) the protein sequences were aligned with known crystal structures of $\lambda$-light chains. Due to this alignment the generation of a three-dimensional (3D) model was possible. The analysis included the identification of amino acid residue positions which putatively form the connection to the constant region of the heavy chain according to Padlan et al. (1986) and the position of detected amino acid residue substitutions within the 3D-molecule. Graphical analyses and figure preparation were compiled with vmd (Humphrey et al., 1996). To demonstrate the accessible surface area, additional $1.4 \AA$ were added to the radii of van der Waals forces (Lee and Richards, 1971).

\subsection{Statistical analyses}

Frequencies of individual IGLC and IGLV genes as well as combinations of both within one breed and among the breeds have been compared by applying non-parametric tests. Such test procedures, i.e. the $\mathrm{Chi}^{2}$-test for analyzing IGLC and IGLV independently and Fisher's exact test for the gene segment $x$ breed contingency table, are implemented in the software package SAS, Version 9.2.

The association between the count variable usage frequency of IGLV and the effects of transcriptional orientation including either all classified IGLV or only the putative functional IGLV was analyzed separately by breed as well as by combining both breeds via mixed model theory. The association study was carried out by defining the usage frequency of IGLV as a dependent variable as well as by modelling transcriptional 
orientation and functionality as fixed effects. Application of linear mixed models considering both fixed and random effects assumes a Gaussian distribution for the variable to be analyzed, whereas the count variable usage frequency of IGLV follows a Poisson-like distribution. Hence, as an extension of linear mixed models a generalized linear mixed model (GLMM), that can be used to analyze data with a Poisson-like distribution, was used for this specific part of the statistical analysis. The main feature of a GLMM is a link function that allows the mean of a population to depend on a linear predictor. The link function $f_{i}$ between the linear predictor ${ }_{i}$ and the observations $y_{i}$ used for these type of count data was a log link defined as $f_{i}=\log _{e}\left({ }_{i}\right)$. The statistical model was defined as follows:

$$
\log \left[y_{r s t}\right]=\eta_{r}=\varphi+\gamma_{s}+\delta_{t}
$$

where $y_{r s t}=$ value for usage frequency of IGLV $r ; \varphi=$ overall mean effect; $\gamma_{s}=$ fixed effect of transcriptional orientation $s ; \delta_{t}=$ fixed effect of functionality $t$. 
Chapter 2: Transcriptional analysis of equine lambda light chains

\section{Results}

\subsection{Amplification of germline IGLJ-IGLC pairs}

To examine the presence of all predicted germline IGLJ-IGLC pairs, specific primers were generated. For functional IGLJ-IGLC pairs, primers based on the sequences of the transcribed $\lambda$-light chain repertoire (IGLJ1-IGLC1, IGLJ4-IGLC4, IGLJ5-IGLC5, IGLJ6/7-IGLC6/7). The genomic contig NW_001867428.1 was used for primer generation of the nonfunctional IGLJ2-IGLC2 and IGLJ3-IGLC3 pairs. Nomenclature of the IGLC-genes refers to Sun et al. (2010). DNA of two RGC animals were not available. All six IGLJ-IGLC pairs were amplified in two RGC animals and one HW animal. IGLJ1-IGLC1 was demonstrated in all RGC animals as well as in eight animals of the breed HW. IGLJ2-IGLC2 was proven in all animals analyzed in RGC, whereas in HW eight animals showed the presence of this IGLJ-IGLC pair. IGLJ3-IGLC3 was amplified in five animals of the breed RGC and in six HW animals, respectively. IGLJ4-IGLC4, which seemed to be nonfunctional due to a single C-insertion within the IGLJ4 exon (Sun et al., 2010), was demonstrated in eight and seven animals of RGC and HW. In four RGC as well as in five HW animals IGLJ5-IGLC5 was amplified. IGLJ6/7-IGLC6/7 was amplified in all RGC and HW animals analyzed. IGLJ6 and IGLJ7 as well as IGLC6 and IGLC7 were not distinguished, because primer hybridization sites were identical due to the high sequence similarities.

\subsection{Transcriptional use of IGLC-genes}

A total of 12 sequences per animal were analyzed to establish potential differences in usage frequencies of IGLC-genes in the breeds RGC and HW. IGLC were differentiated by specific amino acid residue motifs. The analysis of 120 sequences per breed revealed that IGLC-genes were transcribed in different frequencies (Fig. 1a). The isotype IGLC6/7 was predominantly used in both horse breeds examined and was identified in 55\% ( $n=66)$ of the 120 sequences analyzed in each breed. IGLC5 had a lower frequency in RGC $(n=25,20.8 \%)$ than in HW ( $n=42,35.0 \%)$. Twenty-seven sequences $(22.5 \%)$ of RGC were classified to IGLC1, whereas in HW 12 sequences (10\%) were identified as IGLC1. IGLC4 was transcribed in two RGC animals (1.7\%), but not in the breed HW. In the breed RGC highly significant differences $(P<0.001)$ in the transcription were confirmed by the Chi test between IGLC1 and IGLC4, IGLC1 and IGLC6/7, IGLC4 and IGLC5, IGLC4 and IGLC6/7, as well as IGLC5 and IGLC6/7. The same $P$-values were obtained for the statistical comparison of IGLC1 and IGLC5, 
as well as IGLC1 and IGLC6/7 in HW. With $P \leq 0.05$ IGLC5 and IGLC6/7 were significantly different in this breed (Fig. 1a). Statistical analysis did not show significant differences of IGLC usage between the breeds.

In most of the animals more than one IGLC-gene was transcribed (Fig. 1b) but without a significant preference of a specific combination. The isotype combinations IGLC1/IGLC5/IGLC6/7 (four animals) as well as IGLC1/IGLC6/7 (three animals) were preferred in RGC. The combinations of IGLC1/IGLC4/IGLC5 and IGLC1/IGLC4/IGLC5/IGLC6/7 were exhibited by one RGC animal. Exclusive transcription of IGLC6/7 was detected in four animals of the breed HW but in only one RGC animal. Other IGLC-genes transcribed simultaneously were detected in two HW animals (IGLC5/IGLC6/7, IGLC1/IGLC5, IGLC1/IGLC5/IGLC6/7; Fig 1b). 


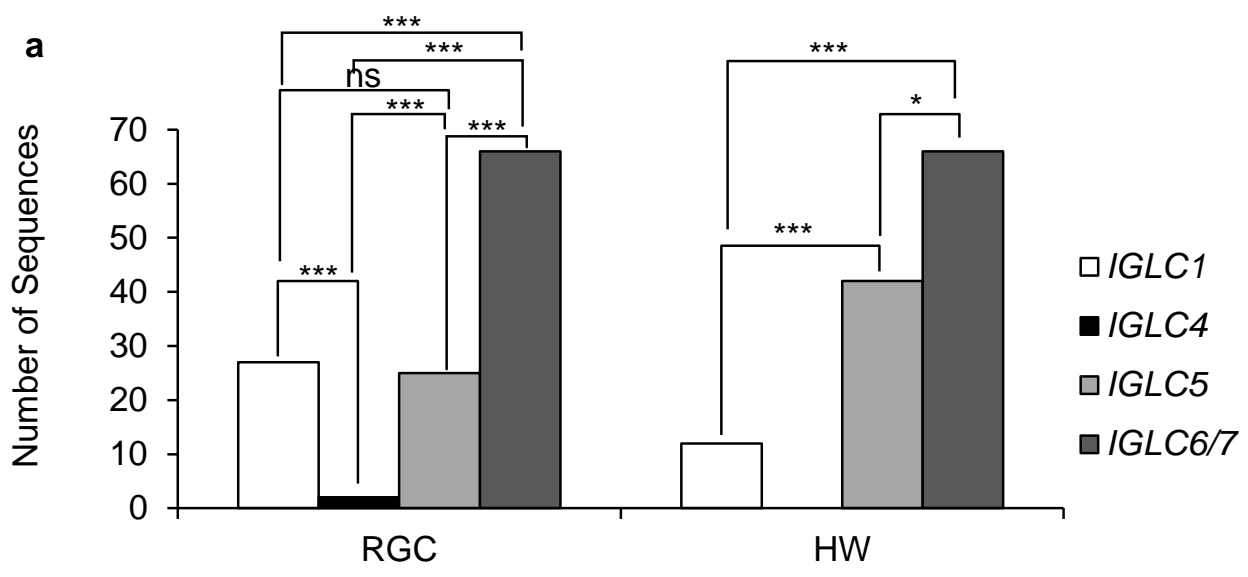

b

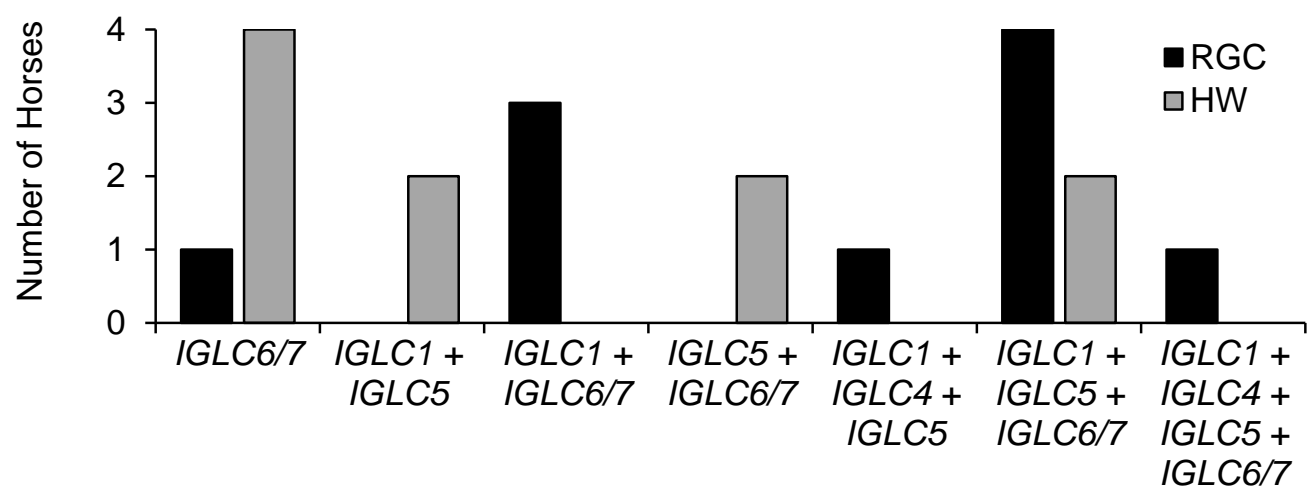

Fig. 1 Transcription of IGLC-genes in the horse breeds Rhenish-German Coldblood (RGC) and Hanoverian Warmblood (HW). a Usage frequency of IGLC-genes in RGC and HW. Significant differences between transcriptional frequency of the four IGLC are indicated by asterisks $\left({ }^{*} P \leq 0.05,{ }^{* *} P \leq 0.01,{ }^{* * *} P \leq 0.001\right)$. b Concurrently transcribed IGLC-genes in RGC- and HW animals. IGLC1, IGLC4, IGLC5, and IGLC6/7 describe immunoglobulin $\lambda$-light chain constant regions that were transcribed in this study. Nomenclature of the IGLC-genes corresponds to a previous study (Sun et al., 2010).

\subsection{Transcriptional use of IGLV-segments}

In analogy to the IGLC-genes, the 120 sequences available per breed were analyzed for transcriptional use of IGLV-segments. With a threshold of at least $80 \%$ sequence identity, all sequences were classified to 11 of 144 genomic IGLV-segments. Those 11 germline IGLV-segments were assigned to subgroup 8 of 11 known IGLV subgroups as previously described (Sun et al., 2010). All eleven IGLV-segments found were transcribed in RGC, whereas nine IGLV-segments were transcribed in HW (Fig. 2). 


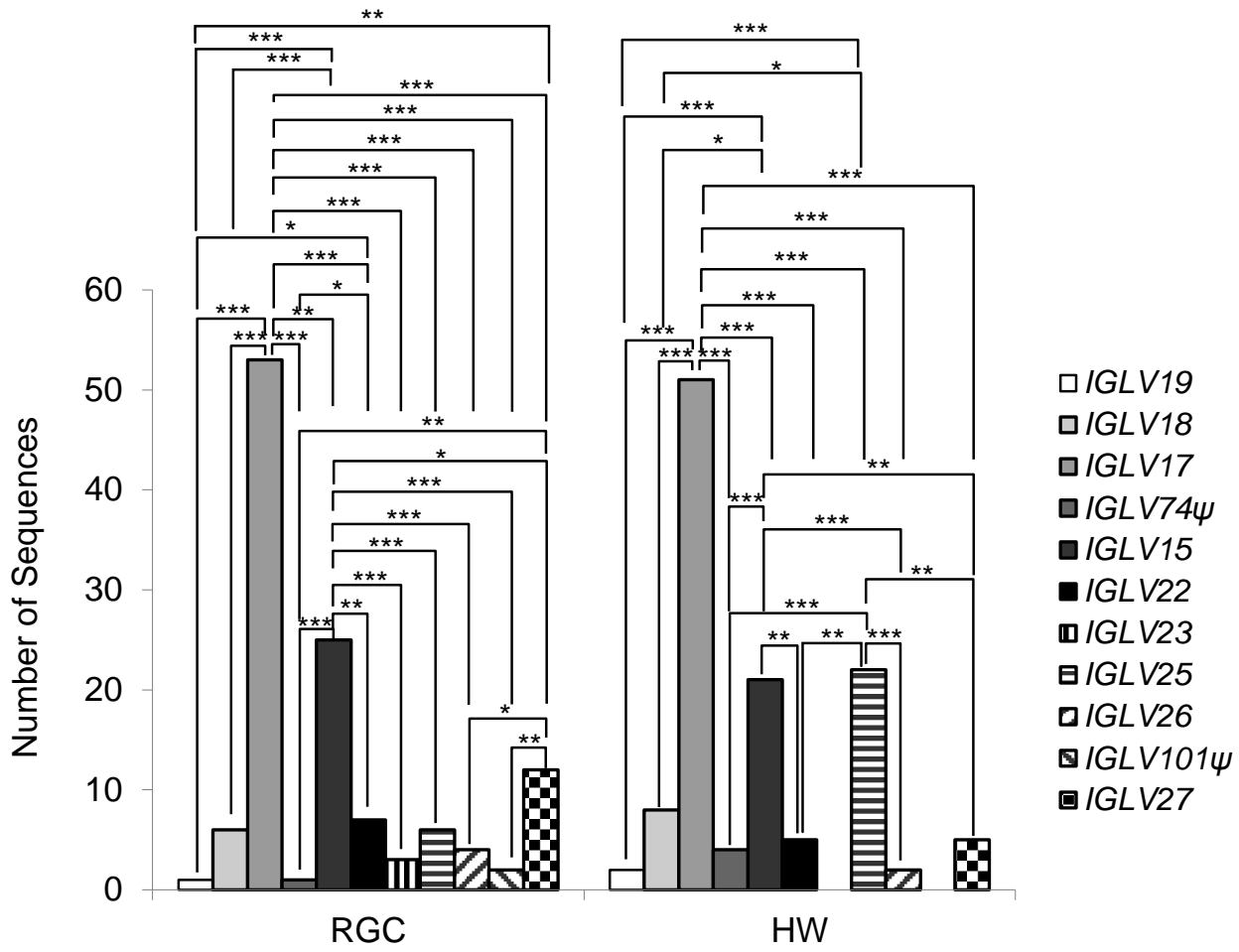

Fig. 2 Usage frequency of IGLV in Rhenish-German Coldblood (RGC) and Hanoverian Warmblood (HW) resulting from transcription analysis. IGLV describe the numbers of immunoglobulin $\lambda$-light chain variable gene segments as designated in a previous study (Sun et al., 2010). Significant differences between transcriptional frequency of the IGLV are indicated by asterisks $\left({ }^{*} P \leq 0.05,{ }^{* \star} P \leq 0.01,{ }^{* * *} P \leq 0.001\right)$. For IGLV25, a significant difference was calculated between the two breeds $(P=0.0233)$.

In both breeds examined, IGLV17 was rearranged preferably (RGC: $n=53,44.2 \%$; HW: $n=51,42.5 \%)$. Similarly, IGLV15 was used with high frequencies in RGC ( $n=25$, $20.8 \%)$ as well as in $\mathrm{HW}(n=21,17.5 \%)$. In both breeds the $\mathrm{Chi}^{2}$ statistics revealed significant differences of the transcriptional use of IGLV15 and IGLV17 in comparison to the other transcribed IGLVs. $P$-values $\leq 0.001$ were obtained for the transcription of IGLV17 compared to IGLV19, IGLV18, IGLV744, IGLV22, IGLV25, IGLV26, and IGLV27 within the both breeds examined. Comparing IGLV17 and IGLV15, we received a $P$-value $\leq 0.01$ for $R G C$ and $\leq 0.001$ for HW. Very high significances $(P \leq 0.001)$ were observed for the use IGLV15 in comparison to IGLV19, IGLV74 $\Psi$, and IGLV26 in RGC and HW animals. The same $P$-values were calculated for IGLV15 and IGLV23 as well as for IGLV15 and IGLV101 $\Psi$ in RGC. Differences in the significance level were obtained between IGLV15 and IGLV18 $\left(P_{\mathrm{HW}}=0.0004, P_{\mathrm{RGC}}=0.0015\right)$ and IGLV15 and IGLV27 ( $\left.P_{\mathrm{HW}}=0.0017, P_{\mathrm{RGC}}=0.0326\right)$. Beside these similarities in transcriptional use of IGLV15 and IGLV17, both breeds differed in usage of further 
Chapter 2: Transcriptional analysis of equine lambda light chains

IGLV-segments. While HW preferred IGLV25 as a third major IGLV-segment $(n=22$, 18.3\%), RGC favored IGLV27 ( $n=12,10 \%)$ as verified by $\mathrm{Chi}^{2}$. In HW significant differences $(P \leq 0.001)$ in transcription frequency were calculated for IGLV25 compared to IGLV19, IGLV74 $\Psi$, and IGLV26. Comparing IGLV25-IGLV18 ( $P \leq 0.05)$, IGLV22, and IGLV27 ( $P \leq 0.01)$ additional significant differences were calculated. In RGC IGLV27

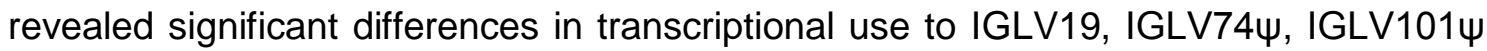
( $P \leq 0.01)$, IGLV23, and IGLV26 ( $P \leq 0.05)$. Statistical analysis further revealed significant differences in the expression of IGLV25 between the breeds $(P=0.0233)$.

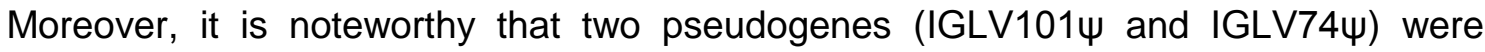
completely transcribed. For IGLV74 $\Psi$ we identified nucleotide variations in CDR1 sequences that started at nucleotide position 96 of IGLV and neutralized the stop codons. These nucleotide variations within CDR1 resulted in a frame shift by what the deduced amino acid sequence changed and replaced the first stop codon by $A$, while $\mathrm{S}, \mathrm{K}, \mathrm{I}$, or R substituted the second stop codon.

In order to describe the impact of the transcriptional orientation on the usage of IGLV-segments two assumptions were examined. The first calculation was based on all classified IGLV independently of their functionality. The second analysis included only the putative functional segments. Both statistical analysis showed that the F-ratios provided in the analysis of variance are identical to the Wald/rank(K) F-statistics as defined by Littell et al. (2004). Those F-statistics have been used to identify the impact of fixed effects on transcriptional orientation and functionality. For both breeds, RGC and $\mathrm{HW}$, the transcriptional orientation has no influence on the transcription frequency of the IGLV-segments.

\subsection{Rearrangement of IGLV-and IGLC-genes}

In order to investigate the $\lambda$-light chain repertoire in the breeds RGC and HW, we analyzed rearrangement frequencies of the transcribed IGLV- and IGLC-genes. In addition, we used non-parametric tests for the analysis of significant differences within the rearrangements in the breeds as well as between the two breeds. The most frequently transcribed IGLC6/7 was rearranged with all of the transcribed IGLV-segments. In RGC, IGLV19, IGLV18, IGLV744, and IGLV1014 were only combined with IGLC6/7 (Fig. 3a), while IGLV26 was combined solely with IGLC6/7 in HW (Fig. 3b). 


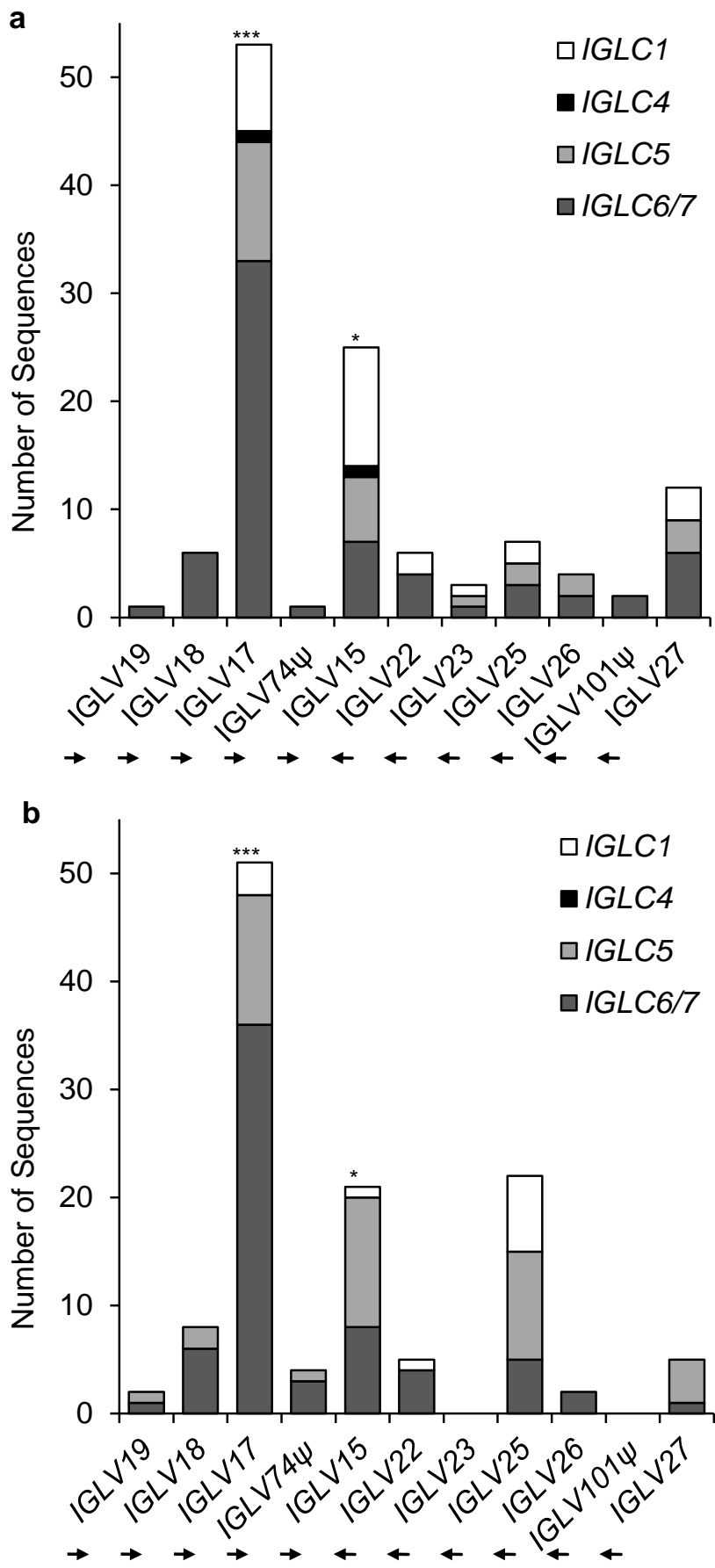

Fig. 3 Frequency of rearrangement of IGLV and IGLC genes in Rhenish-German Coldblood (RGC; a) and Hanoverian Warmblood (HW; b). IGLC1, IGLC4, IGLC5, and IGLC6/7 describe immunoglobulin $\lambda$-light chain constant regions that were transcribed in this study. IGLV describe the numbers of immunoglobulin $\lambda$-light chain variable gene segments as designated in a previous study (Sun et al., 2010). $\Psi$ indicates completely transcribed pseudogenes. Arrows below annotation of the horizontal axis designate transcriptional orientation of IGLV. Arrows pointing to the right hand side indicate the same transcriptional orientation as germline IGLJIGLC cluster. Asterisks show significant differences in IGLC usage within this IGLV $\left({ }^{*} P \leq 0.05\right.$, $\left.{ }^{*} P \leq 0.01,{ }^{* * *} P \leq 0.001\right)$. For IGLV15 there is a significant difference between the breeds $(P=0.011)$. 
Chapter 2: Transcriptional analysis of equine lambda light chains

Furthermore, in RGC IGLV22 and IGLV26 were rearranged either with IGLC1/IGLC6/7 or IGLC5/IGLC6/7, respectively. The IGLV-segments IGLV23, IGLV25, and IGLV27 were rearranged with IGLC1, IGLC5, and IGLC6/7, whereas in RGC IGLV15 and IGLV17 were rearranged with all four transcribed IGLC-genes, wherein in both IGLV IGLC4 was rearranged once (Fig. 3a). 44.0\% of IGLV15 sequences in RGC were rearranged with IGLC1, 24.0\% were joined to IGLC5, and $28.0 \%$ were joined to IGLC6/7. In HW IGLC5 was used preferentially (57.1\%) for recombination with IGLV15. IGLC6/7 (38.1\%) and IGLC1 (4.8\%) were recombined less frequently with IGLV15. Analyses of the sequence numbers showed significant differences $(P=0.011)$ of rearrangements of IGLV15 with the IGLC-genes between both breeds examined. These analyses also revealed significant differences of IGLC rearrangements within the breeds $\left(P_{\mathrm{RGC}}=0.0436 ; P_{\mathrm{HW}}=0.0119\right)$. IGLV17 preferred recombination with IGLC6/7 (RGC: 62.3\%; HW: 70.6\%) followed by IGLC5 (RGC: 20.8\%; HW: 23.5\%) and IGLC1 (RGC: $15.1 \%$; HW: $5.9 \%$ ). For IGLV17 statistical analyses showed highly significant differences of IGLC rearrangements within the two breeds examined $\left(P_{\mathrm{RGC}}<0.0001\right.$; $P_{\text {HW }}<0.0001$ ).

In comparison to RGC, in HW IGLV19, IGLV18, IGLV74 4 , IGLV22, and IGLV27 were rearranged with either IGLC1/IGLC6/7 or IGLC5/IGLC6/7, whereas IGLV17, IGLV15, and IGLV25 were combined with IGLC1, IGLC5, and IGLC6/7 (Fig. 3b).

\subsection{Allelic variants of IGLC1}

In the breeds analyzed, single nucleotide polymorphisms (SNPs) of IGLC1 were detected (Table 2, Fig. 4a). The analyses of the deduced amino acid sequences revealed five allotypic variants denominated as IGLC1 ${ }^{\mathrm{a}}$, IGLC1 ${ }^{\mathrm{b}}, \mid \mathrm{IGLC} 1^{\mathrm{c}}, \mathrm{IGLC}{ }^{\mathrm{d}}$, and IGLC1 ${ }^{\text {e }}$. Germline IGLC1 was set as allotype a. The corresponding sequence can be found in the contig NW_001867428.1 (nucleotides 4144483-4144802) of the equine genome assembly EquCab2.0. SNPs were compared to the positions within this exon. Deduced amino acid residue substitutions were referred to the IMGT nomenclature (Lefranc et al., 2005; Table 2, Fig. 4a). The homology-based modeling showed the closest model for all IGLC1 allotypic variants to the crystal structures of either Fab KOL or Fab NEW derived from human mAbs (IGLC1 $1^{\text {a, e }}$ PDB code 2FB4, IGLC1 ${ }^{\text {b-d }}$ PDB code 1AQK; Faber et al., 1998; Kratzin et al., 1989). Identities of at least $63 \%$ were calculated. 


\section{Table 2}

Allotypic variants of IGLC1. Nucleotide substitutions of allotypes are shown as SNP at corresponding nucleotide positions of IGLC. Resulting amino acid residue substitutions are shown as follows: initial amino acid residue, position, and replacing amino acid residue. Numbering of amino acid residue positions occurred in accordance with the IMGT numbering system for constant domains (Lefranc et al., 2005).

\begin{tabular}{|c|c|c|c|}
\hline Isotype & Allotype/ allele & Position: SNPa & $\begin{array}{c}\text { Position } \\
\text { amino acid } \\
\text { substitution }\end{array}$ \\
\hline \multirow{4}{*}{ IGLC1 } & $b$ & $\begin{aligned} & \text { 7: } C>A \\
& 10: A>C \\
& 146: T>C \\
& 149: G>C \\
& 156: A>C \\
& 189: T>C \\
& 201: G>A \\
& 204: T>C \\
& 217: A>C \\
& 229: G>A \\
& 230: C>G \\
& 316: T>C\end{aligned}$ & $\begin{array}{c}\mathrm{P} 1.3 \mathrm{~T} \\
\mathrm{~T} 1.2 \mathrm{P} \\
\text { I 45.3 T } \\
\text { S 45.4 T } \\
- \\
- \\
- \\
- \\
\text { T } 90 \mathrm{~S} \\
\text { A } 94 \mathrm{~S} \\
\mathrm{~S} 127 \mathrm{P}\end{array}$ \\
\hline & c & 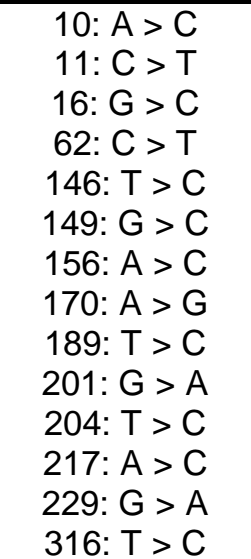 & $\begin{array}{c}\text { T } 1.2 \mathrm{~L} \\
\text { A } 1 \mathrm{P} \\
\text { A } 16 \mathrm{~V} \\
\text { I } 45.3 \mathrm{~T} \\
\text { S } 45.4 \mathrm{~T} \\
- \\
\text { K } 82 \mathrm{R} \\
- \\
- \\
- \\
\text { T } 90 \mathrm{P} \\
\text { A } 94 \mathrm{~T} \\
\text { S } 127 \mathrm{P}\end{array}$ \\
\hline & $d$ & $\begin{array}{l}\text { 16: } G>A \\
\text { 146: } T>C \\
\text { 149: } \rightarrow>C \\
\text { 156: } A>C \\
\text { 189: } T>C \\
\text { 201: } \rightarrow>A \\
204: T>C \\
217: A>C \\
\text { 229: } G>A \\
230: C>G \\
316: T>C\end{array}$ & $\begin{array}{c}\text { A } 1 \mathrm{~T} \\
\text { I } 45.3 \mathrm{~T} \\
\mathrm{~S} 45.4 \mathrm{~T} \\
- \\
- \\
- \\
- \\
\text { T } 90 \mathrm{~S} \\
\text { A } 94 \mathrm{~S} \\
\mathrm{~S} 127 \mathrm{P}\end{array}$ \\
\hline & e & $\begin{array}{l}\text { 149: } G>C \\
\text { 170: } A>G \\
217: A>C \\
\text { 229: } G>A \\
316: T>C\end{array}$ & $\begin{array}{c}\text { S 45.4 T } \\
\text { K } 82 \mathrm{R} \\
\text { T 90 P } \\
\text { A 94 T } \\
\text { S } 127 \mathrm{P}\end{array}$ \\
\hline
\end{tabular}

a Single nucleotide polymorphism. 
Chapter 2: Transcriptional analysis of equine lambda light chains

The allotypic variant IGLC1 ${ }^{\mathrm{b}}$ differed from IGLC1 $1^{\mathrm{a}}$ in two conservative as well as four non-conservative amino acid residue substitutions represented by $\mathrm{P} 1.3 \mathrm{~T}, \mathrm{~T} 1.2 \mathrm{P}$, S45.4T, T90S, A94S, and S127P which are located at the solvent accessible surface (Table 2, Fig. 4b). A fifth non-conservative amino acid residue substitution namely I45.3T was located inside the molecule. The adjoining residues 1.3 and 1.2 are located at the linking area to IGLV. At position 1.3 the hydrophobic $P$ was replaced by the hydrophilic T. Due to the reverse substitution at position 1.2 the polarity in this molecule part was converted. At position 94 the replacement of $A$ by $S$ also led to a polar side chain. Residue 90 was adjacent to the putative interface to $\mathrm{CH} 1$, whereas positions 45.4 and 94 were located at the outer side of the molecule as determined by homologybased 3D modeling (Fig. 4b). Another non-conservative amino acid residue substitution represented by S127P was located at the C-terminal end of IGLC1, leading to the replacement of the neutral and hydrophilic amino acid residue by the stabilizing heterocyclic and hydrophobic P. Altogether 12 sequences of five RGC horses were assigned to allotypic variant IGLC1 ${ }^{\mathrm{b}}$ (JN228100). Three of these five animals transcribed this allotype solely, two were heterozygous with IGLC1 ${ }^{d}$. In HW amino acid residue substitutions regarding $\mathrm{IGLC} 1^{\mathrm{b}}$ were not observed (Table 3 ). 


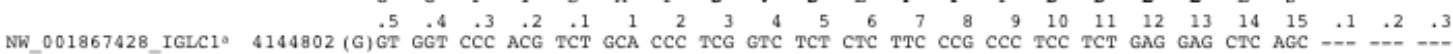
$\operatorname{IGLC1} 1^{b}$

IGLC $1^{\circ}$
IGLC $1^{d}$

IGLC1e

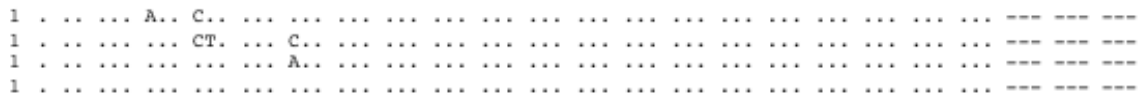

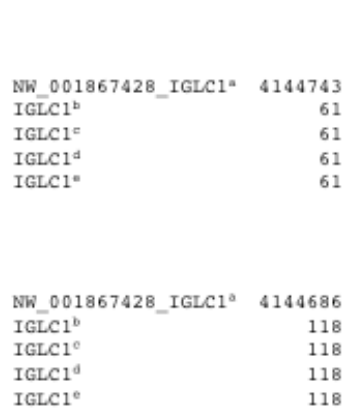

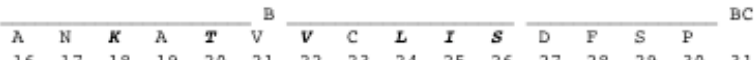

$\begin{array}{lllllllllllllllllllll}16 & 17 & 18 & 19 & 20 & 21 & 22 & 23 & 24 & 25 & 26 & 27 & 28 & 29 & 30 & 31 & 34 & 35 & 36 & 37 & 38\end{array}$

GCC AAC AAG GCC ACA GTG GTG TGT CTC ATC AGT GAC TTC TCC CCC -- --- AGC GAC TTG ACG $\begin{array}{lllllllllllllllllllll}\ldots & \ldots & \ldots & \ldots & \ldots & \ldots & \ldots & \ldots & \ldots & \ldots & \ldots & \ldots & \ldots & \ldots & \ldots & -- & -- & \ldots & \ldots & \ldots & \ldots\end{array}$

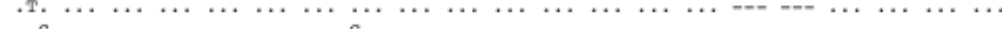

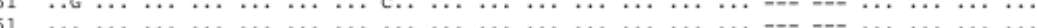

$\begin{array}{lr} & \\ \text { NW_001867428_IGLC1a } & 4144650 \\ \text { IGLC1b } & 154 \\ \text { IGLC1C } & 154 \\ \text { IGLC1d } & 154 \\ \text { IGLC1e } & 154\end{array}$

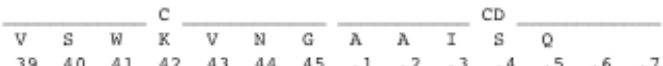

$\begin{array}{llllllllllllll}39 & 40 & 41 & 42 & 43 & 44 & 45 & .1 & .2 & .3 & .4 & .5 & .6 & .7\end{array}$

GGC GCC GCC ATC AGC CAG --- ---

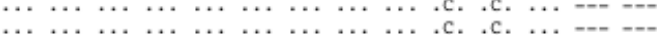

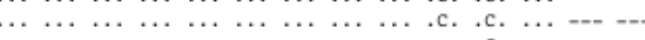

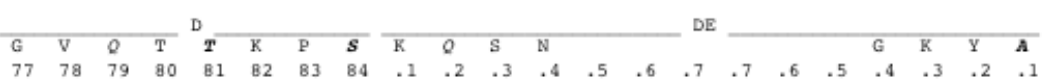

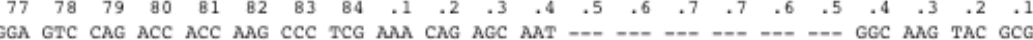

(1)

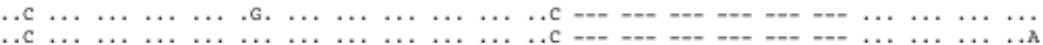

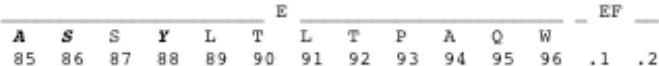

NW 001867428 IGLC1" 4144602 GCT AGC AGC TAC CTG ACG CTG ACC CCC GCC CAG TGG

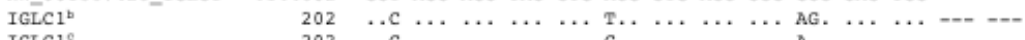

IGLC

IGLC1

$202 \quad \ldots$ c $\ldots \ldots \ldots \ldots \ldots \ldots$... $\ldots \ldots \ldots \ldots \ldots$ A.............

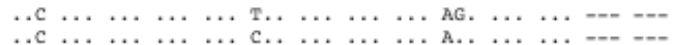

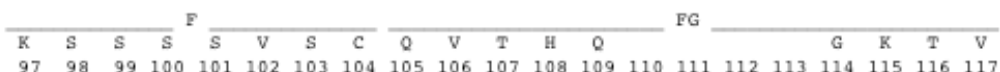

G

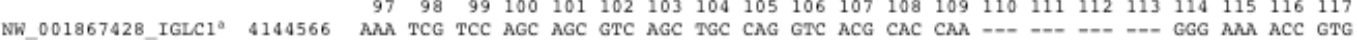

$\mathrm{IGLC1}{ }^{\mathrm{b}}$

IGLC $1^{\circ}$
IGLC10

IGLC1e

$\begin{array}{llllllllllllllllllll}\ldots & \ldots & \ldots & \ldots & \ldots & \ldots & \ldots & \ldots & \ldots & \ldots & \ldots & \ldots & \ldots & --- & -- & -- & \ldots & \ldots & \ldots & \ldots\end{array}$

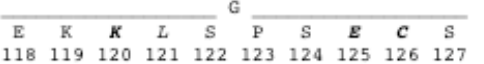

NW_001867428_IGLC1"4144515 GAG AAG AAA CTG TCC CCC TCA GAG TGT TCT TAG

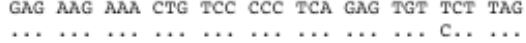

IGLC1

IGLC1 $1^{d}$

$\ldots \ldots \ldots \ldots$

IGLC1*

289

b
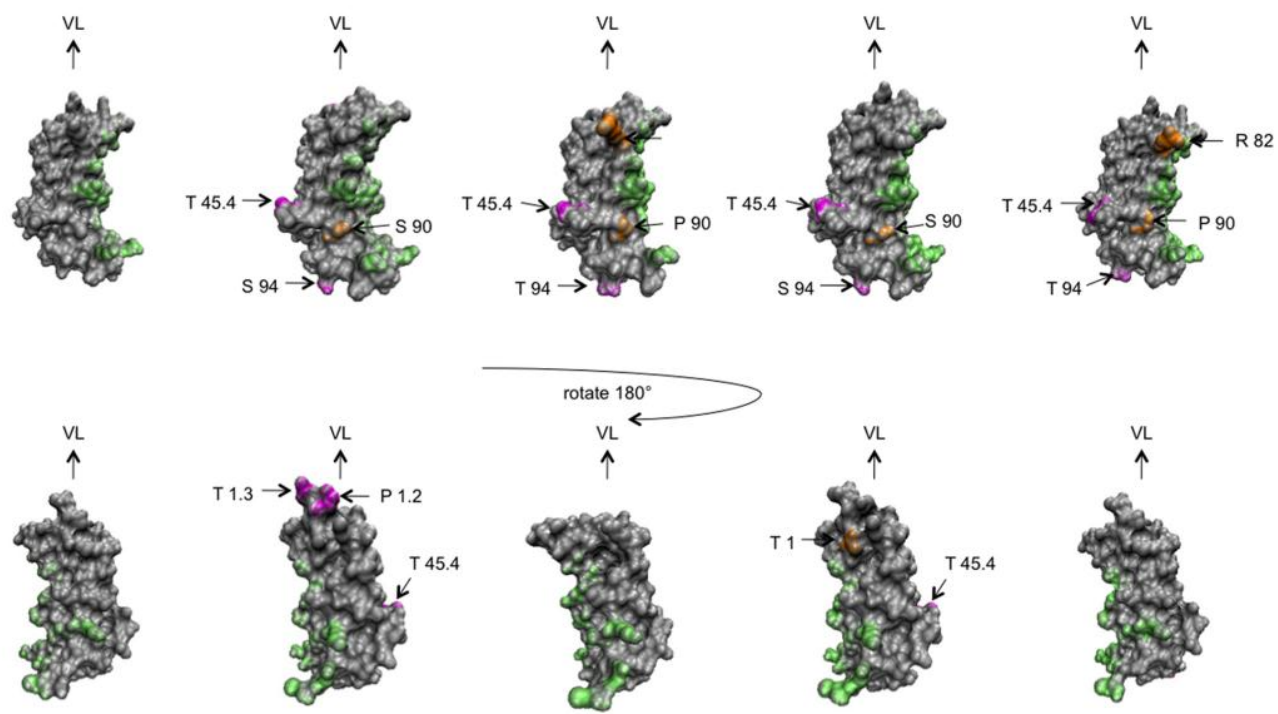

IGLC1 $1^{a}$

IGLC1

IGLC1"

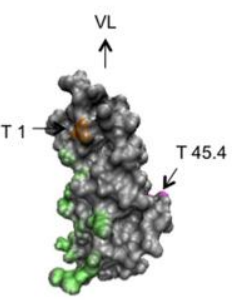

IGLC1 ${ }^{d}$

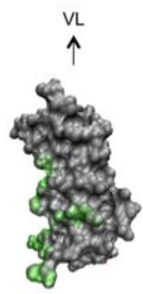

IGLC1 
Chapter 2: Transcriptional analysis of equine lambda light chains

Fig. 4 Comparison of IGLC1 and its allotypic variants. a Alignment of isotype IGLC1 by ClustalW (Thompson et al., 1994) using detected allotypes. Sequences were numbered according to the IMGT numbering system for constant domains (Lefranc et al., 2005). A, B, C, $D, E, F$, and $G$ correspond to the sandwich fold beta strands, whereas $A B, B C, C D, D E, E F$, and FG classify the turns and loops of constant domain immunoglobulin structure. Dots indicate identical nucleotides and dashes indicate gaps. The asterisk marks the stop codon. Amino acid residues providing the contact to $\mathrm{CH} 1$ on the interface between IGHC1 and IGLC in the crystal structure of human mAb NEW are indicated by bold letters and in the crystal structure of human mAb KOL they are indicated by italic letters (Padlan et al., 1986). Bold and italic letters demonstrate interface residues in both of the mAbs. b Homology-based modeling of IGLC isotype IGLC1 and its allotypes. For the predicted 3D structures solvent accessible surfaces are shown from two opposite sides. The images at the bottom are turned by $180^{\circ}$ over the vertical axis compared to those at the top. The putative interface to $\mathrm{CH} 1$ is green. Locations of amino acid residue differences to the basic allotype IGLC1 ${ }^{\text {a }}$ within the interface are marked in orange, whereas the remaining substitutions are marked in magenta. VL indicates the location of the corresponding variable region. Description of the amino acid residues followed the IMGT nomenclature (Lefranc et al., 2005).

$\operatorname{IGLC1}^{\circ}$ showed nine amino acid residue substitutions in comparison to IGLC1 ${ }^{\text {a }}$ (Table 2, Fig. 4b). Seven of them occurred in residues exposed to the surface whereas only four were shown in figure 4 a due to sequence predictions of the homology-based PDB-file. Amino acid residue substitutions at positions 16 and 45.3 were placed inside the molecule. While amino acid residues T45.4 and T94 were adjacent to the putative interface to $\mathrm{CH} 1, \mathrm{R} 82$ and $\mathrm{P} 90$ were located at the outer side of the molecule. The amino acid residue substitutions at positions 45.4 and 82 were conservative, while at position 90 the hydrophilic $T$ was replaced by the hydrophobic $P$ and the hydrophilic $T$ was incorporated instead of hydrophobic A at position 94. There was no change of charge beside the change of hydrophobicity. Allotype IGLC1 (JN228101) was detected in one HW animal as well as in one RGC animal that were heterozygous and also exhibited IGLC1 ${ }^{\mathrm{e}}$ (Table 3).

The fourth allotypic variant IGLC $1^{\mathrm{d}}$ also revealed two conservative amino acid residue substitutions resulting in T45.4 and S90, whereas the hydrophilic $S$ at position 94 replaced the hydrophobic $A$. These positions were located at the surface of the molecule. IGLC1 ${ }^{\mathrm{d}}$ also differed from IGLC1 ${ }^{\mathrm{a}}$ by T45.3. An additional amino acid residue substitution enabling the differentiation of $\mathrm{IGLC} 1^{\mathrm{d}}$ from the other four allotypic variants of IGLC1 was located at amino acid residue position 1. Here, A was replaced by T exhibiting a polar side chain (Table 2, Fig. 4a, b). IGLC1' ${ }^{d}$ (JN228102) was found in three animals of the breed RGC but was not detected in HW. In two animals this allotype was transcribed heterozygously together with IGLC1 ${ }^{\text {b }}$, while it was transcribed homozygously in one animal (Table 3). 
Table 3

Transcriptional occurrence and frequencies of the allotypic variants in the horse breeds Rhenish-German Coldblood (RGC) and Hanoverian Warmblood (HW). Homozygous and heterozygous transcription is indicated by the combination of allotypic variants. Only transcribed allotypic variants are shown in the table.

\begin{tabular}{|c|c|c|c|c|}
\hline & Allotypes/ Alleles & & Horse & breeds \\
\hline & & & RGC & $\mathrm{HW}$ \\
\hline & $b / b$ & & 3 & 0 \\
\hline & $b / d$ & & 2 & 0 \\
\hline IGLC1 & $\mathrm{c} / \mathrm{e}$ & & 1 & 1 \\
\hline & $d / d$ & & 1 & 0 \\
\hline & $\mathrm{e} / \mathrm{e}$ & & 2 & 3 \\
\hline & & $\Sigma$ & 9 & 4 \\
\hline & $\mathrm{b}$ & & 0.44 & 0 \\
\hline & c & & 0.06 & 0.13 \\
\hline Frequencies & $d$ & & 0.22 & 0 \\
\hline & $\mathrm{e}$ & & 0.28 & 0.87 \\
\hline & & $\Sigma$ & 1 & 1 \\
\hline & $a / a$ & & 2 & 5 \\
\hline & $a / b$ & & 0 & 1 \\
\hline IGLC5 & $a / c$ & & 1 & 0 \\
\hline & $a / d$ & & 1 & 0 \\
\hline & $d / d$ & & 2 & 0 \\
\hline & & $\Sigma$ & 6 & 6 \\
\hline & $\mathrm{a}$ & & 0.5 & 0.92 \\
\hline & $b$ & & 0 & 0.08 \\
\hline Frequencies & c & & 0.08 & 0 \\
\hline & d & & 0.42 & 0 \\
\hline & & $\Sigma$ & 1 & 1 \\
\hline & a1/a1 & & 1 & 0 \\
\hline & a1/a2 & & 3 & 7 \\
\hline & a1/a3 & & 1 & 0 \\
\hline & a2/a2 & & 0 & 1 \\
\hline IGLCb// & a2/a3 & & 1 & 0 \\
\hline & a3/a3 & & 1 & 0 \\
\hline & $\mathrm{a} 2 / \mathrm{b}$ & & 1 & 0 \\
\hline & $\mathrm{a} 3 / \mathrm{b}$ & & 1 & 0 \\
\hline & & $\Sigma$ & 9 & 8 \\
\hline & a1 & & 0.33 & 0.44 \\
\hline & a2 & & 0.28 & 0.56 \\
\hline Frequencles & a3 & & 0.28 & 0 \\
\hline & $\mathrm{b}$ & & 0.11 & 0 \\
\hline & & $\Sigma$ & 1 & 1 \\
\hline
\end{tabular}


Chapter 2: Transcriptional analysis of equine lambda light chains

IGLC1 ${ }^{\mathrm{e}}$ revealed five amino acid residue substitutions compared to IGLC1 ${ }^{\text {a }}$ (S45.5T, K82R, T90P, A94T, S127P; Table 2, Fig. 4a). In accordance to IGLC1 ${ }^{\text {b-d }}$, IGLC1 ${ }^{\text {e }}$ differs in S127P IGLC1 ${ }^{\text {a }}$. This stabilizing amino acid substitution is located at the C-terminal end of IGLC1 and also had an influence on hydrophobicity. In HW, three horses were homozygous for IGLC1e as well as two animals of RGC (RGC: JN228103, HW: JN228104). In each breed, one animal showed heterozygous transcription together with IGLC1' ${ }^{\mathrm{C}}$ (Table 3).

In silico analyses of the ESTs database gave evidence to one nucleotide sequence coding for IGLC1 ${ }^{d}$ in a Dartmore Pony (CD470899). For further allotypes no entries were observed matching $100 \%$ sequence identity and coverage. Alleles IGLC1 ${ }^{\mathrm{a}}$, $I G L C 1^{b}, I G L C 1^{c}, I G L C 1^{d}$, and $I G L C 1^{e}$ were at least $96.0 \%$ identical to each other (Online Resource 1).

\subsection{Allelic variants of IGLC5}

For IGLC5, four allotypic variants were identified and were denominated as IGLC5 IGLC5 ${ }^{\mathrm{b}}$, IGLC5 ${ }^{\mathrm{c}}$, and IGLC5 ${ }^{\mathrm{d}}$. The germline sequence of the equine genome assembly EquCab2.0 (NW_001867428, nucleotide no. 4127347-4127666) was set IGLC5a. The allotypic variants were distinguished by the deduced amino acid residue substitutions resulting from 10 different SNPs (Table 4, Fig. 5a). For IGLC5, the deduced amino acid residues of the allotypic variants were aligned and $3 D$ structures of the accessible surfaces were calculated (Fig. 5b). IGLC5 ${ }^{\text {a }}$ shared the best homology with the PDB code 2FB4 (Kratzin et al., 1989). The closest model for IGLC5 ${ }^{\text {b-d }}$ was PDB code 1 AQK (Faber et al., 1998). Homology to selected PDB codes was at least 63\%.

For IGLC5 ${ }^{\mathrm{a}}$ five of ten HW animals were homozygous (JN228106) and one animal also

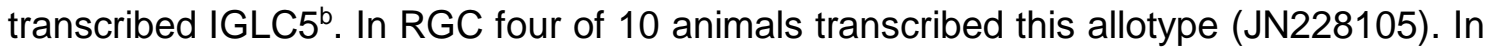
one of these animals IGLC5 ${ }^{a}$ was transcribed heterozygously together with IGLC5 ${ }^{\circ}$. A second RGC animal transcribed IGLC5 (Table 3).

IGLC5 $^{\mathrm{b}}$ differed from IGLC5 ${ }^{\mathrm{a}}$ in three amino acid residue substitutions (Table 4, Fig. 5b). A1P and $\mathrm{K} 107 \mathrm{~T}$ represented conservative amino acid residue substitutions concerning charge and hydrophobicity. In spite of that, the long amino acid side chain of $\mathrm{K} 107$ was replaced by the shorter side chain of $\mathrm{T}$ and a steric influence on the molecule has to be considered. The replacement $\mathrm{T1} .2 \mathrm{~L}$ resulted in a neutral amino acid with hydrophobic side chain which was located at the opposite site of the interface to $\mathrm{CH} 1$. Allotype IGLC5 ${ }^{\mathrm{b}}$ (JN228107) was only identified in five sequences of one HW animal. In this animal IGLC5 ${ }^{\mathrm{a}}$ was also determined (Table 3). 


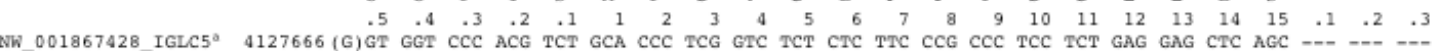
IGLC5 IGLC5

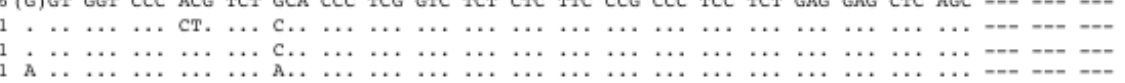

\begin{tabular}{|c|c|}
\hline NW_001867428_IGLC5a & 4127607 \\
\hline IGLC $5^{b}$ & 61 \\
\hline IGLC5" & 61 \\
\hline IGLC $^{d}$ & 61 \\
\hline NW_001867428_IGLC5 a & 4127550 \\
\hline $\operatorname{IGLC} 5^{b}$ & 118 \\
\hline IGLC5 ${ }^{\circ}$ & 118 \\
\hline IGLC5 $^{d}$ & 118 \\
\hline NW_001867428_IGLC5 ${ }^{\circ}$ & 4127514 \\
\hline IGLEC $5^{\circ}$ & 154 \\
\hline IGLC5 ${ }^{\circ}$ & 154 \\
\hline IGLC5 $^{d}$ & 154 \\
\hline
\end{tabular}

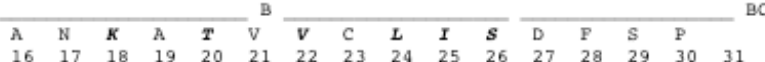

$\begin{array}{lllllllllllllllllllll}16 & 17 & 18 & 19 & 20 & 21 & 22 & 23 & 24 & 25 & 26 & 27 & 28 & 29 & 30 & 31 & 34 & 35 & 36 & 37 & 38\end{array}$

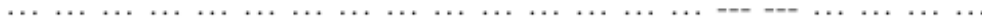

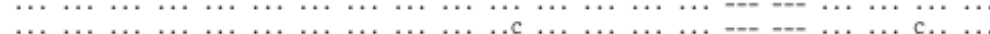

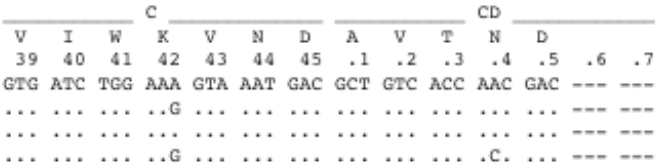

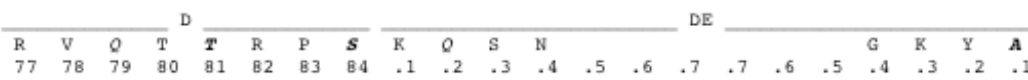
CGC GTC CAG ACC ACC AGG CCC TCG AAA CAG AGC AAC -.- -.. IGLC $^{\circ}$

$\begin{array}{lr} & \\ & \\ \text { NW_001867428_IGLC5 } & 4127466 \\ \text { IGLC5 } & 202 \\ \text { IGLC5 } & 202 \\ \text { IGLC5 }^{\mathrm{b}} & 202\end{array}$

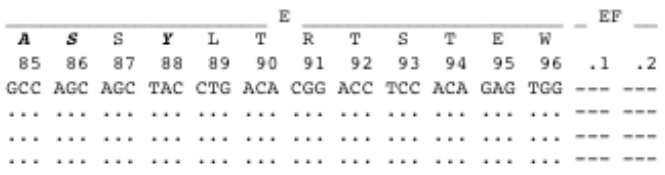

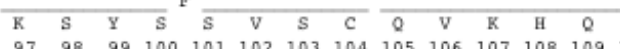
FG

NW_001867428_IGLC5 ${ }^{\circ} 4127430$

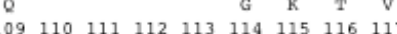

IGLC5 5

IGLC5 $5^{\circ}$

238
238

$\begin{array}{lr} & \\ & \\ \text { NW_001867428_IGLC5 } & 412737 \\ \text { IGLC5 } & 28 \\ \text { IGLC5 } & 289 \\ \text { IGLC5 } & 289\end{array}$

\begin{tabular}{llllllllllllll}
\hline $\mathrm{E}$ & $\mathrm{K}$ & $\boldsymbol{K}$ & $\mathrm{L}$ & $\mathrm{S}$ & $\mathrm{P}$ & $\mathrm{S}$ & $\boldsymbol{E}$ & $\boldsymbol{C}$ & $\mathrm{P}$ &
\end{tabular}

$\begin{array}{lllllllllll}118 & 119 & 120 & 121 & 122 & 123 & 124 & 125 & 126 & 127 & \\ \text { GAG } & \text { AAG } & \text { AAM } & \text { CTG } & \text { TCC } & \text { CCC } & \text { TCA } & \text { GAG } & \text { TGT } & \text { CCT } & \text { TAG }\end{array}$

$\begin{array}{llllllllllll}\ldots & \ldots & \ldots & \ldots & \ldots & \ldots & \ldots & \ldots & \ldots & \ldots & \ldots\end{array}$

b
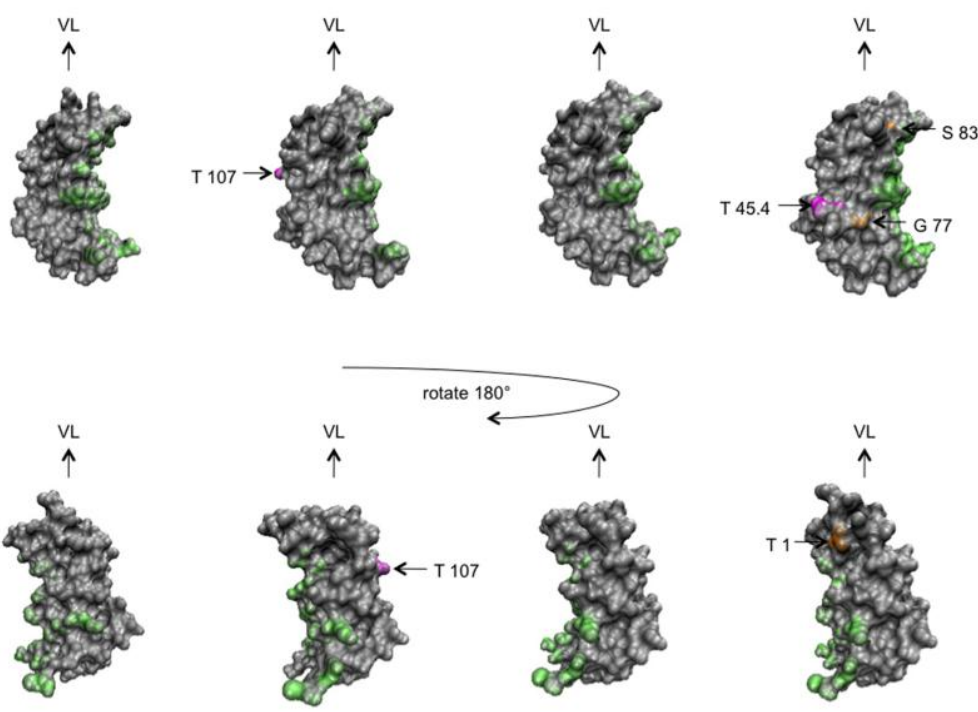

IGLC5
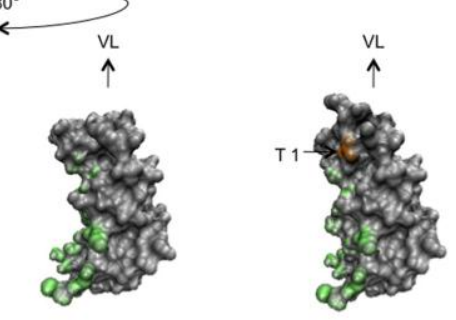

IGLC5a

$191 C 5^{\circ}$

$1 G L 5^{\circ}$ 
Fig. 5 Comparison of IGLC5 and its allotypic variants a Alignment of isotype IGLC5 by ClustalW (Thompson et al., 1994) using detected allotypes. Sequences were numbered according to the IMGT numbering system for constant domains (Lefranc et al., 2005). A, B, C, D, E, F, and G correspond to the sandwich fold beta strands, whereas $A B, B C, C D, D E, E F$, and FG classify the turns and loops of constant domain immunoglobulin structure. Dots indicate identical nucleotides and dashes indicate gaps. The asterisk marks the stop codon. Amino acid residues providing the contact to $\mathrm{CH} 1$ on the interface between IGHC1 and IGLC in the crystal structure of human mAb NEW are indicated by bold letters and in the crystal structure of human mAb KOL they are indicated by italic letters (Padlan et al., 1986). Bold and italic letters demonstrate interface residues in both of the mAbs. b Homology-based modeling of IGLC isotype IGLC5 and its allotypes. For the predicted 3D structures solvent accessible surfaces are shown from two opposite sides. The images at the bottom are turned by $180^{\circ}$ over the vertical axis compared to those at the top. The putative interface to $\mathrm{CH} 1$ is green. Locations of amino acid residue differences to the basic allotype IGLC5a within the interface are marked in orange, whereas the remaining substitutions are marked in magenta. VL indicates the location of the corresponding variable region. Description of the amino acid residues followed the IMGT nomenclature (Lefranc et al., 2005).

IGLC5 $^{\circ}$ (JN228108) revealed a single SNP located near the putative interface to $\mathrm{CH} 1$, which resulted in the conservative amino acid residue substitution A1P (Table 4, Fig. 5b). This substitution was solely observed in one RGC animal which also expressed IGLC5 ${ }^{\mathrm{a}}$ (Table 3).

Three non-conservative amino acid residue substitutions at positions 1,77 , and 83 as well as one conservative amino acid residue substitution at position 45.4 characterized IGLC5 ${ }^{d}$ (Table 4, Fig. 5a). Due to the amino acid residue substitution P83S a new hydrophilic amino acid residue was exposed to the accessible surface. In addition, $\mathrm{R} 77 \mathrm{G}$ converts the charge at this position into neutral as the hydrophobic $\mathrm{G}$ was incorporated instead of the hydrophilic R. Position 77 was adjacent to the putative interface to $\mathrm{CH} 1$. Amino acid residue substitutions at positions 45.4 and 107 were located at the opposite site to the interface to $\mathrm{CH} 1$. As IGLC5 ${ }^{\mathrm{c}}$, allotypic variant IGLC5 ${ }^{\mathrm{d}}$ (JN228109) was only found in one RGC animal that also transcribed IGLC5 ${ }^{\mathrm{a}}$. IGLC5 ${ }^{\mathrm{d}}$ was represented by one sequence in two further animals of RGC (Table 3 ).

For allotypic variants of IGLC5 no EST was observed with $100 \%$ identity and coverage. Alleles IGLC5 ${ }^{\mathrm{a}}$, IGLC5 ${ }^{\mathrm{b}}$, IGLC5 ${ }^{\mathrm{c}}$, and IGLC5 ${ }^{\mathrm{d}}$ showed at least $96.9 \%$ identity to each other (Online Resource 2). 


\section{Table 4}

Allotypic variants of IGLC5. Nucleotide substitutions of further allotypes are shown as SNP at corresponding nucleotide positions of IGLC. Resulting amino acid residue substitutions are shown as follows: initial amino acid residue, position, and replacing amino acid residue. Numbering of amino acid residue positions occurred in accordance with the IMGT numbering system for constant domains (Lefranc et al., 2005).

\begin{tabular}{|c|c|c|c|}
\hline Isotype & Allotype/ allele & Position: SNPa & $\begin{array}{c}\text { Position } \\
\text { amino acid } \\
\text { substitution }\end{array}$ \\
\hline \multirow{3}{*}{ IGLC5 } & b & $\begin{array}{l}10: A>C \\
\text { 11: } C>T \\
\text { 16: } G>C \\
\text { 129: } A>C \\
\text { 269: } A>C\end{array}$ & $\begin{array}{c}\mathrm{T} 1.2 \mathrm{~L} \\
\mathrm{~A} 1 \mathrm{P} \\
- \\
\mathrm{K} 107 \mathrm{~T}\end{array}$ \\
\hline & C & 16: $G>C$ & A1P \\
\hline & $d$ & $\begin{array}{l}16: G>A \\
112: T>C \\
129: A>G \\
149: A>C \\
154: C>G \\
172: C>T\end{array}$ & $\begin{array}{c}\mathrm{A} 1 \mathrm{~T} \\
- \\
- \\
\mathrm{N} 45.4 \mathrm{~T} \\
\mathrm{R} 77 \mathrm{G} \\
\mathrm{P} 83 \mathrm{~S} \\
\end{array}$ \\
\hline
\end{tabular}

a Single nucleotide polymorphism.

\subsection{Allelic variants of IGLC6/7}

The analyses of the predominantly expressed isotypes revealed two allotypic variants

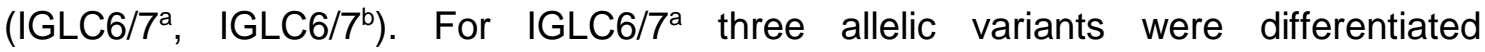

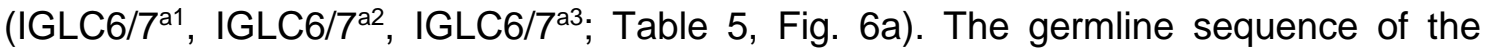
equine genome assembly EquCab2.0 was defined as IGLC6/7a1. The corresponding nucleotides are presented by positions 4120531-4120851 coding for IGLC7 in the contig NW_001867428. IGLC6 and IGLC7 were not distinguished from each other as their sequences differed only in nucleotide position 16 within their genes corresponding to positions 4124306 (G) and 4120837 (A) (NW_001867428). This SNP resulted in different motifs at amino acid positions 1.1 to 3 (SAPS for IGLC6, STPS for IGLC7). In all of our sequences analyzed nucleotide $A$ and therefore amino acid residue motif STPS was determined similarly to germline IGLC7. 
Chapter 2: Transcriptional analysis of equine lambda light chains

\section{Table 5}

Allelic and allotypic variants of IGLC6/7. Nucleotide substitutions of the variants are shown as SNP at corresponding nucleotide positions of IGLC. Resulting amino acid residue substitutions are shown as follows: initial amino acid residue, position, and replacing amino acid residue. Numbering of amino acid residue positions occurred in accordance with the IMGT numbering system for constant domains (Lefranc et al., 2005).

\begin{tabular}{|cccc|}
\hline Isotype & Allotype/ allele & Position: SNPa & $\begin{array}{c}\text { Position } \\
\text { amino acid } \\
\text { substitution }\end{array}$ \\
\hline & a2 & $93: \mathrm{T}>\mathrm{C}$ & - \\
\cline { 2 - 4 } IGLC6/ & $\mathrm{a} 3$ & $112: \mathrm{C}>\mathrm{T}$ & - \\
IGLC7 & $\mathrm{b}$ & $32: \mathrm{T}>\mathrm{C}$ & $\mathrm{L} 6 \mathrm{P}$ \\
& & $93: \mathrm{T}>\mathrm{C}$ & - \\
& & $167: \mathrm{C}>\mathrm{T}$ & $\mathrm{T}$ 81 I \\
\hline
\end{tabular}

a Single nucleotide polymorphism.

Alignments of the deduced amino acid sequences to the crystal structures of human mAb KOL and NEW derived Fabs enabled calculation of the accessible surfaces (Fig. 6b). For IGLC6/7 ${ }^{\text {a, b }}$ the closest model was PDB code 1 AQK (Faber et al., 1998). Homology to this PDB code was at least $63 \%$.

The three alleles were distinguished by SNPs at nucleotide positions 4120760 with T>C (IGLC6/7 $\left.{ }^{\mathrm{a} 2}\right)$ and 4120741 with C>T (IGLC6/7 $\left.{ }^{\mathrm{a} 3}\right)$ within the gene (NW_001867428, Table 5, Fig. 6a). Only one animal of the breeds analyzed showed homozygous transcription of IGLC6/7 ${ }^{\text {a1 }}$ (RGC: JN228110, HW: JN228111). IGLC6/7 $7^{\text {a2 }}$ was found in five RGC animals (JN228112) and in eight horses of HW (JN228113). One HW animal was homozygous for this allele. All remaining animals were heterozygous and transcribed additionally either IGLC6/7 $7^{\text {a1 }}$, IGLC6/7 $7^{\text {a3 }}$, or IGLC6/7 . IGLC6/7 $7^{\text {a3 }}$ (JN228114) was transcribed in four animals of the breed RGC (Table 3).

In comparison to IGLC6/7 , IGLC6/7 is characterized by the two amino acid residue exchanges L6P and T81I. At position six a neutral and hydrophobic amino acid side chain was replaced by an aliphatic and hydrophobic side chain. The amino acid residue substitution resulting in a hydrophobic side chain at position 81 affected one of the conserved residues that were responsible for the interaction between $\mathrm{CL}$ and $\mathrm{CH} 1$ (Fig. 6b). In comparison to IGLC6/7 $7^{\text {a2 }}$ the same SNP like at nucleotide position $4,120,760$ caused a silent mutation in IGLC6/7 .

IGLC6/7 ${ }^{\text {b }}$ was only detected in two animals of the breed RGC (JN228115). In these two animals either IGLC6/7 $7^{\mathrm{a} 2}$ or IGLC6/7 ${ }^{\text {a3 }}$ were observed additionally. For all allotypic variants of IGLC6/7 no EST showed $100 \%$ identity and coverage. The four alleles of IGLC6/7 were at least $98.4 \%$ identical to each other (Online Resource 3). 
NW 001867428 IGIC7 NW 001867428 IGIC6 $^{2}$ IG $\mathrm{C} 6 / 7^{2}$ IGIC6/70 IGIC6/70

WW 001867428 IGIC7 $^{20}$ WW 001867428 IGIC6 IGIC $6 / 7^{22}$ $\operatorname{IGT} 6 / 7^{\circ}$ IG 6 C $6 / 7^{\circ}$

NW 001867428 IGIC7 W 001867428 IGIC6 IGIC6/70 IG $66 / 7^{\circ}$ IGIC $6 / 7$ Th

NW 001867428 IGIC7 $7^{2}$ NW 001867428 IGIC6 $^{2}$ IG 6 C $6 / 7^{20}$ IGIC $6 / 7^{23}$ IGIC $6 / 7$ t

NW 001867428 IGIC7 NW 001867428 IGIC6 IGIC6 $6 / 7^{2}$

IGIC6/70

IGIC $6 / 7 T^{5}$

NW 001867428 IGIC7 $^{2}$ WW 001867428 IGIC6 $\mathrm{IG}\left[\mathrm{C} 6 / 7^{22}\right.$ IGIC6/70 IGIC $6 / T^{\text {th }}$

NW_001867428_IGIC7 NW 001867428 IGIC6 IGIC $6 / 7^{22}$ IGIC6/T

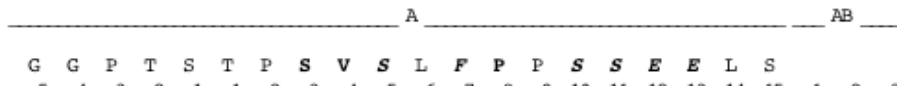

$\begin{array}{lllllllllllllllllllllllllllllll}.5 & .4 & .3 & .2 & .1 & 1 & 2 & 3 & 4 & 5 & 6 & 7 & 8 & 9 & 10 & 11 & 12 & 13 & 14 & 15 & .1 & .2 & .3\end{array}$ 4120850 (G)GT GGT COC AOG TCT ACA COC TOG GIC TCT CIC TIC $\triangle O$ ФOC TOC TCT GAG GAG CIC ACC 4124320

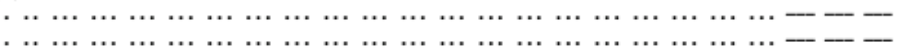

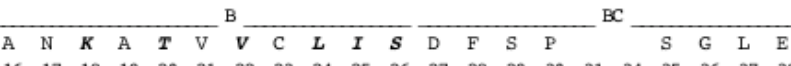

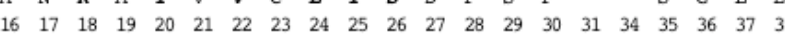
4120791 GOC AAC AAG GOC ACA GIG GIG TGT CTC ATC AGT GAC TIC TOC OOC 4124261

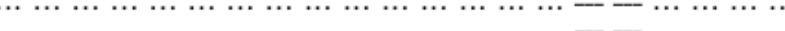

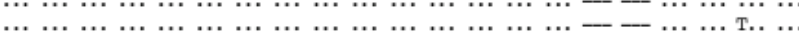
$\ldots \ldots \ldots \ldots \ldots \ldots \ldots \ldots \ldots \ldots, \ldots \ldots \ldots \ldots \ldots,-\ldots \ldots \ldots$

V I W ${ }^{\mathrm{C}} \mathrm{C}$

$\begin{array}{lllllllllllllllll}39 & 40 & 41 & 42 & 43 & 44 & 45 & .1 & .2 & .3 & .4 & .5 & .6 & .7\end{array}$

4120734

4124204

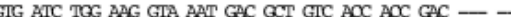

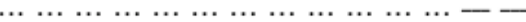

$\ldots \ldots \ldots \ldots \ldots \ldots \ldots \ldots \ldots \ldots \ldots \ldots \ldots+$

$\ldots \ldots \ldots \ldots \ldots \ldots \ldots \ldots \ldots \ldots \ldots \ldots+-$

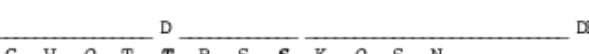
DE

$\begin{array}{lllllllllllllllllllllll}77 & 78 & 79 & 80 & 81 & 82 & 83 & 84 & 1 & .2 & 3 & 4 & .5 & .6 & .7 & .7 & .6 & .5 & .4 & .3 & .2 & .1\end{array}$ 4120698 GOC GIC CAG AOC AOC ACG TOC TOG AAA CAG AOC AAC - - _ $4124168 \quad \ldots \ldots \ldots \ldots \ldots \ldots \ldots \ldots \ldots \ldots \ldots \ldots \ldots \ldots \ldots$ $\ldots \ldots \ldots \ldots \ldots \ldots \ldots \ldots \ldots \ldots \ldots \ldots--\ldots \ldots \ldots \ldots$ $\ldots \ldots \ldots \ldots \ldots \ldots \ldots \ldots \ldots \ldots \ldots \ldots---1 \ldots \ldots \ldots$ 154

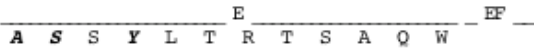
$\begin{array}{llllllllllllll}85 & 86 & 87 & 88 & 89 & 90 & 91 & 92 & 93 & 94 & 95 & 96 & .1 & .2\end{array}$

4120650 GOC AOC AOC TAC CIG AOG COG ACT TOC GCA CAG TOG - -

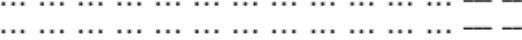

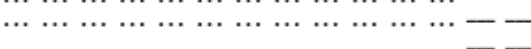

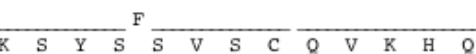

\begin{tabular}{cccc}
$G$ & G & T & V \\
\hline
\end{tabular} AAA TOG TAC AOC AOC GIC AOC TOC CAG GIC AAG CAC CAA - - - GOG AAA AOC GIG

4120614 4124084 $\begin{array}{lllllllllllll} & \ldots & \ldots & \ldots & \ldots & \ldots & \ldots & \ldots & \ldots & \ldots & \ldots & \ldots & \ldots\end{array}$ $\cdots \cdots \cdots \cdots \cdots \cdots \cdots \cdots \cdots \cdots \cdots \cdots$ $\ldots \ldots \ldots \ldots \ldots \ldots \ldots$

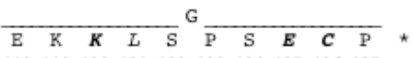

$\begin{array}{llllllllll}118 & 119 & 120 & 121 & 122 & 123 & 124 & 125 & 126 & 127\end{array}$

GAG AAG AAA CTG TOC COC TCA GAG TGT $O C T$ TAC ............ n.
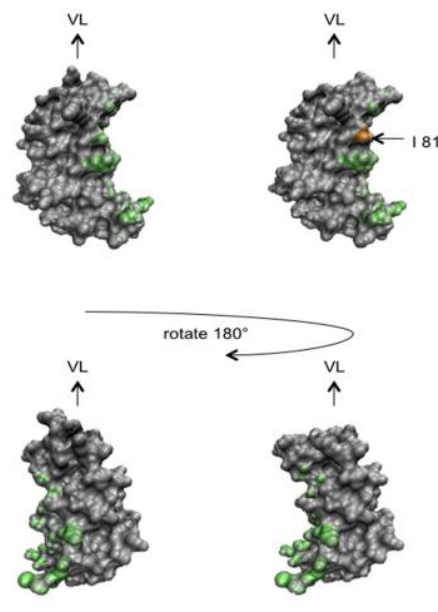

1GLC6/7 $\quad$ 1GLC6/7 
Fig. 6 Comparison of IGLC6/7 and its allotypic variants a Alignment of isotype IGLC6/7 by ClustalW (Thompson et al., 1994) using detected allotypes. Sequences were numbered according to the IMGT numbering system for constant domains (Lefranc et al., 2005). A, B, C, $D, E, F$, and $G$ correspond to the sandwich fold beta strands, whereas $A B, B C, C D, D E, E F$, and FG classify the turns and loops of constant domain immunoglobulin structure. Dots indicate identical nucleotides and dashes indicate gaps. The asterisk marks the stop codon. Amino acid residues providing the contact to $\mathrm{CH} 1$ on the interface between IGHC1 and IGLC in the crystal structure of human mAb NEW are indicated by bold letters and in the crystal structure of human mAb KOL they are indicated by italic letters (Padlan et al. 1986). Bold and italic letters demonstrate interface residues in both of the mAbs. b Homology-based modeling of IGLC isotype IGLC6/7 and its allotypes. For the predicted 3D structures solvent accessible surfaces are shown from two opposite sides. The images at the bottom are turned by $180^{\circ}$ over the vertical axis compared to those at the top. The putative interface to $\mathrm{CH} 1$ is green. Locations of amino acid residue differences to the basic allotype IGLC7 ${ }^{a}$ within the interface are marked in orange, whereas the remaining substitutions are marked in magenta. VL indicates the location of the corresponding variable region. Description of the amino acid residues followed the IMGT nomenclature (Lefranc et al., 2005). 
Chapter 2: Transcriptional analysis of equine lambda light chains

\section{Discussion}

Our study is the first detailed molecular genetic description of allotypic variants within the equine $\lambda$-light chain locus. The present analyses revealed the existence of five IGLC1, four IGLC5, and two IGLC6/7 allotypic variants. For IGLC6/7 ${ }^{\text {a }}$ three alleles were observed. The amino acid residue variations at positions 1.3, 1.2, 1, 45.3, 45.4, 81, 83, 90, 94, 95, and 107 were located at the accessible surface as confirmed by homology-based predicted 3D structural analysis. These modifications are assumed to possess distinct serological properties resulting in allotypic variants.

The transcriptional use of IGLC6/7 was significantly preferred in both breeds analyzed as confirmed by $\mathrm{Chi}^{2}$ statistics. There were highly significant differences in the transcription frequency of the four functional IGLC isotypes within the two breeds. IGLC6 and IGLC7 were distinguished by only one amino acid residue substitution which was caused by one SNP. Due to the similarity of our corresponding sequences it was not obvious whether they were different alleles of IGLC6 or IGLC7 or allotypic variants of one gene. It is noteworthy that the sequences analyzed resembled IGLC7. All allotypic variants identified showed distinct amino acid residue substitutions in all seven $\beta$-sheets according to the IMGT nomenclature (Lefranc et al., 2005). Most of the substitutions were located within the accessible surface area (Padlan et al., 1986). Seven of these substitutions (A1T, R77G, T81I, 82R, P83S, T90S/P) were positioned within the interface between the constant region of the light chain and the first constant region of the heavy chain. These changes might influence the stable connection between both the heavy and light chains. Due to crystal structures of Fabs derived from human mAbs KOL and NEW, six amino acids (F7, E12, E13, T20, V22, and T81) were defined as conserved residues. They are expected for the main interaction of IGLC and IGHC1. The remaining interface residues may be variable (Padlan et al., 1986). Regarding these facts, T81I affected one of the conserved residues for the contact to IGHC1 leading to the conclusion, that the assembly of heavy and light chains could be changed. Otherwise we do not have any information about allelic changes in $\mathrm{CH} 1$ of the heavy chains and this conclusion remains to be verified. In previous studies IGHC1 was related to antibody affinity (Pritsch et al., 1996). IGLC and IGHC1 were found to have an influence on the whole immunoglobulin molecule due to time differences in the assembly of full size antibodies and in the kinetics of antigen binding (Montano and Morrison, 2002). 
Chapter 2: Transcriptional analysis of equine lambda light chains

Amino acid residue substitutions located at the solvent accessible surface area might be distinguished by specific sera and therefore might be used as markers. Specific markers differing in frequencies within human populations were observed in $\lambda$ - and $\mathrm{K}$-light chains as well as in heavy chains of $\lg$ and $\lg \mathrm{A}$ (Calderon et al., 2007; Matsumoto et al., 1984; Schanfield et al., 2008). Due to either direct association or linkage disequilibrium with the causative gene, allotypic markers of human immunoglobulin chains could be related to susceptibility of different infectious diseases (Giha et al., 2009; Granoff et al., 1984; Pandey, 2000; Pandey et al., 1995; Pandey et al., 1979). Influences of bovine allotypes on effector functions, complement activation, and age dependent expression have also been pointed out in previous studies (Bastida-Corcuera et al., 2000; Bastida-Corcuera et al., 1999a; Bastida-Corcuera et al., 1999b; Corbeil et al., 1997). As investigations on these issues are missing in horses, we suppose heterozygotic horses to be advantageous to the humoral immune response.

In addition to IGLC1, IGLC5, and IGLC6/7, a fourth IGLC-gene (IGLC4) was found in one sequence of two RGC horses. Both of them varied from each other as well as from the genomic sequence in the genome assembly EquCab2.0. Since our set criteria for the definition of a new allotype were not fulfilled, this isotype was not analyzed further. This constant region was described as potentially nonfunctional due to a single C-insertion within the corresponding joining gene segment IGLJ4. We observed transcription without the C-insertion within the IGLJ-segment as mentioned in previous studies (Sun et al., 2010).

In our investigation we also analyzed the presence of all predicted germline IGLJ-IGLC pairs. Each of the six distinguishable IGLJ-IGLC pairs was amplified from two RGC and one HW animal. IGLJ5-IGLC5 was detected in four animals of RGC and five animals of HW. In contrast, in previous investigations germline IGLJ5-IGLC5 as well as IGLJ7-IGLC7 were not amplified. In the same study germline IGLC6 and IGLC7 were distinguished by PCR-products amplified with primers that anneal within the genomic introns (Sun et al., 2010). As we used exon specific primer pairs to minimize genomic sequence variability and to avoid false-negative results we could not differentiate between IGLC6 and IGLC7.

Beside the presence of predicted germline IGLJ-IGLC pairs and the transcription frequency of IGLC-genes, we had a closer look at the usage of IGLV-segments of subgroup 8 because this subgroup contains the highest number of functional IGLV. In addition, Sun et al. (2010) showed this subgroup is transcribed preferentially. As there 
Chapter 2: Transcriptional analysis of equine lambda light chains

are nine functional IGLV in subgroup 8, we sequenced 12 clones per animal to find subgroup 8-IGLV transcribed at low frequency in horses, too. Finally, there were two variable segments, IGLV15 and IGLV17, which were preferred in both breeds. For IGLV15, statistical analyses showed significant differences of the rearrangements with the four IGLC-isotypes transcribed. While rearrangement of IGLV17 was highly significant different from the other IGLV-segments in both breeds examined, rearrangement of IGLV15 showed $P$-values of 0.0119 in HW and 0.0436 in RGC. Between both breeds analyzed, only IGLV15 differed significantly in rearrangements with the IGLC-genes $(P=0.011)$. In HW a third variable segment, IGLV25, was transcribed at high frequency. For this segment $\mathrm{Chi}^{2}$ revealed significant differences of transcription frequency within both breeds examined $(P=0.0233)$. These results indicate that the V-domain repertoire is dominated by two breed independent IGLV genes and a third breed specific IGLV gene that are effectively used for combinatorial joining of IGLJ-IGLC-genes and thus for antibody production. Furthermore, two putative pseudogenes (IGLV74 $\Psi$, IGLV101 $\Psi$ ) were transcribed. For IGLV74 $\psi$ a frameshift in CDR1 resulting in two in frame stop codons in FR3 and CDR3 was described (Sun et al., 2010). In addition, no TATA-box was identified. As known for humans and Saccharomyces cerevisiae, the TATA-box is not essential for accurate transcription initiation (Bjornsdottir and Myers, 2008; Yang et al., 2007). Consequently the premature stop codons are seen as reason for nonfunctionality. The isolated sequences did not show premature termination. We identified variations in CDR1 sequences that were introduced by the deletion of $C$, which was present at nucleotide position 96 of germline IGLV74 $\psi$. This deletion resulted in a frame shift replacing the first stop codon by $A$, and the second one either by $S, K$, I, or R. As cDNA sequences were identified as IGLV74 $\Psi$ due to sequence identities between $82.5 \%$ and $90.1 \%$, we suppose individual mutations within the breeds to enable the transcriptional use of pseudogenes. Further we have to consider that germline sequences are prepared from a Thoroughbred, which is a very conserved breed founded by few stallions. So mutations resulting in pseudogenes may be spread within this breed but can be eliminated in other breeds. In RGC IGLV101\%, also caused by a frame shift within the CDR1 of the germline sequence, was identified in two sequences. Transcription of pseudo V-gene segments has already been described for cattle, chicken, and rabbits (Arakawa and Buerstedde, 2009; Parng et al., 1996; Winstead et al., 1999). In these species usage of pseudogenes occurs by gene conversion which is a process contributing to antibody diversity. Our results indicated presence of similar mechanisms 
Chapter 2: Transcriptional analysis of equine lambda light chains

in horses. In addition, we have to take into account that the spacer sequences of the recombination signal sequences shown in Sun et al. (2010) of the transcribed IGLVs seem to be conserved. The spacer sequence in IGLV101\% is identical to that in IGLV17 and IGLV15, which are the mainly used IGLV. As shown in Nadel et al. (1998), spacer sequences contribute to rearrangement of human IGKV segments. In analogy these identical sequences of the spacers are an additional option for the transcription of potential pseudogenes. Moreover, transcriptional use of IGLV19 is limited in comparison to the other IGLV. The spacer of this segment shows three nucleotide substitutions relative to IGLV17 at positions 3,10, and 12 of the spacer which also indicate the spacer to be a determinant factor for rearrangements.

As analyses took place in two breeds with different stud book sizes and breeding goals, some of the results could be linked to breeding. RGC is a quiet conserved breed with approximately 1348 mares and 153 stallions presently in Germany. Animals of this breed are mainly used for agricultural purposes and breed representations (Bremond and Balzer, 2011). In contrast, animals of the breed HW participate in all disciplines of equestrian sports and are internationally well placed. This breed influenced other horse breeds and it presently is one of the biggest horse breeds with 19492 registered mares and more than 522 stallions (Bremond and Balzer, 2011). As there was a large number of founder animals of genetically different breeds, the permission to choose stallions from different sire lines, and modest migration of sires from other breeds is also granted. The inbreeding coefficient in the Hanoverian population is at low level (1.33\%) than those of smaller populations like the Rhenish German Coldblood $1.73 \%$; Biedermann et al., 2002; Hamann and Distl, 2008). Nevertheless, effective management of breeding and therefore restricted use of single stallions is necessary to prevent the random loss of alleles caused by large genetic contributions of few individuals over a long time period (bottleneck effect). Genetic drift and homogenization of genetic information may also occur and have to be controlled as the rate of inbreeding has increased in Hanoverians in the last ten years (Constans et al., 1985; Hamann and Distl, 2008; Simianer and Kohn, 2010).

Our study aimed to characterize genetic and transcriptional differences of $\lambda$-light chain in two horse breeds. Distinct alleles and putative allotypic variants were described for the first time in horses. Future investigations should evaluate the distribution of allotypic markers of immunoglobulin chains in further horse breeds. Moreover, the linkage to infectious diseases such as in humans and cattle should be examined. 


\section{Acknowledgment}

We are grateful to Jessica Olbricht for critical review of the manuscript and thank Tina Henkel as well as Caroline Bierschenk for excellent technical assistance.

\section{Appendix A. Supplementary data}

Supplementary data associated with this article can be found, in the online version, at doi:10.1016/j.vetimm.2011.10.006.

Legends to the Supplemental tables

Supplemental table 1 Percent identity (upper triangle) and divergence (lower triangle) of the detected allotypes of IGLC1 were divided by black boxes. Database entries were indicated by their accession number, calculations were based on the pairwise alignment using ClustalW (Thompson et al., 1994). Percent identity was compared to sequence pairs without regard to their phylogenetic relationships. Divergence was calculated in relation to the phylogeny.

\begin{tabular}{|c|c|c|c|c|c|c|c|}
\hline \multicolumn{8}{|c|}{ Percent Identity } \\
\hline \multirow{8}{*}{$\begin{array}{l}1 \\
2 \\
3 \\
4 \\
5 \\
6\end{array}$} & 1 & 2 & 3 & 4 & 5 & 6 & \\
\hline & & 96.3 & 96.0 & 96.3 & 97.5 & 96.3 & 1 \\
\hline & 3.8 & & 97.5 & 98.8 & 97.8 & 98.8 & 2 \\
\hline & 4.2 & 2.5 & & 97.2 & 98.4 & 97.2 & 3 \\
\hline & 3.8 & 1.3 & 2.9 & & 97.8 & 100.0 & 4 \\
\hline & 2.5 & 2.2 & 1.6 & 2.2 & & 97.8 & 5 \\
\hline & 3.8 & 1.3 & 2.9 & 0.0 & 2.2 & & 6 \\
\hline & 1 & 2 & 3 & 4 & 5 & 6 & \\
\hline
\end{tabular}

IGLC1 $1^{a}$ IGLC1 $^{\text {b }}$ IGLC1 ${ }^{\mathrm{c}}$ IGLC1 $^{\mathrm{d}}$ IGLC1 ${ }^{\mathrm{e}}$ CD470899 
Supplemental table 2 Percent identity (upper triangle) and divergence (lower triangle) of the detected allotypes of IGLC5 were divided by black boxes. Calculations were based on the pairwise alignment using ClustalW (Thompson et al., 1994). Percent identity was compared to sequence pairs without accounting for their phylogenetic relationships. Divergence was calculated in relation to the phylogeny.

\begin{tabular}{|c|c|c|c|c|c|c|c|}
\hline & \multicolumn{6}{|c|}{ Percent Identity } & \multirow[b]{3}{*}{ IGLC5 $^{a}$} \\
\hline & & 1 & 2 & 3 & 4 & & \\
\hline \multirow{5}{*}{$\begin{array}{l}\frac{0}{0} \\
\text { D } \\
\frac{0}{0} \\
\stackrel{2}{0}\end{array}$} & 1 & & 98.4 & 99.7 & 97.5 & 1 & \\
\hline & 2 & 1.6 & & 98.8 & 96.9 & 2 & IGLC \\
\hline & 3 & 0.3 & 1.3 & & 97.5 & 3 & IGLC \\
\hline & 4 & 2.5 & 3.2 & 2.5 & & 4 & IGLC5 \\
\hline & & 1 & 2 & 3 & 4 & & \\
\hline
\end{tabular}

Supplemental table 3 Percent identity (upper triangle) and divergence (lower triangle) of the detected alleles and allotypes of IGLC6/7 were divided by black boxes. Calculations were based on the pairwise alignment using ClustalW (Thompson et al., 1994). Percent identity was compared to sequence pairs without regard to their phylogenetic relationships. Divergence was calculated in relation to the phylogeny.

Percent Identity

\begin{tabular}{|c|c|c|c|c|c|c|c|c|}
\hline & & 1 & 2 & 3 & 4 & 5 & & \multirow{7}{*}{$\begin{array}{l}\text { IGLC7 }{ }^{\mathrm{a} 1} \\
\text { IGLC6 }^{\mathrm{a} 1} \\
\text { IGLC6/772 } \\
\text { IGLC6/773 } \\
\text { IGLC6/7 }\end{array}$} \\
\hline & 1 & & 99.7 & 100.0 & 99.4 & 99.1 & 1 & \\
\hline & 2 & 0.3 & & 99.7 & 99.0 & 98.7 & 2 & \\
\hline$\Phi$ & 3 & 0.0 & 0.3 & & 99.4 & 99.1 & 3 & \\
\hline & 4 & 0.6 & 1.0 & 0.6 & & 98.4 & 4 & \\
\hline & 5 & 0.9 & 1.4 & 0.9 & 1.6 & & 5 & \\
\hline & & 1 & 2 & 3 & 4 & 5 & & \\
\hline
\end{tabular}


Chapter 2: Transcriptional analysis of equine lambda light chains

\section{References}

Almagro, J.C., Martinez, L., Smith, S.L., Alagon, A., Estevez, J., Paniagua, J., 2006. Analysis of the horse $\mathrm{V}(\mathrm{H})$ repertoire and comparison with the human IGHV germline genes, and sheep, cattle and pig $\mathrm{V}(\mathrm{H})$ sequences. Mol. Immunol. 43, 1836-1845.

Arakawa, H., Buerstedde, J.M., 2009. Activation-induced cytidine deaminase-mediated hypermutation in the DT40 cell line. Philos. Trans. R. Soc. Lond. B. Biol. Sci. 364, 639-644.

Arun, S.S., Breuer, W., Hermanns, W., 1996. Immunohistochemical examination of light-chain expression (lambda/kappa ratio) in canine, feline, equine, bovine and porcine plasma cells. Zentralbl. Veterinarmed. A. 43, 573-576.

Bastida-Corcuera, F., Butler, J.E., Heyermann, H., Thomford, J.W., Corbeil, L.B., 2000. Tritrichomonas foetus extracellular cysteine proteinase cleavage of bovine IgG2 allotypes. J. Parasitol. 86, 328-332.

Bastida-Corcuera, F.D., Butler, J.E., Yahiro, S., Corbeil, L.B., 1999a. Differential complement activation by bovine IgG2 allotypes. Vet. Immunol. Immunopathol. 71, 115-123.

Bastida-Corcuera, F.D., Nielsen, K.H., Corbeil, L.B., 1999b. Binding of bovine IgG2a and IgG2b allotypes to protein A, protein G, and Haemophilus somnus IgBPs. Vet. Immunol. Immunopathol. 71, 143-149.

Biedermann, G., Clar, U., Finke, A., Bickel, M., 2002. Analyse der Population des Rheinisch-Deutschen Kaltbluts. Züchtungskunde 74, 237-249.

Bjornsdottir, G., Myers, L.C., 2008. Minimal components of the RNA polymerase II transcription apparatus determine the consensus TATA box. Nucleic Acids Res. 36, 2906-2916.

Bremond, J., Balzer, B., 2011. Zentrale Dokumentation Tiergenetischer Ressourcen in Deutschland (TGRDEU).

Calderon, R., Lodeiro, R., Varela, T.A., Farina, J., Ambrosio, B., Guitard, E., GonzalezMartin, A., Dugoujon, J.M., 2007. GM and KM immunoglobulin allotypes in the Galician population: new insights into the peopling of the Iberian Peninsula. BMC Genet. 8, 37.

Constans, J., Hazout, S., Garruto, R.M., Gajdusek, D.C., Spees, E.K., 1985. Population distribution of the human vitamin $\mathrm{D}$ binding protein: anthropological considerations. Am. J. Phys. Anthropol. 68, 107-122.

Corbeil, L.B., Gogolewski, R.P., Kacskovics, I., Nielsen, K.H., Corbeil, R.R., Morrill, J.L., Greenwood, R., Butler, J.E., 1997. Bovine IgG2a antibodies to Haemophilus somnus and allotype expression. Can. J. Vet. Res. 61, 207-213.

Das, S., Nikolaidis, N., Klein, J., Nei, M., 2008. Evolutionary redefinition of immunoglobulin light chain isotypes in tetrapods using molecular markers. Proc. Natl. Acad. Sci. U. S. A. 105, 16647-16652.

Diesterbeck, U.S., Aboelhassan, D.M., Stein, S.K., Czerny, C.-P., 2011 in press. Detection of new allotypic variants of bovine $\lambda$-light chain constant regions in different cattle breeds. Dev. Comp. Immunol.

Edelman, G.M., 1973. Antibody structure and molecular immunology. Science 180, 830-840.

Emorine, L., Dreher, K., Kindt, T.J., Max, E.E., 1983. Rabbit immunoglobulin kappa genes: structure of a germline b4 allotype J-C locus and evidence for several b4-related sequences in the rabbit genome. Proc. Natl. Acad. Sci. U. S. A. 80, 5709-5713. 
Faber, C., Shan, L., Fan, Z., Guddat, L.W., Furebring, C., Ohlin, M., Borrebaeck, C.A., Edmundson, A.B., 1998. Three-dimensional structure of a human Fab with high affinity for tetanus toxoid. Immunotechnology 3, 253-270.

Gibson, D., 1974. Structural studies on normal horse immunoglobulin light chains. Detection of k-type N-terminal sequences. Biochemistry 13, 2776-2785.

Giha, H.A., Nasr, A., Iriemenam, N.C., Arnot, D., Troye-Blomberg, M., Theander, T.G., Berzins, K., ElGhazali, G., Pandey, J.P., 2009. Antigen-specific influence of $\mathrm{GM} / \mathrm{KM}$ allotypes on $\mathrm{lgG}$ isotypes and association of $\mathrm{GM}$ allotypes with susceptibility to Plasmodium falciparum malaria. Malar. J. 8, 306.

Granoff, D.M., Pandey, J.P., Boies, E., Squires, J., Munson, R.S., Jr., Suarez, B., 1984. Response to immunization with Haemophilus influenzae type b polysaccharidepertussis vaccine and risk of Haemophilus meningitis in children with the $\mathrm{Km}(1)$ immunoglobulin allotype. J. Clin. Invest. 74, 1708-1714.

Hamann, H., Distl, O., 2008. Genetic variability in Hanoverian warmblood horses using pedigree analysis. J. Anim. Sci. 86, 1503-1513.

Hartmann, O., Bruns, E., Hertsch, B., Schade, W., 2006. Pferdezucht. Eugen Ulmer KG, Stuttgart (Hohenheim).

Home, W.A., Ford, J.E., Gibson, D.M., 1992. L chain isotype regulation in horse. I. Characterization of Ig lambda genes. J. Immunol. 149, 3927-3936.

Humphrey, W., Dalke, A., Schulten, K., 1996. VMD: visual molecular dynamics. J. Mol. Graph. 14, 33-38, 27-38.

Kawasaki, K., Minoshima, S., Nakato, E., Shibuya, K., Shintani, A., Asakawa, S., Sasaki, T., Klobeck, H.G., Combriato, G., Zachau, H.G., Shimizu, N., 2001. Evolutionary dynamics of the human immunoglobulin kappa locus and the germline repertoire of the Vkappa genes. Eur. J. Immunol. 31, 1017-1028.

Kelley, L.A., Sternberg, M.J., 2009. Protein structure prediction on the Web: a case study using the Phyre server. Nat. Protoc. 4, 363-371.

Klein, S.L., Strausberg, R.L., Wagner, L., Pontius, J., Clifton, S.W., Richardson, P., 2002. Genetic and genomic tools for Xenopus research: The NIH Xenopus initiative. Dev. Dyn. 225, 384-391.

Korngold, L., Lipari, R., 1956. Multiple-myeloma proteins. III. The antigenic relationship of Bence Jones proteins to normal gammaglobulin and multiple-myeloma serum proteins. Cancer 9, 262-272.

Kratzin, H.D., Palm, W., Stangel, M., Schmidt, W.E., Friedrich, J., Hilschmann, N., 1989. [The primary structure of crystallizable monoclonal immunoglobulin IgG1 Kol. II. Amino acid sequence of the L-chain, gamma-type, subgroup I]. Biol. Chem. Hoppe Seyler 370, 263-272.

Lee, B., Richards, F.M., 1971. The interpretation of protein structures: estimation of static accessibility. J. Mol. Biol. 55, 379-400.

Lefranc, M.P., Pommie, C., Kaas, Q., Duprat, E., Bosc, N., Guiraudou, D., Jean, C., Ruiz, M., Da Piedade, I., Rouard, M., Foulquier, E., Thouvenin, V., Lefranc, G., 2005. IMGT unique numbering for immunoglobulin and T cell receptor constant domains and Ig superfamily C-like domains. Dev. Comp. Immunol. 29, 185-203.

Littell, R.C., Milliken, G.A., Stroup, W.W., Wolfinger, R., 2004. SAS ${ }^{\circledR}$ system for Mixed Models, 6th Edition. SAS Institute Inc, Cary, NC, USA.

Matsumoto, H., Miyazaki, T., Rychkov, Y.G., Zhukova, O., Lebedeva, I.A., Kondik, V.M., Udina, I.G., Spitzin, V.A., Batsuur, J., Shnader, Y.V., et al., 1984. Studies on the human immunoglobulin allotypes in five populations in the USSR. Jinrui Idengaku Zasshi 29, 105-111.

Montano, R.F., Morrison, S.L., 2002. Influence of the isotype of the light chain on the properties of IgG. J. Immunol. 168, 224-231. 
Moxley, G., Gibbs, R.S., 1992. Polymerase chain reaction-based genotyping for allotypic markers of immunoglobulin kappa shows allelic association of $\mathrm{Km}$ with kappa variable segment. Genomics 13, 104-108.

Nadel, B., Tang, A., Escuro, G., Lugo, G., Feeney, A.J., 1998. Sequence of the spacer in the recombination signal sequence affects $V(D) J$ rearrangement frequency and correlates with nonrandom Vkappa usage in vivo. J. Exp. Med. 187, 14951503.

Oli, M.W., Rhodin, N., McArthur, W.P., Brady, L.J., 2004. Redirecting the humoral immune response against Streptococcus mutans antigen $\mathrm{P} 1$ with monoclonal antibodies. Infect. Immun. 72, 6951-6960.

Padlan, E.A., Cohen, G.H., Davies, D.R., 1986. Antibody Fab assembly: the interface residues between $\mathrm{CH} 1$ and $\mathrm{CL}$. Mol. Immunol. 23, 951-960.

Pandey, J.P., 2000. Immunoglobulin GM and KM allotypes and vaccine immunity. Vaccine 19, 613-617.

Pandey, J.P., Elson, L.H., Sutherland, S.E., Guderian, R.H., Araujo, E., Nutman, T.B., 1995. Immunoglobulin kappa chain allotypes (KM) in onchocerciasis. J. Clin. Invest. 96, 2732-2734.

Pandey, J.P., Fudenberg, H.H., Virella, G., Kyong, C.U., Loadholt, C.B., Galbraith, R.M., Gotschlich, E.C., Parke, J.C., Jr., 1979. Association between immunoglobulin allotypes and immune responses to Haemophilus influenzae and Meningococcus polysaccharides. Lancet 1, 190-192.

Parng, C.L., Hansal, S., Goldsby, R.A., Osborne, B.A., 1996. Gene conversion contributes to Ig light chain diversity in cattle. J. Immunol. 157, 5478-5486.

Pritsch, O., Hudry-Clergeon, G., Buckle, M., Petillot, Y., Bouvet, J.P., Gagnon, J., Dighiero, G., 1996. Can immunoglobulin $\mathrm{C}(\mathrm{H}) 1$ constant region domain modulate antigen binding affinity of antibodies? J. Clin. Invest. 98, 2235-2243.

Roschenthaler, F., Hameister, H., Zachau, H.G., 2000. The 5' part of the mouse immunoglobulin kappa locus as a continuously cloned structure. Eur. J. Immunol. 30, 3349-3354.

Sanger, F., Nicklen, S., Coulson, A.R., 1977. DNA sequencing with chain-terminating inhibitors. Proc. Natl. Acad. Sci. U. S. A. 74, 5463-5467.

Schanfield, M.S., Ferrell, R.E., Hossaini, A.A., Gerald Sandler, S., Stevenson, J.C., 2008. Immunoglobulin allotypes in Southwest Asia: populations at the crossroads. Am. J. Hum. Biol. 20, 671-682.

Simianer, H., Kohn, F., 2010. Genetic management of the Gottingen Minipig population. J. Pharmacol. Toxicol. Methods 62, 221-226.

Sitnikova, T., Su, C., 1998. Coevolution of immunoglobulin heavy- and light-chain variable-region gene families. Mol. Biol. Evol. 15, 617-625.

Sun, Y., Wang, C., Wang, Y., Zhang, T., Ren, L., Hu, X., Zhang, R., Meng, Q., Guo, Y., Fei, J., Li, N., Zhao, Y., 2010. A comprehensive analysis of germline and expressed immunoglobulin repertoire in the horse. Dev. Comp. Immunol. 34, 1009-1020.

Thompson, J.D., Higgins, D.G., Gibson, T.J., 1994. CLUSTAL W: improving the sensitivity of progressive multiple sequence alignment through sequence weighting, position-specific gap penalties and weight matrix choice. Nucleic Acids Res. 22, 4673-4680.

Tonegawa, S., 1983. Somatic generation of antibody diversity. Nature 302, 575-581.

Wagner, B., 2006. Immunoglobulins and immunoglobulin genes of the horse. Dev. Comp. Immunol. 30, 155-164.

Winstead, C.R., Zhai, S.K., Sethupathi, P., Knight, K.L., 1999. Antigen-induced somatic diversification of rabbit $\mathrm{lgH}$ genes: gene conversion and point mutation. J. Immunol. 162, 6602-6612. 
Yang, C., Bolotin, E., Jiang, T., Sladek, F.M., Martinez, E., 2007. Prevalence of the initiator over the TATA box in human and yeast genes and identification of DNA motifs enriched in human TATA-less core promoters. Gene 389, 52-65.

Zhou, J., Lottenbach, K.R., Barenkamp, S.J., Reason, D.C., 2004. Somatic hypermutation and diverse immunoglobulin gene usage in the human antibody response to the capsular polysaccharide of Streptococcus pneumoniae Type 6B. Infect. Immun. 72, 3505-3514. 


\section{Chapter 3:}

\section{Bovine Immunoglobulins}

The nature and extent of my contribution to the work was the following:

3. Exceptionally long $\mathrm{CDR} 3 \mathrm{H}$ are not isotype restricted in bovine immunoglobulins

\begin{tabular}{|c|l|}
\hline Nature of contribution & Extent of contribution \\
\hline 1. Scientific design & $50 \%$ \\
\hline 2. Laboratory work & $80 \%$ \\
\hline 3. Evaluation & $70 \%$ \\
\hline 4. Scientific Writing & $65 \%$ \\
\hline
\end{tabular}

4. Development of a bioinformatics framework for the detection of gene conversion and the analysis of combinatorial diversity in immunoglobulin heavy chains in four cattle breeds

\begin{tabular}{|c|l|}
\hline Nature of contribution & Extent of contribution \\
\hline 1. Scientific design & $50 \%$ \\
\hline 2. Laboratory work & $50 \%$ \\
\hline 3. Evaluation & $70 \%$ \\
\hline 4. Scientific Writing & $65 \%$ \\
\hline
\end{tabular}




\section{Bovine immunoglobulin heavy and light chains}

\section{Bovine immunoglobulin heavy chain gene locus}

The bovine immunoglobulin heavy chain gene (IGH) locus was assigned to the Bos taurus autosome (BTA) 21 and spans approximately $250 \mathrm{~kb}$ (Miller et al. 1992; Niku et al. 2012). Southern blot analyses indicated the existence of two IGHV families (boVH1 and boVH2) in the bovine germline repertoire. Before the study of Walther et al. (2013), the expression of only boVH1 genes has been observed and boVH2 was found comprising only pseudogenes (Tutter and Riblet 1989; Berens et al. 1997; Saini et al. 1997; Sinclair et al. 1997; Lopez et al. 1998). The family boVH1 offers a restricted set of functional genes, which shares homologies to the murine Q52 family and human VHII family. The definite number and organization of IGHV has not been determined yet. The latest in silico analysis using blastn on all bovine genome assemblies revealed IGHV on the contigs NW_003104530.1 and NW_003104538.1 on Bos taurus chromosome 21 (BTA21; AC_000178.1), NW_003064289.1, NW_003064290.1, NW_003064296.1, NW_003064297.1, NW_003064298.1, and NW_003064299.1 on BTA7 (AC_000164.1), as well as on several unplaced contigs (Walther et al. 2013). In total, 36 IGHV are identified in the bovine genome assembly. On BTA 21, IGHV1 $\Psi$ and IGHV2 were found at the centromeric region in NW_003104530.1. Eight IGHV located at the telomeric region were described within about $146 \mathrm{~kb}$ on NW_003104538.1

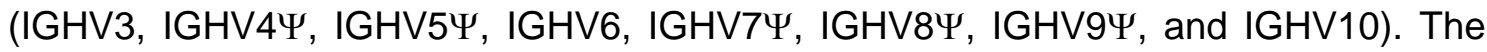
contigs NW_003064289.1, NW_003064290.1, NW_003064296.1, NW_003064297.1, NW_003064298.1, and NW_003064299.1 were annotated to $144 \mathrm{~kb}$ of the centromeric

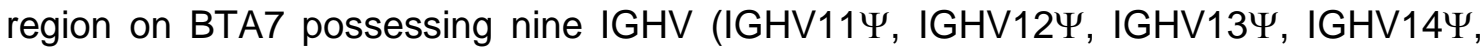

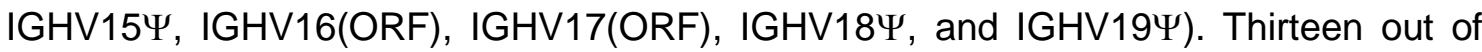
the 36 IGHV segments are putatively functional. Eleven IGHV segment pairs shared sequence identity of $100 \%$ (IGHV3/33, IGHV10/34, IGHV9 $/ 35 \Psi, I G H V 4 \Psi / 32 \Psi$,

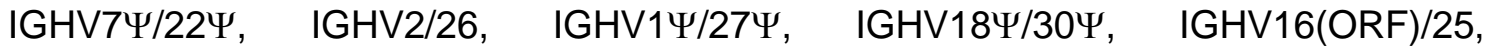
IGHV144/234, and IGHV36/29(F)). Phylogenetic analyses revealed a relation of all functional IGHV to hulGHV2-05, whereas this group corresponds to the bovine IGHV family 1 (boVH1) (Berens et al. 1997; Saini et al. 1997; Sinclair et al. 1997).

In cattle, ten IGHD genes are classified into four families that are organized in subclusters (Koti et al. 2008; Koti et al. 2010). Further, the IGHD exons reveal huge size 
differences (Koti et al. 2008). On the telomeric end of BTA21, the bovine IGHD8 and IGHD4 were localized between IGHV6 and IGHV7Y (Walther et al. 2013). Within the contig NW_003064411.1 on BTA7 one IGHD cluster of $1131 \mathrm{bp}$, encoding IGHD1(ORF) to IGHD3(ORF), was identified. On BTA8 a third location enclosing about $43 \mathrm{~kb}$ possesses five IGHD segments, a $\delta$ chain pseudogene, a $\mu$ chain gene, and six IGHJ segments. In general, loci for the IGHD were observed on three chromosomes (Walther et al. 2013).

By hybridization experiments, six joining segments (IGHJ) were detected on BTA11q23 (Hosseini et al. 2004). The number of IGHJ segments preceding the IGHC genes was described by screening a bovine BAC and Cosmid library. Only two out of six IGHJ are functional (IGHJ1, IGHJ2). IGHJ1 is recombined predominantly while IGHJ2 is rearranged at low frequency (Zhao et al. 2003; Hosseini et al. 2004).

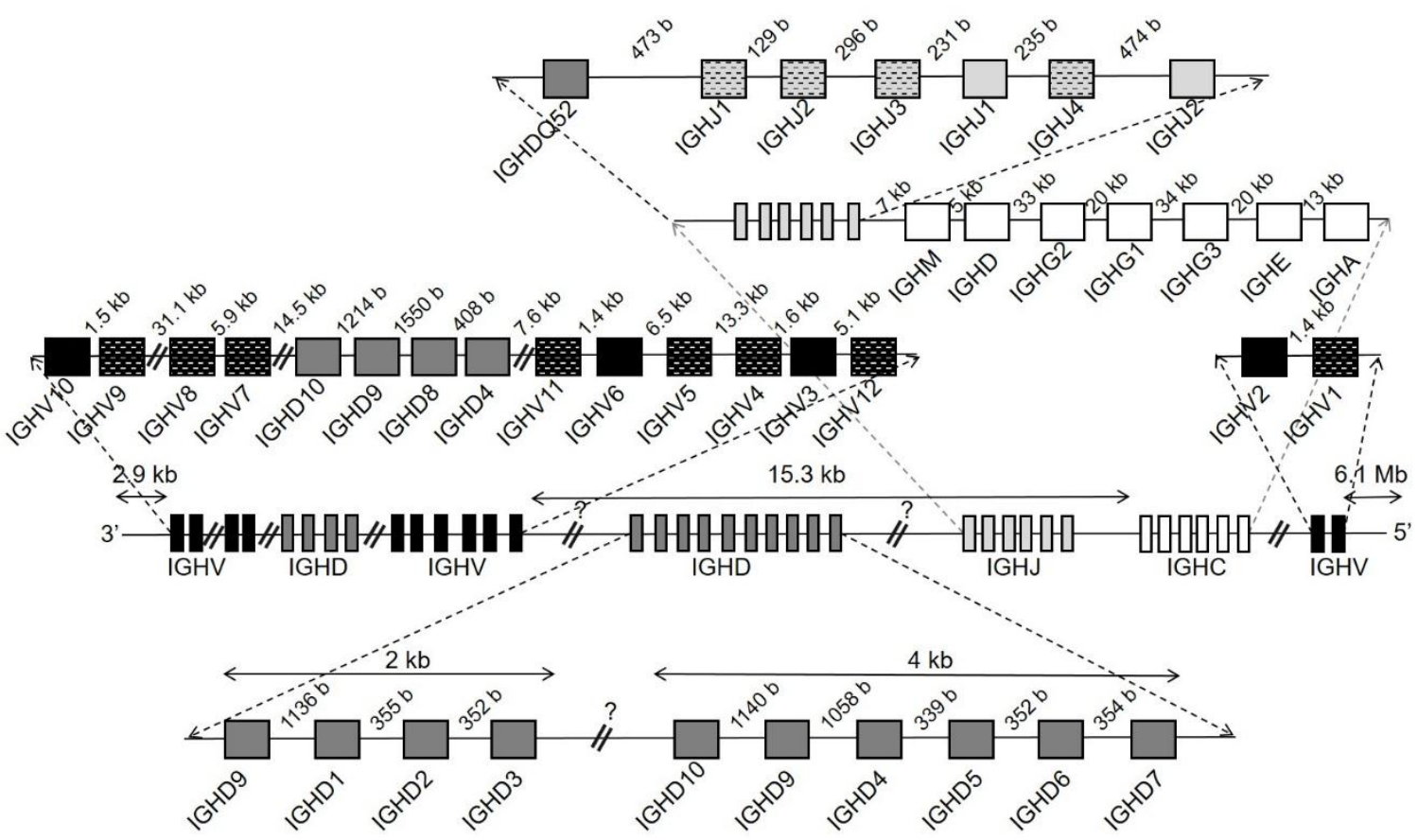

Figure 1: Physical map of the bovine heavy chain locus

The locus is shown in 3'-5' direction on chromosome 21. This figure is adapted from current literature (Zhao et al. 2003; Zimin et al. 2009; Koti et al. 2010; Niku et al. 2012; Walther et al. 2013; Pasman 2014). 
The CDR3H encoded by IGHV-IGHD-IGHJ recombinations varies from 3 to 67 amino acid residues (Berens et al. 1997; Saini et al. 1999; Saini and Kaushik 2002; Saini et al. 2003; Kaushik et al. 2009; Larsen and Smith 2012). These exceptionally long CDR3H are specific for cattle and possess characteristic hydrophilic Glycine and Tyrosine residues. The 148 bp long IGHD2 contributes to those CDR3H (Berens et al. 1997; Shojaei et al. 2003; Koti et al. 2008). Further, a high number of Cysteine residues was described and is demonstrated to promote intra-CDR3H disulfide bonds that contribute to the "knob" domain in exceptionally long CDR3H (Sinclair et al. 1997). In contrast, inter CDR disulfide bonds may exist in mid-length $\mathrm{CDR} 3 \mathrm{H}$ due to one to three Cys residues in CDR3H and one Cys within CDR2H (Saini et al. 1999; Ramsland et al. 2001). Additional mechanisms contributing to the unusual length of bovine CDR3H were studied because the imprecise junction of germline encoded IGHV, IGHD, and IGHJ cannot fully explain the existence of exceptionally long CDR3H in cattle. The insertion of conserved short nucleotide sequences of 13 to 18 nucleotides specifically into the IGHV and IGHD junction was found to contribute to a further elongation of the CDR3H. So far, this mechanism is unique for cattle (Koti et al. 2010).

The genomic organization of the bovine IGHC locus was described by screening a bovine BAC and Cosmid library. All heavy chain isotype classes that have been detected in other mammals were also described for cattle (Knight and Becker 1987; Zhao et al. 2002). Three sub-classes comprise the $\mathrm{\gamma}$-isotype, namely $\mathrm{\gamma} 1, \mathrm{\gamma} 2$, and $\mathrm{\gamma} 3$ (Knight and Becker 1987; Symons et al. 1989). The IGHC locus spans approximately $150 \mathrm{~kb}$ enclosing the genes in the following order: 5 ' $-\mathrm{JH}-7 \mathrm{~kb}-\mu-5 \mathrm{~kb}-\delta-33 \mathrm{~kb}-\mathrm{\gamma} 3-20$

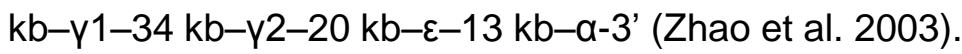

\section{Bovine immunoglobulin M (IgM)}

$\lg \mathrm{M}$ is the major serum antibody in the primary immune response as it provides the first antibody-mediated host defense (Saini and Kaushik 2001; Woof and Burton 2004). IgM combats septicaemia when administered passively to calves and it is an important bactericidal antibody directed against mastitis in cattle and other ruminants (Mousavi et al. 1998). High amounts of lgM are described in colostrum and milk (3 mg/ml) (Butler 1995).

Two loci comprising functional IGHM genes were identified on BTA21 and BTA11q23 by in situ hybridization, whereas the latter is currently assigned to BTA8 (Tobin-Janzen and Womack 1992; Hayes and Petit 1993). The IGHM gene comprises four exons 
(IGHM1-4) encoding the constant domains, as well as two additional exons encoding the transmembrane domain (TM1, TM2) (Mousavi et al. 1998). Three bovine IgM allotypes are described, designated as $\operatorname{lgMa}$, IgMb, and IgMc. While these allotypes are classified by amino acid substitutions within the exons, further IgM variants may result via alternative splicing e.g. inserting in frame codons at the IGHM1 and IGHM2 junction. IGHM2 possesses few proline residues and functions as a hinge restricting flexibility and antigen binding ability of IgM (Saini and Kaushik 2001; Pasman 2014).

\section{Bovine immunoglobulin $D(\lg D)$}

On BTA7 (contig NW_003064411.1), a cluster of 1131 bp was identified encoding exons for IGHD1(ORF) to IGHD3(ORF) and a pseudogene for a $\delta$ chain. The exons 1 and 2 of the IGHD pseudogene are fragmented and exon 6 is missing (codes for the secretory region). Nevertheless, $\lg \mathrm{D}$ is also found in a secreted form although showing lower concentrations than IgG, IgA, and IgM (Preud'homme et al. 2000; Zhao et al. 2002). Further, frame shifts were identified. Genomic information for IGHD4 to 7 and a $\delta$ chain pseudogene were found on the contig NW_001503306.1, while IGHD4 and 8 were detected on NW_001504477.2. A locus involving a putative functional $\delta$ chain gene was detected on NT_186572. In addition to NW_003100387.1, including a $\delta$ chain pseudogene locus, a putative functional $\delta$ chain gene was noted on NW_003100112.1 (Walther et al. 2013).

The bovine, ovine, and porcine germline $\lg \mathrm{D}$ genes are transcriptionally active at low levels and show structural similarities to the human $\lg D$ due to the three constant domains and the hinge region. As phylogenetic analyses revealed the $C \delta$ gene duplicated from the $\mathrm{C} \mu$ gene more than 300 million years ago, the exon of the first constant domain of the IgD (IGHD1) is very similar to the IGHM1 exon. Further, unlike other species, the bovine IGHD has a switch region that may permit class switch recombination (Zhao et al. 2002).

\section{Bovine immunoglobulin $G(\lg G)$}

Immunoglobulin $\mathrm{G}$ is the most abundant isotype in bovine serum as it constitutes more than $75 \%$ of serum immunoglobulins (Saini et al. 2007). It is also found in blood, lymph, peritoneal fluid, and cerebrospinal fluid. Furthermore, IgG contributes to different effector functions. For instance, it is involved in the enhancement of phagocytosis, 
antibody-dependent cellular cytotoxicity, the regulation of cytokine and antibody production, antigen processing and presentation, as well as in the selective transfer via the FcRn-like receptor on alveolar epithelial cells of mammary glands (Burg and Pillinger 2001; Kacskovics 2004; Mestecky 2005; Pentsuk and van der Laan 2009). Three $\lg G$ subclasses (IgG1, $\lg G 2$, and $\lg G 3$ ) were identified in cattle, whereas two subclasses (IgG1 and IgG2) were found in sheep, seven IgG subclasses (IgG1-7) were detected in horses, four subclasses were described in humans, five in swine, and one in rabbits (Knight et al. 1988; Symons et al. 1989; Clarkson et al. 1993; Kacskovics et al. 1995; Kacskovics and Butler 1996; Rabbani et al. 1997; Wagner et al. 2004; Pasman 2014). Depending on characterization methods, two different types of designation exist. IgG1, IgG2a, and lgG2b were used in serological analyses, whereas $\lg$ 1, IgG2, and lgG3 were used in molecular analyses, respectively (Knight et al. 1988). Serum concentrations of $\mathrm{lgG} 1$ and $\mathrm{lgG} 2$ reveal $10 \mathrm{mg} / \mathrm{ml}$ and the concentration of IgG1 can exceed $60 \mathrm{mg} / \mathrm{ml}$ in hyperimmunized animals. In colostrum, IgG1 concentration was also found to exceed $100 \mathrm{mg} / \mathrm{ml}$ (Butler 1995). The bovine IgG3 shows low serum concentrations, which may be caused by the longer hinge region that is a preferred proteolytic site (Rabbani et al. 1997).

These three bovine $C_{\gamma}$ genes ( $\gamma_{1}$, $\gamma_{2}$, and $\gamma_{3}$ ) have been mapped to chromosome $21 q 24$ and are located between the $\delta$ and $\varepsilon$ genes of the heavy chain locus (Gu et al. 1992; Tobin-Janzen and Womack 1992; Chowdhary et al. 1996; Saini et al. 2007). Interestingly, in an in silico study, on the bovine genome assembly BTA20 revealed the genomic information for a $\gamma_{2}$ chain. A genomic order of $\mathrm{\gamma} 3, \mathrm{\gamma} 2$, and $\mathrm{\gamma} 1$ was identified on NT_185580.1. Genes coding for $\gamma 1$ and $\gamma 3$ were found on NW_003100065.1 and NW_003099305.1 (Walther et al. 2013). Gene duplication of bovine IGHG1 led to IGHG2 and IGHG3. Sequence similarities of $85.1 \%$ and $83.4 \%$ exist between the homologues IGHG1 and IGHG3, as well as IGHG1 and IGHG2, respectively (Zhao et al. 2003). For IgG1, four allotypic variants were described (IgG1a-d) (Symons et al. 1989; Jackson et al. 1992; Kacskovics and Butler 1996; Saini et al. 2007). The bovine $\lg G 2$ and IgG3 reveal the two allotypic variants IgG2a and IgG2b (Kacskovics and Butler 1996) and IgG3a and IgG3b (Rabbani et al. 1997), respectively. Significant structural differences within the hinge regions and the $\mathrm{CH} 3$ domain result in different biological effector functions of IGHG1, IGHG2, and IGHG3. Amino acid residue substitutions were also described within the $\mathrm{CH} 1$ and $\mathrm{CH} 2$ domains of these three genes (Saini et al. 2007).

The four known lgG1 allotypic variants are characterized by individual amino acid 
differences at the positions 190 and $192(\mathrm{CH} 1), 218,224,225$, and 226 (hinge region), as well as $281(\mathrm{CH} 2)$, and $402(\mathrm{CH} 3)$, which were designated using the EU numbering system. IgG1a showed the amino acids Gly190, Thr192, Arg218, Thr224, and Thr226, Asp281, Gly402, as well as a deletion of the amino acid at position 225. The most similar allelic variants IgGb and IgGd possess Gly190, Thr192, Thr218, Pro224, Ser225, Pro226, and Asp281. They solely differ by Gly and Ser at position 402. The allotypic variant IgGc shows the amino acid residues Ala190, Ser192, Arg218, Arg224, Pro226, Asn281, Ser402 and a deletion at position 225. Three-dimensional modeling revealed that all substitutions are located on the surface of the $\lg$ molecule and therefore may be recognized by antisera and effector molecules such as cellular adhesion molecules (Symons et al. 1989; Saini et al. 2007). Additionally, the repeating motif Pro-Ala-Ser-Ser at the positions 189-192 and 205-208 within the $\mathrm{CH} 1$ domain of IgG1c may stabilize the core immunoglobulin fold or promote interactions with fibronectin or similar adhesion molecules. The replacement of Thr with Arg residues within the hinge region reduces the number of O-linked glycosylation sites and increases the susceptibility to degradation by protease secreting bacteria (Saini et al. 2007).

The allotypic variants $\lg G 2 a$ and $\lg G 2 b$ differed in amino acid residues at 19 positions. These amino acid residue substitutions were found in the three constant region domains $(\mathrm{CH} 1, \mathrm{CH} 2$, and $\mathrm{CH} 3)$ but also in the hinge region. In $\mathrm{CH} 1$, IgG2a and IgG2b differed by Ser and Ala at position 129 (Ser/Ala129), Cys/Ser131, Lys/Thr135, Gly/Ala190, and Thr/Ser192. Within the hinge region Ser/lle219, Pro/Cys224, Asn/His225, and His/Asn228 were observed. In CH2, Thr/Ser290, Ile/Asn326, and Ser/Pro332 differentiate IgG2a and IgG2b. Val/Leu364, Ile/Thr370, Asp/Ala378, Asp/Asn384, Ala/Thr402, Arg/Glu419, and Thr/Ala422 are the characteristic amino acids described for IgG2a and lgG2b, respectively. These amino acid differences result in different immune responses of the two lgG2 allotypes (Kacskovics and Butler 1996; Corbeil et al. 1997). In comparison to IgG2a, IgG2b activates the complement more than twice (Bastida-Corcuera et al. 1999a).

Previous studies indicated that $\lg G 3 a$ is expressed at a lower level than $\lg G 3 b$. These two allotypic variants differ in six positions. The amino acids Ser/Arg131, Arg/Leu174, and Ser/Thr192 are located in the $\mathrm{CH} 1$ domain, whereas Pro/GIn237 and Lys/Glu238 were found in the hinge region, and Lys/Arg4431 characterizes individual amino acid residues of $\lg \mathrm{G} 3 \mathrm{a}$ and $\lg \mathrm{G} 3 \mathrm{~b}$ in the $\mathrm{CH} 3$ domain (Rabbani et al. 1997). 
The allotypic variants of IgG provide polymorphic immunoglobulin genetic markers with specific abilities. In particular, modified qualities in complement activation, agedependent expression, and influences on the effector function against Haemophilus somnus and Tritrichomonas foetus were made for the allotypic variants of bovine $\lg \mathrm{G} 2$ (Corbeil et al. 1997; Bastida-Corcuera et al. 1999b; Bastida-Corcuera et al. 2000; Saini et al. 2007).

\section{Bovine immunoglobulin $E(\lg E)$}

Whereas $\lg E$ is found in low amounts in the serum, $\lg E$ is mainly found during immune responses against parasite infections mediated by basophiles and mast cells. Furthermore, the presence of $\lg E$ gives rise to type I hypersensitivity reactions such as asthma, conjunctivitis, and rhinitis (Mousavi et al. 1997). The bovine IgE also shows heat labile skin sensitizing ability as the human IgE does (Hammer et al. 1971).

The latest in silico analysis revealed genomic information for an $\varepsilon$ chain on NT_185723.1 (Walther et al. 2013). This finding is consistent with previous studies, where only a single copy of the bovine IgE was identified (Knight et al. 1988). IgE has four exons (IGHE1-4) showing homologies of $87 \%$ to sheep IGHE, 58\% to horse IGHE, $55 \%$ to human IGHE, and 52\% to mouse IGHE. Due to the additional fourth constant domain, IGHE possesses a higher molecular weight than other lg isotypes. The additional domain is supposed to be important for high affinity binding to Fc receptors on mast cells (Mousavi et al. 1997).

\section{Bovine immunoglobulin A $(\lg A)$}

Immunoglobulin $A$ is the dominating isotype on mucosal surfaces such as mammary glands with an important part in the immune response against microbial infections.

A single IGHA gene was identified in the bovine genome and was assigned to the contig NT_185723.1 (Knight et al. 1988; Walther et al. 2013). IgA possesses three IGHA exons (Brown et al. 1997). Two allelic variants were identified by restrictions fragment length polymorphism and serological analyses but were not confirmed by genomic DNA analyses of 50 Swedish cattle (De Benedictis G 1984; Brown et al. 1997; Pasman 2014). 
Chapter 3: Bovine Immunoglobulins

\section{The bovine immunoglobulin light chain gene loci}

In cattle, two distinct immunoglobulin light chain isotypes (lambda $\lambda$ and kappa $\mathrm{k}$ ) have been described (Pilstrom 2002; Das et al. 2008). In serum antibodies, bovine $\lambda$-light chains are predominantly expressed in a proportion of 95\% (Arun et al. 1996). About $5 \%$ of the heavy chains in serum antibodies are connected with functional $\mathrm{K}$-light chains (Arun et al. 1996; Aitken et al. 1999). Similarly, the light chain repertoire is dominated by $\lambda$-light chains in horses (Ford et al. 1994) and sheep (Foley and Beh 1992; Griebel and Ferrari 1994; Broad et al. 1995), although a functional kappa system was described, too (Home et al. 1992). In contrast, human and mice serum antibodies dominantly associate $\mathrm{k}$-light chains (60\% and 95\%) (Chen et al. 2008). Genomic conditions such as genomic complexity of the loci, recombination signal sequences, the ordered rearrangement of $\mathrm{K}$ - and $\lambda$-light chains, as well as antigen selection are supposed to influence differences in the $k: \lambda$ ratio across species (Pasman et al. 2010).

\section{Bovine lambda light chain genes}

The bovine variable, joining, and constant region genes of the $\lambda$-light chain (IGL) are located on Bos taurus autosome 17 (BTA17) (Tobin-Janzen and Womack 1992). The locus spans $412 \mathrm{~kb}$ and contains 25 to 31 variable gene segments (IGLV) whereof 14 to 17 are potentially functional (Ekman et al. 2009). A total of 63 IGLV were identified on ten scaffolds in the current genome assembly (Btau_3.1). Out of these 63 IGLV, 32 were identified on unplaced contigs and 11 of them are also potentially functional (Ekman et al. 2009). The IGLV locus possessed both transcriptional orientations. All IGLV are organized in three clusters separated by $126.8 \mathrm{~kb}$ and $138.3 \mathrm{~kb}$ introns. The three IGLV clusters also define separate IGLV families designated as IGLV1, IGLV2, and IGLV3. The predominantly expressed IGLV1 genes are subdivided into five subfamilies (IGLV1a, IGLV1b, IGLV1c, IGLV1d, IGLV1x) (Sinclair et al. 1995; Saini et al. 2003). These IGLV1 are located within the two 5' subclusters, whereas the minor expressed IGLV2 and IGLV3 genes were identified within the IGLJ-proximal cluster. The subfamilies IGLV1a and IGLV1b are mainly expressed, whereas IGLV1c, IGLV1e, and IGLV1x specifically pair with heavy chains possessing CDR3H with at least 50 amino acid residues (Saini et al. 1999; Saini et al. 2003). Analyses of genomic sequences of the cattle breed Herford revealed 17 IGLV1 genes of which ten are described functional. Further, three out of four IGLV2 genes as well all four IGLV3 genes were designated functional (Pasman et al. 2010). Phylogenetic comparisons revealed that four of six ovine subgroups cluster with the bovine ones, which are 
supposedly ruminant specific genes. Consequently, due to sequence identities of $92.6 \%$ and $88.9 \%$, the bovine IGLV1 and IGLV2 families are closely related with the ovine families IGLVI and IGLVII. The bovine IGLV3 family is related to the unclassified ovine IGLV family (Pasman et al. 2010; Pasman 2014). In addition, the CDR1L length varies from 6,8 , or 9 codons. The length of CDR2L is restricted to 3 or 7 codons. The number of unique CDR1L-CDR2L combinations is lower than in mice and humans (Ekman et al. 2009).

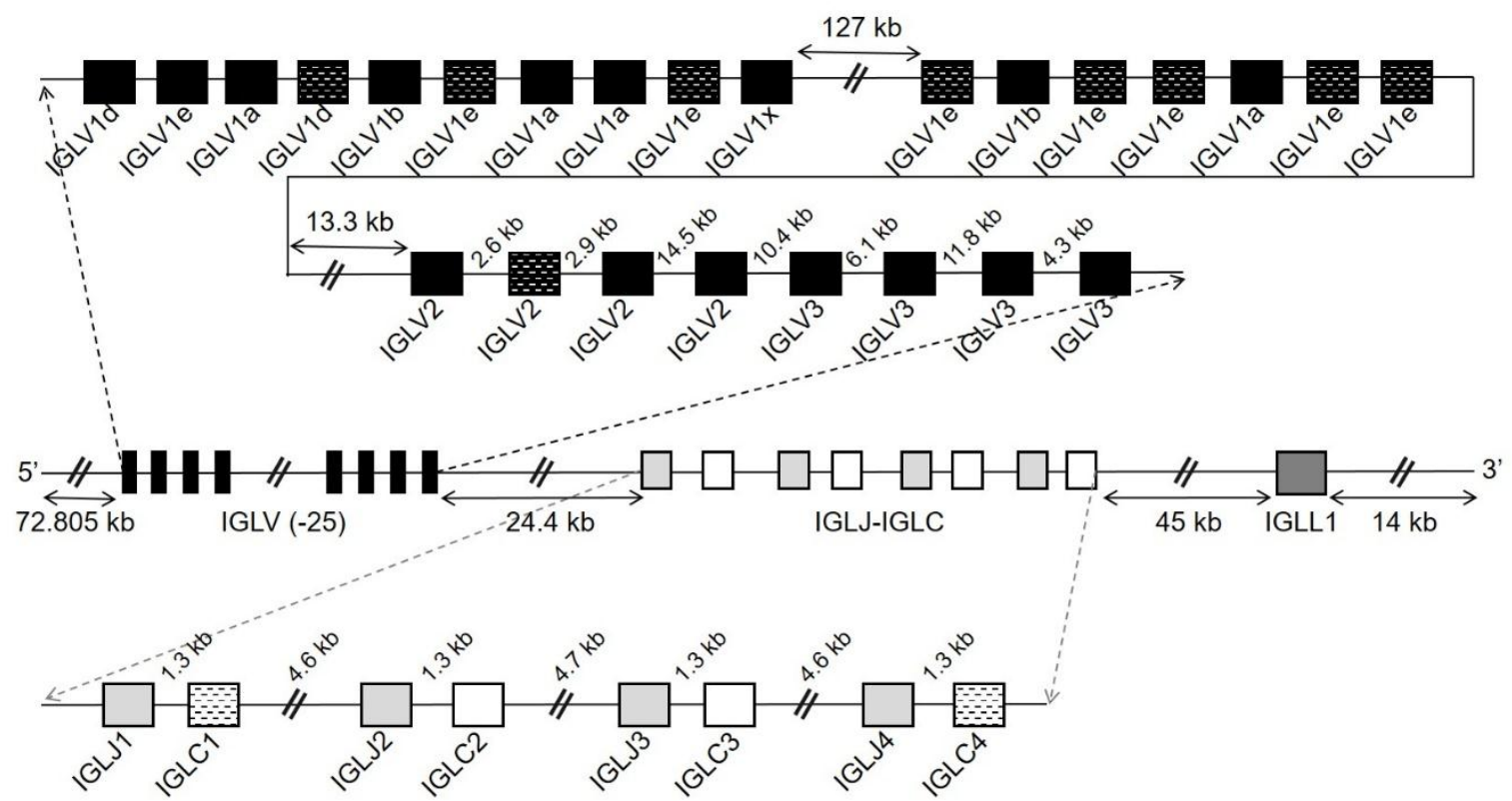

Figure 2: Physical map of the bovine lambda light chain locus

The locus is shown in 5'-3' direction on chromosome 17. This figure is adapted from (Pasman et al. 2010; Pasman 2014).

In cattle, four $\lambda$-light chain constant region (IGLC) genes (IGLC1, IGLC2, IGLC3, and IGLC4) were identified yet, each preceded by a joining gene segment (IGLJ1-4) (Parng et al. 1995; Parng et al. 1996; Chen et al. 2008; Ekman et al. 2009; Pasman et al. 2010). Only IGLC2 and IGLC3 are functional whereas IGLC3 is preferentially expressed during rearrangements. IGLC1 and IGLC4 are pseudogenes (Chen et al. 2008; Ekman et al. 2009; Pasman et al. 2010). A fifth bovine IGLC gene was detected but could not be located on a chromosome until now (Ekman et al. 2009). In comparison, in sheep only two IGLC genes were described (IGLC1 and IGLC2). The 
ovine IGLC1 is functional and in the IGLC2 gene possess a premature stop codon (Jenne et al. 2003). Furthermore, in previous studies seven equine IGLC genes were identified. Three of these genes are functional, whereas the others were described to be pseudogenes (Home et al. 1992; Das et al. 2008; Sun et al. 2010).

Transcription analyses in several cattle breeds revealed single nucleotide polymorphisms (SNPs) for both the bovine IGLC2 and IGLC3 genes resulting in silent mutations or amino acid residue substitutions (Diesterbeck et al. 2012). Within the breeds German Simmental (GS) and Aubrac (A), single nucleotide polymorphisms of IGLC2 were identified. Analyses in the breeds German Black Pied (GBP) and Holstein Friesian (HF) revealed the already known allele and allotype IGLC2a. Two additional alleles, IGLC2b and IGLC2c, were detected and represent putative new allotypes. Amino acid residue substitutions were either conservative or led to charge changes. They were located within the molecule by homology based three-dimensional modeling. Most amino acid residue substitutions were found on the outer side of the molecule in the solvent accessible surface area, within or near the putative interface to IGHC1. Additionally, by in silico analyses IGLC2a was also identified in other cattle breeds such as Angus (DY170709) and Hereford (EH173018). IGLC2a and IGLC2b showed $98.4 \%$ sequence identity while IGLC2C was $97.8 \%$ identical to IGLC2a and 96.9\% identical to IGLC2b (Diesterbeck et al. 2012). Similar to IGLC2, five allelic variants were identified for the predominantly expressed IGLC3 (IGLC3a, IGLC3b, IGLC3c, IGLC3d, IGLC3e) within the same cattle breeds. Amino acid residue substitutions were also determined on the accessible surface of the molecule, as well as in and next to the interface to IGHC1. Reversed charges were observed due to several amino acid residue replacements. Further, nucleotide sequences coding for IGLC3a were found in Hereford (EV679232) and Angus (DY173535), while IGLC3b was only found in Angus (DY149783). IGLC3c was identified both in Holstein Friesian crossbreed (DY145594) and purebred (CK950153). Angus and Holstein Friesian crossbreeds (EV608839, DY216512), as well as Holstein Friesian purebred (CK979405) and Hereford (DT858283) exhibited IGLC3d (Diesterbeck et al. 2012).

\section{Bovine kappa light chain genes}

The bovine $\mathrm{k}$-light chain genes are located on Bos taurus autosome 11 (BTA11) (Ekman et al. 2009) and span approximately $280 \mathrm{~kb}$, although the complete lg k-light chain (IGK) locus encompasses a size of $412 \mathrm{~kb}$ (Pasman et al. 2010). The analysis of the bovine $\mathrm{k}$-light chain locus on Herford genome sequence revealed 22 IGKV, 3 IGKJ, 
and 1 IGKC (Ekman et al. 2009). The IGKV genes are organized in a cluster that covers $150 \mathrm{~kb}$ and which is followed by the three IGKJ genes and the IGKC gene. Out of 22 IGKV, only eight are functional (Ekman et al. 2009). They are present in both transcriptional orientations (Sitnikova and Nei 1998). The 22 IGKV genes are classified into four subfamilies. Twenty-one of them are closely related to the ovine gene families 1,2 , and 4 . Seven of the eight functional segments belong to bovine subgroup 2 (IGKV2), which therefore is supposed to be mainly expressed. This subgroup is phylogenetically related to sheep (91\%), killer whale (84\%), and dolphin (84\%). Bovine IGKV vary from six, ten, or 11 codons within CDR1L and differs from human, mice, and sheep CDR1L possessing 11 codons, whereby CDR2L constantly possesses three codons (Ekman et al. 2009; Ehrenmann et al. 2010; Pasman 2014).

The three IGKJ genes (IGKJ1, IGKJ2, IGKJ3) are clustered within 800 bp on the genome and are followed by one IGKC gene. Although all IGKJ genes are functional, they were all transcribed in different frequencies. IGKJ1 showing the conserved $23 \mathrm{bp}$ RSS, was transcribed predominantly in animals of the breeds GS, HF, A, and GBP (Stein et al. 2012). IGKJ2 was detected in two animals of GS despite of the shortened spacer with a size of $22 \mathrm{bp}$ instead of $23 \mathrm{bp}$ (Ekman et al. 2009). IGKJ3 was also described in each of the four cattle breeds. The distinct cladistic protein motif 'EIK' was found changed in IGKJ3 to 'EIK' (Das et al. 2008).

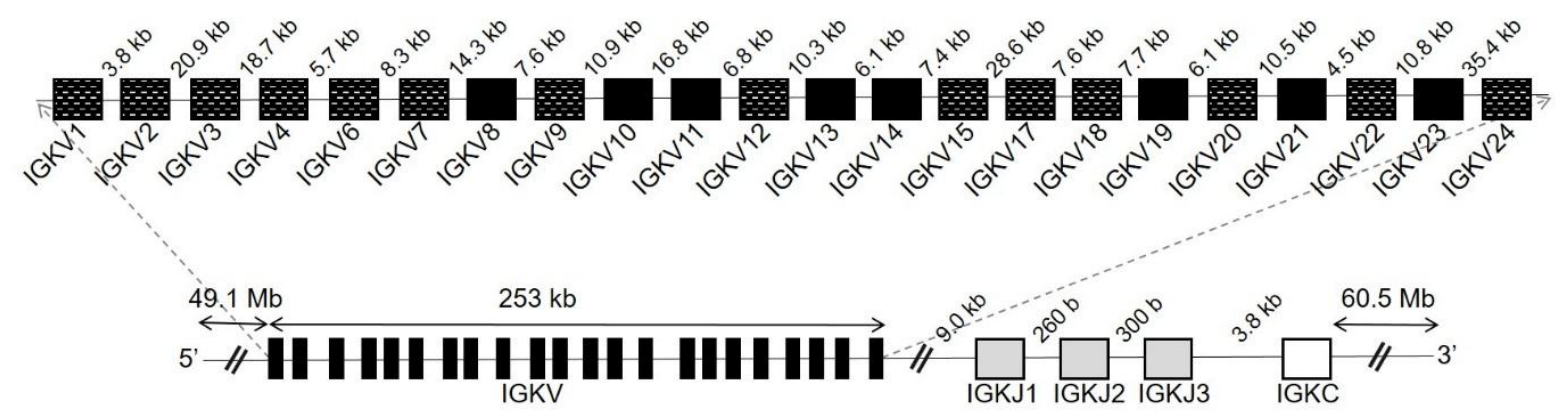

Figure 3: Physical map of the bovine kappa light chain locus

The locus is shown in 5'-3' direction on chromosome 11. The locus is identical to both BTAU 4.6 and UMD 3.1 assembly of the Hereford cow genome. This figure is adapted from Pasman and Kaushik (2014) and Ekman et al. (2009). 
Transcriptional analysis of IGKC within four cattle breeds revealed three alleles distributed in different frequencies (Stein et al. 2012). In animals of HF, GS, and A, all three alleles were identified, whereas in GBP solely IGKCa was found. Additional in silico analyses of the ESTs database revealed allele 1 and 2 of IGKCa in Hereford Shorthorns (CF763228, CF768037). The three alleles differed at four nucleotide positions with two of them leading to amino acid residue substitutions at positions 100 and 116 within the constant region resulting in the putative allotypic variants of IGKCa and IGKCb. The two amino acid residue replacements caused either a change in polarity or a change in electric charge of the amino acid side chain. The amino acid residue substitutions were located at the solvent accessible surface. Also for humans and rabbits three and five allotypes were described, respectively (Emorine et al. 1983; Moxley and Gibbs 1992). In humans, an improvement of immune responses by IGKC allotypes was observed (Pandey et al. 1979; Granoff et al. 1984). Due to the similar substitutions in bovine allotypes, different serological properties are assumed in cattle allotypes, too (Stein et al. 2012).

\section{Development of B cells and the bovine antibody repertoire}

The development of immunoglobulin producing cells already starts in the bovine fetus. While IgM-bearing B-cells have been detected at day 59 of gestation (Schultz et al. 1973), recombinations of heavy and light chain gene segments were observed in splenic B-cells at day 125 of gestation and serum immunoglobulins were detectable at day 145 of gestation (Saini and Kaushik 2002). The perinatal diversification occurs in the ileal Peyer's patches (Yasuda et al. 2004; Yasuda et al. 2006) but $\lambda$-light chain diversification was observed in bovine fetal spleen prior to the diversification in the ileum (Lucier et al. 1998; Aida 2014). Pre-B like cells were also noted in the fetal bone marrow and lymph node indicating $B$ lymphopoieses in parallel to ileal Peyer's patches (Ekman et al. 2012; Aida 2014).

The generation of antibody diversity in vertebrates depends on several mechanisms that are very similar in all vertebrate species. Such processes are the recombination of separated germline gene segments (IGHV, IGHD, and IGHJ; IGLV/IGKV and IGLJ/IGKJ), the imprecise junction of these gene segments due to nucleotide deletions or additions (N, P), the combination of two identical heavy and light chains (Alt and Baltimore 1982; Kurosawa and Tonegawa 1982; Tonegawa 1983; Desiderio et al. 1984), as well as somatic hypermutations (Reynaud et al. 1995; Wagner and 
Neuberger 1996; Berens et al. 1997). The available pool of the germline IGHV, IGHD, and IGHJ segments differs between species. Whereas humans and mice possess a high number of IGHV-IGHD-IGHJ genes, in livestock such as chicken (Reynaud et al. 1989), pigs (Sun et al. 1994), sheep (Dufour et al. 1996), and cattle (Berens et al. 1997; Saini et al. 1997; Sinclair et al. 1997) relatively few genes were detected resulting in a restricted combinatorial diversity. Therefore, several diversifying mechanisms dominate or additional options are employed in such species. For instance, in chicken and bovine $\lambda$-light chains gene conversion is a postrecombinatorial strategy for the generation of the preimmune antibody repertoire (Reynaud et al. 1995; Arakawa et al. 2004). Further, this mechanism is discussed in horses (Sun et al. 2010).

Today, differences in gene usage of bovine fetus and adult animals are known. In fetal IGHV-IGHD-IGHJ recombinations two IGHV are preferentially used (Saini and Kaushik 2002). Furthermore, IGHD5 and IGHD7 dominate both in bovine fetus and adult animals in contrast to IGHDQ52 (Koti et al. 2010). Within the CDRs transition nucleotide substitutions predominate over transversion mutations and in framework region three somatic hypermutations lead to isotype dependent degree of diversification (Kaushik et al. 2009).

In both bovine fetal and adult heavy chains, exceptionally long CDR3H regions contribute to higher variability, which is restricted by the use of only one VH-family (Armour et al. 1994; Saini et al. 1999; Saini and Kaushik 2002; Wang et al. 2013). Those long $\mathrm{CDR} 3 \mathrm{H}$ regions were first described in IgM but were also detected in IgG and in all other isotypes by now and occur in $8-10 \%$ of circulating B cells (Saini et al. 1997; Sinclair et al. 1997; Saini et al. 1999; Saini and Kaushik 2002; Saini et al. 2003; Kaushik et al. 2009; Larsen and Smith 2012; Walther et al. 2013). The length of $\mathrm{CDR} 3 \mathrm{H}$ varies by class. Consequently, the bovine $\mathrm{CDR} 3 \mathrm{H}$ has an average length of $22.7 \pm 3.2$ amino acids, whereas in $\lg M$ there are $21.7 \pm 1.8$ and in $\lg$ G there are $18.2 \pm$ 1.3 amino acids (Almagro et al. 2006; Kaushik et al. 2009; Aida 2014). While the $\mathrm{CDR} 3 \mathrm{H}$ region in human and mice encodes 12 amino acid residues, in cattle it extends up to 67 amino acid residues (Sun et al. 1994; Walther et al. 2013) and up to 30 in chicken (McCormack and Thompson 1990) where this phenomenon is caused by the usage of two IGHD segments within on CDR3H region. Therefore the length of the $\mathrm{CDR} 3 \mathrm{H}$ region is $50 \%$ greater than in human, mice, swine, and rabbit sequences (Butler 1997). The extensive size heterogeneity in CDR3H together with intrachain disulfide bridges results in a configurational diversity of this region (Saini and Kaushik 
2002; Wang et al. 2013). Crystallization of such bovine antibodies revealed a unique 'stalk and knob' structure (Wang et al. 2013). Although both fetal and adult exceptionally long CDR3H originate from identical IGHV-IGHD-IGHJ rearrangements, there are structural differences. In the bovine fetus, there is no addition of CSNS at the IGHV-IGHD junction resulting in a shorter or non-existent stalk. Due to a structural support, bovine heavy chains with exceptionally long $\mathrm{CDR} 3 \mathrm{H}$ always pair with $\lambda$-light chains that possess a conserved Ser at position 90 (Wang et al. 2013).

In contrast to human and mice bovine antibody diversity increases by somatic hypermutation, which may also occur without previous antigen contact (Tomlinson et al. 1996; Berens et al. 1997; Lopez et al. 1998). Furthermore, diversity already exists at the foetal stage as a result of somatic hypermutation.

In bovine light chains, gene conversion and somatic hypermutation are the major mechanisms leading to diversification (Parng et al. 1995; Parng et al. 1996). Initial diversification of $\lambda$-light chains was identified in the early fetal spleen before the establishment of a diverse repertoire in the ileum in cattle and sheep (Lee and Richards 1971). Gene conversion is supposed to be less significant in sheep than in cattle as there is a higher number of IGLV pseudogenes in the bovine genome (Parng et al. 1996; Ekman et al. 2009). This mechanism is also highly relevant regarding light chain diversification in chicken (Arakawa and Buerstedde 2009), as well as in rabbits using gene conversion to diversify Ig heavy chains (Weinstein et al. 1994). In addition, allotypes diversify the immunoglobulin repertoire, which has already been described in species like humans and rabbits. For instance, in humans three allotypic variants of the so called Km 1, Km 1.2, and Km 3 (Moxley and Gibbs 1992) are described. Similarly, rabbits possess five allotypic variants of the $\mathrm{k}$-light chain constant region gene called b4 (a+b), b5, b6, and b9 (Emorine et al. 1983). In cattle, allotypes have been identified for both the heavy and light chains (Bastida-Corcuera et al. 1999a; Bastida-Corcuera et al. 1999b; Diesterbeck et al. 2012; Stein et al. 2012). 
Chapter 3: Bovine Immunoglobulins

\section{References}

Aida Y, Takeshima, S.-N., Baldwin, C. L. and Kaushik, A. K. 2014. Bovine Immunogenetics. in The Genetics of Cattle (ed. DJaR Garrick, A.). CAB International, $\mathrm{CAB}$ International.

Aitken R, Hosseini A, MacDuff R. 1999. Structure and diversification of the bovine immunoglobulin repertoire. Veterinary immunology and immunopathology $\mathbf{7 2 :}$ 21-29.

Almagro JC, Martinez L, Smith SL, Alagon A, Estevez J, Paniagua J. 2006. Analysis of the horse $\mathrm{V}(\mathrm{H})$ repertoire and comparison with the human IGHV germline genes, and sheep, cattle and pig $\mathrm{V}(\mathrm{H})$ sequences. Molecular immunology 43: 1836-1845.

Alt FW, Baltimore D. 1982. Joining of immunoglobulin heavy chain gene segments: implications from a chromosome with evidence of three D-JH fusions. Proc Natl Acad Sci U S A 79: 4118-4122.

Arakawa H, Buerstedde JM. 2009. Activation-induced cytidine deaminase-mediated hypermutation in the DT40 cell line. Philosophical transactions of the Royal Society of London Series B, Biological sciences 364: 639-644.

Arakawa H, Saribasak H, Buerstedde JM. 2004. Activation-induced cytidine deaminase initiates immunoglobulin gene conversion and hypermutation by a common intermediate. PLoS Biol 2: E179.

Armour KL, Tempest PR, Fawcett PH, Fernie ML, King SI, White P, Taylor G, Harris WJ. 1994. Sequences of heavy and light chain variable regions from four bovine immunoglobulins. Molecular immunology 31: 1369-1372.

Arun SS, Breuer W, Hermanns W. 1996. Immunohistochemical examination of lightchain expression (lambda/kappa ratio) in canine, feline, equine, bovine and porcine plasma cells. Zentralblatt fur Veterinarmedizin Reihe A 43: 573-576.

Bastida-Corcuera F, Butler JE, Heyermann H, Thomford JW, Corbeil LB. 2000. Tritrichomonas foetus extracellular cysteine proteinase cleavage of bovine IgG2 allotypes. The Journal of parasitology 86: 328-332.

Bastida-Corcuera FD, Butler JE, Yahiro S, Corbeil LB. 1999a. Differential complement activation by bovine $\operatorname{lgG} 2$ allotypes. Veterinary immunology and immunopathology 71: 115-123.

Bastida-Corcuera FD, Nielsen KH, Corbeil LB. 1999b. Binding of bovine IgG2a and $\lg G 2 b$ allotypes to protein $A$, protein $G$, and Haemophilus somnus IgBPs. Veterinary immunology and immunopathology 71: 143-149.

Berens SJ, Wylie DE, Lopez OJ. 1997. Use of a single VH family and long CDR3s in the variable region of cattle Ig heavy chains. International immunology 9: 189199.

Broad TE, Burkin DJ, Cambridge LM, Jones C, Lewis PE, Morse HG, Geyer D, Pearce PD, Ansari HA, Maher DW. 1995. Six loci mapped on to human chromosome $2 p$ are assigned to sheep chromosome 3p. Animal genetics 26: 85-90.

Brown WR, Rabbani H, Butler JE, Hammarstrom L. 1997. Characterization of the bovine $\mathrm{C}$ alpha gene. Immunology 91: 1-6.

Burg ND, Pillinger $\mathrm{MH}$. 2001. The neutrophil: function and regulation in innate and humoral immunity. Clinical immunology 99: 7-17.

Butler JE. 1995. Antigen receptors, their immunomodulation and the immunoglobulin genes of cattle and swine. Livest Prod Sci 42: 105-121.

Butler JE. 1997. Immunoglobulin gene organization and the mechanism of repertoire development. Scandinavian journal of immunology 45: 455-462. 
Chen L, Li M, Li Q, Yang X, An X, Chen Y. 2008. Characterization of the bovine immunoglobulin lambda light chain constant IGLC genes. Veterinary immunology and immunopathology 124: 284-294.

Chowdhary BP, Fronicke L, Gustavsson I, Scherthan H. 1996. Comparative analysis of the cattle and human genomes: detection of ZOO-FISH and gene mappingbased chromosomal homologies. Mammalian genome : official journal of the International Mammalian Genome Society 7: 297-302.

Clarkson CA, Beale D, Coadwell JW, Symons DB. 1993. Sequence of ovine Ig gamma 2 constant region heavy chain cDNA and molecular modelling of ruminant IgG isotypes. Molecular immunology 30: 1195-1204.

Corbeil LB, Gogolewski RP, Kacskovics I, Nielsen KH, Corbeil RR, Morrill JL, Greenwood R, Butler JE. 1997. Bovine IgG2a antibodies to Haemophilus somnus and allotype expression. Canadian journal of veterinary research $=$ Revue canadienne de recherche veterinaire 61: 207-213.

Das S, Nikolaidis N, Klein J, Nei M. 2008. Evolutionary redefinition of immunoglobulin light chain isotypes in tetrapods using molecular markers. Proceedings of the National Academy of Sciences of the United States of America 105: 1664716652.

De Benedictis G CP, Dragone A. 1984. Identification of an allotypic IgA in cattle serum. Comp Immunol Microbiol Infect Dis 7: 35-42.

Desiderio SV, Yancopoulos GD, Paskind M, Thomas E, Boss MA, Landau N, Alt FW, Baltimore D. 1984. Insertion of $\mathrm{N}$ regions into heavy-chain genes is correlated with expression of terminal deoxytransferase in B cells. Nature 311: 752-755.

Diesterbeck US, Aboelhassan DM, Stein SK, Czerny CP. 2012. Detection of new allotypic variants of bovine lambda-light chain constant regions in different cattle breeds. Developmental and comparative immunology 36: 130-139.

Dufour V, Malinge S, Nau F. 1996. The sheep Ig variable region repertoire consists of a single VH family. J Immunol 156: 2163-2170.

Ehrenmann F, Kaas Q, Lefranc MP. 2010. IMGT/3Dstructure-DB and IMGT/DomainGapAlign: a database and a tool for immunoglobulins or antibodies, T cell receptors, MHC, IgSF and MhcSF. Nucleic acids research 38: D301-307.

Ekman A, Ilves M, livanainen A. 2012. B lymphopoiesis is characterized by pre-B cell marker gene expression in fetal cattle and declines in adults. Developmental and comparative immunology 37: 39-49.

Ekman A, Niku M, Liljavirta J, livanainen A. 2009. Bos taurus genome sequence reveals the assortment of immunoglobulin and surrogate light chain genes in domestic cattle. BMC immunology 10: 22.

Emorine L, Dreher K, Kindt TJ, Max EE. 1983. Rabbit immunoglobulin kappa genes: structure of a germline b4 allotype $\mathrm{J}-\mathrm{C}$ locus and evidence for several b4related sequences in the rabbit genome. Proceedings of the National Academy of Sciences of the United States of America 80: 5709-5713.

Foley RC, Beh KJ. 1992. Analysis of immunoglobulin light chain loci in sheep. Animal genetics 23: 31-42.

Ford JE, Home WA, Gibson DM. 1994. Light chain isotype regulation in the horse. Characterization of Ig kappa genes. Journal of immunology 153: 1099-1111.

Granoff DM, Pandey JP, Boies E, Squires J, Munson RS, Jr., Suarez B. 1984. Response to immunization with Haemophilus influenzae type b polysaccharidepertussis vaccine and risk of Haemophilus meningitis in children with the $\mathrm{Km}(1)$ immunoglobulin allotype. The Journal of clinical investigation 74: 1708-1714. 
Griebel PJ, Ferrari G. 1994. Evidence for a stromal cell-dependent, self-renewing B cell population in lymphoid follicles of the ileal Peyer's patch of sheep. European journal of immunology 24: 401-409.

Gu F, Chowdhary BP, Andersson L, Harbitz I, Gustavsson I. 1992. Assignment of the bovine immunoglobulin gamma heavy chain (IGHG) gene to chromosome 21 q24 by in situ hybridization. Hereditas 117: 237-240.

Hammer DK, Kickhofen B, Schmid T. 1971. Detection of homocytotropic antibody associated with a unique immunoglobulin class in the bovine species. European journal of immunology 1: 249-257.

Hayes HC, Petit EJ. 1993. Mapping of the beta-lactoglobulin gene and of an immunoglobulin $M$ heavy chain-like sequence to homoeologous cattle, sheep, and goat chromosomes. Mamm Genome 4: 207-210.

Home WA, Ford JE, Gibson DM. 1992. L chain isotype regulation in horse. I. Characterization of Ig lambda genes. Journal of immunology 149: 3927-3936.

Hosseini A, Campbell G, Prorocic M, Aitken R. 2004. Duplicated copies of the bovine $\mathrm{JH}$ locus contribute to the Ig repertoire. Int Immunol 16: 843-852.

Jackson T, Morris BA, Sanders PG. 1992. Nucleotide sequences and expression of cDNAs for a bovine anti-testosterone monoclonal IgG1 antibody. Molecular immunology 29: 667-676.

Jenne CN, Kennedy LJ, McCullagh P, Reynolds JD. 2003. A new model of sheep Ig diversification: shifting the emphasis toward combinatorial mechanisms and away from hypermutation. Journal of immunology 170: 3739-3750.

Kacskovics I. 2004. Fc receptors in livestock species. Veterinary immunology and immunopathology 102: 351-362.

Kacskovics I, Butler JE. 1996. The heterogeneity of bovine IgG2--VIII. The complete cDNA sequence of bovine IgG2a (A2) and an lgG1. Molecular immunology 33: 189-195.

Kacskovics I, Wittum TE, Butler JE, Littledike ET. 1995. The heterogeneity of bovine IgG2. VII. The phenotypic distribution of the A1 and A2 allotypes of IgG2a among beef cows with known clinical history. Veterinary immunology and immunopathology 48: 89-96.

Kaushik AK, Kehrli ME, Jr., Kurtz A, Ng S, Koti M, Shojaei F, Saini SS. 2009. Somatic hypermutations and isotype restricted exceptionally long CDR3H contribute to antibody diversification in cattle. Veterinary immunology and immunopathology 127: 106-113.

Knight KL, Becker RS. 1987. Isolation of genes encoding bovine $\lg M, \lg G$, $\lg A$ and $\lg E$ chains. Vet Immunol Immunopathol 17: 17-24.

Knight KL, Suter M, Becker RS. 1988. Genetic engineering of bovine Ig. Construction and characterization of hapten-binding bovine/murine chimeric $\lg \mathrm{E}, \lg \mathrm{A}, \lg \mathrm{G} 1$, IgG2, and IgG3 molecules. Journal of immunology 140: 3654-3659.

Koti M, Kataeva G, Kaushik AK. 2008. Organization of $D(H)$-gene locus is distinct in cattle. Developments in biologicals 132: 307-313.

Koti M, Kataeva G, Kaushik AK. 2010. Novel atypical nucleotide insertions specifically at $\mathrm{VH}-\mathrm{DH}$ junction generate exceptionally long $\mathrm{CDR} 3 \mathrm{H}$ in cattle antibodies. Molecular immunology 47: 2119-2128.

Kurosawa Y, Tonegawa S. 1982. Organization, structure, and assembly of immunoglobulin heavy chain diversity DNA segments. J Exp Med 155: 201-218.

Larsen PA, Smith TP. 2012. Application of circular consensus sequencing and network analysis to characterize the bovine IgG repertoire. BMC immunology 13: 52.

Lee B, Richards FM. 1971. The interpretation of protein structures: estimation of static accessibility. Journal of molecular biology 55: 379-400. 
Lopez O, Perez C, Wylie D. 1998. A single VH family and long CDR3s are the targets for hypermutation in bovine immunoglobulin heavy chains. Immunological reviews 162: 55-66.

Lucier MR, Thompson RE, Waire J, Lin AW, Osborne BA, Goldsby RA. 1998. Multiple sites of $\mathrm{V}$ lambda diversification in cattle. Journal of immunology 161: 54385444.

McCormack WT, Thompson CB. 1990. Somatic diversification of the chicken immunoglobulin light-chain gene. Advances in immunology 48: 41-67.

Mestecky J. 2005. Mucosal immunology, Amsterdam; Boston.

Miller JR, Thomsen PD, Dixon SC, Tucker EM, Konfortov BA, Harbitz I. 1992. Synteny mapping of the bovine IGHG2, CRC and IGF1 genes. Animal genetics 23: 5158.

Mousavi M, Rabbani H, Hammarstrom L. 1997. Characterization of the bovine epsilon gene. Immunology 92: 369-373.

Mousavi M, Rabbani H, Pilstrom L, Hammarstrom L. 1998. Characterization of the gene for the membrane and secretory form of the IgM heavy-chain constant region gene (C mu) of the cow (Bos taurus). Immunology 93: 581-588.

Moxley G, Gibbs RS. 1992. Polymerase chain reaction-based genotyping for allotypic markers of immunoglobulin kappa shows allelic association of $\mathrm{Km}$ with kappa variable segment. Genomics 13: 104-108.

Niku M, Liljavirta J, Durkin K, Schroderus E, livanainen A. 2012. The bovine genomic DNA sequence data reveal three IGHV subgroups, only one of which is functionally expressed. Developmental and comparative immunology 37: 457461.

Pandey JP, Fudenberg HH, Virella G, Kyong CU, Loadholt CB, Galbraith RM, Gotschlich EC, Parke JC, Jr. 1979. Association between immunoglobulin allotypes and immune responses to Haemophilus influenzae and Meningococcus polysaccharides. Lancet 1: 190-192.

Parng CL, Hansal S, Goldsby RA, Osborne BA. 1995. Diversification of bovine lambdalight chain genes. Annals of the New York Academy of Sciences 764: 155-157.

Parng CL, Hansal S, Goldsby RA, Osborne BA. 1996. Gene conversion contributes to Ig light chain diversity in cattle. Journal of immunology 157: 5478-5486.

Pasman Y, Saini SS, Smith E, Kaushik AK. 2010. Organization and genomic complexity of bovine lambda-light chain gene locus. Veterinary immunology and immunopathology 135: 306-313.

Pasman YK, A. 2014. Bovine Immunoglobulin Genetics: Novel Phylogenetic Perspective. in Comparative Immunoglobulin Genetics (ed. AaP Kaushik, Y.). Apple Academic Press, Toronto, Canada.

Pentsuk N, van der Laan JW. 2009. An interspecies comparison of placental antibody transfer: new insights into developmental toxicity testing of monoclonal antibodies. Birth defects research Part B, Developmental and reproductive toxicology 86: 328-344.

Pilstrom L. 2002. The mysterious immunoglobulin light chain. Developmental and comparative immunology 26: 207-215.

Preud'homme JL, Petit I, Barra A, Morel F, Lecron JC, Lelievre E. 2000. Structural and functional properties of membrane and secreted IgD. Molecular immunology 37: 871-887.

Rabbani H, Brown WR, Butler JE, Hammarstrom L. 1997. Polymorphism of the IGHG3 gene in cattle. Immunogenetics 46: 326-331.

Ramsland PA, Kaushik A, Marchalonis JJ, Edmundson AB. 2001. Incorporation of long CDR3s into $V$ domains: implications for the structural evolution of the antibodycombining site. Exp Clin Immunogenet 18: 176-198. 
Reynaud CA, Dahan A, Anquez V, Weill JC. 1989. Somatic hyperconversion diversifies the single Vh gene of the chicken with a high incidence in the D region. Cell 59: 171-183.

Reynaud CA, Garcia C, Hein WR, Weill JC. 1995. Hypermutation generating the sheep immunoglobulin repertoire is an antigen-independent process. Cell 80: 115-125.

Saini SS, Allore B, Jacobs RM, Kaushik A. 1999. Exceptionally long CDR3H region with multiple cysteine residues in functional bovine $\operatorname{lgM}$ antibodies. European journal of immunology 29: 2420-2426.

Saini SS, Farrugia W, Muthusamy N, Ramsland PA, Kaushik AK. 2007. Structural evidence for a new IgG1 antibody sequence allele of cattle. Scandinavian journal of immunology 65: 32-38.

Saini SS, Farrugia W, Ramsland PA, Kaushik AK. 2003. Bovine IgM antibodies with exceptionally long complementarity-determining region 3 of the heavy chain share unique structural properties conferring restricted $\mathrm{VH}+$ Vlambda pairings. International immunology 15: 845-853.

Saini SS, Hein WR, Kaushik A. 1997. A single predominantly expressed polymorphic immunoglobulin $\mathrm{VH}$ gene family, related to mammalian group, I, clan, II, is identified in cattle. Molecular immunology 34: 641-651.

Saini SS, Kaushik A. 2001. Origin of bovine IgM structural variants. Molecular immunology 38: 389-396.

Saini SS, Kaushik A. 2002. Extensive CDR3H length heterogeneity exists in bovine foetal VDJ rearrangements. Scandinavian journal of immunology 55: 140-148.

Schultz RD, Dunne HW, Heist CE. 1973. Ontogeny of the bovine immune response. Infection and immunity 7: 981-991.

Shojaei F, Saini SS, Kaushik AK. 2003. Unusually long germline DH genes contribute to large sized CDR3H in bovine antibodies. Mol Immunol 40: 61-67.

Sinclair MC, Gilchrist J, Aitken R. 1995. Molecular characterization of bovine V lambda regions. Journal of immunology 155: 3068-3078.

Sinclair MC, Gilchrist J, Aitken R. 1997. Bovine IgG repertoire is dominated by a single diversified VH gene family. Journal of immunology 159: 3883-3889.

Sitnikova T, Nei M. 1998. Evolution of immunoglobulin kappa chain variable region genes in vertebrates. Molecular biology and evolution 15: 50-60.

Stein SK, Diesterbeck US, Aboelhassan DM, Czerny CP. 2012. Comparison of joining and constant kappa-light chain regions in different cattle breeds. Animal genetics 43: 776-780.

Sun J, Kacskovics I, Brown WR, Butler JE. 1994. Expressed swine VH genes belong to a small VH gene family homologous to human VHIII. Journal of immunology 153: 5618-5627.

Sun Y, Wang C, Wang Y, Zhang T, Ren L, Hu X, Zhang R, Meng Q, Guo Y, Fei J et al. 2010. A comprehensive analysis of germline and expressed immunoglobulin repertoire in the horse. Developmental and comparative immunology 34: 10091020.

Symons DB, Clarkson CA, Beale D. 1989. Structure of bovine immunoglobulin constant region heavy chain gamma 1 and gamma 2 genes. Molecular immunology 26: 841-850.

Tobin-Janzen TC, Womack JE. 1992. Comparative mapping of IGHG1, IGHM, FES, and FOS in domestic cattle. Immunogenetics 36: 157-165.

Tomlinson IM, Walter G, Jones PT, Dear PH, Sonnhammer EL, Winter G. 1996. The imprint of somatic hypermutation on the repertoire of human germline $\mathrm{V}$ genes. Journal of molecular biology 256: 813-817.

Tonegawa S. 1983. Somatic generation of antibody diversity. Nature 302: 575-581. 
Tutter A, Riblet R. 1989. Conservation of an immunoglobulin variable-region gene family indicates a specific, noncoding function. Proceedings of the National Academy of Sciences of the United States of America 86: 7460-7464.

Wagner B, Miller DC, Lear TL, Antczak DF. 2004. The complete map of the lg heavy chain constant gene region reveals evidence for seven $\lg G$ isotypes and for $\lg \mathrm{D}$ in the horse. Journal of immunology 173: 3230-3242.

Wagner SD, Neuberger MS. 1996. Somatic hypermutation of immunoglobulin genes. Annu Rev Immunol 14: 441-457.

Walther S, Czerny CP, Diesterbeck US. 2013. Exceptionally long CDR3H are not isotype restricted in bovine immunoglobulins. PloS one 8: e64234.

Wang F, Ekiert DC, Ahmad I, Yu W, Zhang Y, Bazirgan O, Torkamani A, Raudsepp T, Mwangi W, Criscitiello MF et al. 2013. Reshaping antibody diversity. Cell 153: 1379-1393.

Weinstein PD, Anderson AO, Mage RG. 1994. Rabbit IgH sequences in appendix germinal centers: VH diversification by gene conversion-like and hypermutation mechanisms. Immunity 1: 647-659.

Woof JM, Burton DR. 2004. Human antibody-Fc receptor interactions illuminated by crystal structures. Nature reviews Immunology 4: 89-99.

Yasuda M, Fujino M, Nasu T, Murakami T. 2004. Histological studies on the ontogeny of bovine gut-associated lymphoid tissue: appearance of $\mathrm{T}$ cells and development of $\lg G+$ and $\lg A+$ cells in lymphoid follicles. Developmental and comparative immunology 28: 357-369.

Yasuda M, Jenne CN, Kennedy LJ, Reynolds JD. 2006. The sheep and cattle Peyer's patch as a site of B-cell development. Veterinary research 37: 401-415.

Zhao Y, Kacskovics I, Pan Q, Liberles DA, Geli J, Davis SK, Rabbani H, Hammarstrom L. 2002. Artiodactyl IgD: the missing link. Journal of immunology 169: 44084416.

Zhao Y, Kacskovics I, Rabbani H, Hammarstrom L. 2003. Physical mapping of the bovine immunoglobulin heavy chain constant region gene locus. The Journal of biological chemistry 278: 35024-35032.

Zimin AV, Delcher AL, Florea L, Kelley DR, Schatz MC, Puiu D, Hanrahan F, Pertea G, Van Tassell CP, Sonstegard TS et al. 2009. A whole-genome assembly of the domestic cow, Bos taurus. Genome biology 10: R42. 


\section{Exceptionally Long CDR3H Are Not Isotype Restricted in Bovine Immunoglobulins}

Stefanie Walther ${ }^{1}$, Claus-Peter Czerny ${ }^{1}$, Ulrike S. Diesterbeck ${ }^{1 *}$

'Department of Animal Sciences, Institute of Veterinary Medicine, Division of Microbiology and Animal Hygiene, Faculty of Agricultural Sciences, Georg-August University Göttingen, Burckhardtweg 2, 37077 Göttingen, Germany

${ }^{*}$ Corresponding author:

U. S. Diesterbeck

Tel.: +49551393375

Fax: +495513913513

E-mail: udieste@gwdg.de

Postal address: Dr. Ulrike Diesterbeck

Department of Animal Sciences,

Georg-August University Göttingen

Burckhardtweg 2

37077 Göttingen, Germany

\section{Published in "PlosOne"}

DOI: 10.1371/journal.pone.0064234

URL: http://journals.plos.org/plosone/article?id=10.1371/journal.pone.0064234 
Chapter 3: Exceptionally long $\mathrm{CDR} 3 \mathrm{H}$ in bovine immunoglobulins

\section{Abstract}

Exceptionally long third complementarity determining regions of the heavy chain $(\mathrm{CDR} 3 \mathrm{H})$ were previously described as a specificity of bovine $\lg G$ and $\lg \mathrm{M}$ immunoglobulins. In addition, the genomic organization of the immunoglobulin heavy chain locus remains to be elucidated with a special focus on the number of variable segments (IGHV).

By analyzing the variable regions according to the isotype-specific PCR using CDNA$\mathrm{PCR}$, we were able to prove the existence of exceptional long CDR3H in all bovine isotypes. The corresponding sequences of three distinct amplicons were grouped according to the length of the CDR3H. Sequences of $\mathrm{CDR} 3 \mathrm{H}$ possessed 5 to 10,12 to 31 or at least 48 amino acid residues. Long and mid-length CDR3H were composed of mainly hydrophilic amino acid residues, while short CDR3H also contained hydrophobic amino acid residues. All sequences with long $\mathrm{CDR} 3 \mathrm{H}$ were related to the germline variable segment 10 .

Using the current genome assembly, Bos taurus NCBI build 6.1, the genomic organization of the bovine immunoglobulin heavy-chain locus was analyzed. A main locus was investigated on BTA21. Exons coding for variable, diversity, and joining segments, as well as for the constant regions of different isotypes, were also localized on BTA7, BTA8, and BTA20. Together with the information from unplaced contigs, 36 IGHV were detected of which 13 are putatively functional. Phylogenetic analysis revealed two bovine IGHV families (boVH1, boVH2). Thus, the existence of the two bovine families suggested was demonstrated, where boVH1 comprises all functional segments.

This study substantially improves the understanding of the generation of immunoglobulin diversity in cattle. 


\section{Introduction}

The generation of antibody diversity in vertebrates is subjected to a sequence of steps such as the recombination of separated germline gene segments for both heavy (V, D, and $\mathrm{J}$ ) and light ( $\mathrm{V}$ and $\mathrm{J}$ ) chains. Furthermore, the imprecise junction of the germline gene segments occurs as a result of nucleotide deletions or additions (N,P), introduced by the terminal deoxynucleotidyl transferase during the recombination process. The assembly of two identical heavy and light chains completes the tetrameric molecule $[1,2,3,4]$. In addition, somatic hypermutations contribute to antibody diversity - dependent or independent of antigen contact $[5,6,7]$. While these general processes of diversification are very similar in all vertebrate species, considerable differences were found in the available pool of the germline V, D, and $\mathrm{J}$ segments. Although humans and mice possess a large pool of VDJ genes [8], livestock such as chicken [9], pigs [10], sheep [11], and cattle $[6,12,13]$ are relatively restricted in the generation of combinatorial diversity. Therefore, species-dependent mechanisms dominate the different diversification steps or additional options are employed. For instance, in chicken gene conversion, the use of pseudogene sequences is a frequent postrecombinatorial strategy for the generation of the preimmune antibody repertoire $[5,14]$. This mechanism was confirmed for $\lambda$-light chains in cattle [15] and is discussed in horses [16].

All heavy-chain isotype classes detected in other mammals were also described for cattle $[17,18]$, whereas the $\gamma$-isotype encompasses three sub-classes, namely $\gamma 1, \gamma 2$, and $\mathrm{Y} 3[18,19]$. The bovine IGH locus was assigned to the Bos taurus autosome (BTA) 21 [20] and localized on the q23-q24 bands [21] or on the q24 band respectively $[22,23]$. An IgM-like chain was assigned to BTA11q23 by hybridization [24,25], which was supported by the detection of six IGHJ segments on the same chromosome [26]. By screening a bovine BAC and Cosmid library, the genomic organization of the IGHC locus was described, as well as the number of the preceding joining segments (IGHJ). Only two out of six IGHJ were classified as functional - of which only one seems to be involved predominantly in the recombination process $[21,26]$. The IGHV itself codes for the complementarity determining regions 1 and $2(\mathrm{CDR} 1 \mathrm{H}, \mathrm{CDR} 2 \mathrm{H})$ and for the $\mathrm{N}$ terminal part of the complementarity determining region $3(\mathrm{CDR} 3 \mathrm{H})$. Bovine IGHV offer a restricted set of genes related to one family (boVH1), which shares homologies to the murine Q52 family and human VHII family. Southern blot analyses indicated one additional IGHV family in the germline repertoire but only expression of boVH1 has 
been observed yet $[6,12,13,27,28]$. The definite number and organization of IGHV remains under further investigation.

Another peculiarity is the organization of the bovine IGHD locus. Ten IGHD genes classified into four families are organized in sub-clusters [29,30]. A comparison of the IGHD exons revealed huge size differences [29]. Cattle antibodies provide exceptionally long $\mathrm{CDR} 3 \mathrm{H}$ consisting of up to 62 amino acid residues (aa) $[6,31,32,33,34,35]$. IGHD2, with $148 \mathrm{bp}$ in size, contributes to those CDR3H and encodes the characteristic hydrophilic Glycine and Tyrosine residues [6,29,36]. The high number of Cysteine residues detected is supposed to promote intra-CDR3H disulfide bonds [13]. Mid-length $\mathrm{CDR} 3 \mathrm{H}$ - containing one to three Cys residues - were almost always accompanied by one Cys residue found in the $\mathrm{CDR} 2 \mathrm{H}$, which may result in intra CDR disulfide bond formation [31,37]. The germline encoded IGHV, IGHD, and IGHJ and their imprecise junction during rearrangement cannot fully explain the remarkable length of the CDR3H. Conserved short nucleotide sequences of 13 to 18 nucleotides are specifically inserted into the IGHV and IGHD junction, leading to a further extension of the CDR3H. This mechanism is unique for cattle [30].

To date, these exceptionally long CDR3H have been attributed exclusively to the $\mathrm{Y1-3-}$ and $\mu$-isotype $[33,35]$. In our study, we demonstrate the expression of exceptionally long $\mathrm{CDR} 3 \mathrm{H}$ in all bovine immunoglobulin isotypes. We were able to observe three distinct groups of $\mathrm{CDR} 3 \mathrm{H}$ sizes, which were related to their genomic origin. Loci of IGHV were determined on BTA7, BTA21 and seven unplaced contigs. 
Chapter 3: Exceptionally long CDR3H in bovine immunoglobulins

\section{Materials and Methods}

\section{In silico Analysis of the Bovine IGHV Segments}

A sequence search was performed with blastn on Bos taurus in the Reference genomic sequences (refseq_genomic) database using the leader and variable region of one mRNA sequence (accession number AY145128). On the identified contigs, the IGHV and their respective leader were annotated together with the recombination signal sequences (RSS). The octamers, TATA boxes, and splicing sites were also noted. The nucleotide sequences of the bovine IGHD1 to 8 and Q52 [30], as well as all IGHJ coding sequences (AY158087, AY149283), were used for a similar alignment approach. The detected IGHV, IGHD, and IGHJ were used in the further analysis of the amplified immunoglobulin sequences. To annotate the constant region locus, IgM (U63637), IgD (AF411240, AF515672), IgG1 (X16701), IgG2 (S82407, X16702), IgG3 (U63638), IgE (AY221098), and IgA (AF109167) bovine coding sequences were applied. Missing transmembrane regions were determined in bovine ESTs (expressed sequence tags). Based on the available sequence data, functionality was defined according to Lefranc [38]. In brief, functional sequences exhibited an open reading frame (ORF) without stop codon, and no defects in the splicing sites, RSS, or in the regulatory elements. If sequence information was missing due to end of contigs or N's introduced in the sequence but the available sequence offered putative functionality, genes were marked with $(F)$. Classification to ORF included either alterations in the splicing site, RSS, regulatory elements, substitutions of conserved amino acid residues (Cys23, Trp41, Leu89, Cys104 within IGHV or IGHC and a Phe/Trp118-Gly119-X120Gly121 motif within IGHJ [39]) or orphons ((ORF)). In this case, orphons are located outside of BTA21 [20,21,22,23]. Pseudogenes $(\Psi)$ were characterized by the presence of stop codons or frameshifts. Fragmented loci were also defined as pseudogenes. Functional recombination assays revealed the spacer lengths, the first three nucleotides of the heptamer as well as three consecutive adenosine residues within the nonamer to be crucial for efficient recombination $[40,41]$.

For the purpose of phylogenetic analysis, the complete nucleotide sequences of bovine IGHV segments were aligned with one member of the human IGHV families 1 to 7 , respectively, using the ClustalW algorithm with the ClustalX 2.1 interface [42]. The phylogenetic tree was calculated using the neighbor-joining method, with the exclusion of gaps. The confidence values were compiled with 1000 bootstrap replicates [43]. To 
root the tree, the sequence of one IGHV segment of the horned shark (accession number X13449) and little skate (X15124) were defined as an outgroup, similar to the method performed by Sitnikova and Su [44] and Almagro et al. [45]. Visualization of the phylogenetic tree was performed using the program NJplot [46].

\section{Ethical Statement}

To collect B-lymphocytes, $20 \mathrm{ml}$ of EDTA blood were taken from the tail vein of a German Simmental bull kept by the Division of Microbiology and Animal Hygiene for demonstrations in claw-treatment within student courses and to study the clinical development of Mycobacteria avium spp. paratuberculosis infection. The bull was owned, because he had acquired a natural infection of MAP and showed positive antibody-titers already with an age of 18 months. Similar to other cattle herds, he has to be tested for cattle diseases periodically. The blood sample was taken from the tail vein during regular investigation of infectious diseases in the bull. The plasma was applied e.g. in an indirect ELISA testing for antibodies against Mycobacteria avium spp. paratuberculosis or BHV-1. Therefore, no specific approval is required.

\section{Isolation of PBMCs and cDNA Synthesis}

Peripheral blood mononuclear cells (PBMCs) were isolated using Ficoll gradients (GE Healthcare Europe $\mathrm{GmbH}$, Germany) according to the manufacturer's protocol. Viable $B$ cells were stained with trypan blue and counted. Total RNA was isolated from $1 \times 10^{7}$ cells using the RNeasy ${ }^{\circledR}$ Mini Kit (Qiagen, Germany). The first-strand cDNA was synthesized using $\mathrm{pd}(\mathrm{N})_{6}$ primers from $3 \mu \mathrm{g}$ of total RNA in a total volume of $20 \mu \mathrm{l}$ (SuperScript ${ }^{\mathrm{TM}} \mathrm{III}$ First-Strand Synthesis SuperMix, Life Technologies $\mathrm{GmbH}$, Germany).

\section{Amplification of Immunoglobulin Heavy-chain Isotype Restricted Variable Regions}

To amplify the variable region restricted to each isotype, a primer set was generated with one primer hybridizing in the leader region, and individual primers with binding sites in the constant region $(\mathrm{CH})$ of the immunoglobulin heavy chains. The primers were based on database entries and their own sequence information (data not shown). For $\alpha, y 1-3$, and $\varepsilon$ isotypes primers anneal to the $\mathrm{CH} 1$. For $\delta$ and $\mu$ isotypes, primers bind within the $\mathrm{CH} 2$ (Table 1). To monitor the integrity and purity of the cDNA, $527 \mathrm{bp}$ 
Chapter 3: Exceptionally long CDR3H in bovine immunoglobulins

of the bovine GAPDH (Glycerinaldehyde 3-phosphate dehydrogenase) were amplified as a positive control. A no template control served as a negative control for the PCR.

Table 1. Primer for the isotype-specific amplification of the complete variable regions.

\begin{tabular}{lllr} 
Forward primer & Reverse primer & Primersequence 5'-3' & $\begin{array}{c}\text { Approximated } \\
\text { product size in } \\
\text { bp }\end{array}$ \\
\hline BoLH_BACK & & ACCCACTGTGGACCCTCCTC & 795 \\
& BolgMCH2_FOR & TGCCGTCACCAGAGAGGCTGT & 805 \\
& BolgDCH2_FOR & TGCGTGCTGACCGCCTTGTT & 536 \\
& BolgG1-3CH1_FOR & GGCACCCGAGTTCCAGGTCA & 467 \\
& BolgECH1_FOR & GCCCAGCCTTACACGGGCTT & 574 \\
GAPDH_for & BolgACH1_FOR & GCCAGCACGGCAGGGAAGTT & 527
\end{tabular}

One universal forward primer was used for annealing within the leader region. For each isotype, a reverse primer was generated for specific amplification. The annealing sites were selected in the first constant region (IGCH1), with the exception of IgM and IgD. Both isotypes share high homologies in the IGCH1 and therefore, specific reverse primers were generated for binding in the second constant region. The IgG subtypes were not distinguished further. Primers for bovine GAPDH served as cDNA quality control.

The total reaction volume of $50 \mu \mathrm{l}$ included $1 \mu \mathrm{l}$ of cDNA, $200 \mu \mathrm{M}$ dNTPs (Bioline, Germany), $5 \mu$ of $10 x$ PCR buffer (75 mM Tris $\mathrm{HCl} \mathrm{pH} \mathrm{9.0;} 2 \mathrm{mM} \mathrm{MgCl}_{2} ; 50 \mathrm{mM} \mathrm{KCl}$; $\left.20 \mathrm{mM}\left(\mathrm{NH}_{4}\right)_{2} \mathrm{SO}_{4}\right), 5 \%$ DMSO (Dimethyl sulfoxide), $0.4 \mu \mathrm{M}$ of each primer pair, and 2 units of DNA polymerase (Biotools, Spain). PCR was performed under cycling conditions of $95^{\circ} \mathrm{C}$ for $5 \mathrm{~min}$, followed by 35 cycles of $95^{\circ} \mathrm{C}$ for $1 \mathrm{~min}, 58^{\circ} \mathrm{C}$ for $1 \mathrm{~min}$, $72^{\circ} \mathrm{C}$ for $2 \mathrm{~min}$, and terminated with elongation at $72^{\circ} \mathrm{C}$ for $10 \mathrm{~min}$. The length and purity of the PCR products were evaluated by means of electrophoresis on $1 \%$ agarose gels.

\section{Cloning and Sequencing of the PCR Products}

The PCR products were purified and concentrated using the DNA Clean \& Concentrator Kit (Zymo Research, USA). Purified products were cloned into the pCR ${ }^{\circledR}$ 2.1-TOPO ${ }^{\circledR} 3.9 \mathrm{~Kb}$ TA vector (Invitrogen ${ }^{\mathrm{TM}}$, Karlsruhe, Germany) and transformed into chemically competent One Shot TOP10 E. coli cells (Invitrogen ${ }^{\mathrm{TM}}$, Karlsruhe, Germany). Transformants were plated on LB agar containing $0.3 \mathrm{mM}$ ampicillin, $40 \mu \mathrm{l}$ $2.44 \mu \mathrm{M}$ X-gal (5-bromo-4-chloro-3-indolyl-beta-D-galactopyranoside), and $40 \mu \mathrm{l} 1 \mathrm{M}$ IPTG (Isopropyl $\beta$-D-1-thiogalactopyranoside) for blue/white selection. After incubation at $37^{\circ} \mathrm{C}$, overnight cultures of randomly selected white transformants were grown in a $5 \mathrm{ml}$ LB-ampicillin broth. Plasmids were isolated using the MiniPrep Kit (Qiagen, 
Germany). In order to assess the insert size, plasmid DNA was cleaved with EcoRI (New England Biolabs, Germany) and DNA sizes were confirmed by agarose gel electrophoresis.

Twenty plasmids of each PCR product were sequenced according to the chaintermination method [47]. The M13 (-20) Forward and M13 Reverse (Invitrogen, Germany) vector-specific primers, as well as the corresponding gene specific primers, were used for sequencing.

\section{Nucleotide and Amino Acid Sequence Analyses}

The genetic information of the VDJ recombinations was used for further analysis. The amplified part of the constant regions served as verification of the respective isotype. The sequences were analyzed using the DNAStar program (GATC Biotech AG, Germany) and aligned by ClustalW [48].

The deduced amino acid residues of the variable parts were aligned to the IMGT nomenclature [39] using the IMGT/DomainGapAlign [49,50]. Framework regions, as well as CDRs, were identified and analyzed with regard to their biochemical properties such as the hydrophobicity, polarity, and charge of the amino acid residues incorporated. The CDR3H regions were classified according to their number of amino acid residues. The amino acid compositions of $\mathrm{CDR} 2 \mathrm{H}$ and $\mathrm{CDR} 3 \mathrm{H}$ were examined for their numbers of Tyr, Gly, aromatic amino acid residues and Cys, since some bovine CDR3Hs are characterized by exceptional length and preferred amino acid residues. 


\section{Results}

\section{Annotation of the Bovine Germline Immunoglobulin Heavy-chain Locus}

For the identification of germline IGHV, a search using blastn on all bovine genome assemblies was performed. The contigs NW_003104530.1 and NW_003104538.1 were identified on Bos taurus chromosome 21 (BTA21; AC_000178.1). NW_003104530.1 was located at the centromeric region with two IGHV (IGHV1Y and IGHV2). A region of about $146 \mathrm{~kb}$ on NW_003104538.1, located at the telomeric

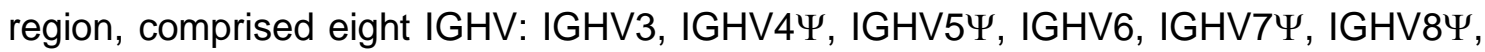
IGHV9Y, and IGHV10. Upstream of them, the exons coding for the $\mu, \varepsilon$ and $\alpha$ chains, were identified. Two IGHD (8 and 4) were localized between IGHV6 and IGHV7Y (Figure 1A).

The contigs NW_003064289.1, NW_003064290.1, NW_003064296.1, NW_003064297.1, NW_003064298.1, and NW_003064299.1 were localized to the centromeric region on BTA7 (AC_000164.1) involving nine IGHV (IGHV114,

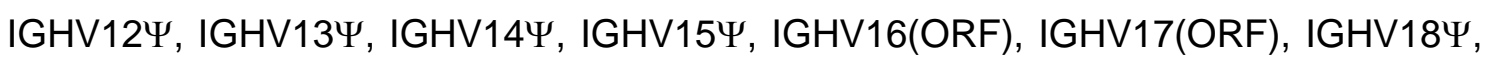
and IGHV194). This cluster spans approximately $144 \mathrm{~kb}$. Downstream on BTA7, a cluster of $1131 \mathrm{bp}$, with exons encoding IGHD1(ORF) to IGHD3(ORF), was identified on contig NW_003064411.1, followed by a pseudogene for a $\delta$ chain. The latter one is characterized by fragmented exons 1 and 2, a deleted exon 6 (codes for the secretory region), and frame shifts (Figure $1 \mathrm{~B}$ ).

A third location comprising five IGHD segments, a $\delta$ chain pseudogene, a $\mu$ chain gene, and six IGHJ segments in about $43 \mathrm{~kb}$ was detected on BTA8. Two loci for the IGHD were observed. IGHDQ52(ORF) was the most downstream segment on contig NW_003066919.1, whereas IGHD4(ORF) to 7(ORF) were found to be the most upstream on contig NW_003066918.1 (Figure 1C). Interestingly, BTA20 revealed the genomic information for a $\mathrm{y}^{2}$ chain (Figure 1D). 
Chapter 3: Exceptionally long CDR3H in bovine immunoglobulins

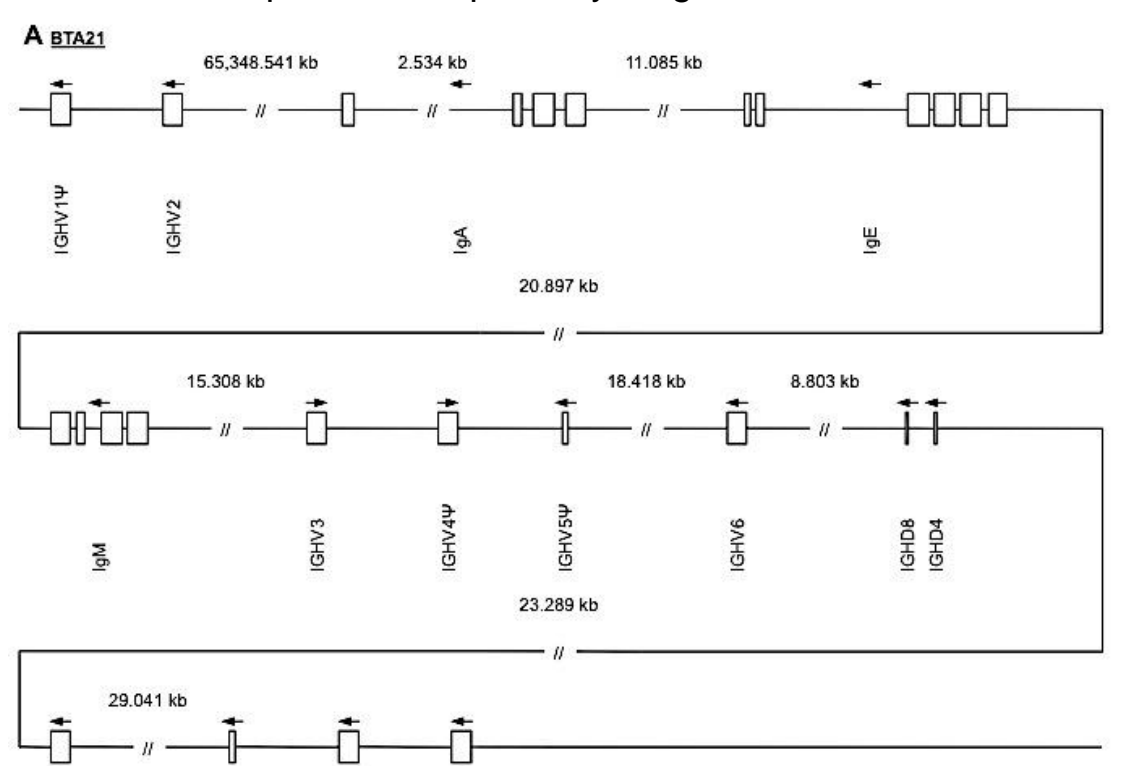

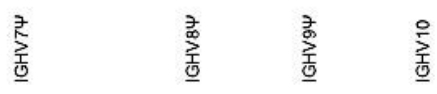

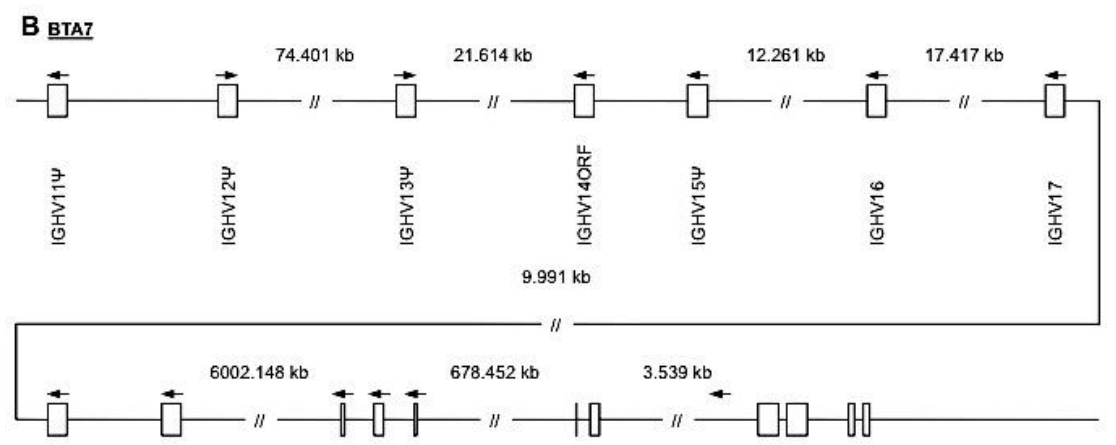

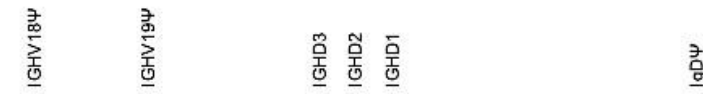

C BTA8

"

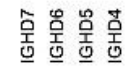

高

$5.437 \mathrm{~kb}$

Hम

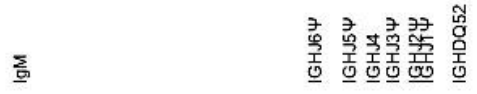

$D_{\text {BTA20 }}$

$\rightarrow \rightarrow[B \quad M$ 
Figure 1. Chromosomal organization of variable (IGHV), diversity (IGHD), joining (IGHJ) segments, and the constant regions of the heavy chains. The physical map displays the order of functional segments $(F)$, pseudogenes $(\Psi)$, and open reading frames (ORF). Classification to "functional" includes an ORF without stop and exhibition of conserved amino acid residues as well as no defects in splicing signals, recombination signal sequences (RSS) or regulatory elements. ORF are defined by alterations in the splicing signals, recombination signal sequences, and/or regulatory elements. In addition, changes to conserved amino acid residues, which may lead to misfolding were included in the ORF classification. Functional elements on orphon localizations are highlighted with ORF in parenthesis (ORF) $[38,39]$. Pseudogenes possessed stop codons, frameshifts or mutations of the spacer lengths within the first three nucleotides of the heptamer as well as in three consecutive adenosines residues within the nonamer abolish the recombination [40,41]. In addition, fragmented loci were also defined as pseudogenes. Arrows indicate the transcription direction.

In addition, unplaced contigs (NT_182448.1, NT_182449.1, NT_183109.1, NT_185036.1, NT_185907.1, NT_186922.1, and NW_003100762.1) were discovered to contain IGHV segments. Likewise, IGHD1(ORF) and 2 genes were localized on NT_186153.1. NW_001494075.1 includes a gene coding for IGHDQ52 downstream of an IGHJ1 to 6 locus. Genes coding for a $\mu$ chain and a $\delta$ chain pseudogene were found most upstream in this contig. The contig NW_001503306.1 comprises the genomic information for IGHD4 to 7 and a $\delta$ chain pseudogene while IGHD4 and 8 were detected on NW_001504477.2. A locus involving IGHJ4ORF to 6ORF and a putative functional $\delta$ chain gene was detected on NT_186572. Additional genomic information for $\alpha$ and $\varepsilon$ chains were discovered on NT_185723.1. A genomic order of $\gamma 3$, $\gamma 2$, and $\mathrm{Y} 1$ was identified on NT_185580.1. Furthermore, a gene coding for $\mathrm{Y} 1$ and $\mathrm{\gamma} 3$ was found on NW_003100065.1 and NW_003099305.1, respectively. Along with NW_003100387.1, including a $\delta$ chain pseudogene locus, a putative functional $\delta$ chain gene was noted on NW_003100112.1.

Thirteen out of the 36 IGHV segments identified are putatively functional (Supplemental Table S1). Eleven IGHV segment pairs shared sequence identity of

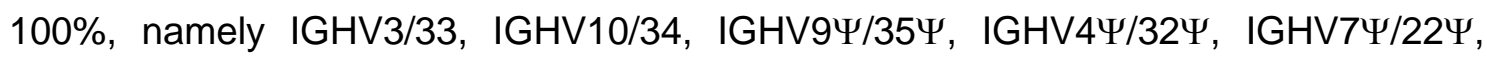

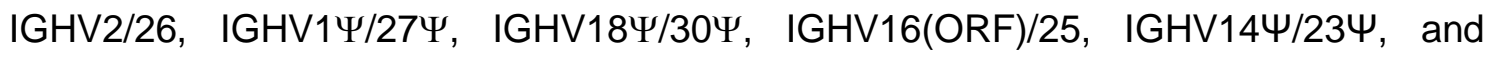
IGHV36/29(F). Since the human IGHV locus has been fully investigated [51], one member of each family was chosen for phylogenetic analysis. The sequences clustered into two distinct branches either with hulGHV2-05 or with hulGHV4-04 and hulGHV6-1. All functional IGHV were phylogenetically related to hulGHV2-05 (Figure 2). This group corresponds to the bovine IGHV family 1 (boVH1) described previously $[6,12,13]$. Multiple alignments revealed two distinct families with identities of at least $80.5 \%$ for boVH1 and $79.5 \%$ for boVH2. IGHV5 $\Psi$ and IGHV8 $\Psi$ represent fragmented

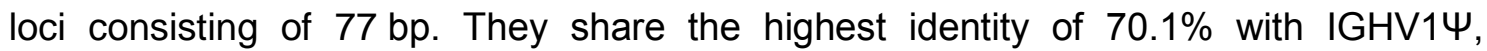




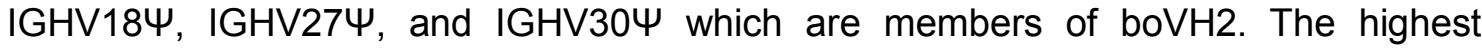
identity between members of both families was calculated to be $69.7 \%$.

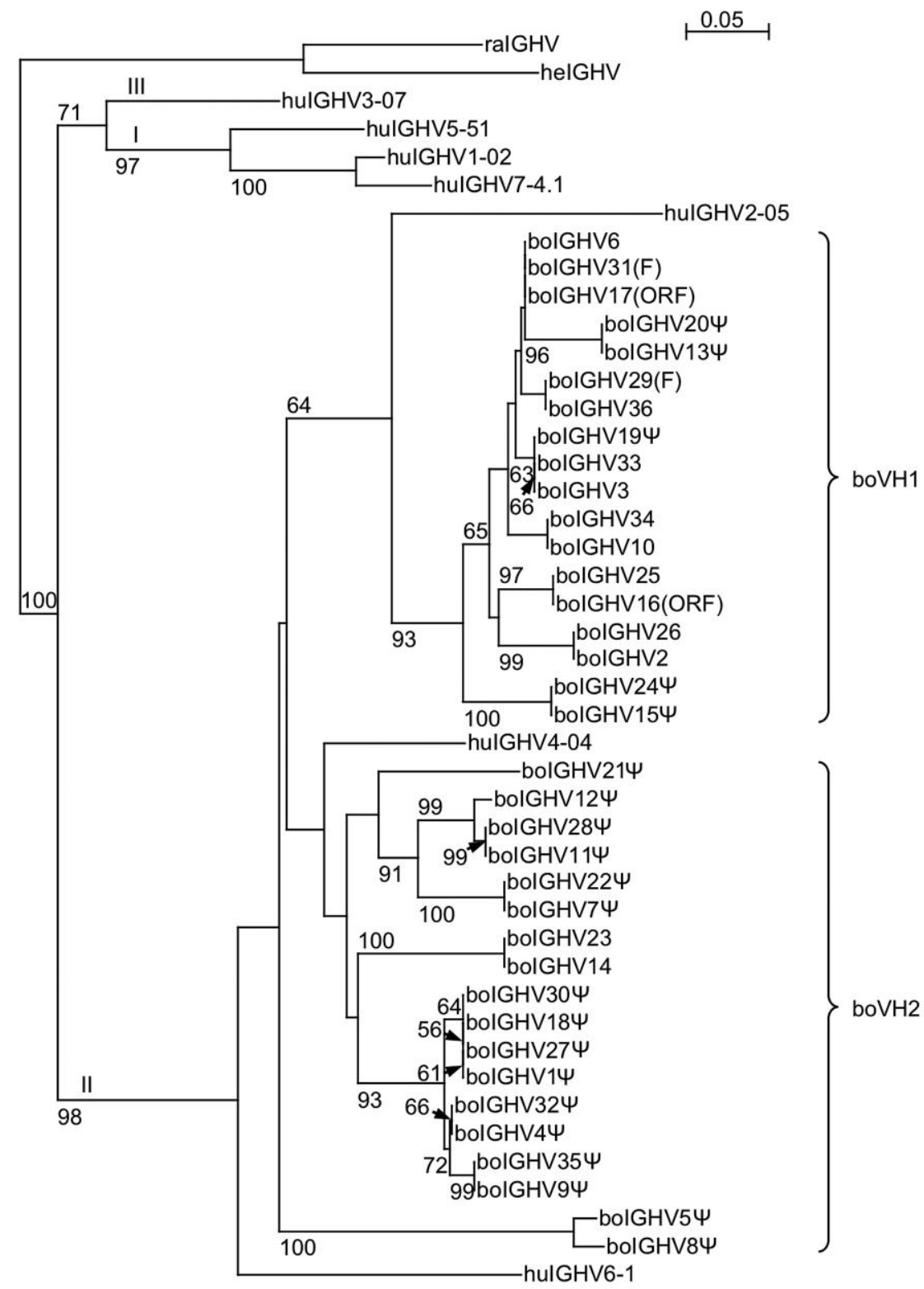

Figure 2. Neighbor-joining phylogenetic tree of the genomic bovine IGHV segments. The complete sequences of the bovine IGHV segment (bolGHV), and one representative sequence of each human family (hulGHV1 to hulGHV7), were used for the comparison. The reliability of the tree was estimated using 1000 bootstrap replicates [43]. Numbers at each node are the percentage bootstrap value and are indicated only when greater than $50 \%$. Arrows mark the respective node. The Roman numerals I, II, and III describe the clans [64]. Two clusters of bovine IGHV were visible and corresponded to two families. The bovine IGHV family 1 (boVH1) comprises all functional segments, whereas boVH2 consists only of pseudogenes. IGHV5 $\Psi$ and IGHV8 $\Psi$ present fragmented loci, which consist of only $77 \mathrm{bp}$. They share $70.1 \%$ sequence

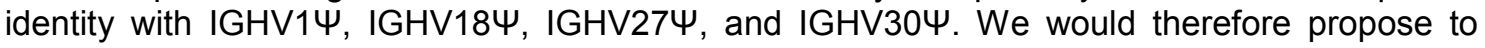
assign IGHV5 $\Psi$ and IGHV8 $\Psi$ to boVH2. Horned shark (helGHV from accession number X13449) and little skate (ralGHV; X15124) represent the outgroup in this analysis, similar to that performed by Sitnikova and Su [44] and Almagro et al. [45]. The scale bar indicates the number of nucleotide substitutions per site. 


\section{Amplification of Isotype-specific Variable Regions and Sequence Analyses}

The immunoglobulin heavy chains were amplified by PCR for each bovine isotype. Three distinct bands became visible following agarose gel electrophoresis of the amplicons of the $\mu, \delta, \gamma 1$ to $3, \varepsilon$, and $\alpha$ isotypes. The expected product sizes of $467 \mathrm{bp}$ $(\lg E)$ to $805 \mathrm{bp}(\lg D)$ corresponded with the lowest band. The isotypes $\mathrm{\gamma} 1$ to 3 , $\varepsilon$, and $\alpha$ revealed the lowest band, always approximately 100 bp below the middle band, which was again $100 \mathrm{bp}$ smaller than the largest band (Figure 3). The dominant product was observed in the middle band. In contrast, a faint lower band was noticed in the products of $\lg \mathrm{M}$ and $\lg \mathrm{D}$ - also with differences in size of $100 \mathrm{bp}$. The middle and upper bands showed strong amplification. The three bands observed per amplified isotype should allow for grouping of the respective CDR3H lengths. After sub-cloning of the purified products, 20 sequences per isotype were evaluated.

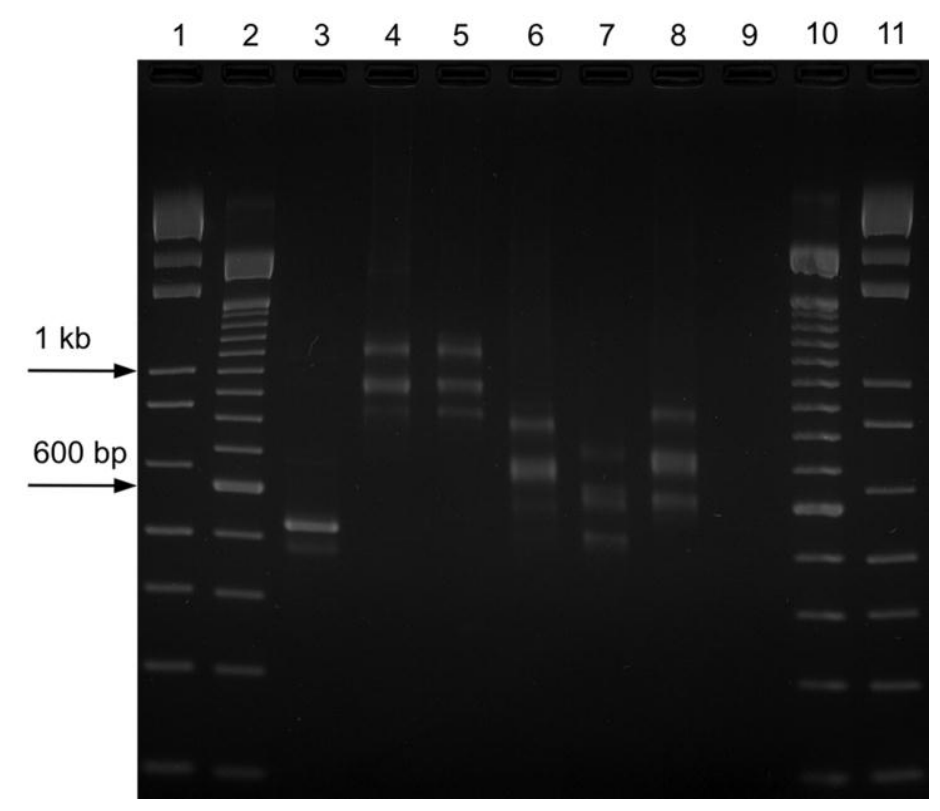

Figure 3. PCR products of the bovine $\mu, \delta, \gamma 1$ to $3, \varepsilon$, and $\alpha$ isotypes.

The amplicons of the heavy chain variable domain of each bovine isotype were resolved by $2.5 \%$ agarose gel electrophoresis and revealed three distinct products. Lanes 1 and $11: 1 \mathrm{~kb}$ ladder, lanes 2 and 10: 100 bp ladder. Lane 1: product of the GAPDH positive control; Lanes 4 and 5 : in the products of $\mu$ and $\delta$, a faint lower band was noticed also with differences in size of $100 \mathrm{bp}$. The middle and upper bands showed strong amplification. Lanes 6 to 8: the dominant product of isotypes $y 1$ to $3, \varepsilon$, and a was observed in the middle with a size difference of about $100 \mathrm{bp}$ compared to both the upper and the lower band. Lane 9 is the no template negative control of the PCR. 
The deduced amino acid sequences were aligned to the IMGT nomenclature for variable domains [39]. The size of the variable regions varied between 111 and 173 amino acid residues (Table 2). The Cysteine residues, forming the intra-chain disulfide bond, were always observed at position 23 and 104. Trp41 and Leu89 were also conserved. These amino acid residue positions are coded by the $V$ segment [39]. A Trp at position 118 and subsequently a Gly119, coded by the J segment, were also found in most sequences analyzed. In four sequences, amino acid residue substitutions were observed at these positions. Two SNPs resulted in the replacement of Gly119 by Asp (SNP: GGC>GAC, in one sequence) or Ser (SNP: GGC>AGC, in two sequences). In one sequence, we found Trp118 substituted by Ser, which was caused by the SNP TTG>TCG.

The CDR1H always comprised eight amino acid residues, whereas CDR2H possessed seven amino acid residues. They consisted of both hydrophobic and hydrophilic amino acid residues.

Table 2. Analysis of the complement determining regions (CDR) of the different isotypes.

\begin{tabular}{lllll} 
Isotype & $\begin{array}{l}\text { Length of the } \\
\text { variable region }\end{array}$ & $\begin{array}{l}\text { Length of the } \\
\text { CDR1H }\end{array}$ & $\begin{array}{l}\text { Length of the } \\
\text { CDR2H }\end{array}$ & $\begin{array}{l}\text { Length of the } \\
\text { CDR3H }\end{array}$ \\
\hline $\operatorname{lgM}$ & $131.65 \pm 14.62$ & $8 \pm 0$ & $7 \pm 0$ & $25.65 \pm 14.62$ \\
$\lg D$ & $138.70 \pm 17.54$ & $8 \pm 0$ & $7 \pm 0$ & $32.70 \pm 17.54$ \\
$\operatorname{lgG} 1-3$ & $138.70 \pm 19.39$ & $8 \pm 0$ & $7 \pm 0$ & $32.70 \pm 19.39$ \\
$\lg E$ & $136.55 \pm 17.97$ & $8 \pm 0$ & $7 \pm 0$ & $30.55 \pm 17.97$ \\
$\lg A$ & $127.05 \pm 12.17$ & $8 \pm 0$ & $7 \pm 0$ & $21.05 \pm 12.17$
\end{tabular}

The average amino acid residue lengths of the complete variable region and the CDR regions, according to the IMGT nomenclature [39], are provided with their standard deviations. The size of the variable regions ranged from 111 to 173 amino acid residues. In total, 20 sequences per isotype were analyzed. 


\section{Composition of the CDR3H with Different Lengths}

The three distinct sizes of the $\mathrm{CDR} 3 \mathrm{H}$ were noted in all bovine immunoglobulin isotypes. A specific composition of amino acid residues was found within the CDR3H, which seems to correlate with the different lengths of CDR3H. The shortest group (group 1) - comprising five to ten amino acid residues according to Lopez et al. [27] was characterized by hydrophobic, as well as hydrophilic amino acid residues, without Cysteines (Figure 4). Twelve to 31 amino acid residues formed the middle size (group 2). Within this group, hydrophilic amino acid residues were incorporated predominantly into the CDR3H. The Gly, Tyr, and Cys-rich, long CDR3H included more than 47 amino acid residues (group 3). Gly and Tyr were frequently found within the $\mathrm{CDR} 3 \mathrm{H}$, whereas Gly dominated in most sequences analyzed. Within the exceptionally long $\mathrm{CDR} 3 \mathrm{H}$, four, six, seven or eight Cys were detected, which accumulated in the middle of the CDR3H. Group 1 and 2 only possessed none, one, or two Cys. In two sequences of $\mathrm{IgE}$, with one Cys in the mid-length $\mathrm{CDR} 3 \mathrm{H}$, one additional Cys was found in CDR2H. Gly, Pro and multiple Cys, as well as aromatic residues, were observed in long and intermediate CDR3H.

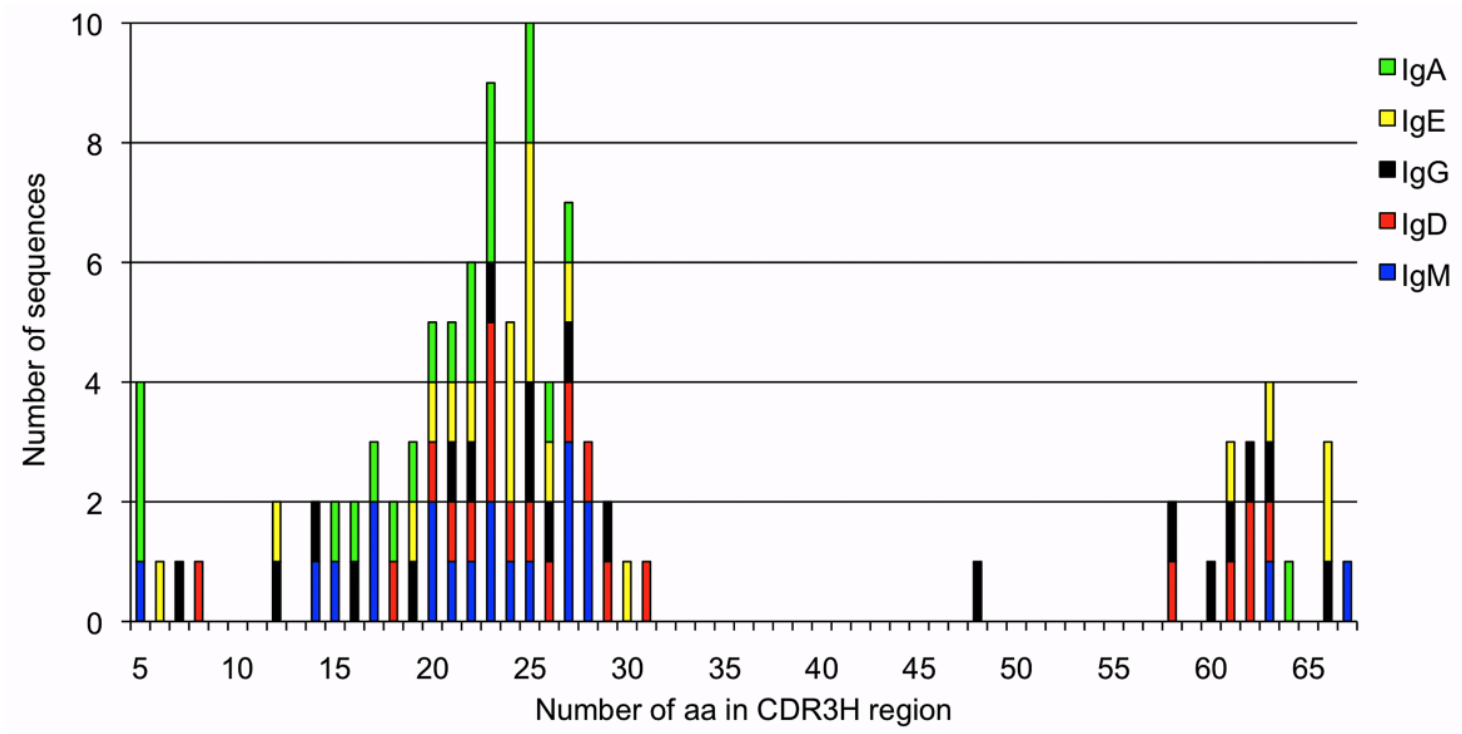

Figure 4. Three distinct sizes of CDR3H in all isotypes. The lengths of CDR3H of all sequences analyzed was divided into three groups. These groups were identified in each isotype, marked in different colors. The numbers of amino acid residues (aa) in CDR3H are indicated on the horizontal axis, whereas the number of sequences possessing each number of aa is provided on the vertical axis. 
The IGHV, IGHD, and IGHJ segments were related to their genomic origin by phylogenetic alignment. In general, IGHJ1 was related to all sequences and heavychain isotypes were analyzed. Although IGHJ2 is also functional, we did not identify this segment. In addition to the exclusive use of one IGHJ segment, six IGHV segments were recombined. For group 1, segment IGHV3 was detected in six out of seven sequences. One sequence showed IGHV6. The segments IGHV2 (1 sequence), IGHV3 (5 sequences), IGHV6 (18 sequences), IGHV10 (6 sequences), IGHV17(ORF) (35 sequences), and IGHV36 (9 sequences) were identified within group 2. These IGHV segments were distributed between the isotypes in similar proportions. Only the segment IGHV10 was identified in all group 3 sequences. The phylogenetic alignment of IGHD segments revealed dissimilarities between recombined and genomic sequences, which resulted in low sequence identities. In addition, there were homologies between the germline IGHD segments ranging from $81.4 \%$ (IGHD2 and IGHD8) up to $97.6 \%$ (IGHD1 and IGHD6). Therefore, it was not possible to unambiguously annotate all $\mathrm{CDR} 3 \mathrm{H}$ regions of the sequences analyzed. Nevertheless, IGHD2 was preferentially recombined in sequences possessing exceptionally long CDR3H. At the IGHV-IGHD junctions, conserved short nucleotide sequences (CSNS) were identified, which are rich in $A$ nucleotides. The inclusion of $N$ and $P$ nucleotides was noted within the IGHD-IGHJ junction. In addition to the presence of CSNS in sequences possessing very long $\mathrm{CDR} 3 \mathrm{H}$, these CSNS were also discovered in sequences with CDR3H of mid-length. IGHD4 was used most often in these group 2 sequences. All other known IGHD segments were recombined less frequently in this group. The short IGHD segments IGHDQ52 and IGHD4(1 and 2) - possessing 15 and 36 nucleotides - were identified in very short CDR3H regions. 


\section{Discussion}

The in silico analyses of the genomic organization of the heavy-chain locus revealed differences from previous mapping and annotation results. The functional locus was mapped to BTA21q23-q24 [21,22,23], where we also detected ten variable and two diversity segments together with the exons coding for $\lg M(F), \lg E$, and $\lg A(F)$. The order of the respective segments highlighted deviations from other fully described mammalian loci [16,52]. Zhao et al. [21] have already described the constant heavychain locus by means of BAC clone analysis, as well as the joining segments organized upstream of $\operatorname{lgM}$. These BAC clones were not introduced into the genomic assembly. Since the UMD2 assembly used mapping data, synteny with the human genome, and paired-end sequence information [53], we did not expect heavy-chain loci on BTA7, BTA8, and BTA20. Upstream of IGHV114, the bovine contig-NW_003064289 on BTA7-harbors the genes for FBXL12 (F-box and leucine-rich repeat protein 12), UBL5 (ubiquitin-like 5), PIN1 (peptidylprolyl cis/trans isomerase, NIMA-interacting 1), and OLFM2 (olfactomedin 2) which share homology with HSA19. The human gene following downstream is COL5A3 (collagen, type 5, alpha 3). No variable segment was found between OLFM2 and COL5A3 on HSA19p13.2. The bovine pseudo $\delta$-chain locus on BTA7 revealed no human equivalent. The human 5pter part is inversely syntenic to BTA20qter [54]. On contig NW_003104522.1, we found the gene ADCY2 (adenylat cyclase 2) as an anchor. The bovine IgG2(ORF) (IGCGAMMA) gene, annotated between AHRR (aryl-hydrocarbon receptor repressor) and PDCD6 (programmed cell death 6), has no homologue on the HSA5pter, although it has been noted that AHRR and PDCD6 overlap on HSA5pter. For IGHD4 to 7, and pseudo $\delta$-chain on BTA8, no specific genes were identified. Human syntenic groups on BTA8 were described for HSA8p and HSA9 [54]. No additional genes were determined on contig NW_003066919, with a complete IgD $\Psi$, IgM(ORF) gene and IGHJ1(ORF) to 6(ORF), as well as IGHDQ52(ORF). Hybridization investigations assigned an IgM-like chain (probe IGHML1) to the syntenic group U16; corresponding to HSA9q [24,55]. Later, IGHML1 was assigned to BTA11q23 by hybridization [24,25], which was supported by the detection of six IGHJ segments on the same chromosome using BAC clone and locus-specific PCR analysis [26]. We were not able to identify an IgM-like locus on BTA11. Based on these results and the fact that we observed a transcribed IGHV from a putative orphon, we concluded an incorrect and incomplete annotation of the bovine immunoglobulin heavy-chain locus, 
which may be solved by the re-sequencing of the described localizations and underpinned by different authors and methods.

Previous studies have already classified bovine IGHV segments into clan II, with the closest homology to the human VH2 family $[6,12,13,44]$. Quite in contrast to the statement made by Tutter and Riblet [28] and Berens et al. [6], none of the genomic sequences clustered with the human clan III family VH3. All of the functional bovine IGHV segments are most closely related to the human $\mathrm{VH} 2$ family represented by IGHV2-05, which explains the exclusive transcription of only one bovine $\mathrm{VH}$ family $[6,13,56]$. The comparison with human sequences was performed by Saini et al. [12] using one VH4 family sequence only. The second bovine $\mathrm{VH}$ family described here consisted only of pseudogenes and clustered with the human VH4 and VH6 family. We were now able to describe the second bovine $\mathrm{VH}$ family (boVH2) previously proposed [6], but we had no indications of the possible gene conversions using boVH2 segments in the sequences investigated, as shown for the bovine $\lambda$-light chains [15]. We would propose that IGHV5 $\Psi$ and IGHV8 $\Psi$ should be assigned to the boVH2 family as they

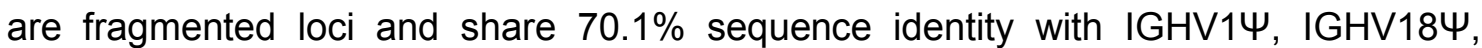

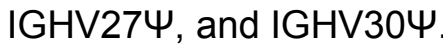

With regard to the description of unusually long $\mathrm{CDR} 3 \mathrm{H}$ in bovine $\operatorname{lgM}$, long and short IGHD segments have already been described. Independent of nucleotide addition during rearrangement they contribute directly to $\mathrm{CDR} 3 \mathrm{H}$ length heterogeneity. Nevertheless, the genomic IGHD segments showed high homologies among themselves, which resulted in the complicated annotation of the transcribed IGHD. In particular, many hypermutations within the recombined IGHD segments led to low sequence identities. Intrinsic hot spots as targets for somatic hypermutations within $\mathrm{CDR} 1 \mathrm{H}, \mathrm{CDR} 2 \mathrm{H}$, and $\mathrm{CDR} 3 \mathrm{H}$ were already found in a bovine fetus. Furthermore, $\mathrm{CDR} 3 \mathrm{H}$ length heterogeneity, junctional flexibility, and somatic hypermutation are thought to contribute solely to $\lg \mathrm{M}$ antibody diversification in both bovine fetus and adult cattle [32]. As we found $\mathrm{CDR} 3 \mathrm{H}$ length heterogeneity in all isotypes, exceptionally long $\mathrm{CDR} 3 \mathrm{H}$ are apparently not primarily generated to compensate the restricted flexibility of IgM caused by reduced Pro numbers within $\mathrm{C} \mu 2$. The most recent study on the IgG repertoire in calves also showed very long CDR3H [35]. Therefore, antigen selection of variable domains and class switch recombination seem to be of higher impact. We did not observe any evidence to suggest that the combination of two different IGHD segments enhances diversity, which was the case for horses [16]. The exceptionally long CDR3H were generated by the direct fusion of a single IGHV 
segment (IGHV10), the longest IGHD segment (IGHD2), and one functional IGHJ segment (IGHJ1), as described previously [30]. In very short $\mathrm{CDR} 3 \mathrm{H}$, we noted a preferred use of the short IGHD segments, IGHDQ52 and IGHD4. In sequences of CDR3H group 1 and 2, no predominant use of one special IGHV segment was determined. According to structural analyses, spatial distances are not thought to contribute to preferred IGHV-IGHD-IGHJ rearrangements, as there are conformational changes of chromatin resulting in the repositioning of the IGHD cluster and the merging of proximal and distal IGHV regions during early B cell development [57]. All IGHV segments identified were found to be functional. Thus, there is no evidence for gene conversion in bovine immunoglobulin heavy chains, which is already known to contribute to the diversity of chicken immunoglobulin heavy chains and bovine $\lambda$-light chains $[15,58]$. Conserved short nucleotide sequences (CSNS), which were inserted into the IGHV-IGHD junction, were found both in intermediate length and very long $\mathrm{CDR} 3 \mathrm{H}$. This novel mechanism, which contributes to antibody diversification, is neither restricted to immunoglobulin heavy chains with exceptionally long $\mathrm{CDR} 3 \mathrm{H}$, nor is it isotype restricted. As the insertion of CSNS is supposed to directly follow antigen exposure during the development of an immune response [30], we conclude class switch recombination to be responsible for isotype-independent, long $\mathrm{CDR} 3 \mathrm{H}$ in cattle. In addition, exceptionally long $\mathrm{CDR} 3 \mathrm{H}$ protrude from the variable domain with support from the $\lambda$-light chains. Thus, there is no conventional combining site and the other two $\mathrm{CDRH}$ do not contribute to antigen binding. Instead, this function is undertaken by side chains that are exclusively contained within long $\mathrm{CDR} 3 \mathrm{H}$ regions, as investigated by structural comparisons with protein toxins [37].

Transcribed CDR1H, CDR2H, and short CDR3H sequences showed both hydrophilic and hydrophobic amino acid residues. In long $\mathrm{CDR} 3 \mathrm{H}$, hydrophilic amino acid residues were represented mainly by repetitive Gly, Tyr, and Ser, whereas Gly dominated in most of the sequences analyzed - which is consistent with previous findings [27]. The major usage of the hydrophilic reading frame was already described in humans [59], mice [60], chicken [58], and rabbits [61]. Their occurrence in antigen-binding loops is thought to enhance flexibility and recruit somatic hypermutations for advantageous antigen binding [59].

Moreover, in accordance with descriptions provided previously, we identified multiple and mainly even numbered Cys within exceptionally long $\mathrm{CDR} 3 \mathrm{H}$, which were accumulated in the middle of the CDR3H $[31,33]$. These Cys are predicted to form intra and inter $\mathrm{CDRH}$ disulfide bonds, rigidifying the combining site or helping to stabilize 
long $\mathrm{CDR} 3 \mathrm{H}$, as demonstrated in the crystallized human Fab Kol [62] and the camel cAb-Lys3 single domain antibody [63]. In this context, additional Cys in CDR2H were noticed before, when there were only one or three Cys in CDR3H [31]. Concerning the IMGT numbering system, we solely identified one additional Cys in $\mathrm{CDR} 2 \mathrm{H}$, when one Cys in CDR3H was found in two sequences of IgE with middle-length CDR3H. When numbering the amino acids according to Kabat et al. [8], ten sequences classified in group 2 possessed one Cys in CDR3H and showed an additional Cys in CDR2H. We did not note analogues for sequences from group 1 or group 3 . In contrast to the findings that there is at least one Cys in CDR3H regions containing more than 12 amino acid residues, and that there is no Cys if the CDR3H possessed less than 10 amino acid residues [27], we also observed CDR3H sequences of intermediate length without Cys residue in $\mathrm{CDR} 3 \mathrm{H}$.

We annotated the bovine immunoglobulin heavy-chain locus, demonstrated the expression of unusually long $\mathrm{CDR} 3 \mathrm{H}$ in the five bovine immunoglobulin isotypes, and specified their genomic origin. Thus, this study reviewed the opinion that exceptionally long $\mathrm{CDR} 3 \mathrm{H}$ are a unique feature of bovine $\lg \mathrm{G} 1-3$ and $\lg \mathrm{M}$.

\section{Note}

Sequences can be found under GenBank Acc.No. KC471523 to KC471622.

\section{Supporting Information}

Table S1 Genomic annotation of the bovine immunoglobulin heavy-chain locus. For a functional gene $(F)$, the complete coding sequence, octamer motif, TATA box, splicing signals or recombination signal sequences (RSS), and poly A motif were identified. Putative functional genes $((F))$ lacked some of the described parts due to end of the contig or N's introduced into gaps for example. ORFs are classified by alterations in the splicing signals, RSS or regulatory elements. The extension (ORF) describes fully functional genes in putative orphons. Pseudogenes $(\Psi)$ revealed stop codons, frameshifts or mutations within the RSS, which lead to abolition of recombination. Fragmented loci were also defined as $\Psi[38,39,40,41]$. 


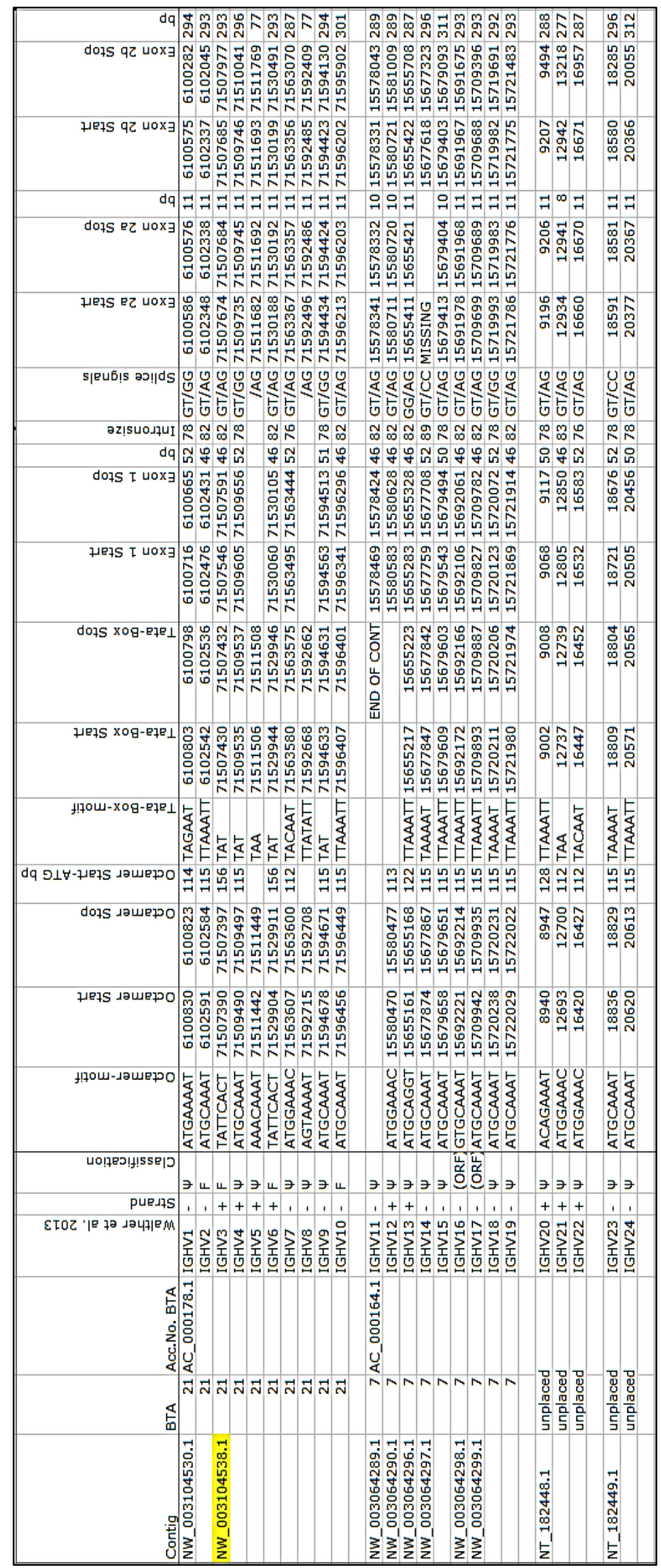




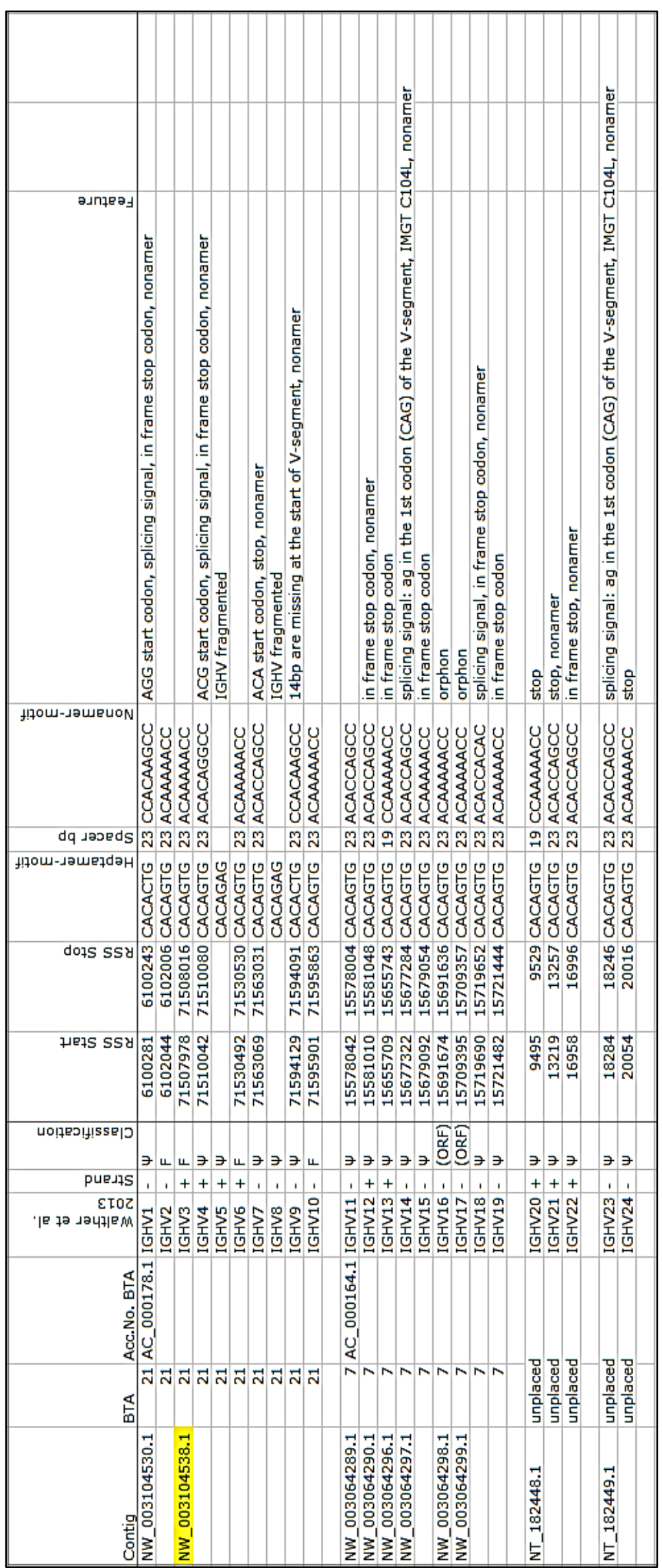




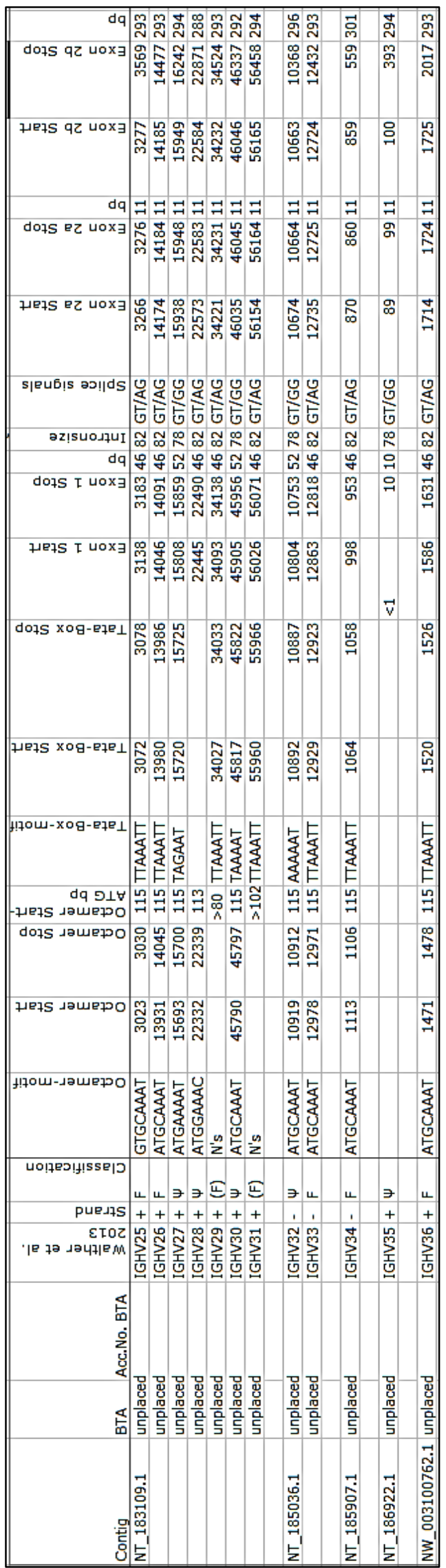




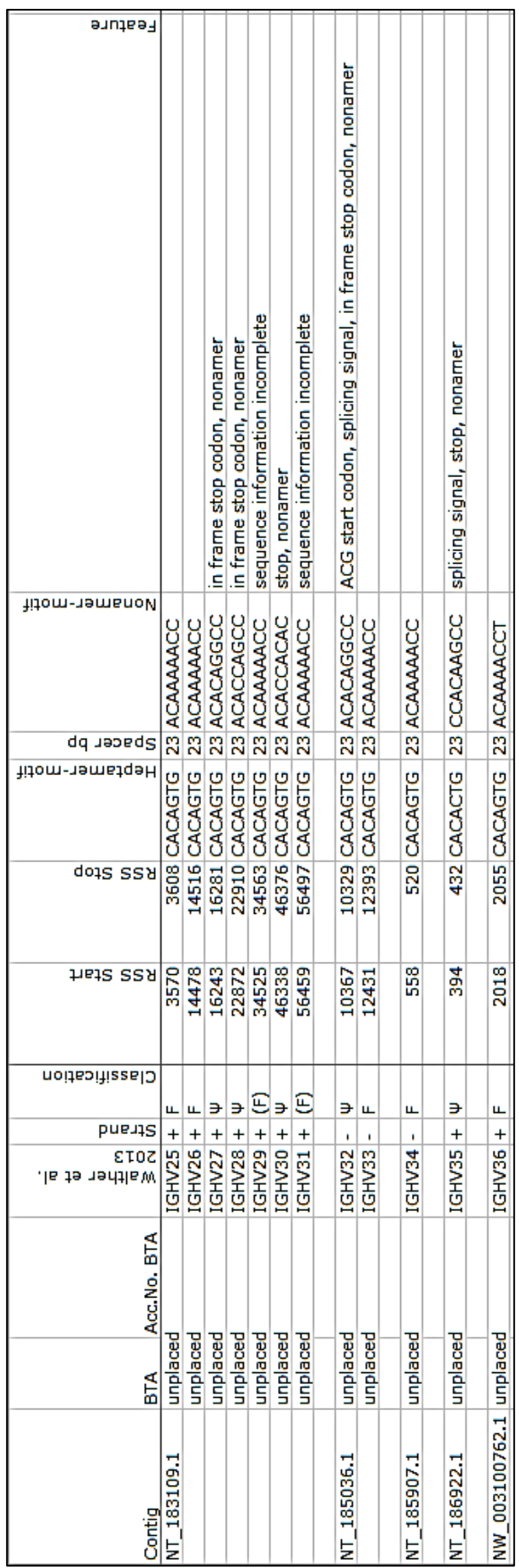




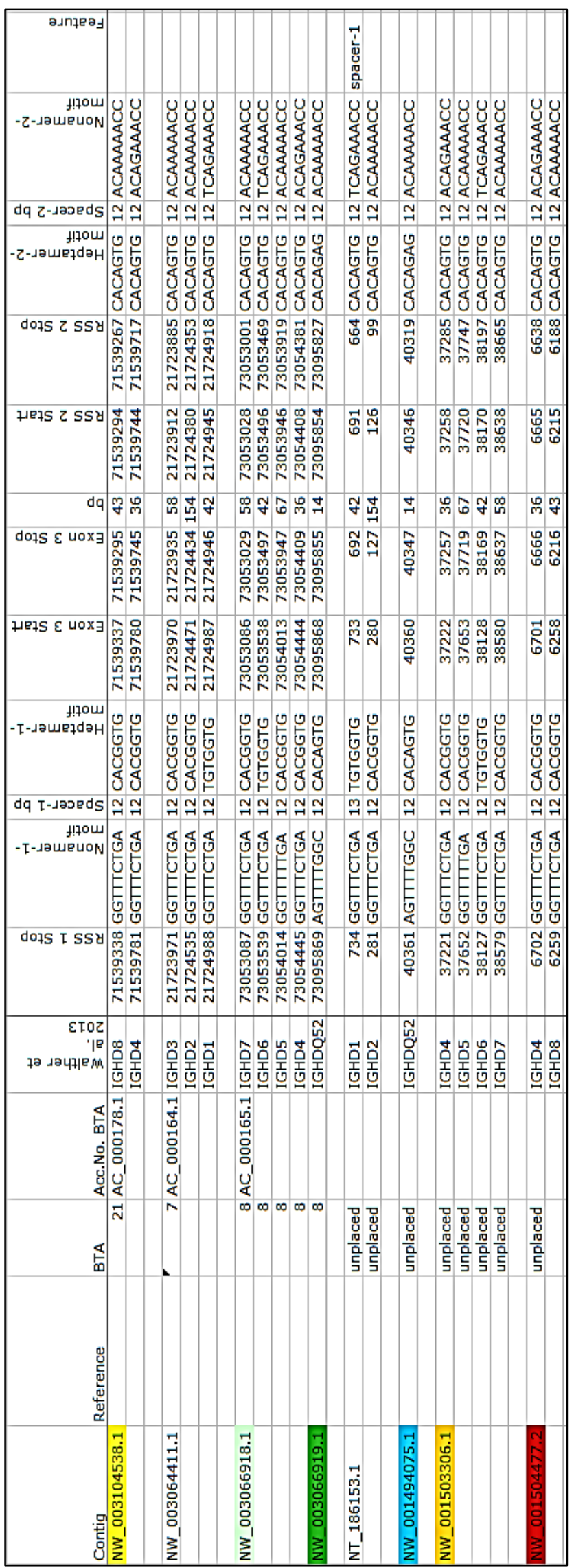




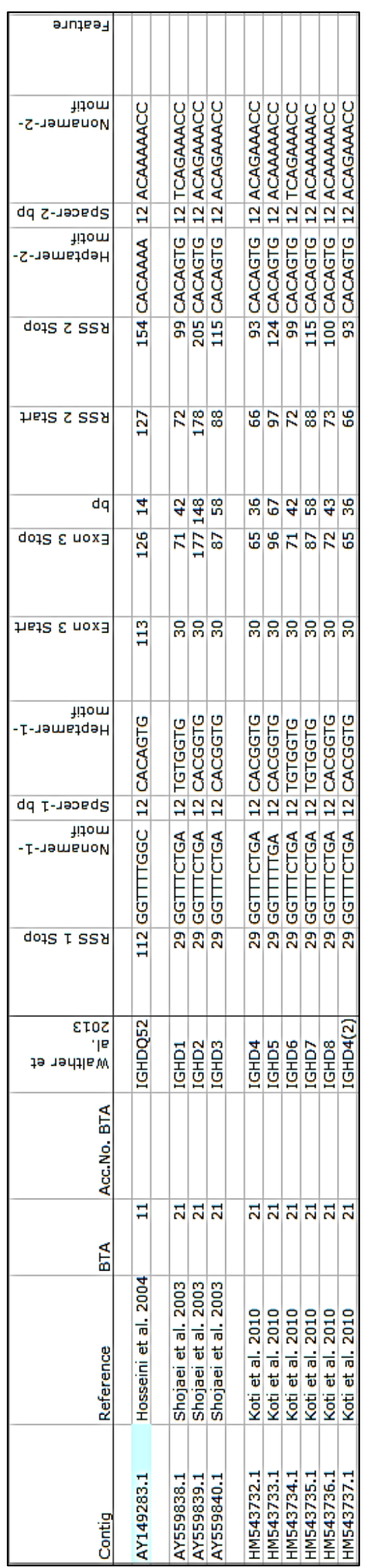




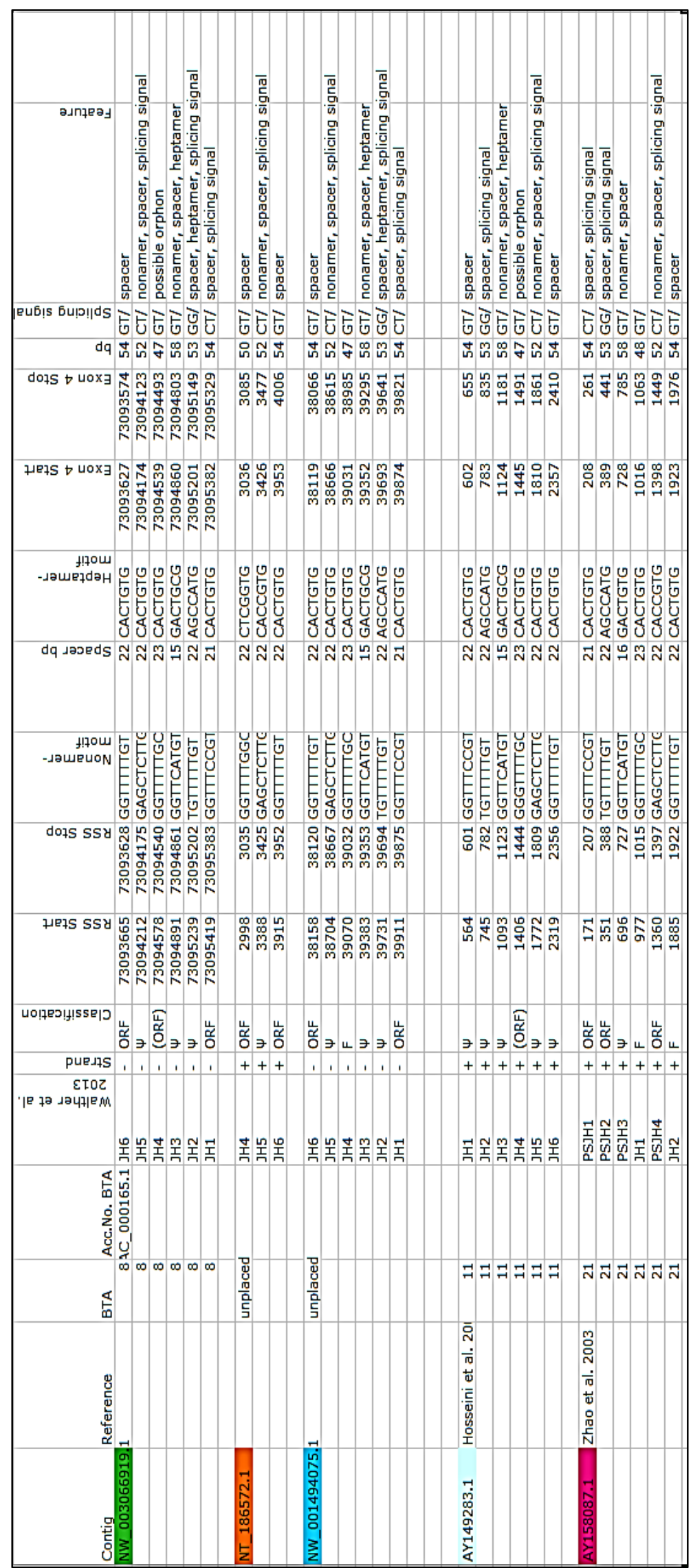


Chapter 3: Exceptionally long CDR3H in bovine immunoglobulins

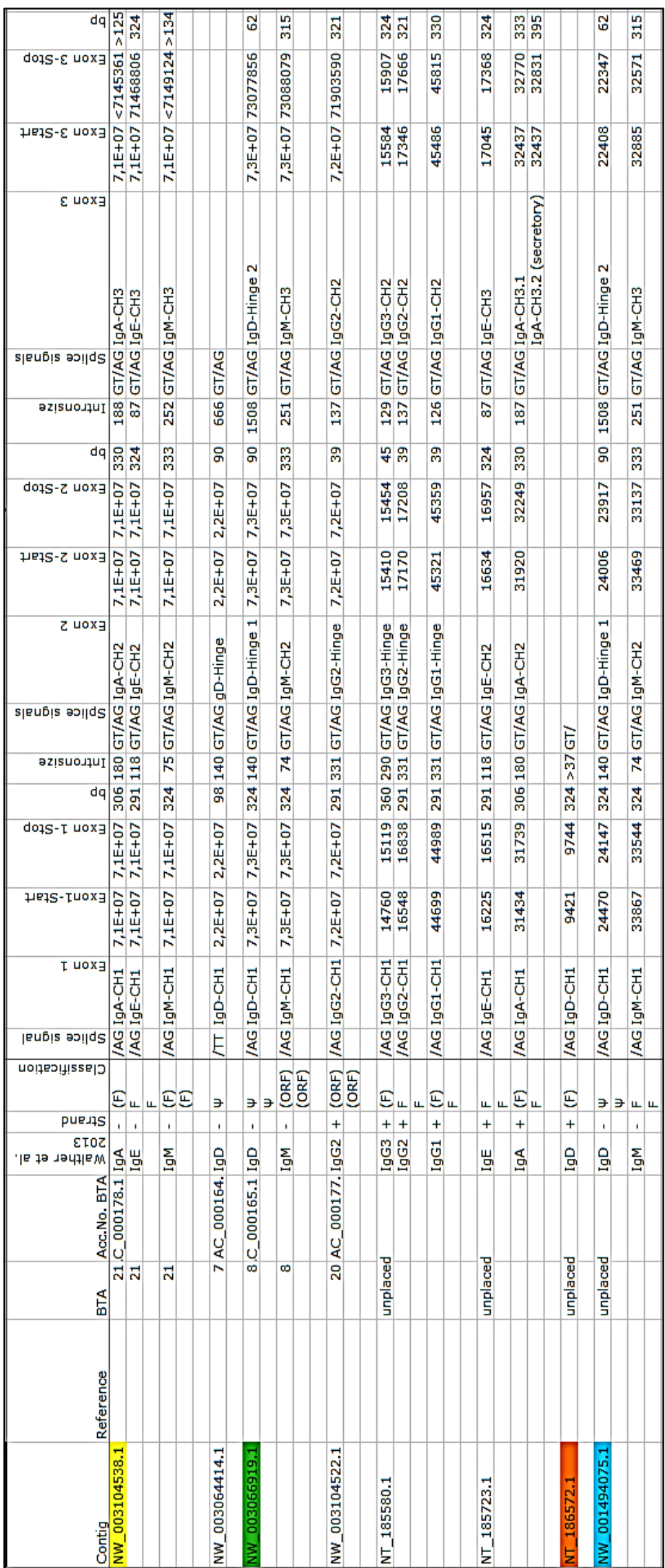




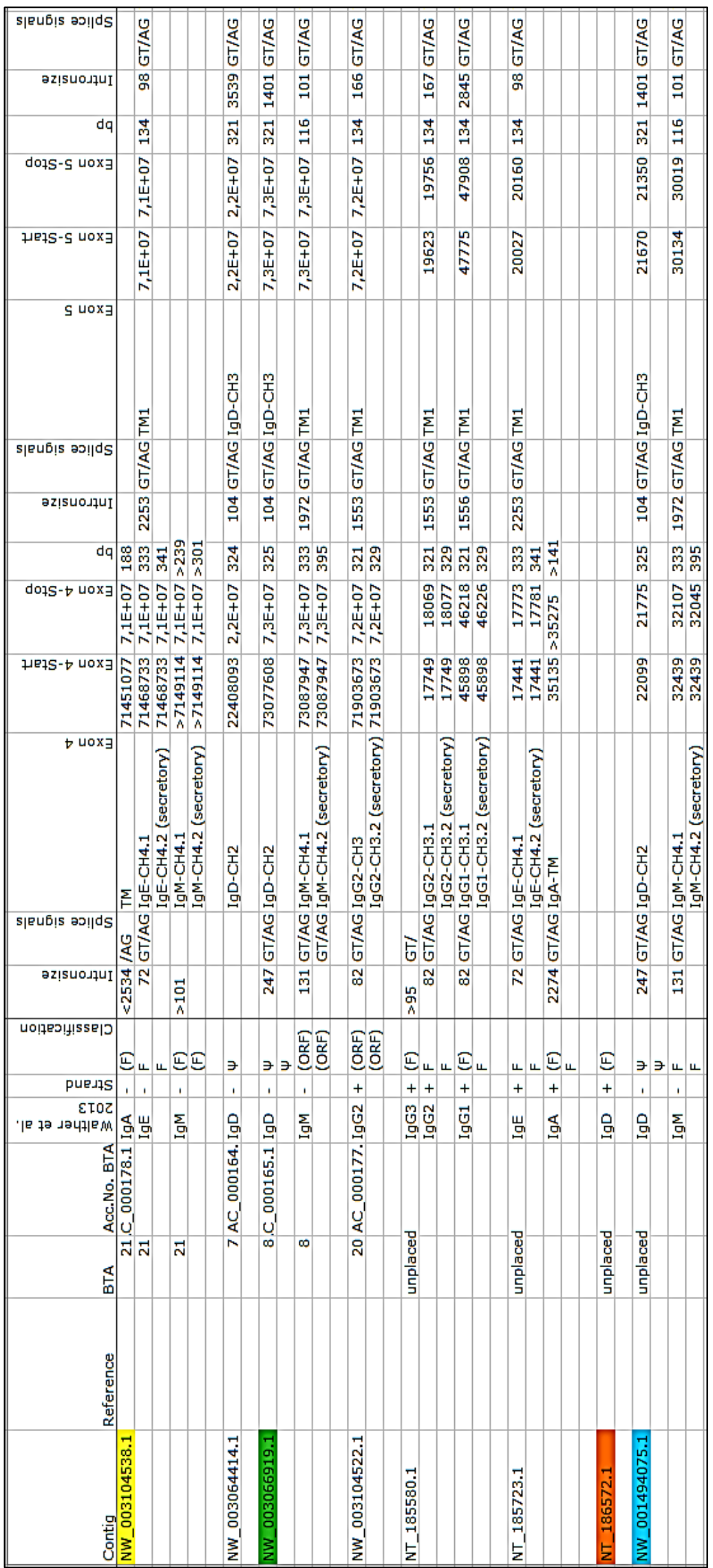


Chapter 3: Exceptionally long $\mathrm{CDR} 3 \mathrm{H}$ in bovine immunoglobulins

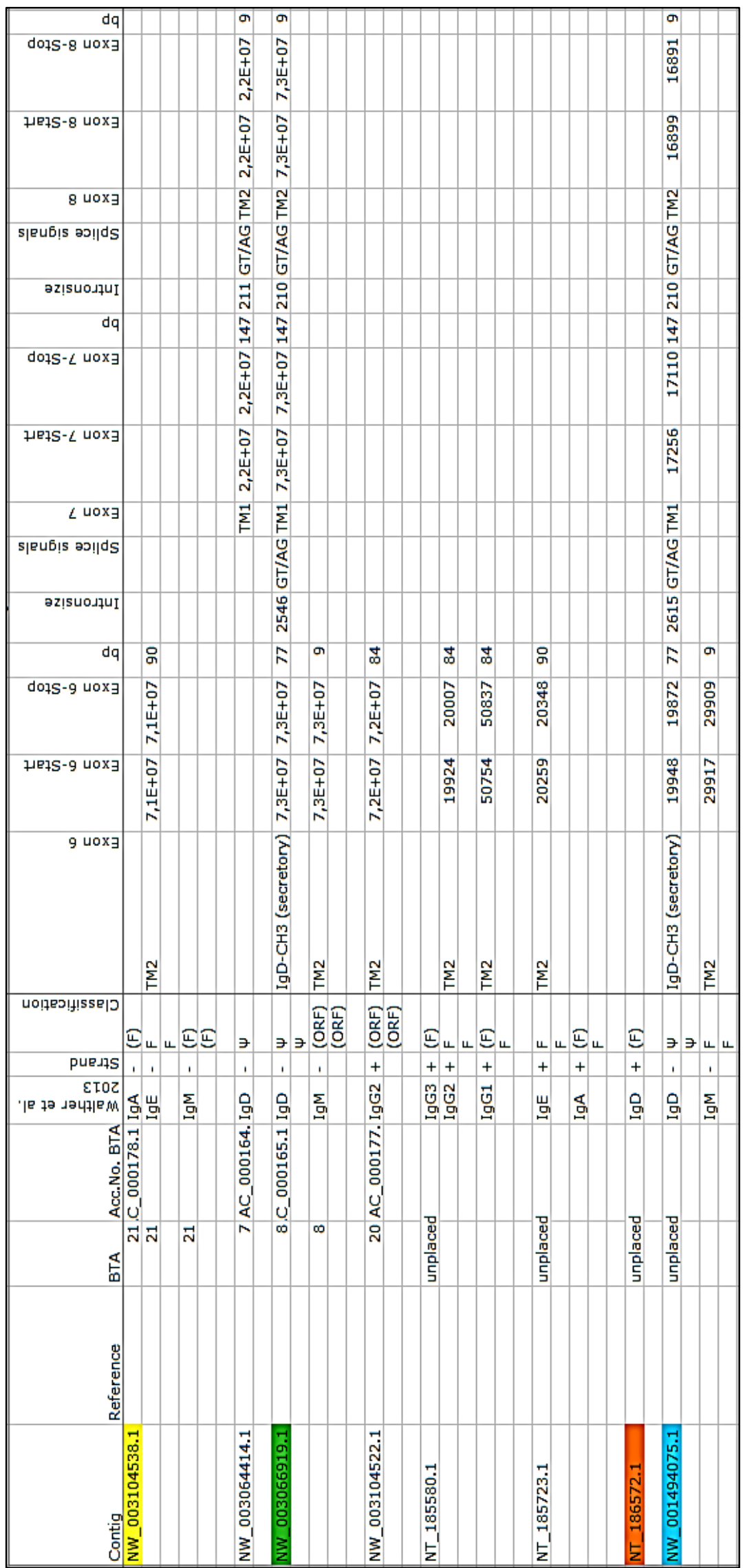




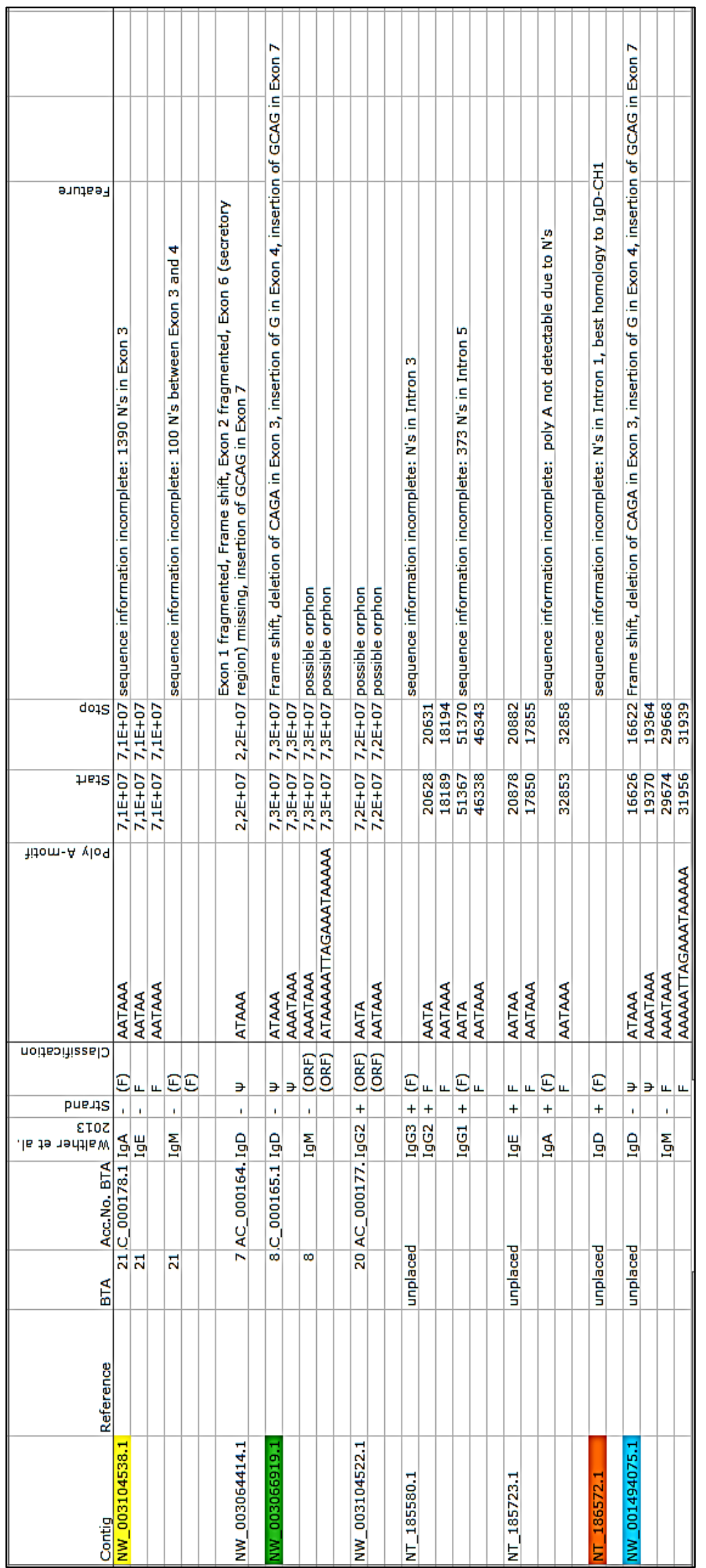




\begin{tabular}{|c|c|c|c|c|c|c|c|c|c|c|c|}
\hline$d q$ & స్లు & 吕 & & $\mathscr{B}$ & m & & 学 & 䄓 & స్ & స్లు & ఫ్లే \\
\hline do7s- $\varepsilon$ uox $\exists$ & ì & ) & & 兽 & 売曽 & & 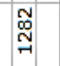 & 量 & : & m్m & $\hat{q}$ \\
\hline 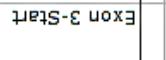 & กิ & 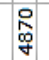 & & 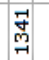 & 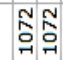 & & 吕 & 足 & 过 & g & 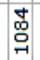 \\
\hline$\varepsilon$ น०X $\exists$ & 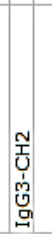 & 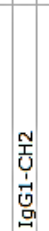 & & 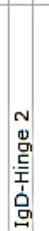 & 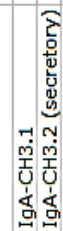 & & 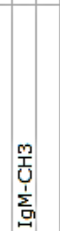 & 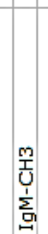 & 矛 & 矛 & 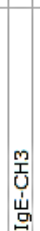 \\
\hline 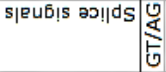 & 黄 & 妾 & & ¿ & 苦 & & 皆 & 量 & 量 & 僦 & 影 \\
\hline әz!suodfuI & స్తి & స్తి & & $\begin{array}{c}\mathrm{G} \\
\mathrm{m} \\
\mathrm{n} \\
\mathrm{n}\end{array}$ & 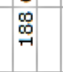 & & 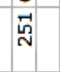 & กู & స్సి & స్సి & $\hat{\infty}$ \\
\hline dq/8 & ร & ติ & & & ల్ల & & m & m & \& & \& & ম్లే \\
\hline do7s-Z uoxق & 点 & $\frac{m}{8}$ & & & œ & 怘 & $\stackrel{0}{i}$ & ָָ & $\frac{0}{10}$ & $\stackrel{\circ}{\circ}$ & ğ \\
\hline 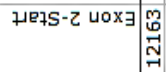 & $\bar{R}$ & \&̊g & & & 志 & $\underset{\infty}{\infty}$ & 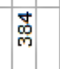 & ดี & N & 总 & $\begin{array}{l}\text { ma } \\
6\end{array}$ \\
\hline 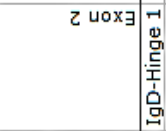 & 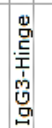 & 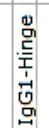 & & & 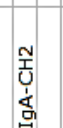 & 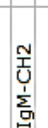 & 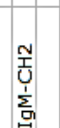 & 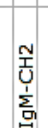 & 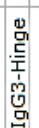 & 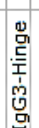 & 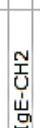 \\
\hline s|Ru6!s әכ!|ds & 全 & 量 & $\vec{G}$ & & 屁 & 亩 & 率 & 屁 & 产 & 量 & 或 \\
\hline әz!sนo.дfuI & ฐి & m & 怘 & & $\stackrel{\circ}{\circ}$ & $\stackrel{n}{\kappa}$ & $\kappa$ & t & g & g & $\stackrel{\infty}{7}$ \\
\hline dq & 总 & กี & 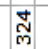 & & : & 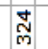 & 总 & ป్ల & 总 & 惫 & ลี \\
\hline 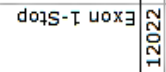 & g & 鱼 & 悹 & & m & $\frac{\pi}{7}$ & 怘 & 苟 & 高 & นึ & 䓌 \\
\hline HE7S-โU0xヨ| & $\vec{్}$ & m & 周 & & $\because$ & 営 & & มू & స్ন & : & 总 \\
\hline 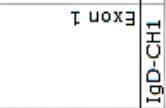 & 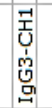 & 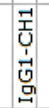 & $\begin{array}{l}\text { 곤 } \\
\text { ! } \\
\text { g }\end{array}$ & & 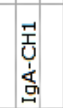 & 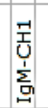 & 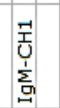 & 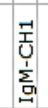 & 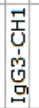 & 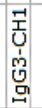 & 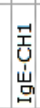 \\
\hline |Ru6!s әכ!|ds $\mid$ E & $\$$ & $\$$ & $\$$ & & $\$$ & $\$$ & & Y & O & ¿ & 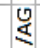 \\
\hline U0!|?Eכ|!!ISSE|J & 近可 & $\mathbb{E}_{4}$ & 뜬 & 7 & $\mathbb{E}_{\Perp}$ & 똔 & 뜬프 & 4 & $\mathbb{E}_{4}$ & $\mathbb{E}_{4}$ & E \\
\hline PUE.75 + & . & + & + & + & + & + & + & + & + & + & $\begin{array}{lll}+ & & l\end{array}$ \\
\hline 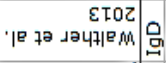 & g & $\vec{\Xi}$ & 㝵 & 曋 & 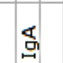 & $\sum_{g}$ & $\sum_{\text {I }}$ & $\sum_{g}$ & M & M & 亗 \\
\hline 摛 & 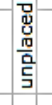 & 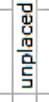 & 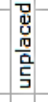 & 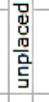 & $\bar{N}$ & $\vec{N}$ & $\bar{N}$ & ळ & $\curvearrowright$ & a & \\
\hline 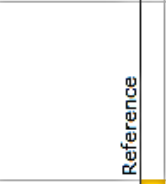 & & & & & 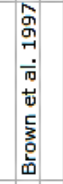 & 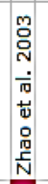 & 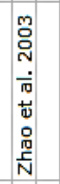 & 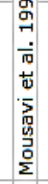 & 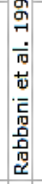 & 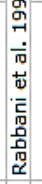 & \\
\hline 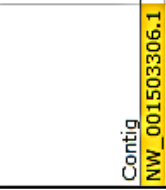 & $\begin{array}{l}\overrightarrow{1} \\
\dot{0} \\
0 \\
0 \\
0 \\
0 \\
0 \\
0 \\
3 \\
3 \\
\end{array}$ & 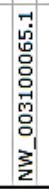 & 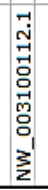 & $\begin{array}{l}7 \\
\vdots \\
0 \\
0 \\
0 \\
0 \\
0 \\
0 \\
0 \\
3 \\
2 \\
\end{array}$ & 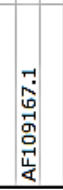 & 氕 & 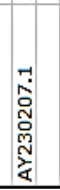 & 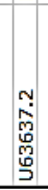 & 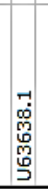 & 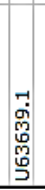 & 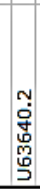 \\
\hline
\end{tabular}




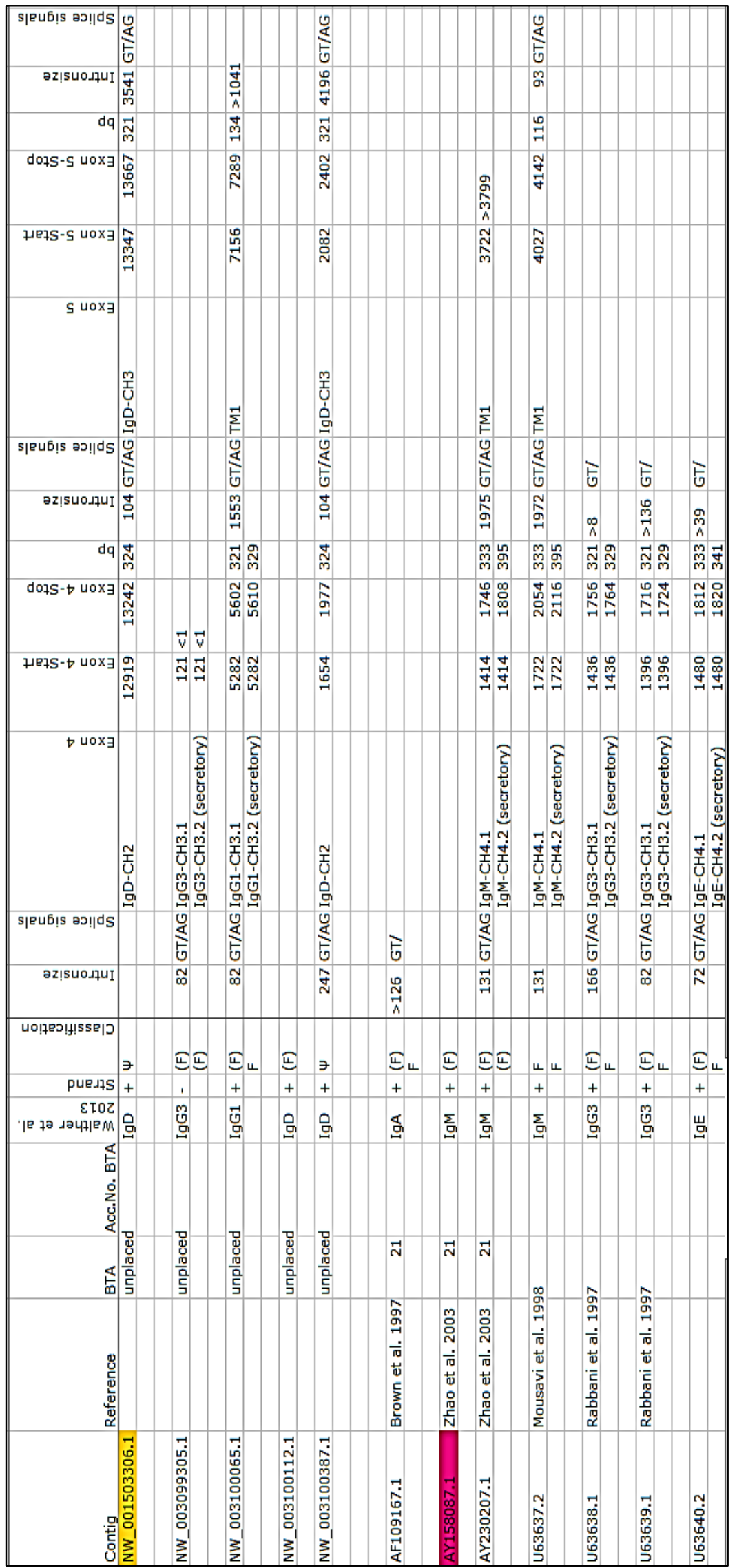


Chapter 3: Exceptionally long CDR3H in bovine immunoglobulins

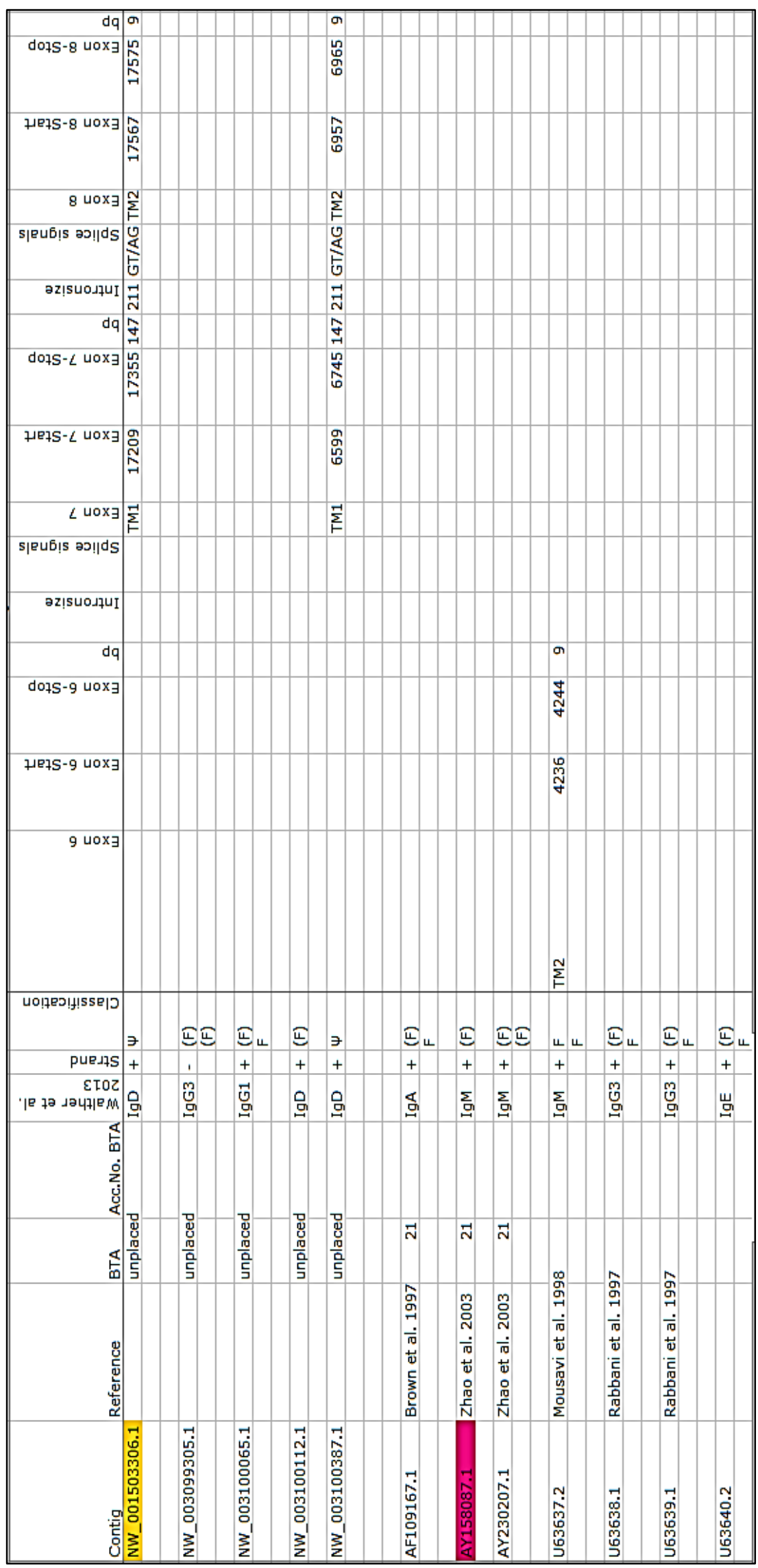




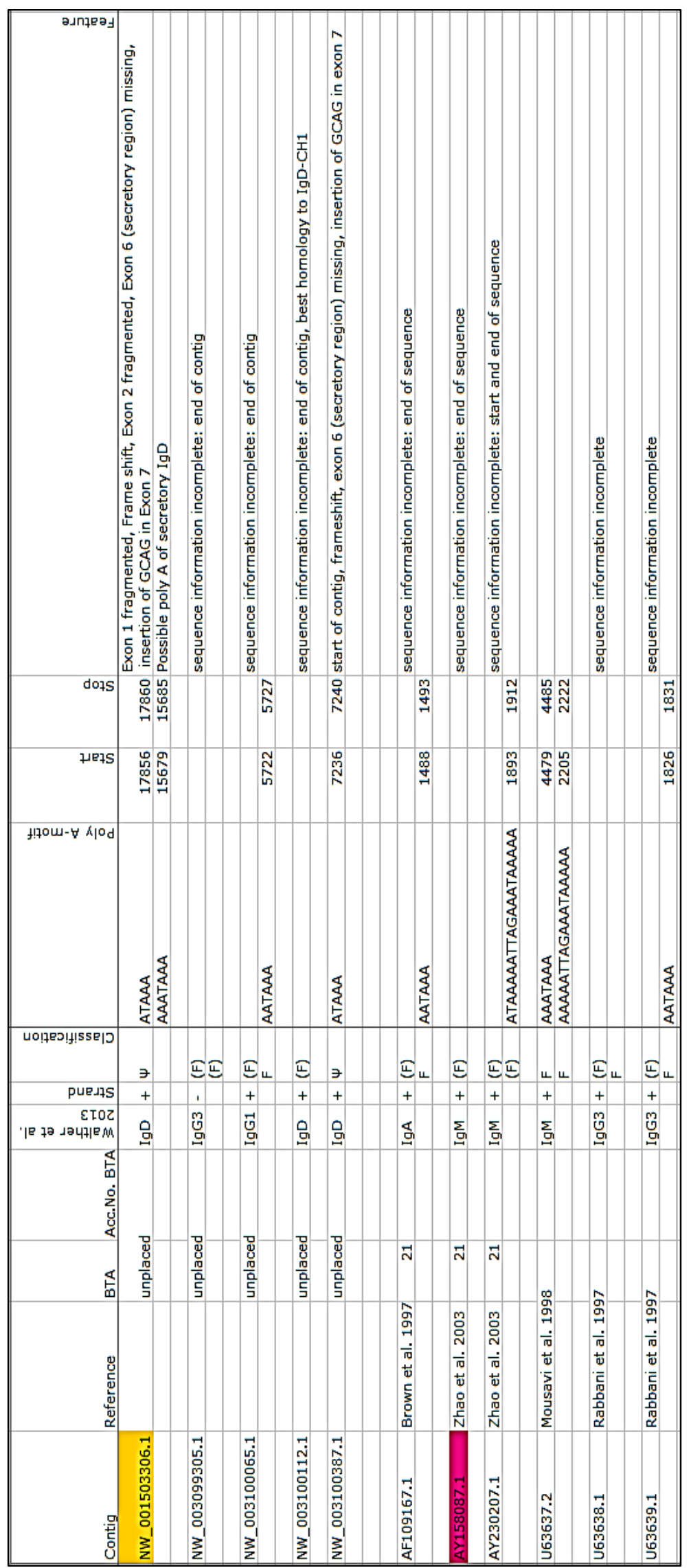



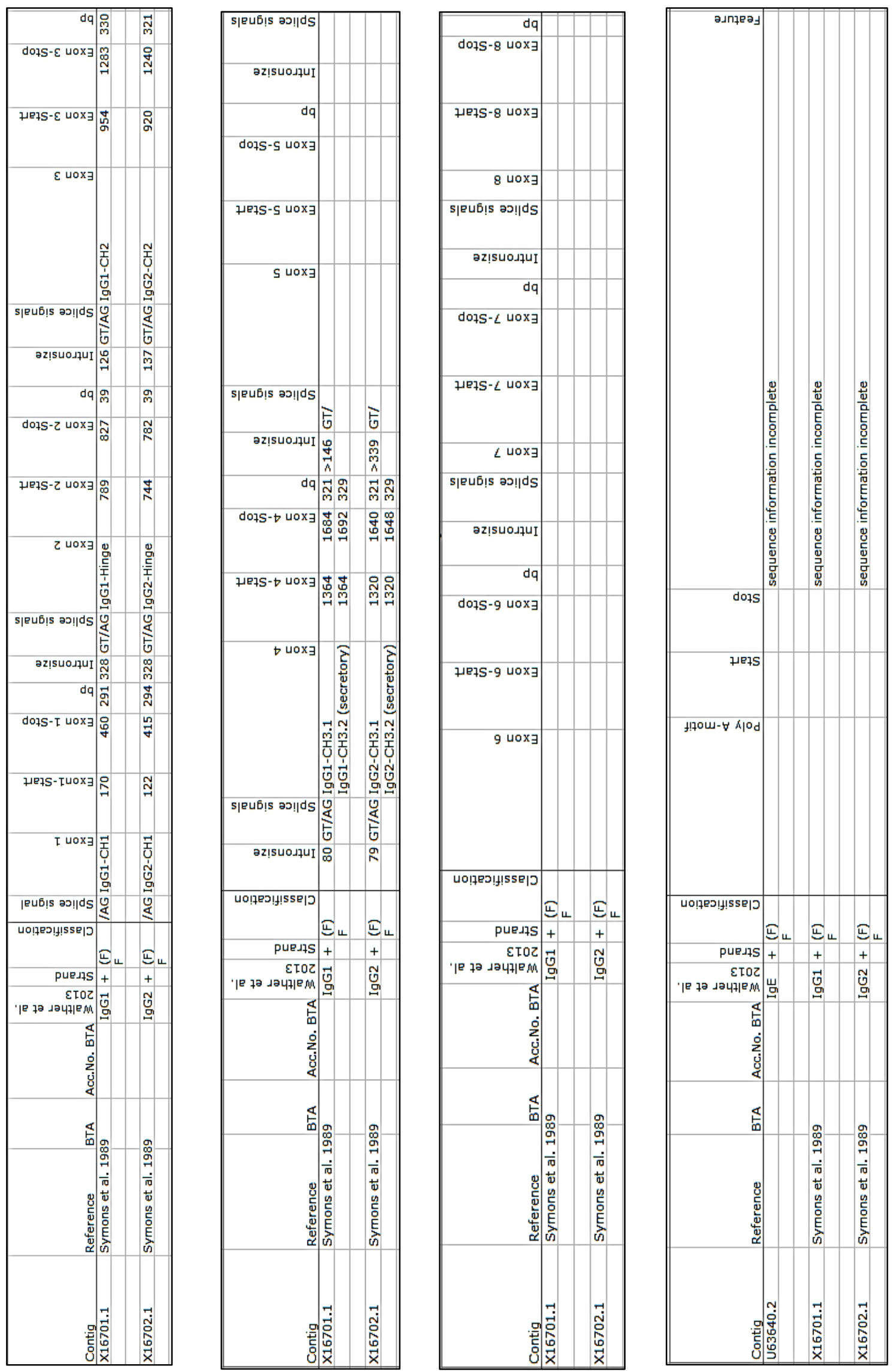


\section{Acknowledgments}

We are grateful to Lynne Armstrong for critical review of the manuscript. We also thank the Göttingen Genomics Laboratory for sample sequencing and Caroline Bierschenk for her excellent technical assistance. 


\section{References}

1. Tonegawa S (1983) Somatic generation of antibody diversity. Nature 302: 575-581.

2. Desiderio SV, Yancopoulos GD, Paskind M, Thomas E, Boss MA, et al. (1984) Insertion of $\mathrm{N}$ regions into heavy-chain genes is correlated with expression of terminal deoxytransferase in B cells. Nature 311: 752-755.

3. Alt FW, Baltimore D (1982) Joining of immunoglobulin heavy chain gene segments: implications from a chromosome with evidence of three D-JH fusions. Proc Natl Acad Sci U S A 79: 4118-4122.

4. Kurosawa Y, Tonegawa S (1982) Organization, structure, and assembly of immunoglobulin heavy chain diversity DNA segments. J Exp Med 155: 201-218.

5. Reynaud CA, Garcia C, Hein WR, Weill JC (1995) Hypermutation generating the sheep immunoglobulin repertoire is an antigen-independent process. Cell 80: 115-125.

6. Berens SJ, Wylie DE, Lopez OJ (1997) Use of a single VH family and long CDR3s in the variable region of cattle Ig heavy chains. Int Immunol 9: 189-199.

7. Wagner SD, Neuberger MS (1996) Somatic hypermutation of immunoglobulin genes. Annu Rev Immunol 14: 441-457.

8. Kabat EA, Wu TT, Perry HM, Gottesmann KS, Foeller C (2006) Sequences of proteins of immunological interest. Bethesda, MD: U.S. Department of Health and Human Services, Public Health Service, National Institutes of Health.

9. Reynaud CA, Dahan A, Anquez V, Weill JC (1989) Somatic hyperconversion diversifies the single $\mathrm{Vh}$ gene of the chicken with a high incidence in the $\mathrm{D}$ region. Cell 59: 171-183.

10. Sun J, Kacskovics I, Brown WR, Butler JE (1994) Expressed swine VH genes belong to a small VH gene family homologous to human VHIII. J Immunol 153: 5618-5627.

11. Dufour V, Malinge S, Nau F (1996) The sheep Ig variable region repertoire consists of a single VH family. J Immunol 156: 2163-2170.

12. Saini SS, Hein WR, Kaushik A (1997) A single predominantly expressed polymorphic immunoglobulin VH gene family, related to mammalian group, I, clan, II, is identified in cattle. Mol Immunol 34: 641-651.

13. Sinclair MC, Gilchrist J, Aitken R (1997) Bovine IgG repertoire is dominated by a single diversified $\mathrm{VH}$ gene family. J Immunol 159: 3883-3889.

14. Arakawa H, Saribasak H, Buerstedde JM (2004) Activation-induced cytidine deaminase initiates immunoglobulin gene conversion and hypermutation by a common intermediate. PLoS Biol 2: E179.

15. Parng CL, Hansal S, Goldsby RA, Osborne BA (1996) Gene conversion contributes to Ig light chain diversity in cattle. J Immunol 157: 5478-5486.

16. Sun $Y$, Wang $C$, Wang $Y$, Zhang T, Ren L, et al. (2010) A comprehensive analysis of germline and expressed immunoglobulin repertoire in the horse. Dev Comp Immunol 34: 1009-1020.

17. Zhao Y, Kacskovics I, Pan Q, Liberles DA, Geli J, et al. (2002) Artiodactyl IgD: the missing link. J Immunol 169: 4408-4416.

18. Knight KL, Becker RS (1987) Isolation of genes encoding bovine $\lg M, \lg G, \lg A$ and IgE chains. Vet Immunol Immunopathol 17: 17-24.

19. Symons DB, Clarkson CA, Beale D (1989) Structure of bovine immunoglobulin constant region heavy chain gamma 1 and gamma 2 genes. Mol Immunol 26: 841-850.

20. Miller JR, Thomsen PD, Dixon SC, Tucker EM, Konfortov BA, et al. (1992) Synteny mapping of the bovine IGHG2, CRC and IGF1 genes. Anim Genet 23: 51-58. 
21. Zhao Y, Kacskovics I, Rabbani H, Hammarstrom L (2003) Physical mapping of the bovine immunoglobulin heavy chain constant region gene locus. $\mathrm{J}$ Biol Chem 278: 35024-35032.

22. Hayes H, Di Meo GP, Gautier M, Laurent P, Eggen A, et al. (2000) Localization by FISH of the 31 Texas nomenclature type I markers to both Q- and R-banded bovine chromosomes. Cytogenet Cell Genet 90: 315-320.

23. Gu F, Chowdhary BP, Andersson L, Harbitz I, Gustavsson I (1992) Assignment of the bovine immunoglobulin gamma heavy chain (IGHG) gene to chromosome 21 q24 by in situ hybridization. Hereditas 117: 237-240.

24. Tobin-Janzen TC, Womack JE (1992) Comparative mapping of IGHG1, IGHM, FES, and FOS in domestic cattle. Immunogenetics 36: 157-165.

25. Hayes HC, Petit EJ (1993) Mapping of the beta-lactoglobulin gene and of an immunoglobulin $\mathrm{M}$ heavy chain-like sequence to homoeologous cattle, sheep, and goat chromosomes. Mamm Genome 4: 207-210.

26. Hosseini A, Campbell G, Prorocic M, Aitken R (2004) Duplicated copies of the bovine $\mathrm{JH}$ locus contribute to the Ig repertoire. Int Immunol 16: 843-852.

27. Lopez O, Perez C, Wylie D (1998) A single VH family and long CDR3s are the targets for hypermutation in bovine immunoglobulin heavy chains. Immunol Rev 162: 55-66.

28. Tutter A, Riblet $R$ (1989) Conservation of an immunoglobulin variable-region gene family indicates a specific, noncoding function. Proc Natl Acad Sci U S A 86: 7460-7464.

29. Koti M, Kataeva G, Kaushik AK (2008) Organization of $D(H)$-gene locus is distinct in cattle. Dev Biol (Basel) 132: 307-313.

30. Koti M, Kataeva G, Kaushik AK (2010) Novel atypical nucleotide insertions specifically at $\mathrm{VH}-\mathrm{DH}$ junction generate exceptionally long $\mathrm{CDR} 3 \mathrm{H}$ in cattle antibodies. Mol Immunol 47: 2119-2128.

31. Saini SS, Allore B, Jacobs RM, Kaushik A (1999) Exceptionally long CDR3H region with multiple cysteine residues in functional bovine $\operatorname{lgM}$ antibodies. Eur $\mathrm{J}$ Immunol 29: 2420-2426.

32. Saini SS, Kaushik A (2002) Extensive CDR3H length heterogeneity exists in bovine foetal VDJ rearrangements. Scand J Immunol 55: 140-148.

33. Kaushik AK, Kehrli ME, Jr., Kurtz A, Ng S, Koti M, et al. (2009) Somatic hypermutations and isotype restricted exceptionally long CDR3H contribute to antibody diversification in cattle. Vet Immunol Immunopathol 127: 106-113.

34. Saini SS, Farrugia W, Ramsland PA, Kaushik AK (2003) Bovine IgM antibodies with exceptionally long complementarity-determining region 3 of the heavy chain share unique structural properties conferring restricted $\mathrm{VH}+$ Vlambda pairings. Int Immunol 15: 845-853.

35. Larsen PA, Smith TP (2012) Application of circular consensus sequencing and network analysis to characterize the bovine IgG repertoire. BMC Immunol 13: 52.

36. Shojaei F, Saini SS, Kaushik AK (2003) Unusually long germline DH genes contribute to large sized CDR3H in bovine antibodies. Mol Immunol 40: 61-67.

37. Ramsland PA, Kaushik A, Marchalonis JJ, Edmundson AB (2001) Incorporation of long CDR3s into $V$ domains: implications for the structural evolution of the antibody-combining site. Exp Clin Immunogenet 18: 176-198.

38. Lefranc MP (1998) IMGT (ImMunoGeneTics) locus on focus. A new section of Experimental and Clinical Immunogenetics. Exp Clin Immunogenet 15: 1-7.

39. Lefranc MP, Pommie C, Ruiz M, Giudicelli V, Foulquier E, et al. (2003) IMGT unique numbering for immunoglobulin and $T$ cell receptor variable domains and Ig superfamily V-like domains. Dev Comp Immunol 27: 55-77. 
40. Akamatsu Y, Tsurushita N, Nagawa F, Matsuoka M, Okazaki K, et al. (1994) Essential residues in V(D)J recombination signals. J Immunol 153: 4520-4529.

41. Hesse JE, Lieber MR, Mizuuchi K, Gellert M (1989) V(D)J recombination: a functional definition of the joining signals. Genes Dev 3: 1053-1061.

42. Larkin MA, Blackshields G, Brown NP, Chenna R, McGettigan PA, et al. (2007) Clustal W and Clustal X version 2.0. Bioinformatics 23: 2947-2948.

43. Felsenstein $J(1985)$ Confidence limits on phylogenies: an approach using the bootstrap. Evolution 39: 783-791.

44. Sitnikova T, Su C (1998) Coevolution of immunoglobulin heavy- and light-chain variable-region gene families. Mol Biol Evol 15: 617-625.

45. Almagro JC, Martinez L, Smith SL, Alagon A, Estevez J, et al. (2006) Analysis of the horse $\mathrm{V}(\mathrm{H})$ repertoire and comparison with the human IGHV germline genes, and sheep, cattle and pig $\mathrm{V}(\mathrm{H})$ sequences. Mol Immunol 43: 1836-1845.

46. Perriere G, Gouy M (1996) WWW-query: an on-line retrieval system for biological sequence banks. Biochimie 78: 364-369.

47. Sanger F, Nicklen S, Coulson AR (1977) DNA sequencing with chain-terminating inhibitors. Proc Natl Acad Sci U S A 74: 5463-5467.

48. Thompson JD, Higgins DG, Gibson TJ (1994) CLUSTAL W: improving the sensitivity of progressive multiple sequence alignment through sequence weighting, position-specific gap penalties and weight matrix choice. Nucleic Acids Res 22: 4673-4680.

49. Ehrenmann F, Kaas Q, Lefranc MP (2010) IMGT/3Dstructure-DB and IMGT/DomainGapAlign: a database and a tool for immunoglobulins or antibodies, T cell receptors, MHC, IgSF and MhcSF. Nucleic Acids Res 38: D301-307.

50. Ehrenmann F, Lefranc MP (2011) IMGT/DomainGapAlign: IMGT standardized analysis of amino acid sequences of variable, constant, and groove domains (IG, TR, MH, IgSF, MhSF). Cold Spring Harb Protoc 2011: 737-749.

51. Matsuda F, Ishii K, Bourvagnet P, Kuma K, Hayashida H, et al. (1998) The complete nucleotide sequence of the human immunoglobulin heavy chain variable region locus. J Exp Med 188: 2151-2162.

52. Matsuda F, Honjo T (1996) Organization of the human immunoglobulin heavychain locus. Adv Immunol 62: 1-29.

53. Zimin AV, Delcher AL, Florea L, Kelley DR, Schatz MC, et al. (2009) A wholegenome assembly of the domestic cow, Bos taurus. Genome Biol 10: R42.

54. Everts-van der Wind A, Kata SR, Band MR, Rebeiz M, Larkin DM, et al. (2004) A 1463 gene cattle-human comparative map with anchor points defined by human genome sequence coordinates. Genome Res 14: 1424-1437.

55. Heuertz S, Hors-Cayla MC (1978) [Bovine chromosome mapping with the cell hybridization technic. Localization on the $x$ chromosome of glucose-6phosphate dehydrogenase, phosphoglycerate kinase, alpha-galactosidase A and hypoxanthine phosphoribosyltransferase]. Ann Genet 21: 197-202.

56. Sinclair MC, Aitken R (1995) PCR strategies for isolation of the $5^{\prime}$ end of an immunoglobulin-encoding bovine cDNA. Gene 167: 285-289.

57. Jhunjhunwala S, van Zelm MC, Peak MM, Cutchin S, Riblet R, et al. (2008) The 3D structure of the immunoglobulin heavy-chain locus: implications for long-range genomic interactions. Cell 133: 265-279.

58. Reynaud CA, Bertocci B, Dahan A, Weill JC (1994) Formation of the chicken B-cell repertoire: ontogenesis, regulation of Ig gene rearrangement, and diversification by gene conversion. Adv Immunol 57: 353-378.

59. Corbett SJ, Tomlinson IM, Sonnhammer EL, Buck D, Winter G (1997) Sequence of the human immunoglobulin diversity (D) segment locus: a systematic analysis 
provides no evidence for the use of DIR segments, inverted D segments, "minor" D segments or D-D recombination. J Mol Biol 270: 587-597.

60. Kaartinen M, Mäkelä O (1985) Reading of $D$ genes in variable frames as a source of antibody diversity. Immunol Today 6: 324-327.

61. Friedman ML, Tunyaplin C, Zhai SK, Knight KL (1994) Neonatal VH, D, and JH gene usage in rabbit $B$ lineage cells. J Immunol 152: 632-641.

62. Schmidt WE, Jung HD, Palm W, Hilschmann N (1983) [Three-dimensional structure determination of antibodies. Primary structure of crystallized monoclonal immunoglobulin IgG1 KOL, I]. Hoppe Seylers Z Physiol Chem 364: 713-747.

63. Desmyter A, Transue TR, Ghahroudi MA, Thi MH, Poortmans F, et al. (1996) Crystal structure of a camel single-domain $\mathrm{VH}$ antibody fragment in complex with lysozyme. Nat Struct Biol 3: 803-811.

64. Ota T, Nei M (1994) Divergent evolution and evolution by the birth-and-death process in the immunoglobulin VH gene family. Mol Biol Evol 11: 469-482. 
Development of a bioinformatics framework for the detection of gene conversion and the analysis of combinatorial diversity in immunoglobulin heavy chains in four cattle breeds

Stefanie Walther ${ }^{1 \uparrow}$, Manfred Tietze ${ }^{2 \pi}$, Claus-Peter Czerny ${ }^{1}$, Sven König ${ }^{2}$, Ulrike S. Diesterbeck $^{1 *}$

${ }^{1}$ Department of Animal Sciences, Institute of Veterinary Medicine, Division of Microbiology and Animal Hygiene, Faculty of Agricultural Sciences, Georg-August University Goettingen, Goettingen, Germany

2Department of Animal Breeding, University of Kassel, Witzenhausen, Germany

${ }^{*}$ Corresponding author:

Email: ulrike.diesterbeck@nih.gov (USD)

IThese authors contributed equally to this work.

Submitted to "PloSOne", manuscript under review 
Chapter 3: Gene conversion and combinatorial diversity in bovine heavy chains

\section{Abstract}

We have developed a new bioinformatics framework for the analysis of rearranged bovine heavy chain immunoglobulin (lg) variable regions by combining and refining widely used alignment algorithms. This bioinformatics framework allowed us to investigate alignments of heavy chain framework regions (FRHs) and the separate alignments of $\mathrm{FRHs}$ and heavy chain complementarity determining regions (CDRHs) to determine their germline origin in the 4 cattle breeds Aubrac, German Black Pied, German Simmental, and Holstein Friesian. Now it is also possible to specifically analyze Ig heavy chains possessing exceptionally long CDR3Hs.

In order to gain more insight into breed specific differences in Ig combinatorial diversity, somatic hypermutations and putative gene conversions of $\lg G$, we compared the dominantly transcribed variable (IGHV), diversity (IGHD), and joining (IGHJ) segments and their recombination in the 4 cattle breeds.

The analysis revealed the use of 15 different IGHV segments, 21 IGHD segments, and 2 IGHJ segments with significant different transcription levels within the breeds. Furthermore, there are preferred rearrangements within the 3 groups of $\mathrm{CDR} 3 \mathrm{H}$ lengths. In the sequences of group 2 (CDR3H lengths $(L)$ of 11-47 amino acid residues (aa)) a higher number of recombination was observed than in sequences of group 1 ( $L \leq 10$ aa) and 3 ( $L \geq 48$ aa). The combinatorial diversity of germline IGHV, IGHD, and IGHJ-segments revealed 162 rearrangements that were significantly different. The few preferably rearranged gene segments within group $3 \mathrm{CDR} 3 \mathrm{H}$ regions may indicate specialized antibodies because this length is unique in cattle.

The most important finding of this study, which was enabled by using the bioinformatics framework, is the discovery of strong evidence for gene conversion as a rare event using pseudogenes fulfilling all definitions for this particular diversification mechanism. 
Chapter 3: Gene conversion and combinatorial diversity in bovine heavy chains

\section{Author Summary}

The humoral immune response is an integral part in the specific pathogen defense. Beside universal processes for the generation of the immunoglobulin diversity applying for all higher vertebrates, species-specific mechanisms exist. Cattle possess less functional gene segments available for combinatorial diversity if compared with human and mice.

Thus, we hypothesized that cattle use pseudogenes for a genetically exchange, the socalled gene conversion. Beside the difficulty of the incomplete annotated bovine heavy chain immunoglobulin locus, we were able to verify gene conversion events fulfilling all conditions like clusters of nucleotide exchanges, at least triplets, origin of the pseudogene upstream of the rearranged segment and a high degree of flanking homology.

In addition, we analyzed the combinatorial diversity and hot spots for hypermutations in four different cattle breeds: Aubrac, German Black Pied, German Simmental, and Holstein Friesian. Here we observed a significant non-random distribution in the usage of the segments as well as in their combinations in Aubrac and German Simmental. The highest variability within the variable region was always observed in the transition of framework regions to complementarity determining regions (CDRH) if compared to the middle of the CDRHs. 
Chapter 3: Gene conversion and combinatorial diversity in bovine heavy chains

\section{Introduction}

The basic genetic mechanism in developing immunoglobulin diversity is similar in all jawed vertebrates. Immunoglobulins (Ig) are Y-shaped hetero-tetramers consisting of two identical heavy chains $(\mathrm{IGH})$ and two identical light chains, either $\kappa$ or $\lambda$ in mammals (IGK, IGL) [1]. Both chains are functionally divided into variable and constant domains that are combined during B-cell development. The variable domain is rearranged by separate heavy and light chain variable (IGHV, IGKV, IGLV), diversity $(I G H D)$, and joining (IGHJ, IGKJ, IGLJ) germline gene segments [2]. In addition, the imprecise junction of the germline gene segments and somatic hypermutations contribute to antibody diversity [3-5].

Species differences were primarily found in the number of germline IGHV/IGKV/IGLV, $I G H D$, and IGHJ/IGKJ/IGLJ segments. In livestock species with restricted combinatorial germline diversity such as chicken [4], pigs [6], sheep [7], and cattle [5, 8, 9], species-dependent mechanisms dominate the different diversification steps. For instance, the use of pseudogene sequence parts is a frequent post-recombinatorial strategy for the generation of the preimmune antibody repertoire in chicken, sheep, and rabbit [4, 10-13]. This phenomenon, called gene conversion, was also confirmed for IGLs in cattle [14] and is assumed to be operative in horses [15].

Gene conversions are difficult to detect especially within a large number of sequences e.g. like those obtained from high throughput sequencing. Gene conversion in immunoglobulins is characterized by clusters of nucleotide changes [14], sometimes only triplets [11], originating from upstream genes of the rearranged segment [4, 13]. High degree of flanking homology of the conversion region ensures the genetic exchange [13], whereby 3 to 5 nucleotides seem to be the minimal overlapping requirement [11].

Detection of gene conversion in bovine IGHV is complicated due to the incomplete IGH locus annotation. The main bovine IGH locus was assigned to the Bos taurus autosome (BTA) 21 but exons coding for variable, diversity, and joining segments were also found on BTA7, BTA8, and BTA20 [16-18]. Thirty-six IGHVs were identified of which 13 are functional and belong phylogenetically to the bovine IGHV family 1 (boVH1). The second bovine IGHV family consists solely of non-functional IGHVs that have not been identified in expression analyses yet. Eleven IGHV segment pairs shared $100 \%$ sequence identity, whereas two of these pairs contain a functional segment and either an ORF or a putative functional segment, respectively [17]. The 
Chapter 3: Gene conversion and combinatorial diversity in bovine heavy chains

high proportion of pseudogene segments leads to the assumption of their use in gene conversion events. Two IGHJ loci possessing six IGHJ segments were detected on BTA11 by BAC clone and locus-specific PCR analysis and were found to rearrange at low frequency while those located on BTA21 rearrange at high frequency. Only two out of these six IGHJ were classified as functional whereas one is involved predominantly in the recombination process $[19,20]$. Fifteen IGHD genes were detected and revealed a sub-cluster organization. IGHD are classified into four families and the IGHD exons revealed huge size differences [21, 22]. The organization of the actual bovine germline repertoire and its possible allelic variants is incomplete and needs to be investigated in more detail [17]. Since, even the organization of the extensively studied human immunoglobulin germline repertoire is questioned and requires ongoing analyses [23]. In all rearranged bovine immunoglobulin isotypes, exceptionally long complementarity determining region 3 of the heavy chain $(\mathrm{CDR} 3 \mathrm{H})$ possessing up to 67 aa were described [17]. Together with IGHD2 and IGHJ1, the germline IGHV10 segment was found to be the only variable segment rearranged in these exceptionally long CDR3Hs $[17,24]$. This mechanism is also not isotype restricted $[17,25]$.

An additional bovine specific mechanism for antibody diversification is the insertion of conserved short nucleotide sequences into the IGHV-IGHD junction, which was found in intermediate and exceptionally long CDR3Hs [24].

Currently available programs like IMGT/Junction Analysis [26], IMGT/V-QUEST [27, 28] and IMTG/HIGHV-QUEST [29], VBASE2 [30], JoinSolver [31], iHMMun-align [32], and IgBLAST [33] allow the annotation of only the entire IGHV sequence to germline IGHV segments. Differentiated analysis of single parts is not directly possible. Most of the databases are focusing on mouse and human immunoglobulin genes (VBASE2, human, [30]). For cattle and other livestock or companion animals, separate databases have to be created (IgBLAST, [33]). Furthermore, the IMGT numbering system does not provide numbering for $\mathrm{CDR} 3 \mathrm{H}$ larger than 31 aa. Placing of bovine intermediate as well as of exceptional long $\mathrm{CDR} 3 \mathrm{H}$ in this numbering system is therefore not possible and consequently does not allow correct analysis of the rearrangement in those immunoglobulins. In addition, only IgBLAST allows the adjustment of parameters for IGHD identification.

Detailed genetic analysis of the pre- and post-immunization humoral immune response is important to describe the developing diversity and the effectiveness of vaccines and to detect possible individual and breed related differences including non-responders. 
As a conclusion those analyses help to develop fast recombinant antibodies for passive vaccination, therapy or diagnostic by genetically pre-selection of newly developed or abundant sequences.

As a first attempt to gain more insight into bovine breed specific differences, we compared the dominantly transcribed and the combinatorial diversity of germline IGHV, $I G H D$, and IGHJ segments as well as somatic hypermutations and putative gene conversions of IgG in the four cattle breeds Aubrac, German Simmental, German Black Pied and Holstein Friesian, by using a newly developed Bioinformatics framework. This new bioinformatics framework combines and extends several analysis tools and takes into account the unique specificities of bovine immunoglobulin sequences of exceptionally long CDR3Hs. In addition, it allows for the adaptation of alignment parameters for the single segments and enables the selective analysis of the different functional regions of the variable domain (namely framework regions and CDRs) to determine putative gene conversions. This new tool should facilitate a fast and detailed analysis of data sets generated by high throughput sequencing. 
Chapter 3: Gene conversion and combinatorial diversity in bovine heavy chains

\section{Results}

For sequence analysis, we developed a new bioinformatics framework using MUSCLE $[34,35]$ for the initial fast and accurate multiple nucleotide sequence alignment. Subsequently, the sequence distances were calculated with ClustalW [36]. For nucleotide alignments of IGHV and IGHJ, default values of MUSCLE were used. To improve the biological significance of the assignment of germline and sample IGHDs, we tested three different procedures using different parameters. To determine the germline origin using the new bioinformatics framework, only the FRHs were aligned to avoid interference with the highly diversified CDRH [18]. To analyze possible gene conversion events, FR1-3Hs and CDR1-3Hs were extracted and aligned separately to the corresponding regions of the IGHV reference sequences to find the most similar germline segment.

Using our bioinformatics tool, we established a sample sequence set for use in detailed analysis of the transcribed bovine immunoglobulin repertoire. Blood samples were taken from 10 animals per cattle breed: Aubrac (A), German Simmental (GS), German Black Pied (GBP), and Holstein Friesian (HF).

In total, $160 \mathrm{lgG}$ heavy chain sequences per breed ( $\mathrm{n}=640$ sequences) were investigated as described above. The variable regions were identified and extracted at the 5 ' end (N-terminal end) using the motif GCCTCCACC/AST (nt/AA) marking the start of the first constant region of all bovine IgGs. Due to premature Stop-codons or incompletely amplified variable regions, 131 sequences were excluded from further analyses. Consequently, 509 sequences were left: 137 in A, 116 in GS, 111 in GBP, and 145 in HF. Sequences analyzed are published under accession numbers KT761498-KT762006. 
Chapter 3: Gene conversion and combinatorial diversity in bovine heavy chains

Transcriptional analyses, assignment of germline gene segments

\section{Assigning the FR1H to FR3H of transcribed IGHV segments to their germline origin}

Ig heavy chain gene usage and identity to germline gene segments was determined by comparing the transcribed sequences with the germline lg heavy chain genes described by Walther et al. [17] and Liljavirta et al. [22]. We found six of the germline IGHVs possess $100 \%$ sequence identity (presented as IGHVx/y) up to the 3 ' end of $\mathrm{FR} 3 \mathrm{H}$. Analysis of transcribed IGHV segments (here: comprising FR1-3H) revealed germline gene usage of IGHV3/33, IGHV6, IGHV10/34, IGHV36/29(F), IGHV17(ORF)/31(F), IGHV1S26, IGHV1S28, IGHV1S32, IGHV1S33, IGHV1S34, IGHV1S35, IGHV1S37, IGHV1S38, IGHV1S39 as well as IGHV1S40 (Fig 1).

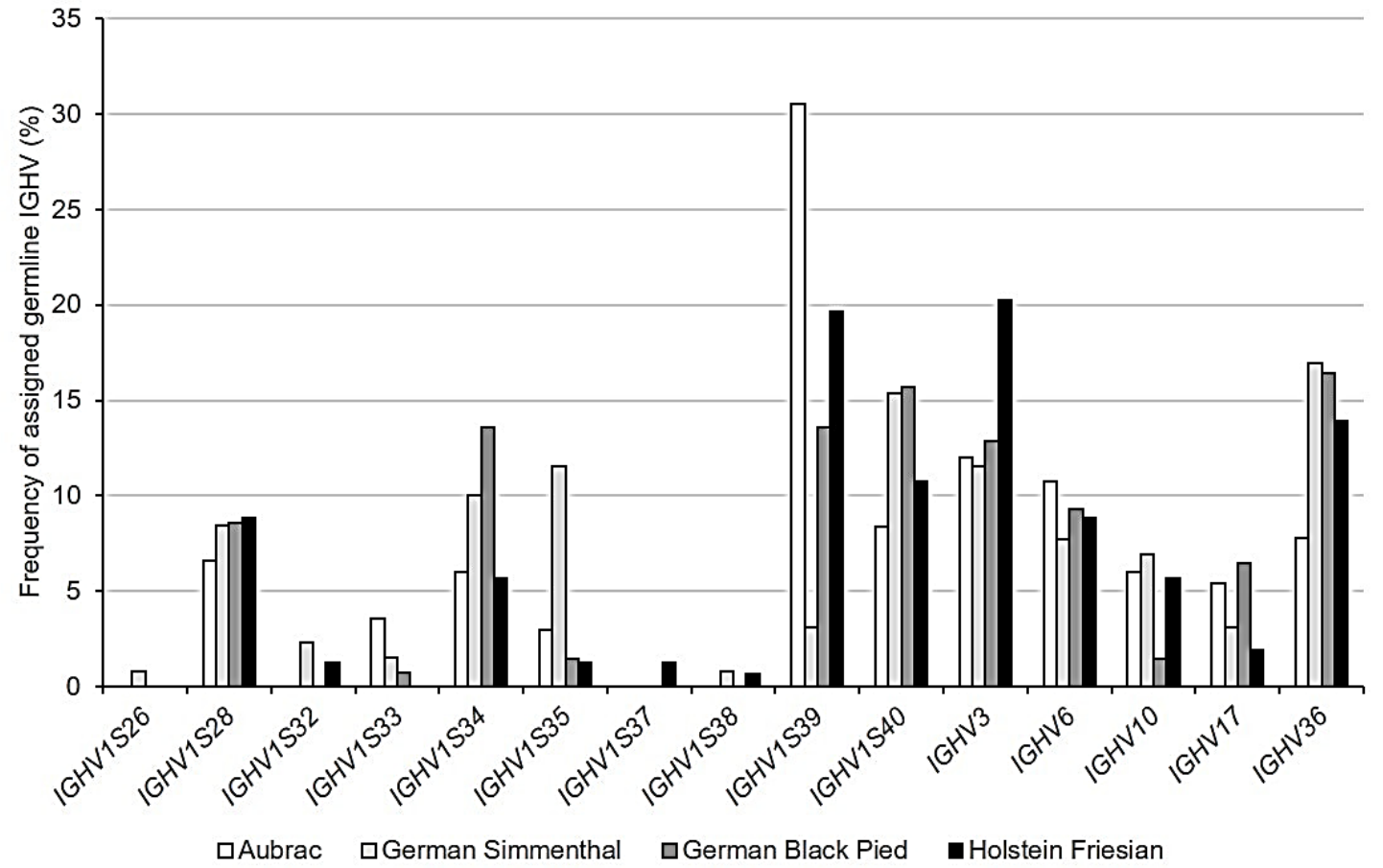

Fig 1. Transcription frequencies of IGHV in four cattle breeds. Transcribed IGHV are shown on the horizontal axis, their relative usage frequencies are indicated on the vertical axis. Each breed is marked by the following color code: Aubrac: white, German Simmental: light grey, Holstein Friesian: black, German Black Pied: dark grey 
Chapter 3: Gene conversion and combinatorial diversity in bovine heavy chains

We were not able to unambiguously assign ten of 116 analyzed GS sequences to one germline IGHV; this was also true for 14 out of 111 GBP sequences, 12 out of $145 \mathrm{HF}$ sequences, and 19 out of 137 analyzed sequences of $A$.

These samples showed equal divergence to at least two germline IGHV which included combinations of IGHV3/33, IGHV6, IGHV10/34, IGHV17(ORF)/31(F), IGHV1S28, and IGHV1S32-40. The most frequent multiple assignment affected IGHV6 and IGHV1S34, followed by IGHV6 and IGHV1S35 in all A, GS, and HF. IGHV6, IGHV1S34, and IGHV17(ORF)/31(F) could not be differentiated in two sequences of GS, one sequence of GBP and two sequences of HF. Ambiguously assigned germline IGHVs and multiple assignments are listed in Table 1.

Table 1. IGHV assigned ambiguously and their frequency. Ambiguous sequences can be assigned to more than one germline IGHV with the same distance.

\begin{tabular}{|l|r|r|r|r|}
\hline Breed & A & GBP & GS & HF \\
\hline Total No. of animals & 10 & 10 & 10 & 10 \\
\hline No. of animals with ambiguous sequences & 9 & 5 & 4 & 6 \\
\hline Total No. of sequences analyzed & 137 & 111 & 116 & 145 \\
\hline No. of ambiguous sequences & 19 & 14 & 10 & 12 \\
\hline Total No. of putative IGHV recombinations & 167 & 140 & 130 & 158 \\
\hline Ambiguous IGHV & & & & \\
\hline IGHV3/IGHV1S33 & 0 & 0 & 1 & 0 \\
\hline IGHV3/IGHV1S39 & 0 & 0 & 0 & 2 \\
\hline IGHV6/IGHV17 & 1 & 0 & 0 & 0 \\
\hline IGHV6/IGHV1S34 & 3 & 3 & 3 & 5 \\
\hline IGHV6/IGHV1S35 & 3 & 0 & 1 & 1 \\
\hline IGHV6/IGHV36 & 0 & 1 & 1 & 1 \\
\hline IGHV6/IGHV1S39 & 1 & 0 & 1 & 0 \\
\hline IGHV1S28/IGHV1S32 & 0 & 0 & 0 & 1 \\
\hline IGHV1S33/IGHV1S39 & 1 & 1 & 0 & 0 \\
\hline IGHV1S33/IGHV1S40 & 1 & 0 & 0 & 0 \\
\hline IGHV1S37/IGHV1S38 & 0 & 0 & 0 & 1 \\
\hline IGHV1S39/IGHV17 & 0 & 1 & 0 & 0 \\
\hline IGHV1S39/IGHV1S40 & 1 & 0 & 0 & 0 \\
\hline IGHV3/IGHV10/IGHV1S39 & 2 & 0 & 0 & 0 \\
\hline IGHV6/IGHV17/IGHV1S34 & 0 & 2 & 2 & 1 \\
\hline IGHV6/IGHV17/IGHV1S40 & 1 & 0 & 0 & 0 \\
\hline IGHV6/IGHV1S32/IGHV1S34 & 1 & 0 & 1 & 0 \\
\hline IGHV6/IGHV1S33/IGHV1S35 & 1 & 0 & 0 & 0 \\
\hline IGHV3/IGHV6/IGHV17/IGHV1S34 & 0 & 2 & 0 & 0 \\
\hline IGHV6/IGHV10/IGHV1S34/IGHV1S39 & 1 & 0 & 0 & 0 \\
\hline IGHV6/IGHV17/IGHV1S28/IGHV1S39 & 0 & 1 & 0 & 0 \\
\hline IGHV6/IGHV17/IGHV1S34/IGHV1S39 & 1 & 1 & 0 & 0 \\
\hline IGHV6/IGHV36/IGHV1S34/IGHV1S39 & 0 & 1 & 0 & 0 \\
\hline IGHV3/IGHV6/IGHV17/IGHV1S34/IGHV1S39 & 1 & 0 & 0 & 0 \\
\hline IGHV6/IGHV17/IGHV1S33/IGHV1S34/IGHV1S35 & & 0 & 0 \\
\hline
\end{tabular}


Chapter 3: Gene conversion and combinatorial diversity in bovine heavy chains

Including the multiple assignments as described above, a total number of 595 possible transcribed germline IGHV were observed. Overall, the most frequent variable gene segment was IGHV1S39; this was identified in $17.65 \%$ of all sequences. This IGHV was used in 51 A sequences $(n=167,30.54 \%)$, in 19 sequences of GBP $(n=140$, $13.57 \%)$, in four sequences of $G S(n=130,3.08 \%)$, and 31 sequences of $H F(n=158$, $19.62 \%$ ) (Fig 1, Table 2). IGHV3/33 was represented by $14.29 \%$ of all sequences. The number of transcribed IGHV3/33 varied from 15 in GS (11.54\%) to 32 in HF (20.25\%) (Fig 1, Table 2). In similar proportions of $13.45 \%$ and $12.27 \%$ germline IGHV36/29(F) and IGHV1S40 were used. IGHV1S28, IGHV1S34, and IGHV6 were transcribed in proportions of $8.07 \%, 8.57 \%$, and $9.24 \%$. The other transcribed IGHVs were identified in minor proportions of $0.17 \%$ to $5.04 \%$, respectively. These rarely used IGHVs were identified once or twice in GS and HF but up to ten times in A (Fig 1). Very high significant differences were calculated for IGHV usage within the breeds and between the breeds $(P<0.0001)$.

Table 2. Percentage of IGHV assigned to sample sequences of four cattle breeds.

\begin{tabular}{|l|r|r|r|r|}
\hline IGHV & $\begin{array}{r}\text { A } \\
\text { (\%; } \mathbf{n = 1 6 7 )}\end{array}$ & $\begin{array}{r}\text { GS } \\
(\% ; \mathbf{n} 130)\end{array}$ & $\begin{array}{r}\text { GBP } \\
(\% ; \mathbf{n = 1 5 8})\end{array}$ & $\begin{array}{r}\text { HF } \\
(\% ; \mathbf{n = 1 4 0})\end{array}$ \\
\hline IGHV1S26 & 0.00 & 0.77 & 0.00 & 0.00 \\
\hline IGHV1S28 & 6.59 & 8.46 & 8.57 & 8.86 \\
\hline IGHV1S32 & 0.00 & 2.31 & 0.00 & 1.27 \\
\hline IGHV1S33 & 3.59 & 1.54 & 0.71 & 0.00 \\
\hline IGHV1S34 & 5.99 & 10.00 & 13.57 & 5.70 \\
\hline IGHV1S35 & 2.99 & 11.54 & 1.43 & 1.27 \\
\hline IGHV1S37 & 0.00 & 0.00 & 0.00 & 1.27 \\
\hline IGHV1S38 & 0.00 & 0.77 & 0.00 & 0.63 \\
\hline IGHV1S39 & 30.54 & 3.08 & 13.57 & 19.62 \\
\hline IGHV1S40 & 8.38 & 15.38 & 15.71 & 10.76 \\
\hline IGHV3 & 11.98 & 11.54 & 12.86 & 20.25 \\
\hline IGHV6 & 10.78 & 7.69 & 9.29 & 8.86 \\
\hline IGHV10 & 5.99 & 6.92 & 1.43 & 5.70 \\
\hline IGHV17 & 5.39 & 3.08 & 6.43 & 1.90 \\
\hline IGHV36 & 7.78 & 16.92 & 16.43 & 13.92 \\
\hline
\end{tabular}


Chapter 3: Gene conversion and combinatorial diversity in bovine heavy chains

\section{Separated analyses of FRH 1-3 and CDRH 1-2 to detect gen conversion events}

In livestock such as chicken, rabbit, and cattle the use of pseudogene segments is known to contribute to immunoglobulin diversity [4, 10, 11, 13, 14, 37, 38]. Gene conversion in immunoglobulins is characterized by clusters of nucleotide changes [14], sometimes only triplets [11], originating from upstream genes of the rearranged segment [4, 13]. A high degree of flanking homology of the conversion region ensures the genetic exchange [13], whereby three to five nucleotides have been shown to be the minimal overlapping requirement [11].

Consequently, FR1-3H and CDR1-2H were analyzed separately to identify mutations within the FR1-3H and CDR1-2H that would indicate possible gene conversion events. The current genomic annotation of germline immunoglobulin segments in cattle makes a correct prediction of $5^{\prime}$ donor segments difficult. Nonetheless, larger contigs have been identified [17] and enabled us to show gene conversion events in bovine immunoglobulin heavy chains variable regions.

For instance, the calculated putative originating germline genes for nucleotide changes

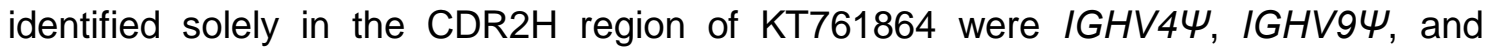
IGHV184 (divergence 0.190). In the calculation covering the whole V-region and using only FR1-3H, IGHV6 was identified as the originating gene for KT761864 (divergence 0.075). All genes but IGHV18 $\Psi$ are located on BTA21, whereas IGHV4 $\Psi$ is located upstream of IGHV6 and is therefore most likely used for the gene conversion. There are two triplets in KT761864 and IGHV4W that are different from IGHV6 due to a transversion mutation in the first changed triplet (from AAT in IGHV6 to TAT in KT761864 and IGHV4Y) and a transition mutation in the second changed triplet (from GAT in IGHV6 to AAT in KT761864 and IGHV44; Table 3).

Table 3. Possible gene conversion in KT761864.

\begin{tabular}{|c|c|c|c|}
\hline Sample/Gene & Sequence in CDR2H & Location & Divergence \\
\hline KT761864 & ATT AAT TAT AAT ... GGA GAC ACC & BTA21 & \\
\hline IGHV44 & ATA TAT TAT AAT ... GGT GAC ACT & BTA21 & 0.190 \\
\hline IGHV9u & ATA TAT TAT AAT ... GGT GAC ACT & BTA21 & 0.190 \\
\hline IGHV184 & ATA TAT TAT AAT ... GGT GAC ACT & BTA7 & 0.190 \\
\hline IGHV6 $^{1}$ & ATA GAT AAT GAT ... GGA GAC ACA & BTA21 & 0.075 \\
\hline
\end{tabular}

${ }^{1}$ Divergence in calculations covering the whole $\mathrm{V}$-region and using only $\mathrm{FR} 1-3 \mathrm{H}$. This gene was not observed in the analysis of the separated CDR2H. 
Chapter 3: Gene conversion and combinatorial diversity in bovine heavy chains

Due to high sequence similarities and because we assumed preferable transcription of functional germline gene segments, we first concentrated on pseudogenes that were calculated as unique origin of the sample sequences considering the particular regions.

In all breeds, IGHV13 $\Psi$ and IGHV17(ORF) were used most often in CDR2H (3.34\% and $1.77 \%)$ as well as in $\mathrm{FR} 2 \mathrm{H}$ and $\mathrm{FR} 3 \mathrm{H}($ IGHV17(ORF) both 1.38\%) and CDR2H (IGHV13\% $1.77 \%$ ), respectively. IGHV15 $\Psi$ was found in $0.59 \%$ of CDR2H in

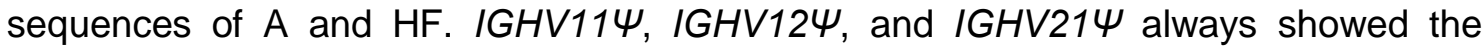
same divergence from GBP and HF sequences but were not solely identified as the

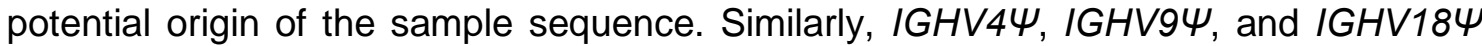
were identified in triplet as possible originating gene segments but only together with functional germline genes. In one sequence of GBP and 2 sequences of GS both in CDR1H and CDR2H pseudogenes were calculated as possible parental IGHVs. In one A sequence only a pseudogene showed lowest divergence from the sample in FR2H and CDR2H (Fig 2).

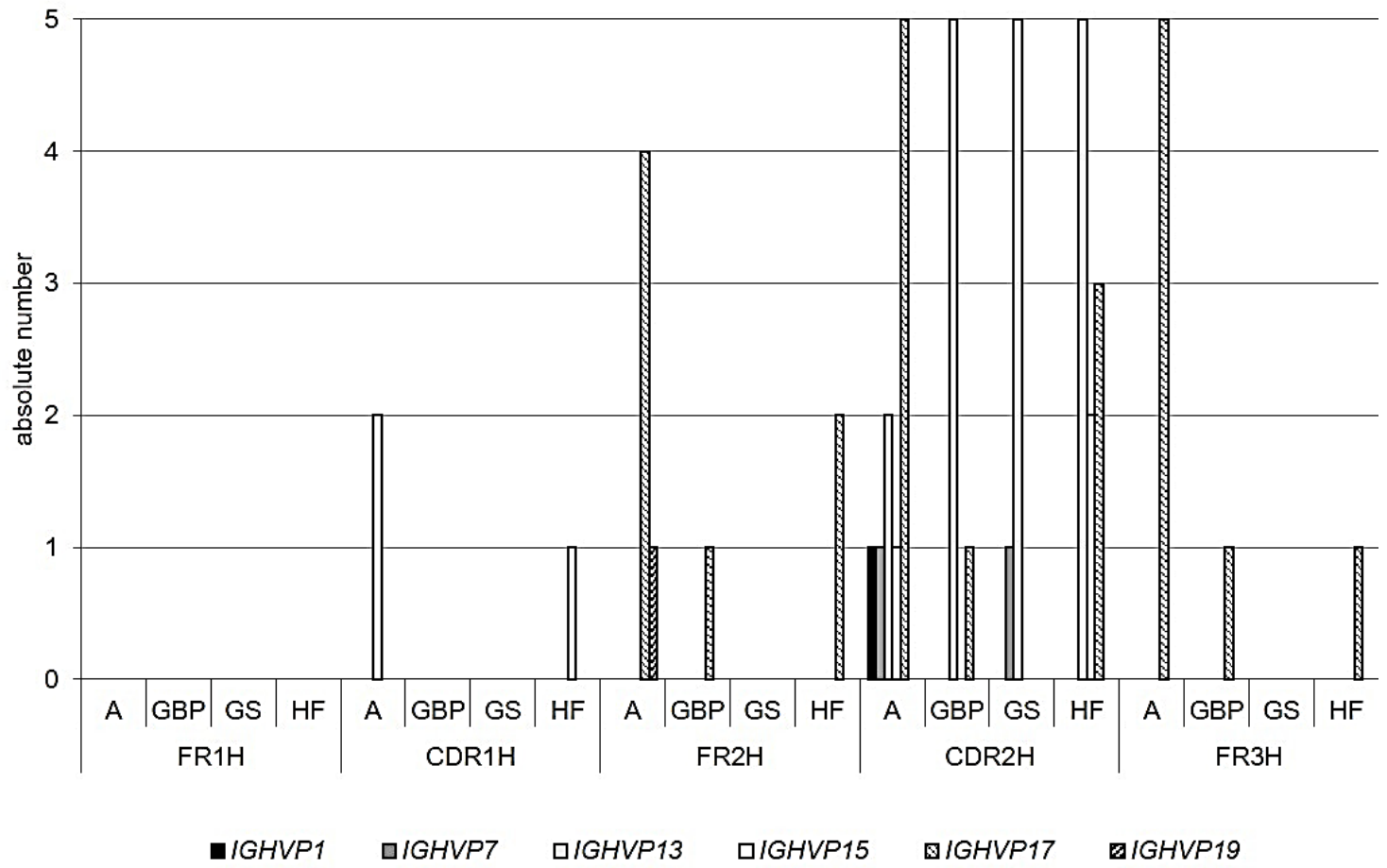

Fig 2. Possible gene conversion in FRH and CDRH. The absolute number of possible gene conversion events is shown for pseudogenes assigned unambiguously to $\mathrm{CDR} 1 \mathrm{H}, \mathrm{FR} 2 \mathrm{H}$, and $\mathrm{CDR} 2 \mathrm{H}$ in the four cattle breeds Aubrac (A), German Black Pied (GBP), German Simmental (GS), and Holstein Friesian (HF). 
Chapter 3: Gene conversion and combinatorial diversity in bovine heavy chains

Our analyses revealed a less distinct assignment to the germline gene segments. Nevertheless, the majority of identified and putative originating germline sequences were IGHV3/33, IGHV6, IGHV10/34, IGHV2/26, IGHV29(F)/36, IGHV16(ORF)/25, IGHV17(ORF)/31(F), and IGHV194, as well as IGHV1S19, IGHV1S23-28, IGHV1S30, IGHV1S32-40 [22] within FRHs and CDRHs. Although these are the same gene segments as determined for the complete IGHV segment, calculations indicate exchanges between the IGHV gene segments within FRHs and CDRHs. Additional

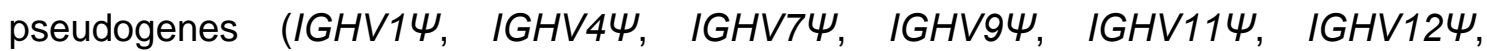

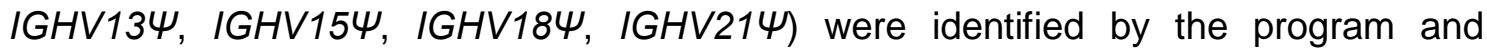

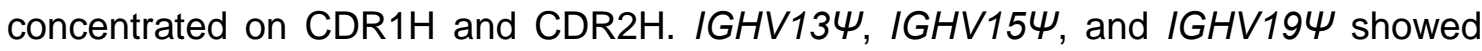
little divergence in $\mathrm{FR} 1 \mathrm{H}$ and $\mathrm{FR} 2 \mathrm{H}$ from sample sequences, too. Within these $\mathrm{FRHs}$, different functional IGHV were possible originating germline sequences (Table 4).

Table 4. Possible gene conversions in FRH and CDRH.

\begin{tabular}{|c|c|c|c|c|c|c|c|c|c|c|c|c|}
\hline & & \multicolumn{2}{|c|}{$\mathbf{A}$} & \multicolumn{2}{|c|}{ GBP } & \multicolumn{2}{|c|}{ GS } & \multicolumn{2}{|r|}{ HF } & \multicolumn{2}{|c|}{ Sum } & Percent \\
\hline & & $a^{1}$ & $\mathbf{u}^{2}$ & $a^{1}$ & $\mathbf{u}^{2}$ & $a^{1}$ & $\mathbf{u}^{2}$ & $a^{1}$ & $\mathbf{u}^{2}$ & $a^{1}$ & $\mathbf{u}^{2}$ & $\mathbf{u}^{2}$ \\
\hline IGHV14 & CDR1H & 1 & $\underline{0}$ & 0 & 0 & 0 & 0 & 1 & 0 & 2 & 0 & 0.00 \\
\hline & CDR2H & 1 & 1 & 1 & 0 & 1 & 0 & 2 & 0 & 5 & 1 & 0.20 \\
\hline IGHV4Y & CDR1H & 1 & 0 & 0 & 0 & 0 & 0 & 1 & 0 & 2 & 0 & 0.00 \\
\hline & CDR2H & 4 & 0 & 1 & 0 & 2 & 0 & 2 & 0 & 9 & 0 & 0.00 \\
\hline IGHV7\% & $\mathrm{CDR} 2 \mathrm{H}$ & 2 & 1 & 1 & 0 & 1 & 1 & 4 & 0 & 8 & 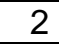 & 0.39 \\
\hline IGHV9U & CDR1H & 1 & 0 & 0 & 0 & 0 & 0 & 1 & 0 & 2 & 0 & 0.00 \\
\hline & CDR2H & 4 & $\underline{0}$ & 1 & 0 & 2 & 0 & 2 & 0 & 9 & 0 & 0.00 \\
\hline IGHV11\% & CDR2H & 0 & 0 & 0 & 0 & 0 & 0 & 2 & 8 & 2 & $\mathrm{c}$ & 0.00 \\
\hline IGHV124 & CDR2H & 0 & 0 & 0 & 0 & 0 & 0 & 2 & 0 & 2 & $\mathrm{c}$ & 0.00 \\
\hline IGHV134 & CDR1H & 18 & 0 & 11 & 0 & 9 & 0 & 19 & 0 & 57 & 0 & 0.00 \\
\hline & FR2H & 1 & 0 & 0 & 0 & 0 & 0 & 0 & 0 & 1 & 0 & 0.00 \\
\hline & CDR2H & 16 & 2 & 20 & 5 & 17 & 5 & 22 & 5 & 75 & 17 & 3.34 \\
\hline IGHV17\% & FR1H & 80 & 0 & 58 & 0 & 63 & 0 & 75 & 0 & 276 & 0 & 0.00 \\
\hline & CDR1H & 20 & $\underline{0}$ & 10 & $\underline{0}$ & 2 & 0 & 10 & 0 & 42 & 0 & 0.00 \\
\hline & FR2H & 8 & 4 & 4 & 1 & 4 & 0 & 6 & 2 & 22 & 7 & 1.38 \\
\hline & $\mathrm{CDR} 2 \mathrm{H}$ & 12 & 5 & 7 & 1 & 3 & 0 & 14 & 3 & 36 & 9 & 1.77 \\
\hline & FR3H & 9 & 5 & 10 & 1 & 9 & 0 & 8 & 1 & 36 & 7 & 1.38 \\
\hline IGHV15\% & CDR1H & 7 & 2 & 3 & 0 & 3 & 0 & 4 & 1 & 17 & 3 & 0.59 \\
\hline & FR2H & 0 & 0 & 0 & 0 & 0 & 0 & 0 & 0 & 0 & 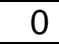 & 0.00 \\
\hline & CDR2H & 3 & 1 & 9 & 0 & 1 & 0 & 9 & 2 & 22 & 3 & 0.59 \\
\hline IGHV184 & CDR1H & 1 & 0 & 0 & 0 & 0 & 0 & 1 & 0 & 2 & c & 0.00 \\
\hline & CDR2H & 4 & 0 & 1 & 0 & 2 & 0 & 2 & 0 & 9 & 0 & 0.00 \\
\hline IGHV194 & FR1H & 80 & 0 & 58 & 0 & 63 & 0 & 75 & 0 & 276 & 0 & 0.00 \\
\hline & CDR1H & 25 & 0 & 13 & 0 & 27 & 0 & 20 & 0 & 85 & 0 & 0.00 \\
\hline & FR2H & 6 & 1 & 1 & 0 & 0 & 0 & 4 & 0 & 11 & 1 & 0.20 \\
\hline & CDR2H & 9 & $\underline{0}$ & 15 & 0 & 9 & 0 & 25 & 0 & 58 & 0 & 0.00 \\
\hline IGHV214 & CDR2H & 0 & 0 & 0 & 0 & 0 & 0 & 2 & 0 & 2 & 0 & 0.00 \\
\hline
\end{tabular}

${ }^{1}$ ambiguously assigned

2 unambiguously assigned 


\section{CDR3H length distribution}

In all four cattle breeds the program identified very short CDR3Hs (less or equal 10 aa, group 1), CDR3Hs of intermediate length (11-47 aa, group 2) as well as exceptionally long CDR3Hs (at least 48 aa, group 3) (Fig 3, Table 5). Very high significant differences were calculated for the number of sequences within the 3 groups of lengths $(\mathrm{P}<0.0001)$ when they were compared between all breeds but also within the breeds (GS, GBP, A, HF: P<0.0001). Fourty-four (7.37\%) sequences possessed a CDR3H length with 10 or less amino acid (aa) residues. The highest amount of sequences within this group were identified in breed A (12.57\%), followed by HF (7.55\%), GBP $(5.00 \%)$ and GS (3.05\%). In the breeds A, GS, and HF one and four sequences possessed only four amino acid residues within the CDR3H. Five amino acid residues were the shortest $\mathrm{CDR} 3 \mathrm{H}$ in sequences of GBP.

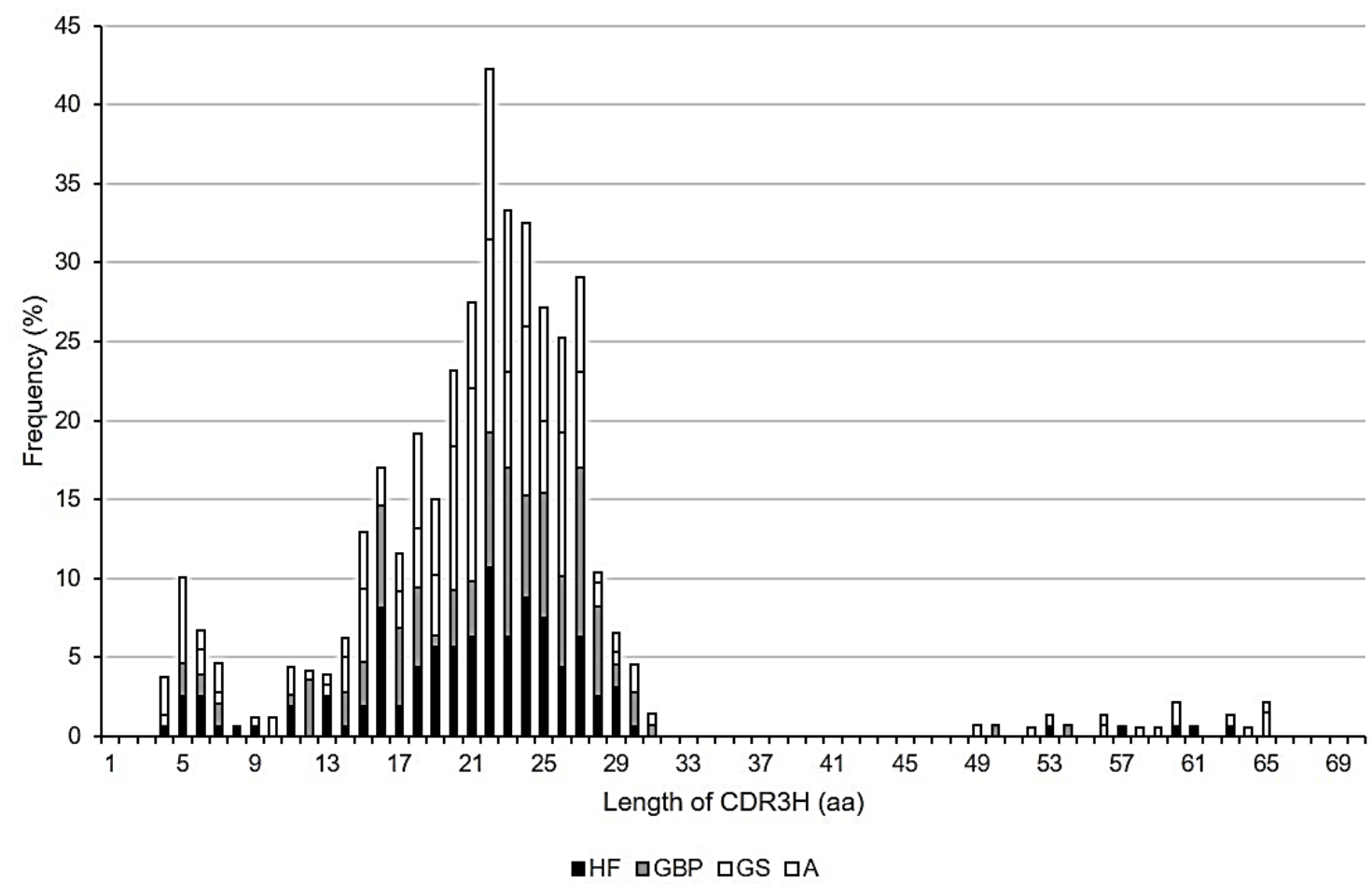

Fig 3. Length distribution of CDR3H in the four cattle breeds. The number of amino acid residues making up the $\mathrm{CDR} 3 \mathrm{H}$ lengths identified are shown on the horizontal axis, their relative usage frequencies are indicated on the vertical axis. Each breed is marked by the following color code: Aubrac: white, German Simmental: light grey, Holstein Friesian: black, German Black Pied: dark grey 
Chapter 3: Gene conversion and combinatorial diversity in bovine heavy chains

Table 5. Length distribution of the CDR3Hs.

\begin{tabular}{|c|c|c|c|c|c|}
\hline $\begin{array}{r}\text { Number of amino } \\
\text { acids within CDR3H }\end{array}$ & $\begin{array}{l}\text { All breeds } \\
(\% ; n=597)\end{array}$ & $\begin{array}{r}\text { GS } \\
(\% ; n=131)\end{array}$ & $\begin{array}{r}\text { GBP } \\
(\% ; n=140)\end{array}$ & $\begin{array}{r}\mathrm{HF} \\
(\% ; n=159)\end{array}$ & $\begin{array}{r}A \\
(\% ; n=167)\end{array}$ \\
\hline 4 & 1.01 & 0.76 & 0.00 & 0.63 & 2.40 \\
\hline 5 & 2.68 & 0.00 & 2.14 & 2.52 & 5.39 \\
\hline 6 & 1.68 & 1.53 & 1.43 & 2.52 & 1.20 \\
\hline 7 & 1.17 & 0.76 & 1.43 & 0.63 & 1.80 \\
\hline 8 & 0.17 & 0.00 & 0.00 & 0.63 & 0.00 \\
\hline 9 & 0.34 & 0.00 & 0.00 & 0.63 & 0.60 \\
\hline 10 & 0.34 & 0.00 & 0.00 & 0.00 & 1.20 \\
\hline 11 & 1.17 & 0.00 & 0.71 & 1.89 & 1.80 \\
\hline 12 & 1.01 & 0.00 & 3.57 & 0.00 & 0.60 \\
\hline 13 & 1.01 & 0.76 & 0.00 & 2.52 & 0.60 \\
\hline 14 & 1.51 & 2.29 & 2.14 & 0.63 & 1.20 \\
\hline 15 & 3.18 & 4.58 & 2.86 & 1.89 & 3.59 \\
\hline 16 & 4.36 & 0.00 & 6.43 & 8.18 & 2.40 \\
\hline 17 & 2.85 & 2.29 & 5.00 & 1.89 & 2.40 \\
\hline 18 & 4.86 & 3.82 & 5.00 & 4.40 & 5.99 \\
\hline 19 & 3.85 & 3.82 & 0.71 & 5.66 & 4.79 \\
\hline 20 & 5.70 & 9.16 & 3.57 & 5.66 & 4.79 \\
\hline 21 & 6.70 & 12.21 & 3.57 & 6.29 & 5.39 \\
\hline 22 & 10.55 & 12.21 & 8.57 & 10.69 & 10.78 \\
\hline 23 & 8.38 & 6.11 & 10.71 & 6.29 & 10.18 \\
\hline 24 & 8.04 & 10.69 & 6.43 & 8.81 & 6.59 \\
\hline 25 & 6.87 & 4.58 & 7.86 & 7.55 & 7.19 \\
\hline 26 & 6.20 & 9.16 & 5.71 & 4.40 & 5.99 \\
\hline 27 & 7.20 & 6.11 & 10.71 & 6.29 & 5.99 \\
\hline 28 & 2.51 & 1.53 & 5.71 & 2.52 & 0.60 \\
\hline 29 & 1.68 & 0.76 & 1.43 & 3.14 & 1.20 \\
\hline 30 & 1.17 & 0.00 & 2.14 & 0.63 & 1.80 \\
\hline 31 & 0.34 & 0.76 & 0.71 & 0.00 & 0.00 \\
\hline 49 & 0.17 & 0.76 & 0.00 & 0.00 & 0.00 \\
\hline 50 & 0.17 & 0.00 & 0.71 & 0.00 & 0.00 \\
\hline 52 & 0.17 & 0.00 & 0.00 & 0.00 & 0.60 \\
\hline 53 & 0.34 & 0.76 & 0.00 & 0.63 & 0.00 \\
\hline 54 & 0.17 & 0.00 & 0.71 & 0.00 & 0.00 \\
\hline 56 & 0.34 & 0.76 & 0.00 & 0.00 & 0.60 \\
\hline 57 & 0.17 & 0.00 & 0.00 & 0.63 & 0.00 \\
\hline 58 & 0.17 & 0.00 & 0.00 & 0.00 & 0.60 \\
\hline 59 & 0.17 & 0.00 & 0.00 & 0.00 & 0.60 \\
\hline 60 & 0.50 & 1.53 & 0.00 & 0.63 & 0.00 \\
\hline 61 & 0.17 & 0.00 & 0.00 & 0.63 & 0.00 \\
\hline 63 & 0.34 & 0.76 & 0.00 & 0.63 & 0.00 \\
\hline 64 & 0.17 & 0.00 & 0.00 & 0.00 & 0.60 \\
\hline 65 & 0.50 & 1.53 & 0.00 & 0.00 & 0.60 \\
\hline
\end{tabular}


Chapter 3: Gene conversion and combinatorial diversity in bovine heavy chains

Complementarity determining regions of group 2 were identified in 532 of all sequences analyzed (89.11\%). 93.57\% of GBP sequences were found to use 11 up to 47 aa. In HF, $89.31 \%$ of the sequences were attributed to this group as well as $83.83 \%$ of $A$ and $90.84 \%$ of $G S$ sequences. The most frequent $\mathrm{CDR} 3 \mathrm{H}$ length was 22 aa, which was found in $10.78 \%$ of $A$ sequences, in $8.57 \%$ of GBP, in $12.21 \%$ of GS, and in $10.69 \% \mathrm{HF}$ sequences. Simultaneously, this length was identified preferably in CDR3H of $A$ and HF. Nevertheless, in GS CDR3H with a length of 21 aa was identified as often as a length of 22 aa (12.21\%). In GBP, CDR3Hs with a length of 23 and 27 aa dominated (10.71\%).

CDR3Hs of group 3 were identified in 21 sequences of all four breeds $(9.22 \%)$. The breed GS showed the highest number of these sequences $(6.11 \%)$ followed by $A$ (3.59\%). German Black Pied and HF sequences possessed smaller proportions of the exceptionally long CDR3H with $3.14 \%$ and $1.43 \%$, respectively. Whereas in GS and $\mathrm{HF}$ sequences with 65 aa were the longest CDR3Hs (1.53\%, 0.6\%), 63 and 54 aa were counted in the longest $\mathrm{CDR} 3 \mathrm{Hs}$ of $\mathrm{A}(0.63 \%)$ and $\mathrm{GBP}(0.71 \%)$, respectively.

\section{Assigning IGHD to their germline origin using 3 different procedures}

We tested three different procedures to assign germline and sample IGHDs in order to improve the biological significance. At first, we applied the default values of MUSCLE, in procedure 2 we changed the penalties for gap opening $(=-4)$ and gap extention $(=-0.3)$, and in procedure 3 we additionally incorporated a modified scoring matrix (match +2$)$ to evaluate transversion and transition mutations.

In all three procedures, the assignment of germline and transcribed IGHD revealed clear results for the sequences analyzed. Nevertheless, using procedure 3 we obtained results matching short and long sample IGHD sequences best to germline short $\mathrm{CDR} 3 \mathrm{H}$ and exceptionally long $\mathrm{CDR} 3 \mathrm{H}$, respectively, whereas procedure 1 and 2 assigned a major number of group $3 \mathrm{CDR} 3 \mathrm{H}$ to germline IGHD of moderate length. Twenty-one different germline IGHD were transcribed, whereby IGHD8 located on BTA21, IGHD3 located on BTA7, IGHD5 as well as the very short IGHDQ52 located on BTA8; these were preferred in all breeds (Fig 4, Table 6). While IGHDQ52, IGHDS10 and 14 [22] were solely transcribed in sense orientation, germline IGHD1 to IGHD8 gene segments were transcribed in antisense direction in 38 sequences distributed over all four breeds investigated. IGHD4 (antisense (as), [21]) was identified the most often, followed by IGHD1 (as, [39]), IGHD3 (as, [39]), IGHD2 (as, [39]), IGHD5 (as, 
[21]), IGHD6 (as, [21]), IGHD8 (as, [21]) and IGHD7 (as, [21]). Very high significant differences were calculated for the IGHD usage within the cattle breeds $(\mathrm{P}<0.0001)$ but not between the breeds $(P=0.06)$.

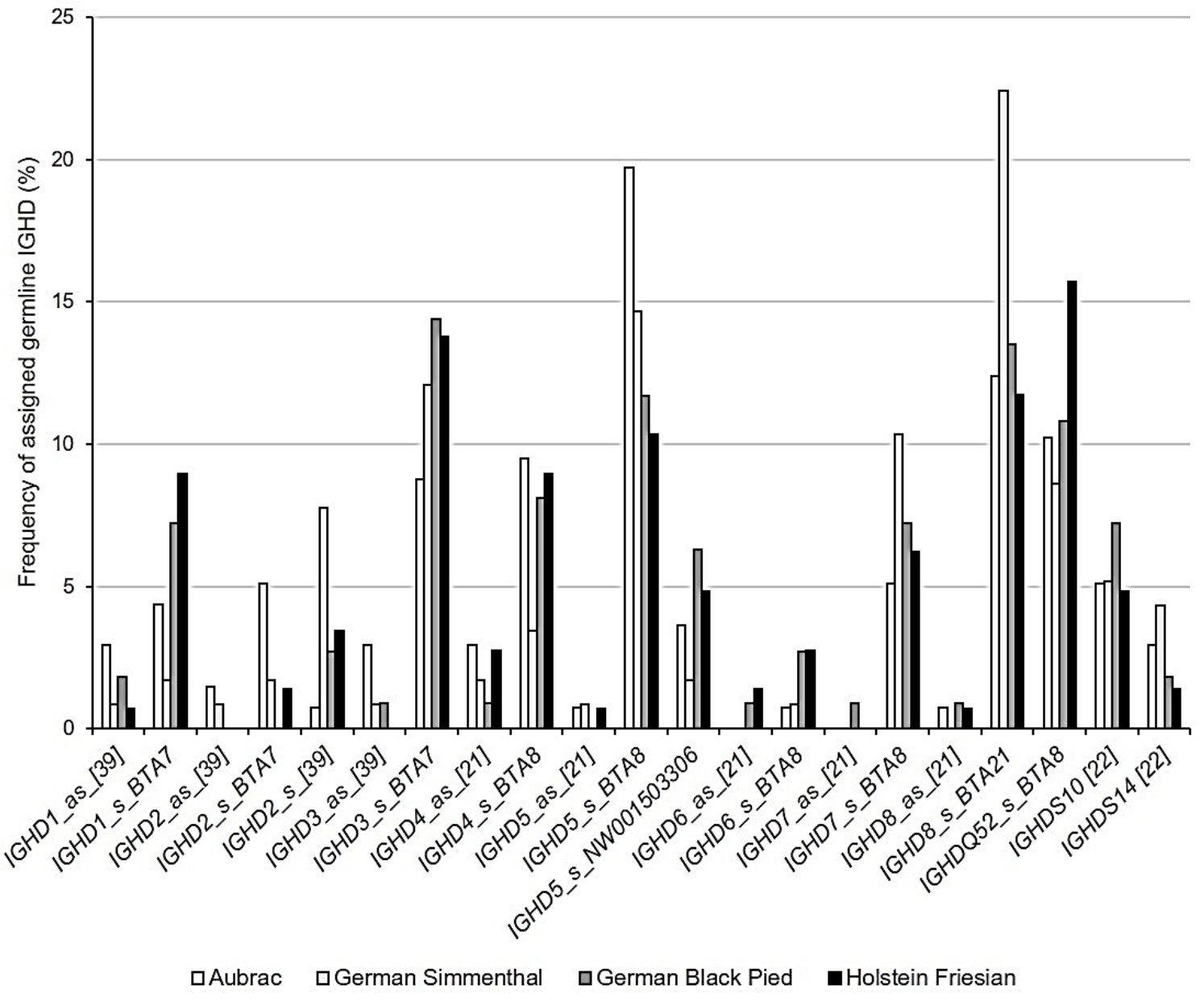

Fig 4. Transcription frequencies of IGHD in four cattle breeds using procedure 3. Transcribed IGHD are shown on the horizontal axis, their relative usage frequencies are indicated on the vertical axis. Calculation occurred after changing the default values for gap opening (-4) and gap extention (-0.3) and a modified scoring matrix (match +2$)$ of MUSCLE. Each breed is marked by the following color code: Aubrac: white, German Simmental: light grey, Holstein Friesian: black, German Black Pied: dark grey 
Chapter 3: Gene conversion and combinatorial diversity in bovine heavy chains

Table 6. Percentage of IGHD assigned to sample sequences of four cattle breeds.

\begin{tabular}{|l|r|r|r|r|}
\hline IGHD & $\begin{array}{r}\text { A } \\
(\% ; \mathbf{n = 1 3 7 )}\end{array}$ & $\begin{array}{r}\text { GS } \\
(\% ; \mathbf{n} 116)\end{array}$ & $\begin{array}{r}\text { GBP } \\
(\% ; \mathbf{n = 1 1 1})\end{array}$ & $\begin{array}{r}\text { HF } \\
(\%=145)\end{array}$ \\
\hline IGHD1_as ${ }^{1}[39]$ & 2.92 & 0.86 & 1.80 & 0.69 \\
\hline IGHD1_s_BTT7 & 4.38 & 1.72 & 7.21 & 8.97 \\
\hline IGHD2_as_[39] & 1.46 & 0.86 & 0.00 & 0.00 \\
\hline IGHD2_s_BTA7 & 5.11 & 1.72 & 0.00 & 1.38 \\
\hline IGHD2_s[39] & 0.73 & 7.76 & 2.70 & 3.45 \\
\hline IGHD3_as_[39] & 2.92 & 0.86 & 0.90 & 0.00 \\
\hline IGHD3_s_BTA7 & 8.76 & 12.07 & 14.41 & 13.79 \\
\hline IGHD4_as_[21] & 2.92 & 1.72 & 0.90 & 2.76 \\
\hline IGHD4_s_BTA8 & 9.49 & 3.45 & 8.11 & 8.97 \\
\hline IGHD5_as_[21] & 0.73 & 0.86 & 0.00 & 0.69 \\
\hline IGHD5_s_BTA8 & 19.71 & 14.66 & 11.71 & 10.34 \\
\hline IGHD5_s_NW001503306 & 3.65 & 1.72 & 6.31 & 4.83 \\
\hline IGHD6_as_[21] & 0.00 & 0.00 & 0.90 & 1.38 \\
\hline IGHD6_s_BTA8 & 0.73 & 0.86 & 2.70 & 2.76 \\
\hline IGHD7_as_21] & 0.00 & 0.00 & 0.90 & 0.00 \\
\hline IGHD7__BTA8 & 5.11 & 10.34 & 7.21 & 6.21 \\
\hline IGHD8_as_[21] & 0.73 & 0.00 & 0.90 & 0.69 \\
\hline IGHD8_s_BTA21 & 12.41 & 22.41 & 13.51 & 11.72 \\
\hline IGHDQ52_s_BTA8 & 10.22 & 8.62 & 10.81 & 15.71 \\
\hline IGHDS10_[22] & 5.11 & 5.17 & 7.21 & 4.83 \\
\hline IGHDS14_[22] & 2.92 & 4.31 & 1.80 & 1.38 \\
\hline
\end{tabular}

1 antisense

2 sense

In comparison, 20 different germline IGHD were transcribed using default conditions of MUSCLE for alignments (procedure 1), whereby IGHD8 being located on BTA21 was the preferred IGHD in all breeds. The very short IGHDQ52 located on BTA8 was transcribed in high frequencies in the breeds A, GBP, and HF (S1 Fig, S1 Table). While IGHDQ52, IGHDS10 and 14, and IGHD6 were solely transcribed in sense orientation, germline IGHD1 to IGHD8 gene segments were transcribed in antisense direction in 23 sequences that were distributed over all four breeds. IGHD4 (as, [21]) was identified the most often. Very high significant differences were calculated for the IGHD usage within the cattle breeds $(P<0.0001)$ but not between the breeds $(P=0.1630)$. 
After changing penalties for gap opening and gap extension (procedure 2), 17 different germline IGHD were transcribed, whereby IGHD8 located on BTA21, was the most observed IGHD in all breeds (S2 Fig, S2 Table). Germline IGHD1, IGHD4, IGHD6, and IGHD8 gene segments were transcribed in as direction in 24 sequences that were distributed over all four breeds. Again, IGHD4 (as, [21]) was identified the most often. Very high significant differences were calculated for the IGHD usage within the cattle breeds $(P<0.0001)$ but not between the breeds $(P=0.6654)$.

\section{Assigning the FR4H and IGHJ to their germline origin}

Located on BTA21, IGHJ1 and IGHJ6 [22] were identified as origin to the transcribed gene segments, which defines the $\mathrm{FR} 4 \mathrm{H}$ within the samples analyzed. IGHJ1 was transcribed preferably in the sequences investigated in all breeds (98.83\%). IGHJ6 [22] $(1.17 \%)$ was detected in only one sequence of each A, GS, and GBP $(0.73 \%, 0.85 \%$, $0.90 \%)$, as well as in three sequences of HF (2.05\%) animals. One sequence of GS and HF, respectively, showed IGHJI and IGHJ6 as a possible originating germline segment (Fig 5, Table 7). Statistical analysis revealed very high significant differences $(\mathrm{P}<0.0001)$ for the usage of IGHJ1 and IGHJ6 [22] within all cattle breeds. No significant differences were calculated between the four breeds.

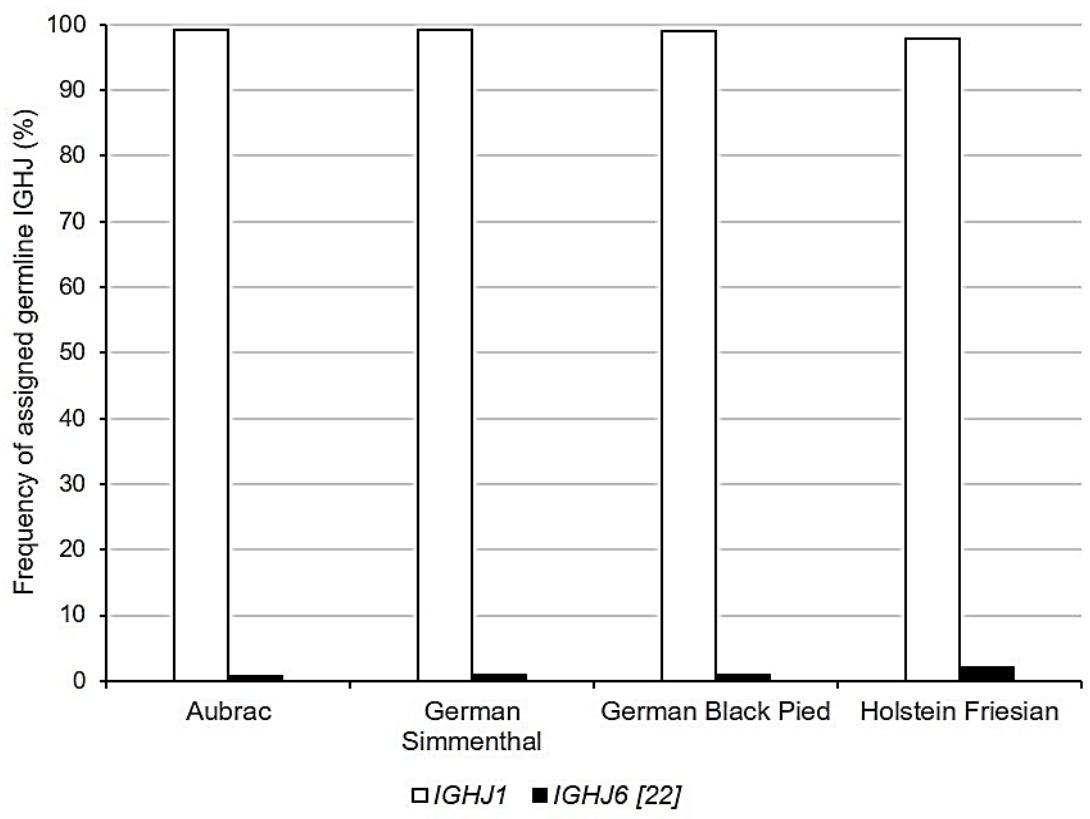

Fig 5. Transcription frequencies of IGHJ in four cattle breeds. Transcribed IGHJ are shown on the horizontal axis, their relative usage frequencies are indicated on the vertical axis. Each breed is marked by the following color code: Aubrac: white, German Simmental: light grey, Holstein Friesian: black, German Black Pied: dark grey 
Chapter 3: Gene conversion and combinatorial diversity in bovine heavy chains

Table 7. Percentage of IGHJ assigned to sample sequences of four cattle breeds.

\begin{tabular}{|l|r|r|r|r|}
\hline IGHJ & $\begin{array}{r}\text { A } \\
(\% ; \mathbf{n = 1 3 7 )}\end{array}$ & $\begin{array}{r}\text { GS } \\
(\% ; \mathbf{n = 1 1 7 )}\end{array}$ & $\begin{array}{r}\text { GBP } \\
(\% ; \mathbf{n = 1 1 1 )}\end{array}$ & $\begin{array}{r}\text { HF } \\
(\%=146)\end{array}$ \\
\hline IGHJ1 & 99.27 & 99.15 & 99.10 & 97.95 \\
\hline IGHJ6 [22] & 0.73 & 0.85 & 0.90 & 2.05 \\
\hline
\end{tabular}

\section{Recombination of IGHV, IGHD, and IGHJ in different cattle breeds using procedure 3}

Recombined IGHV, IGHD, and IGHJ were identified for each sequence and all possible frequencies were analyzed statistically within and between the breeds examined. In total, 597 recombinations were analyzed including double assigned germline origins for IGHV and IGHJ.

Applying procedure 3 for the IGHD assignment, 162 different combinations of IGHV, $I G H D$, and IGHJ were revealed (Fig 6, S3 Table). Most combinations occurred in less than ten sequences. Calculations revealed very high significant differences in usage frequencies of the rearranged gene segments between the breeds investigated $(P<0.0001)$. Eleven rearrangements were observed in ten to 27 sequences. They were observed in sequences of all four breeds. These major rearrangements were: IGHV36IGHD8 (sense (s), BTA21)-IGHJ1 (AY158087) (4.52\%), IGHV1S40-IGHD5 (s, BTA8)IGHJ1 (AY158087) (3.02\%), IGHV1S39-IGHD5 (s, BTA8)-IGHJ1 (AY158087) (2.85\%), IGHV1S40-IGHD3 (s, BTA7)-IGHJ1 (AY158087) (2.35\%), IGHV1S39-IGHD8 (s, BTA21)-IGHJ1 (AY158087) (2.18\%), IGHV3-IGHD5 (s, BTA8)-IGHJ1 (AY158087) (2.01\%), IGHV3-IGHDQ52 (s, BTA8)-IGHJ1 (AY158087) (1.84\%), IGHV36-IGHDQ52 (s, BTA8)-IGHJ1 (AY158087) (1.84\%), IGHV1S39-IGHD4 (s, BTA8)-IGHJ1 (AY158087) (1.68\%), IGHV1S39-IGHD7 (s, BTA8)-IGHJ1 (AY158087) (1.68\%), IGHV3-IGHD8 (s, BTA21)-IGHJ1 (AY158087) (1.68\%). Beside these preferred combinations, 52 minor recombinations were identified solely in one sequence distributed with 19 sequences in A, 14 in GS, six in GBP, and 13 in HF. Six variations of rearranged IGHJ6 were also identified one and two-times. These appear distributed over all four breeds: IGHV3-IGHD3 (s, BTA7)-IGHJ6 [22], IGHV1S28-IGHD4 (s, BTA8)-IGHJ6 [22], IGHV1S39-IGHD3 (s, BTA7)-IGHJ6 [22], IGHV1S40-IGHDS10IGHJ6 [22], IGHV3-IGHD8 (as, [21])-IGHJ6 [22], as well as IGHV10-IGHD5 (as, [21])IGHJ6 [22]. 


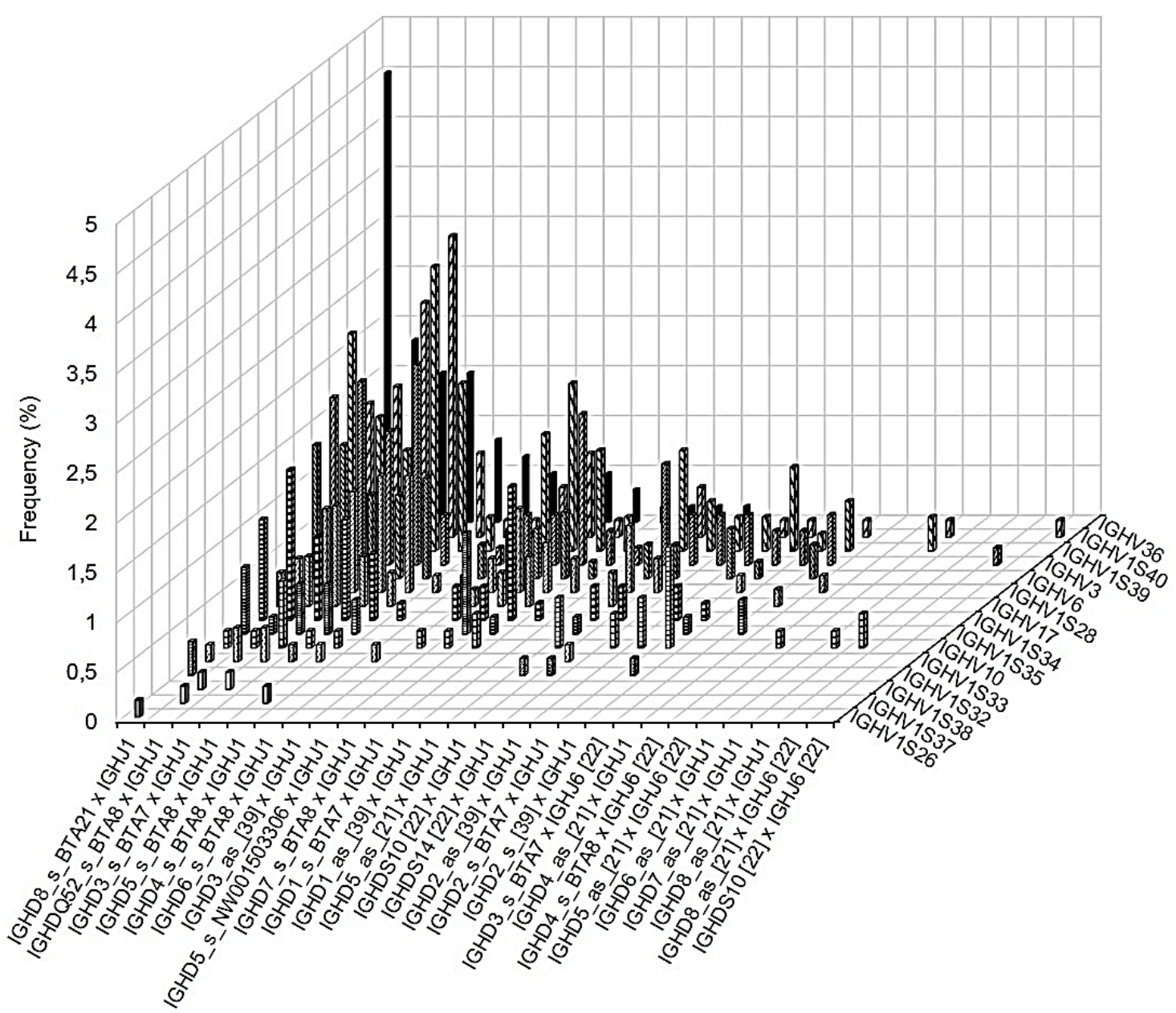

口IGHV1S26ㅁGHV1S37ㅁIGHV1S38日|GHV1S32IGHV1S33日|GHV10 目HV1S35日IGHV1S34

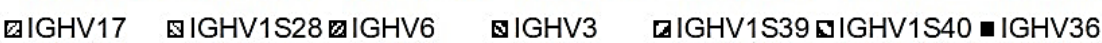

Fig 6. Recombinations of IGHV, IGHD, and IGHJ over all four cattle breeds. In the sequences of all four cattle breeds analyzed 162 different combinations of IGHV, IGHD, and IGHJ were identified. Relative frequencies (\%) of the combinations of the 21 transcribed IGHD and the 2 transcribed IGHJ are shown depending on the rearranged IGHV $(\mathrm{n}=15)$.

Fifteen out of the 162 IGHV-IGHD-IGHJ combinations were identified in all four cattle breeds investigated. In animals of A, 91 different rearrangements were found, whereas in GBP 74 different recombinations were observed. German Simmental revealed 72 combinations and HF showed 85 variations. Within the cattle breed A (167 rearrangements), the combinations IGHV1S39-IGHD5 (s, BTA8)-IGHJ1 (AY158087), IGHV1S39-IGHD8 (s, BTA21)-IGHJ1 (AY158087), and IGHV1S39-IGHD4 (s, BTA8)IGHJ1 (AY158087) were most frequently used in $7.78 \%, 4.79 \%$, and $3.59 \%$, 
Chapter 3: Gene conversion and combinatorial diversity in bovine heavy chains

respectively. Chi square calculations revealed high significant differences in usage frequencies of the recombinations in the breed $A(P=0.0004)$. In animals of HF (159 rearranged sample sequences), the dominant rearrangements were IGHV3-IGHD8 (s, BTA21)-IGHJ1 (AY158087) (4.40\%), IGHV3-IGHDQ52 (s, BTA8)-IGHJ1 (AY158087) (3.77\%), IGHV36-IGHDQ3 (s, BTA7)-IGHJ1 (AY158087) (3.77\%), IGHV3-IGHD5 (s, BTA8)-IGHJ1 (AY158087) (3.14\%), and IGHV3-IGHD1 (s, BTA7)-IGHJ1 (AY158087) (3.14\%). The Chi square test did not show significant usage differences ( $P=0.6689)$ of the different rearranged gene segments in this breed. Very high significant differences were calculated for the recombined IGHV-IGHD-IGHJ in the breeds GS $(P<0.0001)$, while in GBP no significant differences were found $(P=0.8870)$. In $G S$ animals rearranged IGHV36-IGHD8 (s, BTA21)-IGHJ1 (AY158087) (10.69\%), IGHV1S40IGHD3 (s, BTA7)-IGHJ1 (AY158087) (4.58\%), IGHV1S40-IGHD5 (s, BTA8)-IGHJ1 (AY158087) (4.58\%), IGHV1S34-IGHD8 (s, BTA21)-IGHJ1 (AY158087) (3.82\%), and IGHV1S35-IGHD7 (s, BTA8)-IGHJ1 (AY158087) (3.82\%) were observed in at least five sequences (131 rearranged samples). Four rearrangements were preferred in GBP (140 rearranged sequences): IGHV36-IGHD8 (s, BTA21)-IGHJ1 (AY158087) (5.00\%), IGHV1S40-IGHD5 (s, BTA8)-IGHJ1 (AY158087) (3.57\%), and IGHV36-IGHDQ52 (s, BTA8)-IGHJ1 (AY158087) (3.57\%), and IGHV1S34-IGHD1 (s, BTA7)-IGHJ1 (AY158087) (3.57\%).

\section{Recombination of IGHV, IGHD, and IGHJ in different cattle breeds using procedure 1}

With the alignment conditions applied in procedure 1 and 2, different predominantly used recombinations were observed as expected due to the different identified IGHDs. Using the default values of MUSCLE (procedure 1), 147 different combinations of $I G H V$, IGHD, and IGHJ were found (S3 Fig, S4 Table). Most combinations occurred in less than ten sequences. We found very high significant differences in the usage frequencies of the rearranged gene segments between the breeds investigated $(P<0.0001)$. We also observed rearrangements observed in a quantity of ten to 21 sequences that also occurred in sequences spanning of all four breeds. The major rearrangement was IGHV36-IGHD8 (s, BTA21)-IGHJ1 (AY158087) (3.52\%). Beside this preferred combination, 49 were identified solely in one sequence, whereby 12 were identified in A, 15 in GS, nine in GBP, and 13 in HF. We also identified seven variations rearranging IGHJ6 over all animals. 
Twenty-one out of the 147 IGHV-IGHD-IGHJ combinations were identified in all four cattle breeds investigated. In animals of $A, 81$ different rearrangements were found, whereas in GBP 74 different recombinations were observed. German Simmental had 74 combinations and HF had 80 variations. Within the cattle breed A (167 rearranged sample sequences), the combination IGHV1S39-IGHD3 (s. BTA7)-IGHJ1 (AY158087) was the most frequently used in $5.99 \%$. Chi square calculations revealed significant differences in usage frequencies of the recombinations in the breed $A(P=0.0108)$. In animals of HF (159 rearranged sample sequences), the dominant rearrangement was IGHV1S40-IGHD5 (s, BTA8)-IGHJ1 (AY158087) (4.40\%). The Chi square test did not show significant usage differences $(P=0.0546)$ for the different rearranged gene segments in this breed. High significant differences were seen for the recombined IGHV-IGHD-IGHJ in the breeds GS ( $\mathrm{P}=0.0002)$, while in GBP no significant difference was found ( $P=0.9585)$. In GS animals rearranged IGHV36-IGHD8 (s, BTA21)-IGHJ1 (AY158087) (9.16\%) was observed in at least six sequences (131 rearranged samples). Four rearrangements were preferred in GBP (140 rearranged sequences): IGHV36-IGHD8 (s, BTA21)-IGHJ1 (AY158087) (3.57\%), IGHV1S40-IGHD8 (s, BTA8)IGHJ1 (AY158087) (3.57\%), IGHV1S34-IGHD5 (s, BTA8)-IGHJ1 (AY158087) (3.57\%), and IGHV36-IGHD5 (s, NW_001503306)-IGHJ1 (AY158087) (3.57\%).

\section{Recombination of IGHV, IGHD, and IGHJ in different cattle breeds using procedure 2}

When applying procedure 2 with changed values for gap opening and gap extension, we identified 119 different combinations of IGHV, IGHD, and IGHJ (S4 Fig, S5 Table) were identified. As seen for the other procedures, most combinations occurred in less than ten sequences. Calculations revealed very high significant differences in usage frequencies of the rearranged gene segments between the breeds investigated $(P<0.0001)$. Fifteen different rearrangements were observed in a quantity of ten to 42 sequences and were observed across all four breeds. The major rearrangement was IGHV1S39-IGHDQ52 (s, BTA8)-IGHJ1 (AY158087) (7.04\%). Beside the dominant combinations, 43 were identified solely in one sequence, whereby six were identified in A, 13 in GS, 12 in GBP, and 12 in HF. Again, seven variations rearranging IGHJ6 were identified.

Fifteen out of the 119 IGHV-IGHD-IGHJ combinations were identified in all four cattle breeds investigated. For A we found 58 different rearrangements, whereas in GBP 65 
Chapter 3: Gene conversion and combinatorial diversity in bovine heavy chains

different recombinations were observed. German Simmental had 57 combinations and HF had 62 variations. Within A (167 rearrangements), the combination IGHV1S39IGHDQ52 (s, BTA8)-IGHJ1 (AY158087) was the most frequently used and was seen in $11.38 \%$ of the time. Chi square calculations showed significant differences in the usage frequencies of the recombinations for breed $A(P=0.0108)$. In animals of HF (159 rearranged sample sequences), the dominant rearrangement was IGHV1S39IGHDQ52 (s, BTA8)-IGHJ1 (AY158087) (8.81\%). The Chi square test did not show significant usage differences $(\mathrm{P}=0.0546)$ for the different rearranged gene segments in this breed. High significant differences were seen for the recombined IGHV-IGHD-IGHJ in the breed GS ( $P=0.0002)$, while in GBP no significant differences were observed $(\mathrm{P}=0.9585)$. In $\mathrm{GS}$ animals rearranged IGHV36-IGHDQ52 (s, BTA8)-IGHJ1 (AY158087) (11.45\%) was observed predominantly (131 rearranged sequences). The rearrangement IGHV1S40-IGHDQ52 (s, BTA8)-IGHJ1 (AY158087) (9.29\%) was preferred in GBP (140 rearranged sequences).

\section{Recombination of IGHV, IGHD, and IGHJ with different length of CDR3H}

Within the three groups of length of $\mathrm{CDR} 3 \mathrm{H}$ we identified different preferably expressed recombinations of $I G H V$, IGHD, and $I G H J$. Very high significant differences regarding identified rearrangements within these groups were calculated (procedure 1: among the breeds, GS, $A, H F: P<0.0001$; GBP: $P=0.0016$; procedure 2: among the breeds, GS, A, HF, GBP: $\mathrm{P}<0.0001$; procedure 3: among the breeds, A, GBP: $P<0.0001$; GS: $P=0.0003$; HF: $P=0.0085)$.

Using changed penalties for gap opening and gap extension and a new scoring matrix (procedure 3) rearrangements of IGHV3 and IGHJ1 (AY158087) together with IGHD1 (s, BTA7), IGHD2 (as, [39]), IGHDQ52 (s, BTA8), and IGHDS10 [22] dominated (0.5$0.84 \%$ ) in sequences with group $1 \mathrm{CDR} 3 \mathrm{H}$ if breed was not taken into account. The single breeds showed different major recombinations. In A, IGHV6-IGHD2 (s, BTA7)IGHJ1 was calculated for $1.8 \%$ of the sequences, whereas in GS IGHV3-IGHDQ52 (s, BTA8)-IGHJ1 was identified in the same frequencies as IGHV3-IGHD2 (as, [39])IGHJ1, IGHV3-IGHD1 (as, [39])-IGHJ1, and IGHV1S35-IGHD8 (s, BTA21)-IGHJ1 (0.76\%), in GBP IGHV3-IGHDS10 [22]-IGHJ1, IGHV3-IGHDS14 [22]-IGHJ1, IGHV3IGHD3 (s, BTA7)-IGHJ1, IGHV3-IGHD1 (s, BTA7)-IGHJ1, IGHV1S34-IGHDS10 [22]IGHJ1, IGHV1S34-IGHDS14 [22]-IGHJ1 and IGHV6-IGHDS10 [22]-IGHJ1 made up 
Chapter 3: Gene conversion and combinatorial diversity in bovine heavy chains

$0.71 \%$, and in HF IGHV3-IGHDQ52 (s, BTA8)-IGHJ1 was found the most often $(2.52 \%)$.

In samples of all breeds possessing CDR3Hs of group 2 the rearrangement of IGHV36-IGHD8 (s, BTA21)-IGHJ1 was found most often (4.52\%). This is congruent with our findings from the cattle breeds GS and GBP (10.69\%, 5.00\%). In A sequences with rearrangement of IGHV1S39-IGHD5 (s, BTA8)-IGHJ1 were used with the highest frequency (7.19\%) whereas in HF IGHV3-IGHD8 (s, BTA21)-IGHJ1 and IGHV36IGHD3 (s, BTA7)-IGHJ1 dominated (3.77\%).

The recombination of IGHV10-IGHD2 (s, [39])-IGHJ1 was identified in $0.84 \%$ of all sequences with group $3 \mathrm{CDR} 3 \mathrm{Hs}$. This combination was also dominant in sequences possessing an exceptionally long $\mathrm{CDR} 3 \mathrm{H}$ in $\mathrm{HF}$ (1.26\%). In $\mathrm{A}$, the combination IGHV10-IGHD2 (s, BTA7)-IGHJ1 was preferred (1.2\%), whereas in GS, IGHV10IGHDS10 [22]-IGHJ1 was found the most often (2.29\%). In GBP IGHV10-IGHD8 (as, [21])-IGHJ1 and IGHV10-IGHD7 (as, [21])-IGHJ1 were identified (0.71\%).

If only new values for gap opening and gap extension were applied (procedure 2), rearrangements of IGHV3 and IGHJ1 (AY158087) together with IGHD1 (as, [39]) dominated (1.01\%) in sequences with a very short CDR3H if the breed was not taken into account. The single breeds showed different major recombinations. In A, IGHV6IGHD1 (s, BTA7)-IGHJ1 was calculated for $2.4 \%$ of the sequences, whereas in GS IGHV3-IGHDQ52 (s, BTA8)-IGHJ1 was identified in the same frequencies as IGHV3IGHD4 (as, [21])-IGHJ1, IGHV3-IGHD1 (s, BTA7)-IGHJ1, and IGHV1S35-IGHD1 (s, BTA7)-IGHJ1 (0.76\%), in GBP IGHV3-IGHD1 (as, [39])-IGHJ1 made up $1.43 \%$, whereas in HF IGHV3-IGHD1 (as, [39])-IGHJ1 and IGHV3-IGHD4 (s, BTA8)IGHJ1were found the most often (1.26\%).

In samples of all breeds possessing group 2 CDR3Hs, the rearrangement of IGHV1S39-IGHDQ52 (s, BTA8)-IGHJ1 was found the most often (7.04\%). This is congruent with the findings in the cattle breeds A and HF (11.38\%, 8.81\%). In GS sequences showing the rearrangement IGHV36-IGHDQ52 (s, BTA8)-IGHJ1 were used with the highest frequency (11.45\%) whereas in GBP IGHV1S40-IGHDQ52 (s, BTA8)IGHJ1 dominated (9.29\%).

The recombination of IGHV10-IGHD8 (s, BTA21)-IGHJ1 was identified in $1.17 \%$ of all sequences with group $3 \mathrm{CDR} 3 \mathrm{Hs}$. This combination was also dominant in sequences possessing exceptionally long CDR3H in A (2.4\%) and GBP (0.71\%). In GBP IGHV10- 
Chapter 3: Gene conversion and combinatorial diversity in bovine heavy chains

IGHDS10-IGHJ1 was also found at this frequency. In GS, the combinations IGHV10IGHD1 (s, BTA7)-IGHJ1 and IGHV10-IGHD4 (s, BTA8)-IGHJ1 were preferred (2.29\%), whereas in HF, IGHV10-IGHD3 (s, BTA7)-IGHJ1 was found most often (1.26\%).

Using default values (procedure 1) rearrangements of IGHV3 and IGHJ1 (AY158087) together with IGHDQ52 (s, BTA8) as well as IGHV6-IGHD1 (s, BTA7) dominated (1.51 and $0.5 \%$ ) in sequences with a short $\mathrm{CDR} 3 \mathrm{H}$ if the breed was not taken into account. But again the single breeds showed different major recombinations. In A, IGHV3IGHDQ52 (s, BTA8)-IGHJ1 and IGHV6-IGHD1 (s, BTA7)-IGHJ1 were found for $1.8 \%$ of the sequences, whereas in GS IGHV1S35-IGHD4 (s, BTA8)-IGHJ1 was identified at the same frequency as IGHV3-IGHD2 (s, BTA8)-IGHJ1, IGHV3-IGHD1 (as, [39])IGHJ1, and IGHV3-IGHD8 (as, [21])-IGHJ1 (0.76\%), in GBP IGHV3-IGHD1 (s, BTA7)IGHJ1, IGHV3-IGHD2 (as, [39])-IGHJ1, IGHV3-IGHD5 (s, BTA8)-IGHJ1, IGHV3IGHDQ52 (s, BTA8)-IGHJ1, IGHV1S34-IGHD5 (s, BTA8)-IGHJ1, IGHV1S34-IGHD5 (s, NW_001503306.)-IGHJ1 and IGHV6-IGHD5 (s, BTA8)-IGHJ1 made up 0.71\%, and in HF IGHV3-IGHDQ52 (s, BTA8)-IGHJ1 was found most often (3.14\%).

In samples of all breeds possessing CDR3Hs of group 2 the rearrangement of IGHV36-IGHD8 (s, BTA21)-IGHJ1 was found most often (3.52\%). This is congruent with the findings in the cattle breed GS (9.16\%). In HF sequences showing the rearrangements IGHV1S39-IGHD8 (s, BTA21)-IGHJ1 and IGHV1S40-IGHD5 (s, BTA8)-IGHJ1 were used with the highest frequency (4.4\%) whereas in A IGHV1S39IGHD3 (s, BTA7)-IGHJ1 dominated (5.99\%) as well as IGHV1S40-IGHD8 (s, BTA21)IGHJ1, IGHV36-IGHD5 (s, NW_001503306)-IGHJ1, and IGHV36-IGHD8 (s, BTA21)IGHJ1 in the cattle breed GBP (3.57\%).

The recombination of IGHV10-IGHD8 (s, BTA21)-IGHJ1 was identified in $1.01 \%$ of all sequences with exceptionally long CDR3Hs. In sequences possessing those group 3 CDR3H in HF the combinations IGHV10-IGHD4 (s, BTA8)-IGHJ1 and IGHV10-IGHD8 (s, BTA21)-IGHJ1 (1.26\%) dominated. In A, the combination IGHV10-IGHD4 (s, BTA8)-IGHJ1 was preferred (1.2\%), whereas in GS, IGHV10-IGHD2 (s, BTA7)-IGHJ1 and IGHV10-IGHD8 (s, BTA21)-IGHJ1 were found most often (1.53\%), and in GBP only the two recombinations IGHV10-IGHD8 (s, BTA21)-IGHJ1 and IGHV10-IGHD4 (as, [21])-IGHJ1 were identified (0.71\%). 
Chapter 3: Gene conversion and combinatorial diversity in bovine heavy chains

\section{Variability based on amino acid substitutions}

We then counted the amino acid substitutions at each position to calculate variability as described by Wu and Kabat [40]. The results are shown in the variability plots for each breed separately in Figs 8 a-d. The amino acid positions were numbered in accordance to the IMGT numbering systems [41]. Therefore, FRHs and CDRHs are defined by the following amino acid positions: FR1H: 1...26, CDR1H: 27...38, FR2H: 39...55, CDR2H: 56...65, FR3H: 66...104, CDR3H: 105..117, FR4H: 118...128. In cattle there are no amino acids assigned to positions 10, 31-34, 60-62, and 73.

(A)

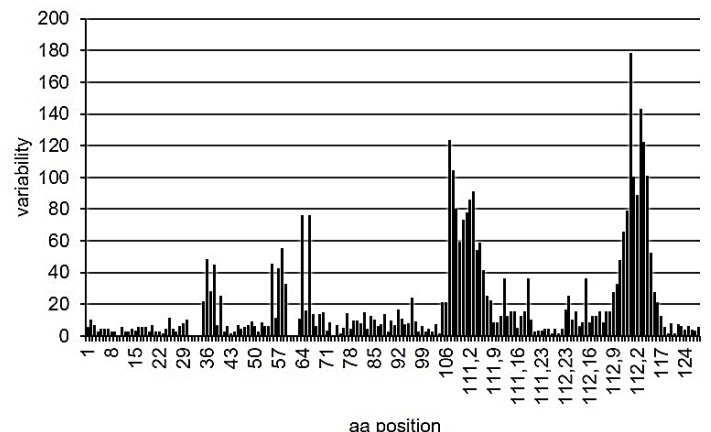

(C)

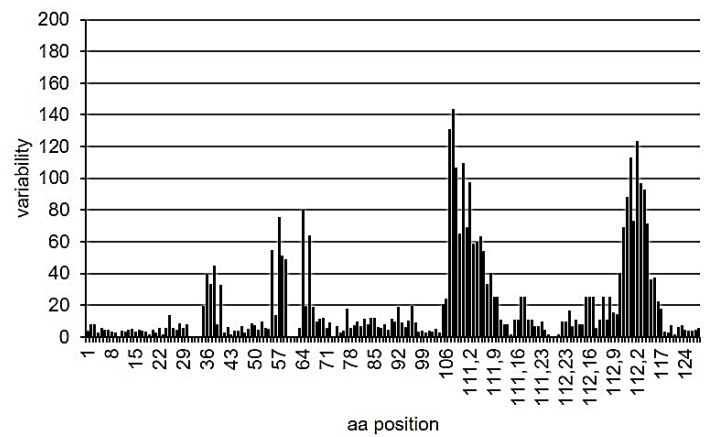

(B)

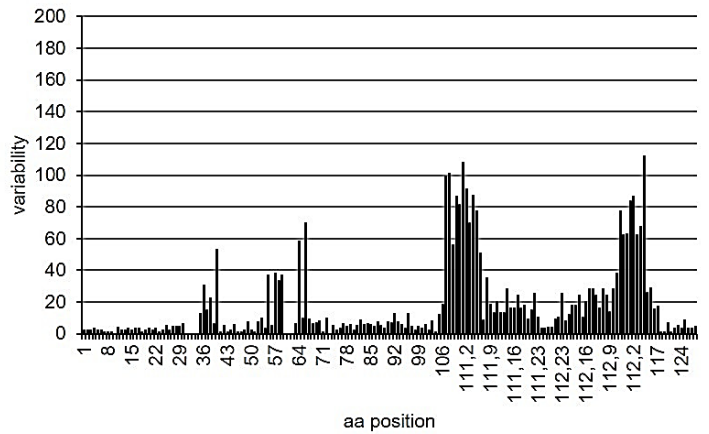

(D)

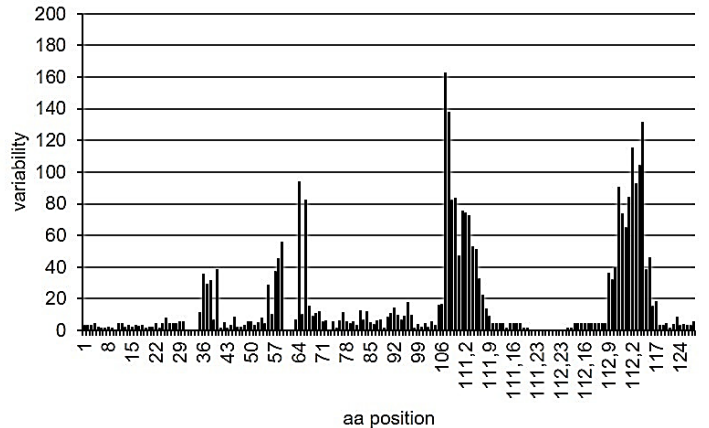

aa position

Fig 7. Variability plots of the heavy chain variable regions in the cattle breeds Aubrac, German Simmental, Holstein Friesian, and German Black Pied. The complete variable region is displayed on the horizontal axis. Positions are numbered in accordance to the IMGT numbering system. Within the $\mathrm{FR} 1-4 \mathrm{H}$, little variability is discernable whereas in CDR1-3H increase in variability is observed. (A) Aubrac, (B) German Simmental, (C) Holstein Friesian, (D) German Black Pied 
Within the FR1-4H little variability is discernable whereas in CDR1-3H an increase in variability is observed as expected. A maximum of variability in $\mathrm{FRHs}$ is calculated for position 96 in $\mathrm{FR} 3 \mathrm{H}$. Variability at this position is 23.7 in A, in HF 19.0, whereas in GS and GBP variability is 12.7 and 17.5, respectively. In CDRHs, variability increases from CDR1H to $\mathrm{CDR3H}$ in all breeds. In CDR1H breed A showed the highest variability. In CDR2H variability varies between 58 in GS and 93 in GBP. GBP also showed highest variability in $\mathrm{CDR} 3 \mathrm{H}$. The lowest variability within $\mathrm{CDR} 3 \mathrm{H}$ was found in GS. Both HF and $A$ showed moderate variability of $\mathrm{CDR} 2 \mathrm{H}$ and $\mathrm{CDR} 3 \mathrm{H}$ in comparison to GBP and GS. Overall, variability was much higher at the transition areas between FRH and CDR than in the middle of the CDRHs. 
Chapter 3: Gene conversion and combinatorial diversity in bovine heavy chains

\section{Discussion}

This study makes a substantial contribution to the analysis and understanding of the development of the transcribed bovine immunoglobulin repertoire. We examined possible gene conversions within the variable region of bovine heavy chains. We investigated the dominantly transcribed IGHV, IGHD, and IGHJ gene segments and their combinatorial diversity using a newly developed bioinformatics framework, which considers the unique specificity of exceptionally long CDR3 group of bovine immunoglobulin heavy chains. During the development of the program, we applied different conditions (procedure 1 to 3 ) to improve the alignments of the single gene segments. The progress of assigning germline IGHV, IGHD, and IGHJ to sample sequences is also shown in this study. Unlike previous studies, we complemented our investigation with the analysis of breed specific differences in the four different cattle breeds Aubrac, German Simmental, German Black Pied, and Holstein Friesian.

Due to the limited germline sequence divergence recent studies on bovine immunoglobulin genetics focused on antibody diversification strategies and the junctional diversity of the antibody repertoire. Specific diversification strategies were identified such as the generation of exceptionally long $\mathrm{CDR} 3 \mathrm{H}[17,42,43]$, the insertion of conserved short nucleotide sequences (CSNS) at the IGHV-IGHD junction [24], the use of pseudogene fragments in lambda light chains as well as gene conversions [14], and somatic hypermutations independent of exposure to external antigens during B-cell development [44]. In addition, more germline gene segments were determined over the last few years $[17,18,22]$. Since the current bovine genome assembly is still incomplete, the full germline repertoire remains under active investigation.

Previous analyses of the bovine immunoglobulin repertoire aimed at identifying rearranged germline gene segments applied various software tools for sequence alignments. As these tools are of limited use for detailed analysis of rearranged immunoglobulin genes due to the difficult and error prone manual assembly of different genes, specialized software tools have been developed. The most familiar ones are IMGT/Junction Analysis [26], IMGT/V-QUEST [27, 28], IMTG/HIGHV-QUEST [29], VBASE2 [30], JoinSolver [31], iHMMun-align [32], and IgBLAST [33]. Only IgBLAST enables the analysis of both nucleotide and protein sequences for FR/CDR and allows the user to either apply the numbering system of Kabat or the IMGT system [41, 45]. Matching germline IGHV, IGHD, and IGHJ genes as well as details at rearrangement 
Chapter 3: Gene conversion and combinatorial diversity in bovine heavy chains

junctions may be analyzed. Searches against germline gene databases and other databases are possible [33]. Tools other than IgBLAST do not provide simultaneous database searches or the analysis of protein sequences. All of these immunoglobulin sequence analysis tools support organisms such as human, mouse, rat, rabbit and rhesus monkey, but representation of livestock is missing or incomplete. We did not apply these analysis tools, as they do not consider the bovine specific occurrence of exceptionally long $\mathrm{CDR} 3 \mathrm{H}$. Therefore, we developed a new bioinformatics framework to address this specific case.

In contrast to the analysis tools mentioned above, our program not only searches our updated bovine specific immunoglobulin germline gene database but also is able to load other pre-designed databases. Matches are identified on the basis of nucleotides as it is the case for all other tools. For the delineation of FR and CDR, we apply the IMGT nomenclature that is currently recommended and most widely used. In addition, we focused on the adjustment of search parameters for IGHV and IGHJ and especially for the identification of $I G H D$.

The analysis of Ig heavy chain variable regions in four cattle breeds revealed the usage of 15 different IGHV segments, 21 IGHD segments, as well as two IGHJ segments. IGHV1S39 was used most frequently followed by IGHV3/33. Rarely used IGHV segments were IGHV1S26, IGHV1S32, IGHV1S33, IGHV1S37, and IGHV1S38. In bovine fetal bone marrow, ileum, and spleen high frequencies of IGHV3/33 (=IGHV1S3) and IGHV1S39 was observed as well as low frequencies of IGHV1S38 and IGHV1S26 [22]. The transcription of IGHV1S32 and S37 has not yet been described. Among the 20,17, and 21 transcribed IGHD (regarding procedures 1-3), IGHDS8, IGHDS5, IGHDS10, and IGHDQ52 (=IGHDS9) were preferred in all breeds. IGHDS1 to IGHDS8 were also found to be transcribed in antisense direction in the third calculation procedure but in low numbers. Using the first procedure, IGHDS6 was not identified in antisense direction, and applying the second procedure IGHDS1, IGHDS4, IGHDS6, and IGHDS8 were shown to be transcribed in antisense orientation. Previous studies also elucidated the transcription of 14 IGHD, where the occurrence of IGHDS5 was the most frequent one and was present in $42 \%$ of the sequences analyzed in bovine fetus [22]. The assignment of the FR4H to germline IGHJ revealed the transcription of IGHJ1, and IGHJ6 [22] with IGHJ1 clearly preferred. In the cattle breed A, procedure 3 confirmed IGHV1S39-IGHD5-IGHJ1 as the most common recombination of gene segments which is identical to the most frequent finding in 
Chapter 3: Gene conversion and combinatorial diversity in bovine heavy chains

bovine fetus [22]. This recombination belongs to immunoglobulins possessing a $\mathrm{CDR} 3 \mathrm{H}$ region of intermediate length. Statistical analyses showed significant different transcription levels of $I G H V$, IGHD, and IGHJ segments within the breeds.

The usage of pseudogene segments has already been described for animals such as chicken [4, 37]. In bovine lambda light chains, fragments of pseudogenes were also shown to contribute to immunoglobulin diversity in a gene conversion process [14]. In the current analysis, possible gene conversion events were identified by the assignments of parental germline IGHV to separate FR1-3H and CDR1-3H. In addition to the IGHV identified for the complete variable region based exclusively on $\mathrm{FR} 1-3 \mathrm{H}$, several pseudogenes were assigned as possible originating germline IGHV in the

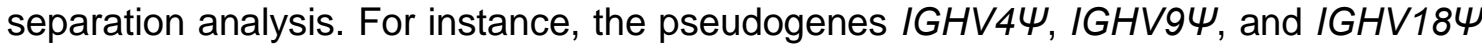
belong to the boVH2 family [17], but seem to contribute to gene conversion events by nucleotide substitutions. In particular, IGHV4 $\Psi$, which was mentioned in the example above meets the criteria for gene conversion such as the location upstream of the rearranged segment [4, 13] and clusters of nucleotide changes [14]. Further, the flanking homology of the conversion region supports the genetic exchange [13] and the separation from IGHV6 by more than $18 \mathrm{~kb}$ on the genome allows looping during rearrangement [17]. In comparison, in chicken the nearest pseudogene is separated by $7 \mathrm{~kb}$ [4]. It should be noted that it is difficult to consider the order of gene segments to evaluate the plausibility of other gene conversions due to the incomplete annotation of the bovine genome $[17,18]$. Finally, our data indicate an exchange between the two bovine $\mathrm{VH}$ families which obviously is rare and which might be an influence of breed or method of analysis when compared to previous results [18].

The length distribution of $\mathrm{CDR} 3 \mathrm{H}$ consists of short CDR3H (group 1), intermediate length $\mathrm{CDR} 3 \mathrm{H}$ (group 2), and exceptionally long CDR3H (group 3) in all four cattle breeds. In the breed GS the highest percentage of group $3 \mathrm{CDR} 3 \mathrm{H}$ was calculated. The longest CDR3H with 65 aa were found in GS and HF sequences. The longest ever detected CDR3H in cattle was 67 aa long using IMGT numbering [17]. In contrast, four amino acids made up the shortest CDR3H in A animals, GS animals, and HF animals. The maximum length of group $2 \mathrm{CDR} 3 \mathrm{H}$ was 22 aa.

The combinatorial diversity of germline IGHV, IGHD, and IGHJ-segments is represented by 162 different rearrangements that were expressed with significant differences (procedure 3). In comparison, 147 and 119 different recombinations of IGHV-IGHD-IGHJ were identified using calculation procedures 1 and 2, respectively. In 
Chapter 3: Gene conversion and combinatorial diversity in bovine heavy chains

the four breeds, different rearrangements were found. In detail, 91 different combinations occurred in A (procedure 1: 81, and 2: 58), 74 in animals of GBP (procedure 1: 74, and 2: 65), 72 in GS animals (procedure 1: 74, and 2: 57), and 85 in the breed HF (procedure 1: 80, and 2: 62). Most of these combinations were observed in less than ten sequences but seven occurred in up to 21 sequences in all four cattle breeds examined (procedure 1: up to 21 , and 2: up to 42 ).

In sequences belonging to group $1 \mathrm{CDR} 3 \mathrm{H}$, combinations of IGHV3 and IGHJ1 (AY158087) together with IGHDQ52 (s, BTA8) dominated over all breeds using procedure 3. As IGHDQ52 is the shortest IGHD segment possessing only four amino acids, these results explain best the origin of short CDR3H. Group $3 \mathrm{CDR} 3 \mathrm{H}$ mostly exhibited IGHV10-IGHD2 (s, [39])-IGHJ1 (procedure 3), or IGHV10-IGHD8 (s, BTA21)IGHJ1 (procedure 1 and 2) in all breeds. Only results from procedure 3 identified biological meaningful combinations of germline IGHV, IGHD, and IGHJ as it gave the best explanation for the origin of group $3 \mathrm{CDR} 3 \mathrm{H}$. IGHD2 is the longest IGHD segment identified so far. Further, IGHV10, which is identical to IGHV1S1 and IGHV1S15, was found to contribute solely to those exceptional lengths $[17,18,22]$. It is assumed, that the "ThrThrValHisGIn" terminal motif of IGHV10, that initiates an ascending $\beta$ strand in the folded antibody enables the formation of the "stalk and knob" structure in addition to inserted conserved short nucleotide sequences (CSNS) [22, 43]. Furthermore, in sequences of group 2 a higher number of recombinations were observed than in sequences of group 1 and 3 . As group $3 \mathrm{CDR} 3 \mathrm{H}$ regions are unique in cattle, the few preferably rearranged gene segments within this group may indicate specialized antibodies.

Variability plots indicated quite similar features within the variable region in all breeds. Nevertheless, amino acid residues at each position vary between the samples and the breeds and within the regions. In FR1-4H little variability was calculated, whereas the variability increased from $\mathrm{CDR} 1 \mathrm{H}$ to $\mathrm{CDR} 3 \mathrm{H}$, which was described already as concentrated areas of diversity in equine heavy and light chain CDRs [46, 47]. The breed $A$ possesses the highest amino acid variations in $\mathrm{CDR} 1 \mathrm{H}$ and $\mathrm{HF}$ in $\mathrm{CDR} 2 \mathrm{H}$ while GS exhibited lowest variability in these two CDRHs. Further, transition areas between FRHs and CDRHs had a higher variability than the middle of CDRHs. Position 96 in FR3H shows the highest variability within the FRHs. This residue is located on the outer surface of the variable region of the immunoglobulin molecule [43] within the area where the constant region is connected to the variable region. The high variability 
Chapter 3: Gene conversion and combinatorial diversity in bovine heavy chains

at this position may indicate an influence on the position of variable and constant region and their sterical orientation, which may affect light chain pairing as heavy chains possessing group $3 \mathrm{CDR} 3 \mathrm{H}$ are connected to a special type of lambda light chains $[43,48]$.

Further analysis revealed that no amino acids were assigned to the IMGT amino acid positions $10,31-34,60-62$, and 73 in cattle. This means, that one amino acid position within $\mathrm{FR} 1 \mathrm{H}$, four positions in $\mathrm{CDR} 1 \mathrm{H}$, three positions in $\mathrm{CDR} 2 \mathrm{H}$, as well as one position in $\mathrm{FR} 3 \mathrm{H}$ were not filled. Consequently, in cattle 8 out of 12 amino acid positions within $\mathrm{CDR} 1 \mathrm{H}$ are covered. In $\mathrm{CDR} 2 \mathrm{H}, 10$ positions are available and 7 are covered. Compared to FR-IMGT and CDR-IMGT lengths of functional and ORF IGHVgenes of human IGHV, mouse IGHV, rat IGHV, arabian camel IGHV, sheep IGHV, and pig IGHV the missing amino acids within FR1H and FR3H are conserved in all animals mentioned $[49,50]$. Averaged eight to ten amino acids were positioned in CDR1H whereas in $\mathrm{CDR} 2 \mathrm{H}$ six to ten amino acids were placed by the IMGT numbering system in human, mouse, rat, camel, sheep, and pig IGHV. Therefore, the positions of missing amino acids are congruent with other species.

In the breed $A$, the highest number of recombinations and variability were observed when compared to the other breeds investigated. GS possessed the lowest number of recombinations and showed less variability except in the middle of the CDR3H region. This finding indicates the contribution of insertions and deletions to diversity in case of few rearrangements [25]. It should be noted that A and GS were kept under the same management in a mixed herd. GBP and HF were kept at different farms. The breeds kept in different areas were consequently exposed to different antigens. Thus, the individual number of rearrangements per breed and differences in variability additionally indicate a specialized immune response as animals on one farm are challenged with the same environment.

The application of the newly developed bioinformatics framework led to important new results. Our analyses demonstrated that the bovine heavy chain diversity is not restricted to the use of a limited number of germline genes although there are preferred rearrangements within the three groups of $\mathrm{CDR} 3 \mathrm{H}$ lengths. We also found strong evidence for gene conversion using pseudogenes. Despite current advances in the understanding of bovine immunoglobulin diversification, future investigations of the germline repertoire are necessary. 
Chapter 3: Gene conversion and combinatorial diversity in bovine heavy chains

\section{Material and Methods}

\section{Detailed analyses of immunoglobulin sequences using a newly developed bioinformatics framework}

For sequence analysis, we developed a new bioinformatics framework using MUSCLE $[34,35]$ for the initial fast but accurate multiple nucleotide sequence alignment and following ClustalW [36] for calculating the sequence distances after deduction of the amino acid residues. Both programs are available as stand-alone algorithms and were implemented into our program. The immunogenetics nomenclature (IMGT) was used to assign framework regions $1-4(\mathrm{FR} 1-4 \mathrm{H})$ and the complementarity determining regions $1-3[41]$.

Therefore, germline nucleotide sequences were imported in FASTA-format (IGHV, $I G H D$, IGHJ $[17,18,22])$. Using the functional IGHV, the nucleotide sequences were translated into amino acids to number the codons of the functional germline IGHV gene segments according to the IMGT system from FR1H to FR3H. This required first the identification of the conserved and preassigned positions of Cys23, Trp41, Leu89, and Cys104 defined by Lefranc et al. [41]. Following, the nucleotide sequences of germline pseudo IGHV gene segments were aligned separately using MUSCLE [34, 35] to obtain the putative open reading frame. The previously defined positions of the codons using the functional genes were transferred onto the pseudogenes. Insertions as well as deletions of nucleotides within the pseudo gene segment sequence were discarded. The last 33 nucleotides of germline IGHJs were then used to define the FR4H. The region between $\mathrm{FR} 3 \mathrm{H}$ and $\mathrm{FR} 4 \mathrm{H}$ is defined as $\mathrm{CDR} 3 \mathrm{H}$. This region was later used to align the sample $\mathrm{CDR} 3 \mathrm{H}$ to germline IGHD segments. Defining $\mathrm{FRHs}$ and $\mathrm{CDRHs}$ allowed, beside the analyses of the complete transcribed genes, the alignment of $I G H V, I G H D$ and IGHJ using different parameters to improve the biological significance as well as the analyses of the single functionally divergent regions to determine putative gene conversion events in those regions. All functional and pseudo gene germline segments are referred as reference sequences.

Framework regions and CDRs of transcribed sequences (designated as sample sequences) were aligned pairwise to the isolated reference sequences after isolation from the first lgG constant region. Following, minimal divergence was used to identify the most similar sample sequence and reference sequence pairing.

For nucleotide alignments of IGHV and IGHJ, default values of MUSCLE were used. We tested three different procedures to assign germline and sample IGHDs to improve 
biological significance. In procedure 1, we applied default values of MUSCLE [34, 35], in procedure 2 we changed the penalties for gap opening to -4 and for gap extention to -0.3 [22], and in procedure 3 we additionally incorporated a new scoring matrix with match $=2$, transversion $=-1$, and transition $=1$ to evaluate transversion and transition mutations, whereby the IUB (international union of biochemistry) code for single and wobble bases was used.

The three procedures were applied to a set of sample sequences of IgG-derived variable regions from four different cattle breeds. Each nucleotide sequence of our sample sequences was aligned separately to the reference sequences to determine the most similar reference sequence as germline origin. Following, the codons were translated into amino acids. Sample sequences possessing premature Stop codons or not covering the full length of the variable region due to incomplete sequencing were eliminated and were not incorporated in further analyses. The remaining sample sequences were annotated in accordance to the IMGT nomenclature. For exceptional long CDR3H no positions are defined in the IMGT system, therefore positions had to be added as required and designated as 111.1-111.x and 112.y-112.1 in accordance to the IMGT numbering system [41]. To determine the germline origin, only the FRHs were aligned to avoid interference with the highly diversified CDRH [18]. To analyze possible gene conversion events, FR1-3Hs and CDR1-3Hs were extracted and aligned separately to the corresponding regions of the IGHV reference sequences to find the most similar one (Fig 8). The results were presented as an html table. 
Chapter 3: Gene conversion and combinatorial diversity in bovine heavy chains

(A)

1. Preparation of the Reference sequences

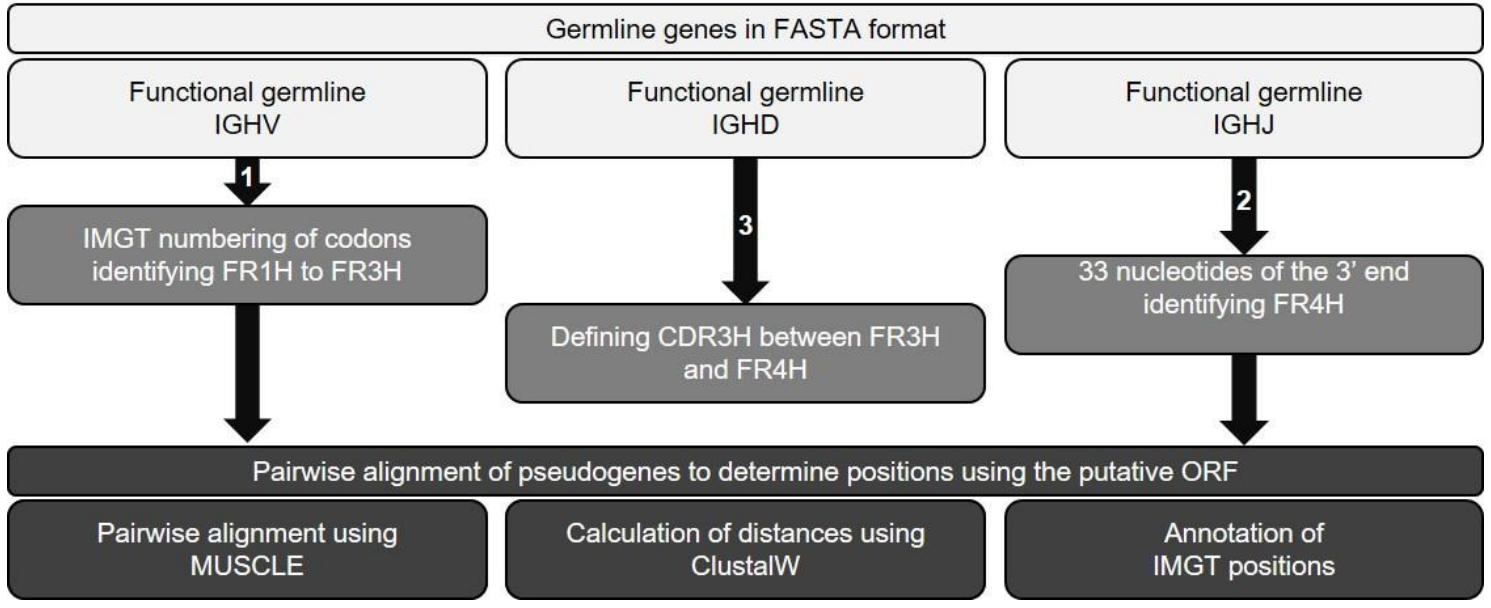

(B)

2. Analyses of Sample sequences

Amplification of transcribed $\lg G 1$ to $\lg G 3$ in 4 cattle breeds

1

Identifying beginning of constant region to extract variable region

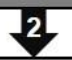

Elimination of variable regions with premature stop or incomplete sequences

3

Pairwise nucleotide alignment to Reference sequences

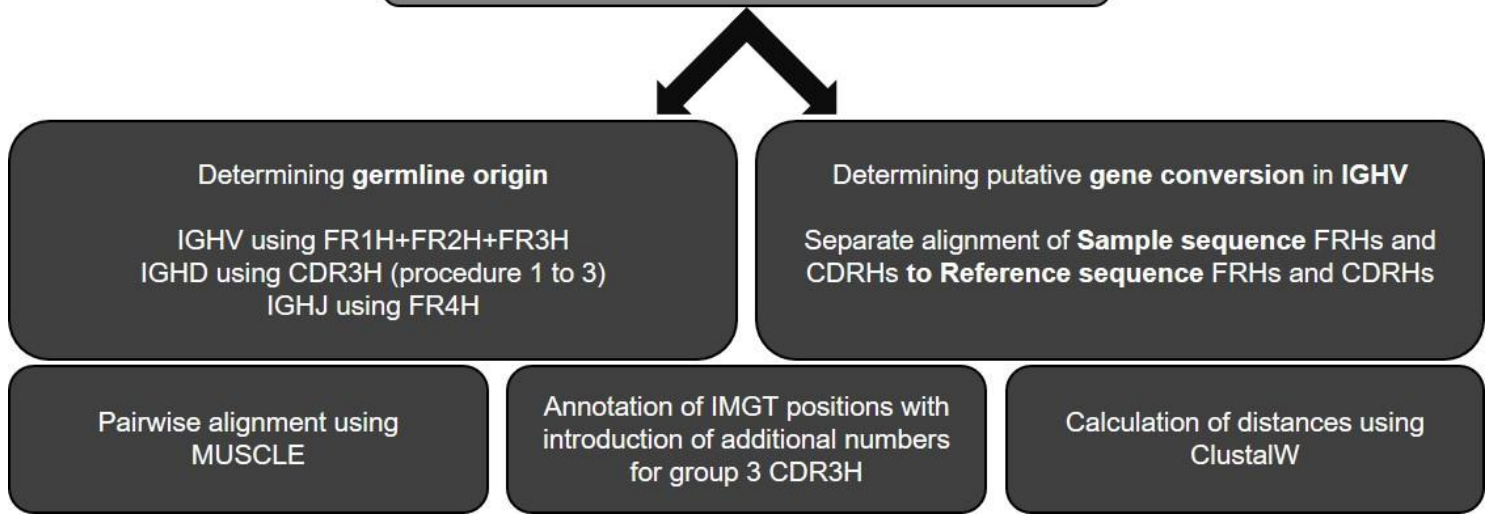


(C)

\section{Analyses of program output}
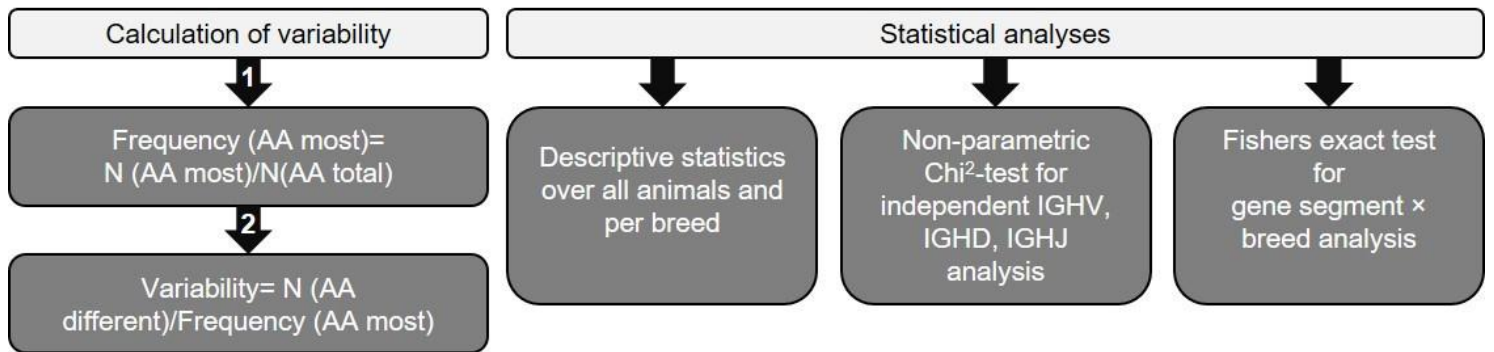

Fig. 8. Graphical presentation of workflow of the developed bioinformatics framework used for analyzing bovine heavy chain IgG. (A) At first the reference sequences were prepared. Using the functional segments the pseudogenes were aligned. (B) The bioinformatics framework analyzed the germline origin of bovine immunoglobulin heavy chain variable segments (IGHV), diversity segments (IGHD), and joining segments (IGHJ). One approach used the framework regions $(\mathrm{FRH}) 1$ to 3 to determine the closest germline IGHV. The second approach analyzed single functional regions $\mathrm{FRH} 1$ to 3 and complementarity determining regions (CDRH) 1 and 2 independently to reveal putative gene conversion events. (C) The last step included the calculation of the variability and the statistical analyses.

To display accumulation of amino acid substitutions in distinct segments of the variable region, variability was calculated as described by $\mathrm{Wu}$ and Kabat [40]. Thus, the frequency of the most common amino acid at a distinct position was calculated first. The number of the most abundant amino acid at a given position was divided by the number of all amino acids observed at this position. This means, only samples possessing an amino acid residue at this position in accordance to the IMGT nomenclature were considered. Subsequently, the number of different amino acids at the given position was divided by the frequency of the most common amino acid residue to determine variability. The variability results were written into a txt-file, which allows further analyses in statistical software.

For the statistical analyses of the distribution of IGHV, IGHD, and IGHJ segments including putative gene conversions within one breed and among breeds have been compared by applying non-parametric tests. Such test procedures, i.e. the $\mathrm{Chi}^{2}$ - test for analyzing IGHV, IGHD and IGHJ independently and Fisher's exact test for the gene segment $\times$ breed contingency table, are implemented in the software package SAS, Version 9.2. 
Chapter 3: Gene conversion and combinatorial diversity in bovine heavy chains

\section{Generation of the sample sequence set}

\section{Breed selection, isolation of PBMCs and cDNA synthesis}

For the analysis of the transcription of IgG heavy chain genes, the four cattle breeds German Black Pied (GBP), German Simmental (GS), Holstein-Friesian (HF), and Aubrac (A) were chosen. The animals selected from the herd of breed $A$ were composed of seven French and three German animals, whereas the sample of breed GS included one Austrian bull. German Black Pied and A represent small populations and have local importance, whereas HF and GS are commonly used in global commercial milk and meat production. German Simmental and A are kept on the same farm under same management conditions and in a mixed herd.

Blood samples were collected from ten randomly chosen animals per breed during routine blood sampling for mandatory examinations in disease control. Peripheral blood mononuclear cells (PBMCs) were isolated using Ficoll gradients (GE Healthcare Europe $\mathrm{GmbH}$, Germany) according to the manufacturer's protocol. Cells were stained with trypan blue and viable cells were counted. Total RNA was isolated from $1 \times 10^{7}$ cells using the RNeasy ${ }^{\circledR}$ Mini Kit (Qiagen, Germany). The first-strand cDNA was synthesized using $\operatorname{pd}(\mathrm{N})_{6}$ primers from $3 \mu \mathrm{g}$ of total RNA in a total volume of $20 \mu \mathrm{l}$ (SuperScript ${ }^{\mathrm{TM} I I I}$ First-Strand Synthesis SuperMix, Life Technologies $\mathrm{GmbH}$, Germany).

\section{Ethical Statement}

To collect B-lymphocytes, $20 \mathrm{ml}$ of EDTA blood were taken from the tail vein during regular investigation of infectious diseases in the cattle herds. The plasma was applied e.g. in an indirect ELISA testing for antibodies against Mycobacteria avium spp. paratuberculosis or BHV-1. Therefore, no specific approval is required.

\section{Amplification of immunoglobulin heavy-chain isotype restricted variable regions}

To amplify the variable regions restricted to $\mathrm{y}^{1-3}$ isotype heavy chains, a PCR was performed with primers binding within the leader region and the 3'UTR (blgG_leader: ATG AAC CCA CTG TGG ACC; blgG_3'UTR: CAG GAG GAA TGC ACA CAG). The primers were based on database sequence information and assigned to accession number X62916. The primer bolgG_leader anneals to position 22-39, and the primer bolgG_3'UTR to position 1518-1535. To monitor the integrity and purity of the cDNA, $527 \mathrm{bp}$ of the bovine GAPDH (Glycerinaldehyde 3-phosphate dehydrogenase) were 
Chapter 3: Gene conversion and combinatorial diversity in bovine heavy chains

amplified as a positive control. A no- template control served as a negative control for the PCR. The total reaction volume of $50 \mu$ included $0.67 \mu \mathrm{l}$ of cDNA, $200 \mu \mathrm{M}$ dNTPs (Bioline, Germany), $5 \mu \mathrm{l}$ of 10x PCR buffer (75 mM Tris- $\mathrm{HCl}, \mathrm{pH} 9.0 ; 2 \mathrm{mM} \mathrm{MgCl}$; $\left.50 \mathrm{mM} \mathrm{KCl} ; 20 \mathrm{mM}\left(\mathrm{NH}_{4}\right)_{2} \mathrm{SO}_{4}\right), 0.4 \mu \mathrm{M}$ of each primer, and 2 units of DNA polymerase (Biotools, Spain). PCR was performed under cycling conditions of $95^{\circ} \mathrm{C}$ for $5 \mathrm{~min}$, followed by $35 \mathrm{cycles}$ of $95^{\circ} \mathrm{C}$ for $1 \mathrm{~min}, 59.4^{\circ} \mathrm{C}$ for $1 \mathrm{~min}, 72^{\circ} \mathrm{C}$ for $2 \mathrm{~min}$, and terminated with elongation at $72^{\circ} \mathrm{C}$ for $10 \mathrm{~min}$. Length and purity of the PCR products were evaluated by means of electrophoresis on $1 \%$ agarose gels.

\section{Cloning and sequencing of the PCR products}

The PCR products were purified and concentrated using the MiniElute Gel Extraction Kit (Qiagen, Germany) in accordance to the manufacturer's protocol except QX1 buffer replaced QG buffer. Samples were eluted with $13 \mu \mathrm{l}$ EB buffer $(10 \mathrm{mM}$ Tris- $\mathrm{HCl}$, pH 8.5) and were stored at $4^{\circ} \mathrm{C}$. Purified products were cloned into the $\mathrm{pCR}^{\circledR} 2.1-\mathrm{TOPO}^{\circledR}$ $3.9 \mathrm{~kb}$ TA vector (Invitrogen ${ }^{\mathrm{TM}}$, Karlsruhe, Germany) and transformed into chemically competent One Shot TOP10 E. coli cells (Invitrogen ${ }^{\text {TM }}$, Karlsruhe, Germany). Transformants were plated on LB agar containing $0.3 \mathrm{mM}$ ampicillin, $40 \mu \mathrm{l} 2.44 \mu \mathrm{M} \mathrm{X}$ gal (5-bromo-4-chloro-3-indolyl-beta-D-galactopyranoside), and $40 \mu \mathrm{l} 1 \mathrm{M}$ IPTG (Isopropyl $\beta$-D-1-thiogalactopyranoside) for blue-white selection. After incubation at $37^{\circ} \mathrm{C}$, overnight cultures of randomly selected white transformants were grown in a $5 \mathrm{ml}$ LB-ampicillin broth. Plasmids were isolated using the MiniPrep Kit (Qiagen, Germany). In order to assess the insert size, plasmid DNA was cleaved with EcoRI (New England Biolabs, Germany) or a colony PCR was performed. Therefore a $25 \mu \mathrm{l}$ mixture containing $2 \mu \mathrm{l}$ cell culture, $0.4 \mu \mathrm{M}$ of vector specific primers M13 (-20) Forward and M13 Reverse (Invitrogen, Germany), respectively, and one PCR-bead (GE Healthcare Europe $\mathrm{GmbH}$, Germany) were used in a hot start PCR at $95^{\circ} \mathrm{C}$ for $5 \mathrm{~min}$, denaturation at $95^{\circ} \mathrm{C}$ for $1 \mathrm{~min}$, annealing at $60^{\circ} \mathrm{C}$ for $1 \mathrm{~min}$, and extension at $72^{\circ} \mathrm{C}$ for 2 min up to a total of 30 cycles. A final extension at $72^{\circ} \mathrm{C}$ for 10 min was included after the final cycle before PCR mixtures were cooled down to $4^{\circ} \mathrm{C}$. The size of the resulting fragments and of the PCR products was confirmed by agarose gel electrophoresis.

Sixteen clones per animal were sequenced according to the chain-termination method [51]. The M13 (-20) Forward (5'-GTA AAA CGA CGG CCA G-3') and M13 Reverse (5'CAG GAA ACA GCT CTG AC-3', Invitrogen, Germany) vector-specific primers, as well as the gene specific primers bolgG_leader, bolgG_3'UTR, bolgG_CH1_for (5'-GCC 
Chapter 3: Gene conversion and combinatorial diversity in bovine heavy chains

TCC ACC ACA GCC CCG AAA G-3'), bolgG_CH3_rev (5'-GAC CTT GCA CTT GAA CTC C-3') and bolgG_CH1_rev (5'-ACG GTC ACC ATG CTG CTG AG-3') were used for sequencing. 
Chapter 3: Gene conversion and combinatorial diversity in bovine heavy chains

\section{Acknowledgments}

We gratefully thank Dr. Dalia Aboelhassan, Sina Stein and Caroline Bierschenk for their technical support in generating the sequences. We also thank Dr. Apostolos Gittis and Dr. Stephen Dollery for critical review of the manuscript. 


\section{References}

1. Edelman GM. Antibody structure and molecular immunology. Science. 1973;180(88):830-40. Epub 1973/05/25. PubMed PMID: 4540988.

2. Tonegawa S. Somatic generation of antibody diversity. Nature. 1983;302(5909):575-81. Epub 1983/04/14. PubMed PMID: 6300689.

3. Wagner SD, Neuberger MS. Somatic hypermutation of immunoglobulin genes. Annu Rev Immunol. 1996;14:441-57. Epub 1996/01/01. doi: 10.1146/annurev.immunol.14.1.441. PubMed PMID: 8962691.

4. Reynaud CA, Dahan A, Anquez V, Weill JC. Somatic hyperconversion diversifies the single Vh gene of the chicken with a high incidence in the $\mathrm{D}$ region. Cell. 1989;59(1):171-83. Epub 1989/10/06. doi: 0092-8674(89)90879-9 [pii]. PubMed PMID: 2507167.

5. Berens SJ, Wylie DE, Lopez OJ. Use of a single VH family and long CDR3s in the variable region of cattle Ig heavy chains. Int Immunol. 1997;9(1):189-99. Epub 1997/01/01. PubMed PMID: 9043960.

6. Sun J, Kacskovics I, Brown WR, Butler JE. Expressed swine VH genes belong to a small VH gene family homologous to human VHIII. J Immunol. 1994;153(12):561827. Epub 1994/12/15. PubMed PMID: 7989761.

7. Dufour V, Malinge S, Nau F. The sheep Ig variable region repertoire consists of a single VH family. J Immunol. 1996;156(6):2163-70. Epub 1996/03/15. PubMed PMID: 8690905.

8. Saini SS, Hein WR, Kaushik A. A single predominantly expressed polymorphic immunoglobulin $\mathrm{VH}$ gene family, related to mammalian group, I, clan, II, is identified in cattle. Mol Immunol. 1997;34(8-9):641-51. Epub 1997/06/01. PubMed PMID: 9393967.

9. Sinclair MC, Gilchrist J, Aitken R. Bovine IgG repertoire is dominated by a single diversified VH gene family. J Immunol. 1997;159(8):3883-9. Epub 1997/10/23. PubMed PMID: 9378976.

10. Reynaud CA, Anquez V, Dahan A, Weill JC. A single rearrangement event generates most of the chicken immunoglobulin light chain diversity. Cell. 1985;40(2):283-91. Epub 1985/02/01. doi: 0092-8674(85)90142-4 [pii]. PubMed PMID: 3917859.

11. Reynaud CA, Anquez V, Grimal H, Weill JC. A hyperconversion mechanism generates the chicken light chain preimmune repertoire. Cell. 1987;48(3):379-88. Epub 1987/02/13. doi: 0092-8674(87)90189-9 [pii]. PubMed PMID: 3100050.

12. Reynaud CA, Garcia C, Hein WR, Weill JC. Hypermutation generating the sheep immunoglobulin repertoire is an antigen-independent process. Cell. 1995;80(1):115-25. Epub 1995/01/13. doi: 0092-8674(95)90456-5 [pii]. PubMed PMID: 7813007.

13. Becker RS, Knight KL. Somatic diversification of immunoglobulin heavy chain VDJ genes: evidence for somatic gene conversion in rabbits. Cell. 1990;63(5):987-97. Epub 1990/11/30. doi: 0092-8674(90)90502-6 [pii]. PubMed PMID: 2124176.

14. Parng CL, Hansal S, Goldsby RA, Osborne BA. Gene conversion contributes to Ig light chain diversity in cattle. J Immunol. 1996;157(12):5478-86. Epub 1996/12/15. PubMed PMID: 8955197.

15. Sun $\mathrm{Y}$, Wang $\mathrm{C}$, Wang $\mathrm{Y}$, Zhang $\mathrm{T}$, Ren $\mathrm{L}$, Hu X, et al. A comprehensive analysis of germline and expressed immunoglobulin repertoire in the horse. Dev Comp Immunol. 2010;34(9):1009-20. Epub 2010/05/15. doi: S0145-305X(10)00103-5 [pii] 10.1016/j.dci.2010.05.003. PubMed PMID: 20466019. 
16. Miller JR, Thomsen PD, Dixon SC, Tucker EM, Konfortov BA, Harbitz I. Synteny mapping of the bovine IGHG2, CRC and IGF1 genes. Anim Genet. 1992;23(1):518. Epub 1992/01/01. PubMed PMID: 1349204.

17. Walther S, Czerny CP, Diesterbeck US. Exceptionally long CDR3H are not isotype restricted in bovine immunoglobulins. PLoS One. 2013;8(5):e64234. Epub 2013/05/30. doi: 10.1371/journal.pone.0064234

PONE-D-13-04746 [pii]. PubMed PMID: 23717573; PubMed Central PMCID: PMC3661452.

18. Niku M, Liljavirta J, Durkin K, Schroderus E, livanainen A. The bovine genomic DNA sequence data reveal three IGHV subgroups, only one of which is functionally expressed. Dev Comp Immunol. 2012;37(3-4):457-61. doi: 10.1016/j.dci.2012.02.006. PubMed PMID: 22369780.

19. Zhao Y, Kacskovics I, Rabbani H, Hammarstrom L. Physical mapping of the bovine immunoglobulin heavy chain constant region gene locus. J Biol Chem. 2003;278(37):35024-32. Epub 2003/06/28. doi: 10.1074/jbc.M301337200

M301337200 [pii]. PubMed PMID: 12829708.

20. Hosseini A, Campbell G, Prorocic M, Aitken R. Duplicated copies of the bovine JH locus contribute to the Ig repertoire. Int Immunol. 2004;16(6):843-52. Epub 2004/05/06. doi: 10.1093/intimm/dxh085

dxh085 [pii]. PubMed PMID: 15126417.

21. Koti M, Kataeva G, Kaushik AK. Organization of $D(H)$-gene locus is distinct in cattle. Dev Biol (Basel). 2008;132:307-13. Epub 2008/09/27. PubMed PMID: 18817318.

22. Liljavirta J, Niku M, Pessa-Morikawa T, Ekman A, livanainen A. Expansion of the preimmune antibody repertoire by junctional diversity in Bos taurus. PLoS One. 2014;9(6):e99808. doi: 10.1371/journal.pone.0099808. PubMed PMID: 24926997; PubMed Central PMCID: PMC4057420.

23. Wang Y, Jackson KJ, Sewell WA, Collins AM. Many human immunoglobulin heavychain IGHV gene polymorphisms have been reported in error. Immunol Cell Biol. 2008;86(2):111-5. doi: 10.1038/sj.icb.7100144. PubMed PMID: 18040280.

24. Koti M, Kataeva G, Kaushik AK. Novel atypical nucleotide insertions specifically at $\mathrm{VH}-\mathrm{DH}$ junction generate exceptionally long CDR3H in cattle antibodies. Molecular immunology. 2010;47(11-12):2119-28. doi: 10.1016/j.molimm.2010.02.014. PubMed PMID: 20435350.

25. Larsen PA, Smith TP. Application of circular consensus sequencing and network analysis to characterize the bovine IgG repertoire. BMC Immunol. 2012;13:52. Epub 2012/09/18. doi: 1471-2172-13-52 [pii]

10.1186/1471-2172-13-52. PubMed PMID: 22978666.

26. Yousfi Monod M, Giudicelli V, Chaume D, Lefranc MP. IMGT/JunctionAnalysis: the first tool for the analysis of the immunoglobulin and T cell receptor complex V-J and V-D-J JUNCTIONs. Bioinformatics. 2004;20 Suppl 1:i379-85. doi: 10.1093/bioinformatics/bth945. PubMed PMID: 15262823.

27. Brochet X, Lefranc MP, Giudicelli V. IMGT/V-QUEST: the highly customized and integrated system for IG and TR standardized V-J and V-D-J sequence analysis. Nucleic Acids Res. 2008;36(Web Server issue):W503-8. Epub 2008/05/27. doi: gkn316 [pii]

10.1093/nar/gkn316. PubMed PMID: 18503082.

28. Giudicelli V, Brochet X, Lefranc MP. IMGT/V-QUEST: IMGT standardized analysis of the immunoglobulin (IG) and T cell receptor (TR) nucleotide sequences. Cold Spring Harb Protoc. 2011;2011(6):695-715. doi: 10.1101/pdb.prot5633. PubMed PMID: 21632778. 
29. Alamyar E, Duroux P, Lefranc MP, Giudicelli V. IMGT((R)) tools for the nucleotide analysis of immunoglobulin (IG) and $T$ cell receptor (TR) V-(D)-J repertoires, polymorphisms, and IG mutations: IMGT/V-QUEST and IMGT/HighV-QUEST for NGS. Methods Mol Biol. 2012;882:569-604. doi: 10.1007/978-1-61779-842-9_32. PubMed PMID: 22665256.

30. Retter I, Althaus $\mathrm{HH}$, Munch R, Muller W. VBASE2, an integrative $\mathrm{V}$ gene database. Nucleic Acids Res. 2005;33(Database issue):D671-4. doi: 10.1093/nar/gki088. PubMed PMID: 15608286; PubMed Central PMCID: PMC540042.

31. Souto-Carneiro MM, Longo NS, Russ DE, Sun HW, Lipsky PE. Characterization of the human Ig heavy chain antigen binding complementarity determining region 3 using a newly developed software algorithm, JOINSOLVER. J Immunol. 2004;172(11):6790-802. PubMed PMID: 15153497.

32. Gaeta BA, Malming HR, Jackson KJ, Bain ME, Wilson P, Collins AM. iHMMunealign: hidden Markov model-based alignment and identification of germline genes in rearranged immunoglobulin gene sequences. Bioinformatics. 2007;23(13):1580-7. doi: 10.1093/bioinformatics/btm147. PubMed PMID: 17463026.

33. Ye J, Ma N, Madden TL, Ostell JM. IgBLAST: an immunoglobulin variable domain sequence analysis tool. Nucleic Acids Res. 2013;41(Web Server issue):W34-40. doi: 10.1093/nar/gkt382. PubMed PMID: 23671333; PubMed Central PMCID: PMC3692102.

34. Edgar RC. MUSCLE: a multiple sequence alignment method with reduced time and space complexity. BMC Bioinformatics. 2004;5:113. doi: 10.1186/1471-2105-5-113. PubMed PMID: 15318951; PubMed Central PMCID: PMC517706.

35. Edgar RC. MUSCLE: multiple sequence alignment with high accuracy and high throughput. Nucleic Acids Res. 2004;32(5):1792-7. doi: 10.1093/nar/gkh340. PubMed PMID: 15034147; PubMed Central PMCID: PMC390337.

36. Thompson JD, Higgins DG, Gibson TJ. CLUSTAL W: improving the sensitivity of progressive multiple sequence alignment through sequence weighting, positionspecific gap penalties and weight matrix choice. Nucleic Acids Res. 1994;22(22):4673-80. Epub 1994/11/11. PubMed PMID: 7984417.

37. Reynaud CA, Bertocci B, Dahan A, Weill JC. Formation of the chicken B-cell repertoire: ontogenesis, regulation of Ig gene rearrangement, and diversification by gene conversion. Adv Immunol. 1994;57:353-78. Epub 1994/01/01. PubMed PMID: 7872160.

38. Thompson CB, Neiman PE. Somatic diversification of the chicken immunoglobulin light chain gene is limited to the rearranged variable gene segment. Cell. 1987;48(3):369-78. Epub 1987/02/13. doi: 0092-8674(87)90188-7 [pii]. PubMed PMID: 3100049.

39. Shojaei F, Saini SS, Kaushik AK. Unusually long germline DH genes contribute to large sized CDR3H in bovine antibodies. Molecular immunology. 2003;40(1):61-7. PubMed PMID: 12909131.

40. Wu TT, Kabat EA. An analysis of the sequences of the variable regions of Bence Jones proteins and myeloma light chains and their implications for antibody complementarity. J Exp Med. 1970;132(2):211-50. Epub 1970/08/01. PubMed PMID: 5508247.

41. Lefranc MP, Pommie C, Ruiz M, Giudicelli V, Foulquier E, Truong L, et al. IMGT unique numbering for immunoglobulin and $\mathrm{T}$ cell receptor variable domains and $\mathrm{Ig}$ superfamily V-like domains. Dev Comp Immunol. 2003;27(1):55-77. Epub 2002/12/13. doi: S0145305X02000393 [pii]. PubMed PMID: 12477501. 
42. Saini SS, MacGlashan DW, Jr., Sterbinsky SA, Togias A, Adelman DC, Lichtenstein LM, et al. Down-regulation of human basophil IgE and FC epsilon RI alpha surface densities and mediator release by anti-lgE-infusions is reversible in vitro and in vivo. J Immunol. 1999;162(9):5624-30. Epub 1999/05/05. PubMed PMID: 10228046.

43. Wang F, Ekiert DC, Ahmad I, Yu W, Zhang Y, Bazirgan O, et al. Reshaping antibody diversity. Cell. 2013;153(6):1379-93. Epub 2013/06/12. doi: S00928674(13)00525-4 [pii]

10.1016/j.cell.2013.04.049. PubMed PMID: 23746848; PubMed Central PMCID: PMC4007204.

44. Lucier MR, Thompson RE, Waire J, Lin AW, Osborne BA, Goldsby RA. Multiple sites of V lambda diversification in cattle. J Immunol. 1998;161(10):5438-44. Epub 1998/11/20. PubMed PMID: 9820519.

45. Kabat EA, Wu TT, Perry HM, Gottesmann KS, Foeller C. Sequences of proteins of immunological interest. 5th ed. Bethesda, MD: U.S. Department of Health and Human Services, Public Health Service, National Institutes of Health; 2006.

46. Tallmadge RL, Tseng CT, Felippe MJ. Diversity of immunoglobulin lambda light chain gene usage over developmental stages in the horse. Dev Comp Immunol. 2014;46(2):171-9. Epub 2014/04/15. doi: S0145-305X(14)00097-4 [pii]

10.1016/j.dci.2014.04.001. PubMed PMID: 24726757; PubMed Central PMCID: PMC4107094.

47. Tallmadge RL, Tseng CT, King RA, Felippe MJ. Developmental progression of equine immunoglobulin heavy chain variable region diversity. Dev Comp Immunol. 2013;41(1):33-43. Epub 2013/04/10. doi: S0145-305X(13)00087-6 [pii]

10.1016/j.dci.2013.03.020. PubMed PMID: 23567345; PubMed Central PMCID: PMC3672396.

48. Saini SS, Farrugia W, Ramsland PA, Kaushik AK. Bovine IgM antibodies with exceptionally long complementarity-determining region 3 of the heavy chain share unique structural properties conferring restricted $\mathrm{VH}+$ Vlambda pairings. Int Immunol. 2003;15(7):845-53. Epub 2003/06/17. PubMed PMID: 12807823.

49. Lefranc MP, Giudicelli V, Ginestoux C, Bodmer J, Muller W, Bontrop R, et al. IMGT, the international ImMunoGeneTics database. Nucleic Acids Res. 1999;27(1):20912. PubMed PMID: 9847182; PubMed Central PMCID: PMC148137.

50. Scaviner D, Barbie V, Ruiz M, Lefranc MP. Protein displays of the human immunoglobulin heavy, kappa and lambda variable and joining regions. Exp Clin Immunogenet. 1999;16(4):234-40. doi: 19115. PubMed PMID: 10575277.

51. Sanger F, Nicklen S, Coulson AR. DNA sequencing with chain-terminating inhibitors. Proc Natl Acad Sci U S A. 1977;74(12):5463-7. Epub 1977/12/01. PubMed PMID: 271968. 


\section{Supporting information}

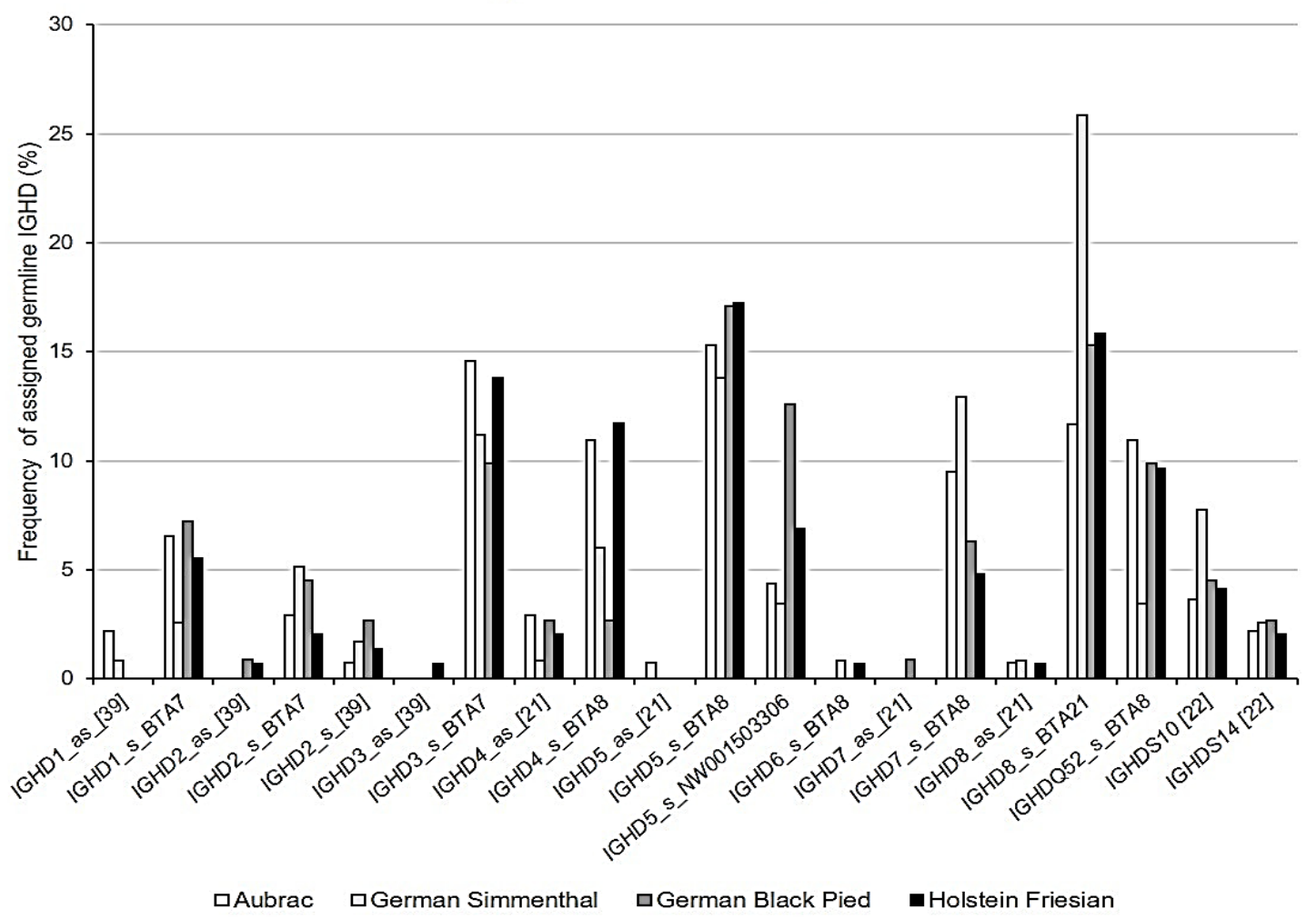

S1 Fig. Transcription frequencies of IGHD in four cattle breeds using procedure

1. Transcribed IGHD are shown on the horizontal axis, their relative usage frequencies are indicated on the vertical axis. Calculation occurred using the default values for gap opening and gap extention of MUSCLE. Each breed is marked by the following color code: Aubrac: white, German Simmental: light grey, Holstein Friesian: black, German Black Pied: dark grey 


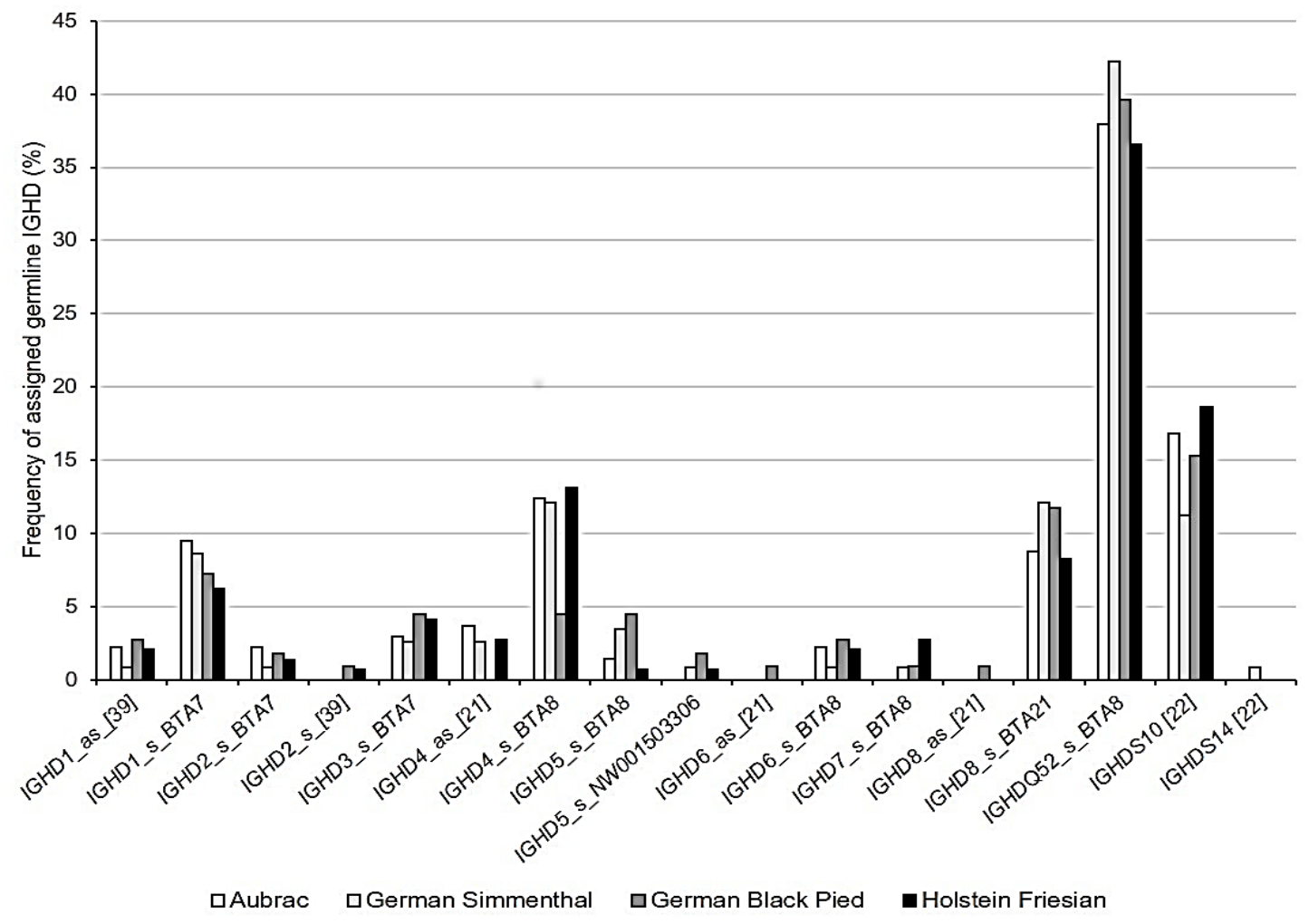

S2 Fig. Transcription frequencies of IGHD in four cattle breeds using procedure

2. Transcribed IGHD are shown on the horizontal axis, their relative usage frequencies are indicated on the vertical axis. Calculation occurred after changing the default values for gap opening (-4) and gap extention (-0.3) of MUSCLE. Each breed is marked by the following color code: Aubrac: white, German Simmental: light grey, Holstein Friesian: black, German Black Pied: dark grey 


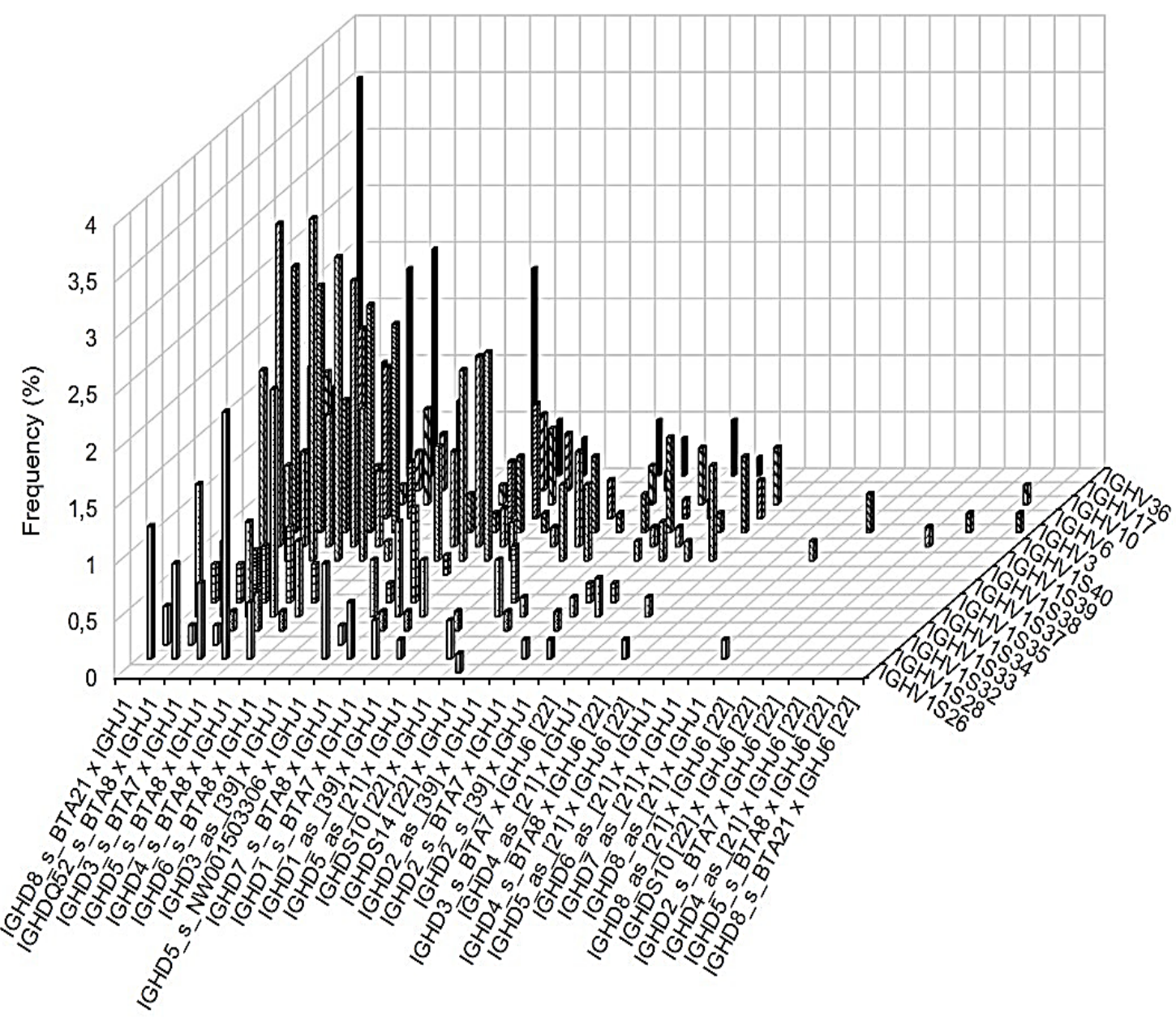

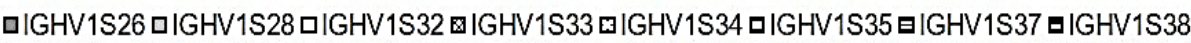

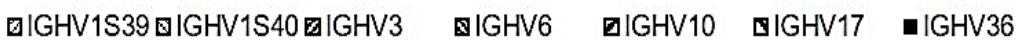

S3 Fig. Recombinations of IGHV, IGHD, and IGHJ over all four cattle breeds using procedure 1. In the sequences of all four cattle breeds analyzed 147 different combinations of $I G H V$, IGHD, and IGHJ were identified. Relative frequencies (\%) of the combinations of the 21 transcribed IGHD and the 2 transcribed IGHJ are shown depending on the rearranged IGHV $(n=15)$. 


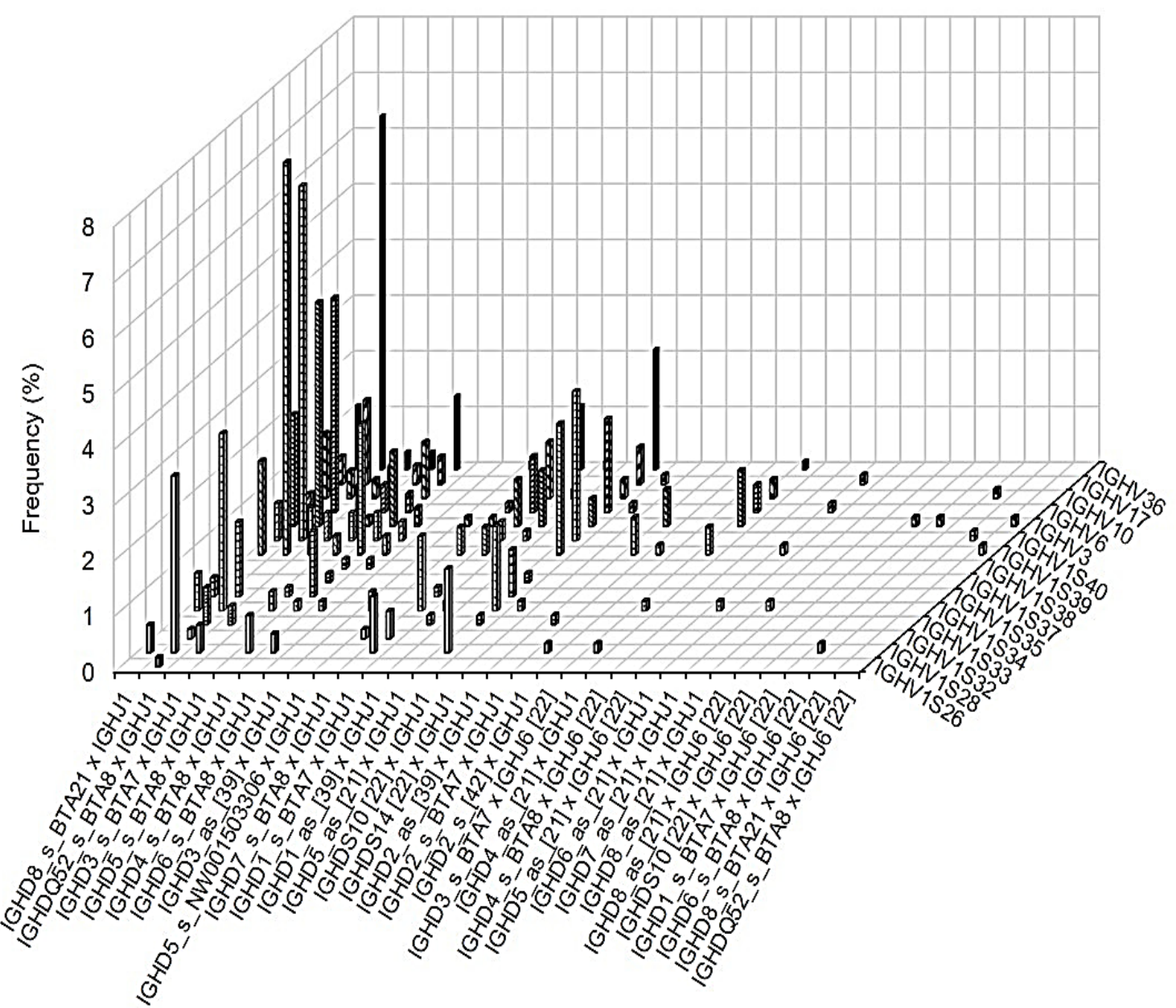

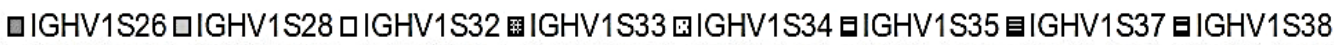

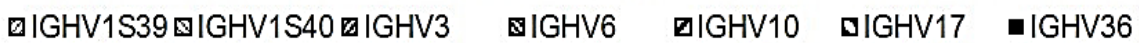

S4 Fig. Recombinations of IGHV, IGHD, and IGHJ over all four cattle breeds using procedure 2 . In the sequences of all four cattle breeds analyzed 119 different combinations of $I G H V$, IGHD, and IGHJ were identified. Relative frequencies (\%) of the combinations of the 21 transcribed IGHD and the 2 transcribed IGHJ are shown depending on the rearranged IGHV $(n=15)$. 
Chapter 3: Gene conversion and combinatorial diversity in bovine heavy chains

S1 Table. Transcription frequencies of IGHD in four cattle breeds using

procedure 1.

\begin{tabular}{|c|c|c|c|c|}
\hline IGHD & $\begin{array}{r}\mathrm{A} \\
(\% ; \mathbf{n = 1 3 7 )} \\
\end{array}$ & $\begin{array}{r}\text { GS } \\
(\% ; n=116) \\
\end{array}$ & $\begin{array}{r}\text { GBP } \\
(\% ; n=111)\end{array}$ & $\begin{array}{r}\text { HF } \\
(\% ; n=145)\end{array}$ \\
\hline IGHD1_as ${ }^{1}$ [39] & 2.19 & 0.86 & 0.00 & 0.00 \\
\hline$I G H D 1 \_s^{2}$ _BTA7 & 6.57 & 2.59 & 7.21 & 5.52 \\
\hline IGHD2_as_[39] & 0.00 & 0.00 & 0.90 & 0.69 \\
\hline IGHD2_s_BTA7 & 2.92 & 5.17 & 4.50 & 2.07 \\
\hline IGHD2_s_[39] & 0.73 & 1.72 & 2.70 & 1.38 \\
\hline IGHD3_as_[39] & 0.00 & 0.00 & 0.00 & 0.69 \\
\hline IGHD3_s_BTA7 & 14.60 & 11.21 & 9.91 & 13.79 \\
\hline IGHD4_as_[21] & 2.92 & 0.86 & 2.70 & 2.07 \\
\hline IGHD4_s_BTA8 & 10.95 & 6.03 & 2.70 & 11.72 \\
\hline IGHD5_as_[21] & 0.73 & 0.00 & 0.00 & 0.00 \\
\hline IGHD5_s_BTA8 & 15.33 & 13.79 & 17.12 & 17.24 \\
\hline IGHD5_s_NW001503306 & 4.38 & 3.45 & 12.61 & 6.90 \\
\hline IGHD6_s_BTA8 & 0.00 & 0.86 & 0.00 & 0.69 \\
\hline IGHD7_as_[21] & 0.00 & 0.00 & 0.90 & 0.00 \\
\hline IGHD7_s_BTA8 & 9.49 & 12.93 & 6.31 & 4.83 \\
\hline IGHD8_as_[21] & 0.73 & 0.86 & 0.00 & 0.69 \\
\hline IGHD8_s_BTA21 & 11.68 & 25.86 & 15.32 & 15.86 \\
\hline IGHDQ52_s_BTA8 & 10.95 & 3.45 & 9.91 & 9.66 \\
\hline IGHDS10 [22] & 3.65 & 7.76 & 4.50 & 4.14 \\
\hline IGHDS14 [22] & 2.19 & 2.59 & 2.70 & 2.07 \\
\hline
\end{tabular}

${ }^{1}$ antisense

2 sense 
Chapter 3: Gene conversion and combinatorial diversity in bovine heavy chains

S2 Table. Transcription frequencies of IGHD in four cattle breeds using

procedure 2.

\begin{tabular}{|c|c|c|c|c|}
\hline IGHD & $\begin{array}{r}A \\
(\% ; n=137)\end{array}$ & $\begin{array}{r}\text { GS } \\
(\% ; n=116)\end{array}$ & $\begin{array}{r}\text { GBP } \\
(\% ; n=111)\end{array}$ & $\begin{array}{r}\text { HF } \\
(\% ; n=145)\end{array}$ \\
\hline IGHD1_as ${ }^{1} \_[39]$ & 2.19 & 0.86 & 2.70 & 2.07 \\
\hline IGHD1_s $\mathrm{s}^{2} \_\mathrm{BTA7}$ & 9.49 & 8.62 & 7.21 & 6.21 \\
\hline IGHD2_s_BTA7 & 2.19 & 0.86 & 1.80 & 1.38 \\
\hline IGHD2_s_[39] & 0.00 & 0.00 & 0.90 & 0.69 \\
\hline IGHD3_s_BTA7 & 2.92 & 2.59 & 4.50 & 4.14 \\
\hline IGHD4_as_[21] & 3.65 & 2.59 & 0.00 & 2.76 \\
\hline IGHD4_s_BTA8 & 12.41 & 12.07 & 4.50 & 13.10 \\
\hline IGHD5_s_BTA8 & 1.46 & 3.45 & 4.50 & 0.69 \\
\hline IGHD5_s_NW001503306 & 0.00 & 0.86 & 1.80 & 0.69 \\
\hline IGHD6_as_[21] & 0.00 & 0.00 & 0.90 & 0.00 \\
\hline IGHD6_s_BTA8 & 2.19 & 0.86 & 2.70 & 2.07 \\
\hline IGHD7_s_BTA8 & 0.00 & 0.86 & 0.90 & 2.76 \\
\hline IGHD8_as_[21] & 0.00 & 0.00 & 0.90 & 0.00 \\
\hline IGHD8_s_BTA21 & 8.76 & 12.07 & 11.71 & 8.28 \\
\hline IGHDQ52_s_BTA8 & 37.96 & 42.24 & 39.64 & 36.55 \\
\hline IGHDS10 [22] & 16.79 & 11.21 & 15.32 & 18.62 \\
\hline IGHDS14 [22] & 0.00 & 0.86 & 0.00 & 0.00 \\
\hline
\end{tabular}

${ }^{1}$ antisense

2 sense 
Chapter 3: Gene conversion and combinatorial diversity in bovine heavy chains

\section{S3 Table. Recombinations of IGHV, IGHD, and IGHJ over all four cattle breeds}

using procedure 3.

\begin{tabular}{|c|c|c|c|c|c|}
\hline IGHV - IGHD - IGHJ & $\begin{array}{r}\text { all } \\
(n=597)\end{array}$ & $\begin{array}{r}A \\
(n=167)\end{array}$ & $\begin{array}{r}\text { GBP } \\
(n=140)\end{array}$ & $\begin{array}{r}\text { GS } \\
(n=131)\end{array}$ & $\begin{array}{r}\text { HF } \\
(n=159)\end{array}$ \\
\hline IGHV1S26 - IGHD8_s'1_BTA21 - IGHJ1 & 0.17 & 0.00 & 0.00 & 0.76 & 0.00 \\
\hline IGHV1S28 - IGHD1_as²_[39] - IGHJ1 & 0.34 & 0.00 & 1.43 & 0.00 & 0.00 \\
\hline IGHV1S28 - IGHD1_s_BTA7 - IGHJ1 & 0.50 & 1.20 & 0.00 & 0.00 & 0.63 \\
\hline IGHV1S28 - IGHD2_s_[39] - IGHJ1 & 0.17 & 0.00 & 0.00 & 0.76 & 0.00 \\
\hline IGHV1S28 - IGHD3_as_[39] - IGHJ1 & 0.17 & 0.60 & 0.00 & 0.00 & 0.00 \\
\hline IGHV1S28 - IGHD3_s_BTA7 - IGHJ1 & 1.01 & 1.20 & 2.14 & 0.00 & 0.63 \\
\hline IGHV1S28 - IGHD4_s_BTA8 - IGHJ6_[22] & 0.17 & 0.00 & 0.00 & 0.00 & 0.63 \\
\hline IGHV1S28 - IGHD4_s_BTA8 - IGHJ1 & 1.17 & 1.20 & 1.43 & 0.76 & 1.26 \\
\hline IGHV1S28 - IGHD5_s_BTA8 - IGHJ1 & 1.17 & 1.20 & 0.71 & 2.29 & 0.63 \\
\hline IGHV1S28 - IGHD5_s_NW_001503306 - IGHJ1 & 0.34 & 0.60 & 0.00 & 0.76 & 0.00 \\
\hline IGHV1S28 - IGHD6_s_BTA8 - IGHJ1 & 0.17 & 0.00 & 0.71 & 0.00 & 0.00 \\
\hline IGHV1S28 - IGHD7_s_BTA8 - IGHJ1 & 0.84 & 0.00 & 1.43 & 1.53 & 0.63 \\
\hline IGHV1S28 - IGHD8_s_BTA21 - IGHJ1 & 0.34 & 0.00 & 0.00 & 0.76 & 0.63 \\
\hline IGHV1S28 - IGHDQ52_s_BTA8 - IGHJ1 & 0.84 & 0.00 & 0.71 & 0.76 & 1.89 \\
\hline IGHV1S28 - IGHDS10 [22] - IGHJ1 & 0.67 & 0.00 & 0.00 & 0.00 & 2.52 \\
\hline IGHV1S28 - IGHDS14 [22] - IGHJ1 & 0.34 & 0.60 & 0.00 & 0.76 & 0.00 \\
\hline IGHV1S32 - IGHD2_s_[39] - IGHJ1 & 0.17 & 0.00 & 0.00 & 0.76 & 0.00 \\
\hline IGHV1S32 - IGHD8_s_BTA21 - IGHJ1 & 0.34 & 0.00 & 0.00 & 1.53 & 0.00 \\
\hline IGHV1S32 - IGHDS10 [22] - IGHJ1 & 0.17 & 0.00 & 0.00 & 0.00 & 0.63 \\
\hline IGHV1S32 - IGHDS14 [22] - IGHJ1 & 0.17 & 0.00 & 0.00 & 0.00 & 0.63 \\
\hline IGHV1S33 - IGHD3_as_[39] - IGHJ1 & 0.17 & 0.60 & 0.00 & 0.00 & 0.00 \\
\hline IGHV1S33 - IGHD3_s_BTA7 - IGHJ1 & 0.34 & 1.20 & 0.00 & 0.00 & 0.00 \\
\hline IGHV1S33 - IGHD4_s_BTA8 - IGHJ1 & 0.17 & 0.00 & 0.71 & 0.00 & 0.00 \\
\hline IGHV1S33 - IGHD5_s_BTA8 - IGHJ1 & 0.17 & 0.00 & 0.00 & 0.76 & 0.00 \\
\hline IGHV1S33 - IGHD8_s_BTA21 - IGHJ1 & 0.17 & 0.60 & 0.00 & 0.00 & 0.00 \\
\hline IGHV1S33 - IGHDQ52_s_BTA8 - IGHJ1 & 0.34 & 1.20 & 0.00 & 0.00 & 0.00 \\
\hline IGHV1S33 - IGHDS14 [22] - IGHJ1 & 0.17 & 0.00 & 0.00 & 0.76 & 0.00 \\
\hline IGHV1S34 - IGHD1_as_[39] - IGHJ1 & 0.17 & 0.60 & 0.00 & 0.00 & 0.00 \\
\hline IGHV1S34 - IGHD1_s_BTA7 - IGHJ1 & 1.34 & 0.60 & 3.57 & 0.76 & 0.63 \\
\hline IGHV1S34 - IGHD2_s_BTA7 - IGHJ1 & 0.34 & 0.60 & 0.00 & 0.00 & 0.63 \\
\hline IGHV1S34 - IGHD2_s_[39] - IGHJ1 & 0.17 & 0.00 & 0.00 & 0.76 & 0.00 \\
\hline IGHV1S34 - IGHD3_s_BTA7 - IGHJ1 & 0.84 & 0.00 & 2.14 & 0.76 & 0.63 \\
\hline IGHV1S34 - IGHD4_s_BTA8 - IGHJ1 & 0.67 & 0.60 & 1.43 & 0.00 & 0.63 \\
\hline IGHV1S34 - IGHD5_s_BTA8 - IGHJ1 & 1.01 & 0.60 & 1.43 & 0.76 & 1.26 \\
\hline IGHV1S34 - IGHD5_s_NW_001503306 - IGHJ1 & 0.34 & 0.00 & 0.71 & 0.00 & 0.63 \\
\hline IGHV1S34 - IGHD6_s_BTA8 - IGHJ1 & 0.17 & 0.00 & 0.00 & 0.76 & 0.00 \\
\hline IGHV1S34 - IGHD7_s_BTA8 - IGHJ1 & 0.34 & 0.60 & 0.71 & 0.00 & 0.00 \\
\hline IGHV1S34 - IGHD8_s_BTA21 - IGHJ1 & 1.01 & 0.00 & 0.71 & 3.82 & 0.00 \\
\hline IGHV1S34 - IGHDQ52_s_BTA8 - IGHJ1 & 1.51 & 2.40 & 2.14 & 0.76 & 0.63 \\
\hline
\end{tabular}


Chapter 3: Gene conversion and combinatorial diversity in bovine heavy chains

\begin{tabular}{|c|c|c|c|c|c|}
\hline IGHV1S34 - IGHDS10 [22] - IGHJ1 & 0.34 & 0.00 & 0.71 & 0.00 & 0.63 \\
\hline IGHV1S34 - IGHDS14 [22] - IGHJ1 & 0.34 & 0.00 & 0.00 & 1.53 & 0.00 \\
\hline IGHV1S35 - IGHD1_s_BTA7 - IGHJ1 & 0.17 & 0.00 & 0.00 & 0.00 & 0.63 \\
\hline IGHV1S35 - IGHD2_s_[39] - IGHJ1 & 0.17 & 0.00 & 0.00 & 0.76 & 0.00 \\
\hline IGHV1S35 - IGHD3_s_BTA7 - IGHJ1 & 0.50 & 0.00 & 0.71 & 1.53 & 0.00 \\
\hline IGHV1S35 - IGHD4_as_[21] - IGHJ1 & 0.34 & 0.60 & 0.00 & 0.76 & 0.00 \\
\hline IGHV1S35 - IGHD4_s_BTA8 - IGHJ1 & 0.34 & 0.60 & 0.00 & 0.76 & 0.00 \\
\hline IGHV1S35 - IGHD5_s_BTA8 - IGHJ1 & 0.50 & 0.00 & 0.00 & 2.29 & 0.00 \\
\hline IGHV1S35 - IGHD7_s_BTA8 - IGHJ1 & 1.01 & 0.00 & 0.71 & 3.82 & 0.00 \\
\hline IGHV1S35 - IGHD8_s_BTA21 - IGHJ1 & 0.67 & 1.20 & 0.00 & 1.53 & 0.00 \\
\hline IGHV1S35 - IGHDQ52_s_BTA8 - IGHJ1 & 0.17 & 0.60 & 0.00 & 0.00 & 0.00 \\
\hline IGHV1S35 - IGHDS10 [22] - IGHJ1 & 0.17 & 0.00 & 0.00 & 0.00 & 0.63 \\
\hline IGHV1S37 - IGHD4_s_BTA8 - IGHJ1 & 0.17 & 0.00 & 0.00 & 0.00 & 0.63 \\
\hline IGHV1S37 - IGHDQ52_s_BTA8 - IGHJ1 & 0.17 & 0.00 & 0.00 & 0.00 & 0.63 \\
\hline IGHV1S38 - IGHD3_s_BTA7 - IGHJ1 & 0.17 & 0.00 & 0.00 & 0.76 & 0.00 \\
\hline IGHV1S38 - IGHDQ52_s_BTA8 - IGHJ1 & 0.17 & 0.00 & 0.00 & 0.00 & 0.63 \\
\hline IGHV1S39 - IGHD1_as_[39] - IGHJ1 & 0.34 & 0.60 & 0.00 & 0.00 & 0.63 \\
\hline IGHV1S39 - IGHD1_s_BTA7 - IGHJ1 & 1.01 & 1.20 & 1.43 & 0.00 & 1.26 \\
\hline IGHV1S39 - IGHD2_as_[39] - IGHJ1 & 0.34 & 1.20 & 0.00 & 0.00 & 0.00 \\
\hline IGHV1S39 - IGHD2_s_BTA7 - IGHJ1 & 0.34 & 0.60 & 0.00 & 0.00 & 0.63 \\
\hline IGHV1S39 - IGHD2_s_[39] - IGHJ1 & 0.84 & 0.00 & 1.43 & 0.76 & 1.26 \\
\hline IGHV1S39 - IGHD3_s_BTA7 - IGHJ6_[22] & 0.17 & 0.00 & 0.00 & 0.00 & 0.63 \\
\hline IGHV1S39 - IGHD3_s_BTA7 - IGHJ1 & 1.01 & 1.20 & 1.43 & 0.00 & 1.26 \\
\hline IGHV1S39 - IGHD4_as_[21] - IGHJ1 & 0.50 & 0.60 & 0.00 & 0.76 & 0.63 \\
\hline IGHV1S39 - IGHD4_s_BTA8 - IGHJ1 & 1.68 & 3.59 & 0.71 & 0.00 & 1.89 \\
\hline IGHV1S39 - IGHD5_s_BTA8 - IGHJ1 & 2.85 & 7.78 & 0.71 & 0.00 & 1.89 \\
\hline IGHV1S39 - IGHD5_s_NW_001503306 - IGHJ1 & 1.17 & 1.20 & 2.86 & 0.76 & 0.00 \\
\hline IGHV1S39 - IGHD6_as_[21] - IGHJ1 & 0.34 & 0.00 & 0.00 & 0.00 & 1.26 \\
\hline IGHV1S39 - IGHD6_s_BTA8 - IGHJ1 & 0.34 & 0.00 & 0.00 & 0.00 & 1.26 \\
\hline IGHV1S39 - IGHD7_s_BTA8 - IGHJ1 & 1.68 & 2.40 & 0.71 & 0.76 & 2.52 \\
\hline IGHV1S39 - IGHD8_s_BTA21 - IGHJ1 & 2.18 & 4.79 & 1.43 & 0.00 & 1.89 \\
\hline IGHV1S39 - IGHDQ52_s_BTA8 - IGHJ1 & 1.34 & 2.99 & 0.00 & 0.00 & 1.89 \\
\hline IGHV1S39 - IGHDS10 [22] - IGHJ1 & 1.01 & 1.20 & 2.86 & 0.00 & 0.00 \\
\hline IGHV1S39 - IGHDS14 [22] - IGHJ1 & 0.50 & 1.20 & 0.00 & 0.00 & 0.63 \\
\hline IGHV1S40 - IGHD1_s_BTA7 - IGHJ1 & 0.17 & 0.00 & 0.00 & 0.00 & 0.63 \\
\hline IGHV1S40 - IGHD2_s_BTA7 - IGHJ1 & 0.17 & 0.60 & 0.00 & 0.00 & 0.00 \\
\hline IGHV1S40 - IGHD2_s_[39] - IGHJ1 & 0.17 & 0.00 & 0.00 & 0.76 & 0.00 \\
\hline IGHV1S40 - IGHD3_as_[39] - IGHJ1 & 0.17 & 0.60 & 0.00 & 0.00 & 0.00 \\
\hline IGHV1S40 - IGHD3_s_BTA7 - IGHJ1 & 2.35 & 0.00 & 2.86 & 4.58 & 2.52 \\
\hline IGHV1S40 - IGHD4_as_[21] - IGHJ1 & 0.17 & 0.60 & 0.00 & 0.00 & 0.00 \\
\hline IGHV1S40 - IGHD4_s_BTA8 - IGHJ1 & 0.84 & 0.60 & 1.43 & 0.00 & 1.26 \\
\hline IGHV1S40 - IGHD5_s_BTA8 - IGHJ1 & 3.02 & 2.99 & 3.57 & 4.58 & 1.26 \\
\hline IGHV1S40 - IGHD5_s_NW_001503306 - IGHJ1 & 0.50 & 0.60 & 0.71 & 0.00 & 0.63 \\
\hline IGHV1S40 - IGHD6_as_[21] - IGHJ1 & 0.17 & 0.00 & 0.71 & 0.00 & 0.00 \\
\hline
\end{tabular}


Chapter 3: Gene conversion and combinatorial diversity in bovine heavy chains

\begin{tabular}{|c|c|c|c|c|c|}
\hline IGHV1S40 - IGHD6_s_BTA8 - IGHJ1 & 0.17 & 0.60 & 0.00 & 0.00 & 0.00 \\
\hline IGHV1S40 - IGHD7_s_BTA8 - IGHJ1 & 0.84 & 0.00 & 1.43 & 1.53 & 0.63 \\
\hline IGHV1S40 - IGHD8_s_BTA21 - IGHJ1 & 1.34 & 1.20 & 2.14 & 0.76 & 1.26 \\
\hline IGHV1S40 - IGHDQ52_s_BTA8 - IGHJ1 & 1.51 & 0.60 & 1.43 & 2.29 & 1.89 \\
\hline IGHV1S40 - IGHDS10 [22] - IGHJ6_[22] & 0.17 & 0.00 & 0.71 & 0.00 & 0.00 \\
\hline IGHV1S40 - IGHDS10 [22] - IGHJ1 & 0.50 & 0.00 & 0.71 & 0.76 & 0.63 \\
\hline IGHV3 - IGHD1_as_[39] - IGHJ1 & 0.34 & 0.60 & 0.00 & 0.76 & 0.00 \\
\hline IGHV3 - IGHD1_s_BTA7 - IGHJ1 & 1.51 & 0.00 & 2.14 & 0.76 & 3.14 \\
\hline IGHV3 - IGHD2_as_[39] - IGHJ1 & 0.50 & 1.20 & 0.00 & 0.76 & 0.00 \\
\hline IGHV3 - IGHD2_s_BTA7 - IGHJ1 & 0.50 & 0.60 & 0.00 & 0.76 & 0.63 \\
\hline IGHV3 - IGHD2_s_[39] - IGHJ1 & 0.34 & 0.00 & 0.71 & 0.00 & 0.63 \\
\hline IGHV3 - IGHD3_as_[39] - IGHJ1 & 0.17 & 0.60 & 0.00 & 0.00 & 0.00 \\
\hline IGHV3 - IGHD3_s_BTA7 - IGHJ1 & 1.34 & 1.20 & 2.14 & 0.76 & 1.26 \\
\hline IGHV3 - IGHD3_s_BTA7 - IGHJ6_[22] & 0.34 & 0.00 & 0.00 & 0.76 & 0.63 \\
\hline IGHV3 - IGHD4_as_[21] - IGHJ1 & 0.50 & 0.00 & 0.71 & 0.00 & 1.26 \\
\hline IGHV3 - IGHD4_s_BTA8 - IGHJ1 & 0.50 & 1.20 & 0.71 & 0.00 & 0.00 \\
\hline IGHV3 - IGHD5_as_[21] - IGHJ1 & 0.17 & 0.60 & 0.00 & 0.00 & 0.00 \\
\hline IGHV3 - IGHD5_s_BTA8 - IGHJ1 & 2.01 & 1.20 & 1.43 & 2.29 & 3.14 \\
\hline IGHV3 - IGHD5_s_NW_001503306 - IGHJ1 & 0.50 & 0.60 & 0.00 & 0.00 & 1.26 \\
\hline IGHV3 - IGHD7_s_BTA8 - IGHJ1 & 0.50 & 0.60 & 1.43 & 0.00 & 0.00 \\
\hline IGHV3 - IGHD8_as_[21] IGHJ6_[22] & 0.17 & 0.60 & 0.00 & 0.00 & 0.00 \\
\hline IGHV3 - IGHD8_s_BTA21 - IGHJ1 & 1.68 & 0.60 & 0.71 & 0.76 & 4.40 \\
\hline IGHV3 - IGHDQ52_s_BTA8 - IGHJ1 & 1.84 & 0.00 & 0.71 & 3.05 & 3.77 \\
\hline IGHV3 - IGHDS10 [22] - IGHJ1 & 1.01 & 2.40 & 0.71 & 0.76 & 0.00 \\
\hline IGHV3 - IGHDS14 [22] - IGHJ1 & 0.50 & 0.00 & 1.43 & 0.76 & 0.00 \\
\hline IGHV6 - IGHD1_as_[39] - IGHJ1 & 0.17 & 0.60 & 0.00 & 0.00 & 0.00 \\
\hline IGHV6 - IGHD1_s_BTA7 - IGHJ1 & 0.67 & 0.60 & 1.43 & 0.00 & 0.63 \\
\hline IGHV6 - IGHD2_s_BTA7 - IGHJ1 & 0.50 & 1.80 & 0.00 & 0.00 & 0.00 \\
\hline IGHV6 - IGHD2_s_[39] - IGHJ1 & 0.17 & 0.00 & 0.00 & 0.76 & 0.00 \\
\hline IGHV6 - IGHD3_as_[39] - IGHJ1 & 0.34 & 0.00 & 0.71 & 0.76 & 0.00 \\
\hline IGHV6 - IGHD3_s_BTA7 - IGHJ1 & 0.84 & 0.60 & 1.43 & 0.00 & 1.26 \\
\hline IGHV6 - IGHD4_as_[21] - IGHJ1 & 0.34 & 0.60 & 0.00 & 0.00 & 0.63 \\
\hline IGHV6 - IGHD4_s_BTA8 - IGHJ1 & 1.01 & 1.20 & 0.00 & 0.76 & 1.89 \\
\hline IGHV6 - IGHD5_s_BTA8 - IGHJ1 & 0.84 & 0.60 & 1.43 & 0.00 & 1.26 \\
\hline IGHV6 - IGHD5_s_NW_001503306 - IGHJ1 & 0.50 & 0.60 & 0.71 & 0.76 & 0.00 \\
\hline IGHV6 - IGHD7_s_BTA8 - IGHJ1 & 0.50 & 0.60 & 0.71 & 0.00 & 0.63 \\
\hline IGHV6 - IGHD8_s_BTA21 IGHJ1 & 1.34 & 1.80 & 0.71 & 3.05 & 0.00 \\
\hline IGHV6 - IGHDQ52_s_BTA8 - IGHJ1 & 1.34 & 1.80 & 1.43 & 0.00 & 1.89 \\
\hline IGHV6 - IGHDS10 [22] - IGHJ1 & 0.34 & 0.00 & 0.71 & 0.00 & 0.63 \\
\hline IGHV6 - IGHDS14 [22] - IGHJ1 & 0.34 & 0.00 & 0.00 & 1.53 & 0.00 \\
\hline IGHV10 - IGHD2_as_[39] - IGHJ1 & 0.34 & 1.20 & 0.00 & 0.00 & 0.00 \\
\hline IGHV10 - IGHD1_s_BTA7 - IGHJ1 & 0.34 & 0.60 & 0.00 & 0.00 & 0.63 \\
\hline IGHV10 - IGHD2_s_BTA7 - IGHJ1 & 0.50 & 1.20 & 0.00 & 0.76 & 0.00 \\
\hline IGHV10 - IGHD2_s_[39] - IGHJ1 & 1.01 & 0.60 & 0.00 & 2.29 & 1.26 \\
\hline
\end{tabular}


Chapter 3: Gene conversion and combinatorial diversity in bovine heavy chains

\begin{tabular}{|c|c|c|c|c|c|}
\hline IGHV10 - IGHD3_s_BTA7 - IGHJ1 & 0.67 & 0.60 & 0.00 & 0.76 & 1.26 \\
\hline IGHV10 - IGHD4_s_BTA8 - IGHJ1 & 0.17 & 0.60 & 0.00 & 0.00 & 0.00 \\
\hline IGHV10 - IGHD5_as_[21] - IGHJ6_[22] & 0.17 & 0.00 & 0.00 & 0.00 & 0.63 \\
\hline IGHV10 - IGHD5_s_BTA8 - IGHJ1 & 0.17 & 0.00 & 0.00 & 0.00 & 0.63 \\
\hline IGHV10 - IGHD5_S_NW_001503306 - IGHJ1 & 0.17 & 0.00 & 0.00 & 0.00 & 0.63 \\
\hline IGHV10 - IGHD7_as_[21] - IGHJ1 & 0.17 & 0.00 & 0.71 & 0.00 & 0.00 \\
\hline IGHV10 - IGHD7_s_BTA8 - IGHJ1 & 0.17 & 0.00 & 0.00 & 0.76 & 0.00 \\
\hline IGHV10 - IGHD8_as_[21] - IGHJ1 & 0.34 & 0.00 & 0.71 & 0.00 & 0.63 \\
\hline IGHV10 - IGHD8_s_BTA21 - IGHJ1 & 0.17 & 0.60 & 0.00 & 0.00 & 0.00 \\
\hline IGHV10 - IGHDQ52_s_BTA8 - IGHJ1 & 0.17 & 0.60 & 0.00 & 0.00 & 0.00 \\
\hline IGHV10 - IGHDS10 [22] - IGHJ1 & 0.50 & 0.00 & 0.00 & 2.29 & 0.00 \\
\hline IGHV17 - IGHD1_s_BTA7 - IGHJ1 & 0.50 & 0.60 & 1.43 & 0.00 & 0.00 \\
\hline IGHV17 - IGHD3_s_BTA7 - IGHJ1 & 1.01 & 1.80 & 0.71 & 1.53 & 0.00 \\
\hline IGHV17 - IGHD4_as_[21] - IGHJ1 & 0.17 & 0.60 & 0.00 & 0.00 & 0.00 \\
\hline IGHV17 - IGHD4_s_BTA8 - IGHJ1 & 0.34 & 0.60 & 0.71 & 0.00 & 0.00 \\
\hline IGHV17 - IGHD5_s_BTA8 - IGHJ1 & 0.50 & 0.00 & 1.43 & 0.00 & 0.63 \\
\hline IGHV17 - IGHD5_s_NW_001503306 - IGHJ1 & 0.17 & 0.00 & 0.71 & 0.00 & 0.00 \\
\hline IGHV17 - IGHD7_s_BTA8 - IGHJ1 & 0.34 & 0.00 & 0.71 & 0.00 & 0.63 \\
\hline IGHV17 - IGHD8_s_BTA21 - IGHJ1 & 0.34 & 0.00 & 0.00 & 1.53 & 0.00 \\
\hline IGHV17 - IGHDQ52_s_BTA8 - IGHJ1 & 0.50 & 1.20 & 0.00 & 0.00 & 0.63 \\
\hline IGHV17 - IGHDS10 [22] - IGHJ1 & 0.34 & 0.60 & 0.71 & 0.00 & 0.00 \\
\hline IGHV36 - IGHD1_as_[39] - IGHJ1 & 0.17 & 0.60 & 0.00 & 0.00 & 0.00 \\
\hline IGHV36 - IGHD1_s_BTA7 - IGHJ1 & 0.34 & 0.60 & 0.00 & 0.00 & 0.63 \\
\hline IGHV36 - IGHD3_as_[39] - IGHJ1 & 0.50 & 0.60 & 0.71 & 0.76 & 0.00 \\
\hline IGHV36 - IGHD3_s_BTA7 - IGHJ1 & 1.51 & 0.00 & 2.14 & 0.00 & 3.77 \\
\hline IGHV36 - IGHD4_s_BTA8 - IGHJ1 & 0.84 & 0.00 & 0.00 & 1.53 & 1.89 \\
\hline IGHV36 - IGHD5_as_[21] - IGHJ1 & 0.17 & 0.00 & 0.00 & 0.76 & 0.00 \\
\hline IGHV36 - IGHD5_s_BTA8 - IGHJ1 & 1.51 & 1.80 & 2.86 & 0.76 & 0.63 \\
\hline IGHV36 - IGHD5_s_NW_001503306 - IGHJ1 & 0.50 & 0.00 & 0.71 & 0.00 & 1.26 \\
\hline IGHV36 - IGHD6_s_BTA8 - IGHJ1 & 0.67 & 0.00 & 1.43 & 0.00 & 1.26 \\
\hline IGHV36 - IGHD7_s_BTA8 - IGHJ1 & 0.50 & 0.60 & 0.00 & 0.76 & 0.63 \\
\hline IGHV36 - IGHD8_s_BTA21 - IGHJ1 & 4.52 & 1.20 & 5.00 & 10.69 & 2.52 \\
\hline IGHV36 - IGHDQ52_s_BTA8 - IGHJ1 & 1.84 & 1.80 & 3.57 & 0.76 & 1.26 \\
\hline IGHV36 - IGHDS10 [22] - IGHJ1 & 0.17 & 0.00 & 0.00 & 0.76 & 0.00 \\
\hline IGHV36 - IGHDS14 [22] - IGHJ1 & 0.17 & 0.60 & 0.00 & 0.00 & 0.00 \\
\hline
\end{tabular}


Chapter 3: Gene conversion and combinatorial diversity in bovine heavy chains

S4 Table. Recombinations of IGHV, IGHD, and IGHJ over all four cattle breeds using procedure 1.

\begin{tabular}{|c|c|c|c|c|c|}
\hline IGHV - IGHD - IGHJ & $\begin{array}{r}\text { all } \\
(n=597)\end{array}$ & $\begin{array}{r}\mathbf{G S} \\
(\mathbf{n}=\mathbf{1 3 1})\end{array}$ & $\begin{array}{r}\text { GBP } \\
(140) \\
\end{array}$ & $\begin{array}{r}A \\
(n=167)\end{array}$ & $\begin{array}{r}\text { HF } \\
(\mathbf{n}=159)\end{array}$ \\
\hline IGHV1S26 - IGHDS14 [22] - IGHJ1 & 0,17 & 0,76 & 0,00 & 0,00 & 0,00 \\
\hline IGHV1S28 - IGHD1_as1_[39] - IGHJ1 & 0,17 & 0,00 & 0,00 & 0,60 & 0,00 \\
\hline IGHV1S28 - IGHD1_s²_BTA7 - IGHJ1 & 0,34 & 0,00 & 0,71 & 0,60 & 0,00 \\
\hline IGHV1S28 - IGHD2_s_BTA7 - IGHJ1 & 0,17 & 0,00 & 0,71 & 0,00 & 0,00 \\
\hline IGHV1S28 - IGHD2_s_[39] - IGHJ1 & 0,17 & 0,00 & 0,71 & 0,00 & 0,00 \\
\hline IGHV1S28 - IGHD3_s_BTA7 - IGHJ1 & 0,67 & 0,76 & 0,00 & 0,60 & 1,26 \\
\hline IGHV1S28 - IGHD4_s_BTA8 - IGHJ6_[22] & 0,17 & 0,00 & 0,00 & 0,00 & 0,63 \\
\hline IGHV1S28 - IGHD4_s_BTA8 - IGHJ1 & 0,50 & 0,00 & 0,00 & 0,60 & 1,26 \\
\hline IGHV1S28 - IGHD5_s_BTA8 - IGHJ1 & 2,18 & 3,82 & 2,86 & 0,00 & 2,52 \\
\hline IGHV1S28 - IGHD5_s_NW_001503306 - IGHJ1 & 0,84 & 0,76 & 0,71 & 1,20 & 0,63 \\
\hline IGHV1S28 - IGHD7_s_BTA8 - IGHJ1 & 0,50 & 0,76 & 1,43 & 0,00 & 0,00 \\
\hline IGHV1S28 - IGHD8_as_[21] - IGHJ1 & 0,17 & 0,00 & 0,00 & 0,00 & 0,63 \\
\hline IGHV1S28 - IGHD8_s_BTA21 - IGHJ1 & 1,17 & 1,53 & 0,71 & 1,20 & 1,26 \\
\hline IGHV1S28 - IGHDQ52_s_BTA8 - IGHJ1 & 0,84 & 0,76 & 0,71 & 1,20 & 0,63 \\
\hline IGHV1S28 - IGHDS10 [22] - IGHJ1 & 0,34 & 0,00 & 0,00 & 0,60 & 0,63 \\
\hline IGHV1S32 - IGHD3_s_BTA7 - IGHJ1 & 0,17 & 0,76 & 0,00 & 0,00 & 0,00 \\
\hline IGHV1S32 - IGHD5_s_NW_001503306 - IGHJ1 & 0,17 & 0,00 & 0,00 & 0,00 & 0,63 \\
\hline IGHV1S32 - IGHD8_s_BTA21 - IGHJ1 & 0,34 & 1,53 & 0,00 & 0,00 & 0,00 \\
\hline IGHV1S32 - IGHDQ52_s_BTA8 - IGHJ1 & 0,17 & 0,00 & 0,00 & 0,00 & 0,63 \\
\hline IGHV1S33 - IGHD1_s_BTA7 - IGHJ1 & 0,17 & 0,00 & 0,00 & 0,60 & 0,00 \\
\hline IGHV1S33 - IGHD2_s_BTA7 - IGHJ1 & 0,17 & 0,00 & 0,00 & 0,60 & 0,00 \\
\hline IGHV1S33 - IGHD3_s_BTA7 - IGHJ1 & 0,17 & 0,00 & 0,00 & 0,60 & 0,00 \\
\hline IGHV1S33 - IGHD4_s_BTA8 IGHJ1 & 0,17 & 0,76 & 0,00 & 0,00 & 0,00 \\
\hline IGHV1S33 - IGHD5_as_[21] - IGHJ1 & 0,17 & 0,00 & 0,00 & 0,60 & 0,00 \\
\hline IGHV1S33 - IGHD5_s_BTA8 - IGHJ1 & 0,34 & 0,00 & 0,71 & 0,60 & 0,00 \\
\hline IGHV1S33 - IGHD7_s_BTA8 - IGHJ1 & 0,17 & 0,76 & 0,00 & 0,00 & 0,00 \\
\hline IGHV1S33 - IGHDS14 [22] - IGHJ1 & 0,17 & 0,00 & 0,00 & 0,60 & 0,00 \\
\hline IGHV1S34 - IGHD1_s_BTA7 - IGHJ1 & 0,50 & 0,76 & 0,71 & 0,60 & 0,00 \\
\hline IGHV1S34 - IGHD2_s_BTA7 - IGHJ1 & 0,17 & 0,00 & 0,00 & 0,60 & 0,00 \\
\hline IGHV1S34 - IGHD2_s_[39] - IGHJ1 & 0,34 & 0,00 & 0,71 & 0,60 & 0,00 \\
\hline IGHV1S34 - IGHD3_s_BTA7 - IGHJ1 & 0,84 & 0,76 & 1,43 & 0,60 & 0,63 \\
\hline IGHV1S34 - IGHD4_as_[21] - IGHJ1 & 0,17 & 0,00 & 0,00 & 0,00 & 0,63 \\
\hline IGHV1S34 - IGHD4_s_BTA8 - IGHJ1 & 0,67 & 0,00 & 2,14 & 0,00 & 0,63 \\
\hline IGHV1S34 - IGHD5_s_BTA8 - IGHJ1 & 2,01 & 3,05 & 3,57 & 1,20 & 0,63 \\
\hline IGHV1S34 - IGHD5_s_NW_001503306 - IGHJ1 & 0,50 & 0,00 & 1,43 & 0,00 & 0,63 \\
\hline IGHV1S34 - IGHD7_s_BTA8 - IGHJ1 & 0,84 & 0,76 & 0,00 & 1,20 & 1,26 \\
\hline IGHV1S34 - IGHD8_s_BTA21 - IGHJ1 & 1,17 & 3,82 & 0,71 & 0,00 & 0,63 \\
\hline IGHV1S34 - IGHDQ52_s_BTA8 - IGHJ1 & 0,67 & 0,00 & 2,14 & 0,60 & 0,00 \\
\hline IGHV1S34 - IGHDS10 [22] - IGHJ1 & 0,50 & 0,76 & 0,00 & 0,60 & 0,63 \\
\hline IGHV1S34 - IGHDS14 [22] - IGHJ1 & 0,17 & 0,00 & 0,71 & 0,00 & 0,00 \\
\hline
\end{tabular}


Chapter 3: Gene conversion and combinatorial diversity in bovine heavy chains

\begin{tabular}{|c|c|c|c|c|c|}
\hline IGHV1S35 - IGHD2_s_BTA7 - IGHJ1 & 0,17 & 0,76 & 0,00 & 0,00 & 0,00 \\
\hline IGHV1S35 - IGHD2_s_[39] - IGHJ1 & 0,17 & 0,76 & 0,00 & 0,00 & 0,00 \\
\hline IGHV1S35 - IGHD3_s_BTA7 - IGHJ1 & 0,50 & 0,76 & 0,00 & 0,60 & 0,63 \\
\hline IGHV1S35 - IGHD4_s_BTA8 - IGHJ1 & 0,34 & 0,76 & 0,00 & 0,00 & 0,63 \\
\hline IGHV1S35 - IGHD5_s_BTA8 - IGHJ1 & 0,67 & 1,53 & 0,00 & 1,20 & 0,00 \\
\hline IGHV1S35 - IGHD5_s_NW_001503306 - IGHJ1 & 0,17 & 0,76 & 0,00 & 0,00 & 0,00 \\
\hline IGHV1S35 - IGHD7_s_BTA8 - IGHJ1 & 0,84 & 3,05 & 0,71 & 0,00 & 0,00 \\
\hline IGHV1S35 - IGHD8_s_BTA21 - IGHJ1 & 0,34 & 0,76 & 0,71 & 0,00 & 0,00 \\
\hline IGHV1S35 - IGHDQ52_s_BTA8 - IGHJ1 & 0,34 & 0,00 & 0,00 & 1,20 & 0,00 \\
\hline IGHV1S35 - IGHDS10 [22] - IGHJ1 & 0,50 & 2,29 & 0,00 & 0,00 & 0,00 \\
\hline IGHV1S37 - IGHDQ52_s_BTA8 - IGHJ1 & 0,34 & 0,00 & 0,00 & 0,00 & 1,26 \\
\hline IGHV1S38 - IGHD7_s_BTA8 - IGHJ1 & 0,17 & 0,76 & 0,00 & 0,00 & 0,00 \\
\hline IGHV1S38 - IGHDQ52_s_BTA8 - IGHJ1 & 0,17 & 0,00 & 0,00 & 0,00 & 0,63 \\
\hline IGHV1S39 - IGHD1_as_[39] - IGHJ1 & 0,34 & 0,00 & 0,00 & 1,20 & 0,00 \\
\hline IGHV1S39 - IGHD1_s_BTA7 - IGHJ1 & 1,84 & 0,76 & 2,86 & 2,40 & 1,26 \\
\hline IGHV1S39 - IGHD2_s_BTA7 - IGHJ1 & 0,17 & 0,00 & 0,71 & 0,00 & 0,00 \\
\hline IGHV1S39 - IGHD2_s_[39] - IGHJ1 & 0,34 & 0,00 & 0,00 & 0,00 & 1,26 \\
\hline IGHV1S39 - IGHD3_s_BTA7 - IGHJ6_[22] & 0,17 & 0,00 & 0,00 & 0,00 & 0,63 \\
\hline IGHV1S39 - IGHD3_s_BTA7 - IGHJ1 & 3,02 & 0,76 & 2,14 & 5,99 & 2,52 \\
\hline IGHV1S39 - IGHD4_as_[21] - IGHJ1 & 0,84 & 0,76 & 0,00 & 1,80 & 0,63 \\
\hline IGHV1S39 - IGHD4_s_BTA8 - IGHJ1 & 1,34 & 0,00 & 0,00 & 3,59 & 1,26 \\
\hline IGHV1S39 - IGHD5_s_BTA8 - IGHJ1 & 2,68 & 0,00 & 2,86 & 5,39 & 1,89 \\
\hline IGHV1S39 - IGHD5_s_NW_001503306 - IGHJ1 & 1,01 & 0,76 & 1,43 & 1,20 & 0,63 \\
\hline IGHV1S39 - IGHD6_s_BTA8 - IGHJ1 & 0,17 & 0,00 & 0,00 & 0,00 & 0,63 \\
\hline IGHV1S39 - IGHD7_as_[21] - IGHJ1 & 0,17 & 0,00 & 0,71 & 0,00 & 0,00 \\
\hline IGHV1S39 - IGHD7_s_BTA8 - IGHJ1 & 1,68 & 0,00 & 0,00 & 3,59 & 2,52 \\
\hline IGHV1S39 - IGHD8_s_BTA21 - IGHJ1 & 1,68 & 0,00 & 0,71 & 1,20 & 4,40 \\
\hline IGHV1S39 - IGHDQ52_s_BTA8 - IGHJ1 & 0,84 & 0,00 & 1,43 & 1,20 & 0,63 \\
\hline IGHV1S39 - IGHDS10 [22] - IGHJ1 & 0,67 & 0,00 & 0,00 & 1,20 & 1,26 \\
\hline IGHV1S39 - IGHDS14 [22] - IGHJ1 & 0,67 & 0,00 & 0,71 & 1,80 & 0,00 \\
\hline IGHV1S40 - IGHD1_s_BTA7 - IGHJ1 & 0,34 & 0,00 & 0,00 & 0,00 & 1,26 \\
\hline IGHV1S40 - IGHD2_s_BTA7 - IGHJ6_[22] & 0,17 & 0,00 & 0,71 & 0,00 & 0,00 \\
\hline IGHV1S40 - IGHD2_s_BTA7 - IGHJ1 & 0,17 & 0,76 & 0,00 & 0,00 & 0,00 \\
\hline IGHV1S40 - IGHD2_s_[39] - IGHJ1 & 0,17 & 0,00 & 0,71 & 0,00 & 0,00 \\
\hline IGHV1S40 - IGHD3_s_BTA7 - IGHJ1 & 1,17 & 2,29 & 1,43 & 0,60 & 0,63 \\
\hline IGHV1S40 - IGHD4_s_BTA8 - IGHJ1 & 0,67 & 0,76 & 0,00 & 1,20 & 0,63 \\
\hline IGHV1S40 - IGHD5_as_[21] - IGHJ1 & 0,17 & 0,00 & 0,00 & 0,60 & 0,00 \\
\hline IGHV1S40 - IGHD5_s_BTA8 - IGHJ1 & 2,35 & 0,76 & 1,43 & 2,40 & 4,40 \\
\hline IGHV1S40 - IGHD5_s_NW_001503306 - IGHJ1 & 0,84 & 0,76 & 2,14 & 0,60 & 0,00 \\
\hline IGHV1S40 - IGHD7_s_BTA8 - IGHJ1 & 1,68 & 4,58 & 1,43 & 1,20 & 0,00 \\
\hline IGHV1S40 - IGHD8_s_BTA21 - IGHJ1 & 2,85 & 4,58 & 3,57 & 1,20 & 2,52 \\
\hline IGHV1S40 - IGHDQ52_s_BTA8 - IGHJ1 & 0,84 & 0,00 & 2,14 & 0,60 & 0,63 \\
\hline IGHV1S40 - IGHDS10 [22] - IGHJ1 & 0,84 & 0,76 & 2,14 & 0,00 & 0,63 \\
\hline IGHV3 - IGHD1_as_[39] - IGHJ1 & 0,17 & 0,76 & 0,00 & 0,00 & 0,00 \\
\hline
\end{tabular}


Chapter 3: Gene conversion and combinatorial diversity in bovine heavy chains

\begin{tabular}{|c|c|c|c|c|c|}
\hline IGHV3 - IGHD1_s_BTA7 - IGHJ1 & 0,67 & 0,00 & 1,43 & 0,00 & 1,26 \\
\hline IGHV3 - IGHD2_as_[39] - IGHJ1 & 0,34 & 0,00 & 0,71 & 0,00 & 0,63 \\
\hline IGHV3 - IGHD2_s_BTA7 - IGHJ1 & 0,84 & 1,53 & 0,71 & 0,00 & 1,26 \\
\hline IGHV3 - IGHD3_s_BTA7 - IGHJ6_[22] & 0,17 & 0,00 & 0,00 & 0,00 & 0,63 \\
\hline IGHV3 - IGHD3_s_BTA7 - IGHJ1 & 1,17 & 1,53 & 2,14 & 0,60 & 0,63 \\
\hline IGHV3 - IGHD4_as_[21] - IGHJ6_[22] & 0,17 & 0,00 & 0,00 & 0,60 & 0,00 \\
\hline IGHV3 - IGHD4_as_[21] - IGHJ1 & 0,67 & 0,00 & 1,43 & 1,20 & 0,00 \\
\hline IGHV3 - IGHD4_s_BTA8 - IGHJ1 & 1,84 & 0,76 & 0,71 & 2,40 & 3,14 \\
\hline IGHV3 - IGHD5_s_BTA8 - IGHJ1 & 2,01 & 2,29 & 2,14 & 0,00 & 3,77 \\
\hline IGHV3 - IGHD5_s_NW_001503306 - IGHJ1 & 0,34 & 0,00 & 0,71 & 0,00 & 0,63 \\
\hline IGHV3 - IGHD7_s_BTA8 - IGHJ1 & 0,17 & 0,76 & 0,00 & 0,00 & 0,00 \\
\hline IGHV3 - IGHD8_as_[21] - IGHJ1 & 0,34 & 0,76 & 0,00 & 0,60 & 0,00 \\
\hline IGHV3 - IGHD8_s_BTA21 - IGHJ6_[22] & 0,17 & 0,76 & 0,00 & 0,00 & 0,00 \\
\hline IGHV3 - IGHD8_s_BTA21 - IGHJ1 & 2,35 & 0,76 & 1,43 & 2,99 & 3,77 \\
\hline IGHV3 - IGHDQ52_s_BTA8 - IGHJ1 & 2,18 & 0,00 & 1,43 & 2,99 & 3,77 \\
\hline IGHV3 - IGHDS10 [22] - IGHJ1 & 0,67 & 2,29 & 0,00 & 0,60 & 0,00 \\
\hline IGHV3 - IGHDS14 [22] - IGHJ1 & 0,17 & 0,00 & 0,00 & 0,00 & 0,63 \\
\hline IGHV6 - IGHD1_s_BTA7 - IGHJ1 & 1,01 & 1,53 & 0,71 & 1,80 & 0,00 \\
\hline IGHV6 - IGHD2_s_BTA7 - IGHJ1 & 0,17 & 0,00 & 0,00 & 0,60 & 0,00 \\
\hline IGHV6 - IGHD2_s_[39] - IGHJ1 & 0,17 & 0,00 & 0,00 & 0,60 & 0,00 \\
\hline IGHV6 - IGHD3_as_[39] - IGHJ1 & 0,17 & 0,00 & 0,00 & 0,00 & 0,63 \\
\hline IGHV6 - IGHD3_s_BTA7 - IGHJ1 & 1,68 & 0,00 & 0,71 & 2,40 & 3,14 \\
\hline IGHV6 - IGHD4_as_[21] - IGHJ1 & 0,34 & 0,00 & 0,00 & 0,00 & 1,26 \\
\hline IGHV6 - IGHD4_s_BTA8 - IGHJ1 & 0,50 & 0,00 & 1,43 & 0,00 & 0,63 \\
\hline IGHV6 - IGHD5_s_BTA8 - IGHJ1 & 1,34 & 0,76 & 2,86 & 1,20 & 0,63 \\
\hline IGHV6 - IGHD5_s_NW_001503306 - IGHJ1 & 0,50 & 0,76 & 0,71 & 0,60 & 0,00 \\
\hline IGHV6 - IGHD7_s_BTA8 - IGHJ1 & 0,50 & 0,00 & 0,00 & 0,60 & 1,26 \\
\hline IGHV6 - IGHD8_s_BTA21 - IGHJ1 & 1,34 & 3,82 & 0,71 & 0,60 & 0,63 \\
\hline IGHV6 - IGHDQ52_s_BTA8 - IGHJ1 & 1,17 & 0,76 & 1,43 & 1,80 & 0,63 \\
\hline IGHV6 - IGHDS10 [22] - IGHJ1 & 0,34 & 0,00 & 0,71 & 0,60 & 0,00 \\
\hline IGHV10 - IGHD1_s_BTA7 - IGHJ1 & 0,67 & 0,76 & 0,00 & 1,20 & 0,63 \\
\hline IGHV10 - IGHD2_s_BTA7 - IGHJ1 & 0,50 & 1,53 & 0,00 & 0,60 & 0,00 \\
\hline IGHV10 - IGHD3_s_BTA7 - IGHJ1 & 0,34 & 0,76 & 0,00 & 0,60 & 0,00 \\
\hline IGHV10 - IGHD4_as_[21] - IGHJ1 & 0,50 & 0,00 & 0,71 & 1,20 & 0,00 \\
\hline IGHV10 - IGHD4_s_BTA8 - IGHJ1 & 0,84 & 0,76 & 0,00 & 1,20 & 1,26 \\
\hline IGHV10 - IGHD5_s_BTA8 - IGHJ6_[22] & 0,17 & 0,00 & 0,00 & 0,00 & 0,63 \\
\hline IGHV10 - IGHD5_s_BTA8 - IGHJ1 & 0,17 & 0,00 & 0,00 & 0,60 & 0,00 \\
\hline IGHV10 - IGHD5_s_NW_001503306 - IGHJ1 & 0,17 & 0,00 & 0,00 & 0,00 & 0,63 \\
\hline IGHV10 - IGHD8_s_BTA21 - IGHJ1 & 1,17 & 1,53 & 0,71 & 0,60 & 1,89 \\
\hline IGHV10 - IGHDQ52_s_BTA8 - IGHJ1 & 0,17 & 0,76 & 0,00 & 0,00 & 0,00 \\
\hline IGHV10 - IGHDS14 [22] - IGHJ1 & 0,34 & 0,76 & 0,00 & 0,00 & 0,63 \\
\hline IGHV17 - IGHD1_s_BTA7 - IGHJ1 & 0,50 & 0,76 & 1,43 & 0,00 & 0,00 \\
\hline IGHV17 - IGHD3_s_BTA7 - IGHJ1 & 0,84 & 0,76 & 0,71 & 1,80 & 0,00 \\
\hline IGHV17 - IGHD4_s_BTA8 - IGHJ1 & 0,50 & 0,00 & 1,43 & 0,00 & 0,63 \\
\hline
\end{tabular}


Chapter 3: Gene conversion and combinatorial diversity in bovine heavy chains

\begin{tabular}{|l|r|r|r|r|r|}
\hline IGHV17 - IGHD5_s_BTA8 - IGHJ1 & 0,34 & 0,00 & 0,71 & 0,60 & 0,00 \\
\hline IGHV17 - IGHD5_s_NW_001503306 - IGHJ1 & 0,17 & 0,00 & 0,71 & 0,00 & 0,00 \\
\hline IGHV17 - IGHD7_s_BTA8 - IGHJ1 & 0,67 & 0,76 & 0,00 & 0,60 & 1,26 \\
\hline IGHV17 - IGHD8_s_BTA21 - IGHJ1 & 0,50 & 0,76 & 0,00 & 1,20 & 0,00 \\
\hline IGHV17 - IGHDQ52_s_BTA8 - IGHJ1 & 0,50 & 0,00 & 0,71 & 1,20 & 0,00 \\
\hline IGHV17 - IGHDS14 [22] - IGHJ1 & 0,17 & 0,00 & 0,71 & 0,00 & 0,00 \\
\hline IGHV36 - IGHD1_s_BTA7 - IGHJ1 & 0,34 & 0,00 & 0,71 & 0,00 & 0,63 \\
\hline IGHV36 - IGHD2_s_BTA7 - IGHJ1 & 0,50 & 0,00 & 0,71 & 0,60 & 0,63 \\
\hline IGHV36 - IGHD2_s_[39] - IGHJ1 & 0,17 & 0,76 & 0,00 & 0,00 & 0,00 \\
\hline IGHV36 - IGHD3_s_BTA7 - IGHJ1 & 1,84 & 0,76 & 1,43 & 1,80 & 3,14 \\
\hline IGHV36 - IGHD4_s_BTA8 - IGHJ1 & 0,67 & 1,53 & 0,00 & 0,00 & 1,26 \\
\hline IGHV36 - IGHD5_s_BTA8 - IGHJ1 & 2,01 & 0,76 & 2,86 & 2,40 & 1,89 \\
\hline IGHV36 - IGHD5_s_NW_001503306 - IGHJ1 & 1,84 & 0,00 & 3,57 & 0,60 & 3,14 \\
\hline IGHV36 - IGHD6_s_BTA8 - IGHJ1 & 0,17 & 0,76 & 0,00 & 0,00 & 0,00 \\
\hline IGHV36 - IGHD7_s_BTA8 - IGHJ1 & 0,50 & 0,00 & 1,43 & 0,60 & 0,00 \\
\hline IGHV36 - IGHD8_s_BTA21 - IGHJ1 & 3,52 & 9,16 & 3,57 & 1,80 & 0,63 \\
\hline IGHV36 - IGHDQ52_s_BTA8 - IGHJ1 & 1,01 & 1,53 & 1,43 & 0,00 & 1,26 \\
\hline IGHV36 - IGHDS10 [22] - IGHJ1 & 0,50 & 0,76 & 0,71 & 0,00 & 0,63 \\
\hline IGHV36 - IGHDS14 [22] - IGHJ1 & 0,34 & 0,76 & 0,00 & 0,00 & 0,63 \\
\hline
\end{tabular}

${ }^{1}$ antisense

${ }^{2}$ sense 
Chapter 3: Gene conversion and combinatorial diversity in bovine heavy chains

\section{S5 Table. Recombinations of IGHV, IGHD, and IGHJ over all four cattle breeds} using procedure 2.

\begin{tabular}{|c|c|c|c|c|c|}
\hline IGHV - IGHD - IGHJ & $\begin{array}{r}\text { all } \\
(n=597)\end{array}$ & $\begin{array}{r}\text { GS } \\
(n=131)\end{array}$ & $\begin{array}{r}\text { GBP } \\
(n=140)\end{array}$ & $\begin{array}{r}A \\
(n=167)\end{array}$ & $\begin{array}{r}\text { HF } \\
(n=159)\end{array}$ \\
\hline IGHV1S26 - IGHDQ52_s'_BTA8 - IGHJ1 & 0,17 & 0,76 & 0,00 & 0,00 & 0,00 \\
\hline IGHV1S28 - IGHD1_s_BTA7 - IGHJ1 & 1,01 & 0,00 & 2,14 & 1,20 & 0,63 \\
\hline IGHV1S28 - IGHD2_s_[39] - IGHJ1 & 0,17 & 0,00 & 0,00 & 0,00 & 0,63 \\
\hline IGHV1S28 - IGHD3_s_BTA7 - IGHJ1 & 0,50 & 0,76 & 0,71 & 0,00 & 0,63 \\
\hline IGHV1S28 - IGHD4_as²_[21] - IGHJ1 & 0,17 & 0,00 & 0,00 & 0,60 & 0,00 \\
\hline IGHV1S28 - IGHD4_s_BTA8 - IGHJ1 & 0,67 & 1,53 & 0,71 & 0,60 & 0,00 \\
\hline IGHV1S28 - IGHD6_s_BTA8 - IGHJ6_[22] & 0,17 & 0,00 & 0,00 & 0,00 & 0,63 \\
\hline IGHV1S28 - IGHD6_s_BTA8 - IGHJ1 & 0,34 & 0,00 & 0,71 & 0,00 & 0,63 \\
\hline IGHV1S28 - IGHD8_s_BTA21 - IGHJ1 & 0,50 & 2,29 & 0,00 & 0,00 & 0,00 \\
\hline IGHV1S28 - IGHDQ52_s_BTA8 - IGHJ1 & 3,18 & 3,05 & 2,86 & 2,40 & 4,40 \\
\hline IGHV1S28 - IGHDS10 [22] - IGHJ1 & 1,51 & 0,76 & 1,43 & 1,80 & 1,89 \\
\hline IGHV1S32 - IGHD1_s_BTA7 - IGHJ1 & 0,50 & 1,53 & 0,00 & 0,00 & 0,63 \\
\hline IGHV1S32 - IGHD7_s_BTA8 - IGHJ1 & 0,17 & 0,00 & 0,00 & 0,00 & 0,63 \\
\hline IGHV1S32 - IGHDQ52_s_BTA8 - IGHJ1 & 0,17 & 0,76 & 0,00 & 0,00 & 0,00 \\
\hline IGHV1S33 - IGHD1_as_[39] - IGHJ1 & 0,17 & 0,00 & 0,00 & 0,60 & 0,00 \\
\hline IGHV1S33 - IGHD2_s_BTA7 - IGHJ1 & 0,17 & 0,76 & 0,00 & 0,00 & 0,00 \\
\hline IGHV1S33 - IGHD3_s_BTA7 - IGHJ1 & 0,34 & 0,00 & 0,00 & 1,20 & 0,00 \\
\hline IGHV1S33 - IGHDQ52_s_BTA8 - IGHJ1 & 0,67 & 0,76 & 0,71 & 1,20 & 0,00 \\
\hline IGHV1S33 - IGHDS10 [22] - IGHJ1 & 0,17 & 0,00 & 0,00 & 0,60 & 0,00 \\
\hline IGHV1S34 - IGHD1_as_[39] - IGHJ1 & 0,17 & 0,00 & 0,71 & 0,00 & 0,00 \\
\hline IGHV1S34 - IGHD1_s_BTA7 - IGHJ1 & 1,34 & 0,76 & 1,43 & 2,99 & 0,00 \\
\hline IGHV1S34 - IGHD4_as_[21] - IGHJ1 & 0,17 & 0,00 & 0,00 & 0,60 & 0,00 \\
\hline IGHV1S34 - IGHD4_s_BTA8 - IGHJ1 & 0,17 & 0,00 & 0,71 & 0,00 & 0,00 \\
\hline IGHV1S34 - IGHD5_s_BTA8 - IGHJ1 & 0,34 & 0,76 & 0,71 & 0,00 & 0,00 \\
\hline IGHV1S34 - IGHD5_s_NW_001503306 - IGHJ1 & 0,34 & 0,76 & 0,71 & 0,00 & 0,00 \\
\hline IGHV1S34 - IGHD6_as_[21] - IGHJ1 & 0,17 & 0,00 & 0,71 & 0,00 & 0,00 \\
\hline IGHV1S34 - IGHD6_s_BTA8 - IGHJ1 & 0,17 & 0,00 & 0,71 & 0,00 & 0,00 \\
\hline IGHV1S34 - IGHD8_as_[21] - IGHJ1 & 0,17 & 0,00 & 0,71 & 0,00 & 0,00 \\
\hline IGHV1S34 - IGHD8_s_BTA21 - IGHJ1 & 0,67 & 1,53 & 0,71 & 0,00 & 0,63 \\
\hline IGHV1S34 - IGHDQ52_s_BTA8 - IGHJ1 & 3,18 & 5,34 & 2,86 & 1,80 & 3,14 \\
\hline IGHV1S34 - IGHDS10 [22] - IGHJ1 & 1,51 & 0,00 & 3,57 & 0,60 & 1,89 \\
\hline IGHV1S34 - IGHDS14 [22] - IGHJ1 & 0,17 & 0,76 & 0,00 & 0,00 & 0,00 \\
\hline IGHV1S35 - IGHD1_s_BTA7 - IGHJ1 & 0,17 & 0,76 & 0,00 & 0,00 & 0,00 \\
\hline IGHV1S35 - IGHD4_s_BTA8 - IGHJ1 & 1,17 & 3,82 & 0,71 & 0,00 & 0,63 \\
\hline IGHV1S35 - IGHD5_s_BTA8 - IGHJ1 & 0,17 & 0,76 & 0,00 & 0,00 & 0,00 \\
\hline IGHV1S35 - IGHD8_s_BTA21 - IGHJ1 & 0,34 & 1,53 & 0,00 & 0,00 & 0,00 \\
\hline IGHV1S35 - IGHDQ52_s_BTA8 - IGHJ1 & 1,34 & 3,82 & 0,00 & 1,20 & 0,63 \\
\hline IGHV1S35 - IGHDS10 [22] - IGHJ1 & 0,84 & 0,76 & 0,71 & 1,80 & 0,00 \\
\hline IGHV1S37 - IGHD4_s_BTA8 - IGHJ1 & 0,17 & 0,00 & 0,00 & 0,00 & 0,63 \\
\hline
\end{tabular}


Chapter 3: Gene conversion and combinatorial diversity in bovine heavy chains

\begin{tabular}{|c|c|c|c|c|c|}
\hline IGHV1S37 - IGHDS10 [22] - IGHJ1 & 0,17 & 0,00 & 0,00 & 0,00 & 0,63 \\
\hline IGHV1S38 - IGHD4_s_BTA8 - IGHJ1 & 0,17 & 0,00 & 0,00 & 0,00 & 0,63 \\
\hline IGHV1S38 - IGHD6_s_BTA8 - IGHJ1 & 0,17 & 0,76 & 0,00 & 0,00 & 0,00 \\
\hline IGHV1S39 - IGHD1_s_BTA7 - IGHJ1 & 0,50 & 0,76 & 0,00 & 1,20 & 0,00 \\
\hline IGHV1S39 - IGHD2_s_BTA7 - IGHJ1 & 0,67 & 0,00 & 0,71 & 1,20 & 0,63 \\
\hline IGHV1S39 - IGHD2_s_[39] - IGHJ1 & 0,17 & 0,00 & 0,71 & 0,00 & 0,00 \\
\hline IGHV1S39 - IGHD3_s_BTA7 - IGHJ1 & 0,84 & 0,00 & 1,43 & 1,20 & 0,63 \\
\hline IGHV1S39 - IGHD4_as_[21] - IGHJ1 & 0,50 & 0,00 & 0,00 & 1,80 & 0,00 \\
\hline IGHV1S39 - IGHD4_s_BTA8 - IGHJ1 & 2,35 & 0,00 & 0,71 & 5,39 & 2,52 \\
\hline IGHV1S39 - IGHD5_s_BTA8 - IGHJ1 & 0,34 & 0,76 & 0,00 & 0,60 & 0,00 \\
\hline IGHV1S39 - IGHD6_as_[21] - IGHJ1 & 0,17 & 0,00 & 0,71 & 0,00 & 0,00 \\
\hline IGHV1S39 - IGHD6_s_BTA8 - IGHJ1 & 0,34 & 0,00 & 0,00 & 1,20 & 0,00 \\
\hline IGHV1S39 - IGHD7_s_BTA8 - IGHJ1 & 0,50 & 0,00 & 0,71 & 0,00 & 1,26 \\
\hline IGHV1S39 - IGHD8_s_BTA21 - IGHJ1 & 1,68 & 0,00 & 2,14 & 2,40 & 1,89 \\
\hline IGHV1S39 - IGHDQ52_s_BTA8 - IGHJ6_[22] & 0,17 & 0,00 & 0,00 & 0,00 & 0,63 \\
\hline IGHV1S39 - IGHDQ52_s_BTA8 - IGHJ1 & 7,04 & 1,53 & 5,00 & 11,38 & 8,81 \\
\hline IGHV1S39 - IGHDS10 [22] - IGHJ1 & 2,35 & 0,00 & 1,43 & 4,19 & 3,14 \\
\hline IGHV1S40 - IGHD1_as_[39] - IGHJ1 & 0,17 & 0,00 & 0,00 & 0,60 & 0,00 \\
\hline IGHV1S40 - IGHD1_s_BTA7 - IGHJ1 & 0,34 & 0,00 & 1,43 & 0,00 & 0,00 \\
\hline IGHV1S40 - IGHD3_s_BTA7 - IGHJ1 & 0,50 & 1,53 & 0,71 & 0,00 & 0,00 \\
\hline IGHV1S40 - IGHD4_s_BTA8 - IGHJ1 & 0,50 & 0,00 & 0,00 & 0,00 & 1,89 \\
\hline IGHV1S40 - IGHD5_s_BTA8 - IGHJ1 & 0,50 & 0,76 & 1,43 & 0,00 & 0,00 \\
\hline IGHV1S40 - IGHD6_s_BTA8 - IGHJ1 & 0,34 & 0,00 & 0,71 & 0,60 & 0,00 \\
\hline IGHV1S40 - IGHD8_s_BTA21 - IGHJ6_[22] & 0,17 & 0,00 & 0,71 & 0,00 & 0,00 \\
\hline IGHV1S40 - IGHD8_s_BTA21 - IGHJ1 & 0,67 & 0,76 & 0,00 & 1,80 & 0,00 \\
\hline IGHV1S40 - IGHDQ52_s_BTA8 - IGHJ1 & 6,37 & 6,87 & 9,29 & 3,59 & 6,29 \\
\hline IGHV1S40 - IGHDS10 [22] - IGHJ1 & 2,68 & 5,34 & 1,43 & 1,80 & 2,52 \\
\hline IGHV3 - IGHD1_as_[39] - IGHJ1 & 1,01 & 0,00 & 1,43 & 1,20 & 1,26 \\
\hline IGHV3 - IGHD1_s_BTA7 - IGHJ6_[22] & 0,17 & 0,76 & 0,00 & 0,00 & 0,00 \\
\hline IGHV3 - IGHD1_s_BTA7 - IGHJ1 & 0,84 & 1,53 & 0,00 & 0,60 & 1,26 \\
\hline IGHV3 - IGHD2_s_BTA7 - IGHJ1 & 0,67 & 0,76 & 0,71 & 0,60 & 0,63 \\
\hline IGHV3 - IGHD4_as_[21] - IGHJ1 & 1,01 & 1,53 & 0,00 & 1,20 & 1,26 \\
\hline IGHV3 - IGHD4_s_BTA8 - IGHJ1 & 1,34 & 1,53 & 0,00 & 0,60 & 3,14 \\
\hline IGHV3 - IGHD5_s_BTA8 - IGHJ1 & 0,17 & 0,00 & 0,71 & 0,00 & 0,00 \\
\hline IGHV3 - IGHD5_s_NW_001503306 - IGHJ1 & 0,17 & 0,00 & 0,00 & 0,00 & 0,63 \\
\hline IGHV3 - IGHD6_s_BTA8 - IGHJ1 & 0,34 & 0,00 & 0,00 & 0,60 & 0,63 \\
\hline IGHV3 - IGHD7_s_BTA8 - IGHJ1 & 0,17 & 0,76 & 0,00 & 0,00 & 0,00 \\
\hline IGHV3 - IGHD8_S_BTA21 - IGHJ1 & 2,01 & 1,53 & 2,86 & 0,60 & 3,14 \\
\hline IGHV3 - IGHDQ52_s_BTA8 - IGHJ6_[22] & 0,17 & 0,00 & 0,00 & 0,00 & 0,63 \\
\hline IGHV3 - IGHDQ52_s_BTA8 - IGHJ1 & 4,02 & 2,29 & 5,71 & 4,19 & 3,77 \\
\hline IGHV3 - IGHDS10 [22] - IGHJ6_[22] & 0,17 & 0,00 & 0,00 & 0,60 & 0,00 \\
\hline IGHV3 - IGHDS10 [22] - IGHJ1 & 2,18 & 1,53 & 1,43 & 1,80 & 3,77 \\
\hline IGHV6 - IGHD1_s_BTA7 - IGHJ1 & 1,01 & 0,00 & 0,71 & 2,99 & 0,00 \\
\hline IGHV6 - IGHD3_s_BTA7 - IGHJ1 & 0,50 & 0,00 & 1,43 & 0,00 & 0,63 \\
\hline
\end{tabular}


Chapter 3: Gene conversion and combinatorial diversity in bovine heavy chains

\begin{tabular}{|c|c|c|c|c|c|}
\hline IGHV6 - IGHD4_as_[21] - IGHJ1 & 0,50 & 0,00 & 0,00 & 0,60 & 1,26 \\
\hline IGHV6 - IGHD4_s_BTA8 - IGHJ1 & 0,34 & 0,00 & 0,00 & 0,60 & 0,63 \\
\hline IGHV6 - IGHD5_s_BTA8 - IGHJ1 & 0,50 & 0,76 & 0,71 & 0,00 & 0,63 \\
\hline IGHV6 - IGHD6_as_[21] - IGHJ1 & 0,17 & 0,00 & 0,71 & 0,00 & 0,00 \\
\hline IGHV6 - IGHD7_s_BTA8 - IGHJ1 & 0,17 & 0,00 & 0,00 & 0,00 & 0,63 \\
\hline IGHV6 - IGHD8_s_BTA21 - IGHJ1 & 0,34 & 0,00 & 0,71 & 0,00 & 0,63 \\
\hline IGHV6 - IGHDQ52_s_BTA8 - IGHJ1 & 3,85 & 6,11 & 3,57 & 4,19 & 1,89 \\
\hline IGHV6 - IGHDS10 [22] - IGHJ1 & 1,68 & 0,00 & 1,43 & 2,40 & 2,52 \\
\hline IGHV6 - IGHDS14 [22] - IGHJ1 & 0,17 & 0,76 & 0,00 & 0,00 & 0,00 \\
\hline IGHV10 - IGHD1_as_[39] - IGHJ1 & 0,17 & 0,00 & 0,00 & 0,00 & 0,63 \\
\hline IGHV10 - IGHD1_s_BTA7 - IGHJ1 & 1,01 & 2,29 & 0,00 & 1,20 & 0,63 \\
\hline IGHV10 - IGHD3_s_BTA7 - IGHJ1 & 0,34 & 0,00 & 0,00 & 0,00 & 1,26 \\
\hline IGHV10 - IGHD4_as_[21] - IGHJ1 & 0,34 & 0,00 & 0,00 & 1,20 & 0,00 \\
\hline IGHV10 - IGHD4_s_BTA8 - IGHJ1 & 1,01 & 2,29 & 0,00 & 1,20 & 0,63 \\
\hline IGHV10 - IGHD6_s_BTA8 - IGHJ6_[22] & 0,17 & 0,00 & 0,00 & 0,00 & 0,63 \\
\hline IGHV10 - IGHD8_s_BTA21 - IGHJ1 & 1,17 & 1,53 & 0,71 & 2,40 & 0,00 \\
\hline IGHV10 - IGHDQ52_s_BTA8 - IGHJ1 & 0,50 & 0,76 & 0,00 & 0,00 & 1,26 \\
\hline IGHV10 - IGHDS10 [22] - IGHJ1 & 0,34 & 0,00 & 0,71 & 0,00 & 0,63 \\
\hline IGHV17 - IGHD3_s_BTA7 - IGHJ1 & 0,34 & 0,00 & 0,71 & 0,60 & 0,00 \\
\hline IGHV17 - IGHD4_s_BTA8 - IGHJ1 & 0,50 & 0,00 & 0,00 & 1,80 & 0,00 \\
\hline IGHV17 - IGHD5_s_BTA8 - IGHJ1 & 0,34 & 0,76 & 0,71 & 0,00 & 0,00 \\
\hline IGHV17 - IGHD6_as_[21] - IGHJ1 & 0,17 & 0,00 & 0,71 & 0,00 & 0,00 \\
\hline IGHV17 - IGHD8_s_BTA21 - IGHJ1 & 0,50 & 0,00 & 0,71 & 0,00 & 1,26 \\
\hline IGHV17 - IGHDQ52_s_BTA8 - IGHJ1 & 1,51 & 0,76 & 2,86 & 1,80 & 0,63 \\
\hline IGHV17 - IGHDS10 [22] - IGHJ1 & 0,67 & 0,76 & 0,71 & 1,20 & 0,00 \\
\hline IGHV17 - IGHDS14 [22] - IGHJ1 & 0,17 & 0,76 & 0,00 & 0,00 & 0,00 \\
\hline IGHV36 - IGHD1_as_[39] - IGHJ1 & 0,17 & 0,76 & 0,00 & 0,00 & 0,00 \\
\hline IGHV36 - IGHD1_s_BTA7 - IGHJ1 & 1,17 & 0,00 & 0,71 & 0,60 & 3,14 \\
\hline IGHV36 - IGHD3_s_BTA7 - IGHJ1 & 0,34 & 0,00 & 0,71 & 0,00 & 0,63 \\
\hline IGHV36 - IGHD4_as_[21] - IGHJ1 & 0,17 & 0,76 & 0,00 & 0,00 & 0,00 \\
\hline IGHV36 - IGHD4_s_BTA8 - IGHJ1 & 1,34 & 1,53 & 0,71 & 0,60 & 2,52 \\
\hline IGHV36 - IGHD5_s_BTA8 - IGHJ1 & 0,34 & 0,00 & 0,71 & 0,60 & 0,00 \\
\hline IGHV36 - IGHD5_s_NW_001503306 - IGHJ1 & 0,17 & 0,00 & 0,71 & 0,00 & 0,00 \\
\hline IGHV36 - IGHD8_s_BTA21 - IGHJ1 & 1,17 & 1,53 & 1,43 & 0,00 & 1,89 \\
\hline IGHV36 - IGHDQ52_s_BTA8 - IGHJ1 & 6,37 & 11,45 & 7,14 & 4,19 & 3,77 \\
\hline IGHV36 - IGHDS10 [22] - IGHJ1 & 2,18 & 0,76 & 4,29 & 1,80 & 1,89 \\
\hline
\end{tabular}


Chapter 4:

General Discussion 


\section{General Discussion}

Since the resistance of antigens to antibiotics remains a significant problem in livestock production due to considerable economic impacts, research in the alternate treatment of infectious diseases increased. The investigation of special genetic aspects of immunoglobulins has attracted higher attention during the last few decades. New high throughput technologies are available that facilitate and advance the experimental workflow. The price for analyses of the immunoglobulin repertoires is descending. Initial studies on the immunoglobulin repertoire were performed on humans and mice but successful techniques were applied to various other animals such as chicken, rabbit, cattle, horses, and zebrafish, too. Nevertheless, investigation in immunoglobulin genetics still needs more attention to complete previous information about fundamentals, such as the number of genomic available gene segments, gene families, and allotypes of different isotypes of the immunoglobulin heavy and light chains in cattle and horses.

The primary objective of this thesis was to analyze the genomic available and transcribed immunoglobulin heavy and light chain gene segments to contribute to a more detailed understanding of immunoglobulin diversity in cattle and horses.

Similar to most vertebrates, bovine and equine immunoglobulins possess two identical heavy and light chains that consist of a variable region and a constant region. The variable regions are created by the random fusion of germline variable (IGHV/IGLV/IGKV), diversity (IGHD), and joining gene segments (IGHJ/IGLJ/IGKJ) that are combined with a constant region gene (IGHC/IGLC/IGKC) (Tonegawa 1983). The gene segments are found in species-specific numbers in the genome. The immunoglobulin repertoire is generated by several processes of combinatorial and junctional diversity comprising the imprecise joining of the single gene segments and non-templated or palindromic nucleotide insertions between two adjacent gene segments, as well as somatic hypermutations. Secondary mechanisms such as gene conversion and isotype switch may increase the diversity further. Highly specialized, complementarity determining regions form a perfect counterpart to the antigen epitope and are stabilized by conserved framework regions (Kabat and Wu 1991).

In mammals, two types of light chains are described - the lambda and kappa light chains (Korngold and Lipari 1956). They are expressed in species-specific ratios. In 
contrast to humans and mice, where the kappa isotype dominates in serum antibodies, the lambda isotype is predominantly found in cattle and horses (Arun et al. 1996; Almagro et al. 1998). In addition to what is already known about the general structural features of both bovine and equine immunoglobulins and their function, this thesis on diversity gave some important baseline information and contributes to the scientific background for the production of highly specific and effective recombinant antibodies or antibody libraries. The findings will also be of future importance in analyzing seroconversion data after infection or vaccination, as well as determining breed specific differences to select healthy, robust animals.

Since the late $19^{\text {th }}$ century, horse antibodies in terms of anti-sera were closely connected with the treatment of human diseases. Today, equine immunoglobulins are also used to support immunosuppression after organ or stem cell transplantation, or to manage autoimmune diseases in humans (Leleu et al. 2006; Zand 2006). In addition, equine antibody applications gain in importance in order to prevent or treat equine infectious diseases. Amongst others they can be regarded as potential alternatives to antibiotic therapy in the near future. Therefore it is necessary to investigate the fundamental immunoglobulin genetics underlying the equine immunoglobulin immune response.

The understanding of the organization of equine immunoglobulin genes has increased significantly in recent years (reviewed in publication 1). For equine heavy chains, 52 IGHV, 40 IGHD, 8 IGHJ and 11 IGHC were determined. With 40 IGHD identified, horses belong to the mammalian species that possess the most IGHD. Likewise, in guinea pig and the African elephant 41 and 87 IGHD gene segments are known so far (Guo et al. 2011; Guo et al. 2012). Seven of the equine IGHCs are gamma chain genes ( $\lg G$ ). Only in the porcine immune system a similarly high number of eleven genomic constant region genes representing six putative subclasses has been described (Butler et al. 2009).

Using biochemical and serological methods, the subclasses $\lg G a, \lg G b, \lg G c, \lg G(T)$, and $\lg \mathrm{G}(\mathrm{B})$ were identified and characterized (Rockey et al. 1964; Sandor et al. 1964; Klinman et al. 1965; Rockey 1967; Widders et al. 1986; Sheoran and Holmes 1996). Later, seven IgG isotypes were identified by the analysis of a BAC library. The previous subclasses were renamed. Multiple duplications, gene conversions, and crossovers are supposed to explain these seven IgG isotypes (Wagner et al. 2006). The individual 
IgG subclasses possess specific roles in protective immunity. Thus, the FcyR and complement-binding isotypes $\lg G 1$, lgG4, and $\lg G 7$ (former $\lg G a$ and $\lg G b$ ) contribute to the protection against several equine pathogens such as equine influenza virus (Nelson et al. 1998; Breathnach et al. 2006), Streptococcus equi (Sheoran et al. 1997) and Rhodococcus equi (Lopez et al. 2002). In addition, systemic and mucosal IgG responses were described to play an important role in limiting the spread and severity of equine herpes virus 1 (Kydd et al. 2006). The first recombinant versions of all seven equine $\lg G$ subclasses (mouse $\lambda$-light chains, horse IGHG1-7) were expressed in Chinese Hamster Ovary cells to analyze their individual physical and biological properties (Lewis et al. 2008). Further, seven distinct monoclonal equine antibodies ( $\lg M$, IgG1, $\lg G 3$, $\lg G 4 / 7$, $\lg G 5$, $\lg G 6$, and $\lg E$ ) were produced in equine-murine heterohybridomas to be used for quantification of isotypes in diagnostic testing and immunological research (Keggan et al. 2013).

The combinatorial and junctional levels of IGHV-IGHD-IGHJ sequences in equine fetus were comparable to those of adult horses and a similar set of variable gene segments was used during fetal and post-natal life stages (Tallmadge et al. 2009; Tallmadge et al. 2013). Few IGHV segments were used predominantly at all ages. The 250,000 bases between the expressed gene segments suggest that the entire IGHV locus is available throughout equine life (Tallmadge et al. 2013). Similarly, in fetal piglets the IGHV usage was described to be independent of the genome position although there is a limited number of IGHV representing the Ig repertoire, too (Eguchi-Ogawa et al. 2010; Butler et al. 2011). In contrast, the biased usage of germline IGHV in the mouse fetus was explained with different accessible positions within the IGHV locus (Jeong et al. 1988). The incorporation of more than one IGHD into the IGHV-IGHD-IGHJ rearrangement resulting in IGHV-IGHD-IGHD-IGHJ was observed (Sun et al. 2010). In horses, sequence diversity and length variation further increased in complementarity determining region $1(\mathrm{CDR} 1 \mathrm{H})$ and $\mathrm{CDR} 2 \mathrm{H}$, and framework regions in accordance to somatic hypermutation (Tallmadge et al. 2013). Within the CDR3H of an adult horse, at least 5 amino acid residues were observed, whereas 25 amino acid residues were counted in the longest $\mathrm{CDR} 3 \mathrm{H}$, which was identified in an equine neonate (Tallmadge et al. 2013). In comparison, recent studies showed the length heterogeneity of very short CDR3H (5-10 amino acids), midlength CDR3H (11-31 amino acids) and exceptionally long CDR3H (more than 47 amino acids) in all isotypes in both bovine fetuses and adult cattle. Very long CDR3H contribute to diversity by uniquely folded 
small domains (Saini et al. 1999a; Saini and Kaushik 2002; Shojaei et al. 2003; Koti et al. 2010; Walther et al. 2013; Wang et al. 2013).

The equine kappa light chain contains 60 IGKV, 5 IGKJ and 1 IGKC, whereas there are 144 IGLV, 7 IGLJ, and 7 IGLC for the lambda light chain. Each of the 7 IGLJ is preceded by one IGLC and there are two clusters of IGLV possessing different transcriptional orientations. Within each IGLV cluster there are functional genes and pseudogenes (Sun et al. 2010). Similarly, pseudogenes are described in different species such as chicken, where it is already known that pseudogenes are used for gene conversion (Reynaud et al. 1985). During age development, a decrease in IGLVs is noted, although nucleotide diversity and significant differences in gene usage increased. As in all previous studies different methods were used for designation, the standardization of the existing nomenclature of immunoglobulin genes is suggested.

The study of allotypic variants within the equine $\lambda$-light chain locus (publication 2) was the first detailed molecular genetic description. Five IGLC1, four IGLC5, and two IGLC6/7 allotypic variants, as well as three alleles for IGLC6 $/ 7^{\text {a }}$ were observed. Especially for the transcriptional use of IGLC6/7, significant differences were calculated by $\mathrm{Chi}^{2}$ tests.

Several amino acid residue variations were located at the accessible surface as confirmed by homology-based predicted 3D structural analysis. Distinct serological properties resulting in allotypic variants due to these modifications are assumed. All allotypic variants showed distinct amino acid residue substitutions in all seven $\beta$-sheets according to the IMGT nomenclature (Lefranc et al. 2005). Most of them were located within the solvent accessible surface area (Padlan et al. 1986). Seven of these substitutions were positioned at the interface to the first constant region of the heavy chain (IGHC1). As one of these amino acid substitutions affected one of the conserved residues for the main interaction of constant region of the light chain (IGLC) and IGHC1, the stable assembly of heavy and light chains could be changed. We do not have any information about allelic changes in IGHC1, thus this assumption remains to be verified. Amino acid residue substitutions located at the solvent accessible surface area might be distinguished by specific sera and therefore might be used as markers. Previously, allotypic markers of human immunoglobulin chains were related to the susceptibility of different infectious diseases due to either direct association or linkage disequilibrium with the causative gene (Pandey et al. 1979; Granoff et al. 1984; Pandey 
et al. 1995; Pandey 2000; Giha et al. 2009). Furthermore, influences of bovine allotypes on effector functions like complement activation, and age dependent expression have also been shown (Corbeil et al. 1997; Bastida-Corcuera et al. 1999a; Bastida-Corcuera et al. 1999b; Bastida-Corcuera et al. 2000). Specific investigations on functional differences of allotypic variants are missing in horses, but similar to cattle heterozygotic horses are thought to possess an advantage in their humoral immune response.

Beside the presence of predicted germline IGLJ-IGLC pairs and the transcription frequency of IGLC-genes, the usage of IGLV-segments of subgroup 8 was further analyzed. This subgroup contains the highest number of functional IGLV and was shown to be transcribed preferentially (Sun et al. 2010). The variable segments IGLV15, IGLV17, and IGLV25 were preferably transcribed. Significant differences were calculated for the rearrangements with the four IGLC-isotypes transcribed within and among the breeds. Consequently, the V-domain repertoire is dominated by two breed independent IGLV genes and a third breed specific IGLV gene that are productively used for combinatorial joining of IGLJ-IGLC-genes and thus for antibody production.

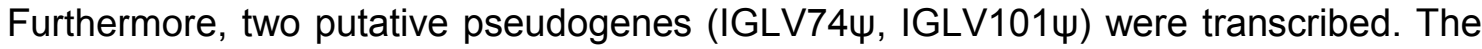
isolated sequences showed variations in CDR1 sequences resulting in a frame shift replacing the premature stop codons of germline IGLV74 $\Psi$. Individual mutations within the breeds are supposed to enable the transcriptional use of pseudogenes. In addition, in silico germline sequences are prepared from a Thoroughbred, which is a conserved breed founded by few stallions. Hence, mutations resulting in pseudogenes may be segregate within this breed but can be functional in other breeds. The transcription of pseudo V-gene segments has already been described in species such as cattle, chicken, and rabbits and occurs by gene conversion (Parng et al. 1996; Winstead et al. 1999; Arakawa and Buerstedde 2009). The results of this study indicated the presence of similar mechanisms in horses. Moreover, the spacer sequences of the recombination signal sequences of the transcribed IGLVs seem to be conserved (Sun et al. 2010). In IGLV101\%, the spacer sequence is identical to that in IGLV17 and IGLV15, which are the dominantly used IGLV. These identical sequences of the spacers are an additional option for the transcription of potential pseudogenes as was already shown in human IGKV segments (Nadel et al. 1998).

Some of the results could be linked to breeding because two breeds with different stud book sizes and breeding goals were examined. While Rhenish German Coldblood is a 
quiet conserved and small breed mainly used for agricultural purposes and breed representations (Bremond and Balzer 2011), animals of Hanoverian Warmblood belong to one of the biggest horse breeds and participate in all disciplines of equestrian sports (Bremond and Balzer 2011). Although the inbreeding coefficient in the Hanoverian population (1.33\%) is at lower level than those of smaller populations like the Rhenish German Coldblood (1.73\%; (Biedermann et al. 2002; Hamann and Distl 2008), effective management of breeding is necessary to prevent the random loss of alleles caused by large genetic contributions of few individuals over a long time period (bottleneck effect). Genetic drift and homogenization of genetic information may also occur and have to be controlled as the rate of inbreeding has increased in Hanoverians in the last ten years (Constans et al. 1985; Hamann and Distl 2008; Simianer and Kohn 2010).

This study characterized genetic and transcriptional differences of $\lambda$-light chain in two horse breeds. For the first time distinct alleles and putative allotypic variants were described in horses. Future investigations should evaluate the distribution of allotypic markers of immunoglobulin chains in further horse breeds. Moreover, the linkage to infectious diseases such as in humans and cattle should be examined.

The in silico analyses of the genomic organization of the bovine heavy-chain locus revealed differences from previous mapping and annotation results (publication 3). The functional locus was mapped to BTA21 (Gu et al. 1992; Hayes et al. 2000; Zhao et al. 2003). Additional bovine heavy-chain loci were detected on BTA7, BTA8, and BTA20, unexpectedly. The order of the respective segments deviated from other fully described mammalian loci (Matsuda and Honjo 1996; Sun et al. 2010). Further, the constant heavy-chain locus described by means of BAC clone analysis (Zhao et al. 2003), were not introduced into the genomic assembly. Several genes upstream of the heavy-chain locus on the bovine contig-NW_003064289 on BTA7 share homology with HSA19 but some did not show any human equivalent. Similarly, on HSA5pter no equivalent was found to the bovine IgG2(ORF) gene, although the adjacent genes were identified. Hybridization experiments assigned IGHML1 to BTA11 (Tobin-Janzen and Womack 1992; Hayes and Petit 1993), which was supported by the detection of six IGHJ segments on the same chromosome using BAC clone and locus-specific PCR analysis (Hosseini et al. 2004). Nevertheless, in silico analyses did not identify an IgMlike locus on BTA11. Consequently, the bovine immunoglobulin heavy-chain locus 
seems to be annotated incorrectly and incompletely. This problem may be solved by the re-sequencing of the described localizations and underpinned by different authors and methods.

All of the functional bovine IGHV segments are most closely related to the human clan II family $\mathrm{VH}$, which explains the exclusive transcription of only one bovine $\mathrm{VH}$ family (Sinclair and Aitken 1995; Berens et al. 1997; Sinclair et al. 1997). The second bovine $\mathrm{VH}$ family consists only of pseudogenes and clustered with the human VH4 and VH6 family. The description of the second bovine $\mathrm{VH}$ family (boVH2) previously proposed was now possible (Berens et al. 1997), but there are still no indications of possible gene conversions using boVH2 segments (Liljavirta et al. 2014) in the sequences investigated, as shown for the bovine $\lambda$-light chains (Parng et al. 1996). For further studies the assignment of fragmented loci of bovine IGHV to boVH1 or boVH2 based on sequence identity is recommended.

In bovine IgM, three groups of CDR3H defined by their number of amino acids were described. Independent of nucleotide addition during rearrangement long and short IGHD contribute directly to CDR3H length heterogeneity. Nevertheless, annotation of the transcribed IGHD is complicated due to high homologies among the genomic IGHD segments. In particular, CDR3H length heterogeneity, junctional flexibility, and somatic hypermutation within the recombined IGHD segments led to low sequence identities. Furthermore, intrinsic hot spots targeting somatic hypermutations to $\mathrm{CDR} 1 \mathrm{H}, \mathrm{CDR} 2 \mathrm{H}$, and $\mathrm{CDR} 3 \mathrm{H}$ were thought to contribute solely to $\lg \mathrm{M}$ antibody diversification in both bovine fetus and adult cattle (Saini and Kaushik 2002).

$\mathrm{CDR} 3 \mathrm{H}$ length heterogeneity was fond in all bovine immunoglobulin isotypes. Hence, exceptionally long $\mathrm{CDR} 3 \mathrm{H}$ are apparently not primarily generated to compensate the restricted flexibility of $\operatorname{lgM}$. As the most recent study on the $\lg G$ repertoire in calves also showed exceptionally long CDR3H (Larsen and Smith 2012), antigen selection of variable domains and class switch recombination seem to be of higher impact. The exceptionally long $\mathrm{CDR} 3 \mathrm{H}$ were generated by the direct fusion of a single IGHV segment (IGHV10), the longest IGHD segment (IGHD2), and one functional IGHJ segment (IGHJ1), as described previously (Koti et al. 2010). In short CDR3H, the preferred use of the short IGHD segments, IGHDQ52 and IGHD4 was observed. All IGHV segments identified were found to be functional. Thus, analyses do not reveal evidence for gene conversion in bovine immunoglobulin heavy chains, which contributes to the diversity of chicken immunoglobulin heavy chains and bovine $\lambda$-light 
chains (Reynaud et al. 1994; Parng et al. 1996). Both in intermediate and exceptionally long $\mathrm{CDR} 3 \mathrm{H}$ conserved short nucleotide sequences (CSNS) were inserted into the IGHV-IGHD junction. This mechanism contributes to antibody diversification in all immunoglobulin heavy chain isotypes and CDR3H lengths. As the insertion of CSNS is supposed to directly follow antigen exposure (Koti et al. 2010), class switch recombination is supposed to be responsible for isotype-independent exceptionally long $\mathrm{CDR} 3 \mathrm{H}$ in cattle. In addition, exceptionally long $\mathrm{CDR} 3 \mathrm{H}$ do not have a conventional antigen binding site because these $\mathrm{CDR} 3 \mathrm{H}$ protrude from the variable domain with support from the $\lambda$-light chains. Antigen binding is undertaken by side chains that are exclusively contained within long $\mathrm{CDR} 3 \mathrm{H}$ regions, as investigated by structural comparisons with protein toxins (Ramsland et al. 2001).

As described in humans (Corbett et al. 1997), mice (Kaartinen and Mäkelä 1985), chicken (Reynaud et al. 1994), and rabbits (Friedman et al. 1994), mainly hydrophilic amino acid residues such as Tyr, Ser, and Gly were identified in the bovine exceptionally long $\mathrm{CDR} 3 \mathrm{H}$. The occurrence of the hydrophilic reading frame in antigenbinding loops is thought to enhance flexibility and recruit somatic hypermutations for advantageous antigen binding (Corbett et al. 1997).

In accordance with previous descriptions, multiple and mainly even numbered Cys accumulated in the middle of the exceptionally long CDR3H were identified (Saini et al. 1999a; Kaushik et al. 2009). They are predicted to form intra and inter CDRH disulfide bonds that rigidify the combining site or help to stabilize long $\mathrm{CDR} 3 \mathrm{H}$, as demonstrated in the crystallized human Fab Kol (Schmidt et al. 1983), the camel cAb-Lys3 single domain antibody (Desmyter et al. 1996), and the bovine Fabs BLV1H12 and BLV5B8 (Wang et al. 2013). Former observations of an additional Cys in CDR2H when there were only one or three Cys in CDR3H (Saini et al. 1999a), or at least one Cys in $\mathrm{CDR} 3 \mathrm{H}$ regions containing more than 12 amino acid residues and no Cys in CDR3H with less than ten amino acids (Lopez et al. 1998) were verified with some reservations. The numbering system had an influence on the number of additional Cys in $\mathrm{CDR} 2 \mathrm{H}$ and some sequences with intermediate CDR3H lengths did not possess any Cys.

The bovine immunoglobulin heavy-chain locus was annotated, the expression of exceptionally long $\mathrm{CDR} 3 \mathrm{H}$ in the five bovine immunoglobulin isotypes was demonstrated, and their genomic origin was specified. Thus, this study reviewed the opinion that exceptionally long CDR3H are a unique feature of bovine $\lg M$ and $\lg G 1-3$. 
The new bioinformatics framework enables more detailed analyses of bovine immunoglobulin heavy chains and substantially contributes to the understanding of the development of the transcribed bovine immunoglobulin repertoire (publication 4). It is now possible to analyze the unique exceptionally long $\mathrm{CDR} 3 \mathrm{H}$ group of bovine immunoglobulin heavy chains. Possible gene conversions within the variable region of bovine heavy chains were examined, too. Further, the dominantly transcribed IGHV, IGHD, and IGHJ gene segments and their combinatorial diversity were investigated. In contrast to previous studies, this investigation extended analyses to breed specific differences in the four cattle breeds Aubrac (A), German Simmental (GS), German Black Pied (GBP), and Holstein Friesian (HF).

Recent studies on bovine immunoglobulin genetics focused on antibody diversification strategies and the junctional diversity of the antibody repertoire. Bovine specific diversification mechanisms were identified such as the generation of exceptionally long CDR3H (Saini et al. 1999b; Walther et al. 2013; Wang et al. 2013), the insertion of conserved short nucleotide sequences (CSNS) at the IGHV-IGHD junction (Koti et al. 2010), the use of pseudogene fragments in lambda light chains, as well as gene conversions (Parng et al. 1996), and somatic hypermutations independent of exposure to external antigens during B-cell development in lambda light chains (Lucier et al. 1998). In addition, new germline gene segments were determined in the recent years (Niku et al. 2012; Walther et al. 2013; Liljavirta et al. 2014). Nevertheless, the current bovine genome assembly is still incomplete and the full germline repertoire remains under active investigation.

Previous analyses of rearranged immunoglobulin germline gene segments applied various software tools for sequence alignments. Due to the difficult and error prone manual assembly of different genes, specialized software tools have been developed such as IMGT/Junction Analysis (Yousfi Monod et al. 2004), IMGT/V-QUEST (Brochet et al. 2008; Giudicelli et al. 2011), IMTG/HighV-QUEST (Alamyar et al. 2012), VBASE2 (Retter et al. 2005), JoinSolver (Souto-Carneiro et al. 2004), iHMMun-align (Gaeta et al. 2007), and IgBLAST (Ye et al. 2013). Only IgBLAST provides different settings for nucleotide and protein sequences, the numbering system of Kabat or the IMGT system (Lefranc et al. 2003; Kabat et al. 2006), and germline gene database searches (Ye et al. 2013). Matching germline IGHV, IGHD, and IGHJ genes, as well as details at rearrangement junctions may be analyzed. Organisms such as human, mouse, rat, 
rabbit and rhesus monkey are supported but the representation of livestock is missing or incomplete. In particular, the bovine specific occurrence of exceptionally long $\mathrm{CDR} 3 \mathrm{H}$ is not taken into account. For more detailed analyses of the origin of the immunoglobulin diversity considering species-specific diversification mechanisms, a new bioinformatics framework was developed. Three different procedures were applied to improve biological significance. Consequently, the program is based on nucleotide sequences, searches our updated bovine specific immunoglobulin germline gene database, and is able to load other pre-designed databases. The currently recommended and most widely used IMGT nomenclature is used for the delineation of FR and CDR. The adjustment of search parameters for IGHV an IGHJ and especially for the identification of IGHD is also possible.

The analysis of Ig heavy chain variable regions in the four cattle breeds revealed the usage of 15 different IGHV segments, 21 IGHD segments, as well as two IGHJ segments. Within the breeds, statistical analyses showed significant different transcription levels of IGHV, IGHD, and IGHJ segments. IGHV1S39 and IGHV3/33 were used most frequently. The segments IGHV1S26, IGHV1S32, IGHV1S33, IGHV1S37, and IGHV1S38 were rarely used. Both findings were consistent with findings for IGHV1S39, IGHV3/33, IGHV1S26, and IGHV1S38 in bovine fetal bone marrow, ileum, and spleen (Liljavirta et al. 2014). The transcription of IGHV1S32 and S37 was described for the first time. In all breeds IGHDS8, IGHDS5, IGHDS10, and IGHDQ52 (=IGHDS9) were preferred. IGHDS1 to IGHDS8 were transcribed in antisense direction, too. In previous studies 14 IGHD were transcribed, where IGHDS5 was the most frequent one in $42 \%$ of the sequences analyzed in bovine fetus (Liljavirta et al. 2014). The germline IGHJ1 was clearly preferred but IGHJ6 (Liljavirta et al. 2014) was transcribed in small proportions. In Aubrac, procedure 3 confirmed IGHV1S39IGHD5-IGHJ1 as the most common recombination of gene segments which is identical to the most frequent finding in bovine fetus (Liljavirta et al. 2014). This recombination belongs to immunoglobulins possessing a $\mathrm{CDR} 3 \mathrm{H}$ region of intermediate length.

Possible gene conversion events were identified. For instance, the pseudogene IGHV4 $\Psi$ seem to contribute to gene conversion events by nucleotide substitutions. This gene segment meets the criteria for gene conversion such as the location upstream of the rearranged segment (Reynaud et al. 1989; Becker and Knight 1990) and clusters of nucleotide changes (Parng et al. 1996). The flanking homology of the conversion region also supports the genetic exchange (Becker and Knight 1990) and 
the separation from IGHV6 by more than $18 \mathrm{~kb}$ on the genome allows looping during rearrangement (Walther et al. 2013). In comparison, in chicken the nearest pseudogene is separated by $7 \mathrm{~kb}$ (Reynaud et al. 1989). Nevertheless, it is difficult to consider the order of gene segments to evaluate the plausibility of other gene conversions because of the incomplete annotation of the bovine genome (Niku et al. 2012; Walther et al. 2013). Finally, a rare exchange between the two bovine VH families is indicated. These results might be an influence of breed or method of analysis when compared to previous results (Niku et al. 2012).

In the $\mathrm{CDR} 3 \mathrm{H}$, three different groups of lengths were examined in all four cattle breeds: short CDR3H (group 1, $\leq 10$ aa), intermediate length CDR3H (group 2, 11-47 aa), and exceptionally long $\mathrm{CDR} 3 \mathrm{H}$ (group $3, \geq 48$ aa). The breed GS possessed the highest percentage of group $3 \mathrm{CDR} 3 \mathrm{H}$. In this study, the longest $\mathrm{CDR} 3 \mathrm{H}$ with 65 amino acids were found in GS and HF sequences, whereas the longest ever detected CDR3H in cattle was 67 amino acids long using IMGT numbering (Walther et al. 2013). Four amino acids made up the shortest CDR3H in A animals, GS animals, and HF animals.

The combinatorial diversity of transcribed germline IGHV, IGHD, and IGHJ-segments was represented by 162 different rearrangements. They were expressed with significant differences (procedure 3). Different rearrangements were found in the four breeds, whereas 91 different combinations occurred in A, 74 in animals of GBP, 72 in GS animals, and 85 in the breed HF. Most of these combinations were observed in less than ten sequences but seven occurred in up to 21 sequences in all four cattle breeds examined.

In sequences of group $1 \mathrm{CDR3H}$, the combination of IGHV3-IGHDQ52 (sense, BTA8)IGHJ1 (AY158087) dominated over all breeds using procedure 3. As IGHDQ52 possesses only four amino acids, these results explain best the origin of short CDR3H. Sequences of group 3 CDR3H mostly exhibited IGHV10-IGHD2 (sense, (Shojaei et al. 2003))-IGHJ1 (procedure 3) in all breeds. Only results from this procedure identified biological meaningful combinations of germline IGHV, IGHD, and IGHJ, and gave the best explanation for the origin of group $3 \mathrm{CDR} 3 \mathrm{H}$. IGHV10 contributed solely to those CDR3H of exceptional lengths (Niku et al. 2012; Walther et al. 2013; Liljavirta et al. 2014). The "Thr-Thr-Val-His-GIn" terminal motif of IGHV10 that initiates an ascending $\beta$ strand in the folded antibody, is assumed to enable the formation of the "stalk and knob" structure together with inserted conserved short nucleotide sequences (CSNS) (Wang et al. 2013; Liljavirta et al. 2014). Further, IGHD2 is the longest IGHD segment 
identified so far. In contrast, sequences of group 2 showed a higher number of recombinations than sequences of group 1 and 3 . The few preferably rearranged gene segments within group $3 \mathrm{CDR} 3 \mathrm{H}$ may indicate specialized antibodies because group 3 regions are unique in cattle.

Variability plots indicated quite similar features of varying amino acid residues at each position within the variable region in all breeds. From $\mathrm{CDR} 1 \mathrm{H}$ to $\mathrm{CDR} 3 \mathrm{H}$ variability increased, which was described already as concentrated areas of diversity in equine heavy and light chain CDRs (Tallmadge et al. 2013; Tallmadge et al. 2014). In transition areas between FRHs and CDRHs variability was higher than in the middle of $\mathrm{CDRHs}$. The highest variability within the $\mathrm{FRHs}$ was identified at position 96 in $\mathrm{FR} 3 \mathrm{H}$. This residue is located on the outer surface of the variable region of the immunoglobulin molecule (Wang et al. 2013) within the area where the constant region faces the variable region. The high variability at this position may indicate an influence on the position and sterical orientation of variable and constant region. This may affect light chain pairing because heavy chains possessing group $3 \mathrm{CDR} 3 \mathrm{H}$ are connected to a special type of lambda light chains (Saini et al. 2003; Wang et al. 2013).

The highest number of recombinations and variability were observed in the breed $A$, while GS possessed the lowest number of recombinations and showed less variability except in the middle of the $\mathrm{CDR} 3 \mathrm{H}$ region. Therefore, the contribution of insertions and deletions to diversity is indicated in case of few rearrangements (Larsen and Smith 2012). The breeds $A$ and GS were kept under the same management in a mixed herd, whereas GBP and HF were kept at different farms. Consequently, the breeds kept in different areas were exposed to different antigens. Thus, the individual number of rearrangements per breed and differences in variability additionally indicate a specialized immune response as animals on one farm are challenged with the same environment.

Important new results were gained by the application of the newly developed bioinformatics framework. Analyses demonstrated that the bovine heavy chain diversity is not restricted to the use of a limited number of germline genes although there are preferred rearrangements within the three groups of $\mathrm{CDR} 3 \mathrm{H}$ lengths. We also found strong evidence for gene conversion using pseudogenes. Despite current advances in the understanding of bovine immunoglobulin diversification, future investigations of the germline repertoire are necessary. 


\section{Conclusions}

This thesis contributed essentially to the more detailed understanding of the expressed immunoglobulin repertoire in cattle and horses.

Allotypic and allelic variants have been described in equine lambda light chains for the first time. Individual mutations within the breeds are supposed to enable the transcriptional use of pseudogenes. Several amino acid residue substitutions were located at the accessible surface as confirmed by homology-based predicted 3D structural analysis. These modifications are supposed to result in distinct serological properties of the allotypic variants. Further, one of the conserved residues for the main interaction of IGLC and IGHC1 was affected and, therefore, the stable assembly of heavy and light chains might be changed.

Specific investigations on functional differences of allotypic variants are missing in horses, but similar to cattle heterozygotic horses are thought to possess an advantage in their humoral immune response.

Significant differences in transcription frequencies of IGLV and IGLC were observed within and between the two horse breeds examined. As these breeds with different stud book sizes and breeding goals were kept under different conditions, the results could be linked to breeding and an influence of the exposure to antigens is strongly indicated.

The bovine immunoglobulin heavy-chain locus seems to be annotated incorrectly and incompletely because inconsistent germline gene segments were identified on various bovine autosomes. Consequently, future investigations of the germline repertoire are necessary.

The CDR3H length heterogeneity was found in all bovine immunoglobulin isotypes. Hence, exceptionally long $\mathrm{CDR} 3 \mathrm{H}$ are not primarily generated to compensate the restricted flexibility of $\lg \mathrm{M}$ and class switch recombination contributes to the bovine specific long $\mathrm{CDR} 3 \mathrm{H}$ in all immunoglobulin isotypes.

Previous analyses of rearranged immunoglobulin germline gene segments applied various software tools for sequence alignments. As underlying databases did not support the bovine specific occurrence of exceptionally long CDR3H and more detailed analyses of the transcribed gene segments was required, a new bioinformatics 
framework was developed. Within the breeds, statistical analyses showed significant different transcription levels of IGHV, IGHD, and IGHJ segments as described for equine lambda light chains. In the $\mathrm{CDR} 3 \mathrm{H}$, three different groups of lengths were examined in all four cattle breeds. Thus, exceptionally long $\mathrm{CDR} 3 \mathrm{H}$ are neither restricted to isotypes nor to cattle breeds. The combinatorial diversity of transcribed germline IGHV, IGHD, and IGHJ-segments showed significant differences. Only few of these rearrangements were preferred within group $3 \mathrm{CDR} 3 \mathrm{H}$ and may indicate specialized antibodies because group 3 regions are unique in cattle. Although the annotation of the bovine germline repertoire is incomplete, possible gene conversion events were identified within the variable region of bovine heavy chains.

The highest variability within the FRHs was identified at position 96 in FR3H may indicate an influence on the position and sterical orientation of variable and constant region.

The breeds A and GS were kept under the same management in a mixed herd, whereas GBP and HF were kept at different farms. Consequently, the breeds kept in different areas were exposed to different antigens. Breed specific numbers of recombinations and differences in variability were observed and may indicate a specialized immune response as animals on one farm are challenged with the same environment. 


\section{Future Prospects}

Further research in this field should concentrate on more detailed analyses of both the germline repertoire, as well as the expressed immunoglobulin repertoire in cattle and horses. Allelic or haplotypic differences for both variable and constant region genes should be investigated and linked to individuals or breeds. For instance, in vitro analyses of bovine IgG already determined the influences of genetic variations on the immune response (Corbeil et al. 1997; Bastida-Corcuera et al. 1999a; BastidaCorcuera et al. 1999b; Bastida-Corcuera et al. 2000). Similar in vitro studies of the effector functions of recombinant versions of the seven equine $\lg G$ subclasses also revealed different effects on the immune response (Lewis et al. 2008). Subsequent studies in genetic, structural, and configurational properties of bovine and equine immunoglobulins, as well as analyses of the immunoglobulin repertoire after infection or immunization might offer opportunities for the development of new antibody-based therapeutics. The results will contribute to the analysis and generation of synthetic recombinant antibodies, which replace the production of recombinant antibodies from animals. In addition, unfavorable side effects of conventional therapeutics may be excluded and higher antigen specificity will be achieved. Consequently, recombinant antibodies and antibody fragment related products are important tools for research, diagnostics and therapy (Hust and Dubel 2004). However, only few engineered species-specific antibodies have been developed against veterinary pathogens (Koti et al. 2014). A very promising outcome from immunoglobulin research might be the construction of tailor-designed antibodies based on the format of single chain fragments variable (scFv). In these antibodies $\mathrm{VH}$ and $\mathrm{VL}$ domains are linked together. Therefore, naïve or immunized immunoglobulin libraries have to be constructed. Then, phage display can be used to identify highly affine and neutralizing recombinant ScFv molecules against any desired viral, bacterial, and parasitic antigenic site, as well as toxins. Through genetic manipulation or the fusion of an appropriate constant domain, a promising scFv-molecules can be enhanced, for example to scFv-FCGRT (FcRn) fusion proteins (Kontermann 2011; Koti et al. 2014) or to complete lgG antibodies from different species as established for human antibodies (Moutel et al. 2009). A further advantage is the elongated half-life time and clearance of scFvs in vivo, that also achieves effector functions such as opsonization, complement-dependent cytotoxicity (CDC), and antibody-dependent cellular cytotoxicity (ADCC). 
In addition, analyses of antigen/antibody binding using X-ray crystal structures (Saini et al. 1999a; Saini and Kaushik 2002; Wang et al. 2013) and sequencing data from highthroughput next generation sequencing will give valuable information on antigen/antibody interactions. Subsequent amino acid replacement, especially in the CDRregions may lead to recombinant antibody molecules with enhanced binding efficiency. 


\section{References}

Alamyar E, Duroux P, Lefranc MP, Giudicelli V. 2012. IMGT((R)) tools for the nucleotide analysis of immunoglobulin (IG) and T cell receptor (TR) V-(D)-J repertoires, polymorphisms, and IG mutations: IMGT/V-QUEST and IMGT/HighV-QUEST for NGS. Methods Mol Biol 882: 569-604.

Almagro JC, Hernandez I, Ramirez MC, Vargas-Madrazo E. 1998. Structural differences between the repertoires of mouse and human germline genes and their evolutionary implications. Immunogenetics 47: 355-363.

Arakawa H, Buerstedde JM. 2009. Activation-induced cytidine deaminase-mediated hypermutation in the DT40 cell line. Philosophical transactions of the Royal Society of London Series B, Biological sciences 364: 639-644.

Arun SS, Breuer W, Hermanns W. 1996. Immunohistochemical examination of lightchain expression (lambda/kappa ratio) in canine, feline, equine, bovine and porcine plasma cells. Zentralblatt fur Veterinarmedizin Reihe A 43: 573-576.

Bastida-Corcuera F, Butler JE, Heyermann H, Thomford JW, Corbeil LB. 2000. Tritrichomonas foetus extracellular cysteine proteinase cleavage of bovine IgG2 allotypes. The Journal of parasitology 86: 328-332.

Bastida-Corcuera FD, Butler JE, Yahiro S, Corbeil LB. 1999a. Differential complement activation by bovine $\lg 2$ allotypes. Veterinary immunology and immunopathology 71: 115-123.

Bastida-Corcuera FD, Nielsen KH, Corbeil LB. 1999b. Binding of bovine IgG2a and IgG2b allotypes to protein A, protein $G$, and Haemophilus somnus IgBPs. Veterinary immunology and immunopathology 71: 143-149.

Becker RS, Knight KL. 1990. Somatic diversification of immunoglobulin heavy chain VDJ genes: evidence for somatic gene conversion in rabbits. Cell 63: 987-997.

Berens SJ, Wylie DE, Lopez OJ. 1997. Use of a single VH family and long CDR3s in the variable region of cattle Ig heavy chains. International immunology 9: 189199.

Biedermann G, Clar U, Finke A, Bickel M. 2002. Analyse der Population des Rheinisch-Deutschen Kaltbluts. Züchtungskunde 74: 237-249.

Breathnach CC, Clark HJ, Clark RC, Olsen CW, Townsend HG, Lunn DP. 2006. Immunization with recombinant modified vaccinia Ankara (rMVA) constructs encoding the HA or NP gene protects ponies from equine influenza virus challenge. Vaccine 24: 1180-1190.

Bremond J, Balzer B. 2011. Zentrale Dokumentation Tiergenetischer Ressourcen in Deutschland (TGRDEU).

Brochet X, Lefranc MP, Giudicelli V. 2008. IMGT/V-QUEST: the highly customized and integrated system for IG and TR standardized V-J and V-D-J sequence analysis. Nucleic Acids Res 36: W503-508.

Butler JE, Sun X, Wertz N, Lager KM, Chaloner K, Urban J, Jr., Francis DL, Nara PL, Tobin GJ. 2011. Antibody repertoire development in fetal and neonatal piglets $\mathrm{XXI}$. Usage of most $\mathrm{VH}$ genes remains constant during fetal and postnatal development. Molecular immunology 49: 483-494.

Butler JE, Wertz N, Deschacht N, Kacskovics I. 2009. Porcine IgG: structure, genetics, and evolution. Immunogenetics 61: 209-230.

Constans J, Hazout S, Garruto RM, Gajdusek DC, Spees EK. 1985. Population distribution of the human vitamin $D$ binding protein: anthropological considerations. Am J Phys Anthropol 68: 107-122. 
Corbeil LB, Gogolewski RP, Kacskovics I, Nielsen KH, Corbeil RR, Morrill JL, Greenwood R, Butler JE. 1997. Bovine IgG2a antibodies to Haemophilus somnus and allotype expression. Canadian journal of veterinary research $=$ Revue canadienne de recherche veterinaire 61: 207-213.

Corbett SJ, Tomlinson IM, Sonnhammer EL, Buck D, Winter G. 1997. Sequence of the human immunoglobulin diversity (D) segment locus: a systematic analysis provides no evidence for the use of DIR segments, inverted D segments, "minor" D segments or D-D recombination. J Mol Biol 270: 587-597.

Desmyter A, Transue TR, Ghahroudi MA, Thi MH, Poortmans F, Hamers R, Muyldermans S, Wyns L. 1996. Crystal structure of a camel single-domain VH antibody fragment in complex with lysozyme. Nat Struct Bio/ 3: 803-811.

Eguchi-Ogawa T, Wertz N, Sun XZ, Piumi F, Uenishi H, Wells K, Chardon P, Tobin GJ, Butler JE. 2010. Antibody repertoire development in fetal and neonatal piglets. $\mathrm{XI}$. The relationship of variable heavy chain gene usage and the genomic organization of the variable heavy chain locus. Journal of immunology 184: 3734-3742.

Friedman ML, Tunyaplin C, Zhai SK, Knight KL. 1994. Neonatal VH, D, and JH gene usage in rabbit $B$ lineage cells. $J$ Immunol 152: 632-641.

Gaeta BA, Malming HR, Jackson KJ, Bain ME, Wilson P, Collins AM. 2007. iHMMunealign: hidden Markov model-based alignment and identification of germline genes in rearranged immunoglobulin gene sequences. Bioinformatics 23: 15801587.

Giha HA, Nasr A, Iriemenam NC, Arnot D, Troye-Blomberg M, Theander TG, Berzins K, EIGhazali G, Pandey JP. 2009. Antigen-specific influence of GM/KM allotypes on $\mathrm{IgG}$ isotypes and association of $\mathrm{GM}$ allotypes with susceptibility to Plasmodium falciparum malaria. Malar J 8: 306.

Giudicelli V, Brochet X, Lefranc MP. 2011. IMGT/V-QUEST: IMGT standardized analysis of the immunoglobulin (IG) and $T$ cell receptor (TR) nucleotide sequences. Cold Spring Harb Protoc 2011: 695-715.

Granoff DM, Pandey JP, Boies E, Squires J, Munson RS, Jr., Suarez B. 1984. Response to immunization with Haemophilus influenzae type b polysaccharidepertussis vaccine and risk of Haemophilus meningitis in children with the $\mathrm{Km}(1)$ immunoglobulin allotype. The Journal of clinical investigation 74: 1708-1714.

Gu F, Chowdhary BP, Andersson L, Harbitz I, Gustavsson I. 1992. Assignment of the bovine immunoglobulin gamma heavy chain (IGHG) gene to chromosome 21 q24 by in situ hybridization. Hereditas 117: 237-240.

Guo Y, Bao Y, Meng Q, Hu X, Meng Q, Ren L, Li N, Zhao Y. 2012. Immunoglobulin genomics in the guinea pig (Cavia porcellus). PloS one 7: e39298.

Guo Y, Bao Y, Wang H, Hu X, Zhao Z, Li N, Zhao Y. 2011. A preliminary analysis of the immunoglobulin genes in the African elephant (Loxodonta africana). PloS one 6: e16889.

Hamann H, Distl O. 2008. Genetic variability in Hanoverian warmblood horses using pedigree analysis. J Anim Sci 86: 1503-1513.

Hayes H, Di Meo GP, Gautier M, Laurent P, Eggen A, lannuzzi L. 2000. Localization by FISH of the 31 Texas nomenclature type I markers to both Q- and R-banded bovine chromosomes. Cytogenet Cell Genet 90: 315-320.

Hayes HC, Petit EJ. 1993. Mapping of the beta-lactoglobulin gene and of an immunoglobulin $M$ heavy chain-like sequence to homoeologous cattle, sheep, and goat chromosomes. Mamm Genome 4: 207-210.

Hosseini A, Campbell G, Prorocic M, Aitken R. 2004. Duplicated copies of the bovine $\mathrm{JH}$ locus contribute to the Ig repertoire. Int Immunol 16: 843-852. 
Hust M, Dubel S. 2004. Mating antibody phage display with proteomics. Trends in biotechnology 22: 8-14.

Jeong HD, Komisar JL, Kraig E, Teale JM. 1988. Strain-dependent expression of VH gene families. Journal of immunology 140: 2436-2441.

Kaartinen M, Mäkelä O. 1985. Reading of $D$ genes in variable frames as a source of antibody diversity. Immunol Today 6: 324-327.

Kabat EA, Wu TT. 1991. Identical $V$ region amino acid sequences and segments of sequences in antibodies of different specificities. Relative contributions of $\mathrm{VH}$ and VL genes, minigenes, and complementarity-determining regions to binding of antibody-combining sites. J Immunol 147: 1709-1719.

Kabat EA, Wu TT, Perry HM, Gottesmann KS, Foeller C. 2006. Sequences of proteins of immunological interest. U.S. Department of Health and Human Services, Public Health Service, National Institutes of Health, Bethesda, MD.

Kaushik AK, Kehrli ME, Jr., Kurtz A, Ng S, Koti M, Shojaei F, Saini SS. 2009. Somatic hypermutations and isotype restricted exceptionally long $\mathrm{CDR} 3 \mathrm{H}$ contribute to antibody diversification in cattle. Veterinary immunology and immunopathology 127: 106-113.

Keggan A, Freer H, Rollins A, Wagner B. 2013. Production of seven monoclonal equine immunoglobulins isotyped by multiplex analysis. Veterinary immunology and immunopathology 153: 187-193.

Klinman NR, Rockey JH, Karush F. 1965. Equine Antihapten Antibody li. The GammaG(7s-Gamma) Components and Their Specific Interaction. International journal of cancer Supplement = Journal international du cancer Supplement 47: 51-60.

Kontermann RE. 2011. Strategies for extended serum half-life of protein therapeutics. Curr Opin Biotechnol 22: 868-876.

Korngold L, Lipari R. 1956. Multiple-myeloma proteins. III. The antigenic relationship of Bence Jones proteins to normal gammaglobulin and multiple-myeloma serum proteins. Cancer 9: 262-272.

Koti M, Kataeva G, Kaushik AK. 2010. Novel atypical nucleotide insertions specifically at $\mathrm{VH}-\mathrm{DH}$ junction generate exceptionally long $\mathrm{CDR} 3 \mathrm{H}$ in cattle antibodies. Molecular immunology 47: 2119-2128.

Koti M, Saini SS, Sachan A, Kaushik A. 2014. Engineered Bovine Antibodies in the Development of Novel Therapeutics, Immunomodulators and Vaccines. Antibodies 3: 205-214.

Kydd JH, Townsend HG, Hannant D. 2006. The equine immune response to equine herpesvirus-1: the virus and its vaccines. Veterinary immunology and immunopathology 111: 15-30.

Larsen PA, Smith TP. 2012. Application of circular consensus sequencing and network analysis to characterize the bovine IgG repertoire. BMC immunology 13: 52.

Lefranc MP, Pommie C, Kaas Q, Duprat E, Bosc N, Guiraudou D, Jean C, Ruiz M, Da Piedade I, Rouard M et al. 2005. IMGT unique numbering for immunoglobulin and $\mathrm{T}$ cell receptor constant domains and Ig superfamily $\mathrm{C}$-like domains. Dev Comp Immunol 29: 185-203.

Lefranc MP, Pommie C, Ruiz M, Giudicelli V, Foulquier E, Truong L, Thouvenin-Contet $V$, Lefranc $G$. 2003. IMGT unique numbering for immunoglobulin and $T$ cell receptor variable domains and Ig superfamily V-like domains. Dev Comp Immunol 27: 55-77.

Leleu X, Terriou L, Duhamel A, Moreau AS, Andrieux J, Dupire S, Coiteux V, Berthon C, Micol JB, Guieze R et al. 2006. Long-term outcome in acquired aplastic anemia treated with an intensified dose schedule of horse antilymphocyte globulin in combination with androgens. Ann Hematol 85: 711-716. 
Lewis MJ, Wagner B, Woof JM. 2008. The different effector function capabilities of the seven equine IgG subclasses have implications for vaccine strategies. Molecular immunology 45: 818-827.

Liljavirta J, Niku M, Pessa-Morikawa T, Ekman A, livanainen A. 2014. Expansion of the preimmune antibody repertoire by junctional diversity in Bos taurus. PloS one 9: e99808.

Lopez AM, Hines MT, Palmer GH, Alperin DC, Hines SA. 2002. Identification of pulmonary T-lymphocyte and serum antibody isotype responses associated with protection against Rhodococcus equi. Clin Diagn Lab Immunol 9: 12701276.

Lopez O, Perez C, Wylie D. 1998. A single VH family and long CDR3s are the targets for hypermutation in bovine immunoglobulin heavy chains. Immunological reviews 162: 55-66.

Lucier MR, Thompson RE, Waire J, Lin AW, Osborne BA, Goldsby RA. 1998. Multiple sites of $\mathrm{V}$ lambda diversification in cattle. Journal of immunology 161: 54385444.

Matsuda F, Honjo T. 1996. Organization of the human immunoglobulin heavy-chain locus. Adv Immuno/ 62: 1-29.

Moutel S, El Marjou A, Vielemeyer O, Nizak C, Benaroch P, Dubel S, Perez F. 2009. A multi-Fc-species system for recombinant antibody production. BMC biotechnology 9: 14.

Nadel B, Tang A, Escuro G, Lugo G, Feeney AJ. 1998. Sequence of the spacer in the recombination signal sequence affects $V(D) J$ rearrangement frequency and correlates with nonrandom Vkappa usage in vivo. J Exp Med 187: 1495-1503.

Nelson KM, Schram BR, McGregor MW, Sheoran AS, Olsen CW, Lunn DP. 1998. Local and systemic isotype-specific antibody responses to equine influenza virus infection versus conventional vaccination. Vaccine 16: 1306-1313.

Niku M, Liljavirta J, Durkin K, Schroderus E, livanainen A. 2012. The bovine genomic DNA sequence data reveal three IGHV subgroups, only one of which is functionally expressed. Developmental and comparative immunology 37: 457461.

Padlan EA, Cohen GH, Davies DR. 1986. Antibody Fab assembly: the interface residues between $\mathrm{CH} 1$ and $\mathrm{CL}$. Molecular immunology 23: 951-960.

Pandey JP. 2000. Immunoglobulin GM and KM allotypes and vaccine immunity. Vaccine 19: 613-617.

Pandey JP, Elson LH, Sutherland SE, Guderian RH, Araujo E, Nutman TB. 1995. Immunoglobulin kappa chain allotypes (KM) in onchocerciasis. The Journal of clinical investigation 96: 2732-2734.

Pandey JP, Fudenberg HH, Virella G, Kyong CU, Loadholt CB, Galbraith RM, Gotschlich EC, Parke JC, Jr. 1979. Association between immunoglobulin allotypes and immune responses to Haemophilus influenzae and Meningococcus polysaccharides. Lancet 1: 190-192.

Parng CL, Hansal S, Goldsby RA, Osborne BA. 1996. Gene conversion contributes to Ig light chain diversity in cattle. Journal of immunology 157: 5478-5486.

Ramsland PA, Kaushik A, Marchalonis JJ, Edmundson AB. 2001. Incorporation of long CDR3s into V domains: implications for the structural evolution of the antibodycombining site. Exp Clin Immunogenet 18: 176-198.

Retter I, Althaus HH, Munch R, Muller W. 2005. VBASE2, an integrative $\mathrm{V}$ gene database. Nucleic Acids Res 33: D671-674. 
Reynaud CA, Anquez V, Dahan A, Weill JC. 1985. A single rearrangement event generates most of the chicken immunoglobulin light chain diversity. Cell 40: 283-291.

Reynaud CA, Bertocci B, Dahan A, Weill JC. 1994. Formation of the chicken B-cell repertoire: ontogenesis, regulation of Ig gene rearrangement, and diversification by gene conversion. Adv Immunol 57: 353-378.

Reynaud CA, Dahan A, Anquez V, Weill JC. 1989. Somatic hyperconversion diversifies the single Vh gene of the chicken with a high incidence in the D region. Cell 59: 171-183.

Rockey JH. 1967. Equine antihapten antibody. The subunits and fragments of antibeta-lactoside antibody. The Journal of experimental medicine 125: 249-275.

Rockey JH, Klinman NR, Karush F. 1964. Equine Antihapten Antibody. I. 7s Beta-2aand 1os Gamma-1- Globulin Components of Purified Anti-Beta-Lactoside Antibody. The Journal of experimental medicine 120: 589-609.

Saini SS, Allore B, Jacobs RM, Kaushik A. 1999a. Exceptionally long CDR3H region with multiple cysteine residues in functional bovine IgM antibodies. European journal of immunology 29: 2420-2426.

Saini SS, Farrugia W, Ramsland PA, Kaushik AK. 2003. Bovine IgM antibodies with exceptionally long complementarity-determining region 3 of the heavy chain share unique structural properties conferring restricted $\mathrm{VH}+$ Vlambda pairings. Int Immunol 15: 845-853.

Saini SS, Kaushik A. 2002. Extensive CDR3H length heterogeneity exists in bovine foetal VDJ rearrangements. Scandinavian journal of immunology 55: 140-148.

Saini SS, MacGlashan DW, Jr., Sterbinsky SA, Togias A, Adelman DC, Lichtenstein LM, Bochner BS. 1999b. Down-regulation of human basophil IgE and FC epsilon RI alpha surface densities and mediator release by anti-lgE-infusions is reversible in vitro and in vivo. J Immunol 162: 5624-5630.

Sandor G, Korach S, Mattern P. 1964. 7s Globulin, Immunologically Identical to 19s Gamma-1 (Beta-2)-M-Globulin, a New Protein or Horse Serum. Nature 204: 795-796.

Schmidt WE, Jung HD, Palm W, Hilschmann N. 1983. [Three-dimensional structure determination of antibodies. Primary structure of crystallized monoclonal immunoglobulin IgG1 KOL, I]. Hoppe Seylers Z Physiol Chem 364: 713-747.

Sheoran AS, Holmes MA. 1996. Separation of equine lgG subclasses (IgGa, IgGb and $\lg G(T))$ using their differential binding characteristics for staphylococcal protein $A$ and streptococcal protein G. Veterinary immunology and immunopathology 55: 33-43.

Sheoran AS, Sponseller BT, Holmes MA, Timoney JF. 1997. Serum and mucosal antibody isotype responses to M-like protein (SeM) of Streptococcus equi in convalescent and vaccinated horses. Veterinary immunology and immunopathology 59: 239-251.

Shojaei F, Saini SS, Kaushik AK. 2003. Unusually long germline DH genes contribute to large sized CDR3H in bovine antibodies. Mol Immunol 40: 61-67.

Simianer H, Kohn F. 2010. Genetic management of the Gottingen Minipig population. $J$ Pharmacol Toxicol Methods 62: 221-226.

Sinclair MC, Aitken R. 1995. PCR strategies for isolation of the $5^{\prime}$ end of an immunoglobulin-encoding bovine cDNA. Gene 167: 285-289.

Sinclair MC, Gilchrist J, Aitken R. 1997. Bovine IgG repertoire is dominated by a single diversified VH gene family. Journal of immunology 159: 3883-3889.

Souto-Carneiro MM, Longo NS, Russ DE, Sun HW, Lipsky PE. 2004. Characterization of the human $\mathrm{lg}$ heavy chain antigen binding complementarity determining 
region 3 using a newly developed software algorithm, JOINSOLVER. $J$ Immunol 172: 6790-6802.

Sun Y, Wang C, Wang Y, Zhang T, Ren L, Hu X, Zhang R, Meng Q, Guo Y, Fei J et al. 2010. A comprehensive analysis of germline and expressed immunoglobulin repertoire in the horse. Developmental and comparative immunology 34: 10091020.

Tallmadge RL, McLaughlin K, Secor E, Ruano D, Matychak MB, Flaminio MJ. 2009. Expression of essential B cell genes and immunoglobulin isotypes suggests active development and gene recombination during equine gestation. Dev Comp Immunol 33: 1027-1038.

Tallmadge RL, Tseng CT, Felippe MJ. 2014. Diversity of immunoglobulin lambda light chain gene usage over developmental stages in the horse. Dev Comp Immunol 46: 171-179.

Tallmadge RL, Tseng CT, King RA, Felippe MJ. 2013. Developmental progression of equine immunoglobulin heavy chain variable region diversity. Dev Comp Immunol 41: 33-43.

Tobin-Janzen TC, Womack JE. 1992. Comparative mapping of IGHG1, IGHM, FES, and FOS in domestic cattle. Immunogenetics 36: 157-165.

Tonegawa S. 1983. Somatic generation of antibody diversity. Nature 302: 575-581.

Wagner B, Miller WH, Morgan EE, Hillegas JM, Erb HN, Leibold W, Antczak DF. 2006. $\lg \mathrm{E}$ and $\lg \mathrm{G}$ antibodies in skin allergy of the horse. Vet Res 37: 813-825.

Walther S, Czerny CP, Diesterbeck US. 2013. Exceptionally long CDR3H are not isotype restricted in bovine immunoglobulins. PLoS One 8: e64234.

Wang F, Ekiert DC, Ahmad I, Yu W, Zhang Y, Bazirgan O, Torkamani A, Raudsepp T, Mwangi W, Criscitiello MF et al. 2013. Reshaping antibody diversity. Cell 153: 1379-1393.

Widders PR, Stokes CR, Bourne FJ. 1986. Investigation of the antigenic relationship between equine $\operatorname{lgG}$ and $\operatorname{lgGT}$. Veterinary immunology and immunopathology 13: 255-259.

Winstead CR, Zhai SK, Sethupathi P, Knight KL. 1999. Antigen-induced somatic diversification of rabbit $\mathrm{IgH}$ genes: gene conversion and point mutation. $J$ Immunol 162: 6602-6612.

Ye J, Ma N, Madden TL, Ostell JM. 2013. IgBLAST: an immunoglobulin variable domain sequence analysis tool. Nucleic acids research 41: W34-40.

Yousfi Monod M, Giudicelli V, Chaume D, Lefranc MP. 2004. IMGT/JunctionAnalysis: the first tool for the analysis of the immunoglobulin and $T$ cell receptor complex V-J and V-D-J JUNCTIONs. Bioinformatics 20 Suppl 1: i379-385.

Zand MS. 2006. B-cell activity of polyclonal antithymocyte globulins. Transplantation 82: 1387-1395.

Zhao Y, Kacskovics I, Rabbani H, Hammarstrom L. 2003. Physical mapping of the bovine immunoglobulin heavy chain constant region gene locus. The Journal of biological chemistry 278: 35024-35032. 
Chapter 5:

Appendix 


\title{
Curriculum Vitae
}

\section{Personal Details}

$\begin{array}{ll}\text { Name } & \text { Stefanie Walther } \\ \text { Date of birth } & 16 \text { June } 1985 \\ \text { Place of birth } & \text { Sangerhausen } \\ \text { Nationality } & \text { German }\end{array}$

\section{Education}

\author{
Name of school/ college Subject \\ Geschwister-Scholl \\ General qualification for university entrance \\ Gymnasium, \\ Sangerhausen \\ University Bayreuth \\ Bachelor of Science in Biochemistry \\ Thesis: Analysis of the interactions of the \\ amino acid residues 33,34 , and 37 , as well \\ as their influence on the stability of the $G \beta 1$ \\ protein of Streptococcus
}

Date

07/2005

10/2005 -

$09 / 2008$

Georg-August-University

Master of Science in Equine Sciences

$10 / 2008-$

Göttingen

Thesis: Genomic organisation and transcription analysis oft he equine lambda and kappa immunoglobulin genes in the horse breeds Rhenish-German Coldblood and Hanoverian Warmblood

Georg-August-University

Ph.D. Program for Agricultural Sciences

10/2011 -

Göttingen

Thesis: Organization and transcription

$05 / 2016$

Division of Microbiology and Animal Hygiene, Institute of Veterinary Medicine, Department of Animal Sciences analyses of the immunoglobulin genes in cattle and horses 